\title{
ON THE PATHOLOGY OF HODGKIN'S DISEASE
}

$$
\text { by }
$$

ROBERT IAN SHAW DUNN, B.SC., M.B., Ch.B.

THESIS PRESENTEP TO THE UNIVERSITY OF GLASGOW

FOR THE DEGRES OF DOCTOR OF MADICINE 
ProQuest Number: 13838874

All rights reserved

INFORMATION TO ALL USERS

The quality of this reproduction is dependent upon the quality of the copy submitted.

In the unlikely event that the author did not send a complete manuscript and there are missing pages, these will be noted. Also, if material had to be removed, a note will indicate the deletion.

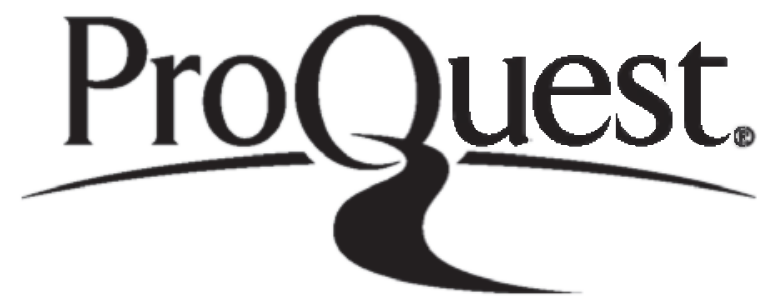

ProQuest 13838874

Published by ProQuest LLC (2019). Copyright of the Dissertation is held by the Author.

All rights reserved.

This work is protected against unauthorized copying under Title 17, United States Code Microform Edition (C) ProQuest LLC.

ProQuest LLC.

789 East Eisenhower Parkway

P.O. Box 1346

Ann Arbor, Ml $48106-1346$ 


\section{ARRANGEMENT OF TEE WORK}

TEXT OF THESIS

PROTOCOIS AND REFERENCES

PHOTOGRAPHIC ILIUSTRATIONS

DRAWINGS, DIAGRAMS AND GRAPHS
VOLUME I

VOLUME II

VOLUME III

VOLUME IV 


\section{FOREWORD}

Hodgkin's disease is the central interest in this study. It is difficult to confine researches strictly to the avowed object because in this disease wider issues obtrude continually. It is common knowledge that Hodgkin's disease affects the reticulo-endothelial system, or more closely, the lymphatic system. These general considerations must be respected and this involves the presentation of summaries of relevant knowledge to form a background to the study of the disease itself.

The work is divided into five main parts. There is first a brief account of the reticulo-endothelial system and its diseases, which is followed by remarks on the lymphatic system. In the third part Hodgkin's disease is reviewed and observations on its morphology and nosological affinities are offered. Next, certain evidences drawn from other diseases are presented in support of arguments and propositions advanced previously, and the final part is reserved for the experimental work on the disease.

There are two appendices of protocols recording the observational work performed. Illustrations supporting the several parts are given, and a final summary concludes the study. 


\section{APOLOGIA}

'Gutta cavat lapidem non semel sed semper cadendo'

In the warfare that is medicine Hodgkin's disease is an insistent challenge. Figuratively, this uncompromising fortress stands unreduced on the far side of the no-man's-land of pathology, while the redoubts of other diseases succumb to investigation. Those who study Hodgkin's disease will agree that here is no Castra Romana defended by vallum and fossa, but a formidable example of the celebrated third system of Vauban. Every approach is curtained and enfiladed, and the ground is beset with fougasses and chevaux-de-frises. Those who would attack it have chiefly a tradition of failure for their encouragement, yet this should be tempered by reflection on the fates of impregnable fortresses as recent as Festung Europa and Monte Cassino. For most, the assault must be carried out through the smoke of ignorance. It is a recurrent experience that this screen clears as the first bastion is reached, and reveals the objective to have been a mirage, and the raiding party to be still floundering in the fieldworks. This is almost a true similitude but ground is being won, albeit piecemeal. It is a 'salient fact that almost all real knowledge and understanding of this disease has been gained by morbid anatomists who, if the simile be maintained, are the infantry of pathology. The view that properly supported infantry can do anything has long been a classical axiom in ralitary science, and until the equivalents of atomic weapons become available in pathology, morbid anatomy will be the basis of success.

It is submitted that so far as can be foreseen at present final understanding of Hodgkin's disease is more likely to be derived from 
the consolidation and integration of the morbid anatomists myriad small facts than by any other method.

The present work is primarily an observational study made for this purpose. It is supplemented by information from other diseases and by limited empirical animal experimentation. While it is premature to submit what is properly relegated to the sumrnary, at this stage, the complete failure of the new experimental method tried-out reinforces the decision which was taken. 
VOLUME I

TABLE OF CONTENTS

PART I THE RETICUIO-ENDOTHELIAL SYSTEM.

PAGE 1

PART II THE IMMPHATIC SYSTEM.

PAGE 57

PART III HODGKIN'S DISEASE.

PAGE 115

PART IV FIBROSIS AND EOSINOPHILIA.

PAGE 239

PART $\checkmark$ EXPERIMEHTAL.

PAGE 272

SUMMARY.

PAGE 288

ACKNOWLEDGMENTS.

PAGE 291 
0.

\section{PART I}

THE REPICULO-ENDOTHELIAI SYSTEA

The structure of the reticulo-endothelial system.

p. 1-11

Introduction to diseases of the system.

p. $12-21$

Primary reticulosis.

p. $22-32$

Thmours of the system.

p. $33-56$

Illustrations.

Volume III Figs. I-3

Volume IV p. $3-5$ 


\section{THE STRUCTURE OF THE RETICULO-PNDOTHELIAL SYSTEM}

Considered collectively as an organ, the reticulo-endothelial system may be described as composed of a stroma and a parenchyma, Piney (1925). This concept is reasonable and helpful, but stereotyped ideas of the stroma and parenchyma relationship in organs like the liver, or salivary glands \& etc. must be abandoned for its appreciation. The relation of the neuroglia to nerve cells in the central nervous system presents an approximate homology.

The Stroma

The stromalmoiety is fundamentally connective tissue. This may be mesenchyme, general connective tissue, or reticular tissue. Mesenchyme is composed of mutually identical small cells with scanty cytoplasm which are uniformly dispersed. The cells have slender branching processes which are continuous from one cell to another forming a fine open mesh. In adult human material strictly homologous tissue is rare and scanty. A near approach is said to be seen in the nucleus pulposus of the intervertebral discs. These structures, numbering twenty three in all, have received scant attention until comparatively recently but the work of Schmorl confirms the widely quoted report that they are, in part at least, derived from the notochord. Duval, (1900) gave a brief account of their microscopical structure. Collins (1949) described them as consisting of myxoid or chondroid basophile matrix traversed by a few collegenous fibres, and sometimes containing stellate cells like those of a myxoma, or large vesiculated balloon cells resembling those or chordoma. 
In my own studies the nuclei pulposi recovered from elderly people have usually been devoid of cells, but they were demonstrable in children. Physaliphorous change is commonly visible, but tissue morphologically close to mesenchyme is not. A closer approach to embryonic mesenchyme is seen in some myxomata but these are very rare tumours - many supposed examples are merely dropsical degeneration, true mucoid material being absent. It is stated that mesenchymal cells are to be found easily in the vicinity of blood vessels, Robb-Smith (1938). These cells are also described as the adventitial cells of Marchand, resting wandering cells (Maximow), pericytes \& etc. I have seldom succeeded in convincing myself of their identity in ordinary routine sections.

The facts are that the methods and material of ordinary human morbid anatomy do not readily yield convincing evidence of persistent mesenchyme in adult tissues. Confirmation of this phenomenon calls for facilities which are not readily attainable, namely absolutely fresh tissue, tissue cultures and much time.

In the adult the mesenchyme is represented by the loose connective tissue and the reticular tissue. The former is composed of fibroblasts or fibrocytes, and histiocytes, with a variable amount of collagen and elastic fibre. Mast cells and pigment-laden cells - probably histiocytes, are sometimes also present. In adipose tissue the fat cells are derived from fibroblastic cells.

The ubiquitous general connective tissue of the body can be regarded as relatively generalised and undifferentiated tissue, preserving in some degree an embryonic potentiality for undergoing differentiation into more 
highly organised supporting structures, generally by some form of fibre production. To this extent it has been regarded as a persistence of the embryonic mesoderm (mesenchyme).

The reticular tissue is nearer to the parent mesenchyme. The same general arrangement obtains but the cells are larger and the mesh shows local differences. Although the tissue is described as net-like it is more accurately represented by a sponge which is the skeletal remnant of a porifer, (genus Euspongia). The mesh is formed of protoplasm and a variable amount of fine areyrophile fibre called reticulin. This fibre material is often nanifestly in direct continuation with collagen but unlike the latter its fibres run singly, anastomose freely, and are of uneven thickness. Triturated collagen is argyrophile, its component fibrillae take on the silver. It is unlikely that reticulin is a specific substance, and the argyrophile property is certainly not an exclusive possession of these fibres. Flastic fibres (elastin) are not present in reticular tissue itself. In some texts the term retiform tissue is used and in others the words reticulum or pulp are employed as equivalents. The last named is apt to lead to confusion.

In parafin sections stained with haematoxylin and eosin it is usually difficult to identify this ground tissue in normal lymph nodes, spleen, bone marrow \& etc. Silver impregnation is often difficult to interpret. This can be helped by substituting the orthodox counterstains saffranin or neutral red, with haematoxylin of light intensity. Another device is to mount two adjacent sections on the same slide, one being impregnated with silver, and the other stained with haematoxylin and 
eosin. Comparison is thus easily made. It must be emphasized that the silver impregnation is only a partial picture since the ground protoplasm is not stained. The most convincing demonstration of the protoplasmic continuity of reticular tissue is obtained by the old device of 'pencilling', Renvier (1889); Jolly (1922). These preparations are easily seen unstained, but light aniline stains may be used.

The proportion of stromal tissue varies in different sites, it is relatively high in the spleen and lymphoid tissue and low in the bone marrow and liver.

The component 'cells' of the reticular tissue, which is properly a a plasmodium, are usually termed reticulym cells. There are many other names as will be seen later. There is certainly confused thinking concerning the reticulum cell. It is obvious that many authors refer to reticulum cells when they actually mean reticulum cell nuclei. This error is understandable because the cytoplasm is generally so inconspicuous and faint-staining. The usage is common and is apparently condoned. The characterisation of this cell is difficult because although quite precise definition is available from many sources the title has a certain omnibus propensity. The term, actually as 'reticular cell', was first introduced by Ribbert (1889). He applied it to the true reticulum cells of lymphoid tissue to distinguish them from the modified reticulum cells of the lymph sinuses which were formerly more definitely termed endothelial cells. It is convenient to allow a degree of elasticity in the descriptive morphology of this cell, otherwise an host of pro- and meta- forms etc. and many named variants 
complicate the issue. Thus, in lymphoid tissue the 'cells' of the pale centres of germinal follicles, the reticular cells of Ribbert, the proliferating cells of Warchand, the 'endothelial' or 'Iittoral' cells of the sinuses are all reticulum cells, despite their individual differences. Similarly the various primitive blood cells, e.g. haemohistioblast, haemocytoblast, common lymphoid stem cell, lymphoidocyte \& etc. are all considered as reticulum cells. This wide application is open to criticisms but if the varieties of reticulum are imagined as siblings of a family - mutually similar and yet dissimilar at the same time, the paradox is acceptable.

\section{The Parenchyma}

This part of the complex rather defies attempts to consider it as a whole because the individual scions of it subserve very different functions. There are however several features which encourage some reflection.

This component of the system is entirely cellular. Forrad elements of cells, at least in the generally understood sense, do not contribute. The minor exceptions alluded to are the surface films which may be shed from macrophages, lymphocytes, or plasma cells, in immunity development. The cells of the reticulo-endothelial parenchyma are less wutually coherent than any others in the body, many indeed are entirely free. This characteristic is interesting because it is balanced by a functional individuality, the morphological and functional versatility of the cells in this system far surpassing that observed in any other tissue. The majority of the free forms have independent mobility, an archaic property which betokens much; even the relatively fixed cells 
have potential to move provided an appropriate stimulus is offered. The parenchyma is conveniently described for the purposes here under four main heads. These are the erythron (Boycott), the leukon, the macrophageion, and the Iymphocytogeion. (Approbation of these last three neologisms is not anticipated but they avoid unwieldy circumlocutions).

There is general agreement that series of cells can be recognised Which untimately form erythrocytes and myeloid series leucocytes. Specialisation in function and morphology is carried to the extreme in the former and it seems certain that a stage of differentiation is soon attained in the progenitors whereby erythrocytes only can be produced thereafter. The myeloid leucocyte is similar but specialisation is not carried nearly so far. The end-cell still retains its power of independent motion, of phagocytosis and is nucleated.

The macrophageion is nearest of all to its fundamental prototype. The parent tissue is not defined so closely as that of the former two, neither in topography nor structure. It is further complicated by the undoubted contribution of functionally identical cells from nonmesodermal sources.

Finally there is the lymphocytogeion. This is the least understood of all. It will be discussed more fully later but the extraordinary mystery of lymphoid tissue which collectively would yield a mass approaching the liver in size (Drinker), and which is so deceptively inert in appearance is a most baffling enigma. 
Indeterminate cells also contribute to the parenchyma. To observers who are ordinary mortals, their proportion is usually uncomfortably high. Nost are doubtless transition forms in exasperating disguises but others may be visitors, child-cells lost in the crowd, or captives.

The mutual relationships of the cells in this system are difficult to study. The problems are inherently very complex and are not amenable to easy investigation. A very confusing nomenclature has developed which makes the consolidation and integration of individual researches most difficult. It is important to appreciate that with the exception of blood-formation, little has been contributed on these problems in the human system. Also, by virtue of the paradox that the anatomists with the deepest experience of human tissues are morbid anatomists, more is understood concerning some aspects of the system in disease than in health. The identity and nomenclature of many of the imnature cells of the system are not yet satisfactorily agreed. The mutual identities of cells which might be regarded as equivalent in different species of animals are also far from established. The subject has prompted a great amount of work much of which is highly controversial. Adding to these difficulties inconsistency pervades even the best writings. These soon impress the tyro who makes an honest and critical approach to the subject, and unless he is exceptionally fortunate he also discovers that very few can help him.

Certain peculiar features attend the origin and cellular maintenance of the reticulo-endothelial system. It is axiomatic that each human 
individual starts existence as the fusion product of two haploid cells and therefore every cell in the body must own to this common ancestor. Specific differentiation of almost all descendants of this first cell follows with eventual production of all the different cells of the adult. This differentiation is accomplished remarkably early and thereafter the increase is chiefly on the homoplastic plan; that is to say muscle increases by proliferation of pre-existing muscle cells \& etc. In many tissues these fully differentiated cells are end-products; the nerve cells of the central nervous system enjoy an impregnable reputation for immutability, even to the absence of propagation.

The connective tissue from which the reticulo-endothelial system springs deviates from this general plan, but the difference is not so much one of kind as one of degree. There is good evidence that environment and functional demands determine the individual morphological varieties of cell produced, the genetic influence being less concerned with the ultimate form of the cells than with endowing them with ability to react to a wide functional spectrum.

It is a general rule that cells which differentiate to subserve a particular function lose some of their ability to de-differentiate or metamorphose, and this loss is increased as that function becones more complex or specialised. This law is evaded by the reticulo-endothelial system cells on a remarkable scale; the erythrocytes and polymorphonuclear leucocytes are probably the only two real end-cells of the entire system. Neither is capable of cell-division and so far as the body economy is concerned they are expendable. The remainder, even the 
mature fibrocyte and the ever-incongruous lymphocyte can probably adopt the habits of most other cells of the macrophage system. This lability must be regarded as a system characteristic. One important outcome of these curious properties is that in tissue of this system a departure is made from the stroma and parenchyma relationship observed in ordinary organs. In the reticulo-endothelial system the parenchyma arises from the stroma. This is a fundamental peculiarity, and remains a central fact even if the issue is complicated by the contentions of the polyphyletic or monophyletic schools. These latter arguments have acquired undue advertisement; what they chiefly seek to determine is whether there are maturation levels in the earliest precursor cells after which homoplastic generation of one type of cell is finally determined. Good evidence exists in favour of both theories. The inflammatory cellular response, the totality of aplastic anaemia, and the phenomenon of myeloid metaplasia favour the monophyletic view; the extreme rarity of mixed leukaemias, the polyphyletic one. It is reasonable to accept both as correct, they are in reality far less irreconcilable than is often supposed. One can merely presume that under appropriate circumstances of which knowledge is very restricted - one or other path is taken. Comprehensive studies on the genetic relationships of the reticuloendothelial cells have been made. Among the most valuable are those of Maximow and his colleagues. Much of their work has consolidated and integrated in Maximow' well-known review in 1924. It is to be noted however that these findings were almost exclusively derived from fresh material, a method which has very restricted application to man. It is 
apparent that in some quarters uncritical transference of these results to man has been made with the result that generalisations have been formulated without a really satisfactory basis.

The recognition of the fundarnental cells of the system in man presents much difficulty to the beginner because the discrepancies between authoritative descriptions are serious, e.g. Robb-Smith (1938), and Hadfield and Garrod (1943), describe undifferentiated mesenchymal cells and reticulum cells so differently that their mutual identities are quite irreconcilable. Wany schemata describing the inter-relationships of the cells of the system have been prepared, e.g. Laximow (1924), Hadfield and Garrod (1943), and Gillman, Gillman, and Gilbert (1949), but it is difficult to assess how much can be applied to man. The metamorphoses believed to occur in the human reticulo-endothelial system which are of particular interest in the study of lymphoid tissue are summarized in the following table. 


\begin{tabular}{|c|c|c|}
\hline Precursor cell. & Differentiation product. & Authority. \\
\hline Reticulum cell. & Fibroblast, fibrocyte & General. \\
\hline & Epithelioid (or endothelioid) & \\
\hline & cell. & $"$ \\
\hline & Littoral cell. (Lymph sinus). & $"$ \\
\hline & Macrophage. & $"$ \\
\hline & Monocyte (histiocyte). & $"$ \\
\hline & Lymphocyte series. & $"$ \\
\hline Lomphocyte. & Plasma cell (Warshalko type). & $"$ \\
\hline & Eosinophil. & Dominici; Pullinger. \\
\hline & Blood vascular endothelium. & Moschowitz. \\
\hline & Macrophage. & Downey and Stasney. \\
\hline & Erythron. & Jordan; Yoffee. \\
\hline Macrophages. & Fibroblast, fibrocyte. & General. \\
\hline
\end{tabular}

This table outlines some of the simpler relationships for which evidence has been found; many other genealogies are derived, but are less well endorsed for the human species. 
INTRODUCTORY RELARKS ON THE DISEASTS OF THE

\section{RET ICULO-ZNDOTHELIEL SYOTHU}

It is established practice in Special Pathology to study the diseases of the individual systems separately. The method is generaliy satisfactory, even if it sometimes loses its applicubility because of its exclusiveness. In practice it serves well and is much easier to apply than the older and more precise system of the textures.

On general grounds the application of the principle to the reticuloendothelial system is quite orthodox. The system now emancipated from its restrictive functional definition, has, despite its anatonical diffuseness, a defined topography.

In systemic diseases it is cormon to find a degree of localisation of the roorbid process. Indeed it happens occasionally that the lesion is solitary and confined alnost rigidly to one site. This extreme state of affairs is illustrated readily in examples of peptic ulceration of the alimentary tract. Here it is connon to find a single lesion, and the remainder of the syster often natural.

On other occasions while a disease apparently affects one part predominantly it is in reality much more generalised. Formerly endocarditis was stressed as the all-inportant lesion of rheumatic diseases of the circulatory system, valvular defects resulting therefrom were considered the most significant results. Later the discovery by sachoff that the connective tissue interstitium of the heart was affected, together with the clinical insiatence on gross myocardial dysfunction realised the concept of pancarditis. This in turn has given way to the 
idea that the disease is primarily a general connective tissue or even a diffuse collagen affection; a belief supported by the results of careful scrutiny which disclosed blood vessel and other inconspicuous lesions which had been previously overlooked. On the other hand systemic disease may be truly generalised. A familiar example is arteriosclerosis of the ordinary type which invariably affects all the arterial vessels. In like manner several of the primary muscular dystrophies involve all the muscles in some degree.

Analagous variations of involvement are exhibited in disease of the reticulo-endothelial system but in general, systemic participation in the morbid process is regarded as the rule. Numerically the vast majority of alterations in the retidulo-endothelial system are secondary in character; hardly deserving the appellation disease but rather falling into the category of reactive or adaptive change. The universality of participation of this widely scattered system in these reactive states cannot usually be assessed, in man at any rate, with much accuracy. This is particularly true when the causes evoking the reaction are transient or trivial. Pronouncements such as 'general reticulo-endothelial hyperplasia' while reasonable on theoretical grounds are in actual practice little more than a shrewd guess. Even where the stimulant factor is known, or presumed on good grounds, and is operative over a protracted period the interpretation of the results in the system is not easy. Thus in the peculiar metabolic maladies, the lipoidoses; in chronic haemolytic anaemia; in pernicious anaemia; in haemochromatosis \& etc., considerable individual variation of the 
reaction pattern is well attested fact. These anomalies have not received the attention they deserve, they are probably of considerable significance. The individual response of different people's macrophage systems is as bewildering as the apparently fortuitous distribution of metastases in individual instances of malignant disease.

The primary diseases of the system are the chief concern in this work. Here they may be defined as non-altruistic proliferative conditions arising in it which are not obviously brought about by disease in any other system, and for which it is not possible to assign any specific cause. This category will manifestly include true tumours but there are also conditions which at present do not enjoy universal recognition as neoplasm despite their tumour-like character. These are usually termed primary reticuloses. There is an impression prevalent that the primary diseases of the system have a basic comnon morbid anatomy related to the sites of location of irmature mesenchyme. Thus enlargement of lymph nodes, enlargement of the spleen, involvement of bone rarrow and enlargement of the liver are frequently all present. As a working generalisation this is probably fairly true but at the same time it is an overworked hypothesis. Topical variation of involvenent is quite evident in the primary maladies of the reticulo-endothelial system just as in other systems.

To the pathologist the problem of greatest interest presented by primary disease of the reticulo-endothelial system is unquestionably its status. This question is complex and very difficult, and it is pertinent at this juncture to discuss the meaning of tumour, and to 
present some general points concerning tumour growth in this system. Tumour, in its proper pathological sense is very difficult to define. Nicholson (1925) maintained, That it is impossible to define a tumour. Whenever we look we see that tumours exhibit no differences in kind but only differences in degree - and these often of the slightest - from the other tissues of the body. I have tried for years to formulate a definition, but have failed. Others nave been bolder. I shall not weary you with their definitions every one of which breaks down at one or more points'. This is a discouraging reflection but at the same time it overlooks the limitations inherent in definition, it is hard to believe that all experience of an idea or thing can ever be accurately expressed in a few neat words. In any event it would tax even a mind like Nicholson's to define what constitute differences of kind and differences of degree; the nuance can be very delicate. If this limitation is acknowledged then Nicholson is correct and it would be futile to attempt definition; on the other hand it is possible by collating a descriptive account of observed facts to render an imperfect definition which has practical value. This in turn may be expressed in quintessence avoiding at least the grosser errors.

Commencing with Cohnheim a long series of definitions have been submitted and it is interesting to examine some representative examples. Cohnheim maintained that a tumour was 'a circumscribed atypical production of tissue from a matrix of superabundant or erratic deposit of embryonic elements'. This became obsolete when the 'cell-nest' theory was proved inapplicable to tumours in general. Further it is doubtful 
whether circurnscription can be taken to mean more than apartness. Iubarsch included 'all spontaneously arising tissue growths which while typical in form differ in histological structure from the originating tissue, and, in spite of organic connection with the body, pursue an autonomous course, rarely to the benefit of the whole body'. As far as the practical morbid anatomist is concerned this is a good definition but with the advent of newer knowledge it is losing its value. (Still, few experimental pathologists would be ready to assign specific causes in individual cases of human cancer). It is unlikely that Iubarsch referred only to histiomata in his usage 'tissue growths'.

Ribbert regarded tumours as 'self-contained, dependant upon the organism for their nourishment but otherwise largely if not quite independant; corresponding more or less but never absolutely with the tissues of the natural body and presenting no typical limit of growth'. Serious doubt is now cast on whether tumours are self-contained; their non-correspondence with natural tissues may be theoretically true but sometimes their morphological identity can be treacherous. The paradoxical term benign metastasizing goitre is not retained for nothing, and granulation tissue in bone is only separable from sarcoma with assurance - by the really clever - or the foolish. The phrase 'no typical limit of growth' is extremely neat because it allows for the spontaneous cessation which is sometimes apparent. Birch-Hirschfields' definition is similar to Ribbert's but introduces the idea of progressive new growth. Ziegler submitted that 'a tumour is a new formation of 
tissue possessing an atypical structure; not exercising any function of service to the body and presenting no typical limit of growth'. This approaches closer to the modern definitions.

Powell-ithite defined a tumour as follows - 'A tumour proper is a mass of cell tissues or organs resembling those normally present in the body but arranged atypically. It grows at the expense of the body without at the same time subserving any useful function'. This fails to distinguish tumours from malformations (fillis, 1948). But this objection assumes that the latter are distinguishable with certainty, which it is respectfully submitted is sometimes a moot point. The limited capacity for aberrant growth displayed by Albrecht's hamartoma could be regarded as a type of link between the two.

Prudden, Jwing, and Luir concur in the definition of tumour as being 'any autonomous new growth'. This brevity has been condemned, yet X autonymity does seem the only feature common to all neoplasms. It has the advantage of course that the less one says, then the less one can be held to, and evades the issue of defining autonymity. This autonymity is very complex, it means that the new growth is a law unto itself in innumerable ways. To interpret it as meaning that tumour disregards the 'laws of normal growth' - which are neither formulated nor understood - is a serious understatement.

Willis submits the most recent authoritative definition namely, 'A tumour is an abnormal mass of tissue; the growth of which exceeds and is unclordinated with that of the normal tissues and persists in the same excessive manner after cessation of the stimuli which evoked the change'. 
Host emphasis is laid on this last feature which willis terms the irreversible neoplastic habit of growth. This is in accordance with the facts of experimental carcinogenesis and the author leads good evidence that the time lag in human cancer development after exposure to known carcinogenic hazards is dependant on the same principle. On the other hand the aetiology of most human cancers is still guess-work.

It is important to acknowledge the extraordinary arnount of experience, learning, and ingenuity displayed by the originators of these definitions. With a few exceptions all of these statements of what constitutes tumour are basically the same, but different facets of the peculiarity of neoplasm are emphasized.

It is invidious to be forced to choose and then permanently adopt any particular one. Wh interest in tumours is prirarily concerned with the ability to recognise them and while I admit much attraction towards the old definitions especially those of Iubarsch and Ziegler, I believe that luir's 'any autonymous new growth' is the safest. Peculiar new growth is the central idea. It can be applied widely - to tissue, cells, or free cells displaying the change, and displaying it in any degree. Finally, there is a useful and indeed much used term - 'tumour-like'. So far as I am aware no one atternpts to define this beyond calling it blastomatoid, which is like calling the Barth a Geoid. We all labour under the judgment of Ruskin - that it is the hardest thing in the world one ins rus. to put into words exactly what you mean. This, with a sombre background of ignorance, inexperience, and imperfect observation, makes the task even harder. The eye and the brain can recognise appearances accurately 
without us being actively conscious of the processes involved. A normally intelligent and interested child can be taught to recognise the mushroom (Agaricus campestris) with infallibility in a matter of a few days; and the only reasons he can offer for his identification is that it is a mushroom. Analogous situations, on a more sophisticated level, apply to the morbid anatomist. 'Yes, it is tumour'. To which the cynical colleague says 'Well, would you stand up in a court of law and defend that statement?' At this point frailty and abject inability to give reasons which sound convincing begin their pernicious work, and a compromise, 'tumour-like' may be substituted.

This is not to be condemned too harshly, provided it is not simply used as an easy way out of every difficulty. The diagnosis of tumour, malignant tumour, is far too serious a matter for doubt. The criteria must be met properly every time. Even so - disease does not always play fair. The gist of the matter is that microscopically tumours nearly all have a 'look' about them which is peculiarly difficult to describe but which the initiated appreciate.

There are several aspects of tumour growth which are particularly worthy of reflection in relation to tumours of the reticulo-endothelial apparatus.

As Willis (1948) has pointed out, Cohnheim's cell-nest hypothesis, despite its abandonment, has left two persistent corollaries. The first is the belief in unicentric origin. For a long period this was accepted as axiomatic and apparently with good reason, the majority of tumours in many organs and tissues are single. On the other hand many 
typically multiple tumours exist, for example duct papillomata of the breast, cancers arising in polyposis coli, and myomata of the uterus. A further evidence of fields of origin is afforded by recurrence of tumour growth when ricroscopical examination proves the initial resection to have been adequate. It is sugsested that fields of origin can explain autochthonous growth. This phenomenon is highly characteristic of tumours of the reticulo-endothelial system. If the lymphoid tissue collectively is envisaged as an organ which is virtually a single mass of identical tissue it is not difficult to rationalise the idea.

The second corollary is that tumours are purely intrinsic. In human pathology it is certainly rare to encounter sarcomatous change in the stroma of epithelial tumours but lesser degrees of 'taking up' of normal tissue are very probable, and, as lillis maintains, such a phenomenon is explicable on the potential present in a field of origin. That this occurs in lymphoid tissue is certain. It is unorthodox to recognise local Hodgkin's disease in part of a lymph node but it does occur. An explanation for these two facts lies in this hypothesis.

Metastases are common in human malignant neoplastic disease. There is considerable understandine of how they occur but very little interest in the reason for them. It is moderately true to regard tumours as parasites. These sycophantic forms of life will take the most fantastically prodigal steps to ensure their own survival. Possibly lymphoid tissue tumours are more cunning than the others - they do not need to metastasize, they rely on autochthonous growths to further their distribution. (On occasion the whim to metastasize by orthodox 
mechanisms may be exercised).

One of the commonest forms of tumour is the spheroidal nodule and it is hoped to shew that this primitive formation is common in many types of lymphoid tissue tumour even if ephemeral and elusive.

There are two further points of general interest. The first is that the process of mitosis is relatively fast, it is estimated that it occupies I-2 hours in man. Accordingly even one mitotic figure in every high power field reflects considerable growth activity. This proportion is about the minimurn observed in many reticulo-endothelial system tumours.

The other point is that it is doubtful whether cells in mitosis can be recognised with certainty. It is perhaps a naive reminder but they often look singularly alike. Ellermann (1923) led evidence that specific mitotic angles characterised each type of cell, this criterion is however very hard to apply.

The cause of death in neoplastic disease is sometimes obvious enough, e.g. haemorrhage, inflammation, starvation, suffocation, \& etc. Often it is not. It is suggested that in lymphoid tissue tumour, death is often the result of a virtual ablation of the lymphoid tissue. The normal lymphoid tissue is reduced to a level incompatible with the maintenance of life, by either anatomical or functional destruction. 


\section{PRIHIARY RETICULOSIS}

The notion that a morbid process might exist intermediate between tumour and inflammation was first advanced by Craigie in 1828. This interjection appeared in his considerations on 'Vascular sarcoma'; the description of the hard variety of this condition has been interpreted by Fox (1926), and others as possibly a reference to Hodgkin's disease. The account however was anecdotal. In the second edition of his 'ilements' (1848), significant new matter was not added concerning this entity, nor was the idea elaborated. This writer has a further claim to priority in this subject which is little known. In 1845 he contributed a paper on two cases of 'Puriform absorption from the Spleen'. He gave a good account of the clinical course and morbid anatomy of two cases which were undoubtedly examples of leukaemia. He noted that in the terminal phase of the illness a severe exacerbation developed and he attributed this to a suppurative episode. Arguing from this, and the fact that abscess of the spleen is of great rarity, he expressed the belief that the 'puriform blood', which he had confirmed by microscopy, originated in that organ and emanated therefrom instead of remaining local. It is remarkable how closely this result, furnished by reasoning, anticipated the true state of affairs. The second of Craigie's patients, John Menteith, formed the basis of a paper published synchronously in the same Journal by John Hughes Bennet, whom llacCallum (1928) quoted as the discoverer of leukaemia. Bennet described the instance as one of 'Suppuration in the blood'. In this he reverted to the old idea of Piorry of an haematitis and clearly supported this curious hypothesis. Later in the same year 
Virchow described similar cases and recognised the lymphoid nature of the cells in one type and their granular character in the other. In addition he separated the general syndrome into leukaemic and aleukaemic groups. Later, in 1865, Cohnheim described the latter as pseudoleukamie. It is interesting that Virchow considered that leukaemia was an expression of a perverted erythropoiesis, whereby the invariably attendant anaemia was neatly explained, a view tenable only so long as this metamorphosis passed as a physiological postulate. This erroneous premiss was abrogated by Neumann, (1879); particular credit for Neumann's recognition of the bone narrow hyperplasia in myeloid leukaemia was awarded by Cohnheim in the second edition of his lectures.

By this time, 1882, Cohnheim was manifestly advocating recognition of systematized hyperplasias of the haematopoietic tissues as the underlying cause of this group of diseases. Horeover it seems very probable that his invention of 'pseudo-leukamie' reflected the application of this idea. The equivalent French terms, 'Iymphocytémies frustes', 'formes frustes' Jolly, (1898) convey the same sense. Thile most cases referred to under this head were probably aleukaemic lymphatic leukaemia, Hodgkin's disease was specifically included by Cohnheim himself. In 1900 Dorninici put forward the brilliant sugestion that the 'metastases' in these conditions were due to the local growth of cells at these sites; these cells having powers of differentiation which were evoked in the disease. After this point was gained at a time when much authoritative belief (Ehrlich et alii)/favoured the idea that leukaemia and allied disorders were orthodox neoplasms; the morbid process reticulosis appears to have 
attracted recognition but doubtful acceptance. The French certainly appear to have had a shrewd percipience of these peculiar aleukaemic hyperplasias; records preserved from this period at certain Paris hospitals include provisional classifications closely similar to those advocated much later in accessible literature. This is a sidelight on the parochial proclivity of the Catholic Gaul! Kastiras, (1945).

The term reticulosis requires elucidation. This form, reticulose or reticulosis, was introduced by Letterer in 1924. Etymologically it is unsatisfactory, fillis, (1948), but is taken to mean proliferation of reticulum. Other forms, some with restricted meanings e.g. leukosis, myelosis, lymphadenosis, reticulo-endotheliosis etc. have not come into wide use.

The following abstracts from British and Empire literature are quoted to indicate the progressive attempts to formulate a definition of reticulosis.

MacCallum, (1928), did not make use of the term but employed the older 'aleukaemic lymphadenosis', and throughout he used the word hyperplasia unqualified.

Pullinger, (1932), did not actually define reticulosis but suggested that 'a group of disease of the reticulum existed in which proliferation was possible into one or several of the possible progeny'.

Rose, (1933), did not define the condition, but, following MacCallum, referred to 'the systemic hyperplasias of the blood-forming organs'. 
Robb-Smith, (1938), submitted the following definition.

'A progressive hyperplasia of reticular tissue with differentiation to one or more cell types. It is commonly systematized, that is to say it affects throughout the body tissue homologous to that affected in the lymph node. Further free cellular elements may be present in the circulating blood (leukaemia). Owing to the proliferation arising at many points there may be apparent infiltration but stromal destruction is not seen and dissemination by embolism (proved by emboli in vessels) is rare'.

It is seen at once that definition is difficult and even in the full exposition of Robb-Smith there is still doubt about the specificty of the process.

The morbid process is unique, it occurs solely in tissue of the reticulo-endothelial system. Disease processes restricted to one system are rare in general pathology, the only common example being dental caries, to which there seems no analogous change elsewhere. The rare primary inyopathies, in so far as they have been studied and understood, furnish another possible exception. Nental disease may represent isolated systemic disease, but it is too abstract to discuss in the present state of knowledge. The old tetrad; malformation, trauma, inflammation and tumour cover most pathological contingencies in all the systems. The invocation of a new special morbid process is therefore hazardous. The reticulo-ondothelial system is certainly unique, but it does not follow that it has pathological changes of its own. The ordinary pathological changes may be merely rendered unfamiliar by reason 
of the peculiarity of the systern.

It is equivocal what is meant by progressive hyperplasia. The word hyperplasia was invented by Virchow to mean an increase in the number of the cells of a part in response to derand. In ordinary usage this is tacitly accepted as physiological and purposeful or at least altruistic. Further it seems generally understood to connote homoplastic generation. It $\mathbf{s}$ quite properly applied to haenopoietic tissues, e.g. the increase of red bone marrow after haemorrhage; and so must cover the maturation aspect of this process as well. In the definition it is not clear whether progressive neans perpetual or autonyrnous. There is not any indication whether it differs from the continual new blood formation which is maintained throughout life or if it means that the process, once inititated is self-maintaining (i.e. without continued action of the genetic stimulus). If the latter, it is reasonable to say it is tumour growth. Mlost protagonists of reticulosis avoid close discussion about this kind of hyperplasia; indeed certain writers, e.g. Gorer, (1946), avoid the tern hyperplasia, substituting the safer word 'proliferation'.

It is in the character of systematisation that one of the nost difficult inconsistencies in the idea of reticulosis lies. Now while it is admitted that in Robb-Smith's definition that this is to be expected only commonly - not invariably, it is understood by many protagonists as an inherent feature. With a few exceptions, the lesions in this group of diseases do not readily affect the general connective tissue of the body where reticular tissue abounds, nor are they to be found in those 
inconspicuous deposits of persistent early mesenchyme which the adult body still harbours. Wy chief interest has been concentrated on Hodgkin's disease. In my necropsy series of cases of this disease, the omentum, which is a rich, indeed a classical source of reticular tissue, was never affected except in definite lymphoid tissue therein. I examined the nuclei pulposi of the intervertebral discs on a limited scale in the series and never found deposits of the new tissue in them. Fascial planes were equally negative. The rarity of skin lesions is well accredited, and yet collectively the integument contains an enormous number of reticulo-endothelial cells. Goldman and Victor (194j), recorded skin nodules in only two cases out of a total of 319, (0.62 per cent). Further observations on the location of the lesions will be offered later but the view that real systematisation occurs is not impressively illustrated in the majority of reticuloses.

The meaning of apparent infiltration is dubious and rests chiefly on personal interpretation. There is, in certain cases, very real difficulty in deciding whether true infiltration has oocurred; but it is equally difficult to prove that it has not. In ordinary tumours infiltration is one of the most significant microscopical criteria of malignancy. Here the ability of all leucocytes to move individually complicates interpretation. Likewise their undoubted local development in ectopic sites may invalidate an opinion that they are truly metastatic.

It has long been assumed that the entrance of tumour cells into the lumina of vascular channels is a natural and exclusive consequence of their vital property of invasive growth. 
A corollary of practical value is that the finding of turnour cells in blood or lymph vessels reinforces the diagnosis of malignancy. However embolism of normal tissue cells is well known, (Young and Griffiths, 1950). Familiar examples are seen where trophoblastic elements are caught up in the lungs, and where liver cells are seen lying free in the hepatic vein radicles.

This reduces the absolute value of the finding in tumour diagnosis.

It is still true however that blood-borne dissemination is a very important feature of many, if not most, malignant tumours, whatever mechanism precipitates entry. Thus an admission that reticuloses may spread by such a route is suspect.

The relationship with acknowledged rumour is very close. It is certain that individual examples of reticulosis undergo change into frank malignant tumour. This has been recorded in many cases by the study of serial biopsies and subsequent necropsy. At what point is one to draw a line between the two processes? - or, is there a line at all? It might reasonably be held that all tumours are malignant by virtue of their being autonomous new growths but the grade of malignancy can range from exceedingly low to very high. This is justifiable because from a simple point of view all tumours compete with the host's tissues, some in the mildest fashion possible, others with aggressive avidity.

The status of certain reticuloses stands in acknowledged doubt. The disease described first by Brill, Baehr, and Rosenthal in 1925 is a good example. This initially bore the non-committal title 'splenomegalia lymphatica hyperplastica'. (Some pathologists refer to it as Brill's 
disease; in so far as eponymic titles are an excellent idea this is good, but confusion may arise with the Rickettsial disease of the same name, cf. the medley of von Recklinghausen's diseases). At present in Great Britain it is better known as Iymphoid follicular reticulosis or giant follicular reticulosis, but in the U.S.A. the terms follicular lymphoma or follicular lymphoblastorna are used instead. Hore remains to be said concerning Hodgkin's disease, but only a few enthusiasts really call it fibro-myeloid medullary reticulosis and very few fibro-myeloidhistiocytic medullary reticulosis. Mallory's title for it (1913), scirrhous lymphoblastoma, is also little used, not because the tumour theory is unsupported but for the good reason that convention has narrowed down the import of lymphoblast. Lymphogranuloma is however still widely used.

In many respects the leukaemias conform better than most other reticuloses to the postulates in the definition. The peculiarities of these diseases are conducive to this. Chronic leukaemia is much commoner than the acute variety. In the former the majority of the leucocytes, whether granular or lymphocytic are usually very close to normal cells in morphology. Hyperplasia is therefore used with much justification to account for their supply. In chronic myeloid leukaemia it is exceptionally rare to encounter localised tumour (chloroma) and the gross generalised granulopoiesis is definite enough in most cases. On the other hand the inherent migratory properties of these leucocytes may conceivably account for this absence of localised tumours.

In chronic lymphatic leukaemia the apparent identity of the 
lymphocytes with normal ones is even closer. Here the generalised lymphadenopathy and splenomegaly could be reasonably interpreted as the result of hyperplasia. But, if a section of one of these lymph nodes is examined one pathologist will report it as medullary reticulosis of lymphoid type and another as lymphosarcoma. There is not any reliable criterion offered to tell the two apart; it is open to question whether these are not simply two histological names for the same thing.

In the acute leukaemias the supporters of reticulosis do not press their claims; the balance is too heavily weighted in favour of tumour. In the rnyeloid form localised 'tumour' of a sort is occasionally encountered. Whitby and Britton/ (1942) refer briefly to rare cases in which chloroma preceded leukaemic manifestations but unfortunately they do not give any details or references. The paradoxical aleukaemic leukaemias are not really additive to the reticulosis hypothesis since so little is known about what determines the entry of the cells into the blood. If leukaemia is regarded as neoplastic then the aleukaemic types or phases might be considered a reasonable parallel to those instances of ordinary malignant disease where metastases at necropsy are either exceedingly restricted or even not demonstrable.

A very rare condition termed histiocytic medullary reticulosis was described by Scott and Robb-Smith, (1939). This was characterised clinically and pathologically by these authors and identified with six similar cases reported previously. This disease appears admirably qualified to be considered a near-perfect example of reticulosis. In the four cases they studied a true generalisation, even to the formation 
of macrophage-producing foci in the skin was observed. The perverted hyperactivity of the macrophages (histiocytes) produced an intense anaemia which hastened death in each case. It is possible however to regard the growth as tumour which was lethal by virtue of the erythrophagocytosis before it evinced pathological features indicative of that status.

The Papal principle 'quicquid novum, falsun' is usually sound. It must be admitted that reticulosis has been accepted rather uncritically and until recently there has been a tendency to force diseases into the reticulosis schema without adequate justification. As a puthological category it is obviously more elastic than tumour but it is really questionable whether it has deserved its enthusiastic welcone.

Attempts to classify the reticuloses have been many. Four representative schemata by British workers are cited here as they reflect the successive developments of the idea over a period of about 20 years. These works are all readily accessible and need not be repeated, viz. Piney (1925), MacCalium (1928), Ross (1933), and Robb-Smith (1938) and (1944).

It is not proposed to discuss these schemata in detail individually but a few observations are appropriate.

Piney's schema adhered to his concept of the reticulo-endothelial system. The classification was simple and admitted considerable elasticity. The histiocytic (macrophage) scion was not included at all.

MacCallum's classification was on his own admission theoretical. Ross's design was much more an attempt to draw up observed and recorded 
facts into a semblance of order and she nade a complete distinction between the true and storage reticuloses. It is regrettable that this last feature has not been naintained in later attempts by others. Robb-Smith's system (1938), is widely known, (and also a further elaboration, 1944). One new principle was introduced - the functional medulla. This will be discussed more fully later, but it is equivocal whether it really behaves so independently as the classification suggests. It has been argued that the more elaborate classifications are too rigid and that like the Ellis classification of Bright's disease they break down at the individual case. This is true but they were primarily an attempt to systematise knowledge out of a chaos of poorly understood entities, and for this they are worthy of much admiration. 
TULIOURS OF THE RETICULO-ENDOTHELIAL SYSTEM

This is a difficult and complex subject. The peculiarities of the system in normal growth are retained when it assumes the neoplastic habit. While tumour growth in the tissue may sometimes apparently simplify the problems inherent in it, it more often complicates them in a capricious fashion.

Parts of the system have enjoyed separate anatomical description for a considerable time and even now in systematic studies it is still the practice to treat of these separately. It is convenient to speak of diseases of the blood, diseases of the lymphoid tissue, diseases of the spleen, \& etc., but the perpetuation of this method militates against the grander idea of reticulo-endothelial disorders. Notwithstanding, this older method is appropriate in many respects, and within limits emphasizes the weak parts of the system. It has come about therefore to describe tumours of the bone marrow, of lymphoid tissue, of the spleen, and recently of the dermal histiocytes. The representative of the system in the liver is not considered separately, which fact is curious since this part is probably the largest. Exceedingly rare tumours morphologically identical with reticulum cell sarcomata are described from time to time in unorthodox sites e.g. the thyroid gland, testis \& etc.

The multiplicity of names given to the tumours of the system is remarkable. This serves to confuse the subject even more. The timehonoured association of botany with anatomy seems to have transferred itself to morbid anatomy, in particular the love of artificial genera. The application of the Natural Orders in classification is only now /comrnencing. 
commencing.

The identity of these growths has often been obscured by well-meant efforts to systematise knowledge by the introduction of better names; which disadvantage is not always off-set by the pictorial presentations of their advocates.

Up till fairly recently tumours of the bone marrow and the lymphoid tissue were recognised in a restricted sense. Newer knowledge with its resultant appreciation of the basis of histogenesis in these tissues has introduced a curious inconsistency which is apparent in retrospect. Thus, while myeloma, chloroma and polycythaemia vera were recognised, entities like diffuse endotheliona of bone (Awing's tumour) and nonocytic leukaemia did not enjoy inclusion. In lymphoid tissue the lymphosarconata have long been known but the tumours more closely related to the reticulum are recent additions. It is singularly unfortunate that this has occurred because it has led to the artificial separation of these newly described neoplasms. In fact, they alone are considered as the tumours of the reticulo-endothelial system in several works. There is not any generally accepted classification yet, but many tentative proposals have been made. Before discussing these, several 'scrap-heaps' require attention.

Wost of us are reluctant to abandon the valuable labels round-cell sarcoma and mixed-cell sarcoma. The title, 'round-cell sarcoma', sometimes qualified as larg̈e or small, has much to justify its use in actual practice. However, it has now been shewn that true round-cell turnours 
rarely arise from mesoblastic tissues other than reticulo-endothelial and visceral muscle derivatives. (Certain authorities e.g. Boyd (1944) include glial tumours). The foremost supply the majority. Accordingly most of these growths are lymphosarcomata and myelomata. In much the same fashion the omnibus 'spindle-cell' and 'mixed-cell' sarcomata have acted as cloaks for turnours, some of which would now be termed reticulumcell sarcomata. Actually the tumour lymphosarcoma was recognised a considerable time ago, and as far back as 1893 Kundrat sought to differentiate it from Hodgkin's disease; on the other hand the vague round-cell sarcoma was apparently seldom identified with it.

That pathological bete-noir - the endothelial problem - makes its inevitable intrusion. Curiously enough this time the black sheep served to augment the family fortune not a little! In a very careful study in 1913, Ewing produced convincing evidence of the existence of 'endotheliomata' of lymph nodes. In his initial review he referred to several antecedent accounts of 'primary carcinoma' or 'carcinoma alveolare epitheliodes' of these structures. Since reticulum cell growths do sometimes appear epithelial and in contact with one another, these erroneous designations were perfectly understandable mistakes. Bwing was fully aware of the danger of misinterpreting metastatic carcinoma as a primary tumour in a gland and went to great lengths to substantiate that the neoplasms in question were in fact primary. This work was slow to gain credence. It is of interest that Iwing also sugesested that the tumours arose from the node reticulum, indeed he even referred to them as reticulum cell sarcomata. This was nine jears before Downey (1922) 
finally settled the identity of lymph node sinus endothelium with reticulum cells. The concept of reticulum cell sarcoma became further realised. In 1925 Goormaghtigh, in a brief and lucid communication imparted the idea of 'reticulo-endotheliome'. This antedates the widely quoted work of Roulet by five years. His article is illustrated partly in colour which has doubtless aided its popularity, he suggested the term Retothel-sarcom. After this time reticulo-sarcomata were diagnosed so freely that Delbet was prompted to suggest that there was an intoxication in the air responsible!

The status of these tumours is debatable, but when full allowances are made for the extraordinary properties of the component cells of this system, their fantastic interchangeability, their peculiar location and their essential unity, the problem becomes much more straight-forward. Localised tumour is not a necessary qualification for the application of Muir's definition of neoplasm which is adopted. Therefore the leukaemias and polycythaemia vera are properly tumours, the circulatory blood changes merely an inconstant, even incidental, expression of the primary growth in the progenitor cells. Autochthonous growth, the multicentric origin feature which most pathologists emphasize, is nothing more than what is to be expected in a tumour field which happens by nature to be dispersed and worked on a sort of feudal strip system.

The majority of the tumours are malignant. This is peculiar, but not without precedent. Malignant is a relative term and this elasticity is in accord with these growths which show a wide range of behaviour in this respect. This preponderance of malignancy may be related to the essential prinitiveness of the cells of the system whereby 
even slight augmentation of dedifferentiation at once constitutes frank cytoma. Benign tumours undoubtedly exist; the rare pure myxoma is the best known, even if poorly accredited, example. Stout (1949) is to be congratulated for emphasizing this growth as the important benign tumour of primitive mesenchyme.

Before considering the classifications in more detail it is pertinent to draw attention to the need for certain arbitrary limits to what constitute reticulo-endothelial system tumours. Almost any cell can be phagocytic under appropriate circunstances, but this does not imply that all cells are reticulo-endothelial cells. In the same way connective tissue tumours, e.g. fibroma, chondroma, osteorna, lipoma and chordoma and their malignant counterparts, are not ordinarily included as reticulo-endothelial system tumours. Notwithstanding, the claim of certain fibro-sarcomata and chordomata to inclusion is reasonable, indeed, far more reasonable than the inclusion of histiocytoma (derrnatofibroma) which is probably not a true tumour but an hamartoma. If these restrictions are not made then the group becomes unmanageably large.

In this present work emphasis is centred on Hodgkin's disease and its relation to lymphoid tissue tumour. Àcordingly these interests receive most attention. The classification of tumours of the reticulo-endothelial system.

Most schemata suggested present essentially a summary of the growths encountered most frequently. Some workers attempt to qualify these recordings with the clinical aspects, others prefer a purely cellular morphology basis. I few are highly speculative systems of considerable 
theoretical interest but like Adami's ingenious lepidoma and holoma theory, extremely difficult to apply in practice. The subject has manifestly proved attractive to numerous pathologists, and it is possible therefore to discuss only a limited number. The examples chosen represent an eclectic series recovered from the writings of the last twenty years. It has already been emphasized that old established portions of the reticulo-endothelial system are often described separately, and certain authors cited below restrict their observations to certain parts. In scme cases abstracts from comprehensive writings have been collated for my purpose and this does not imply that the authority from whom the several accounts are drawn supports this.

Ewing (1929) (1941), in his classical text, gives highly critical and profound infornation on the tumours of the system, but unfortunately it is diffuse and obscure. His classifications are complicated, particularly by the inclusion of granulomata of neoplastic type and the identification of clinical varieties. The full value of his writings is only appreciated after some experience has been gained; they are then recognised as singularly penetrating. It is well known that Ewing retained the term endothelioma despite the difficulties and objections inherent in it. This has caused him to be criticized adversely but it is frequently overlooked that he was far more cognisant of those difficulties than most oncologists. Criticism of the name endothelioma simply because it is no longer fashionable is as unreasonable as criticism of the large lymphocyte of Waximow. Iwing never abandoned the view that Hodgkin's disease was inflammatory and his famous aphorism 
'tuberculosis follows Hodgkin's disease like a shadow' reveals where his bias lay. Figuratively Hodgkin's disease can be examined under the enlightening rays of a shadowless lamp, and yet, who can deny that a penumbra lurks is some of the crannies? This concept of neoplastic granuloma pervades his work and has caused great difficulty and misunderstanding. After much thought I have come to interpret it as Ewing's way of stating the very real dilemmas that puzzle honest pathologists, especially those who have studied many doubtful lymph nodes, namely, is it inflammatory?, is it tumour?

The classifications he offered were tentative. In lymphoid tissue tumour he considered that the lymphocyte might give rise to a lymphocytoma which may take the form of a benign circumscribed growth, in certain cases inititated by tuberculous infection, a malignant lymphocytoma or lymphatic leukaemia, and pseudo-leukaemia (probably Hodgkin's disease is meant). The reticulum cells of the same tissue were credited with giving rise to large round-cell hyperplasia or neoplasia expressed as a granuloma malignum, myeloid leukaemia, large cell lymphosarcoma or Hodgkin's sarcoma. The endothelial cells were accorded specificity in this schema and their overgrowth or neoplasia acknowledged separately. It should be noted, however, that in the relevant text and in his 1913 paper, the identity of lymph node reticulum cells (Ribbert's type) and lymphatic endothelium are virtually admitted. His rare diffuse endothelioma of bone (endothelial myeloma) corresponds to reticulum cell sarcoma in that site.

Warthin (1931) did not actually submit a classification (he had /already 
already done so in 1904), but this work is highly relevant as it is the first reasoned discussion on the generic affinities of Hodgkin's disease and mutations between certain lymphoid tissue tumours. The hypothesis that Hodgkin's disease is neoplastic was well propounded, and a full descriptive account of the morphology of Hodgkin's sarcona was given. The triad, Hodgkin's disease, Iymphosarcoma and the less well characterized mycosis fungoides, were shewn to merge imperceptibly into one another. These transitions are to be sought in the study of examples of these diseases which present in an atypical form. This communication is deficient in actual presentation of illustrated instances, but it is offered by an undoubted authority.

Callender (1934) produced a comprehensive integrated system of classification of the tumours of the reticulo-endothelial system. This was based on a complex analysis of 380 cases collected over a period of seven years by the Lymphatic Tumour Registry. The classification is derived from the presumed progenitor cells of lymphocytes, granular leucocytes, erythrocytes and monocytes. The first category contains lymphatic leukaemia, Iymphatic pseudo-leukaemia and Iymphosarcoma. Callender observed that true lymphoma, meaning a benign circumscribed tumour of lymphocytes, was not represented and probably did not exist; accordingly he held that it was illogical to call the malignant counterpart lymphoblastoma. For this reason he equates Iymphatic leukaemia with 'Iymphocytoma, Ieukaemic'. His Iymphatic pseudoleukaemia is equivalent to 'Iymphocytoma, aleukaemic'. This corresponds in his view to two diseases viz. aleukaemic lymphatic leukaemia (diffuse lymphatic /pseudoleukaemia) 
pseudoleukaemia) and giant follicular hyperplasia with splenomegaly, Brill's disease, (nodular lymphatic pseudoleukaemia). Lymphosarcoma in this schema is characterized by the development of true metustases and is typically aleukaemic. The latter feature nay be lost in some cases.

The characterisation of the varieties listed is very good and full appreciation of their lability is acknowledged. On the other hand, criticism of the nomenclature adopted is justified, particularly the use of pseudoleukaemia which has long been a synonym for Hodgkin's disease. In addition, the clinical features which are itemised for the various forms are insufficiently stable to reinforce the subdivisions very much.

Under the granular leucocyte heading are listed leucocytosis, myeloid leukaemia, aleukaemic myelocytoma and myelosarcoma. Myeloid leukaemia is termed 'myelocytoma, leukaemic', the aleukaemic form being identified with single or multiple myeloma. Since Callender asrees that the commonest form of the latter is the plasma cell type, the propriety of including this turnour here is open to question. Hyelosarcoma is recorded as possibly occurring in an aleukaemic form and in a leukaemic form which is also termed myelocytic leukosarcoma. These categories include the green tumour, chloroma. Callender believes that the members of this group are more stable entities than the lymphocyte tumours and that the most difficult problem is the establishrnent of satisfactory criteria to recognise sarcomatous change among them. This however, he admits, is not of much practical importance.

The erythrocyte series is represented by the polycythaemias including the symptomatic forms. Polycythaemia vera is considered 
dubiously homologous to leukaemia and the doubtful erythrocytoma is mentioned.

The last group is collected under the heading 'Reticulocyte'. The usage is confusing, the reticulum cell or monocyte being better equivalents. The monocytoses of specific infections and the storase diseases are classed together as reactive reticulum cell hyperplasias. Under the sub-heading of reticulocytoma, leukaemic and aleukaemic varieties are described. The first corresponds to monocytic leukaeraia. It is to be noted that Callender manifestly leans to Naegli's interpretation of this disease, the Schilling type is only doubtfully acknowledged. The aleukaemic form is equivalent to the reticulum cell sarcoma of other authors. This latter identification is anongst the first statements of this idea.

Hodgkin's disease is considered as belonging to a special category but still under the general reticulocyte heading. Two forms are described, one corresponding to the classical malady and another apparently the counterpart to acute Hodgkin's disease. Hodgkin's sarcoma is a further special category, being regarded as a reticulum cell sarcoma which is characterized by an high degree of pleiomorphism. In his notes on this section, Callender criticises the view that transition occurs as much as is suggested by some writers, e.g. Herbut, Warthin \& etc., and makes a plea for careful studies of the natural evolution of diseases in the group. The schema has beon criticised widely but it is a very considered and thoughtful exposition. Ehrlich and Gerber (1935), studied a series of 18 cases of lympho/sarcomatosis 
sarcomatosis in considerable detail. The examples were examined fully at necropsy and most of these patients had had antecedent biopsies performed. These workers decided that the tumours fell into three main categories using as a basis for this separation the morphological characteristics of the predominating cell type. These cell types were found to correspond in their essential morphology to the immature, intermediate and mature cells resulting from abnormal differentiation of the cytoplasmic reticulum along lymphopoietic lines. This hypothesis of reticulum cells giving rise to lymphatic series cells is one of the main contentions of these authors, and they frequently invoke the authoritative work of Klemperer in support of it. So fär as I am aware, this is the first acknowledgment of the 'bipotential stem-cell' idea in reticulo-endothelial system tumour. They classified their tumour as follows:-

1. Cases in which lare pale cells in reticular arrangement predominated. Formation of new reticulin fibrils was minimal or absent. This variety they termed 'reticular'. It appears to correspond with 'stem-cell Iymphoma' and 'lymphoblastic lymphosarcoma'.

2. Intermediate type. A group in which mixed cells occur, partly reticular (syncytial) and partly free. Some new reticulin formation was characteristic.

3. Iymphocytic type. A group in which the tumours were composed predominantly of free cells either of the immature or mature lymphocytic type. This growth seems to correspond to the lympho- 
cytic reticulum cell sarcoma of Robb-Smith (1938) but they add that new reticulin is formed in considerable amount. It is this last feature which is difficult to accede to readily if the neutral path of orthodoxy is followed.

This subdivision does reflect what occurs - the need for the recognition of intermediate mixed reticulum cell/lymphocytic cell growth is very real.

In their discussion the mode of growth is fully 2 and, I believe, very ably presented, in particular their views regarding partial - almost focal-involvement of lymph nodes in early stages are most welcome. One interesting peculiarity is the rarity with which their cases exhibited splenic involvement ( 2 cases); this they observe may be related to the fact that this organ is not true lymphoid tissue since it lacks lymphatics. Limited systematisation of the process is another feature they emphasize and contend that this is in contrast to hyperplasia ('Iymphadenosis'). Their views are extended to Hodgkin's disease in which they hazard that both fibroblastic and lymphopoietic potentialities are developed, the former being the major expression. Brill's disease they regarded as a link between lymphadenosis and lymphosarcomatosis. Dawson, Innes \& Harvey (1937), in the second of their admirable "Debatable Tumours" series, discuss the same problem in detail. They define their subject as, 'lymphosarcoma or reticulum cell sarcoma, a radio-sensitive tumour of lymphoid tissue arising from the embryonal stem cells of that tissue'. They state that in the fully developed tumour the normal micro-architecture of the lymphoid tissue is lost, and 
the cellular appearance becomes more uniform. The tumour cells are invariably larger than small lymphocytes according to these authors, (correspondingly they reject 'lymphocytic reticulum cell sarcoma' as a true neoplasm). In form the cells may be polyhedral, ovoid or spheroidal with very variable cytoplasm content. Hitoses are always to be found. The nucleus is described as somewhat vesicular and containing more chromatin than the parent reticulum cells. Characteristically the tumours are locally invasive and though some deposits are undoubtedly autochthonous, blood borne metastases are probable.

The histogenesis is discussed very fully on the basis of their summary of normal lymph node structure which is a particularly lucid and acceptable account. They give the following schema indicating the relationships between the cells of the reticulo-endothelial system:-

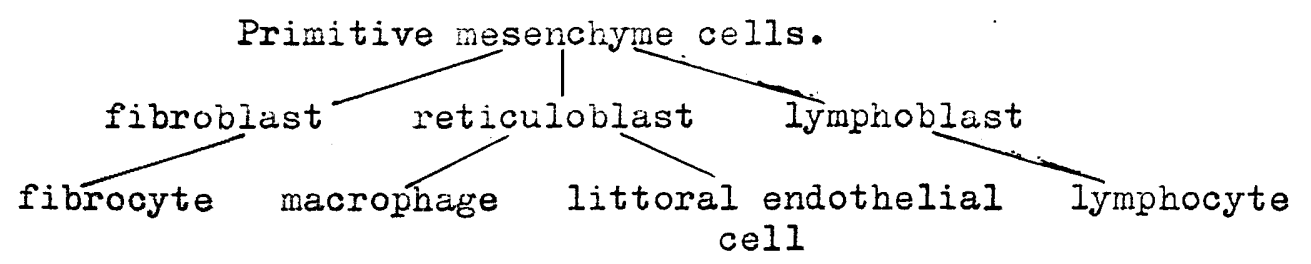

and suggest that 'mesenchymal' should be substituted for 'reticuloendothelial'. This might have advantages, but the former word is used in different senses and the idea is too late. The bi-potential stemcell idea is fully endorsed; they write 'reticulum cell and lymphopoietic lymphoblast we consider the same cell or at least only slightly separated from the stem cell'. The illustrations though small, are excellent.

Towards the end of the paper Hodgkin's disease is discussed and /curiously 
curiously treated. It is insisted that a sharp distinction is to be made between the two lesions yet at the same time it is admitted that this is constantly a source of difficulty. Thereas in the earlier part of the study, multinucleated giant cells, which answer perfectly to those of Hodgkin's disease, are described as occasionally to be seen in their reticulum cell sarcoma; and fibrosis, represented by variable reticulin increase is held to be common, they seem to recant suddenly and by-pass the only logical solution: Namely, they are variants of the same process. In fairness it is only right to the authors to recognise that they did not set out to settle this last question. This is an highly instructive communication and establishes the generic reticulum cell sarcoma beyond reasonable doubt.

Robb-Smith/ (1938), put the whole reticulosis and reticulo-sarcoma problem on a sophisticated basis which has rightly prompted universal admiration. Reticulosis has already been discussed and here it will suffice to consider the latter. At the outset of the second part, this author stresses the peculiar difficulties attendant on distinguishing true tumour from primary reticulosis and advances the phenomenon of stromal destruction as the only reliable criterion. In the classification of the true reticulo-sarcomatain lymph nodes he all but abandons the follicular, sinus and medullary theme and reverts to a cytological basis. The schema resulting introduced a much more comprehensive concept of reticulo-sarcoma than had been contemplated previously, or at least integrated by earlier workers. 


\section{Reticulo-sarcoma.}

A. Undifferentiated reticulo-sarcoma (syncytial).

1. Diffuse.

2. Trabecular (stroma reaction).

B. Differentiation to histioid cells.

1. Dictyosyncytial (fibrillo-syncytial) reticulo-sarcoma.

2. Dictyocytic (fibrillary) reticulo-sarcoma.

c. Differentiation to haemic cel Is.

1. Iymphocytoma.

(i) Lymphoblastic sarcoma.
(a) Medullary.
(b) Follicular.

(ii) Lumphosarcoma.

2. Plasmocytoma.

3. (Wonocytoma).

4. Myeloblastoma.

5. Erythroblastoma.

D. Mixed type (polymorphic reticulo-sarcoma).

9. Differentiation of sinus lining cells.

(a) Undifferentiated cell type (reticulo-endothelio-sarcoma).

(b) Differentiated cell type (histiocytoma).

The growths classified in $A$. and $B$. are essentially variants which are very closely related, the distinction probably not being of very deep significance except theoretically.

C.I(i) corresponds to the familiar large cell lymphosarcoma, the 
sub-divisions (a) and (b) connote the type of origin or presumed origin; (b) indicating development from a previous Brill's disease. c.l(ii) is the small cell lymphosarcoma - the growth to which Dawson et al (1937) take exception. The remainder, C.2., C.3., C.4., and C.5., since the classification is primarily descriptive of lymph node tumours, are rather hypothetical. The author does not discuss them beyond a brief note. The omission of $\mathrm{C} .3$ is interesting since Callender (1934) considered monocytic leukaemia as a reticulum cell sarcoma of lymph nodes, etc.

Category D., Robb-Smith holds, corresponds to many cases of Hodgkin's sarcoma or malignant Hodgkin's disease. This question will receive further attention later.

Category $\mathrm{I}$. is again chiefly included for completeness. (The omission of myxoma is curious considering that bistiocytoma is included).

This schema is open to rany criticisms when tried out in practice. A common observation is that several variants may often be found in one and the same case, or even node. On the other hand the classification is only what it is stated to be - a histological one, it does not pretend to be a cut and dried résumé of what actually happens. At the same time it is not entirely speculative and is of extreme theoretical interest because it has a catholicity fully reflecting the potentialities of reticulo-endothelial system cells.

In 1941 Narren and Picena published a valuable contribution to the subject of reticulum cell sarcoma in lymph nodes. In particular they gave an excellent critical review of the criteria advanced from time to 
time to establish the identity of these tumours and gave it as their opinion that the diagnosis of reticulum-cell sarcoma was probably too readily and too frequently made. The writers adhere to Oberling's definition of réticulo-sarcoma indifferencié (1922). This definition is considered rather restricted by some workers but is very helpful nevertheless. 'The new growth is formed of syncytial masses of an undivided or slightly fenestrated protoplasm whose limits are illdefined and connected with zones of more or less differentiated lymphoid tissue. The fenestration, by exaggerating the internuclear spaces, may give a reticular structure. In these undivided masses of protoplasm there are many irregularly distributed oval or idented nuclei, with granules, and one or two prominent nucleoli. The nuclei often appear almost empty. Mitoses are observed and some isolated tumour giant cells are common. It is not rare to see some lymphocytes scattered through the syncytium'.

In their own studies these workers record very variable impregnation results and significantly they set little store on its value. They suggest the following classification:-

1. Reticulum cell type - undifferentiated or syncytial. reticular (dictyocytic).

2. Mixed type - combination of reticulum cells and lymphoid cells.

3. Lymphoblastic - large cell (lymphoblastic lymphosarcoma). 4. Iymphocytic - small cell (lymphocytic lymphosarcoma). In so far as it goes this classification is good because these /variants 
variants do really happen, in particular their category 'mixed type' is welcome. In their discussion they state 'our concept of the normal lymphoid tissue is a network of undifferentiated cellular reticulum (stem tissue) in whose interstices lie lymphoid cells; (Iymphoblasts, prolymphocytes and lymphocytes), the offspring of the former. Thus, the complex of reticulum cells and lymphoid cells cannot be destroyed without destroying the concept of the essential unity of lymphoid tissue. Proliferation of one almost invariably causes variations in the other'.

They give several very convincing, well illustrated examples bearing out the truth of this belief and include an ingenious drawing depicting the subtle transition of predorninant reticulum cell neoplasm to predominant sinall cell lymphosarcoma.

Although restricted to lymph node growth, the essential unity of lymphoid tissue is emphasized and the generic lymphoid tissue sarcoma is clearly envisaged in this paper.

Gall and Mallory, (1942), give a discursive and exhaustive analytical account of a series of lymphoid tissue sarcomata (malignant lymphoma) extending to 618 cases. They uphold the cytological basis of classification exclusively and criticise Callender adversely. Their definition of malignant lymphoma, 'a generic term to designate those maladies which are characterised clinically by progressive tumour-like enlargement of lymphoid tissue with eventual death', is interesting as it covers the reticuloses. This latter concept does not appear to have gained much approbation in the U.S.A.

In their studies they concluded that these growths could almost all /be 
be classified as follows:-

1. Where the histological pattern is simple. (This appears almost equivalent to one-cell-type tumours).
(a) Stem cell lymphoma.
Incidence. 56 cases
(b) Clasmatocytic lymphoma.
$"$
$71 "$
(c) Lymphoblastic lymphoma.

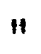
85 "
(d) Lymphocytic Iymphoma
$"$
135 "

2. Where the histological pattern is complex.
(e) Hodgkin's Iymphoma.
$n$
193 "
(f) Hodgkin's sarcoma.
$n$
$36 "$
(g) Follicular lymphoma.
$n$
$42 "$

Total $\underline{\underline{618}}$

Note: Stem cell lymphoma is the undifferentiated reticulum cell sarcoma of other writers; the clasnatocytic growth is a tumour of cells morphologically equivalent to monocytes and marcophages. In subdivision 2, Hodgkin's lymphoma is ordinary Hodgkin's disease.

It is notable that all of the series could be fitted into one of the seven different categories. This postulates a definite elasticity or their series must be unique; I collected only 220 and in finer details anyway, I could devise almost as many categories!

The most significant aspect of this classification is the inclusion of Hodgkin's disease. As will be seen later, the view that Eodgkin's disease is tumour is as old as its discoverer and therefore no new thing, but here it is openly included in a schema of lymphoid tissue sarcoma for the first time. fnother important view of these authors is that within the categories above there is great constancy of morphology and whilst admitting variations do occur, they insist that these are practically 
limited to degree of differentiation without significant transition between one major Eroup and the other. In 84 of their cases either repeated biopsies or subsequent necropsy, or both, were carried out. In 56 of these patients there was an interval of at least one month between the procedures. Analysis yielded the following results:- 


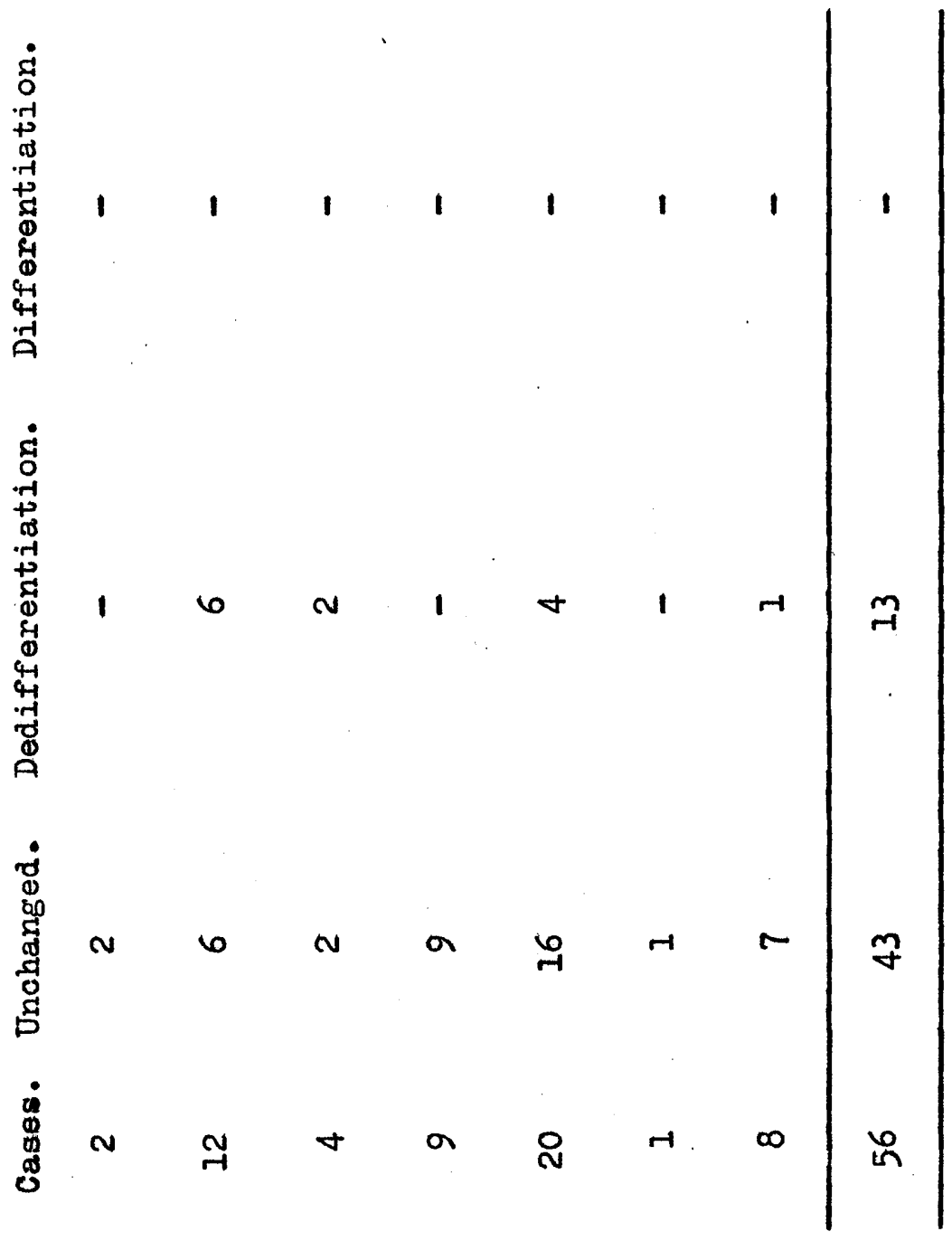

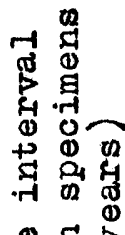

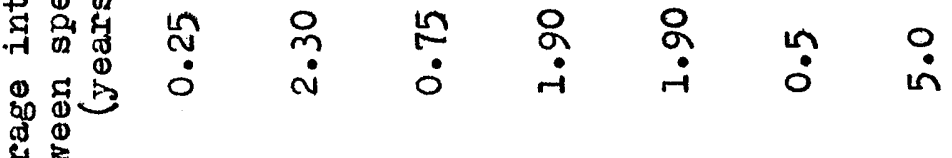

क्ष

要

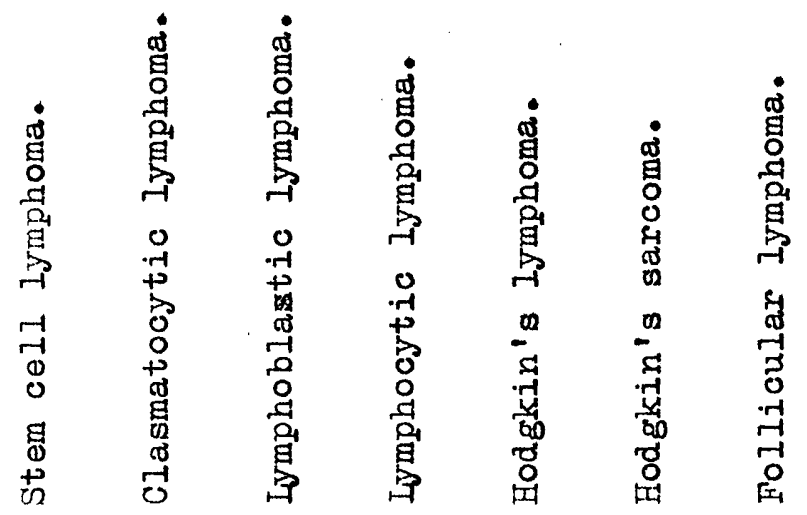




\section{$54 \cdot$}

It is seen that $43(76.82$ per cent) retained the original structure and 13 (23.2 per cent) shewed dedifferentiation. It is noteworthy that all the alterations recorded were atavistic. This latter figure is too large to be dismissed lightly and lends support to the view that the growths are potentially labile phases of lymphoid tissue sarcoma and not fixed entities.

Except for refinements or broad generalisations, the classification of lymphoid tissue tumours has not been altered much in the last decade. The unity has been fairly generally accepted, Hodgkin's disease and Brill's disease have been included, and a degree of transition from one morphological variant to another has been admitted. It is concerning the last phenomenon that appreciable advance has been made in this period.

Custer and Bernhard, (1948), reinforced this last belief. Their paper includes an excellent critical account of the gradual development of the hypothesis that Eodgkin's disease is neoplastic and a comprehensive study of the mutations encountered in a very large series of cases of lymph node tumour. Their series consisted of 200 necropsy cases, 138 of which had had antecedent biopsies; together with 500 biopsy examples. In the latter material 431 were single specimens, 60 were two specimens at intervals, and 9 were three specimens at intervals. 33 per cent of the total cases studied shewed alterations in the course of their natural history. The following schema indicates the nature of the alterations observed:- 

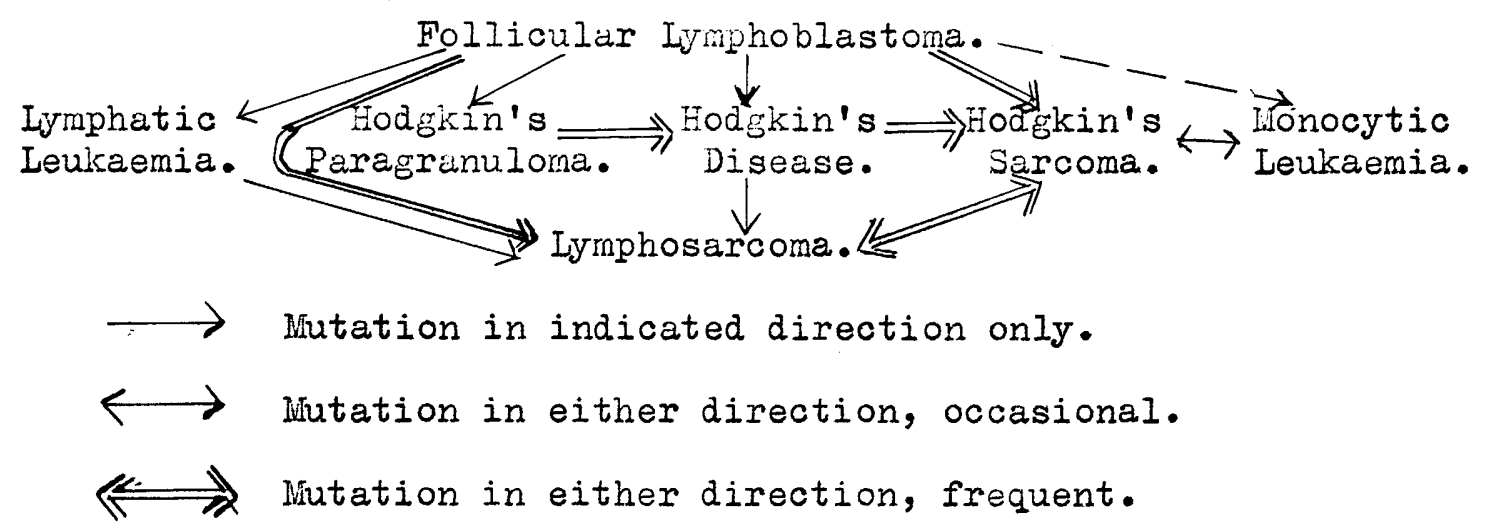

Their experience determined that follicular lymphoblastoma was the most labile variant of the group. They were unable to confirm that X-ray treatment had any influence in causing the transformations noted. The illustrations to this paper are copious and excellent.

It is overwhelmingly convincine that so far as the variants scheduled are concerned, there is not any doubt whatever that lymphoid tissue tumour is to be regarded as a single neoplastic entity which may be expressed in different histological patterns. The one regrettable feature of this work is the absence of full characterisation for the diagnosis of Hodgkin's paragranuloma and Hodgkin's sarcoma.

Willis (1948) in a masterly chapter simplifies the problem of lymphoid tissue tumour in an emphatically practical manner. In essence his views include a central concept of lymphoid tissue sarcoma, which tumour conforms broady to one of four main morphological expressions. His schema is as follows:- 


\section{Lymphoid tissue sarcoma.}

Wain variants:-

(a) Follicular lymphoma.

(b) Lyrnphosarcoma, with or without leukaemia.

(c) Hodgkin's disease.

(d) Reticulum cell sarcoma.

A simple classification admits of much elasticity and it is partly for this very reason that fillis prefers it. The previous more complex schemata tended to emphasize unduly the expressions of lymphoid tissue tumour as entities, which is misleading. On the other hand they exemplify the most praiseworthy endeavours which must of necessity precede the broad generalisations - namely the study of things caused. 


\section{PART II}

\section{THE LYMPHATIC SYSTEM}

Foreword.

The lymphatics and their functions.

Note on development of lymphatics.

The lymphoid tissue, general anatomy of lymphoid tissue structures.

The biological significance of lymphoid tissue.

The lymph nodes, general anatomy.

Involutionary changes in lymph nodes.

Iymphocyte depopulation,

Fat replacement,

Fibrosis. p. 59

p. $\quad 60-71$

p. $71-72$

p. 73-81

p. $81-85$

p. 86-100

p. 100-103 
58.

The development of lymph nodes in man.

p. 104-108

Fimbryology,

Neogenesis.

The numbers of the Iymph nodes, a study on the p. $109-114$ axillary nodes.

Illustrations.

Volume III Figs. 4-44, Figs. 236-246.

Volume IV p. 2-17, p. 58, 59. 


\section{THE IYIPHATIC SYSTEM}

Foreword:

This body system has long enjoyed the distinction of separate consideration in Anatomy. Despite the advent of its parent, the reticulo-endothelial system, it is still conmon practice to accord it separate attention. The selection of a portion of a diffuse and complex system suggests that simplification would result but this unfortunately does not follow. The reasons include inseparability of sibling from sire and a failure to define the lymphatic system satisfactorily.

In most standard anatomical texts the definitions of the lymphatic system are brief and insufficiently comprehensive. Le Gros Clark (1945) acknowledging the deficiencies, defines it thus:- The lymulatic system in its narrower sense consists of a system of fine absorptive vessels permeating most of the tissues of the body and emptying their contents ultimately into the venous system. Along the course of these vessels are groups of lymph nodes ...... localised masses of lymphoid tissue of different types closely related in structure to these are also to be found in other parts of the body ....'

It is proposed to offer some account of the system as defined by Le Gros Clark and to augment this by observations on the human lymph nodes. 


\section{THE LYIPHATICS with observations on some of their functions.}

The structure of the lytaphatic vessels is simple. The typical lymphatic capillary has a wall made up of a single layer of endothelial plates. The lymphatic trunics are lined by a similar intima surrounded by scanty connective tissue. Smooth muscle is interposed between these tunicae in the largest trunks but rarely forms a perfect media. In all animals which do not possess lymph hearts the lymphatic trunks and larger lymphatics are valved, and these structures follow one another at much more frequent intervals than in veins.

Iymphatic vessels are not present in tissues which are devoid of a blood supply; namely epidermis, hyaline cartilage, nail, enamel, and the cornea. One further certain exception is the central nervous systen, despite its high vascularity. It has been suggested by ioolard (1924), that the Virchow-Robin spaces act as lymphatics in this situation. In support of this view, which incidentally implies the assignment of lymphlike properties to the cerebrospinal fluid, is the fact that it drains into the venous system via the Paccionian bodies. On the other hand pathological increase of protein in the fluid is apparently reduced very slowly. This phenumenon is well seen in patients recovering from anterior poliomyelitis where high protein levels persist long after the pleiocytosis has regressed. In ordinary lymphatics the protein level is hardly ever more than half the blood protein level.

It is now established that the serous cavities, the pleural and peritoneal sacs, joints and bursae are not part of the lymph vascular system as von Recklinghausen believed. Representatives of the 
lymphatics are very doubtfully present in bone and muscle apart from the perivascular channels. In certain internal organs lymphatic vessels have a restricted distribution. In the liver they are confined to the portal canals and in the lungs to the immediate vicinity of the bronchial arborisation. Similarly in the spleen lymphatics are confined to the sheaths of blood vessels in the trabeculae, and then only in the region of the hilar intrusion.

Elsewhere they are disposed in superficial and deep plexuses. The former are exceedingly larğe and are related particularly to surfaces, where they form vast mutually intercommunicating networks almost comparable in extent to the blood capillary mesh.

The anatomical discovery of this inconspicuous vascular system was accomplished over 300 years ago. The lymphatic vessels were first observed in 1627 by Gasparo Asellius, Professor of Anatomy at the University of Padua, who noted them in the mesentery of the intestine of a well-fed dog. He also appreciated their association with the mesenteric lymph nodes which in this animal, as in the cat and rabbit, form a tolerably discrete conglomerate mass, and are appropriately known as Asellius' pancreas. (The purist may complain that a second pancreas is a bad idea to perpetuate, but after all the word means 'all meat', and is not so very apposite for the sweetbread which in man very commonly shews some degree of fat replacement). It is noteworthy that their discovery was related to their being filled with opaque chyle at the time of the historic observation. It seems fit to record in honour of Asellius that this well known and undeniable task performed by the 
lymphatics, viz., the absorption of fat from the intestine is still an almost solitary agreed fact. This property does not appear at first to have much connection with what may be their function elsewhere, nevertheless there is one small point of comparison which is seldom considered. This concerns the dimensions of the fat particles absorbed. Although this subject appears a dangerously Physiological problem for one schooled to think in terms of dead issues, courage is gained from the following statement. The mechanism of fat absorption is a highly controversial subject' Samson Wright (1945). A frank admission of this order from authority is tantamount to saying that one guess is as good as another. The proposal that fat is directly absorbed in very highly divided form is attracting serious attention once more. The original observation of $\mathrm{Krehl}$ in 1890 - when osmic acid $\left(\mathrm{O}_{\mathrm{SO}} \mathrm{O}_{4}\right)$ enjoyed a higher reputation for specificity than it does now - purported to shew how exceedingly minute droplets of fat appeared in the intestinal epithelial cell cytoplasm, and by coalescence formed larger globules. These then made their way into the villus lacteal, the transit being effected by leucocytic activity. This latter has long been a disputed mechanism and still merits the good Scottish verdict of Not Proven. This theory fell into disrepute after it was decided that hydrolysis of fat was the initial step in the absorption process. Later when it became popular to visualise the fatty acids as little fir-tree-like molecules in which only the 'rootportion' - the carboxyl group was soluble, the problem of their entrance had still to be considered. The configuration of the long chain fatty acids does not appear conducive to an easy passage, even with lecithin 
as a lubricant. Holecular distortion in proteins is an established phenomenon but is not reported to occur in fatty acids. The emulsion of fat in the upper small intestine reduces the particles to chylomicra which have been estimated by Gage and Fish (1924-5) to be $0.5-1.0 \mu$ (500 - $1000 \mathrm{~m} \mu)$ diameter. These dimensions are well within the scope of ordinary microscopical method but in terms of physical chemistry such particles are enormous. It is probable that the fat elements that actually traverse the villus cell wall are much smaller. With a good microscope it is possible to see but not resolve, particles as small as $0.074 \mu(74 \mathrm{m \mu})$. (They can indeed be resolved by Barnard's ultraviolet light microscope, and of course offer little theoretical difficulty to fuller examination by electron microscopy). In dark ground examination of a fine emulsion e.g. milk, it is possible to see particles which are probably fatty which are much smaller than $0.5-1.0 \mu$, and furthermore it is probable they are spherical. If good histological preparations of intestine during fat absorption fixed with osmium tetroxide are examined with the oil immersion objective particles of osmophile material well below $1.0 \mu$ diameter are discernible.

Elkes, Frazer, and Stewart (1938), made measurements of chylomicra in human blood and reported that the majority lay in three ranges of the orders of $1 \mu, I / 3 \mu$ and $35 \mathrm{~m} \mu$. They also considered that all the evidence available supported the belief that the particles in question were fat. Frazer (1946) in a review on the theories of fat absorption, maintained that provided emulsification was sufficiently complete even paraffin was absorbed. This fact which is capable of demonstration 
indicates that particle size is alnost certainly an operative factor.

The evidence for the existence of exceedingly finely divided fat is reasonably convincing and this brings the phenomenon of fat absorption by the intestinal lymphatics nearer to the functions of other lymphatics than is at first obvious. This connection relates to certain aspects of protein metabolism in which the lymphatic system is concerned. The link lies in the reasonable comparability of particle size between the ultimate forms of fats and proteins. The size of protein molecules is of an extremely small order. Lloyd and Shore (1938) have abstracted a large series of papers relating to protein molecular size expressed in absolute terms. The molecules of pepsin, trypsin, ovalbumin, insulin and muscle haemoglobin are reported to be spheroidal with diameters of $4.4 \mathrm{mp}$. Haemoglobin and serum albumin have not spheroidal molecules but calculated as spheres they would be $6.8 \mathrm{mp}$. It is further stated that one milligram of protein (type not specified) will present an area of one square metre. This has been calculated to correspond to the surface area presented by a set of spheres of radius about $2.2 \mathrm{mp}$. Svedberg (1937) found that nost proteins form homodispersed solutions with molecules which are spheroidal or at least approach that form. Regarding their size and molecular weight he decided that they fall into two classes. A group with a diameter of 2-4 mp with nolecular weights approximating to a multiple of $34-36,000$ and a group of $72 \mathrm{mp}$ diameter with molecular weights of the order 400,000 . It is widely agreed that natural proteins usually exist in multimolecular aggregates, which postulate is in accord with their colloid properties. Thus there is 
some justification for believing that no great disparity exists between the fat and protein particles at certain metabolic levels.

The lymphatics are concerned with two main functions relating to the transit of protein in the body. The first pertains to the conservation of the integrity of the vascular system and the second to defence mechanisms of the body.

While ideas concerning the functions of the lymphatic stream generally are still relatively meagre, it has gradually come to be postulated that it is a vascular contrivance which off-sets certain defects of blood vascular systems. This role is emphasized by Drinker and Yoffee, (1941) and the concept is reinforced by Le Gros Clark (1949). In essence this function is the transport of extravasated protein back to the blood. In animals with an highly developed blood vascular system the blood stream subserves the majority of the metabolic functions. In the process of nutrition food substances and oxygen are yielded to the tissues at capillary level. The gaseous exchanges are not relevant here, but other metabolites in dialysable form traverse the capillary impelled by osmotic and hydrostatic mechanisms. It appears that rninute amounts of plasma protein inevitably leak and the raised colloid osmotic pressure at the venous end of the capillaries is not sufficiently powerful to recapture it. It is believed that this material and fluid sufficient to retain tissue-fluid at an inconsiderable level - are absorbed by the lymphatics. Thus in one view the system may be regarded as a compensatory apparatus to correct deficiencies which are inherent in blood vascular systems utilizing high pressure. Phylo- 
genetically lymphatic systems are old but blood vascular systems are older so this idea is reasonable.

In general terms there is evidence that the working pressure of the blood vascular system rises as the phylogenetic scale is ascended, and concurrently the lymphatic system becomes more complex and efficient. The carriage of protein by the lymph is well established fact yielded by direct examination of the fluid in efferent trunks in experimental animals. This is readily performed in the frog which has easily accessible lymph hearts. In birds and mamals it is a protracted and difficult procedure calling for the highest skill and patience. It entails not only identifying the lymph trunk chosen but cannulating it, initiating a lymph flow in it, and maintaining that flow long enough to serve the purpose.

In man comparable experiments are not applicable and opportunities to study similar circumstances arising from injury or disease are rare. Drinker (1946) in a most thoughtful and delightful contribution to a symposium on lymph, draws attention to the following remarkable instance which is fully recorded by Crandall, Barker and Graham (1943).

A negress aged 30 years sustained a gun-shot wound of her neck whereby her thoracic duct was severed. The lymph fistula resulting did not heal spontaneously, an unusual event, and a careful study was made of its discharge (lymph), and the blood, together with recordings of the patient's weight over a period of six weeks. It was found that a weight loss of $5 \mathrm{lb}$. a week resulted and that the plasma proteins fell to $3.5 \mathrm{gm}$. per cent in a month. High protein feeding resulted in a slight 
rise of plasma protein (to $4.6 \mathrm{gm}$. per cent in thirteen days). At this point the thoracic duct was ligated and the patient began to regain weight. Drinker points out how with the lymph lost to the blood a progressive depletion of protein resulted which unchecked would probably have been fatal.

I have personal experience of a similar case. During the assault an Sicily in 1943 the Unit I served in received casualties by air from the beaches. A young German paratrooper who had been repeatedly bayonetted in the chest was admitted within 12 hours of wounding. He came under the care of It/Col. d'Abreu R.A.in.C. who established the diagnosis of haemothorax. Paracentesis thoracis revealed several pints of blood in the left chest which were drained and the patient was treated by blood transfusion. The following day signs of fluid had reappeared in the left chest and paracentesis disclosed approximately seven pints of pale pink wilky fluid. This drainage alleviated his disconfort very considerably. The fluid was shewn to contain fat and formed a gel on standing, protein estimations however were not done. It remained sterile on culture. It was found necessary to aspirate the chest daily to combat cyanosis and embarrassment. His general condition deteriorated and he became oedematous and died about ten days after admission. At necropsy healing wounds of the lung and liver were confirmed and the thoracic duct was found to be severed; the cut ends were retracted and everted recalling the appearances of bowel wounds when missiles traverse them. The injury had been caused by a glancing blow of the weapon striking the spine. In this case it is doubtful whether 
Iyraph loss alone accounted for death since the duration was brief, clinically the mechanical effects of the fluid appeared the chief derangement operative.

At this point it is appropriate to allude to the extraordinary mystery of how the lymphatics work, and what they are capable of accomplishing.

In the frog the lymphatic system is a conspicuous fraction of the vascular apparatus and it has been studied extensively. In this animal the system is not closed but communicates by means of stomata with the tissue spaces. An anecdotal and amusing fact with regard to these stomata is recorded by Klein. The cells lining them become ciliated in the winter time - but only in the female of the species! These animals have in addition lymph hearts, four in number. Thus, in the female frog cilia at the radicles of the system, and hearts in the course of it, serve to propel the lymph back to the blood, in the winter; presumably lacking the ciliary aid in the suminer. This näive account is not offered very seriously but a sururce of motive force is at least apparent in it, and inductive portals of entry are exhibited.

In the higher vertebrates, including man, it is very generally agreed that the developed Iymphatic system is a closed one, Drinker and Yoffele (1941) and Le Gros Clark (1945). Further, lymph hearts do not exiat. The lacteals are the only lymphatics in man which shew typical contractility. Yet material of relatively enormous molecular weight effects entry readily via non-alimentary lymphatics. Barns and Trueta $/(1941)$ 
(1941), investigating the route of absorption of venorn and toxins found that Black Tiger snake venom, molecular weight over 20,000 and Russel viper venom, molecular weight at least 30,000, were almost entirely absorbed by lymphatics. Diphtheria toxin with a molecular weicht about 70,000 was also absorbed exclusively by these channels. This latter is an important observation since plasma albumin has practically the same molecular weight $(67,500-70,000+)$, Harrison (1934). Cobra venom with a molecular weight between 2500 and 4000 enters the blood stream directly at about the same rate as via the lymphatics.

Exactly how this 'absorption' is effected is obscure. The comnon term applied to the lymphatic channels of a part, namely the lymph drainage, suggests a sophisticated sewage system with the thoracic duct naught else but a thinly disguised Cloaca haxima. Admittedly the pressure at this Ostia is low, probably in the order of $5-15 \mathrm{~cm}$. water, but it is not much lower than the blood capillary pressure, Nright (154j).

In a patient with general chronic venous congestion this pressure (capillary) may rise to twice the latter figure, i.e. $30 \mathrm{cms}$. Water, but lymph still drains. If it did not he would assuredy die, as a frog does when its lymphatics are obstructed, and it is a fact that in cases of the above nature protracted chronic dropsy is not uncomnon. This diminishes the strength of the argument which assumes that a pressure gradient is responsible for the shift. In any event it is decidedly peculiar for the effluent of drains to proceed uphill! The presence of valves in the lymph channels (beyond the capillaries) stimulates the imagination and one might envisage a kind of hydraulic ram mechanism /effecting 
effecting the lift of lymph from say the foot to the shoulder. This however is a proposition with little to support it - where is such an intermittent force to come from? The arterial pulse does not extend down to the true capillaries and the rhythmical opening and closing of these channels is a gentle affair.

In experimental studies of lymph production in mammals it is invariably difficult to obtain a flow in the cannulated lymph trunixs of the anaesthetized animal. The flow can be augmented by producing passive movements in the beast and ingenious mechanical devices to effect this have been elaborated. As a corollary it is supposed that muscle rovement is a potent factor in promoting lymph flow.

This factor is undoubtedly important particularly in visceral muscle activity. It seems conceivable that the lethal effects of paralytic ileus are related to the lymph stasis resulting. On the cther hand patients with paraplegia or bed-ridden people do not become oedematous by reason of muscle inactivity alone. The invocation of incidental muscle movement as a factor in the transport of body fluids is ingenious but suspect. In the case of venous return it is merely subsidiary, the left ventricle effecting most of the shift.

The above remarks do not much contribute real understanding of the crucial problem, namely how do such enormous particles get into the closed lymphatic. It has been shewn, Clark and Clark (1926), that a poor kind of phagocytosis is a property of lymphatic endothelium. This however can be rejected here because the endothelium studied was recently repaired and the process wis very slow. Personally I find the 
situation quite incomprehensible. The entry of microbes does not present so much difficulty if the generally accredited mechanism is the only one. It assumes that the micro-organisms are ingested first by microphages or macrophages and that these cells then enter the lymphatic in the same mysterious fashion in which they enter or leave blood capillaries. The entry of viruses is in all probability direct. In most cases their dimensions, $10-300 \mathrm{mp}$, are conducive to belief in this hypothesis.

Note on the Development of the Lymphatic System

This subject has received little attention in the study of human embryology, most of the restricted work performed has been conducted on the lower animals, notably the chick and the pig.

Frazer, (1931), states that lymphatics first appear in the human embryo at or just after the $10 \mathrm{mril}$ stage, i.e. In the sixth week of intrauterine life. The origin of the lymphatic vessels is still a disputed problem. Two main views have been held, both ably supported by competent observers, and both open to certain criticisms. The one idea is that the lymphatics arise from veins, and the other that they arise de novo in the mesenchyme. The former view is more favourably regarded, Le Gros Clark, (1945). In this connection it is significant that permanent intercommunication between the lymphatic vessels and the veins is constantly established at the terminations of the thoracic and right lymphatic ducts. It is difficult to imagine why an highly complex subsidiary vascular system of lymphatics should come into being independently and then acquire anastomoses at these curious loci with 
such regularity. On the other hand the arguments of Huntingdon (1914), and critical support of LcClure, (1951, 1921) deserve a just appraisal. There is not any doubt that injection technique in embryological material is peculiarly susceptible to artefact and the meticulous presentations of Sabin (1902, 1911, 1913) seem almost impossibly fortunate.

If the venous origin of lymphatics is accepted, then their assistance in the return stream of fluid from the periphery is certainly in accord with behaviour which could be expected from modified veins. There is no doubt that the lynphatic endothelium acquires a morphological specificity very soon. In subsequent growth in the normal course of events, or in the phenomenon of injury requiring repair, the new lymphatics arise from pre-existing lymphatics by a building process. In this way they conform to the normal principle of homoplastic regeneration. Clark and Clark (1937), using the transparent chamber technique have established beyond doubt that new lymphatics do not arise de novo in the connective tissue in extra-uterine life in the rabbit. 


\section{TEE LYIPHOID TISSUE}

The definition of lymphoid tissue is not precise. Any tissue consisting predominantly of Iymphocytes has been held to constitute it $y^{\prime}$ Drinker and Yoffe' (1941). The other components vary in character and in proportion. In all but the smallest aggregates of lyrophocytes, connective tissue and reticulo-endothelial elements are present, and vascular tissue, either lymphatic or blood, is almost as consistent in its presence. In animals below the mammalia the tissue is characteristically more coherently integrated with the myeloid tissue; this association is less evident in the adult higher forms, but is a notable phenomenon in them in certain morbid states. Iven in normal man the separation is not complete in an anatomical sense, since lymphoid tissue nodules are probably constantly present in the bone marrow, Dominici (1902). Later the relation of lymphocytes to myeloid cells will be discussed more fully, here it suffices to state that some evidence points to the existence of a much closer relationship than is generally accorded.

The definition of lymphoid tissue can be confined more closely without error as tissue consisting predominantly of lymphocytes together with their progenitor cells and other close relatives set in a vascular stroma. It has already been emphasized in Part I that in reticuloendothelial organs the parenchyma arises from the stroma. This interpretation is a general one and applies more particularly to the cellular maintenance of the organ. In the first origin of lymphoid tissue it might be supposed that the stroma developed first but this has never 
been confirmed, the origin of the first lymphocyte is still unsolved. It is akin to the fanous riddle one encounters first in the nursery, 'which came first, the chicken or the egg?' The sophisticated reply, namely, 'the egg - but it was a reptilian egg', only half solves the difficulty but it supplies an analogy for comprehension of the primordial anlage of lymphoid tissue.

The term lymphoid tissue is preferred. It is older than other names suggested and it is very widely adopted. According to Erich, $(1929, a)$ Aschoff maintained that the word lymphatic should relate to the tissue in question when germinal centres were present and the term lymphoid restricted to formations without them. It is doubtful whether anything usefuI is gained by this distinction, besides it reflects too static a concept of the morphology.

In the study of Iympioid structures it is irnediately obvious that the appearances shew topical variation. Deposits of the tissue may be circunscribed or diffuse, the cellularity of the formations may be high or low, in particular the density of lymphocytes may be uniform or the reverse. Recently it has become fashionable to draw a broad distinction between loose lymphatic tissue and dense lymphatic tissue but many special terms are used in addition. Generalisations about the lymphoid tissue are few because it is exceptionally difficult to study it comprehensively. It is exceedingly diffuse and widespread so that total ablation experiments cannot be applied with precision.

Aschoff considered that the lymphoid tissue organ could be regarded as distributed in three main groups. 
1. The tissue in the lyrnph nodes. This part has afferent and efferent lymphatic vessels related to it and lies chiefly in the lymph stream. 2. That in the mucous menbranes. Efferent lymphatics only are present and the tissue is located in the fluid streams going from the mucosae into the interior of the organism.

3. That in the spleen, where neither efferent nor afferent lymphatic vessels exist but the tissue lies in the blood-stream.

Quantitatively the bulk of the lymphoid tissue is accounted for in this preliminary schema, but not all of it. The facts are that at present there is insufficient knowledge and agreement for any more comprehensive account. An attempt is made below to describe the various moieties briefly.

Thile the Eeneral principles of this classification are adhered to, some tentative inclusions will be added.

At the outset several jeneral renarks are offered. Iymphatics and lymphoid tissue are present in all vertebrates. They are first recognised in the Selachii (Elasmobranch fishes). It is probable that the fundamental reasons for the system lie in the attainment of increasingly better blood and blood vascular apparatuses. The spleen and the organ of Leydig are the oldest representatives of the actual lymphoid organs and in this primitive animal the spleen occupies its original site in very close proximity to the bowel. This has been interpreted as reflecting the old association of phagocytes with the coelome, and is a significant phylogenetic principle. Iymphoid tissue seems destined to 
form round tubes, not only the alimentary tube, but later "round other tubular structures in which there is flow. As the phylogenetic scale is ascended migration of lymphoid tissue fron the old para-enteronic sites cornmences and reaches its hichest expression in marnmals, where, although the bulk of the tissue is still probably in the primitive position (disregarding the peculiar migration of the spleen), much is out towards the periphery in the form of lymph nodes. The old lymphoid organ - the paleo-lymphocytogeion and the new, the neo-lymphocytogeion are relatively distinct in many ways and these include properties of interest to the pathologist. It is a general principle that disease has most chances of success in attacking more recently developed structures and functions; the old ones are more immune. This is particularly well illustrated in certain acquired diseases of the central nervous system, e.g. dementia paralytica. Examples can be cited for the lymphoid organs. Exclusive or almost exclusive involvement of the para-enteronic lymphoid tissue in primury tumour is exceedingly rare. The pseudoleukaemia gastrointestinalis of Briquet (1838) is so little known that under ten cases have been described (Symers). (The original case is depicted in Cruveillier's Atlas (1835-42). By contrast Hodgkin's disease without extensive lymph node lesions is extremely rare, and intestinal or gastric lesions are recorded in under 5 per cant of cases in most series. Further, Hodgkin's disease is the commonest primary growth in lymphoid tissue and is characteristically a lymph node affection.

The break up of large lymphoid tissue masses into smaller separate parts is generally held to be an indication of phylogenetic maturity. 
Thus it is thought that the dispersal of the pancreas of Asellius into several hundred mesenteric lymph nodes is a higher development.

The schema adopted to describe the lymphoid tissue is as follows.

1. The lymph nodes.

2. The sub-epithelial lymphoid apparatus which includes:

(a) i. The lymphoid tissue related to the intestinal tract. ii. The lymphoid tissue related to the respiratory tract. (b) The lymphoid tissue of the integument.

3. The isolated follicles.

4. The thymus gland.

5. The spleen and haemal lymph nodes.

6. The ectopic Iymphoid tissue.

1. The lymph nodes.

The term lymph node is replacing the older one, lymph gland. This is an advantage because true glands are typically epithelial structures and characteristically they elaborate secretions. Iymph nodes are not epithelial and do not secrete in the ordinary sense of the term. From its derivation the word 'node' can be accepted as referring to a lymphatic channel knot or morass of Gordian complexity. (The French ganglion lymphatique conveys the same idea). Incidentally the word gland means an acorn and was orifinally adopted to describe the glans penis. Iymph nodes seldom resemble acorns; if they must be characterised generally, they are bean-shaped. These structures are situated in the lymph stream; as a corollary it is reasonable to suppose that they bring about modifications in the lymph itself and this is being demonstrated with increasing conviction. 
In quintessence they are situated in the path of the circulating lymph. Topographically they are mostly organised in groups which are related to the larger blood vessels. It appears that afferent lymph is required to traverse a node after it has completed part of its journey to the blood. Large aggregates of nodes are found at the base of the neck, the roots of the limbs and the lymphatic hila of viscera or groups of viscera. Peripheral to these sites formed lymph nodes are rare and where present they are swall. The actual disposition and identification of the nodes or groujs of nodes present much difficulty. Despite statements to the contrary, it is certain that individual variation is considerable, indeed capricious. The meticulous demonstrations pictured by Poirier and Charpy, Cuneo and Marceille, and Jamieson and Dobson in Gray's Anatomy must be either reflective of individual cases or refer to an ideal. The number of lymph nodes in man is doubtful. Sappey (1869) hazarded that between 600 and 700 might be recovered by ordinary dissection but that many small ones might be overlooked. Drinker and Yoffee (1941) suggest the true number is probably noarer 2000 .

2. (a) i. The para-enteronic lymphoid tissue is developed in relation with the epithelial lining of the alimentary tract. It is represented by the pharynceal tonsils of Luschka, the palatine tonsils, the Peyer's patches and the less coherent or solitary lymphold foll10les of the entire tract. It is believed that these deposits are with fow exceptions related to the lymphatic system solely by efferent ohannels. The generalisation is derived chiefly from studies of small representative 
parts. The solitary follicles may be relatively closely grouped as in the tongue, or dispersed as in the stomach and the colon. The aggregate of the latter is often impressively shewn in enteric fever and may be demonstrated in the normal by clearing the bowel in lactophenol. Orsk $\phi$ (1901) using this method computed their number to be between 2000 and 3000 .

2. (a) ii. The lung parenchyma is normally devoid of lymphatics and lymphoid tissue but both are present in the immediate vicinity of the bronchial tree. The para-bronchial lywphoid tissue is generally diffuse and scanty but comparatively large, well-formed intra-pulmonary lymph nodes may occur. Embryologically the lungs are formed by outgrowth from the primitive pharynx and accordingly this component of the lymphatic system is virtually para-enteronic. An elaborate lymphatic plexus is situated in the visceral pleurae and occasionally small laminate lymph nodes are encountered here.

2. (b) The lymphoid tissue of the integument is probably considerable but very few studies have been made on it. Among the better known representatives are the solitary follicles of the conjunctiva (Bruch's glands). The problem of differentiating 'chronic inflamnatory foci' from normal lymphoid fornations is frequently difficult but it is possible that many foci of this description in the skin are in fact natural. Their relation to the lymphatics is unknown. 3. The isolated follicles. Very small deposits of lymphoid tissue are regularly encountered in the bone marrow, the uterine endometrium, the salivary glands, and the breast. The significance of these islets 
is obscure. It has been suggested by Jordan and others that the bone marrow lymphoid tissue gives rise to the blood cells but this view does not yet enjoy much popularity. The recent work of Yoffee promises interest in this subject. The endometrial follicles may possibly have led to the opinion formerly widely held that the endometrial stroma was of lymphoid character.

4. The thymus gland is sometimes considered along with the epitheliolymphoid apparatus. There is not complete agreement however that the tissue is truly lymphoid. For long the early development of the gland was disputed but it is now widely agreed that an entodermal outgrowth forms the anldgge. Whether a proportion of cells become physiologically equivalent to reticulum cells or whether the mesenchyme cells at the locus do this is not settled though the latter interpretation is better supported. Liorphologically the thymocytes have not any specificity by which they can be differentiated from lymphocytes, their mutual identity is probable. Serologically they are the same cells and the thymocyte's high sensitivity to $x$-rays is exactly parallel to that of ordinary lymphocytes. The gland does not appear related to lymphatics in any particular fashion and lymphatic sinus tissue is absent from it. (Iymphatics are present in the stroma trabeculae and drain to the deep cervical and mediastinal nodes). This anomalous and peculiar structure is probably the most bewildering and labile lymphoid organ. Among the few facts known about it, one of the most striking is its dramatically rapid shrinkage in response to infection, a process which is almost akin to autolysis. Atrophy and necrosis sometimes subserve physiological 
needs, a familiar instance is the universal death of the superficial part of the skin, which phenomenon enhances the insulative properties of the integument enormously. It is attractive to speculate whether or not acute thymic involution has some equally valuable function but the issue is an extremely complex one.

5. The spleen and haemal lymph nodes are unique representatives of the lymphoid tissue. The former was originally a haemopoietic organ and even in man an ephemeral phase of this activity is shewn in foetal life. Other functions have been superimposed but it cannot be said that any of them appear very vital. This lymphoid tissue is virtually in the blood stream and constitutes the largest single deposit in man. Although developmentally para-enteronic it migrates peripherally and characteristically exhibits a tendency to react along with the lymph nodes in disease. The comparative anatory of the organ is peculiarly inconsistant, at least two types of spleen are described in the mamnalia. In the less common one, represented broadly by the spleen of ungulates the intravascular position is much less definite. It is tempting to wonder if this viscus is a kind of unfinished experiment in morphology and function. The existence of true haemal lymph nodes in man is not universally acknowledged. Originally described by Gibbes in 1884 they have evoked desultory attention. The original description is poor but later publications have remedied this deficiency: Warthin (1901) gave an excellent account. They are small, inconspicuous, and usually about as difficult to find as the organ of Zuckerkandl or the superior parathyroid glands. The classical site to attempt their recovery is in the 
inconsiderable areolar tissue between the renal artery and vein. It is improbable that they are of much importance and personally I am only doubtfully convinced of their specificity; erythrocytes accumulate so readily in ordinary lymph node sinuses as the result of minimal trauma. 6. Ectopic Iymphoid tissue is a phrase coined to describe deposits of the tissue in sites where it is not fenerally recorded. This phenomenon will be more fully discussed later, it includes problems related to the neo-formation of Iymphoid tissue, including lymph nodes: and the significance of the phenomenon.

The biological significance of lymphoid tissue

The essential component of the tissue is the mass of lymphocytes. These cells are among the most mysterious in the body, singularly little is really known about them. Drinker (1946) emphasizing this, stated that the two acknowledged facts of importance regarding them are their high sensitiveness to $x$-rays and their indispensibility in the life process. The first characteristic is common to all lymphocytes in whatever species they nay be found. The anatomical diffuseness of lymphoid tissue militates against the successful resection of it in entirety, although the skilful and ingenious operations of Sanders and Florey (1940) go close to realising it. The method of irradiation has been suggested as an alternative; but experimentation by this device has not been successful since the dosage required is lethal. Jolly (1924), reported that this sensitiveness could be considerably depressed if the irradiated lymphoid tissue were simultaneously deprived of its blood supply, which suegests that the susceptioility was not intrinsic. 
It is a general principle that an actively proliferating tissue is more vulnerable to $x$-rays than an indolent one and a reasonable corollary derived from the lymphocytes behaviour is that it must be an actively growing tissue. The postulate is however difficult to reconcile with the apparent inertia so commonly observed in ordinary histological preparations of Iymphoid tissue. Evidence has been adduced that the lymphocyte population of the blood vascular system is renewed daily or even several times a day. These results are derived from counts made of Iymphocytes in the thoracic duct lymph. This at once raises the question - Where do thej EO? A satisfactory answer is not yet available. It has been suggested that some are returned to the lymphoid tissue, others to the bone marrow, many to the intestinal lumen (yet they still disappear as readily in an animal previously deprived of its gut!), some to the skin to form epidernal epithelium, and some to the tissue spaces. This last site seems probable since lymphatic endothelium appears to resent the re-entry of Iymphocytes. The idea that they are destroyed in the blood stream now receives less support.

The indispensibility of the lymphocytes is emphatic. It has long been suspected that the lymphoid tissue is part of the body's defence mechanism. This appears to have originated from the supposed efficacy of the filtering property lymph nodes exhibit. This effect is well shewn in nodes draining tattoo marks, or anthracotic lungs. The filter is not perfect because distant lymph nodes may be pigmented and even the spleen may become anthracotic. Then the jarticles are really small e.g. bacteria or viruses the filtration is far from perfect. It is 
established however that lymph nodes are important sites of antibody formation though the rôles of the various cells concerned is unsettled; Oakley, Varrack, and Batty (1949). Ingenious interpretations have been offered e.g. the nacrophage component elaborates the antibody which it then sheds in surface films, this material, claimed to be $\gamma$ globulin, then becomes the cytoplasm of lymphocytes which is identified as $\gamma$ globulin too, and these on their dissolution yield this fraction to the blood plasma. Other theories hold the lymphocytes to be directly concerned with the whole process and more recently the plasna cell has come into favour. This problem has prompted a great deal of work in the last few years and it is still too early to assess it.

Winy clinical facts seem inconsistent with the too ready acceptance of an effectual defensive rôle on the part of lynphoid tissue. Inlarged tonsils and adenoids are not convincingly associated with high resistance to infection, nor is the florid para-enteronic lymphoid tissue of the child related to singular freedom from bowel infections.

The rneaning of lymphocytic infiltration round cancerous growths is quite obscure. The phenomenon is very cominon but not invariable. I have to record one curious exception. In the series which I have studied of mucoid cancer deposits in organs or lymphoid tissue the phenomenon has been usually strikingly absent. In one relatively early metastasis in a lymph node the mucoid material was seen lying in the marginal sinus and in the vicinity a process of lymphocyte disappearance was evident.

The elementary function of lymphoid tissue is sometimes stated to 
be the production of lymphocytes. Superficially this might appear axiomatic but it is exceedingly hard to prove. In morphological studies with fixed human tissue the best reasons for believing it are that they do not eppear to be produced elsewhere, and adhering to the principle that cells are best recognised by the company they keep. Since the primary interest in this work as a whole is Hodgkin's disease, emphasis will be laid on the more directly relevant problem of lymphoid tissue which the disease raises. 


\section{THE LYY DTODOS}

The structure of lymph nodes with observations on physiological human nodes

Appreciation of morbid lymph node histology presupposes full knowledge of the normal structure. The acquisition of this axiomatic requisite is difficult because good descriptions are few, and the latitude of normal appecirances presented by fymph nodes is wide. In many standard anatomical texts illustrations of several easily procured mammalian nodes have long been offered as adequate. His (1860), a pioneer of descriptive microscopical anatomy based his structural schema solely on the lymph nodes of the ox, and certain of his drawings were repeated at intervals in other books for about fifty years. His described the lymph node as composed of a peripheral cortex of dense lymphoid tissue enclosing a sinusoidal medulla. Von Recklinghausen (1871) first disputed the correctness of this and over the next thirty years better descriptions became available. Ispecially valuable were the works of Ranvier, Jolly, Flemming, Dominici, Gulland and Bunting. At the sume time deficiencies still attended the descriptions; species differences were commonly overlooked and generalisations persisted without criticism. There has been relatively little published which applies exclusively to human lywph nodes until comparatively recently. Hellman (1913) and Heudorfer (1921) published very good accounts and illustrations of human nodes but the texts are not very accessible. In English, Bremner and Weatherford (1948) also used human material but the section is brief. A further anomaly was justly criticised by Job (1922-3) 
who pointed out that the structure given in most text-books related to an ideal with little indication that all nodes were not the same.

The conventional description of a lymph node includes the following features. The node is invested by a capsule which is usually invaginated at one or more points to form a hilar intrusion; trabeculae spring from the inner aspect of this envelope. It is emphasized that with the possible exception of the Australian opossum the trabeculae do not characteristically segment the node. (In the Jutheria, the pig is often erroneously credited with the possession of septate nodes). In the hilar intrusion the major blood vessels arrive and leave and the efferent lymphatics accompany them. The multiple afferent lymphatics enter at any point of the capsule piercing it directly or after running a short distance obliquely. Under the capsule is the marginal or peripheral sinus. This is a sub-capsular lake from which the trajectorial sinuses which penetrate the node substance are given off. Subsequently these drain via the nedullary sinuses into the efferent channel. The lymphatic tissue is disposed as pulp and follicular tissue. Some authorites refer to the julp as medulla, an unfortunate convention since it is not anatomically the medulla, other terms are the parenchyma, or loose lymphatic tissue.

In the anatomical medulla follicular tissue is much less in evidence and the sinuses are separated by columns of loose lymphatic tissue which are described as the lymphatic cords. The sinus tissue is usually clear of lymphocytes, except in the trajectorial paths, it is lined and fenestrated by modified reticulum cells variously termed littoral cells 
or endothelial cells. The former term is supplanting the latter. Before describing the individual tissues listed above a general statement of node structure is appropriate.

Fundarnentally a lymph node is a circumscribed plasmodium of reticulurn confined and partly segmented by condensations of the fibrous elements of the surrounding areolar tissue. This locally condensed vascular connective tissue constitutes the capsule and trabeculae, the latter are generaliy very imperfect in man. The reticulum symplasma shews local attenuations and condensations and corresponding with these loci morphological modification of the reticulum cells is seen. An argyrophile filigree of reticulin is intimately reluted to this plasmodium and it too exhibits local alterations including focal deficiencies. In most of this basic framework ure inserted lymphocytes and their precursors, together with a variable but small content of other cells. This statement appears at first to have little relation to what is seen in an ordinary haematoxylin and ôsin stained paraffin section. None the less it is correct, but suitable material and suitable methods are required to rationalise the identity. The general reticulum of nodes is most convincingly demonstrated by the old device of pencilling. A young lymph node is obtained as fresh as possible and without prior fixation is sliced, and macerated in Ranvier's alcbol-en-tiers (33 per cent spirit), for 24-48 hours. Frozen sections are then cut in gum arabic. These are placed in the dilute spirit in a watch glass over a dark background and gently tapped with a moist soft camel's hair brush. As this is done a faintly opalescent fluid cones away with the medium, 
this is a suspension of the mechanically dislodged cells, chiefly lymphocytes. The finished preparation is mounted in glycerin. Perfect results are difficult to obtain, but in parts of most specimens the continuity of the reticulum of sinuses, follicles and pulp is well shewn. In the centres of the follicles the reticulum is extremely delicate and this portion is almost always damaged or lost. The argyrophile reticulin filigree is demonstrated by silver impregnation. The method of Witchell and fislocki, (1944), gives extremely good results but is expensive. The reticulin mesh is closer in the anatomical cortex of the node than in the medulla and this pattern persists in old age, (Denz, 1947). It is customary to counterstain these preparations with diffuse stains e.g. saffranin, neutral red, \& etc, but these are substituted with light haematoxylin with advantage. The mesh is deficient in the centres of follicles. It is to be noted that the walls of small blood vessels are also outlined by these silver methods and further it is nearly always possible to trace optical continuity of the reticulin with the collagen of trabeculae which stain various tints of brown. Hallory's phosphotungstic acid haematoxylin, Lieb's haematoxylin, van Gieson's stain, and picro-fuchsin will also display some of the reticulin mesh in most cases. These methods supplemented by serial sections prepared by orthodox histological methods enable a satisfactory understanding of lymph node structure to be gained. The Capsule and Trabeculae Nodes are invariably set in areolar tissue and it is usual to /encounter 
encounter anchoring strands of cornective tissue running between this adjacent areolar tissue and the capsule. The capsule is conveniently regarded as a condensation of the peri-nodal connective tissue and not as intrinsic to the node. This interpretation is prompted by consideration of the embryonic development. In human nodes the envelope is composed almost entirely of white fibrous tissue which fibrifies progressively as it matures but rarely is the process complete. It is generally four to ten cells thick but variationswith local thickening, extreme attenuation or even partial disappearance are seen, especially in old nodes. In its substance run blood vessels, lymphatics and less constantly nervous stmuctures. Small islets of adipose tissue and lymphoid tissue are also sometimes encountered. The smooth muscle of blood vessels and elastic fibres from the same source are the only contrioutions of these elements in human nodes. Nerve fibres are fairly common in node capsules but are not apparently distributed in the node itself, paccinian corpuscles and ganglia are found rarely. The trabeculae are derived from the hilar intrusion and from the general capsule. In form they are very irregularly fenestrated laminae. This can best be appreciated from the study of serial sections and from corrosion specimens. The basal area from which they spring is very irregular in extent.

The blood supply is distributed from the hilar intrusion and trabeculae. These septa act as scaffoldings for the vessels in their earlier course. In the medullary tissue of nodes vessels may be seen running almost free of support in the lumina of lymph sinuses, having 
left their perivascular connective tissue sleeves. Small branches leave these sterns and enter the node pulp. A complex pre-capillary and venule resh is formed by the terminal vessels. Alound follicles, either of resting or pale centre type, an anastomotic basket-work is common, and a central arteriole generally supplies the centres of follicles. The blood vascularity of most nodes is seen to be remarkably high if measures are taken to ensure its visibility. The simplest method is auto-injection. As soon after death as possible the cadaver is placed so that passive hypostasis engorges the nodes it is desired to study. Within a few hours, when the subject comes to necropsy the node vessels are often satisfactory filled with blood.

The sinus tissue

After traversing the capsule the afferent lymphatics open into the subcapsular sinus. (This term is more descriptive than the alternatives peripheral or marginal since these suggest it exists only in one place). This space is rarely so complete as conventional description indicate; in many old nodes the underlying lymphoid tissue is in direct contact with the inner aspect of the capsule. The subcapsular sinus is continuous with sinuses which surround the fibrous trabeculae and these following a centripetal course traverse the anatomical cortex. The actual path taken may be tortuous and this is commonly due to displacement by the follicular tissue. In addition to juxta-trabecular sinuses others make their way independently in sirnilar fashion. In the anatomical medulla the sinus system is usually more conspicuous because the lymphoid density is less. The efferent lymphatios effect junction 
with these medullary channels and often also with the subcapsular sinus, and leave in the hilar intrusion. The sinus systern is best seen in nodes related to the abdominal viscera especially the stonach, because in these glands the celiular structure is looser and this often is further enhanced by oedema, and physiological activity. The sinuses are lined by nodified reticulum cells (specialised endothelial cells, littoral cells), and traversed by very similar cells arranged in retiform fashion. It is eraphasized that these cells are but slight local modifications of reticulum cells. Downey (1922), in a very exhaustive paper demonstrated this beyond all doubt, and abrogated the mistaken view that they are true lymphatic endothelial cells. Liacrophages, lymphocytes, plasma cells, granulocytes, and erythrocytes are commonly present in small numbers.

Reticulin strands continue across the sinuses in addition to forming a fenestrated wall. In developing nodes and in old inactive ones these fibres frequently appear naked, and constitute most of the internal structure of the sinuses.

Studies of morphology alone have limitations but it is probable that sinus tissue has not the perranence which might be imagined, it is further likely that sinus tissue can develop locally in response to appropriate stimuli. Conway (1937) led evidence that the sites of follicles were not constant, and while this is not universally accepted e.g. Denz (1947), it is a reasonable belief and reinforces this idea. The Iymphoid tissue

This exists in two primary forms, the pulp or loose lymphatic /tissue, 
tissue, and the follicles. In adult human lymph nodes the former may be the sole expression, at least at the level studied.

The pulp is composed of the general reticulum in the interstices of which are inserted lymphocytes and other cells. This free cellular component exhibits considerable variation in its composition dependent on the activity of the node. In a quiescent or inactive node the cells are nearly all mature small lymphocytes; whereas in the reactive state their proportion falls due to the appearance of lymphocyte precursors, proliferating endothelial cells of lifarchand, plasna cells, and cells in mitosis, with occasional addition of granulocytes, giant-cells, \& etc.

This groundwork ly:lphoid tissue displays peculiar focal aggregates of cells at intervals in its existence. These are terned lymphoid follicles, or less accurately lymph follicles. Unfortunately the different varieties have a confused nomenclature, and for so small structures they have roused much controversy. Two varieties are well recognised and can be demonstrated easily in human material. The first is the solid or resting follicle. These consist of closely packed small Iymphocytes which form aggregates of elastic size. They are by far the commoner formation visible in human lymphoid tissue, being present throughout life, even long before birth. The conglomeration of small lymphocytes decreases in density peripherally and merges insensibly with the loose lymphatic tissue. The outline of these formations is conventionally circular in section but ovoid or bilobed configuration is common. Careful scrutiny reveals minute blood vessels and scanty reticulum cell nuclei in the lymphocyte mass. They are not traversed by 
sinus tissue as a rule. Witotic figures and pyknotic nuclei can generally be identified if sought. In comnon with most cells in mitosis their identity is usually a matter for speculation. The pyknotic fragments often present a bi-valve appearance which is peculiar to lymphocytes. In a silver impregnation deficiency in the reticulin fibres is generally present centrally, and the surrounding mesh is typically ragged in demarcation. These follicles are also termed secondary nodules because at one time the phrase primary nodule was used to indicate the segmented division of the lymphoid tissue produced by the capsular trabeculae. In recent works they are often termed the solid secondary follicles of Groll and Krampf.

The second conspicuous follicle is one in which there is a circumscribed spheroidal centre of large leptochromatic nuclei with symplasmic cytoplasm. The relative pallor of this formation in stained sections has led to it being termed a pale centre. This name, sanctioned by usage is not very imaginative, but carries less implication than others. The demarcation of this pale centre varies from remarkably sharp to indistinct. It is surrounded by a marginal zone of small lymphocytes which may encircle it uniformly or with increased density locally. In the latter event the maximum density is typically cap-shaped and orientated towards the capsule. These follicles have a long and interesting history in microscopical anatomy.

They were first described in detail by Flemrning in 1885. He characterised them as possessing 'light centres with a dark shell in which the reticulum is often arranged concentrically'. He termed thern /secondary 
secondary nodules in a morphological sense and physiologically 'germinal centres' or places of origin of lymphocytes. He pointed out that locally the pale centre was a site where mitotic figures were more numerous than elsewhere; that the displacement of the previously evenly distributed reticulum indicated an outward growth of cells from the centre and surmised that the daughter cells were driven out by a slow centrifugal pressure through the spaces of the reticulum. In addition he drew attention to minute stainable bodies of uncertain nature in between or in the cells of the pale centre. For some obscure reason nearly everyone prefers to call these by their German name viz. 'tingible korper'. In this country Bunting (1904, 1905), in his excellent account of the histology of lymph nodes added further details. He recorded that germinal centres were very rare or absent in the new born, and sometimes absent in adults. He noted their relative profusion at the periphery of nodes and paucity in the deeper parts. He observed too, that in active nodes the capsule might be mammilated by florid growths of nodules of this variety. In pencilled sections he observed that the germinal centres tended to drop out and adduced evidence of an entirely protoplasmic reticulum in the pale centre. Among the cells of the pale centres he recognised two main varieties. One had a large oval pale staining vesicular nucleus, and the other was smaller and darker. Occasional phagocytic cells, 'angular reticulum cells', were also present and he emphasized the very dubious cytoplasmic demarcation of all cells in the centre. He supported Flemming's contention that the centres were lymphocytopoietic by primitive micro-dissection methods. Thile this view is probably still the rost widely supported by authoritative 
opinion today, the sceptics began to question it soon. Ihrich (1929) recorded that the first healthy doubt was that of Warchand in 1913, and in that year and the following, Hellman refuted their lymphopoietic properties with some very convincing facts. This last writer, who has considerable claim for attention, observed that the marginal zone of small lymphocytes did not appear to augment or decrease in regular fashion with coincident activity of the pale centre; that there was no definite relationship between the number of mitoses and the size of the marginal zone, and that convincing transition form between the centre cells and small lymphocytes simply did not exist.

Further to these he added the fact that typical germinal centres did not appear in the young until several months after birth. (In a later publication he modified this after finding that they sometimes arose in cases of foetal infection). His final reason was and is overwhelmingly convincing to a norbid anatomist, namely pale centres are not found in lymphatic leukaemia. In his later work Hellman, with Heidelberg and others introduced the idea that the pale centres were 'reaction centres' related to irritation.

Latta (1921) studying the development of the intestinal tonsils in lepus discovered that the first free cells were mainly small lymphocytes which arose by differentiation of fixed mesenchymal cells, and in a well balanced argument decided that the so-called germinal centre was not a centre of proliferation of lymphocytes. He was among the first to submit that the 'tingible korper' of Flemning were nuclear remnants of degenerating lymphocytes. In the same year Nakahara and Iurphy (1921) 
attempted to solve tie problein by ingenious experimental methods. They found that after a brief exposure to dry heat there followed a sharp initial fall of circulating lymphocytes which was closely followed by a rise, often up to $200 / 300$ per cent above the level in the intact animal. Study of the lymph nodes and spleen at this point revealed numerous dead cells in all parts of the lymphoid tissue except the pale centres. By 48 hours numerous mitoses appeared in the latter and this activity persisted. They also found that if mice were injected with an ernulsion of homologous living tissue ten days before infoculation with a cancer graft that they were rendered relatively resistent thereby and shewed a fall in the number of takes. This immunity was associated with a distinct lymphocyte rise in the blood and the level often rose again when the tumour inoculum was introduced. Parallel with these phenomena two related phases of mitotic activity in the pale centres were demonstrated. In further experiments using very small doses of $x$-rays similar changes were produced. They concluded that these findings were evidence of lymphocytopoietic function of the pale centres; it is convincing up to a point, but the crucial question of how the pale centre mitoses account for the lymphocyte rise was not met.

Jolly (1922) accepted Flemming's hypothesis without demur, and added some interesting matter. After a formal description of the chief cells of the pale centre he stated 'beside these clear nuclei are found scanty small lymphocytes and cells which are larger with basophile and scanty protoplasn which resemble the germinative lymphoid cells of the embryo and the myeloblasts of the bone marrow. They are of the same nature and 
here are called lymphoilasts or leucoblasts'. A foot-note explains that 'these are the same cells as celis called large lymphocytes in many recent works - this just leads to confusion'. (This reference is probably to liaximow). He proceded - 'Some authors have advanced the view that the mitoses of the clear centres belong only to the conjunctive framework' (Ribbert in Ziegler's Bietrdgge, VI.V889), but Jolly insisted that mitoses could be seen in manifestly lymphoid cells.

A reconciliation between germinal centre and reaction centre is accepted by some authorities, indeed this is endorsed by Aschoff. West (1924) believed that the pale centres were lymphocytopoietic and at the same time centres of cell destruction.

Maximow's work is very well known and need not be repeated. A frequently quoted suminary is available in Cowdray's text-book of Special Cytology (1932). Allowing for the nomenclature anomalies the relation of small lymphocytes to certain cells of the pale centres is parallel to that given by Jolly.

This work carried out over many years has met with fairly general approval. It is however worthy of mention that cat and rabbit material were used in preference to human in which haximow himself agreed it was difficult to identify the cells named. One interesting finding of Maximow is that the centre can be traced through a cycle of different recurring phases.

Two further authorities deserve mention. Bhrich (1929a,b) clearly supported Heliman's idea. In addition he described a third variety of lymphoid nodule as well as recognising and characterising transition 
stages between the solid and pale centre types. This new formation he called a pseudo-secondary nodule and he outlined a tentative schema indicating the relations between the different types:-

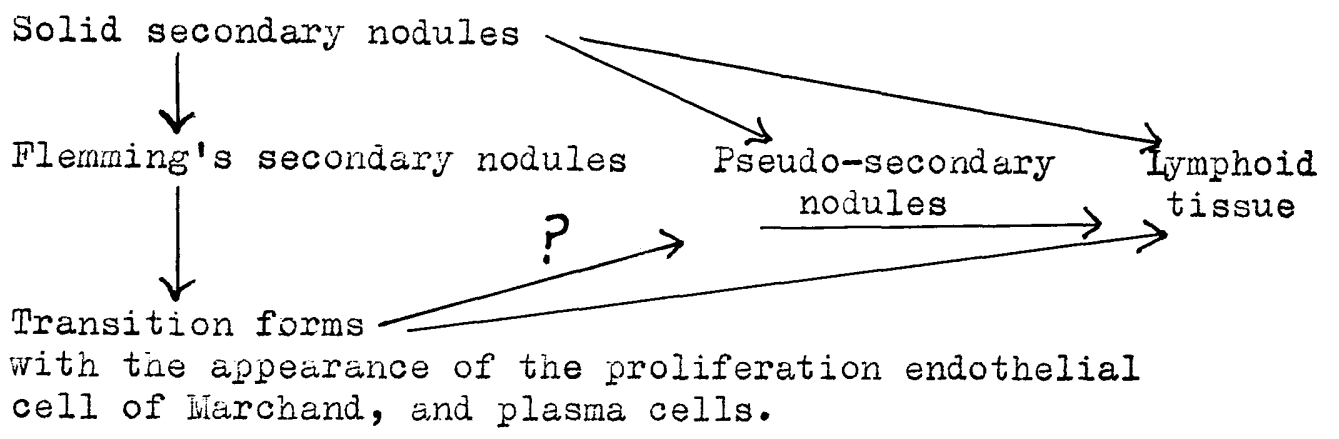

The pseudo-secondary nodule is a large formation, of the order of $3 \mathrm{~mm}$. diameter, with indistinct outlines and best appreciated with very low-power examination.

The importance of the proliferating endothelial cells of Marchand in lymphocytopoiesis was stressed. These cells are well characterised and their recognition is easy. Ehrich is probably correct regarding their nature viz. precursors of lymphocytes, but I have not been able to convince myself of the entrance of lymphocytes into the peculiar veins which he described. Drinker and Yoffef (1941) in their highly critical study incline towards the orthodox view but readily admit the inconsistencies inherent in it.

The foregoing summary is necessarily eclectic, the subject has an enormous literature. In the main my studies have been limited to morphology and I shall not atternt to survey the immunological and irradiation methods which have been employed to solve the problem beyond stating that I have not encountered any convincing proof that the pale /centres 
centres in man are producers of lymphocytes. Involutionary changes in human lymph nodes.

In the practical study of adult human lymph nodes it is remarkably common to find evidence of atrophy. Cowdray (1942) has drawn attention to the fact that lymphoid tissue shews a disproportionate rate of ageing.

It is established that in man lymph nodes reach their most florid development in late cinildhood or in early adolescence. Phereafter they are prone to undergo atrophy. This phenomenon is seen earlier and more consistently in the peripheral nodes. A detailed study of the problem, covering sone 300 nodes from the axillae and nesenteries has been perforned by we for another purpose and here only a brief account of the findings will be given.

There are two common expressions of atrophy in lymph nodes. The first which is most characteristically seen in para-enteronic nodes is a progressive depletion of lymphocytes. This process is apparently the result of a failure of supply, and it progresses slowly, rarely is the de-population anything like complete. Simultaneous with the fall of lymphocytes the proportion of reticulum cell nuclei appears to rise and often sinus tissue is thrown into prominence. Follicles with pale centres are rarely seen in this form of atrophy and often secondary follicles are absent. In most case there is also regression of the connective tissue of the node and the trabeculae become fragmented or tenuous.

The other form which is well seen in axillary nodes is best described as fat replacement. Fat cells replace tine lymphoid tissue in 
two main ways. They may either appear as a diffuse irregular sprinkling of individual fat cells, or by the development of a conical process of adipose tissue which originates near or in the hilar cleft and excavates the node. This fat cone may be formed by local coalescence of fat cells of the first type but is usually a separate formation. In many cases both mechanisns are visible, the individual fat cells lying ahead of the fat cone. The lyuphoid tissue recedes absolutely passively in front of the advancing fat. Sinus tissue appeurs slightly more resistant and these structures are commonly out-flanked till the reticulin gives way and then they disappear. Iventually the capsule is reached, this membrane persists till all the lymphoid tissue and sinus tissue have disappeared, it then also succumbs. In general the node does not tend to gastrulate or collapse, the capsule retains its position till it is extinguished piecemeal. On the other hand the lymphoid tissue falls back onto capsule while it persists, thus conforming to the general shrinkage pattern described by Thompson (1942). Ultirnately the node may disappear almost entirely except for a tiny residual erescent of surviving lymphoid tissue and capsule. There is evidence that the fat is a new product. It is paler than the adult yellow fat in the perinodal areolar tissue and often the cells are larger and more uniform in size, recalling the tissue of lipoma. In my studies I am led to the conclusion that the nutrition of the subject bears a close relation to the degree of tine phenomenon and its recurrence. It has probably more influence than age alone. It occurs earlier and much more consistently in the obese, especially in women. The process can be almost complete 
and yet the locus is still a potential lymph node. It is not uncomron to find carcinoma metastases in the relics.

Regeneration is commonly observed in response to appropriate stimuli. This is well shewn in the axillary nodes of obese middle aged women who develop mammary carcinoma. This repopulation is simply a reversal of the atrophic process and was admirably described by ReubensDuval and Fage (1909).

Neither of these atrophic processes is inevitable. Even in elderly people, over 80 years of age, remarkably intact lymph nodes can sometimes be found. Follicles with pale centres may even be present.

Recognition of this atrophic process is important in the present work because one very remarkable and consistent finding is that it is very rare indeed in lymph nodes which are the seat of primary tumour. Whach less comronly atrophy with fibrosis is encountered. In cervical, axillary, and mediastinal nodes this is definitely rare, but minor degrees are more often seen in mesenteric, retroperitoneal, and groin nodes. This fibrosis is vascular in origin and is primarily a hyalinisation of blood vessels. Only rarely is it extreme. In groin nodes particularly, augmentation of the hilar intrusion connective tissue contributes. It is emphasized that this fibrosis does not arise from the general reticulum.

Fibrosis arising in the reticulum is common, but fortunately the unimportant forms have usually an obvious cause. It is very common in nodes invaded by carcinoma metastases and in scrofula, both conditions which are senerally easy to recognise. Despite statements to the 
contrary, fibrosis in sinus tissue or general node reticulum due to prolonged sinus catarrh or chronio non-specific inflammation is extremely rare. As a practical corollary the finding of reticulum fibrosis without obvious cause must be viewed with the greatest concern. 


\section{THE DEVELOPMONT OF LYMPH NODES}

Lymph nodes do not appear until the lymphatic vascular system is tolerably complete. They are first observed in human embryos at or after the $40 \mathrm{~mm}$. stage (10 weeks), Hellman (1931); Le Gros Clark (1945); Bremner and Weatherford (1948). The process of their development in man receives little attention in many standard works on embryology; Frazer (1931), does not offer information and Arey (1941) gives only a formal synoptic account. Gulland, (1894), studied the problem in considerable detail but his naterial included sheep, guinea pig, and rabbit tissues in addition to human specimens. Briefly the mode of origin which he traced was as follows. The first indication of node formation was the development of a freely anastomosing plexus of lymphatic at the locus. (It is interesting to note that in Gulland's drawings of this stage, the nuclei of the lymphatic endothelium are identical with the connective tissue (mesenchyme) nuclei in the vicinity). The anlage of the node consisted of an ill-defined islet of condensed connective tissue surrounded by these lymph channels. 'Leucocytes' then appeared between the condensed connective tissue cells; these were not described but it appears likely that lymphocytes were meant, since their origin was ascribed to the thymus. While this mode of population is doubtful in the light of more recent work, it was a convenient and safe shelving of the problem since the thymus appears first in the $8 \mathrm{~mm}$. stage ( 5 weeks). In a human foetus of 3 inches ( $75 \mathrm{~mm}$, about $3 \frac{1}{2}$ months) the nodes still retained a primitive appearance, there were not any hila, the marginal sinus was not highly organised, and deep sinuses could not be recognised. Noteworthy changes at this stage included increased cellularity of the 
node and the appearances approximated to the structure of a lymphoid follicle. Further illustrations were given by reference to preparations from foetuses of $90 \mathrm{~mm}$. and $115 \mathrm{~mm}$. In the former trabeculae were seen in the process of formation. The smaller trabeculae arise from the slips of intervening general connective tissue between the individual lymphatics forming the marginal sinus; the larger were attributed to the folding of the capsule due to unequal growth. In the latter specimen the new definitive blood supply, an arteriole, was depicted entering in the substance of a large trabecula. The node capsule, and this deserves special emphasis, was merely a condensation of the surrounding connective tissue. This important fact aids materially in the proper appreciation of capsular changes in morbid lymph nodes. The capsule is a pressure condensation product of the environmental connective tissue and not an integral part of the node. The development of the deep sinuses of lymph nodes is only briefly treated in most texts. Downey/(1922), in a most comprehensive paper, described their origin in the lymph nodes of the pig. The first developments were spaces in the general reticulum of the node, as these increased in number and size the lining reticulum cells took on endothelial characters. The original non-endothelial character is very well indicated in Downey's drawing No.6 where comparison with two normal blood vessels in the field emphasizes the point. Connection of these developing deep sinuses with the lymph channels of the plexus in which the node is developing is secondary. A corollary of much importance derives from this interpretation, namely lymphoid tissue has vaso- 
formative ability. Loschowitz, (1950), has arrived at the same conclusion by further morphology studies in relation to chronic inflammation.

In my own studies I have relied on human material collected personally from post-mortem and surgical specimens. These sources have provided embryos of $22 \mathrm{mrn}, 46 \mathrm{~mm}, 65 \mathrm{~mm}, 78 \mathrm{~mm}$, and foetuses of $94 \mathrm{~mm}$, and $210 \mathrm{~mm}$. In addition specimens were obtained from still-births and infants dying in the neonatal period, some being premature. The youngest specimen (22 mm. embryo) was poorly preserved, but the remainder were moderately good. So far as the limited material permits the sequence of development follows Gulland's thesis reasonably closely.

In the most mature specimens in the group the appearances shew little mutual difierence. Between the neonatal period and the end of the first year is a span I have been unable to study adequately, due to paucity of material. The main interest in this phase concerns the appearance of germinal centres. The general consensus of experience indicates that these are very rare before six months of age. Fith the exception of isolated examples, the childhood and adolescent epochs were also not represented in the series collected.

It will be appreciated that this mode of formation of the lymph node is unduly formalised. Then serial sections are studied it is at once obvious that the appearance of a ring of contiguous or reasonably adjacent lymphatic channels is only manifest at certain levels of the node anlage. It is indeed doubtful whether this 'ring' is essential. It would appear that when the process is resolved to its simplest generalisation, the fundamental fact is that lymphoid tissue develops at 
loci immediately adjacent to lymphatics. Acceptance of this principle carries inplications which will be exploited later but I believe it to be correct for the following reasons. In embryonic material lymph node development is seen without the orthodox plexus formation; it tends to occur in the axil of a lymphatic vessel dichotomy or where nearby blood vessels, capillaries as a rule, form an analogous enclosure with a lymphatic channel. This mode of origin was depicted by Klein in Quain's Anatomy, (1912 Edn.) The development of most of the paraenteronic lymphoid tissue is evolved without any afferent channels assisting the process, and in adult life it is quite common to find lymphoid tissue developed in the immediate vicinity of lymph nodes, either in or just outwith the capsule. This phenomenon is common in nodes which are functionally active, hyperplastic, or undergoing reactive regenerative change. Recognition of this process is afforded little attention, Jolly (1922) is among the few who appreciate it.

It is not certainly known when lymph node production ceases. Gulland surmisea that there were three orders of lymph nodes which developed successively, and invoked the last generation to account for apparent new formation of nodes in adult life. Jolly (1923) expressed a similar conclusion. Neo-formation of lymph nodes is not generally accorded much recognition but has been reported by Bayer (1885) and MacCallum (1928). The former writer maintained that nodes could arise de novo in adipose tissue, but as MacCallum pointed out this is unlikely. MacCallum suggested that they might develop in relation to pre-existing lymphatics. It will be appreciated that this is a difficult subject to /investigate 
investigate but in personal observations reasonably convincing evidence for HacCallum's theory has been found.

Liorphological studies indicate that the first development is a focal lymphocytic infiltration which forms a circumscribed nodule. The cells at this early stage are almost all small lymphocytes. Reticulum cell nuclei then appear in this aggregate and pale centre tissue is subsequentiy developed. The size of the aggregate appears to determine these progressive changes. The site is usually very close to blood and lymphatic vessels and the latter becomes gradually more intimately related to the lymphoid mass and ultimately forms the marginal sinus. The propriety of regarding this new formation as a lymph node depends on the fact that carcinoma metastases may occur in it. (In the illustrations of this present work there is a record of these findings). An indirect method of supporting the fact of neogenesis is to enumerate the nodes in presumed normals and compare them with cases where they are increased. This I have been able to perform only once and while in this case an apparent two-fold increase was demonstrated, the method carries less conviction. 


\section{THE NUMBERS OF THE LYIPH NODES}

Estimates of the total numbers in the body have already been indicated. The establishment of this figure is a very formidable task, at the same time it would be extremely interesting to know it. The significance which attaches to this enumeration is considerable, it might lead not only to a far better appreciation of the biological import of lymphoid tissue throughout life but might also furnish a means of determining whether neo-genesis of lymph nodes occurs.

While it has been impracticable to attempt this full scale enumeration, it was considered that even on a restricted regional basis some absolute figure might be useful. This section records an attempt to establish the number of lymph nodes recoverable from the human axilla throughout life. The dissections on which the findings are based are tedious performances and the total number is small, however they give some indication of the probable ranges to be found.

The axilla was chosen as suitable for the purpose. This region has the advantages of being readily accessible to ordinary post-mortem dissection where mutilation must be avoided, and of being tolerably circumscribed anatomically. In addition the nodes are less superficial and exposed to trauna than the inguinal groups. The left side was selected empirically. Identification of the Axillary Iymph nodes

Johnston (Gray's Anatomy (1935)) gives the following information. The nodes are relatively large and vary in number from twenty to thirty. They may be divided into five groups which are not sharply demarcated 
from each other.

Schema, after Gray

I. A lateral group of from four to six nodes lies medial to and behind the axillary vein.

II. An anterior or pectoral group of four or five nodes lies along tine lower borier of the $m$. pectoralis minor in relation with the lateral tioracic vessels.

III. A posterior or subcapsular group of six or seven is placed along the lover margin of the posterior wall of the axilla in the course of the subcapsular vessels.

IV. A central group of three or four large nodes is imbedded in the fat near the jase of the axilla.

V. An apical group of six to twelve nodes is situated partly posterior to the upper portion of the m. pectoralis major and partly above the upper border of this muscle and extends upwards into the apex of the axilla along the medial side of the axillary vein.

This schema is not easy to follow in the actual process of dissection. In the practical study the text of Taylor and Nathanson (1942) proved more valuable. These writers have considered the recovery of lymph nodes as a practical surgical problem and two features of their anatomical studies deserve particular mention. The first is the use of veins as indicators of lymph node locations. The veins are analogous to the axis of advance used by a division in battle, the nodes being related paravenously. Laintaining the military simile, just as 
the component brigrades and supports may be disposed in line, column or echelon, in similar fashion the nodes along tributary veins are conveniently described. The second advantage is a simplification of nomenclature which is really an outcome of the first idea. The subgroups are named according to the vein to which they are immediately related.

Schema, after Taylor and Nathanson (1942)

1. The axillary vein group, a variable but considerable group, they extend along the vein from the outer border of the m. pectoralis major to the apex.

2. The subcapsular vein group, a few lymph nodes lying on the axillary surface of the vein, the teres and $m$. latissimus dorsi; they are usually related to the subcapsular nerve as well.

3. The lateral thoracic vein group, a chain of nodes lying approximately in the mid-axillary line in close relation to the digitations of $\mathrm{m}$. serratus anticus, often between the bellies from about the fifth rib up to the apex. They are related to the lateral thoracic nerve of Bell.

4. The thoracic-acromial vein group, the highest group in the axilla, essentially coinciding with the axillary vein group. They coincide with the entrance of the cephalic vein into the axillary vein.

In Gray's system the numbers given yield a minimum of 23 and a maximum of 34. Taylor and Nathanson do not submit figures. It will be seen that the two systems are almost synoptic the groups corresponding 
as follows.

Gray

Part of IV + I

II

Part of IV + III

IV

V corresponds to

"

11

"

11

\section{Taylor \& Nathanson}

1

3

2

4

The contents of the left axilla were recovered from twenty routine necropsies. The patients' ages varied from I day to 81 years, eleven were females and nine were males. All cases were free of primary lymph node disease and did not suffer from cancer or scrofula. The methods adopted were simple. The axilla was rendered accessible with the widest reflection permissible and the arm extended at right angles to the body. The entire axillary fat was then disseoted out. In the final clearing the m. pectoralis minor was reflected and a segment of the axillary vein removed. The larger nodes were readily appreciable with the bare fingers and easily picked out. This form of blunt dieseotion proved the best particularly where advanced degrees of fat replacement obtained. In these cases the slightly enhanced tenseness of a node and its just appreciable relative pallor proved valuable. Smaller nodes were also recoverable by palpation. The smallest nodes presented more difficulty. In cases where the fascia was not heavily fat laden stretching it often brought thern into view. ihere much fat was present they were best identified by compressing the teased specimen between two sheets of thick perspex and examining it against a strong light. Many of these nodes are very small, of the order, $1.0 \mathrm{~mm}$ diameter. Finally 
histological confirmation was obtained by making a single composite block of pieces from the presumed nodes recovered. Results

\begin{tabular}{|c|c|c|c|}
\hline No. of Case & Sex & Age & $\begin{array}{c}\text { Lymph Nodes recovered } \\
\text { by dissection }\end{array}$ \\
\hline 1. & $F$ & I day & 11 \\
\hline 2. & $\mathbf{M}$ & 6 days & 10 \\
\hline 3. & $\mathbb{M}$ & 30 days & 23 \\
\hline 4. & $M$ & 2 years & 31 \\
\hline 5. & $\mathbb{M}$ & 5 years & 32 \\
\hline 6. & $F$ & 13 years & 31 \\
\hline 7. & $\mathbb{M}$ & 18 years & 30 \\
\hline 8. & $F$ & 22 years & 26 \\
\hline 9. & M & 29 years & 29 \\
\hline 10. & $F$ & 43 years & 21 \\
\hline 11. & $F$ & 47 years & 24 \\
\hline 12. & $M$ & 51 years & 20 \\
\hline 13. & $F$ & 56 years & 23 \\
\hline 14. & $F$ & 61 years & 12 \\
\hline 15. & M & 65 years & 15 \\
\hline 16. & $\mathbf{F}$ & 66 years & 5 \\
\hline 17. & $F$ & 69 years & 9 \\
\hline 18 & $F$ & 70 years & 11 \\
\hline 19. & $\mathbb{M}$ & 72 years & 8 \\
\hline 20. & $F$ & 81 years & 12 \\
\hline
\end{tabular}


Summary of Findings

The series recorded extend over the life span but the representation of successive periods is not uniform. This is primarily due to lack of suitable material, specimens from the first three decades were difficult to obtain.

In so far as the findings permit analysis the following observations are offered. At jirth and in the early neonatal period the total number of nodes is in the region of 12 . This low figure is attributable chiefly to the smallness of the structures, even at one month their size is zuch larger making their identification easier. Throughout childinood till the end of the third decade figures near the classical meximu are found. Thereafter there appears to be a steady slow decline. The figures are too small to assess sexual differences; it is my impression however that in the elderly, women have fewer nodes and this appears related to obesity which is commoner. Adiposity is apparentis comducive to fat atrophy of lymph nodes $\left(q \cdot v_{\bullet}\right)$ besides which it renders surviving nodes very inconspicuous. Perfect illustration of the results would require photographs of all the nodes from each case, this was too expensive and only half the cases have been so pictured. 


\section{PART III}

\section{HODGKIN'S DISEASE}

\section{HISTORICAL}

Biographical note on Thomas Hodgkin, M.D.

The Contribution of Thomas Hodgkin.

Confirmation of the identity of the disease.

Other claims of priority.

Nomenclature of the disease.

The subsequent history of the disease. p. $118-121$

p. $122-132$

p. $133-139$

p. $140-142$

p. $143-144$

p. $145-158$ 
PATHFOLOCGI

Tte of Hodgin's disease.

p. $158-174$

Thelmoiffication of Hodgkin's disease.

p. $174-178$

Thote om Hodifkim"s sarcoma.

p. $178-180$

Cemenarl morbid anatom.

p. $181-184$

Distifribution of Iesions.

p. 185-193

/ over. 
The necropsy series.

Protocols, Volume II

Biopsy series, Appendix A. p. 1-222

Necropsy series, Appendix B. p. 223-267

\section{Illustrations.}

Volume III. Figs. 45-116

Volume IV. p. 20-57. 


\section{BIOGRAPHICAL NOTE ON THOMAS HODGKIN, M.D. (EDIN.)}

Thomas Hodgkin was born at Pentonville, London, on August $17 \mathrm{th}$, 1798. His father John Hodgkin, to whom he was much attached, was a member of the Society of Friends and a paedogogue by profession. Thomas, who was the third of his four sons, was a premature child and was never robust in health. He was educated at home by his father who was no mean scholar, and acquired an enviable knowledge of Latin and Greek. Concerning his medical training little is recorded. Part of his course was performed at Guy's Hospital, but he also studied abroad, in Paris. He graduated H.D. from Edinburgh in 1823, a matter for delectation. His thesis was entitled 'De absorbendi functione' and he received special commendation for his Latin - a graceful recognition for an Englishman - from a Scottish University. In 1825 he joined the staff of Guy's Hospital, undertaking the offices of Curator of the lifuseum and Lecturer on Morbid Anatomy. These he performed with distinction for a period of twelve years, at which point a senior post of physician beome vacant at. that institution. Rather unexpectedly he was passed over and one of his colleagues, Dr. Babbington, obtained the situation. This circumstance was a considerable disappointment to him and he left the hospital. Some years later, in 1842, he was invited to undertake the task of reorganising the medical School of St. Thomas's Hospital in which he was appointed Lecturer on the Practice of Physic. Significantly enough, this hospital had previously been linked with Guy's - in fact Hodgkin's first appointment there was a consequence of this disruption. A lesser man might readily have exploited this state of affairs to cause. 
an unpleasant rivalry but Hodgkin was not so minded.

On the formation of the University of London he was elected to the Senatus Academicus and proved a proficient and wise counsellor.

As a man Hodgkin was a Christian gentleman and one of the Salt of the Earth. Like his father he was a sincere member of the Society of Friends and adopted their dress and opinions throughout his life. He was one of those precious individuals who cannot remain apathetic observers of injustice and inhumanity: and it seems that much of his spare time was spent in advocating and supporting reforms. His work in this direction was essentially philanthropic in character and not political as some critics have suggested. Indeed, it is interesting in these days to note that he foresaw and warned against the tyrannies unprincipled trade unionism would exert on individual liberty. Altruistic principles seldom exist in those with worldly wisdom and Hodgkin had only the former.

His nedical works include his Catalogue of the Guy's Hospital luseum, (1829); his paper entitled, 'On some morbid appearances of the absorbent glands and spleen', (1832); 'Lectures on the means of promoting and preserving the health', (1835); and his Lectures on the Morbid Anatomy of the Serous and Irucous Membranes, (1836-40). More is related concerning the first two entries elsewhere. His lectures on the health are dignified and sound. They must represent fuller expositions than the original verbal comrnunications made at the Mechanics' Institute, since the book which contains three lectures with appended notes, takes several days to read! The last publication 
unhappily was never finished; it was used as a standard text for some years and is superior to contemporary British works. It is really a general morbid anatomy text book because the subjects of the title are amplified considerably by appended observations and notes. It is not easy to read, but I was brought up to believe that anything worth while was invariably hard. The English translation of Rokitansky's Morbid Anatomy (1846) is far worse. His writings have been criticised as uninspiring and too diffuse, Hale-White (1944). The former charge is possibly true where the reader is a pharmacologist, but they do not leave a morbid anatomist unmoved. The second objection may be tenable in the sense that they are not written in the stilted and abbreviated scientific jargon of today. Theirs is the diffuseness of scholarship and there is in them a refreshing clarity of expression. The strong discipline of the Humanities of Hodgkin's early schooling is reflected in them. This renders them a trifle pedantic perhaps - even unconscious humorous at times, but I for one am delighted to meet such old friends as the English equivalents of ut, quin, quominus and causa!

Particulars of Hodgkin's private life are anecdotal, one fragment which delights me personally is that at one point he owned a velocipede - a modern equivalent of which vehicle I myself possess. It is recorded that he was wont to travel from Lewes to Brighton on this machine.

He married comparatively late in his life, in 1850. There were no children of this union. He died from an attack of dysentery in /Jaffa, 
Jaffa, Palestine, in April 1866 while accompanying his friend Sir Moses Liontefiori on an expedition to relieve the plight of destitute Jews. This gentleman erected an obelisk over his burial place - 'Humani nihil a se alienum putabat'. 
THE CONTRIBUTION OF THOMAS HODGKIN

It is almost certain that priority must be conceded to Hodgkin for the initial characterisation of the disease which now bears his name.

In the introductory apology for his original observations he modestly disclaimed this honour. Indeed he suggested that the morbid alterations he was about to describe were already recognised and as likely to have been succinctly summarized. This circunstance has prompted several writers to sponsor the claims of previous observers, and certain of these will be considered below.

At the time of his now famous publication he was liorbid Anatomist at Guy's Hospital. This paper was read before the Medico-Chirurgical Society of Iondon in two parts; the first on 10th January 1832, and the second a fortnight later. Hodgkin himself was not a member of this society and the communication was made verbally by his colleague Dr. Lee who was the Secretary. Despite certain limitations, especially the absence of immediate microscopical study this is a work of conspicuous merit. It has pleased several to emphasize its deficiencies and even to argue that Hodgkin is lucky to have been remembered at all. The blunt statement that Hodgkin 'undoubtedly had no conception that in one, or possibly two of his cases he was dealing with a peculiar and rare disease', Reed, (1902), and the patronizing account of Hale-White, (1924), are both unjust.

The study in question is based on the findings in six personally observed cases and a seventh reported from St. Louis Hospital, Paris, by H. Iugol. (In addition a further series of seven cases not relating to /this 
this particular entity are recorded in the second half of the paper.) The identities of the cases, after Hodgkin.

I. Joseph Sinnott, a child about 9 years old, in Lazarus' ward under Mr. J. Morgan. Dated 2nd November, 1826.

II. Ellenborough King, aged 10 years, in Luke's ward under Dr. Richard Bright. Dated 24th September, 1828.

III. William Burrows, aged about 30 years, in Naaman's ward under Jir. J. Morgan. Dated 28th November, 1829.

IV. Thomas Westcott, aged apparently about 50 years, in Clinical ward under Dr. Addison. Dated 8th January, 1830.

V. 'A middle aged man who had latterly been a patient of Dr. Back' previously under Dr. Bright. Not dated.

VI. Thomas Black aged about 50 years, in Barnabas' ward . Under Dr. Bright. Not dated.

VII. (Reported case 'of a man who died in the St. Louis Hospital, Paris in April' (?1831).

In the interests of brevity, ease of comparison, and ready recognition of the salient facts it is expedient to present these findings in a more formal manner than the original. The relevance of several of the cases has been seriously questioned on many occasions. The exercise of critical study is reasonable, the responsibility of rejection resting with the individual. Accordingly an eclectic series will be given which represent the examples which I believe are true cases of the disease. 
Case I. Joseph Sinnott

History. He had been ill for about nine months, complaining of pain in the back which extended round to the abdomen. His brother, a bed-fellown died of phtrisis a few months previously. On admission he was found to have ascites and oederna of the prepuce and scrotum. (There is no account of the patient's death).

Sectio Cadaveris

Head. The arachnoid mater contained a few 'opake' spots and there was serous effusion under it. The pia was very thin and pallid. The Brain was soft and flabby; no local morbid change was present in it.

Chest. The Heart was natural.

Pleurae Rt. Numerous old and tough adhesions were found. There was evidence of recent pleurisy in addition. Lt. A very few adhesions were noted.

Iungs. In tire - ht organ there was a doubtful tuberculous cicatrix at the apex. 'but he substance of both lungs was generally light and crepitant with a very few exceedingly small tubercles scattered through them' Lucosa of bronchi, engorged. The bronchial glands vere greatly enlarged and indurated.

ibdomen. Peritoneum. A sero-purulent effusion was present. 'The viscera were universally overlaid with a very soft light yellow coagulum, too feeble to affect their union though evidently having a tendency to do so'

The mucosa of the stomach was very pale.

The contents of tine intestine were uninealthy.

The hesenteric glands were enlarged, several reaching the size of a pigeon's egg. They resented semi-cartilaginous hardness and were streaked with black natter.

The Liver was normal on the whole but dissection revealed a few tubercles somewhat larger thar peas. These were white, semi-cartilasinous and of uneven surface.

The Pandreas was firner than normal.

* The Spleen was large, and contained many tubercles.

The Kidneys, both were mottled with a light colour but free from induration.

Para-cortic Lymph nodes (he adds) 'A continuous chain of much enlarged indurated absorbent glands of a light colour accompanied the aorta throughout its course, they were closely adherent to the bodies of the vertebrae and extended along the iliac arteries too'.

Thoracic duct. Large: the coats were transparent and nornal. 
Case II. Illenborough King

History. Namitted 6th kusust 1828. Patient was the youngest of six children, the others being alive and well. He was fit till about thirteen months ago when his strength, flesh, and healthy appearance began to fail. it tumour was noted in the left hypochondrium, corresponding to the spleen. The glandulae concatenatae on the right side were also enlarged, they fluctuated somewhat in size, this was attributed to treatment while in hospital. His appetite was good and he had not any haemorrhagic tendency. He became dropsical before death.

Sectio Cadaveris

Head. Not opened.

Neck. The glands in the neck had assumed the form of large smooth ovoid masses connected merely by loose cellular membrane and minute vessels: when cut into they exhibited a firm cartilaginous structure of a light colour and very feeble vascularity; but with no appearance of softeniner or suppuration'.

Chest. The pericardium contained more fluid than nornal, the sac itself Was not diseased. The Heart was normal. Pleurae. Old adhesions were noted.

The Iungs were generally healthy.

The bronciial and nediastinal glands presented the same enlargement and induration as those in the neck.

Abdomen. Peritoneum. Ascites was present.

The stornach and intestines were normal.

The mesenteric glands were mostly normal, some were slightly enlarged.

The para-aortic glands were grossly enlarged, and so were the juxta splenic and those accompanying the iliac vessels.

The liver was natural.

The spleen was enlarged to at least four times its normal size; 'its surface mammilated and its structure sprinkled with tubercles' presenting the same structure as the enlarged glands'. 
History. Admitted ten days previously with a conspicuous lymphadenopathy affecting all the accessible glands, especially those in the neck, axillae, and groins. They were moderately firm and many were as large as pigeons egos. Abaominal distension was present. The functions of the brain had been somewhat disturbed and the vision of the left eye was imperfect. He died very suddenly.

Sectio Cadaveris ( $4 \frac{1}{2}$ hours post mortem)

Fxternal. The veins of the head and neck were turgid.

Head. The arachoid mater was remarkably thick and opaque. The brain itself was normal; the cerebellum was small. The right optic nerve was smaller than the left.

Neck. Cervical lymph nodes. These were greatly enlarged, in general the deepest being maximal in size. They were set in loose cellular tissue and when separated were smooth and white. In consistence they compared with a testicle. The cut surfaces were white and homogeneous, vascularity being low. Degenerative change was not apparent in them.

Chest. Pericardium. Natural. Heart. The organ was much hypertrophied and dilated. The cardiac muscle itself appeared healthy. The agonal thrombus was very poorly formed.

Pleurae. Normal, cavities free from effusion.

lungs. Both were pale, crepitant and mildly emphysematous.

The bronchi contained some thick mucus.

Mediastinal lymph nodes. Those along the subclavian arteries and about the roots of the bronchi were much enlarsed.

Axillary Iymh nodes. These were considerably enlarged and shewed precisely the same features as those in the neck.

Abdomen. The peritoneum was not remarkable.

The mucous inembrane of the stomach and intestines shewed no pathological features of note.

The liver was very large, pale and slightly granular.

The Spleen was very greatly enlarged, measuring approx. 9 x 5 inches and proportionately thick. 'Its colour was lighter and redder than is natural,

and more firm and close'. The cut surface was speckled with innumerable little white nodules of irregular outline, they were not tubercles. The Pancreas was large and pale but otherwise healthy.

The abdominal lymph nodes. Those at the lesser curvature of the stomach, several in Glisson's capsule, and the para-aortic groups were great $7 y$ enlarged. The mesenteric glands though enlarged, bore no comparison in size to the former specimens.

(Note. Hodgkin is emphatic on this point, the disparity was remarkable). The lymph glands of the groins were greatly enlarged and bore a close resemblance to the axillary groups. 
Case VI. Thomas Black.

History. Admitted nineteen days previously. He was affected with large tuberose swellings of considerable firmness on both sides of the neck, in both axillae and both groins. He was pale, cachectic, and dysphoeic. The abdomen was greatly distended. The cervical tumours were of about two years duration.

Sectio Cadaveris

The head wis not opened.

Neck. Cervical lyaph nodes. (See note below).

Chest. Pericardium and heart. (No record).

Pleurae. There was evidence of recent inflammation. Serous effusion was present. Iungs. (No record).

Lediastinal lymph nodes. The glands accompanying the carotid, subclavian, and internal mamary arteries were greatly enlarged. The posterior group were less conspicuous, and the bronchial glands did not appear affected.

Abdomen. The peritoneal cavity contained copious yellow serum mixed with sorne flakes of lymph.

Stomach and intestines. (iTo record).

The Liver was small and pale with an irregular and uneven surface. Two or three white nodules which resembled 'fungoid tubercles' were visible at the surface.

The spleen was normal.

The pancreas was embedded in grossly enlarged lymph nodes but intact. The kidneys were livid and congested.

(The cervical, axillary, para-aortic and inguinal lyraph nodes were reported collectively) sic:-

The tumours in this case very nearly resembled each other in structure; there was a little difference in firmness. They varied in size from that of a horse-bean to that of a hen's egg. They were mutually adherent in light fashion in their respective groups. The capsules proper were inseparable from the gland substance. The cut surfaces were pale and slightly translucent, some were of semicartilaginous hardness'. 
Brief notes on the cases excluded

Case III. Milliam Burrows.

(The findings in this case, which were first reported by H.Peacock, Esq., will not be set out in detail. The patient was known to suffer from syphilis. The morbid anatomy corresponds with this disease and the lymph node changes, which were confined to the lower half of the body, did not resemble the other examples).

Case V. A middle a ged man.

(The identity of this case is unknown, the history is vague and the necropsy report does not indicate sufficiently characteristic findings to warrant its inclusion. Careful reading of the text rouses an uneasy suspicion that the record 7 as not made as soon as it might have been ego quoque isiles sun).

\section{Case VII.}

'Sancer cerebriformis of the lymphatic glands and spleen'. (This case did not come under personal scrutiny by Hodgkin and is therefore omitted). 
The conclusions expressed by Hodgkin

Certain of these, which are quoted in full, not only reflect Hodgkin's shrewdness and acumen but shew an astonishing insight into the unique entity he had discovered.

He wrote 'It may be observed that notwithstanding some differences in structure, to be noticed hereafter, all these cases agree in the remarkable enlargement of the absorbent flands accompanying the larger arteries, namely the fiandulae concatinatae in the neck, the axillary and inguinal slands and those accompanjing the aorta in the thorax and abdomen, that as far as could be ascertained from observation or from what could be collected from the history of the cases the enlargement of the glands appeared to be a prinitive affection of these bodies rather than the result of an irritation propogated to them from some ulcerated surface or other inflamed texture through the medium of their inferent vessels, and that although, in some instances the glands so enlarged may contain a little concrete inorganisable matter such as is known to result from what is called scrofulous inflanmation it is obvious that this circumstance is not an essential character but rather an accidental concomitant to the idiopathic interstitial enlargement of the absorbent glandular structure throughout the body. That unless the word inflammation be allowed to have a more indefinite and loose meaning than is generally assigned to it this affection of the glands can scarcely be attributed to that cause since they are unattended with pain heat and other ordinary symptoms of inflammation and are not necessarily accompanied by any alteration in the cellular or other surrounding 
structures, and do not shew any disposition to go on to the production of pus or any other acknowledged product of inflamation, except where, as in the cases above alluded to inflammation may have supervened as an accidental affection of the hypertrophied structure'.

- It appears in nearly all cases to consist of a pretty uniform texture throughout and thus rather to be the consequence of a general increase of every part of the gland than of a new structure developed within it, and pushing the original structure aside as when ordinary tuberculous matter is deposited in these bodies'.

Concerning the deposits in the spleen he added '... has been found more or less diseased and in some thickly pervaded with defined bodies of various sizes, in structure resembling that of the diseased glands. We might from this circumstance be induced to suspect that these bodies in the spleen, like the anlarged glands themselves are the result of a morbid enlargement of a pre-existing structure'.... (Here he quoted Walpighi (1660) who considered the acini or granulations in the spleen to be glands). ...'Hence we may conclude that if, as I conceive to be the case, there be a close connection between the derangement of the glands and that of the spleen, the latter is a posterior effect, and on this account may not always have been produced when that of the glands or some other disease carried off the patient'.

Sumrarizing, his conclusions were: that it was a primitive affection of the lymph glands, without any obvious primary inflammatory cause; that it could not properly be called inflammatory but seemed the consequence of a general increase of every part of the glands and that caseation in the affected nodes was due to secondary infection (scrofula). 
Finally he considered that the splenic changes were of the same nature and probably usually followed the gland changes.

It is submitted that most pathologists who believe that this disease is neoplastic or a prinary reticulosis would have little difficulty in accepting these conclusions at the present day.

Several minor features are of interest. It is to be noted that all the cases were males, and two were children. Dropsy is recorded in five of the seven and there is occasional reference to black pigmentation in the affected glands. This last finding is rarely alluded to in works after 1900, it may refer to products of haemoglobin destruction. 
Note on Critical Analyses of Hodgkin's Series

Several authorities have perforned these and awarded various identifications. These are briefly summarized in the appended table. SULMARY OF ANALYSES OF HODGKIN'S SERIES

\begin{tabular}{|c|c|c|c|c|c|c|}
\hline \multirow[b]{2}{*}{ CASE } & \multicolumn{4}{|c|}{ AUTHORITY } & \multirow[b]{2}{*}{$\begin{array}{l}\text { FOX } \\
1926\end{array}$} & \multirow[b]{2}{*}{$\begin{array}{c}\text { NAEGLI } \\
1932\end{array}$} \\
\hline & $\begin{array}{l}\text { VILKS } \\
1865\end{array}$ & $\begin{array}{l}\mathrm{REED} \\
1902\end{array}$ & $\begin{array}{l}\text { SYMNGRS } \\
1924\end{array}$ & $\begin{array}{c}\text { HALE-WHITE } \\
1924\end{array}$ & & \\
\hline$I$ & + & $?$ & $?$ & $?$ & $\begin{array}{c}\text { (Tubercul- } \\
\text { osis) }\end{array}$ & 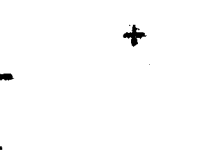 \\
\hline$I I$ & + & $?$ & $?$ & $?$ & + & + \\
\hline III & (Iues) & $?$ & $?$ & $?$ & (Ines) & + \\
\hline$I V$ & + & $?$ & $?$ & $?$ & + & + \\
\hline V & - & $?$ & $?$ & $?$ & - & + \\
\hline VI & + & $?$ & $?$ & $?$ & - & + \\
\hline VII & - & $?$ & $?$ & $?$ & + & + \\
\hline TOTAL & 4 & $\begin{array}{c}1 \text { or } \\
\text { possibly } 2 \\
+=\text { Consid } \\
-=\text { Not co } \\
\dot{P}=\text { No com } \\
=\text { No ind }\end{array}$ & $\begin{array}{l}\quad 2 \\
\text { ed Hodgkir } \\
\text { idered Hod } \\
\text { nt. } \\
\text { ation give }\end{array}$ & $\begin{array}{l}4 \\
\text { s disease. } \\
\text { kin's disease } \\
\text { - }\end{array}$ & e. & $\begin{array}{l}\text { 'probably } \\
\text { alI' }\end{array}$ \\
\hline
\end{tabular}




\section{THE CONFIRKATION OF THE IDENTITY OF THE DISTASE}

This is a fascinating chapter in the history of the malady. By a most fortunate set of circurnstances some of the material from the original cases on which the paper was based has been preserved. The post-mortem histology has been merely delayed.

In most institutions post-mortem material does not command the priority accorded to the surgical specimen routine. A lapse of ninetyfour years is nevertheless exceptional and it is of interest to trace how it came about.

It is remarkable how comparatively recently the subject of paleontology has been exploited. Iven as late as the eighteenth century the recognition of cerauniae as prinitive tools of Jarly Man was sporadic and hesitant.

In the middle of the ensuing century Boucher de Perthes gained acceptance of his claims and the study rapidly expanded. After this general sanction was won, attention was turned to physical anthropology and gradually the subject of paleopathology came into being. It is a neglected subject on the whole, particularly in this country, contributions to it being scanty and anecdotal. The Americans exploited it better, because, like archeology it is an expensive form of enquiry. For obvious reasons much of it concerns persistent structures like the bones and teeth. Various studies have been made from time to time concerning these; Inoodie, (1923), gives many references. Wuskens, (1926), in his huge treatise on epilepsy depicted a series of trephined neolithic skulls and suggested that the apertures were surgically 
for the relief of that malady. In several specimens evidence is present which suggests survival of the operation, at least long enough for a suppurative osteitis to develop. Malunited fractures of long bones have often been described, and it has been claimed that syphilitic and tuberculous bone disease has been recognised in neolithic man, though this is not universally accepted.

The next logical refinement was the application of histological methods. This is of course possible only in comparatively recent material.

Oliver rendell Jolnes in one of his whimsical asides (1859) wrote there were jars in rows where interesting cases outlived the grief of widows and heirs in alcoholic immortality - for your preparation jar is the true monumentum aere perennius'

So far as I can discover the late Professor John Teacher of this University was the first to take advantage of this possibility - but it is not claimed that he did so as a result of reading 'Ilsie Venner'! In 1894 Teacher became under-keeper of the anatomical and pathological department of the Hunterian Iuseurn (Hilliam Hunter 1718-1783). Six years later he produced the valuable and complete catalogue (1900). In the case of the tumours, Teacher prepared sections and remarked how surprisingly successful this proved, despite the fact that the specimens had been preserved in spirit for 120-150 jears. It is quite gratifying to realise that this work anticipates American labours in the same field by at least twenty years! Ruffer (192I) is generally credited as the first to attempt this: his studies were related primarily to mummified tissues from the Igyptian excavations. On the whole the experiment was 
not successful, the najority of the tissues being too brittle, even after special treatment. Shaw (1938) reported the results of histological examination of the muing of an Egyptian singer, Har-mose who lived in the 18th Dynasty (circa 1490 B.C.) The results in this case were exceptionally good.

In 1926 Fox examined several original specimens from Hodgkin's cases in Guy's Hospital Museum by modern histological methods. It is interesting to note in passing that Bright's disease as well as Hodgkin's has come under microscopical scrutiny, Osman (1937). It is greatly to be regretted that only three renal specimens of Bright have come down to us, all unhappily soundly injected with red lead. (One shewed changes of amyloid disease, another subacute extracapillary glomerulo-nephritis and the third chronic glomerulo-nephritis).

The identity of Hodgkin's material is not entirely straightforward. Since this is so fundanental an issue it will receive separate attention.

The source of the original material

The specimens which have been examined histologically by Fox (1926) and NacCallum (1928) were recovered from the museum at Guy's Hospital. This institution was founded by Thomas Guy in 1725 but for the greater part of a century it was under the aegis of St. Thomas's Hospital which also enjoyed a large share of the same patrons discreditably amassed fortune. Shortly before Hodgkin joined the staff, Guy's had becone fully independent and its energetic secretary, Harrison, was launching forth schemes for its improvement. He purchased a collection of morbid anatomical specimens from a private source and this formed the nucleus 
of a museum which Hodgkin rapidly augmented. Hodgkin related how in the space of four years the collection amounted to over three thousand, a great increase on the original five hundred. In 1829 he published his catalogue. Like all catalogues it represented an enormous amount of thankless work and considering it was the first proper inventory it is a very creditable achievement. It represents one of the best classification systems of its time, shewing a great advance on the famous museurn at Leyden where only five headings were considered, viz. Ossa Horbosa, Partes molles morbosae, Calculi, llonstra, and Varia.

The entries vary much in completeness: some have clinical notes and full descriptions; others are very brief, e.g. 'another of the same'. In a quiet way it ranks high in entertainment value. It is delightful to know that It./Col. Eerriot of the 22nd Regiment of Foot presented the museum with the skeleton of an Ilephant! (943). Minseurns were miseums in those days, not merely a set of teaching bottles! (Possibly it was the sort of gift that could not very well be refused). Hodgkin used a simple consecutive number system to identify the specimens. This nethod has limitations. The most obvious difficulty being that the arrangenent of cognate subjects in groups with serial numbers is impossible; the addition of fresh naterial introduces numbers not in the series already allotted. However, it is doubtful whether the adoption of nodern library methods is much better because classifications themselves are continually liable to change. The important principle is that once any particular sjsten is introduced it should not be altered - usually a pious hope. 
The identification of Hodgkin's specimens

The sources of information available include Hodgkin's catalogue (1829), Nilks' papers of 1856 and 1865, and Fox's paper (1926).

The information yielded by a critical study of these may be sumarized as follows.

1. Hodgkin's Catalogue. 1829

There is direct evidence of specimens from Case I, Joseph Sinnot. The relevant entries are 1558 and 2009. On the negative side there could not be any record of specimens from Case IV, Thornas ilestcott or Case VI, Thomas Black since both are dated after 1829, the date of this edition. Case $V$ cannot be identified at all since there is not any name, number nor date. It is unlikely that any specimens from Case III, William Burrows would be recorded here since it is dated late 1829 (November 28th). Absence of information concerning Case II, Ellenborough King, is curious since necropsy was performed in the previous year, presumably the specimens were still being prepared.

2. Samuel Wilks' paper of 1856

In this communication dilks gave his cases hith own serial numbers. Some of these are identified further, among them Cases 4I, 42, and 44. From the text there is definite evidence of specimens being present in the museum at that date from Cases I. Joseph Sinnot (Wilks 4I); II. Ellenborough King (

3. Samuel Viliks' paper of 1865 .

In this paper there is evidence of specimens from five of Hodgkin's original series. 
Case I. Joseph Sinnot.

Case II. Ellenborough King.

Case III. William Burrows.

Case IV. Thomas Westcott.

Case VI. Thomas Black.
Specimens 1558 and 2009.

Specimens $1541 \cdot 12$. cervical lymph nodes $2009 \cdot 50$. spleen.

Specimen $2005 \cdot 50$.

Specimens $1538 \cdot 50 \cdot ; 1555^{\circ} 29 \cdot$; and $1558 \cdot 50 \cdot$; All of glands.

Specimens $1543^{\cdot 32} ; 1543^{\cdot 64}$; and $1858 \cdot 30$. nature not stated.

4. Fox. (1926)

Fox was unable to identify the reference numbers given by Wilks 61 years previously, with specimens presently in the museum. He learned however from Nir. Burne, Curator of the Royal College of Surgeons and Dr. Beadle, Curator for Pathology, that one of Hodgkin's cases was in Guy's museum No. 1523 - under the name of Dr. Richard Bright. This statement is difficult to reconcile with Hodgkin's Catalogue entry No. 1523, which refers to a case of fungoid testis. The number must have been re-used, presumably between 1829 and 1856. This specimen, Fox declares, can be well identified with Hodgkin's Case II because its description coincides. An extract from the present (1926) catalogue identifies it as spleen and lymph nodes from Case II, Bllenborough King, and adds:- 'See Insp. 6.P.156 and Prep.1541'12 Sec Edit' - this last number is the same as Wilks' 1865 reference to the cervical lymph nodes from the same case. Fox further learned that the present specimen 4768 corresponds to $1541^{12}$.

He also obtained two further pieces from tissue studied by Hodgkin. These were present number 4769, identified with Case IV, Thomas Westcott, and present number 4770 , identified with Case VI Thomas Black. 
It is presumed that material from I, and III no longer exists. The results of $\overrightarrow{H i s t o l o g i c a l}$ examination

The spleen and lymph nodes from Case II Ellenborough King shewed a typical microscopical picture of Hodgkin's disease as presently defined and understood. The photograph made by Fox is unmistakable and convincing. Jackson and Parker, (1949), employed a closely similar photograph, probably from the same source, as a frontispiece to their book.

The preparations from Case IV, Thomas Westcott, were not so satisfactory but still strongly favoured the diagnosis. In Case VI, Thomas Black, Fox regarded the lesion as an example of 'The large lymphoid cell sarcoma type of growth' but he also declared that coarse fibrosis was present in the specimen. Eosinophiles are not mentioned in this case perhaps it is too speculative, but might this not be an example of Warthin's type II Hodgkin's sarcoma?

MacCallum(1928), also confirmed the identity of the disease and stated that it shewed the characteristic morphology brilliantly.

This tangible evidence is curiously satisfying and vindicates the pioneer efforts of Thomas Hodgkin. At its lowest it fully meets the minimal assessment of Reed, (1902), at its highest it anticipates the kinship of lymphoid tissue sarcomata. 


\title{
NOTE ON SUPPOSID REFERINCE TO THE DISEASE BY
} TRITIRS PREVIOUS TO HODGKIN

It has long been the custom in medical writings to introduce a subject with germane historical facts. These may be a genuine history but often they are thinly. disguised premisses for later argument, or provocative allusions to stimulate interest. Hodgkin's disease made its début so quietly that it was a comparatively long time before it too gained the distinction of a history. Now we have the authority of Holy Writ for the observations 'that of books there is no end', and 'Is there anything whereof it may be said - See, this is new, 10! - it hath been of old time which was before us'. Hodgkin, good quaker that he was had these facts in mind even in the first sentence of his paper. This anticipation was apparently rapidly rewarded because he adds a footnote on page 97 sic. - 'shortly after the reading of this paper, I was favoured with the following communication from my friend G.0. Heming of Kentish Town:-

\section{'Dear Sir,}

You will, I am sure, be pleased with the following extract from Malpighi.

\author{
Yours truly, \\ G.0. HOHING'
}

'In homine difficilius emergunt (speaking of the granules in the spleen): si tamen ex morbo universum glandularum genus turgeat, manifestiores redduntur, aucta ipsarum magnitudine, ut in defuncta puella observavi, in qua lien globulis conspicuis racematim dispersis totus scatebat'. 
(Hodgkin did not need to be told this, he was faniliar with Halpighi's work and in fact alluded to this reference on p.88). The exact context of this information is nearly always given wrongly, it appeared first in 'De Viscerum Structura exercitatio anatomica', Chapter 5, page 124. These figures apply to the original edition printed at Bonn in 1666 - and not the London edition which appeared three years later. It is also available in lialpighi's 'Opera Omnia' which was printed in 1687 (Figuris elegantissimis in aes incisis illustrata!) in London. It appears on page 111 in Tom II of this edition.

Gowers (1879) stated that Malpighi was the first writer to mention this association of general enlargement of the lymphatic glands with nodules in the spleen. It is very questionable if this can be assumed to mean anything more than exactly what it said. The characterisation is far too incomplete to sustain the claim that this refers to Hodgkin's disease. Several other diseases could produce just such a picture. Writers previous to Hodgkin had alluded to, or described instances of general enlargement of the lymphatic glands, but most of these were regarded as scrofulous or carcinomatous. A remarkable example of the former is attributed to llorgagnif (1752).

A claim is also submitted from time to time on behalf of Craigie. In the first editions of this authors 'Plements of Morbid Anatomy' (1828), under the heading of 'vascular sarcoma' or 'enlargement with induration' of the glands, is distinguished a form of lesion which could represent Hodgkin's disease. ...'The great hardness and the malignant tendency of this growth have procured for it from most authors the ominous names of 
scirrhus and cancer. Though correct enough for all practical purposes these epithets are not justified by the anatomical characters'. (In the 2nd Edition of the work which appeard in 1848 there is not any significant addition to this statement). Fox (1926) interpreted this account to reflect recognition of a morbid process possessing features of both scrofula and neoplasm. Wilks (1856) ; though possibly unaware of Craigie's idea, restated the same reflections - as a dilemma. This particular book of Craigie's dealt with disease in a rather abstract fashion, actual cases were not cited to any extent. Had it been otherwise the sponsorship might well have been Scottish. 
The Nomenclature of the Disease

If Hodgkin defaulted in this respect - the deficiency has been remedied by others. Wallhauser writing in 1933 collected fifty one synonymous titles which have been employed to connote it. Many are trivial variants, but several are still in current use. The eponymic Hodgkin's disease is very widely known, even if it is not universally used.

The French equivalent La Maladie de Hodgkin and the German, Die Hodgkinsche Krankheit, are less widely used than formerly. In English this old nare has a stark arresting quality with a certain aura of mystery. It has the advantage of being non-committal, and has undoubted claim to priority.

A common alternative is Lymphadenoma. This is relatively old, having been suggested by Wunderlich in 1866. Etymologically it is doubtful and there is little to commend it. Some authors (Robb-Smith et al) sometimes add 'verum'. If this is necessary then the word must be condemned. In point of fact Wilks and Moxon (1875) defined lymphadenoma as 'any tumour having a lymph gland-like structure' but this has either been abandoned or forgotten.

Iymphogranuloma or Iymphogranulomatosis enjoys a certain popularity among continental and American writers. It suggests that the disease is granulomatous in nature, a theory which is still not proven, and it is insufficiently specific. Malignant granuloma conveys an idea to which Hwing has contributed so much; this concept is discussed elsewhere but as a name it is a difficult hybrid which is liable to confuse those 
unacquainted with it. Malignant lymphoma, Bilroth, or the less antithetical lymphoblastoma of some American writers is too vague since many other growths could properly constitute it. The names suggested by those who regard the disease as a reticulosis have been considered already. 


\section{THE SUBSEQUENT HISTORY OF THE DISEASE}

An enormous amount of work has been performed on the subject of Hodgkin's disease. It is impossible to give a comprehensive account here, and particularly in an outline, the just apportionment of credit to those who followed Hodgkin in the study of the malady is very difficult. It has already been noted that the disease has masqueraded under different names and this adds further obstacles in the path of historical research. Suffice it to say that the contributions which have accrued over a period of more than a century are overwhelmingly numerous. Popular interest in the subject has its crests and troughs as its nature, cause, and remedy are alternately discovered or discredited.

Lany accounts of the main facts are available in text-books, reviews and papers. In examples of the foremost, in English, a very good summary is that of Gowers (1879), and another of merit is that by Murray (1909). Nore recent contributions of this character are those of Gordon, Gow and Rolleston (1937) and Jackson and Parker (1947).

Circumspect and unprovocative reviews have had a certain popularity, particularly in the United States of America, but to a lesser extent in this country. Widely quoted publications of this variety were offered by Longcope (1903); Rolleston (1925); Simmonds (1926); The Rose Research on Iymphadenoma (1932); and Wallhauser (1935). A lengthy review supported by nearly six hundred references was produced in 1948 by Hoster, Dratman, Craver, and Rolnick. This is possibly the most comprehensive of its kind as an amassment of information, but it is so 
non-committal and uncritical that it loses much of its appeal.

Individual papers, particularly when they are the work of one man undoubtedly form the most stimulating source of information. This is because the writer is an individualist with a purpose, and he who goes alone goes furthest. The articles by the following are conspicuous because reasoned criticism is brought to bear on the historical aspects related. Wilks, (1856 and 1865); Reed (1902); Gibbons (1906); Ziegler (1911); Oliver (1913); Vueller (1921); Varthin (1930); Ginsberg (1934); Krumbhaar (1934); Symmers (1948); Custer and Bernhard (1948) and Jackson and Parker (1949). This by no means exhausts the list of valuable contributions and others will be referred to later, but as in nost controversial subjects soine dismally bad writings exist, inaccurate and stilted assemblies of second-hand material where even the references are unreliable.

In the necessarily discursive reading undertaken for the purposes of this work many little publicised points of information have emerged. Below I shall endeavour to offer an account of the title of this section in the form of an eclectic summary with criticisms. It is reasonable that I should disclose my bias since this will facilitate my later purposes but for this I am naturally personally responsible. I believe that the history of Hodgkin's disease is best presented in three parts which cover respectively the periods 1832-1865, 1866-1903, and 1904 - to date.

\section{Period I 1832-1865}

Up till the close of this period the history is anecdotal. The publication of its sponsor was manifestly not received with much 
enthusiasm. This was partly due to the fact that medical journals had a more restricted and sulaler circulation than nowadays, and also because Hodgkin did not suggest a distinctive name for the disease. This trivial short-coming was in full accord with his lack of worldly wisdom but was a severe impediment to its advertisement. A name has remarkable power - witness the notoriety the 'Pancoast syndrome' has gained. Hodgkin's work excited scant recognition even from his own colleagues. There was however one heartening exception. Richard Bright, writing in 1838, gave full credit to Hodgkin; and judging from his remarks he appreciated the reality of this unique disease. The value of this acknowledgment is emphasized by reflection on the exceptional ability of Bright. This minor episode of loyalty is as solitary as the single company of infantry which Platea sent to Marathon and it seems to have suffered a similar fate.

It has already been suggested that tentative recognition of the disease anticipated Hodgkin's description. Malpighi (1666), and Morgasni (1752), possibly; and Craigie (1828) certainly knew of it. It seems improbable that it was a new disease like Trench Nephritis or von Economo's encephalitis lethargica. Although this postulate is speculative, it appears reasonable. The incidence of Hodgkin's disease is low but apparently fairly constant, and therefore it was liable to be recognised sooner or later by careful observers even in the absence of a conventional description. It seems very likely that Velpeau (1840-1) recognised it independently in France. This writer discussing the aetiology and pathology of lymphoid tumours in a lecture-demonstration 
exposition clearly appreciated the existence of primary growths unrelated to infective disease or ordinary cancer. In the case described, a young woman, the indications are that it was the same morbid process.

In 1845 Craigie of idinburgh described leukaemia and several months later, Virchow (1845) also did the same. It is probable that both writers knew of Hodgkin's disease but at this period it was still in the aleukaemic leukaemia group which had not been fully investigated.

In 1853 the disease reappeared in British literature. Markham described a case under the heading, 'Fibrinous deposits in the Spleen, thoracic glands \& etc.' These specimens were removed from the body of a man aged 30 years. The naked eye morbid anatomy was well described, particularly that of the spleen which was of the hard-bake variety and weighed I Ib. $10 \frac{1}{2}$ oz. ( $\left.740 \mathrm{~g} \cdot\right)$ This material was submitted to microscopical study by Bristowe at the same meeting. A fresh unstained specimen was examined and the report which stressed the great fibrosis present is strongly suggestive.

Three years later (1856) Wilks' first paper was published. This was entitled 'Cases of Lardaceous disease and some allied affections, with renarks'. This is a long article, of interest chiefly because of the evidence it furnishes concerning the preservation of material from Hodgkin's cases I, II, and IV. (Cases 39, 4l, 42 and 44 of Wilks' series; case VI is reported). He described 'Hodgkin's disease' fairly well and certainly knew a good deal about it. As Hale-ihite (1924) insists, it stands greatly to filks' credit that he acknowledged Hodgkin's priority. (The cynic might point out that both Hodgkin and 
Bright were still alive at this date).

A belated recognition of the disease was recorded by Pavy (1859), he wrote:- The malady is so striking and yet so peculiar that when carefully studied it is almost impossible to mistake its identity'. One case, occurring in a man aged 30 years was described briefly.

In 1865 Wilks' second paper appeared. This included a critical analysis of Hodgkin's cases with an assessment of their accuracy. He added other eleven but they are not all included in the text. A little microscopy was given in some of the cases, and one feels that he almost realised the features several times sic:- They were firm and composed of tough fibro-nucleated tissue' - 'composed of cells which somewhat resemble those of tubercle'. Unfortunately his discussion was vague but in sumarising he observed shrewdy that the disease presented, 'some features of tubercle, some of cancer'. The hardships which Hodgkin strove so much to mitigate were still present, 'Case 12, Sarah P- aet.13. Under Dr. Gull, February 13, 1864. Her parents were dead, and she had consequently been very badly fed...' An important immodiate outcome of this paper was that the disease received its classical name.

\section{Period II 1866-1903}

In the first period it has been seen that after a somewhat precarious transit the disease has arrived at the stage of being an entity complete with a name and patron.

In this second phase the real trouble starts and yet it is from here, despite the confusion, that it will emerge in its full dignity and preposterous ambiguity. In the same year in which Wilks' second paper 
appeared Cohnheim identified some cases of aleukaemic leukaemia with what he termed pseudo-leukaemia (pseudo-leukamie). This concept, or perhaps more accurately this name, has come in for much adverse criticism. The central idea however was excellent because it emphasized the unity of the primary morbid process - and literally it meant a leukaemia that played false. Since it is now widely appreciated that leukaemia is but an inconstant expression of certain reticulo-endothelial system diseases the idea is justified. Rather than despise it as a 'mischmasch' it is better to consider it as premature. The usage persisted especially in the German Schools, (Paltauf, Langhans, etc.). Cohnheim himself in 1870 identified 'certain cases' of Hodgkin's disease as pseudo-leukaemia and these doubtless corresponded to the 'hard' variety of Paltauf. In this year also, Cornil collected a series of cases of Hodgkin's disease, of which the description was now current, and added two more of his own with a careful study of the morbid anatomy. Trusseau (1868) introduced the term 'L'adénie' as a Gallic synonym for the malady. The word is variously rendered as 'adénie', 'adenia', etc. by other authors. His contribution was primarily clinical, the morbid anatomy was indifferently recorded.

After this point the main interest lies in the deternination of the microscopical appearances by modern histological methods. Although histology was the earliest refinement of anatomy and pathology it was relatively elementary till this era. (Hassel's microscopical anatomy (1849), while praiseworthy to a degree, is comparatively elementary). In 1872 Langhans described the results of microscopy in a case of pseudo-leukaeria; the account gave some details of the multinucleate 
giant cells and dense fibrous tissue in the lesion but it was brief and not illustrated. Six years later, in 1878, Greenfield of Dainburgh gave a further description. This certainly has priority, a point which is understandably emphasized by Alumni of the Capital (Muir 1924, van Rooyen 1938, and Ogilvie 1940), and renarkably enough by dwellers in Albania (now Londoniana), including Robb-Smith (1938). I have studied this paper carefully and frankly I feel it falls a little short of its reputation. It recounted precisely and carefully the clinical features of five instances of the disease. It is of interest that one of these, Case I, a man of 26 years, was under the care of Murchison who eight years previously had described the recurrent fever of Hodgkin's disease. (This original case was a child of six years who remained under observation for several months before death). Symmers, (1945)/ is to be congratulated for taking the Profession to task for attributing this discovery to the Dutch and German physicians, Pel and Ebstein. The mode of preparation which Greenfield used is not described but it seems probable, judging by contemporary articles, that the sections were stained with logwood. The multinucleate cells were well described and line drawings depicted them; Greenfield considered they were particularly related to the trabeculae of the new fibrous tissue. He stressed fibrosis as integral to the complex - The growth has an essential tendency to induration' and he observed the frequency of perivascular fibrosis. Hany critics have insisted that Greenfield maintained that increase of lymphocytes, a 'simple' lymphoid hyperplasia was one of the first evidences of the disease. There is not any direct statement to this effect in the text. A carefully worded account of the changes in 
adjacent lymph nodes beginning to enlarge was given but the stress was unquestionably - and perfectly correctly too - on the incipient fibrosis. In the description of the splenic lesions the black pigment recorded by Hodgkin was also noted by Greenfield. He also observed that in the liver the disease began in the portal canals. Greenfield appeared to draw a distinction between Hodgkin's disease and Iymphadenoma. (This interpretation is antithetical to that of Jackson and Parker (1949)). The latter term was widely employed in a generic fashion at this date although it had been suggested by Wunderlich as a specific term for the disease twenty years previously. Dr. F.I. Reynolds a former assistant with the late Professor Greenfield tells me that Greenfield was too good a pathologist to venture into print lightly, so it is probable that he said much less than he knew.

Doubtless as the result of limitations of staining methods, the eosinophile cells in the new tissue escaped notice till 1892, when they were discovered and fully depicted by Goldmann.

The remaining contributions of importance in this period are those of Sternberg (1898), Andrews (1902), Reed (1902) and Longcope (1903). These writers it will be noted belong to three different nationalities and anyone who reads much on the subject of Hodgkin's disease cannot fail to be impressed by the partisan tendencies of their respective compatriots. The complete lack of paeans for Andrews is to be regarded as a sort of negative self-effacement characteristic of the British. At the same time Andrews deserves the Palm for one of the most lucid and accurate descriptions of the microscopical features of the disease that has ever been written. His communication was 
contributed to a symposium on the subject, called primarily to settle whether tuberculosis was an aetiological factor or not.

The paper was written in a refreshing personal style, a little repetitive perhaps, but evidencing a clear insight into the disease from his own personal experience. He commenced his study with a definition which is worth quoting - I mean by it a disease characterised by a progressive enlargement of the lymph glands and lymphoid tissue of certain internal organs, notably the spleen and liver; unattended by the indiscriminate metastases of sarcoma and characterised by what I believe to be a definite histological change in the lymphatic tissues affected'. Then followed an excellent description of a normal lymph node so arranged that the pathognomonic changes in the malady were most aptly compared and contrasted. Among the features described ho emphasized the following:-

The general plan of the structure of the node was obscured and simplified, the distinction between cortex and medulla being almost or entirely abolished so that an unwonted homogeneity resulted. Iumphocytes were reduced in number to an extent which rendered the framework of the node more ceadily visible. Corresponding with this there was hyperplasia of the franework and the fibrillar reticulum became conspicuous, this change amounting to actual fibrosis. The endothelial cells in connection with it were more numerous and more obvious. Scattered individual cells of this description attained a very large size with two, four, or nore nuclei. These cells were especially numerous in the soft rapialy growing forms of the disease, and were quite different from Langhan's giant-cells. They most closely 
resembled the large cells found scattered throughout many rapidly growing sarcomata. Eosinophiles were sonetimes but not always greatly increased. The affected nodes were singularly exempt from degenerative changes but these did occur occasionally.

In his ultimate summary he compared the lesions with tuberculosis, and, whilst inclining to the view that it was a granuloma insisted that this was in a confined sense, a point being reached when all similarity ceased. This account is necessarily abbreviated, there are many small points raised in the original text which revealed an appreciation of the peculiarities of the malady which was singularly penetrating.

Sternberg (1890) gave a very complete description of the pathological findings in fifteen cases, thirteen of which were considered to be in the category pseudo-leukaemia. The clinical histories are meagre in the extreme. The histological findings concurred mutually. In eight (i.e. out of thirteen) positive evidence of tuberculous disease was present in one or more organs, and Sternberg concluded that the disease was a peculiar form of tuberculosis. Necropsy was performed in only one case in the relevant series. (It may interest readers that this publication is exceedingly difficult to obtain. I am indebted to Nr. Archibald Goodall, F.R.C.S., F.R.F.P.S.G. for procuring a micro-film copy of it from U.S.A. The illustrations were extremely good).

In 1902 Reed published her paper. This work is very widely known and probably the most freely quoted in relation to the histological study of Hodgkin's disease. It is difficult to suppress one's feelings entirely and for this reason the severity of the authoress, and her irritating style detract from the undoubted merit of the work. It 
must be stated that the description is very good, and the deductions, inferences, and speculations are sound. Several interesting new observations are added. These include, the apparent increase in vascularity in the nodes in the earliest stage, the occasional presence of Touton type giant-cells and the mode of fibrosis. In her series of eight cases three died and necropsies were performed. Reed discounted entirely the role of tuberculosis as an agent of the disease but believed that it was the commonest mode of death.

The publication with which this phase closes is that of Longcope. This is one of the most thorough expositions and is probably the best of its kind. It includes detailed protocols of his eight cases, experimental work, and so far as I am aware the first systematic examination of the bone marrow to ascertain whether the eosinophiles emanated from thence. In three of nis cases out of four that came to necropsy he reported evidence of a response to the demand for eosinophile leucocytes but rightly cautioned against too sanguine an interpretation of findings in a tissue which is difficult to sample accurately. His efforts to transfer the growth to monkeys failed completely. He presented a careful and thoughtful sumary emphasizing the anomalies inherent in accepting the disease as either inflammatory or neoplastic but was unable to solve the dilema. It is interesting to learn from this author that Langhans, (1872), was the first to conceive of the extra-nodal deposits of Hodgkin's disease as being autochthonous in character. This is an unexpected priority - antedating Dominici (1900) by almost thirty years. 
Iith the close of the second period there followed a dissemination of the characterisation of the disease which enabled others to assist in the researches which it prompted. Almost every aspect has now been examined with competence and thoroughness. It is an unhappy truth that the last fifty years of study have contributed refinement but only limited progress. Thile some discoveries have been added, others of previous time have been forgotten. Much of the knowledge gained is negative, but this is not equivalent to useless.

In this section, the subsequent history will be traced on a restricted basis with particular attention to problems which most directly concern the pathologist. These include contributions to the morbid anatomy, attempts to elucidate the nature of the disease, and classifications of the disease with corollaries developing from them. Advances in liorbid inatomy

Additions to the morbid anatomy are few in this period. In English the most important are the studies furnished by Symmers, (1924), and Pullinger, (1932).

Symmers' work was a very thorough examination of 14 necropsy cases with interesting and thoughtful interpretations of the findings. Among the points brought out was emphasis on how the true lymph nodes bore the brunt of the disease in contradistinction to the submucosal lymphoid tissue which generally escaped. Symmers envisaged an 'auxiliary Iymphoid tissue' which normally was inconspicuous in amount, but which augmented greatly in Hodgkin's disease and frequently provided new loci in which the lesion might develop. He was among the first to consider 
$x$ some of these man west have been chromic

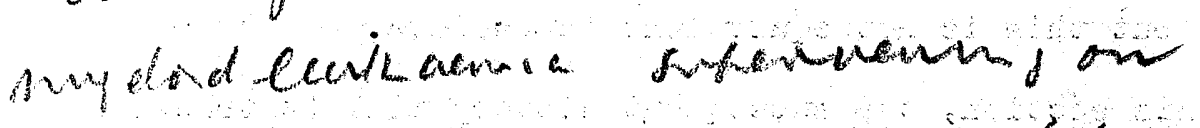

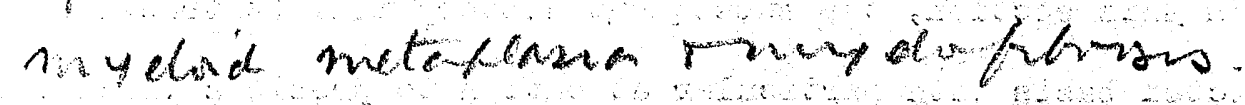
have seen the? 
that while an enlarged cervical lymph node might be the first clinical sign of the disease, this often betokened established lesions in the deep mediastinal nodes. The analysis of the findings in various sites including the spleen and liver, conformed to the general experience. His speculations and arguments on the nature of the disease were reasonable and it is interesting to record that he anticipated liedlar, (1931), in his recognition of the morphological similarity between the marrow in $X$ chronic myeloid leukaemia and the cellular complex of Fodgkin's disease. Since he regarded the fibrosis component in terrns of residual inflammatory scarring the absence of collagen from the marrow picture did not impress him. (His two photographs of these lesions admittedly shew convincing similarity, but single high power fields are not a conclusive comparison).

Pullinger's contribution to the Rose Research on Lymphadenoma, (1932), comprised an exceptionally complete examination of the microscopical features of the disease together with observations on atypical examples. The evolution of the lesion from its incipient stages to maturity was traced and described in detail. Pullinger followed the topical development of eosinophiles from lymphocytes in the lesions using Mann's stain. This netamorphosis she considered as indicative of a myeloid affinity in the new tissue. She reinforced this thesis by drawing attention to the almost invariable presence of polymorphonuclear leucocytes in addition to eosinophiles. She was the first to emphasize this feature and also described how the neutrophiles were often visible as small coherent aggregates. Pullinger's study is undoubtedly the 
most meticulous woris which has been offered on the microscopy of this disease. The illustrations are copious and extremely good, and the text is very well written.

Potter, (1935), re-examined the microscopical features of the Hodgkin lesion; her additions of interest were a fuller description of the typical multinucleate giant-cells for which she suggested abandonment of the older eponymic titles and substitution by 'Hodgkin giantcells'; she also gave interesting characterisations of the early stages of the disease.

Many reviews of large series of cases appeared in the latter part of this period. Relatively little of factual importance was added in respect of the typical disease. (These studies will be further alluded to later).

The Nature of Hodgkin's Disease

There is still widespread indecision on the nature of the disease. While this situation is representative of much authoritative orthodox thought, several hypotheses exist. The main theories advanced are that it is either an influmatory lesion, or a neoplasm, or a reticulosis. In addition several less accredited ideas are, or have been, entertained. A later part of this present work is devoted to support of one of the main contentions listed, here a brief sumary of the chief theories is given.

1. The Theory that Hodgkin's Disease is Inflammatory in Character.

This view survives despite a protracted series of failures to prove it exclusively tenable. Inherent in it is the interpretation that the 
lesion is a granuloma, which in turn derives from clinical and pathological observations. Wheh of the evidence adduced in favour of this hypothesis is actually negative and consists of observations against accepting the lesion as tumour. The view is supported by many capable authorities and an enormous, if diffuse, literature. It is not practicable to construct a chronological narrative of the development of the inflamatory thesis because, till comparatively recently, systematic additions to, and analyses of, the evidence have been wanting. The idea has persisted almost since the discovery of the disease; the best considered appraisals of it are found in reviews and books devoted to the subject. In the former class the works of symmers, (1924), and Krumbhaar, (1934), are highly critical; and in the latter the texts of Chevallier \& Bernara, (1929), and Jackson \& Parker, (1947), are among the best. A further valuable contribution is made by Iwing, (1929), though not as a special monograph. The arguments brought forward are conveniently listed under the headings of clinical findings, morbid anatomy, and bacteriological results.

In the first category it is pointed out that many cases are encountered in young adults and children, eras in human life in which neoplasms are uncomron. The greater incidence in males is in keeping with the distribution usually observed in infective disease. Fever, a common accompaniment of infective illness is frequent, notably in cases with extensive lesions and in the later stages. This has long been known; Wurchison, (1870), first described the peculiar protracted relapsing fever characteristic of the disease. Severe sweating nay be observed, and tachycardia disproportionate to the temperature rise is 
common. The secondary anaemia and wasting which develop as the diseaso progresses are also seen in chronic infective conaitions such as tuberculosis. In common with nany chronic infections there may be spontaneous remissions and exacerbations throughout the relatively protracted course of the illness. The invariably fatal outcome is not inconsistent with inflamnation since chronic infections like leprosy and torulosis are very lethal. Hodgkin's disease may present in an acute form, where lymph node enlargement is generalised from the start and toxic manifestations are conspicuous. In these the similarity to virulent infection is reasonably parallel.

The morbid anatomy of Hodgikin's disease unquestionably presents anomalies which preclude a straightforward acceptance of it as tumuur. The restriction to lymphoid tissue is remarkable. There is a lack of the indiscriminate inetastases which characterises sarcoma, the growth is only feebly aggressive, and its rate of proliferation is relatively slow. Effusions into the serous sacs are very commonly seen but rarely are they haemorrhagic like those in neoplastic disease. At microscopical level it is held that despite the pleiomorphism of the component cells there is a degree of orderliness foreign to tumour and that the anaplasia is limited. It is also pointed out that the component cells are for the most part identical to those which partake in inflammatory reactions. In the early stages hypervascularity is often discernible but regresses as fibrosis develops, which is parallel to an inflammation with residual scarring.

The disease has been submitted to bacteriological study in a most exhaustive manner. At the outset it is pertinent to emphasize that 
lymph nodes lie in the lymph strean and among their functions is the filtration of this fluid which is probably seldom sterile. Bloomfield, (1915), draxing attention to this fact, exanined lymph nodes from presumed normal cases, instances of Hodgkin's disease, and miscellaneous diseases. His findings fully justified his scepticism regarding the significance of positive cultures from nodes, and his further investigations of the microbes recovered failed to identify any pathogens. He considered that morbid nodes were more likely to afford resting places for fortuitous contaminants, and this view was reinforced by Jymmers, (1924).

Since Delbet, (1893), first isolated 'Bacillus Hodgkini' from a single case, and claimed to have reproduced the disease in a dog, several groups of organisms have enjoyed short-lived reputations as the casual wgent. Proescher \& ihite, (1507), found a spirochaete in four out of five cases of the disease. This microbe, Spirochaeta lymphatica, was soon abandoned however, as successive attempts at confirination failed. From 1913 to 1916 diphtheroids were reported by Negri \& lieremit, (1913), Bunting \& Yates, (IS14), and Torrey, (1916). Bunting and Yates produced lesions in Hacacus rhesus monkeys with their Corynebacterium Hodgkini but the similarity was not close to the human one. Cunninghem, (1917), and Twort, (1930), reinvestigated the role of diphtheroids very critically and may be said to have disproved the theory beyond doubt. Another microbe which engaged much attention for several years recently is Brucella. This work was first done in North Carolina, U.S.A. by Parsons, Poston, \& Vise, (1939). Initially confirmation appeared to be forthcoming, but soon it was appreciated that this mas 
local only. Jones, (1946), pointed out that the localisation of the phenomenon was significant because brucellosis was endemic in the same areas. The view is now fairly generally discredited.

In 1922 Kofoid, Boyers, \& Swezy, advanced the view that some of the morbid mononuclear reticulum cells in the lesion of Hodgkin's disease were in fact examples of Endanoeba dysenteriae. In the recognition of this identity morphology was the sole criterion adopted and the original communication related to two cases. Confirmation has not been forthcoming, and the interpretation has very little in its favour.

Simple fungi, dimorphic micro-organisms, and yeasts, have also been isolated from Hodgkin's disease lesions. In some cases full investigation including tests of pathogenuity have revealed them to be saprophytic contaminants e.g. the series reported in the Rose Research on Iymphadenoma, (1932). On the other hand exumples of torulosis lesions comparable to those of Hodgkin's disease have been found, most cases are reported from U.S.A. Professor Symmers (1953) related to me a case of presumed fungal infection which produced an histological picture of the true disease so perfectly that all the nembers of a panel of morbid anatonists concurred in its recosnition. The raycelium was cultured on one occasion and the patient has survived for longer than compatible with Hodgkin's disease.

Fitchett \& Weidmann, (1934), discuss the role of torula critically and while nothing definite can be proved it is clear that torula can sometimes mimic the lesion very closely in lymph nodes. The relationship of tuberculosis with Hodgkin's disease has been examined fairly consistently over the greater part of this period. The amount of work 
devoted to this problem alone is prodigious. The association of tuberculous infection with Hodgkin's disease was first emphasized by Sternberg in 1898, and he initially regarded the latter as a peculiar form of tuberculosis. This view he later abandoned, but the interim period being long - nearly forty years - it prompted very numerous attempts to incriminate 1 ycobacterium tuberculosis as the agent. It must now be conceded that tuberculosis is not an aetiological factor. For a short while the work of L'isperance, (1929), (1931), revived the theory by suggesting that avian strains of $\mathrm{M}$. tuberculosis were responsible for the lesion. General failure to confirm her findings and the reminder by $\operatorname{van}$ Rooyen, (1937), that avian infection though rare in man produces the usual form of tuberculous lesion have abrogated the theory. That there is an association of the two diseases is unquestionable and the many ingenious hypotheses to explain it are discussed by fallhauser, (1933). In general terms the nost reasonable conclusions are triat firstly tuberculosis is a common infection, and secondly that a patient with Hodgkin's disease is more susceptible to'it. This proposition may with propriety be related to the progressive reduction of physiologically normal lymphoid tissue and lymphocytes which is a definite result of Hodgkin's disease.

Up to the present there has not been any convincing evidence that a virus is responsible. Gordon, in 1932, discovered that if lymph nodes affected with the disease were suitably emulsified and injected intracerebrally into rabbits the animals developed an encephalitis. Serial transmission of the postulated virus proved impossible and subsequent /work, 
work, Friedemann, (1934), Edwards, (1938), has indicated that the concephalitogenic agent is not a virus. It is noteworthy that up till his death Gordon and some of his associated were reluctant to abandon the virus hypothesis.

In 1950 Debré and his associates described an interesting condition under the attractive title of 'La Maladie des Griffes de Chat'. This newly-described disease is characterised by the development of a primary lesion in the skin; the result of trauma and presumably infection, inflicted by the cat. (It is also recorded that in some cases plant thorns were the vector). The regional lymph nodes enlarge and in the earlier stages may present a microscopical picture closely resembling Hodgkin's disease. Further work by Rollaret, Reilly, Bastin \& Tournier, (1950), has demonstrated sensitization phenomena which appear specific but its viral nature is unproved. The disease runs a brief course terminating with spontaneous resolution, on occasion abscess is developed before this; it is essentially benign.

Hodgkin's Disease Regarded as Neoplastic in Character

This view is undoubtedly gaining ground against all the others. Progress has been gradual, every gain has been small and has had to be consolidated. The hypothesis is as old as Hodgkin's discovery but the means to support it have been slow in developing. At the outset it is admitted that there is not any single great decisive fact which proves the case to the satigfaction of all, rather are there many fragrnents of evidence which collectively can resolve the lesion as neoplasm. Iven then the decision resenbles a mechanism of conversion, Sargent (1951), it /requires 
requires intuitive belief. After its adoption a retrospect at the evidences and their corollaries confirns the decision and rationalises the difficulties.

The first supported contentions for tumour status for the disease were made in the opening years of the period and now at the close of it endorsement is accorded by nany pathologists including some of the leading oncologists of the present day.

The facts collected in favour of the theory derive from extensive studies covering a wide field. They include the clinical and morbid anatomical findings in the common form of the disease and additional facts bearing on the problem which are litent in unusual forms of the disease or in disenses having affinities with it.

liany clinical aspects of ordinary Hodgrin's disease are coinpatible with the tumour hypothesis. The disease is commoner in males in the proportion of approxinately $2: 1$., in children the ratio is even more pronounced in favour of males, e.g. Smith, (1934), submits the ratio 4:1. This disproportion conforms to analogous figures in neoplastic disease. Age is a more capricious factor. In most series recorded the incidence in children and youths is notable and most figures stress the vulnerability of these eras. This inpression is not supported by all observers. Gall \& Wallory (1942), reporting a large series gave the average age at initial biopsy as 36 years and Vilis (1949), decided that the disease is comnonest in the fourth and fifth decades of life. Geographically the disease is widespread. It appears comoner in highly civilised communities but this is probably due to the fact that its certain diafnosis is a sophisticated attainment. Typical 
cases have been reported frow Nigeria. Garven (1952), however, encountered very few cases during a prolonged stay in North China. Ipidemiolosically it exhibits the apparently fortuitous incidence of malignant disease, fanilial incidence is extremely rare. There is not any doubt that the disease is invariably fatal though intercurrent illnesses or complications may precipitate death. Untreated the disease runs a progressive course which seldom exceeds three jears, individual cases may be very brief or unexpectedly protracted. It is attended by localised tumour, the development of weakness and secondary anaemia and in the late stages by cachexia. All remedies devised so far appear merely palliative in effect, its response to therapy being closely parallel to that of inoperable malignant disease.

Features in the morbid anatony which favour the tumour theory are many. Those which have been made the subject of personal study will be amplified later. Here a synopsis of better authenticated facts is offered, together with observations, and areuments to meet some of the objections.

The Iirst deliberate contention that Hodgkin's disease is a tumour was made by Giboons in 1906. Study of this text reveals that although the writer failed to substantiate all of his thesis, he was anong the first to recognise the origins of the new tissue and its affinities to lymphosarcoma. He considered it arose by proliferation of cells of the germinal centres, the endothelium lining the sinuses, the fibrous tissue cells of the capsule, blood vessels and node framework. (Ginsberg (1934), quotes Gibbons as employing 'reticulum cells', this is erroneous, the term fibrous tissue was employed). Three of Gibbons' cases were /Hodgkin's 
Hodgkin's disease with malignant features, in which unusual aggressiveness was displayed by the growth. He observed rupture of node capsules and new concentric formation opposite the breaches. He also drew attention to localised outgrowths of the new tissue from the periphery of nodes which tissue was confined by capsular tissue drawn out with it. Gibbons regarded the extra-nodal deposits as metastases, but did not offer convincing evilence of their transit. On the other hand he emphasized their lack of encapsulation and distinctly infiltrative tendencies, especially in the lung parenchyma distant from the bronchial tree. Read in the light of present day knowledge, Gibbons was far ahead of his time. He also deserves credit for emphasizing the feature of capsular thickening which Reed (1902), failed to appreciate. it this period lymphosarcoma was probably a larger category than it is now. (It is interesting to note that as early as 1893 Kundrat had attempted to separate Hodgkin's disease from lymphosarcoma, but his differentiation was very poor.

Coley (1908), supported the tumour hypothesis emphatically but his reasons were confined to generalisations. These included apt comparisons of the clinical features of the disease with lymphosarcoma, and insistence on the difficulty of separating some cases from exampies of sarcoma of lymph nodes.

In 1911 an important side-issue came into being, namely Hodgkin's sarcoma. This influenced the development of the tumour hypothesis; more account of it will be given later.

Oliver, (1913), produced a well-reasoned discourse on the nature of the disease and introduced the unitarian idea of the origin of endo- 
thelioma of lymph nodes, lymphosarcoma and Hodgkin's disease. Strongly influenced by the contemporary work of Ewing on the foremost subject, she interpreted all three as different expressions of one neoplastic process.

Dight years later Nueller, (1921), reopened the question. Two special cases from a series were described. These illustrated the principle of matation of microscopical picture in lymphoid tissue tunour and furthered the ideas of coley. In his conclusion hueller wrote 'Wialignancy as it expresses itself in the destructive invasion of the neighbouring tissue and in metastases, is in these chronic diseases of the lymphatic system apparently not connected with a particular anatomical structure'. This observation reflected a mature appreciation of the peculiarity of lymphoid tissue tumour which is insufficiently advertised.

In 1931 Nedlar gave a new and unexpected interpretation of the tumour hypothesis. This publication has bewildered most readers and some critics have even sugrested that the author, who is no mean authority, meant it to be provocative. Briefly his conclusions were that Hodgkin's disease was a tumour arising from megalkaryocytes and that the complex of cells characteristic of the growth represented a developmental cycle of the type-cell. Acceptance of the interpretation necessitated a complete reorientation of ideas about the disease, and while a new approach to an old problem is a salutory procedure this one has a speculative ingenuity verging on the incredible. By Nedlar's theory primary malignant turnour arises in the megakaryocytes - a megakaryoblastoma - and metastasises to the lymph nodes and other tissues outside the bone marrow. A fundamental difficulty to most patholosists 
is the morphological dissimilarity between the megakaryocytes and the Hodgkin's giant-cell. In addition the aliost exclusive location of metastases in lymph nodes and gradual successive involvernent of the node groups are very difficult to explain.

The material from which this study was derived deserves criticism. A considerable background of experience of Hodgkin's disease, human tuberculous lymphadenitis and of experimental work were interpreted on the basis of the findings in a single biopsy specimen of a skin nodule from a case of chronic nyeloid leukaemia. This latter specimen was certainly studied intensively, but it is questionable whether this was adequate for the inferences arawn. Hedlar was prepared to accept lesions without fibrosis or eosinophile infiltration as examples of the disease. This dilution of criteria is open to question; Hodgkin's disease without fibrosis is very dangerous ground. On the other hand in Hedar's favour is the fact that it is a practical impossibility to examine all the bone marrow in a human body, a postulated latent primary is therefore impossible to refute.

This tumour theory has not attracted confirmatory work.

In this year also Varthin produced an important paper which enlarged and consolidated the concept of lymphoid tissue sarcona. The material for this study comprised 506 cases represented chiefly by Hodgkin's disease including atypical forms of it, together with exanples of lymphosarcoma, leukaemia, mycosis fungoides and a small group of unclassified cases. The neoplastic status of Hodgkin's disease was expounded with emphasis, and Varthin insisted that the allied disorders named above vere closely related to it. (The further contents of tris 
paper are referred to below under the heading Hodgkin's sarcona. q.v.) Fraser \& irekie, (1933), in a curiously written paper - it reads like a sermon - also accorded the rank of tumour to the disease, but new matter was not idded to the argument.

A further study which included an excellent critical analysis of previous work was submitted by Ginsberg, (1934). The writer collated a variety of evidences of the biological similarities which could be demonstrated between Hodgkin's disease and lymphosarcoma. He also emphasized the importance of crediting reports of confused diagnosis, as these served as evidence of interchangeability of the lesions. His own work was an apt comparison of the remarkable similarities presented in the course, spread and morbid anatory of two cases. In each instance the patient was a middle aged woman. In one patient the lesion was Hodgkin's disease throughout, in the other a large-celled lymphosarcoma.

In the last decade up till 1950, several important works have appeared which directly or indirectly support the tumour theory. These include the publications varren \& Picena, (1941), Gall \& Mallory, (1942), Herbert, willer \& Erf, (1945), and Custer \& Bernard, (1948). The first paper was essentially a description of the variants of sarcoma arising in lymphoid tissue, emphasizing their extremely close kinship. This schema did not include Hodgkin's disease; but Gall and Mallory integrated the latter into the generic group lymphoid tissue sarcoma. Their authors maintained at the outset that constancy of the individual members of the group was a characteristic feature, but at the same time they recorded that in 84 of their cases with serial biopsies, the original structure was maintained in 77 per cent of cases and showed de- 
differentiation in 23 per cent. They wrote 'it is believed reasonable to expect this degree of de-differentiation in any group of cytomata followed over an extended period of time. In this particular instance the occasional transition from one form to another is a feature lending credence to the belief that these tumours are essentially of tumour origin'. Herbert, Iiller, and Irf, reported six cases in which the transition phenomenon was unusually well substantiated and demonstrated. The final work quoted provided additional evidence of the same character on a very large scale. The material studied covered 700 cases of lymph node tumour. Their results revealed that 39 per cent of the cases exhibited mutation of histological picture in the period studied. The following diagram sumarized the findings.
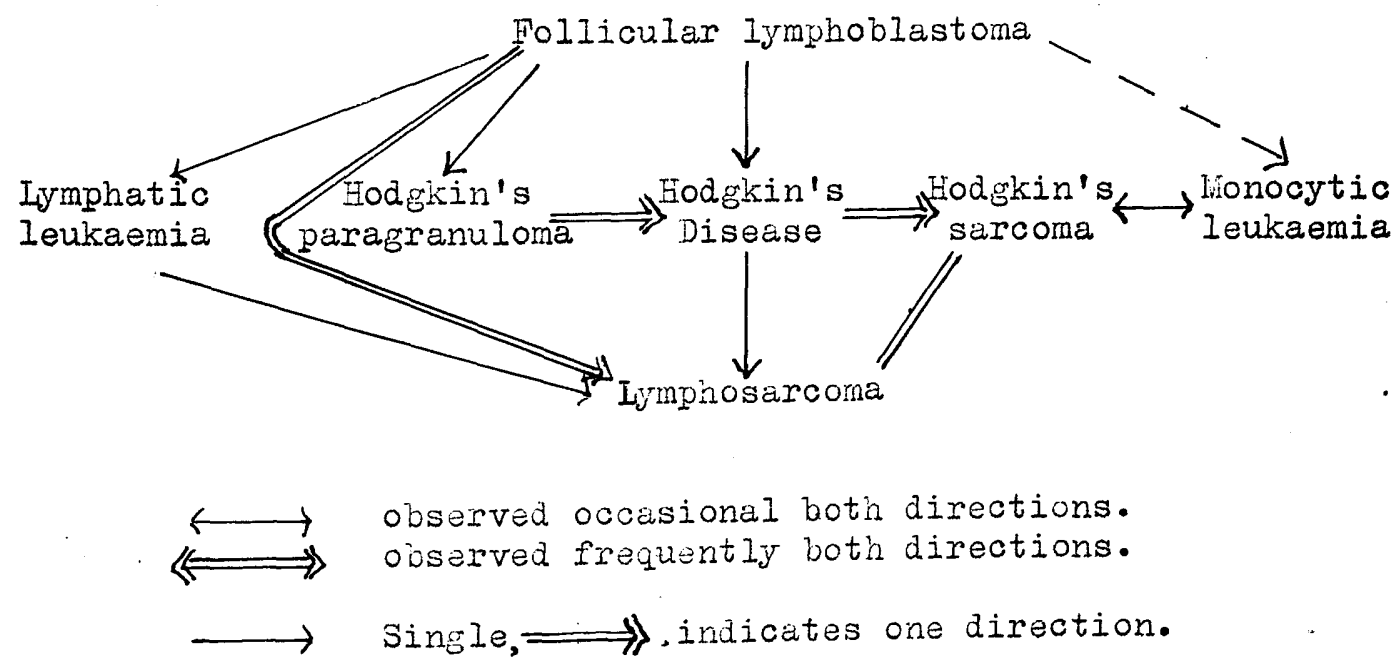

Experimental investigation of the disease will receive full attention in part IV. Very little has been found out however which indicates the nature of the lesion. Laser, (1951), investigated the metabolism of Hodgkin tissue and reported that it showed a type of metabolism characteristic of but not specific for tumour growth. 


\section{Hodghin's Disease Regarded as a Reticulosis}

Piney, (1925), was the first to suggest that Hodgkin's disease was an example of reticulosis, (reticulo-endotheliosis). In 1932 Pullinger developed this theory and modified the name to fibro-myeloid reticulosis in order to emphasize the predominant differentiation tendencies in the growth. Induration by fibrosis is extremely characteristic of the lesion but the myeloid affinities are less emphatically inherent. Pullinger observed the development of eosinophile granules in lymphocytes in the lesion and was able to identify progressive development of this phenomenon till cells morphologically the sume as eosinophile leucocytes were produced. This she interpreted as a local mjeloid metaplasia and supported the argument by assigning to the neutrophile leucocytes of the lesion a jossibly sinilar local origin. The former metamorphosis has precedents; the formation of eosinophile leucocytes from lymphocytoid cells was derionstrated by Opie, (1904a, and 1904b), and by Dominici, (1909). It is curious that confirmatory reports of Pullinger's work on the eosinophiles of the Hodgkin's disease lesion have not been offered. Personal studies on this problea will be given later. Here it suffices to state that I have baen able to confirm this transformation, but only in relatively very few samples of the disease. Further, topical eosinophilia is not invariably demonstrable in Hodgkin's disease. The eosinophiles may be so few that their presence can hardy be significant. Ross, (1933), supported Pullinger's thesis but relegated Hodgkin's disease to the 'unrestricted differentiation' category in her scheme. Robb-Smith, (1938), adopted Pullinger's nane for Hodgkin's disease. He included the malady in his reticulosis classifij'cation 
cation but, sisnificantly, he admitted that it ind features anomalous anong the other reticuloses. Since the lesion usually presents first in the pulp of the lymph node it was classed as a redullary reticulosis. Arong recent writers Hadfield, (1949), has supported the reticulosis theory.

In this country the reticulosis concept was quietly accepted by most pathologists and clinicians, and in many continental countries similar or nore enthusiastic approbation has been given to it. In contrast the idea has never been popular in the U.j.A. Lately there has been a partial retreat from reticulosis. This is because its initial exploitation was too rapid and uncritical, and because of tendencies to fit the diseases to the theory instead of the reverse. Other Comments on the Nature of the Disease

At the outset it was stated that a wide body of opinion existed which was non-comittal on the nosological categrory of the malady. Even though ultimate indecision is expressed it is usually possible to detect bias in favour of one particular theory in the writings of contributors in this group. It is my impression that most of the more recent authoritative reviews give timorous support in favour of inflammation. This trend is still apparent but prior to this period tumour status was favoured more definitely, particularly in continental works.

In conclusion mention is made of several very poorly accredited theories regarding this disease. They have very little claim because most are merely guesses. It has been suggested that it is an inflammatory manifestion dependent on allerey, that it is a metabolic 
disease, that any chronic inflaming agent can cause it, and that it is a unique morbid process. The evidence is simply the vritten word. The Classification of Hodgikin's Disease

In all fields of study attempts to catalocue the material have a strong appeal. The purpose is to group the objects of study within a defined field so as to produce a precise and rational separation which has true value. Idealiy classifications should exhibit mutually exclusive classes which are each defined by unique characteristics.

In biology it is difficult for these postulates to be met because the data are usually very complex and often incomplete. Thus most classifications in pathology are necessarily provisional, but any which may be advocated or adopted should have justification beyond being merely elegant refinement.

There is definite vindication for attempts to classify the lesions of Hodgkin's disease, even if it is adequate to regard it as an exclusive category in itself for clinical purposes.

The main support for this endeavour is that any procedure which improves the diagnosis of Hodgkin's disease is worth while. The disease is a very serious one and it is a fict that its microscopical diagnosis is not particularly well performed. Its comparative rarity determines that perfectly typical examples are not commonly seen. A classification enforces closer inspection of specimens and a better appreciation of the latitude of apearance which the lesion may present. The disease is thus better defined and other diseases are less likely to be confused with it. Failure to recognise Hodgkin's disease in its less familiar forms is quite common, yet this is a fault which is 
usually corrected later; on the other hand migf-identifyine it is also not rare, and is probably a more serious accident because the modern treatments of irradiation and cytotoxic drug therapy are in themselves potentially nocent.

Less obvious adventages may also derive from a classification. In time experience may relate certain variants to prognosis and likely reaction to treatment. The stability, mutation, or progression of a lesion way all be more accurately assessed when variants are recognised; and the mind becomes better conditioned to exploit the chance anomaly which is a potential key. Unexpected and interesting side-issues also emerge from this type of work, e.g. In my own studies I have encountered examples of cancer mimicking Hodgkin's disease almost to perfection, a circumstance of some importince.

Finally in attempts at classification it is common for 'scrap-heaps' to develop. These indeterminate groups though often immediately embarrassing can form the basis for new knowledge. The doubtful lymph nodes are among the nost terrible treasures the morbid anatomist collects in his sojourn. Their ability to stultify the worthiest efforts, whilst humiliating, is a salutory reminder that the subject is by no means worked-out.

A really comprehensive classification of the disease is a prodigious task. It has been attempted, and setting aside the very reasonable objection that it is easy to criticise, it must be conceded that the results have not been very helpful. Ziegler, (1911), attempted it and described nine varieties. The subdivisions however are unsatisfactorily vasue, being partly anatomical, partly clinical and partly 
microscopical. In several the evidence of identity is so meagre that they are almost hypothetical, e.g., the splenic and osteitic forms. On the restricted basis of microscopical structure several tentative classifications have been evolved. Besides formal classifications, many writers have offered tentative schemata. Andrews, (1902), recognised hard, soft and intermediate varieties; Gibbons, (1906), referred to malignant Hodgkin's disease as well as the ordinary form; and Nelch, (1910), first described Hodgkin's sarcoma. (This latter will be fully discussed later, it is noted here that the term is often loosely used).

Varthin, (1931), recognised typical and atypical Hodgkin's disease, and defined Hodgkin's surcoma. Htypical Hodgkin's disease he reported to be characterised by relative or absolute lack of Dorothy Reed cells, and he added that subsequent study of these cases often revealed development of the typical picture. Kettle, (cited by Pullinger, 1932), was wont to recognise typical Hodgkin's disease and place all examples falling short of requirement for this category in 'Hodgkin Group'. Inclusion was restricted to lesions exhibiting multinucleate giant-cells and uninuclear reticulum cells. Pullinger herself described a soft cellular variety in which Hodgkin giant-cells were sparse, the reticulum cell proliferation marked, and eosinophiles few or lacking. She noted, moreover, that areas of this character were often seen in otherwise typical lesions. Jackson, (1937, 1939), submitted a simple classification which has been widely and rather uncritically adopted. He contended that the disease might present in three forms viz. Hodgkin's paragranuloma, Hodgkin's granuloma and Hodgkin's sarcoma. In the 
initial description the first form was described as affecting single or multiple nodes. The predominant cells were small lymphocytes, and few or rarely many 'Sternberg-Reed' cells; eosinophiles, fibrosis, and necrosis being absent. In later publications with Parker, (1944, 1949), the descriptions differ a little, chiefly by conceding that fibrosis occurs. This microscopical entity has since been very much better described by Harrison, (1950). Hodgkin's granuloma corresponds to ordinary Hodgkin's disease. Hodgkin's sarcoma is poorly described, these authors considered it highly malignant.

Bersack, (1943), introduced another histolocical classification. He found he was able to segregate three varieties readily viz. Hodgkin's lympho-reticuloma, Hodgkin's granulorna, and Hodgkin's lymphoma. The first, from the description and photosraphs, appears to correspond with Pullinger's soft cellular variaty or Gall and fallory's stem-cell lymphoma. The 'granuloma' is ordinary Hodgkin's disease, and the last variety is like Jackson's paragranuloma. The discussion in this paper is disappointing, one has the impression it has been drastically edited. A year later Bersack advocated subdivision of the reticuloma and Iymphoma groups into typical and atypical. The matter of this comranication is interesting but is highly speculative.

It is common experience that varieties of Hodgkin's disease are recognised which never reach the dignity of print. These include nany slightly atypical exumples. The experienced, having encountered them before, can allay the tyro's concern with assurance.

There is one peculiar group in this category where the diagnosis is arrived at $b_{J}$ a kind of reductio ad absurdum procedure - 'It must be /Hodgkin, 
Hodgkin, nothing else can account for this appearance'. Note on Hodgkin's Sarcoma

The characteristics of ordinary Hodgkin's disease are widely agreed and well known. In contrast there is much less understanding and agreement concerning 'Hodgkin's sarcoma'. The title is not in wide use and receives doubtful acknowledgement in most standard text-books of pathology. It is reported rarely and the descriptions of it vary considerably.

The legend has been used in different senses and often loosely. Tases of Hodgkin's disease may present highly cellular and active lesions which prompt the description 'frankly sarcomatous'; again examples are encountered where besides an otherwise typical picture unequivocal malignant characteristics are prominent. These include cases where there is active invasion and destruction of node capsules, entrance into blood vessels, and conspicuous deposits outwith the normal sites of lymphoid tissue. From the general behaviour of such examples the term Hodgkin's sarcoma has reasonable justification.

In a more restricted meaning the title is applied to a lesion which may be regarded as a further stage of, or an unusual sequel to, ordinary Hodgkin's disease. Even in this usage niceties of distinction exist and these merit further attention. Yamasaki, (1904), is frequently quoted as being the first to employ the term. This is correct but his characterisation of the lesion was unsatisfactory, the title itself being the chief claim to priority. Welch, (1910), is properly credited as being the first to give a satisfactory report of the phenomenon. Ewing, (1929), Eave some account of this original case and afforded 
full recognition to the entity. A protagonist of the view that ordinary Hodgkin's disease is granulomatous in nature he put forward the ingenious suggestion that it bore the same relation to Hodgkin's disease as squamous cell carcinoma may to a lupus scar. ining considered that Hodgkin's sarcoma arose most frequently in the mediastinal form (Ziegler) of the disease. The structure of the tumour varied from a close counterpart of the ordinary disease to a growth composed almost exclusively of large round cells with faintly staining granular cytoplasm, and moderately large chromatic vesicular nuclei. Large round giant-cells with multiple or multilobed nuclei might predominate in some cases. He allowed that the histological signs of malignancy were usually not pronounced, the perforation of node capsules being slow. Jackson \& Parker, (1947), followed Jing in their interpretation of the lesion. They stated that microscopically the tumour was composed of cells two to tinree times the size of a normal lymphocyte. The nuclei were round or ovoid and generally possessed prominent nucleoli. Multinucleate giant-cells of Sternberg-Reed type were always present. They added that besides these, scattered lymphocytes, scanty granulocytes, plasma cells and reticulum cells were also seen. The reticulin mesh was generally increased.

In a second group are those writers who, supporting the neoplastic theory of Hodgkin's disease, employ the sarcoma term in a descriptive usage for certain morphological pictures.

Warthin, (1931), considered that the sarcomatous transformation of the disease proceeded in one of two directions, to a lymphosarcoma or to a large-celled form with abundant reticulum and numerous giant-cells 
('Reticulo-endothelioblastona'). In this latter form the lymphoid cells became reduced in number, the eosinophiles disappeared and the najority of the cells came to be large polymorphic cells with abundant cytoplasm and many large hyperchromatic nuclei. He added that numerous cells of the 'myeloid type' occurred; though exactly what was meant was not further explainea. Both lesions were depicted by single photographs. Callender, (1934), characterised Hodgkin's sarcoma as identical with reticulum cell sarcoma in which pléfomorphistn was prominent beyond the degree exhibited by typical tumours of this cell. The above synopses represent most of the descriptive work on the tumour.

The transformation to frankly neoplastic growth is the most significant feature. There is reason to believe that the change is commoner than generally supposed and partial transition is definitely frequent. 


\section{MHE GENBRAL MORBID ANATOMY OF HODGKIN'S DISEASE}

There are many good analyses of tha general morbid anatomy of Hodgkin's disease. Some are individual studies of small series in which the accounts are detailed, others represent the collated results of exhaustive abstracts. Representing both groups papers by the following are noteworthy: Symons 1903; Ziegler 1911; Cunningham 1915; Symmers 1917, \& 1924; Vallhauser 1933; Smith 1934; Goldman 1940; Jackson and Parker 1947; and Hoster et al 1948.

In the following general account these works and several others. will be drawn on, and supplemented by the findings in a series of 49 cases encountered at necropsy in Glasgow Royal Infirmary in the period $1900-1951$.

Frequency and distribution of Hodgkin's disease

The disease is distinctly rare. In Sweden Uddstromer (1934) recorded an incidence of 0.54 per 100,000 living persons. In the U.S.A. Isaacs (1944) quoted a frequency of 1.3 per 100,000 for 1921, and 2.1 per 100,000 for 1934-6. Robb-Smith gave the figure for Great Britain (1947) as 2.3 per 100,000 living persons.

Figures from hospital mortality reports give much higher values since the population is a selected one, and this despite the fact that patients with Hodgkin's disease often die at home.

Table. 
Table.

Source.

Symmers

Barron

Ciechanowski

Jackson and Parker

Glasgow Royal

Infirmary

(unpublished)
Total Consecutive

Cases. necropsies. Percentage.
(1924)
14
8,485
0.16

(1926) 24

7,253

0.32

(1938) 204

59,704

0.34

(1947)

63

18,668

0.34

(1951)

49

0.26

Geographically the disease has been recorded in most countries. Itwill be appreciated that its certain diagnosis is a sophisticated attainment, with the result that its incidence is difficult to assess in backward countries. Hoster et al (1948) record that it has been reported in Arontina, Brazil, South Africa, Australia, New Zealand and Japan. To this list may be added Nigeria, Smith (1935). Sex incidence.

The disease is definitely commoner in males, this discrepancy being greater in children.

Table.

Source. Trales. Females. Ratio.

$\begin{array}{lrrrrl}\text { Barron } & (1926) & 16 & 8 & 2: 1 & \\ \text { Wallhauser } & (1933) & 1,009 & 438 & 2.3: 1 & \\ \text { Smith } & (1934) & 88 & 20 & 4: 1 \quad \text { (Children). } \\ \text { Goldman } & (1940) & 143 & 69 & 2: 1 \\ \text { G.R.I. } & (1951) & 35 & 14 & 2.5: 1 \\ \text { Agge. } & & & & \end{array}$

Figures for the age incidence shew considerable variation. Vallhauser gave the maximum in the two decades between 18 and 38 years, and 
this is probably the most general experience. On the other hand studies of smaller series, Willis (1948), and the G.R.I. series reflect a higher incidence at a later age. In the latter the maximum fell in the fifth decade.

Duration.

This is difficult to determine since the onset is usually uncertain. The figures for the G.R.I. series gave an average of 2 years 2 months from diagnosis. Some authorities give a period of 3 years as average but most are agreed that individual variation is to be expected, cases as brief as seven weeks have been recorded, others as long as 20 years have been known.

In one of the G.R.I. series (P.I. 12966) the history stated that a swelling had been present in the right axilla for 20 years and that diagnosis was made from biopsy of this tumour.

other features.

Congenital cases have been encountered. A well substantiated instance is that reported by Priesel and Winkelbauer (1926). Rarely it has been met with in siblings but these reports are isolated. 
Tables.

G.R.I. Series Necropsies. 1900-1951.

Total Number of Cases. 49.

Sex

Males.

Females.

Ratio

35

14

$2.5: 1$

Age. Decades. 1

\begin{tabular}{|c|c|c|c|c|c|c|c|}
\hline Cases. & 0 & 7 & 6 & 8 & 14 & 9 & 4 \\
\hline $\begin{array}{l}\text { Sex of } \\
\text { Cases. }\end{array}$ & • & All m. & All m. & $\begin{array}{l}5 \mathrm{~m} . \\
3 \mathrm{f} .\end{array}$ & $\begin{array}{r}10 \mathrm{~m} . \\
4 \mathrm{f} .\end{array}$ & $\begin{array}{l}4 \mathrm{~m} . \\
5 \mathrm{f} .\end{array}$ & All $\mathrm{m}$. \\
\hline
\end{tabular}

Note - Relatively few children came to necropsy.

Duration of the disease from presumed onset clinically till death

2 years 2 months (25.8 months).

(From data of 40 cases where records were adequate). 


\section{DISTRIBUTION OF LESIONS}

1. Reticulo-endothelial System

(a) The lymph nodes.

It is axiomatic that lymph nodes are affected. To establish the absence of lymphadenoid lesions would require the recovery and microscopical examination of all the nodes in the body, a task which attracts few. Reasonably well accredited cases without apparent lymph node lesions have been recorded but they are very rare, sone further account will be given where apposite.

Involvement is commonly assumed to be universal. This is certainly erroneous; normal lymph nodes can be found at necropsy in most cases if sought assiduously. The most likely sites include the mesenteries, the antecubital fossae and the popliteal fossae. The clinical and morbid anatomical firdines often suggest that the lymph node groups become involved successively. The old observation that the disease appears first in the neck, usually on the left side, has much support, but it does not justify the postulate that the disease actually starts in this site. Symmers (1924) emphasized that deep mediastinal or retroperitoneal nodes were often conspicuously enlarged while the peripheral ones were small. In some cases all nodes appear to enlarge simultaneously (Callender 1934) but these cases are very rare and are not typical Hodgkin's disease. Restriction of involvement is comroner than might be expected, its interpretation is very difficult. The most obvious cause, namely premature death of the patient cannot always be invoked to explain it. In one case observed personally a single lymph node focus appeared to be the only lesion. (Case G.R.I. P.M.100/54). 
This patient was an elderly man of solitary habit who was found moribund in his lodging. Necropsy revealed a large cerebral softening. An incidental finding was a solitary hard white tumour nodule in the right side of the neck which was thought to be a carotid body tumour. The lymph nodes generally were not remarkable naked-eye, none were sectioned. Microscopy of the cervical nodule revealed typical sclerosing Hodgkin's disease in a lymph node.

Affected lymph nodes are almost invariably enlarged. The increase in size is sometimes prodigious, few conditions besides Hodgkin's disease are associated with so gross an hypertrophy. Untreated, the nodes tend to remain discrete.

Different grades of maturity of the lesion are common, and alterations of the microscopical picture are also frequent. This change may amount to frank sarcomatous metamorphosis, in a later part of this work the phenomenon is studied.

Table.

Analysis of lymph nodes found to be involved at necropsy in 49 cases of Hodgkin's disease. G.R.I. series. 1900-1951.

$\begin{array}{lcc}\text { Site. } & \text { Number of Coses. } & \text { Percentage. } \\ \text { Cervical. } & 39 & 80 \\ \text { Axillary. } & 30 & 61 \\ \text { Inguinal. } & 24 & 38 \\ \text { Mediastinal. } & 39 & 80 \\ \text { Retroperitovieal. } & 36 & 73 \\ \text { Mesenteric. } & 22 & 45\end{array}$


(b) The spleen.

In most cases the spleen is ultimately involved. Hodgkin's proposition of the sequence has been quoted, it is the general experience. The incidence of morbid change in this organ is variously reported between 56 per cent and 78 per cent of cases, Hoster et al (1948). It is clear from study of these references that distinction between enlargement and histological evidence of deposits of the new tissue is not always made. A further difficulty is that some figures are drawn from clinical studies during life.

In the G.R.I. series spleen weights were recorded in 36 cases. The average was $638 \mathrm{~g}$. With extremes of $170 \mathrm{~g}$. and $3,200 \mathrm{~g}$. This is close to Symmers' series which gave an averase of $802 \mathrm{~g}$.

In 43 of the G.R.I. series the naked eye appearances were described, all shewed visible lesions i.e. 88 per cent of the full series.

The form of the lesion varies. In most studies the classical hard-bake is the cormonest. Karsner (1910) however insisted that miliary deposits were the most frequent. On section the spleen is usually firmer than normal and the cut surface is more or less studded with yellowish-grey or whitish-grey nodules which vary in size from a millet seed to a walnut. These nodules have a subtle individuality. Their outlines are not so rounded as metastatic cancer nodules but are irregularly angular. They are at once incongruous. They are sharply demarcated from the red pulp, small satellite nodules may be seen round large ones. If the slice is allowed to oxygenate a deep crimson line develops round the edge of the almost white nodules producing one of the beautiful pictures of morbid anatomy. 
Necrosis in splenic deposits is commoner than in any other site. The remarkable appearance of the organ has prompted the true Pathologists - to whom all life is pathology (Boycott) - to draw names for it from that vital source of inspiration and simile - the kitchen. 'Hardbake' spleen is a term of some antiquity ranking in dignity with 'sago' spleen; yet the fancied resemblance to this confection is less apt than the 'bauenwurst' of Jenz! (In Benda's house the kitchen door may have been open so that his comparison was drawn from the porphyry of the back steps).

Table.

G.R.I. Series (1900-51). Splenic lesions in 43 cases.

Form of lesion.

Miliary nodules.

Solitary or scanty large nodules.

Typical Hard-bake.
Number of cases.

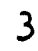

4

36

It has been held that the disease may occur solely in the spleen or at least arise primarily in it. Exclusive involvement has been reported by Symmers (1909), Zieglex (1911), Wade (1913), and Isaacson, Spatt and Grazzel (1947). None is really satisfactory. Ziegler's contention was not properly supported. Symmers' case and Wade's case both presented with splenomegaly during life; Hodgkin's disease was discovered to be the cause, but in neither did the patient come to necropsy. The last reference is a more satisfactory account but necropsy revealed doubtful lesions in the liver and definite ones in the bone marrow.

(c) The bone marrow.

It is difficult to assess the frequency of Hodgkin's disease lesions in 
in the bone marrow. Complete examination of the bones is a practical impossibility in ordinary necropsies. Steiner (1943), in support of the interpretation that the disease was a reticulo-endothelial disorder rather than a lymphoid tissue one, insisted that marrow lesions were commoner than supposed. He supported this by many high figures drawn from the literature. Figures recorded vary between 6 per cent and 23 per cent but it has been postulated that the upper limit would be nearer 100 per cent if search were adequate. It is the opinion of most observers that bone marrow lesions are relatively late in occurence. In practical study of this problem it is pertinent that the lesion can be extremely difficult to identify with certainty microscopically. Bvidence of secondary anaemia is common and at necropsy extensive gelatinous degeneration is a feature in many cases.

It is almost certainly exceptional for the bone marrow to be replaced by Hodgkin tissue to a significant degree.

\section{(d) The liver.}

This organ is fairly commonly affected. Collated statistics give a frequency between 38 per cent and 60 per cent. In the G.R.I. series the data are unfortunately incomplete. In 30 cases weights were given and yield an average of $1868 \mathrm{~g}$. According to Shennan (1927) the average weight for the liver is $1600 \mathrm{~g}$. , thus the increment is about onefifth. Visible deposits of the new tissue were seen in 29 cases (59 per cent), in the remaining 20 cases there was not any record. The appearance of the deposits is similar to the splenic lesions but necrosis is rare.

In small microscopical deposits the location is almost invariably in the portal tracts. This suggests that these loci favour origin of 
the new tissue, and argues against the reticulo-endothelial cells of the liver (Kupffer cells) being the originating cells, otherwise a midzonal or central initiun would be equally represented. The other systems.

The lesions of Hodgkin's disease have been reported in almost every organ and tissue of the body. It is very probable that the deposits develop in pre-existing lynphoid tissue in these sites, but this tissue itself may be ectopic. Ectopic lymphoid tissue is comon, and may be developed as a compensatory formation when the normal lymphoid tissue is diseased or ablated. There is evidence that this joes not readily occur under experimental conditions (Saunders and Florey 1940). In Hodgkin's disease the morbid encroachrent is relatively protracted and occurs in a measured piece-meal fashion; this time factor favours the chances of development of new lymphoid tissue.

The table appended below sumarises the location of lesions outwith the reticulo-endothelial system encountered in the G.R.I. series. 
Table.

Organs or tissues found to be involved at necropsy in 49 cases of Hodgkin's disease. G.R.I. 1900-1951.

Organ or Tissue

Lomph nodes.

Spleen.

Liver.

Bones.

Stomach.

Srnall bowel.

Gall bladder and ducts.

Peritoneum, omentum.

(Ascites).

Bronchi)
Lungs
Pleura.
Diaphragm.

Kidneys.

Ureters.

Pericardium.

Veins.

Hypophysis cerebri.

Thymus gland.

Adrenal gland.

Skin.

Dura mater.

Brain.
Number of cases

49

43

29

19

\section{2}

2

1

3

4

9

5

8

1

1

1

1

1

1

1

1

1
Percentage

100

88

59

39

4

4

2

6

8

18

10

10

16

2

2

2

2

2

2

2

2

2

Note on findings in the other systems.

Alimentary system. The liver excepted, this system is relatively immune. Lesions are reported in the tract in 1 to 5 per cent of cases, the majority are gastric. The deposits usually commence as sub-mucosal nodules which mature to form malignant ulcers. Microscopically these lesions are usually more cellular than nodal ones. Ascites is common 
but rarely is the effusion haemorrhagic.

Respiratory system. Involvement of the bronchi, lungs and pleurae is relatively common, sometimes this is obviously due to extension from mediastinal nodes. Figures from the literature vary from 15 to 33 per cent. Effusion is very comon, and like the ascites in this disease, blood is rare in the fluid. Extension of the disease from the diaphragmatic pleura into the diaphragm is common, in fact this is the chief expression of infiltration of muscle observed.

Genito-urinary system. Renal deposits are reported in 8 to 16 per cent of cases, even the lower frequency is in marked contrast to the incidence of cancer metastases in renal tissue. Ureteric involvement is rare.

Cardiovascular system. The pericardium is probably the commonest site, occasionally with extension into the myocardium. Invasion of veins has been reported (Ross 1933).

Endocrine system. Reticulo-endothelial tissue is located in the vessels of the hypophysis cerebri and adrenal glands. However the incidence of deposits in these is low. Thymus is commonly reported in children as a locus, but rarely in adults.

Integument. Deposits are unquestionably rare although café-au-lait pigmentation and pruritus are common.

Central nervous system. Deposits are very rare in the brain, less so in the meninges. (In the G.R.I. case reported extension was from the nose).

In the preparation of the foregoing survey it became obvious that there was much variation between the standards of different pathologists. /These 
These discrepancies inevitably modified the results. As one becomes increasingly interested in a subject one's standards improve and one becomes correspondingly critical. The satisfactory antidote to this enhanced scepticism is to perform the task oneself. This has been done and the following sections embody the work and results of this endeavour. 


\section{THE BIOPSY SERIES}

'Nulla est alia pro noscendi via, nisi quam plurimas et morborum et dissectionum historias tam aliorum proprias collectas habere et inter se comparare'.

9

INorpagni; De sedibus et causis morborum Lib XIV

This classical advice is difficult to follow. There are many practical obstacles to the managenent of large series and the ultimate analyses, whilst ostensibly objective are dependent in some measure on subjective discrimination. Full appreciation of the morphological affinities of Hodgkin's disease would require a comprehensive series of primary lymphadenopathies, entailing a formidable amount of material and study; attainments beyond the reach of those whose research is necessarily a spare-time occupation. The present series is an atternpt to implement Morgagni's advice so far as it has been possible for me to accomplish. The series is primarily a collection of biopsy preparations of Hodgkin's disease. It includes less familiar and atypical forms of the lesion, and also a few cases of various lymphoid tissue tumours in which I attempt to discern some homology with Hodgrin's disease. In addition an inevitable miscellaneous group is included; this embraces lymphoid tissue disease of indeterminate nosology, remarkable reactive changes in lymph nodes, metastatic carcinoma nasquerading as Hodgkin's disease, and several unsolved problem nodes.

The collection.

Altogether a total of 222 cases were studied. The intention was to restrict each serial number to an individual patient. In most cases including those with serial biopsies or later necropsy, this was /effected. 
this was effected. In three patients however serial biopsies have been given separate serial numbers viz. Ross; 71 and 76. , Topping; 75 and 81., and Finnigan; 73 and 135. The series was almost complete when these irregularities were discovered and they have been allowed to stand so as to avoid re-numbering so many protocols blocks and slides. Sources. of the collection

Serials 1-58 inclusive refer to a collection built up by my father, the late Professor John Shaw Dunn. These slides were found in the house after his death and were passed to me by my mother. The information available for most is restricted to identity and occasional abbreviations on the labels. Serials 1-19 are from patients at the Western Infirmary of Glasgow in the period 1910-1913. The remainder are derived from Birmingham, itanchester and several other hospitals. (Several private cases and some of unknown origin are interpolated among this hospital series).

The serials 59-210 were collected by myself. These are chiefly from the morbid anatomy routine of Glasgow Royal Infirmary but some are from outside.

Note on the material and methods

Many of the serials 59-222 were prepared by myself. For fixation formol-corrosive, saturated corrosive sublimate, Heidenhain's Susa, or Bouin's solution with reduced acetic acid ( $I$ per cent instead of 5 per cent) were used. The mercury fixatives were found generally superior for satisfactory staining but the modified Bouin's fluid was very good and the tissue cut well. Ten per cent formol-saline or formol-water was found to be poor, especially when the tissues were fresh and warm. 
Zenker's fluid without acetic acid was tried but the well-known technical difficulties it produces reduced its value. It was found better to slice nodes at right angles to their long axes rather than adopt the orthodox method; distortion was less and the whole circumference of the capsule was more readily studied.

In all the serials $1-58$ at least one section stained with haematoxylin and eosin was available and usually there were several. In the latest numbers of this group some connective tissue stains and silver impregnations were available. In the majority of the remainder sections stained by haematoxylin and eosin, van Gieson's method, and impregnated with silver were prepared. In a limited number Dominici's stain, Mann's stain, dilute eosin, Mallory's blue and orange, Masson's trichrome and other special stains were used. There was not any doubt that good haematoxylin and eosin surpassed all other methods in general usefulness. Eosin is an excellent polychrome stain and often indicated phenomena which special stains confirmed later. Van Gieson's stain was the most valuable adjuvant, it is more delicate than the aniline blue methods. Silver impregnation proved of doubtful value, it rarely added to what simpler methods already shewed, and introduced a new set of artificial standards.

For this study a foolscap pro-forma was made so as to record systematic descriptions of each specimen which would be mutualy comparable. The following is a facsimile, reduced and condensed. 
Available data.

Specimen.

Persistence of normal structure.

\section{Identity.}

Capsule. Thickening, hyalinisation, concentric re-formation,

Habit of growth. infiltration.

Fibrosis. Prabecular, Peri-vascular, diffuse fibril, fibriliar, symplasmic transformation, coralline.

Reticulum cell proliferation, epithelioid, morbid mononuclear, multinuclecte giant-cells, typical, atypical.

Josinophiles. Plasina cells. Neutrophiles. Lymphocytes. Witoses. Necrosis.

Diagnosis, affinities.

This schema was evolved after a preliminary survey and study. The scope and meaning of the various headings will be fully explained in the analyses of the results.

These original protocols being unsuitable for presentation, comprehensive synopses of each have been made (See Appendix A.). It will be noted that throughout the work interpretation has been governed by the hypothesis that Hodgkin's disease is the central expression of tumour in Iymphoid tissue. The same pattern of scrutiny has been applied to all specimens. For each serial a diagnosis was recorded and, where relevant, explanatory remarks and etc. were added. The serials have been re-examined repeatedly over the last five years and the ultimate assessments embody the results of these revisions.

Histories where available or traceable are included. The clinical features are always of value, exactly as naked-eye appearances are helpful. Yet it is remarkable how often the history recounted little more than a painless swelling at a lymph node site. The fate of cases which I encountered personally were traced as far as possible but it is admitted that this proved singularly difficult. In this institution, (Glasgow Royal Infirmary), patients pass through so many departments that follow-up attempts by one individual are exasperatingly hard. In 
fact what may be termed the administrative aspect of the series proved the most tedious and frustrating task.

The microscopical examination of each specimen was as thorough as possible. The slides were first examined under very low power, a $5 \mathrm{X}$ hard lens, and then under a magnification of $20 \mathrm{x}$. These low power studies proved of value in many unexpected ways. Jach section was then submitted to scrutiny with the ordinary low and high-power objectives using a Zeiss 4 eyepiece. To examine a whole section thus, occupied about 2 hours. This is fatiguing, and it was found that oil illumination was far superior to electricity for these protracted observations. The restfulness of oil illumination is remarkable, it is probably related to the constant slight movement of the flame which reduces fatigue. Another old-fashioned device was helpful namely the use of centre-stops in the condenser. These can be made to produce a quasi dark-ground effect which helps to detect fibrillae \& etc. Filters were an aid. By the use of a pale blue filter, Wratten No. $78 \mathrm{AA}$, inconspicuous eosinophiles were more readily detected, and conversely by using a red filter, Wratten's tricolour red, the granules of eosinophiles disappeared permitting study of the nuclei. Defects in staining, e.g. in old preparations, could be partly remedied on this principle. In an effort to assess activity the numbers of mitoses were counted in standard fields. The size of the high power field was adjusted with the draw tube till it was $0.1 \mathrm{sq} . \mathrm{mm}$. Five random fields of this size were surveyed and the average taken. Findings and analysis of the series

The task of presenting a well-bälanced and critical account of the 
findings in tha series has proved formidable.

Inherent in microscopy is a lack of objectivity, and there are practical limits to illustration. Truthful and convincing generalisations are therefore difficult to make and present.

Hodgkin's disease is above all capricious, the elasticity of appearance is remarkable and except for the characterization of important variants, classification is somewhat futile. In the belief that descriptions of the major variants might at least help in the microscopical diagnosis of the disease this has been done. So far as possible use has been made of existing categories, since to introduce new ones from studies of an already eclectic series is absurd. In the application of the classification evolved some overlap was common and at points decisions had to be arbitrary

It is not advocated that the analysis offered should be adopted but rather that it should serve to indicate the appearances Hodgkin's disease may present and point out criteria and pit-falls in diagnosis. It is urged that study of the protocol synopses in Appendix A. will give the reader the best appreciation of the work. 


\section{TYPICAL HODGKIN'S DISEASE}

In the following serials all diasnostic criteria are net. i. 3. 6 .

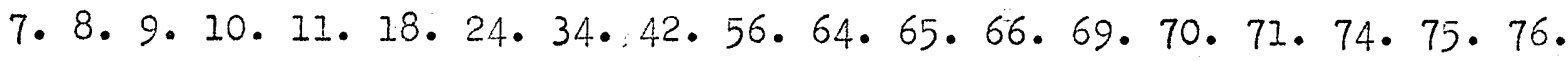
79. 81. 83. 89.91.92.99. 105. 106. 113. 114. 115. 116. 120. 122. 126. 132. 138. 184. 188. 195. 201. 211. 212. 216. 217.

Total 48 examples from 46 cases; with the exception of Serial 81; all are node lesions and represent all grades of maturity. Features of the typical lesion

(A) Eabit and mode of growth

The initium of the new tissue is commonly nodular in form, and this characteristic may persist. It is usual for it to develop in the deep pulp of the node but it is occasionally cortical in position. This primary focus of the disease is generally single. As it grows it rapidy tares up the surrounding lymphoid tissue and also displaces it; in this study this has been terned the exodic growth phenomenon. The derarcation between the periphery of the expanding nodule and the surrounding lymphoid tissue is best appreciated by low-power examination of the order 5-20 diameters. In many cases high-power scrutiny can confirm that the peripheral lymphoid tissue shews only physiological reactive hyperplasia. In 22 cases this partial or incomplete involvement of the node was a feature. The surviving lymphoid tissue may contain solid follicles, follicles with pale centres, and even sinus tissue. In many othermise totally involved nodes snall aggregates of normal small lymphocytes, survival islets, were found.

In 10 cases a further phenomenon related to this expanding nodule conformation was detected namely the development of a swathe or swathes 
of new connective tissue roughly garallel to the true cupsule. This his been temed concentric re-fornation of the capsule.

As the lasion matures all or almost all of the orifinal tissue of the node is replaced by the new tissue. In these established lesions a more or less segmental or folliculoid pattern is common. This was detected in 24 examples. Ultimately the picture is one of irregular islets of the new tissue set in a coarse fibrous matrix.

\section{(B) The new tissue}

This consists of the products of proliferation and fibroblastic transfornation of reticulum cells, mature and inmature lymphocytes, eosinophiles, neutrophiles and plasma cells. Even in the earliest stages all components are present. It can be stated categorically that the earliest recognisable stage of typical Hodrkin's disease is the presence of this characteristic new tissue, which it has already been seen may be only focal. Fictures of reticulum cell proliferation without fibrosis, Iynjoid hyperplasia, or general loss of node architecture alone are not ever safely labelled early Hodgkin's disease.

\section{Reticulum cell proliferation}

This is invariable and produces several characteristic cells. Sometimes it is distinctly focal, especially where the gross pattern ia strongly folliculoid. The pulp reticulum cells are prinarily involved but the others may partake. Four main cell-types can be described.

(a) Morbid mononuclear reticulum cells.

These are always present, they are at least twice the size of normal reticulum cells, certainly in a nuclear respect. They are characterised by large plump nuclei which are usually oval or spheroidal and posess a 
well developed nuclear mernbrane. Nucleoli are comon and may stain as plasmasomes or as Karyosomes. The chronatin is generally aggregated.

(b) Hodgin eiant-cells.

These are invariably present. Mitoses are rarely seen in these cefls and it is comnon for them to be lost in the course of preparation. Their form is remarkably protecu.

(c) Ipithelioid cell-types.

These can generally be found but are sometimes absent. They correspond closely to the epithelioid cells of early tuberculous lesions or to macrophages. The nuclei are characteristically smaller than those of (a). There present these cells are often seen in closely set clusters.

(d) Fibroblastic cell types.

These are always found. They correspond to the plump mildiy pleiomorphic fibroblasts of active granulation tissue, but are usually larger. 2. Fibrosis.

True collagenous fibre production is invariable. There may or may not be reticulin increase. Fibrosis is expressed in several ways.

(a) Augmentation of pre-existing fibrous tissue in the node. capsule and trabeculae. It is frequently gross. The mechanism is difficult to follow but it appears in some cases to derive from cells (a) above.

(b) The appearance of fibrillae.

This occurs in the new tissue pulp. The origin of these most delicate collagen fibres may be traced to morbid reticulum cells of types (a), (b) and (d), apparently forming in the cell cytoplasm. A 
small number of fibrillae can be traced to type (c) cells. Here they appear as condensations of contiguous cell margins. This mechanism has been termed symplasmic transformation. It is very similar to the fibrosis process in tuberculous lymphadenitis.

(c) The development of fibrils.

(The term fibrilla is used as a diminutive). These originate as fibrillae and are regarded as condensation and accretion products of them.

(d) The formation of coralline deposits of collagen.

These may take the form of tortuous cylinders which have many outlines or of sieve-like structures resembling red coralf (Corallum rubrum). They may be related to antecedent fibrils but are often seen well apart from them. Their origin is somewhat mysterious, they are very rarely of vascular source.

(C) Perivascular lamellation.

This form is very characteristic and particularly affects arterioles. In transverse section the vessel is seen surrounded by concentric, but often incomplete, collagenous lamellae.

Analysis of the studies made reveals that the most constant forms of fibrosis in the lesion are fibrillae and fibrils. In general terms the more cellular the lesion the less is the fibrosis. The remaining types are comnoner in slowly evolving examples of the disease. It may be added that post-infarction scarring is occasionally seen, it appears to result from granulation tissue formation round necrotic foci, the process is rarely complete.

3. Iymphocyte series cells

The normal lymphocytes of the node always diminish. The decrease 
may be slow or rapid and is roughly proportionate to the rate of frouth of the new tissue. It is extremely com to find a proportion of irmature lympocytes, including the exriest precursors. These imature linnocytes nay virtualiy replace the norul ones. Their immaturity is reflected by appreciable enlargement and leptochromicity of their nuclei.

4. تosinophilia.

In the typical disease eosinophiles are present in sufficient numbers to arrest attention at once. In most cases they are typical binucleate blood-stream forms. The cranules are fewer and coarser but this is due to histological as oposed to cytolopical preparation. Ihey are very capricious in distribution, often tending to be focal. One fairly charucteristic site is in the interstices of new young connective tissue. Josinophiles with single lymphocytoid nuclei are sometimes present. In 7 cases ( 14.5 per cent) these cells were denonstrable and transitions could be traced with some conviction. Special methods were essential for the purpose, the best results being obtained with Dominici's stain.

5. Neutrophiles and plasma cells.

These cells can always be found. Nost often they are scattered diffusely through the new tissue but may form small ageregetes. Neutrophiles are occasionally sufficiently numerous to indicate superadded inflamation and mav also be notable round necroses.

\section{Necrosis.}

In 22 cases of the series necrosis was absent in any form. In 9 cases it was limited to scattered ndividual cells and in the remainder 
occurred in small foci. It is generally a quiet necrosis of coagulative fibrinoid or caseous type, which evokes very little reaction. 7. Wtotio activity.

The results of this survey proved disappointing, the figures recovered pretend in acouracy which is false. In 37 cases 1 or less nitotio fidires presented in each standurd high power field, but in severul cases where multipia sections vere exained great discrepancies apoecred.

II. ALOST TYPIGAL HODGKIN'S DISEASE.

Due to the strict apulication of criteria the following serials were excluded from the category Typical Hodgkin's disease. 14. 16. 17. 96. 98. 101. 107. 110.121. 129.196.

Total 11 examples from 11 cases.

It is emphasized that deviation from the characteristic lesion was minimal.

The group is constituted as follows. Celluiarity above average.

6 cases (14. 16. 17. 96.

$$
\text { 101. 196.) }
$$

Presence of rare Lanğhan!s giant-cells. I case. (98). Läre morbid reticulum cells numerous. I case. (107). Josincphizes below averaze. 1 case. (110). Conspicuous epithelioid cell foci. 1 case. (121). Anomalies attributable to site.

1 case. (129). mesenteric node. 
II. ATYPICAI HODGKIN'S DISEASE.

This is a large category. In all 75 examples were encountered in the series. The atypism varied from comparatively minor deviations from the typical lesion, to relatively major alterations. Cases representing these extremes are easily separated but analysis of the intermediate ones is difficult because of the lack of consistent objective criteria. In general terms the whole group is considered under two main headings viz., major and minor variants. These subgroups are then divided on a basis of the most obvious mutual differences.

A. Miajor variants.

I. Soft cellular and tumour-like Hodgkin's disease.

In this form the lesion is still recognisable as Hodgkin's disease. In some examples this recognition depends on much experience, and in others the lesion is passing to another category. A proportion of these cases shew strong morphological affinities to other lymphoid tissue tumours.

This category is divisible into two depending on whether eosinophiles are retained or not.

(i) Eosinophiles retained in normal or slightly reduced numbers. Serials. $\quad 31.32 .40 \cdot 60.62 \cdot 63 \cdot 87 \cdot 100 \cdot 111 \cdot 112 \cdot 118 \cdot 119$. 131. 193. 198. Total 15 cases.

In serials 111. 119. 193. the lesion was cellular but not tumour-like; in serials 40.60.63. 100. 112. 118. 131. 198. tumour-like features were more pronounced; in serials 31.32 .62 .87 . neoplastic features were extreme.

Features. Persistence of normal lymphoid tissue is less than in the /typical 


\section{7.}

typical disease but the Eeneral habit of gronth is the sume. The eoxdic growth phenomenon is less common and so is concentric re-foruation of the capsule. The node cupsule does not usually siow narked hyaline thickening and it is sometimes infiltrated with the new tissue.

Fibrosis is characteristically much less in total amount and is expressed chiefly by fibrillary and diffuse fibril forration. It is mainly on this decrease of fibrosis that the cellular appearance depends.

The reticulum cell proliferation is usually florid and is productive of smaller cells than in the typical disease. Horbid mononuclear cells and Hodgkin giant-cells are both affected. It is not uncomon for this cell proliferation to be focal. Josinophiles while persisting tend on the whole to be reduced. The lyraphocytes are comonly large and imature in type. Plasma cells and neutrophiles are little affected but necrosis especially on an individual cell basis is comrnon. Witotic activity is either normal or increased, these lesions are rarely indolent.

The tumour-like designation is applied where an unwonted homogeneity is apparent in the new tissue. All grades are encountered to a point where it is equivocal whether reticulum cell sarcoma is not an equally correct microscopical diagnosis. It is noted that where eosinophiles persist netamorphosis to other lymphoid tissue tumours is not seen.

\section{(ii) Josinophiles reduced or lost.}

Serials. 57. 67. 72. 77. 80. 97. 102. 103. 127. 128. 130. 143. 154. 159. 161. 176. 180. 181. 185. 205. 207. 213. 218. Total 23 examples. In serials. 57. 72. 80.127. 130. 143. 154. 176. 180. 181. 205. 
the lesion was cellular with relatively unimpressive tumour-like appearance; in Serials 67. 77. 97. 102. 103. 128. 159. 161. 185. 213. 207. 218. neoplastic features were pronounced.

Features. These are essentially the same as in the previous heading. It is noted that the total encountered is larger and that proportionate more of the cases qualify for tumour-like affinities. While the general direction of affinity is towards reticulum cell sarcoma other variants are seen. In Serials 72. 127. 180. 207. an approach to the microscopical features of lyrnphosarcoma is notable, and in Serial 80 some features of Brill!s disease are observed. 2. Diffuse fibroblastic Hodgin's disease.

This uncomaon variant was present in Serials 124. 140. 153. 168. 219. 220. Total 6 cases.

This variant is characterised by a striking increase of fibroblastic cells, the majority of the cells present being of this character. Typical morbid mononuclear and Hodgkin's giant-cells are present but may be absent in individual sections. Eosinophilia is absent or less than usual. Small lymphocytes are scanty. In some cases whorling of the new fibroblastic tissue is a feature recalling indolent fibro-sarcoma. There appears to be little tendency for the fibrillae and fibrils to augment by accretion so that these lesions often present an openwork appearance. In two of the cases epithelioid cells were conspicuous.

This variant is treacherous, in Serial 168 the diagnosis was only made after many sections had been taken. Thile six cases is a very limited series it appears that these cases always do bady. They react 
very poorly to radiotherapy, and most of these patients were dead within six to twelve months of diagnosis. Clinically they also presented difficulties; lymphadenopathy was often slight, and weakness was usually the chief complaint.

\section{Lymphoblastic Hodgkin's disease.}

Serials. $53.85 \cdot 158.214$. Total 4 cases.

This is not a very satisfactory category because lymphoblastic differentiation was not the sole abnormality nor is it restricted to these cases. However in these four examples the phenomenon was pronounced.

Features. Undue prominence of immature lymphoid series cells is seen in all. The appearances indicate that the reticulum cell proliferation is given over particularly to production of lymphocyte precursors. Fibrosis is slicht and in all except one, eosinophilia is lacking. All are considered nearer to Hodgkin's disease than any other variant of lymphoid tissue tumour, but confusion with lymphosarcoma or Brill's disease could arise. (See protocols of Serial 85. 214). 4. Benign Hodgkin's disease.

The microscopical appearances described for this entity were observed in Serials 2. 54. 137. Total 3 cases.

In only one case, Serial 54, was a history of relatively long survival recorded. Thile there is little doubt that this recently described lesion is real enough it is dangerous to diagnose because it is very rare. The essential features appear to be conspicuous fibrosis of the capsule and trabeculae with resultant segmentation of the node, little if any fibril-type fibrosis and an indolent slightly immature 
Iymphoote population in which rare morbid reticulum cells are seen. (The relevant protocols should be consulted for details).

In Jerials 23. (30). 33. Iesions which were interpreted as links between typical Hodgkin's disease and the above variant were encountered. Features. In these cases the ribrosis is widely scattered but not so diffuse as in the typical lesion. The reticulum cell proliferation is below average und pleionorphisn is reduced. Fosinophiles are low and the shall lyphocytes are nature tjpes. (Serial 30 is anomalous, it is nearer to the typical disease but is a very early lesion).

5. Atypical Hodgkin's disease with prominent epithelioid cells. Serials. 47. 68. 117. Total 3 cases.

In these cases epithelioid cells were very conspicuous but were not tire only aunorual feature.

Features. In this form islets of epithelioid cells occur in patchy fashion throughout the new tissue. Symplasmic transformation fibrosis is comonly seen in relation to them. Differentiation to this cell appears to occur at the expense of morbid rononuclear and Hodgkin giantcells. Fibrosis of the usual form is slight and eosinophiles are few. Differentiation from endothelial tuberculosis can be very difficult. 6. Miscellaneous atypical Hodgkin's disease lesions.

The following did not correspond to any of the variants listed above. Serials $44 \cdot 59 \cdot 206$.

Serial 44 represents an extraordinary example of the disease. All components of the normal complex are present but the lesion is a parody of the disease.

Serial 59 is Hodgkin's disease growing in the integument. Pseudosuppuration is present and granulation tissue complicates the picture. 
Sertal 206. The appearances in this case strongly favour a sinus origin of the lesion. Lorbid reticulum cell production is seen in sinus sites but is not restricted to them. (Josinophiles are also much reduced).

B. Minor atypical features in Hodgkin's disease.

1. The absence of eosinophiles in otherwise typical lesions.

This wus observed in Jerials 15. 30.70. 80. 134. 162. 176. 181. 219. Total 9 cases.

In otherwise typical lesions this anowaly does not invalidate the diagnosis of Hodgkin's disease; eosinophilia is characteristic but not essential. It may sometimes be absent at the level first examined but present in further sections. In late lesions, especially after irradiation, a marked reduction of eosinophiles is common.

2. The presence of abnormal nultinucleate giant-cells.

These were seen in Jerials 7. 13. 21. 53. 124. 153. Total 6 cases.

In berials 7.13 .21 . the cells ware typical Langhan's type giantcells and constituted the only aberrant feature. Ividence of tuberculosis was lacking.

In Serial 53 the lesion was tumour-like and the cells corresponded closely to foreign-body giant-eells. In Serials 124. 153. the lesions were also atjpical and the cells of Langhan's type.

This phenomenon possibly reflects the ability of some reticulum cell descendents to react physiologically, there is a little evidence that antagonism exists between the Langhan's cells and Hodgkin giant-cells. 


\section{FALSE HODGRII'S DISTIST.}

1. Netastatic carcinoma masuuerading as Hodgkin's disease.

This was encountered in Serials 95. 174. 222. Total 3 cases.

Features. This hazard in diagnosis is rare. It is most likely to cause difficulty where carcinomatous invasion of a ljmph node is aiffuse, the individual tunour cells lying out of contact with one another. Topical eosinophilia may accompany infiltration of this variety and brings the similarity to Hodgkin's disease closer. Diffuse fibroblastic reaction to the invading tumour also mimics the true disease. Study of further sections is the nost helpful measure in establishing: the true diagnosis.

In Jerial 95 the diäbnosis remains open. The case has been followa for almost four jeurs without any development of a pathognomonic evolution. This latency is unusual for primary or secondary tumour but the appearance are microscopically outwith the linits of reactive change. In Serials 174. 222. the diagnosis of metastatic carcinoma is sustained. Note. Metastatic nelanoma in lymph nodes may mimic Hodgkin's sarcoma. In these cases the tumour is unpigmented and the large cells closely simulate morbid reticulum cells.

2. Pronounced physiological reaction simulating Hodgkin's disease.

In the following serials marked physiological reactive change was encountered. Serials. 84. 86. 94. 148. 152. 155. 157. 165. 171. 178. 182. 183. Total 12 cases.

Features. In the majority of these cases the mimicry is not close and is often restricted to single characteristics. In four examples however, Serials 94. 148. 155. 171., real difficulty in separation from early or atypical Hodgkin's disease is present. 
In these reactive hyperplastic states there is a more or Iess conspicuous proliferation of reticulum cells. The majority of cells resulting have smaller nuclei than those in the proliferation characterising Fodgin's disease and giunt-cells are very rare. ft the same time a few typical morbid nononuclear types are not uncommon. Attending this hyperplasia there is some general loss of definition of the normal architecture but it is never complete. Fibrosis other than capsular and trabecular thickening is unusual, and this deficiency is most helpful in differentiation. Topical eosinophilia may be present but is rarely up to the normal level observed in Iymphadenoma.

In Serial 94. difficulty arises because of apparent fibrosis in the pulp of the node but this interpretation is false as the connective tissue can be traced to the hilar intrusion.

In Serial 148. the size of the node was considerably above normal which is a very suspicious feature. The subsequent history of this patient is worth relating because it justifies the rejection of the orifinal diagnosis. After being told the nature of his malady and advised to attend for X-ray therapy, he promptIy entered a recruiting office and was accepted for the Amy without demur. He went overseas with his unit and served three yeurs with the Colours in the Far East. On his return to the United Kingdom he was relegated to the Reserve and took up his old work. Through the courtesy of the family physician, Dr. Boyle, who has helped me most generously, I have been able to trace the patient to date and can confirm that he is alive and well.

Serial 155 is an interesting lesion probably attributable to X-ray therapy. Endarteritic thickening of the blood-vessels in contradistinction to perivascular thickening is notable. 
Serial 171 is an example of reactive change in which some follicles are very large. Superficially the appearances recall Brill's disease and changes suggestive of early tuberculous inflamnation are also seen. 


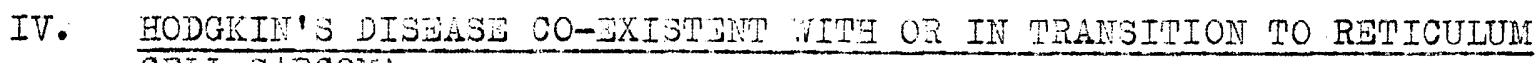
CEII 3LRCONA.

These cases were found in the folloving Jerials and grouped thus.

1. Recognisable typical or atypical Hodgkin's disease accompanying some variety of reticulum cell sarcoma.

Serials. 22. 190. 191. 210. Total 4 cases.

2. Lesions where a process of transition between Hodgkin's disease and reticulum cell sarcorna is apparent.

Serials. 93. 125. 160. 188. 199.202. 204. 209. Total 8 cases.

3. Hodgkin's disease co-existent with Brill's disease.

Serial 113 .

Features. 1. The form of reticulum eell sarcoma may be either predowinantly a sten-cell growth, Serials 190. 191. 210., or Hodgkin's sarcona type 2 of Tarthin (reticulo-endothelioblastoma), Serial 22.

The lesions may be seen together in the same node or separately in different nodes. Iransition is discernible in three cases, Serials 22. 191. 210.

2. In this group the lesion is Hodginin's sarcoma in all the cases except one, (Serial 204), where further transition to a stem-cell type of growth was recorded later.

The most important changes in this development of Hodgkin's sarcoma are a aisporportionate increase in morbid reticulum cells, repression of eosinophilia, reduction of lymphocytes and increase in grosth rate. The pleiomorphism of Hodgkin's disease is retained partly.

3. This example is unique, representation of the lesions is about equal. 
V. BETIOULUH CELL SARCOLA.

This tumour was diagnosed in the following groups.

A. Fure reticulum cell sarcoma.

Serials. 5. 25. 26. 35. 39. 43. 48. 52. 61. 73. 78. 82. 109. 133. 135. 141. 142. 156. 221. Total 19 cases.

B. Intermediate reticulum cell sarcoma.

Serials. 12. 19. 1. 49.50.90.163. 194. 200. Total 9 cases. inalyses of both groups was made with particular reference to features in wich they shewed morphological affinities to Hodgkin's disease.

Features, Group s. Folliculoid or segmental habit is seen in 7 cases but is absent in the remainder. Fibrosis in the forms displayed in Hodgrin's disease is detectuble in 15 cases. In all, morbid mononuclear reticulun cells own be found, though the actual number present may be very small. Giant-cells identical to or closely resembling Hodgkin giant-cells are seen in 17 cases. Fosinophiles are present in only. 1. It is considered that the morphological affinities to Hodgkin's disease are near in 3 cases, woderute in 2 and renote in the remainder. Three of the cuses shew affinity with Brill's disease.

Group B. Folliculoid or segmental habit is seen in 5 cases, and Hodgkin's disease types of fibrosis occur in 6 cases. In all, morbid mononuclear reticulum cells are detected, and Hodgkin fiant-cells are encountered in 7 cases. In 3 cases eosinophiles are present but never in large numbers. It is considered that the affinities of these growths to Hodgkin's disease are near in 4 cases, moderate in $I$ and remote in the rest. Two cases shew sorne affinity to lymphosarcona and three to Brill's disease. 
Note. In both these groups the affinity to Hodgkin's disease is assessed either by the surn of the corresponding features or by the finding of microscopical fields which simulate the disease. 
Analysis of 19 cases of pure reticulum cell sarcoma. Biopsy Series.

\begin{tabular}{|c|c|c|c|c|c|}
\hline Serial. & $\begin{array}{l}\text { Hodgrin } \\
\text { fibrosis. }\end{array}$ & $\begin{array}{l}\text { Mononuclear } \\
\text { morbid } \\
\text { reticulum } \\
\text { cells. }\end{array}$ & $\begin{array}{l}\text { Hodgkin } \\
\text { giant- } \\
\text { cells. }\end{array}$ & Eosinophils. & $\begin{array}{l}\text { Affinity to } \\
\text { Hodgkin's } \\
\text { disease. }\end{array}$ \\
\hline 5 & $P$ & $P$ & $P$ & A & $\mathrm{R}$ \\
\hline 25 & $P$ & $P$ & $P$ & A & $\mathbb{N}$ \\
\hline 26 & A & $P$ & $P$ & $A$ & $\mathrm{~N}$ \\
\hline 35 & $\mathrm{P}$ & $P$ & $P$ & $\mathrm{~A}$ & $\mathbb{N}$ \\
\hline 39 & $P$ & $P$ & $P$ & A & $\mathbf{R}$ \\
\hline 43 & A & $P$ & $P$ & A & $\mathbb{M}$ \\
\hline 48 & $P$ & $P$ & $\mathrm{P}$ & $A$ & B \\
\hline 52 & $\mathbb{A}$ & $P$ & $\mathrm{P}$ & A & $\mathbf{R}$ \\
\hline 61 & $P$ & $\mathrm{P}$ & $P$ & $A$ & $\mathrm{~N}$ \\
\hline 73 & $P$ & $P$ & $P$ & A & $\mathbb{N}$ \\
\hline 78 & $\mathrm{P}$ & $P$ & $P$ & $P$ & $\mathrm{~N}$ \\
\hline 82 & $P$ & $P$ & $P$ & $A$ & $\mathrm{R}$ \\
\hline 109 & $A$ & $P$ & $P$ & A & $\mathrm{B}$ \\
\hline 133 & $\mathrm{P}$ & $P$ & $P$ & A & MI \\
\hline 135 & $P$ & $P$ & A & $A$ & $\mathbf{R}$ \\
\hline 141 & $P$ & $P$ & $P$ & $A$ & $\mathbf{R}$ \\
\hline 142 & $\mathrm{P}$ & $\mathrm{P}$ & $P$ & A & $\mathrm{N}$ \\
\hline 156 & $P$ & $P$ & $\mathrm{P}$ & $A$ & $\mathrm{~N}$ \\
\hline 221 & $P$ & $P$ & $\mathbf{A}$ & A. & R \\
\hline \multicolumn{2}{|c|}{$\mathbf{P}=$ present } & $N=$ near & \multirow{2}{*}{\multicolumn{2}{|c|}{$\mathbf{R}=$ remote }} & \\
\hline$A=a b s e$ & & $\mathbb{M}=$ moderate & & & \\
\hline
\end{tabular}


Analysis of 9 cases of intermediate reticulum cell sarcoma. Biopsy series.

\begin{tabular}{|c|c|c|c|c|c|}
\hline Serial. & $\begin{array}{l}\text { Eodgisin } \\
\text { fibrosis. }\end{array}$ & $\begin{array}{l}\text { Mononuclear } \\
\text { morbid } \\
\text { reticulum } \\
\text { cells. }\end{array}$ & $\begin{array}{l}\text { Hodgkin } \\
\text { giant- } \\
\text { cells. }\end{array}$ & Eosinophiles. & $\begin{array}{l}\text { Affinity to } \\
\text { Hodgkin's } \\
\text { disease. }\end{array}$ \\
\hline 12 & $P$ & $\mathrm{P}$ & $P$ & $A$ & $\mathbb{N}$ \\
\hline 19 & $P$ & $P$ & $P$ & A & $\mathrm{R}$ \\
\hline 41 & $P$ & $P$ & $P$ & $A$ & $M$ \\
\hline 49 & F & $\mathrm{P}$ & $P$ & $P$ & $\mathbf{R}$ \\
\hline 50 & $\dot{s}$ & $\mathrm{P}$ & A & $P$ & $\mathrm{R}$ \\
\hline 90 & $\dot{P}$ & $\mathrm{P}$ & $\Delta$ & A & $\mathrm{R}$ \\
\hline 163 & $\Delta$ & $P$ & $\mathbf{P}$ & $A$ & $\mathrm{~N}$ \\
\hline 194 & $\mathbf{P}$ & $P$ & $\mathbf{P}$ & $\Delta$ & $\mathbb{N}$ \\
\hline 200 & A & $P$ & $P$ & $P$ & $\mathrm{~N}$ \\
\hline \multicolumn{2}{|c|}{$P=$ present } & $=$ near & \multirow{2}{*}{\multicolumn{2}{|c|}{$\mathrm{R}=\operatorname{remot} \theta$}} & \\
\hline$A=a b s$ & & moderate & & & \\
\hline
\end{tabular}


VI. BRILL'S DISEASI.

This was recorded in the following, Serials 20.27. 28. 36. 38. 45 . 46. 51. 104. 108. 145. 172. 173. 203. Total 14 cases.

It is well-know that this entity is the least stable variant of lymphoid tissue tumour. This inconstancy evidenced by incipient metamorphosis was confirmed among these examples, and in several the growth was atypical but morphologically nearest to Brill's disease. Features. Folliculoid habit is present in all but early loss of the characteristic perfection is appurent in several.

In 10 of the group fibrosis is notable. This is generally perifollicular but sometines diffuse in the inter-follicular pulp. In the other 4 the phenomenon is insignificant. Although visible in 12 of the cases morid mononuclear reticulurn cells are always fer; similarly Hodjikin oitant-cells are hard to find and often atypical. They are seen in 8 cases. In nost of these tumours the type-cell is closely similar to that of the internediate reticulum cell sarcoma. Eosinophiles are present in only 1 case.

Affinity to Hodglrin's disease is less in evidence. Only in 4 is it near, in $I$ it is moderate and in the remainder remote or absent.

In 3 of these tumours affinities to lymphosarcoma are noted, in 1 conversion is in progress. 
Analysis of 14 cases of Brill's disease. Biopsy Series.

\begin{tabular}{|c|c|c|c|c|c|}
\hline Serial. & $\begin{array}{l}\text { Hodgkin } \\
\text { fibrosis. }\end{array}$ & $\begin{array}{l}\text { Iononuclear } \\
\text { norbid } \\
\text { reticulum } \\
\text { cells. }\end{array}$ & $\begin{array}{l}\text { Hodigkin } \\
\text { giant- } \\
\text { cells. }\end{array}$ & Eosinophiles. & $\begin{array}{l}\text { Affinity to } \\
\text { Hodgkin's } \\
\text { disease. }\end{array}$ \\
\hline 20 & $P$ & $P$ & $P$ & A & $\mathbb{N}$ \\
\hline 27 & $P$ & $P$ & A & A & $\mathrm{R}$ \\
\hline 28 & $A$ & $A$ & A & $A$ & $A$ \\
\hline 36 & $P$ & $P$ & $P$ & A & M \\
\hline 38 & $P$ & $P$ & $P$ & $P$ & $\mathbb{N}$ \\
\hline 45 & $P$ & $P$ & $P$ & A & M \\
\hline 46 & $A$ & A & A & $A$ & $A$ \\
\hline 51 & $\mathrm{P}$ & $P$ & $P$ & $A$ & $\mathrm{~N}$ \\
\hline 104 & $P$ & $P$ & $P$ & A & $N$ \\
\hline 108 & $P$ & $P$ & $P$ & $\Delta$ & R \\
\hline 145 & $P$ & $P$ & $\Delta$ & $\Delta$ & R \\
\hline 172 & A & $P$ & $\Delta$ & A & $\Delta$ \\
\hline 173 & $\mathrm{~A}$ & $P$ & $P$ & $A$ & A \\
\hline 203 & $P$ & $P$ & A & $A$ & M \\
\hline \multicolumn{2}{|c|}{$P=$ present } & $N=$ near & \multirow{2}{*}{\multicolumn{2}{|c|}{$R=\operatorname{remot} \theta$}} & \\
\hline$A=a b s \epsilon$ & & $\mathbb{M}=$ moderate & & & \\
\hline
\end{tabular}


VII. IMIPHOSARCONA.

This tumour was encountered in the following forms.

1. Small or internediate cell lymphosarcoma.

Serials. 123. 144. 149. 150. 151. 169. 170. 175. 186. 192. 197. 208. Total 12 cases.

2. Larse cell lymphosarcoma.

Serials. 136. 147. Total 2 cases.

These tumours have least morphological affinity with Hodgkin's disease, the characteristics that are parallel are usually minimal expressions.

Features. Folliculoid habit is detectable in 8 , and in 3 of these the tumour is probably an evolution from antecedent Brill's disease. Fibrosis in forms characteristic of Hodgkin's disease is seen in half of the group but the total amount of collagen is small. Relatively isolated morbid mononuclear reticulum cells are present in 12 cases but Hodgkin giant-cells are only found in 6. With the exception of 1 case eosinophilia is conspicuously absent. These findings are reflected in the assessments of affinity of these growths to Hodgkin's disease, I shews moderate affinity, 3 remote likeness and 10 none at all. 
Analysis of 14 cases of lymphosarcoma. Biopsy Series.

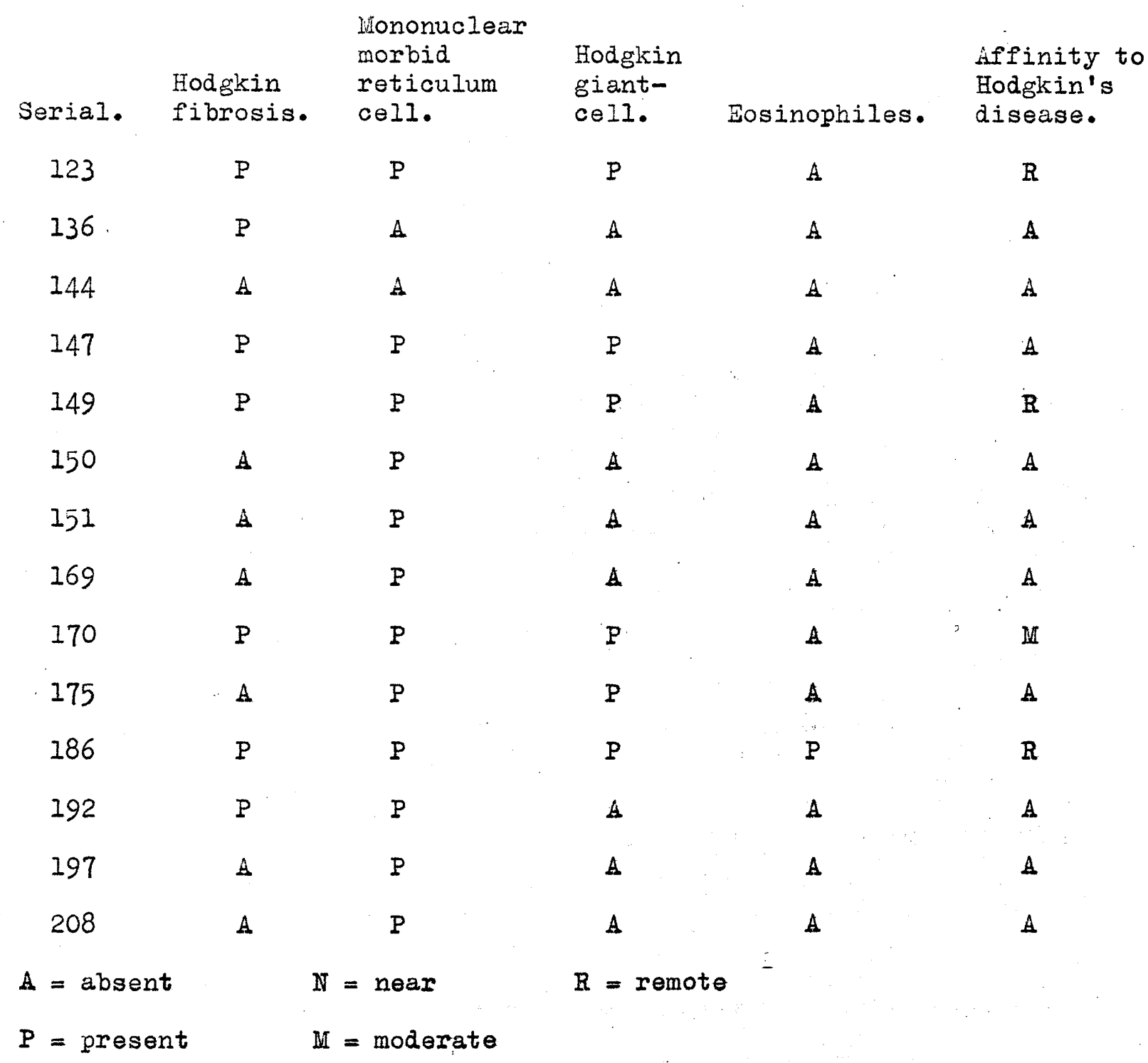


VIII. LYHPH NODES II MYELOID LOUKADNIA.

Two examples were encountered, Serials 179 and 189.

These cases are of much interest because in both a tentative diagnosis of acute or atavistic Hodgkin's disease was made from lymph nodes before blood examination established the true diagnosis. These diagnoses were invented to indicate that the impressive features were the immaturity of the cells of the complex, and the tenuous light fibrosis. It has already been recorded that this identity between myeloid leukaemia and Hodglin's disease has convinced Symmers and Nedlar. It is ironic that I should have rejected the hypothesis of these writers and then have subscribed to it unwittingly. Further, I repeated the error within six months. The lessons to be learned are that blood examination should be made before diagnosis of unusual variants of Hodgkin's disease is completed, and that specuiative morbid anatomy is unsound.

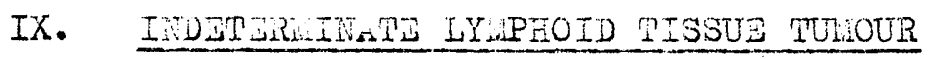

This unsatisfactory diagnosis was necessary in the following, Serials 29. 37. 55. 58. 88. 139. 146. 164. 166. 167. 177. 187. 215. Total 13 cáses.

Thile the characteristics of these lesions did not permit diagnosis in existing categories, they are provisionally grouped thus:-

1. Nearer to Hodgin's disease than other variants

Serials. 29. 146. 167. 187. 215. Total 5 cases.

2. Indeterminate Iymphoid tissue tumour

Serials. 55. 58. 88. 139. 166. 177. Total 6 cases.

3. DoubtfuI

Serials. 37. 164. Total 2 cases. 
Analysis beyond this is not profitable. The original protocols should be consulted for details of individual cases. 


\section{THE NECROPSY SERIES}

It is difficult to obtain necropsies in cases of Hodgkin's disease. This limitation of material enforces the exploitation of any that may be obtained, and consequently the threshold of acceptance tends to be lower than in eclectic series of comnoner diseases. The present collection comprises 13 cases gathered over a period of five years. Nost of these necropsies were performed personally and single-handed; in two cases I an indebted to colleagues who permitted me to re-examine the cadavera after routine necropsy. All the naterial from every case was processed, cut, and stained by myself. This entailed much work since each case required 50-70 blocks, many of which had to be kept mutually separate. Inevitably some inaterial has been badly chosen, incomprehensibly forgotten, lost at the list monent, or ais-identified. Similarly as experience increased more extensive and sophisticated surveys developed. These factors diminish the completeness and mutual comparability from the ideal conterplated but not too seriously. The specinens selected for microscopy varied in freshness from 2 hours to 2 days, most were under 12 hours. The standard of histology attained was reasonably good, the exceptions, where post-mortem change was advanced, shewed the usual capricious relationship to the actual time involved.

The main interests in this study were the distribution, location, and extent of the lesions. In addition the stability of the lesion and several subsidiary issues have received attention. Material.

The series consist of twelve cases collected from the routine necropsies in Glasgow Royal Infimary, and the Dastern District Hospital, 
Glasgow, and one cuse from Law Hospital, Carluke.

IDINTITY OF THE CASES.

Code

No. Letter.

1.

2.

$3 \cdot$

$4 \cdot$

$5 \cdot$

6.

7 .

8.

$9 \cdot$

10.

11.

12.

13.

A.

B.

c.

D.

E。

F.

G.

H.

I.

J.

I.
K. Marie Traynor.

John Young.

Wrs. Lirgaret Royal.
Age.

(years) Reference.

62

Е.16:49

G.R.I.201:49

G.R.I.193:50

G.R.I.332:50

ت.35:51

G.R.I.61:51

Law. 131:52

31

G.R.I.155:52

217

41

G.R.I.119:53

191

23

G.R.I.274:53

220

18

G.R.I.415:53

219

58

G.R.I.418:53

209

72

Biopsy

None

188

117

72

57

None

218

General principles of selection of material for microscopy.

Lymph nodes. Representative samples from all accessible groups were taken including specimens from both sides in the case of paired groups. There nodes were very large, pieces only were taken. Those from the face and base of the skull are difficult to extract without mutilation and attempts were limited. The nodes in the antecubital, epitrochlear and popliteal regions were investigated in all except the earliest cases reported.

Organs (chiefly viscera, but including some outwith the large body cavities). 
The selection was aeterined primarily by the results of naked eye scrutiny. In such viscera as lunE, liver, spleen and kidney it was found valuable to cool the tissue till it was rendered appreciably rigid and then slice it closely. In cases there naked eye lesions were absent random sanples were taken.

Skeleton. Thorough examination of the bone marrow is an impossibility in ordinary necropsy practice because it produces serious mutilation besides being exceptionally tedious. In nost cases examination was perforce linited to the skull, sternum, ribs, bodies of the vertebrae, and one femur. The nuclei pulposi were examined in 8 cases. The method found most useful was to dissect the structure after it had been partly approached by the tangential saw-out made to exumine the vertebral bodies. (IIicroscopy was unstisfactory in these structures, they almost irvariably disintegrated during processing). Inteuanent und fuscial pianes. The entire skin was inspected and palpated, any suspicious irregularity or siveling being renoved en bloc. Examination of superficial and deep fascia was restricted to the anterior thoracic and abdominal representative. The omenta and mesenteries and the fascia of the lower limb opened were also examined closely. Wethods.

Fixation: In most cases 10 per cent formol-corrosive was employed as a routine. Iany hard lymph nodes however were fixed in Bouin's solution with reduced acetic acid ( 1 per cent), since this was found to give better results. Bone marrow specimens were fixed in 'Susa', the decalcifying properties of this acid solution being generally adequate to soften small spicules of bone included in these specimens while at the same time giving excellent results in staining. 
Dehydration and clearing were effected with the usual reasents including toluol. The tissues were enbedaed in paraffin and sections 5. $\mu$. thicis ivare cut.

Stains. all sections were stained ath Earris' haenatoxylin and water soluble eosin. In selected cases further sections were stained by connective tissue stains principally van Gieson's stain and lallory \& Orange $G$. These were supplemented by silver impregnations (Gordon \& Sweets 1936) and (Iitchell \& islocki, 1944). The latter method gives beautiful results but is expensive to use. In a few cases, where indicated, Ziehl-Neelsen's stain, Lieshman's stain and Dominici's stain were employed.

\section{RDSULTS}

Horphological findings are difficult to present briefly. In each case a full necropsy report of the naked-eye and microscopical findings has been prepared; these are supplemented by homunculus diagrams recording the reticulo-endothelial systen findings. In addition a series of photo graphs relevant to each case has been added. The full protocols are in bypendix' 'B'.

Here the results are confined to analyses of the subjects referred to in the introduction above.

1. Distribution of the lesions.

To facilitate the presentation of the results of this survey each necropsy has been perforined and recorded on a definite plan.

Appended is a schema which covers nearly all of the morbid anatomy studied. 
Schema of the basis of analysis of the necropsy series.

I

Reticulo-endothelial system

A. Reticulo-endothelial organs.

1. Iymph nodes.

2. Spleen.

3. Bone marrow.

B. Reticulo-endothelial elements situated in organs.

1. Liver.

2. Adrenal grlands.

3. Hypophysis cerebri.

C. Dispersed elements of the reticulo-endothelial system.

1. Skin.

2. Onenta.

3. Fascial planes.

4. (Nuclei pulposi of intervertebral discs, persistent mesenchyme).

II Respiratory system. (excluding intra-pulmonary lymph nodes).

II Alimentary system. (excluding adnate mesenteric lymph nodes).

IV Serous Hembranes.

1. Leninges.

2. Pericardium.

3. Pleurae.

4. Peritoneum, other than mesenteries and omenta.

$V \quad G e n i t o-u r i n a r y$ system.

VI Cardiovascular system.

VII Skeletal muscle system.

VII Endocrine glands. 


\section{Systematic survey.}

Is. 1. Iymph nodes.

Whe results are recorded in the following table.

TABLS SHOVING INOIDINCE AND DISTRIBUTION OF LYMPH NODE LESIONS

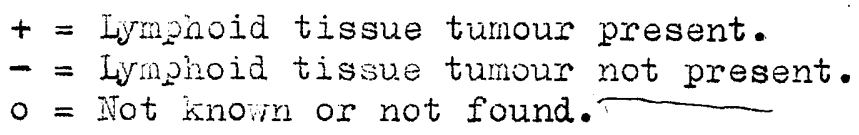

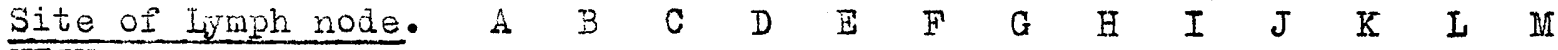
NECK. Left

Right

$$
\begin{array}{llllllllll}
+ & + & + & + & + & + & + & + & + & + \\
+ & + & + & + & + & + & + & + & + & +
\end{array}
$$

ARR.

I. Axilla

R.Axilla

L. Antecubital

R. Antecubital

$$
\begin{array}{llllllllllllll} 
& + & + & + & - & + & + & + & - & + & + & + & + & + \\
+ & + & + & + & + & + & - & 0 & - & + & + & + & + \\
& 0 & 0 & 0 & 0 & 0 & 0 & - & 0 & 0 & 0 & 0 & 0 & 0 \\
0 & 0 & 0 & 0 & 0 & 0 & - & 0 & 0 & 0 & 0 & 0 & 0
\end{array}
$$

THORAX.

Stibbes nodes

Para-tracheal

Hediastinal

I. Hilar

R.Hilar

I. Intra-pulmonary

R.Intra-pulmonary

$\begin{array}{lllllllllllll}+ & + & + & + & + & + & - & + & + & + & + & + & + \\ + & + & + & + & + & + & - & + & + & + & + & + & + \\ + & + & + & + & + & + & - & + & + & + & + & + & + \\ + & + & + & - & + & + & - & + & + & + & + & + & + \\ + & + & + & - & + & + & - & + & + & + & + & + & + \\ - & + & 0 & 0 & + & - & - & + & - & 0 & + & + & + \\ 0 & + & 0 & 0 & + & + & - & + & + & 0 & + & + & +\end{array}$

ABDOLEND.

Mesenteric.

Lesser omentum.

Greater omentum.

lesentery S.I.

Hesentery I.I.

Retro-peritoneal.

Juxta-panereatic

Para-aortic.

I. Pelvic.

R. Pelvic.

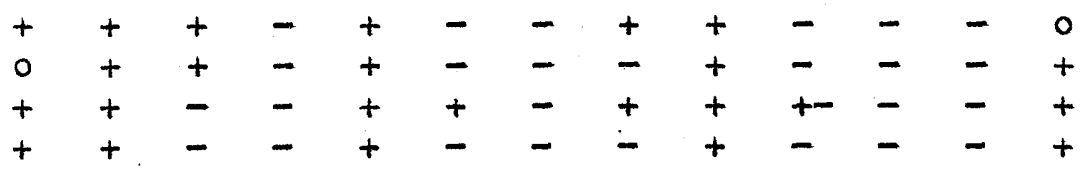

LEG.

I. Groin.

R. Groin.

L. Popliteal.

R. Popliteal.

$\begin{array}{lllllllllllll}+ & - & + & - & + & + & - & + & + & - & - & - & + \\ + & + & + & + & + & + & + & + & + & + & + & + & + \\ + & + & + & - & + & 0 & 0 & + & + & + & 0 & - & + \\ + & + & + & - & + & 0 & + & + & + & + & 0 & - & +\end{array}$ 
The term lymphoid tissue tumour covers Hodrgin's disease, Hodgikin's sarcoma and reticulum cell sarcoma. In nearly all the cases noninvolvement was confirmed microscopically. In the majority of cases the nodes of the antecubital fossae could not be found; search for those in the popliteal fossae met with rather better success. In all the cases except case $\mathbb{3}$. at least one and occasionally many persisting non-involved nodes were found. These nodes were generally atrophic, fut atrophy and lymphocyte depletion being very common. A number have been photographed.

I.A.2. The spleen

In ten cases lesions were identified naked-eye and confirmed by aicroscopy. All the deposits vere in the form of notules of which the smallest were consistently sited in the red pulp. In the remaining three cises nakel-eye scruting was negative and microscopy confirmed the absence of the disease. Necrosis and haemosiderin deposit were frequently notable in the foci in this locus. I.A.3. The bone marrow.

Deposits of the new tissue nere identified with certainty in six of the cases. In two of the positive cases it was restricted to one and two samples respectively. There deposits were found, they were always focal, consisting essentially of nodules of the new tissue. I.B.I. The Liver.

The disease was manifest in this organ in six cases, in one case, P.II. 'D', a large spheroidal tumour in the liver appeared the chief expression of the disease, and in another, P.I.'. 'I', direct extension into the viscus from neighbourine tumour was the node of involvenent. 
In the remining cases scattered nodules enerally small and relatively scanty constituted the form of the disease. The majority of siall deposits vere in the portal tracts but in one case, P.I. 'G', minute nodules were seen in the lobules.

I.B.2. The adrenal glands.

In only one case, F.l. 'I', was deposit of the growth encountered in the gland. Here there was copious surrounding tuinour tissue and the lesion wus too advanced to disclose whether the intra-adrenal deposits were prifurily intrinsic in the gland or the result of extraneous infiltration. In two cases, P.ifs. ' $B$ ' and ' $K$ ', glands were invested by Hodgkin tissue but vere not the seat of the diseuse. I.3.3. The Hypophisis Cerebri.

The gind wos not examined in three cases, F.Ms. 'A', 'G', and 'G'. In the rewaining ten it wis invariably free from deposits. I. $0.1-4$.

The lesion was not encountered in the skin nor fascial planes in any case. Further deposits were never found in the omenta other than in organised lymphoid tissue (nodes) in this site. - In eight cases a nucleus pulposus was exanined since these structures are reputed to represent persistent primitive mesenchyme. The lesion was not present in any.

II. Respiratory System.

In nine cases deposits were present in the lungs. In most it was impossible to surmise where the initium was located. Centrally in the lesions the normal lung tissue was destroyed but peripherally the new tissue appeared to grow out into the alveolar spaces using the alveolar 
Walls as a scuffolding. The dppearances often recalled a chronic interstitial pneumonia. Necrosis was common. In four of these cases nodular deposits mere present in the submucosa of the trachea or bronchi. In one case nodules pere found in the air passages but not in the lungs. III. hlimentary System.

In three instances deposits vere present in the stomach, in one case a deposit was partly oesophageal as well as gastric. In each case the disease foci presented as small malignant ulcers usually with a few satellite nodules. Persistent but decayed lymphoid tissue was common in this system (para-enteronic Iymphoid tissue). IV. Serous Membranes.

The aeninges wo not involved in any of the cases where the head was exwined. In six cuses the pericardium contained deposits and in: six the ileura mas siailarly affected. Peritoneal deposits were observed in four cases. V. Gonito-urinary Gystem.

Renal deposits were encountered in two cases. In another a ureter contained the growth in the submucosa. The bladder was involved in two instances and the prostate rland in one. (All these lesions were mutually unreluted). The ovary contained a deposit in one case. VI. Tardiovascular jystem.

Extension from the epicardium into the myocardium was observed in one case. VII. Skeletal Muscle System. In two cases infiltration of the diaphragm was present; in one the right psoas muscle was also affected. 
VIII. Endocrine Glands. (Iess hypophysis cerebri and adrenal glands). In one case the thyroid gland was apparently completely replaced by tumour. Stability of the lesion.

In the section devoted to the description of typical Hodgkin's disease it was accepted that the microscopical complex generally followed a sequence of maturation in which it retained its identity. While the appearances observed in this process shew variation they have limits. In some of the biopsy series alteration beyond this reasonable latitude was encountered and here further observations on this type of metamorphosis are made. In eleven of the cases appropriate comparison with antecedent biopsy findings can be made and in all the cases the necropsy material itself can bo similarly studied.

The decision whether alteration is evidenced or not is dependent on interpretation and judgment. Accordingly there is inevitably an element of abritrary distinction in these results. The criteria of definition have been applied rigidly; this is reflected in the fact that in the whole series the biopsy of only one case conforms to the ideal typical Hodgkin's disease. The remainder are atypical. In most the atypism is slight and insufficient to assail the diagnoses as practical and truthful reports. In two cases the deviation is more pronounced and in one, the lesion, while simulating cellular tumour-like Hodgkin's disease, must be regarded as belonging to the category of reticulum cell sarcoma. An analysis of the findings in the series is given in table 
Case. Biopsy Findings

A. (None)

B. Slightly atypical Hodgkin's disease, eosinophiles reduced, cellularity and pleiomorphism enhanced.

c. Slightly atypical Hodgkin's disease, eosinophiles low, many foci of epithelioid cells.

D. Slightly atypical Hodgkin's disease, mostly small cells, the lesion is early.

E. Slightly atypical Hodgkin's disease. Eosinophiles few. Focal reticulum cell proliferation.

F. (None)

G. Atypical Hodgkin's disease, no eosinophiles, tumour-like.

H. Typical Eodgkin's disease.

I. (a) Atypical Hodgkin's disease, soft, cellular, eosinophiles low.

(b) Reticulum cell sarcoma.

J. Atypical Hodgkin's disease. Diffuse fibroblastic type, very cellular.

K. Atypical Hodgkin's disease. Diffuse fibroblastic type.

I. Atypical Hodgkin's disease. Highly cellular, tumour-like, almost Hodgkin's sarcoma.

M. Reticulum cell sarcoma, close affinity to cellular Hodgkin's disease.
Necropsy Findings

(a) Typical mature Hodgkin's disease

(b) Hodgkin's sarcoma.

Very similar to biopsy.

Similar to biopsy but eosinophiles further reduced, near to Hodgkin's sarcoma.

(a) Typical Hodgkin's disease.

(b) Hodgkin's sarcoma.

(a) Atypical Hodgkin's disease, as in biopsy.

(b) Cellular tumour-like Hodgkin's disease, eosinophiles retained.

(a) Typical Hodgkin's disease.

(b) Hodgkin's sarcoma.

Very similar to biopsy.

Highly cellular tumour-like Hodgkin's disease.

(a) Very small component of mature Hodgkin's disease.

(b) Reticulum cell sarcoma.

Very similar to biopsy.

Very similar to biopsy.

Very similar to biopsy.

Very similar to biopsy. 
It is seen that in seven of the thirteen cases there was significant alteration in the microscopical appearances of the lesion. In two of these, Cases A. and F. previous biopsy had not been performed but in both the necropsy material displayed at least two reasonably distinct pictures. In these cases the inference is that the Hodgkin's sarcoma form arose on a basis of ordinary Hodgkin's disease since transitions between the two were observed. Of the other five cases shewing alteration of form, three, Cases D., E. and I., shewed similar mixed pictures. In the remaining two cases, C. and H. the alteration was less in degree and incomplete.

In the six cases where metamorphosis was not a feature it is notable that in four, P.Ms. G.J.K. and L., biopsy already revealed tumour-like appearances of the lesion. In one case, B., the atypism was slight. The final case was already frank tumour. The nature of the metamorphosis.

In general terms this is chiefly expressed by the disproportionate augmentation of morbid reticulum cells in the lesion. This increase affects the morbid mononuclear types, and the multinucleate giant cells. In most cases fibrosis persists but is less in amount than in the classical mature Hodgkin's disease lesion. The eosinophiles diminish to the point of disappearance and frequently the lymphocytes are markedly decreased.

In the earlier part of this work (p.178.) the lesion, which is probably correctly regarded as a morphological variant of the typical form, received the name Hodgkin's sarcoma. These present studies support this view. There is not convincing evidence of any enhancement 
of malignancy where this results. On the other hand when the metamorphosis yields a reticulum cell sarcoma in which pleiomorphism is less evident, e.g. case I., truly malignant features are displayed.

The time over which these metamorphoses develop is difficult to assess. In the five cases where the dates of biopsy are known an average period of three-and-a-half years elapsed, but the minimum time was three months and the maximum nine years. In one of the cases, A., specific treatment was not given; all the remainder received X-ray therapy.

The chief effect exerted by X-ray therapy on the lesion is the destruction of lymphocytes and their immediate precursors. Note on the incidence of tuberculosis.

Histological evidence of tuberculosis was found in four cases. In each, the lungs always shewed foci and in two spread to other organs had resulted. In none was tuberculous disease seriously extensive. There spread beyond the lungs was observed the lesions were mostly miliary and probably represented terminal dissemination. Note on Oedema.

Dropsy was very common. In only two cases was it absent. Effusion into the serous sacs affected principally the pleurae, then the peritoneum and least the pericardium. Anasarca was found in nine cases. 
PART IV

FIBROSIS AND EOSINOPHILIA

SECTION A.

The phenomenon of fibrosis with observations

p.240-262

on its tumour-like character in certain

diseases.

Illustrations.

Volume III. Figs. 206-212. 


\section{OBSERVATIONS ON FIBROSIS AND DOSINOPHILIA}

DRAIN FROL GENERAL PATHOLOGY

\section{Foreword}

In the previous part fibrosis and topical eosinophilia in Hodgkin's disease have received attention. It appeared relevant to enquire into these phenomena in the field of general pathology to try and derive information of any comparable findings in other neoplastic or tumourlike diseases.

This has entailed the study of several diseases in which fibrosis appears integral, and an analysis of the incidence of eosinophilia in a series of carcinomata.

\section{Introductory remarks on Fibrosis}

Despite wide and common application in Pathology, fibrosis is a process which it is difficult to define comprehensively and accurately. Its etymology does not yield particularly satisfactory understanding. The suffix '-osis' implies an increase of cells, tissues, or stored substancesf Powell-ihite (1927); and, compounded with the root word, fibre, indicates simply an increase of fibres. The exact nature of these fibres is rarely stated fully, but it is generally accepted that they are collagenous, and possibly also fibroglialy Nallory (1913). Mallory also considered that elastic fibres were elaborated by the same cells as the foregoing, but there is reluctance among more recent writers to acknowledge this c.f. Cowdry, (1932), Le Gros Clark (1947). Furthermore, the term elastosis has come into wide use to connote augmentation or undue prominence of these fibres and it is customary to regard them separately. In ordinary reparative fibroblastic activity these fibres 
are generally conspicuously absent.

Reflection on the manifestations of the phenomenon of fibrosis regarded as a process innediately disturbs the aptness of the simple definition indicated above. Mile it seems true in the main to postulate that collagen fibres are a maturation product of proliferating fibrocyte precursors this is not always obvious. In the healing wound the process is patently related to a preliminary proliferation of fibroblasts. In most orthodox teachings it is stated that these cells arise from pre-existing fibrocytes at the locus by an initial

de-differentiation followed by homoplastic multiplication of these more primitive cells. These mature as healing proceeds and pari passu fibrosis occurs.

On the other hand the exact mechanism of fibrosis in many of the slowly evolving varieties of replacement fibrosis is less easy to follow. In many examples of ischaenic fibrosis or in arteriosclerosis without hypertonus the sequences in the process are postulated rather than demonstrated. The fibrosis of atrophy, especially in the process of ageing, sugEests a retrogressive change by metamorphosis of existing elements rather than by new formation of connective tissue.

Fibrosis is certainly not the exclusive property of the orthodox fibroblasts. Under certain conditions other mesenchymal cells can produce collagen. The lymph node reticulum and homologous tissue elsewhere is quite reasonably and properly regarded as peculiar connective tissue, and as a corollary its fibre product, reticulin, is simply collagen in a special form, or pre-collagen. The difference between collagen fibres and reticulin fibres, frequently made an issue of much 
importance is probably over-emphasized. Reticulin increase is demonstrable in certain incipient fibroses but it is not apparently an invariable stage. The fact that reticulum cells can produce collagen is well established and moreover it appears that in sone morbid conditions they do so characteristically.

Collagen may arise from non-mesoblastic sources. This phenomenon does not enjoy universal recognition yet, but authoritative opinion in some quarters favours it. Wasson considers that the collagen produced in certain tumours or experimentally induced tumour-like growths, of the peripheral nerves is properly attributed to the neurilemmal cells. These cells arise by differentiation from the tissue of the early neural crest and also are reported to emerge from the neural tube by way of the ventral roots, Arey (1941). The exact origin of the neural crest is not agreed (i.e. Whether it is simple ectodermal or neurectodermal) but whichever view is more correct it is still fundamentally ectodermal. Masson insists that the only mesoblastic collagen in these growths is that in close relation to the blood vessels.

The application of tissue culture to tumours arising in relation to nerves supports the view that neurilemal tissue has specific characters and can form collagen, Hurray and Stout, (1942). If these and Hasson's interpretation are accepted the principle is important, since it enhances the evidence against the older postulates of germinal layer specificity •

There is some evidence that under exceptional circumstances collagen may be formed by endothelial cells e.g. the serosal cells, Cappell, (1952). 
The exact relation of that vague but unquestionably valuable entity terned 'hyaline', with collagen, is equivocal. Collagen certainly becomes hyalinised, from a microscopical point of viev, and is usually considered a degenerative change; at the same time many other sources of apparently identical hyaline exist. It is very common for example in smooth muscle cells in blood vessels, in the ageing uterus and in the prostate gland. In this particular cell it usually appears as a central thread extending along the lons axis of the fibre. In skeletal nuscle hralinisution can produce an appearance identical with collagen.

The current impression of the significance of the phenomenon is that it is cornensatory, this term being used in its widest sense. Whilst the untowid effects of its results are widely recognised, the process of fibrosis itself is pretty generally hailed with enthusiasm, much as laudable pus was formerly greeted. It is possible however that too much emphasis has been laid on this rôle. It is continually stressed how important fibrosis is in relation to repair, to inflarmation, and how it forms a readily available and cheaply maintained substitute for more specialised tissues. Inevitably one comes to think of it as a simple altruistic process of a benign, plain, worka-day tissue with no character of its own. The comparative rarity of fibro-sarcoma certainly reflects credit on its general good behaviour. Nevertheless, the process of fibrosis sometimes deviates from the altruistic path without getting into serious trouble. Little systematic study his been accorded to these biologicel flutters as a group, although idami (191I), and Powell-thite (1927), offered some tentative 
outlines. An innate perversity to do the wrong thing first probably exists in cells as vell as huge collections of cells. Illustrative of this wayward propensity are a few peculiar fibroses which reflect an unwonted degree of enterprise on the part of this rather stodgytissue. At the sume time these growths of fibrous tissue do not receive unanimous approval as examples of neoplasm.

The particular observations offered are admittedly a somewhat quaint collection. They defy any rigid classification, and some indeed are almost anecdotal. However there are reasonable grounds to associate them because in some degree they all shew a process of fibrosis in which some autonymity obtains. This type of fibrosis belongs to that inysterious no-man's-land or borderland dim (Boyd) where the answers are 'well, yes and no'.

\section{MYI POOULIAR IBIROSES}

It is considered that in the following conditions this quasineoplastic propensity is evident.

$$
\begin{aligned}
& \text { 1. Cheloid. } \\
& \text { 2. Desmoid tumour. } \\
& \text { 3. Dermatofibroma. } \\
& \text { 4. Riedel's struma. } \\
& \text { 5. Polyserositis. } \\
& \text { 6. Hyelofibrosis. } \\
& \text { 7. Neurofibromatosis. }
\end{aligned}
$$

It is appreciated that these are diverse instances of disease and that many authorities would demur at their consideration as a group. It is not advocated here that they are related beyond evidencing this intrinsic peculiarity in varying degree. In the first four examples the lesion is of local character and in the remainder it is of more general distribution. 


\section{CEISIOID}

In accordance with ifeigert's ingenious Law - that the degree of reparation of injured tissue always exceeds the dernand - fibrosis in the healing process is always more abundant than is really necessary. In the nornal case this excess is insignificant, but in some individuals there exists a congenital and sometimes familial tendency for this increment to become embarrassing. This results in abnormal but selflimiting overgrowtin of fibrous tissue and constitutes the well-known cheloid or keloid. (The word is variously derived - the former on better etymological grounds). The new fibrous tissue matures rapidly to form large cylindrical deposits of collagen interspersed with rows of nuclei and merges imperceptibly with the adjacent normal connective tissue.

The intrinsic cause of this remarkable predisposition in the subjects of cheloid is obscure, many writers consider that is particularly common in negroes. The tendency becomes apparent after the operation of some form of trauma. This may take many forms, such as burns, surgical wounds, scratches, and etc. and it has been observed after very trivial injuries e.g. vaccination. It is probable that spontaneous development of cheloid is to be related to insignificant traumata operating where the threshold is very low.

In most ordinary texts pronouncements on the interpretation of cheloid are evasive. Authoritative statements from the sources indicated below reflect the diversity of opinion. Adami (1909), included it under the head of proliferative fibrosis, a category in which he placed encapsulating fibrosis, the fibrosis of infective granulomata, 
and post-inflammatory fibrosis. Mallory (1913), considered it to be hypertrophied scar, in a category by itself, but he described it in association with fibrous tissue tumour. Fwing (1929) allowed that, 'The neoplastic properties in cheloid are not pronounced'. It seems inferred that it is a neoplasm yet he qualified this implication adroitly by attributing it to chronic disturbance of nutrition. Donaldson (1931) described cheloid as a form of fibrous tumour. Willis (1949), decided in favour of some non-neoplastic disturbance of reparative growth. Morbid Anatomy of Cheloid

By general definition cheloid is limited to the integument, but homologous formations are observed in bowel, vagina, and possibly other organs.

The naked eye appearance shew considerable variation. Hinimal manifestations of the phenomenon are not very rare in striae gravidarum. In the common form encountered in linear wounds, they form elevated flat broad thickenings of the scar which nay spread slowly locally, or generally. The overlying sisin is often smooth shing and thin, with loss of elasticity. The colour tends to remain pink longer than normal scar because devascularisation is slower. In some cases, especially after burns, the growtins are more exuberant and form large polypoid masses. Several examples of this gross manifestation are impressively illustrated by liallory (1913) and Ewing (1929).

Microscopically the established cheloid consists of large interwoven bundles of fibres composed of collagen; these are often hyalinised and compacted into a cylindrical form reminiscent of skeletal muscle. 
Elastic fibres are not represented. The nuclei are disposed chiefly in the interstices in linear fashion. In form they approximate closely to those of fibroblasts but are larger and often slightly hyperchromic. The periphery of the deposit is characteristically poorly demarcated; it is usually more cellular and nerges imperceptibly with the adjacent normal tissue. The blood vessels are well formed and small, but generally nurnerous. The glands of the dermis and the hair follicles tend to be displaced rather than overrun; the overlying skin is likewise devoid of these structures.

It is submitted that the mild atypism of the components, the purposeless nature of the formation, the tendency to recur and its very limited capacity to infiltrate, warrant its inclusion in the series listed above.

DESLOID TUT:OUR

Desmoid tumour is probably the most widely used term to connote the growth which was formerly described as recurrent fibrous tissue tumour of the abdominal wall. The first recorded cases are attributed to Macfarlane (1832) a former surgeon in this Institution, Stewart and Mow at (1924). The name desmoid tumour was introduced by shnger in 1884, and derives from the supposed similarity of the cut surface to tendon. The main facts regarding these formations are well known and classical treatises have been offered by Pfeiffer in 1904 and Stewart and Howat twenty years later. Briefly these distinctive and rare growths are recorded chiefly in parous women in the third and fourth decades. Iorbid anatomy of desmoid tumour

The growths are believed to arise from the muscular aponeuroses of the 
the abdomen, enerally thit of the $\mathrm{m}$. rectus abdominis. The majority are situated below the umbilicus and almost invariably away from the mid-line. Their naked-eje appearance was well described by lacfarlane (1832). In his first patient the tumour was the size of a lemon, greyish, not unlike half-bleached wax, in some parts half transparent, and exhibited a smooth compact texture its centre being fibrous'. Microscopically they resemble fibroma but in some cases their structure gives rise to difficulty in deciding their true character. They may sirnulate leiomyorna or neurofibroma closely. The growing edge is devoid of a capsule and the overrun skeletal muscle undergoes atrophy. Commonly syncytium-like masses with numerous nuclei are to be seen. This is a well-known phenomenon in damaged muscle and was first fully described by Durante (1902). Adhesions to local intra or extra peritoneal structures are connon and probably initiated the belief of Huginer (1860) that they arose from periosteum. This view was long supported by the French. The tendency to recur is possibly overemphasized and is a relic of the results of less satisfactory surgery. In Stewart and Howat's series of seven cases recurrence was not recorded but the observation periods were mostly short. In three examples of the lesion studied personally recurrence was not observed. (One of these cases had been treated by irradiation after surgical resection of the main tissue mass).

There is general statement that these growths are tumours; Stewart, Ewing, willis, Donaldson, Boyd, Nuir and many other subscribe to this view. It would be impertinent to question this opinion but the entity deserves some special qualifications. The relation to trauma is remarkably well attested, possibly better than in cheloid. The general 
relation of most tunours to trauma is less clear cut. The absence of a capsule is unusual in benign tumours. This of course applies to the established neoplasm, the initium is presumably unencapsulated and the capsule develops in the host tissue in response to the growth of the tumour. Lit what point this occurs or the factors deternining it are matters for speculation. Jiving (1929) obviously favoured the idea that some degree of aggressiveness was essential to wall-building resentment of the host tissue. I have encountered several 'fibromata' of ovary in which the appearances were typical apart from the lack of a capsule, indeed the difficulty of recovering a fibroma suitable for class purposes is notorious.

It is the usual experience that this vigorous fibrous tissue in desmoid does not assume true malignant status. Willis (1949) reported an instance in wich one of those growths co-existed with a fibrosarcoma elsewhere and submitted that this might be argued as evidence of a tendency to fibroblastic neoplasia. It is submitted that this growth has some affinities to cheloid but is generally a more aggressive expression of the process.

DERIATTOEIBROWA

This peculiar growth has recently enjoyed renewed interest, in fact it is almost true to say that it has been rediscovered.

It is an unquestionably rare lesion and has been recognised under different names over a considerable time.

Among the titles which have been used to designate it are:sclerosing angioma, fibroxanthosarcoma, benign dermatofibrosarcoma, fibrome-en-pastille, dermatofibroma, histiocytoma and fibromatoid 
granulona. With the exception of the last synonym, which incidentally is said to have been the first name, all the remainder infer etymologically that the growth is a tumour. It is felt that there are grounds for disputing whether this lesion is a true neoplasm and some of these originate from the derivations of the alternative labels which have been suggested to connote it.

The names which acknowledge relationship to angioma cannot imply tumour status on that account. linost present day opinion is hostile to the belief that angioma is more than a nalformation, (the very rare angiosarcoma is excepted), accordingly the category hamartorna would be more appropriate. In several further respects this idea has claims to attention. The tumour endothelioma has already received attention (Part I), it will be remembered that while the lucid criticisms of rillis are generaliy sustained, reasons for sympathy with it were offered. Amongst these was the submission that the tendency to form whorls could be interpreted as a property beyond simple functional modification. It could be regarded as an atavistic trait of endothelial cells. In the few examples of dermatofibroma which I have encountered at firsthand this tendency has been discernible in some degree. The production of collagen by endothelial cells of capillaries has been demonstrated by Corner (1920). In most dermatofibromata sclerosis is a prominent feature but vaso-formative habit is far less in evidence so that sclerosing angioma is a far-fetched nomenclature for the growth.

Xanthoma is a neoplasm which has come down in the world. The name frequently appears in inverted commas, a slight on its individuality, or it is joined with another or several other syllables giving high-sounding 
compound titles which are no longer popular. It has an extensive and confusing literature in the older dermatological annals and in the modern oncological works it receives scant attention. The inference gained from the latter is that it is a very dubious neoplasm. That aggregates of xanthoma cells appear in certain tumours is widely known but there is always hesitation to ascribe neoplastic properties to them.

The view that the growth is a variety of fibroma or benign sarcoma of fibrous tissue has certain claims. An unencapsulated fibroma of ovary is widely recognised and has been alluded to earlier in this section; the other alternative, benign sarcoma while it is paradoxical doubtless springs from recognition of this anomaly.

Noringer's term, histiocytoma, (1931) is probably the most popular name at present. This title was prompted by the observation that cells of the growth store iron and lipoid. It has been supported by the experimental finding that cells of the growth could ingest and store colloidal iron injected into the tissues in their vicinity. Since the growth scleroses and fibrifies as it matures it follows that histiocytes (macrophages) form collagen. This is an undoubted fact in experimental work, Curran, (1951), Nicol and Abou-Zirky, (1953), but most human morbia anatomy texts are hesitant to postulate the process with assurance in natural disease. The principle is very reasonable, in a healing wound the distinction between fibroblasts and macrophages can be so difficult that their identity is the only logical interpretation.

An interesting corollary of accepting Noringer's hypothesis is that we are furnished with an example of benign tumour of the reticuloendothelial system. The rarity of these neoplasms has already been 
alluded to in Part I. where the claims of myxoma were upheld. Further to this exceptional status is added the fact that the deposit is almost always solitary. In the rare histiocytic reticulosis (of both sinus and medullary type, Robb-Smith, 1938) systematization or at leist very wide diffusion was the key-note.

The nane fibromatoid granuloma is credited with being the oldest. Except in the sense in which iwing employs the word, granuloma is an unsatisfactory term, like tuberculoma or syphilona its employment is chiefly sanctioned by usage. As ordinarily understood it assumes an inflammatory porcess. The lesion in question here does not suggest inflammation if only on account of the total absence of chronic vascular dilatation.

Worbid anatomy of dermatofibroma

The lesion is invariably benign, it is nearly always solitary, of very indolent growth and although often circumscribed, it may be diffuse. In the fresh specimen the intracutaneous site, the remarkable induration, the absence of capsule and the faint flat yellowish-grey colour of the cut surface are characteristic. The exact nature of the colouration is obscure, it is unlikely that haemosiderin accounts for it. Microscopically it generally presents as an imperfectly circumscribed proliferation of cells. The bulk of these has that superficial appearance of uniformity which is common in tumour. This noiety is generally composed of stout spindle-shaped cells closely resembling hyperchromic fibroblasts, frequently the nuclei shew distortion and the cells then appear stellate. They are applied to or set in a mesh of collagen fibres. In this groundwork tissue and at the periphery xanthoma cells 
may be identified either singly or in groups. A variety of giant cell the Touton cell (1885) is comrnonly present, this is believed to be a fusion product of xanthoma cells. Haemosiderin is nearly always denonstrable in some areas of the Erowth.

It is important to appreciate that much of the theory regarding this tumour-like formation is speculative; the precise histogenesis has not yet been satisfactorily proved. The synovioma or benign giant-cell tumour of tendon sheath presents a fairly close similarity in structure to dermatofibrona but its claims to tumour status are much more justified.

In sumnary while the growth does present some tumour-like propensities its pretensions in this respect are low, and it may be regarded as a link between the peculiar fibrosis under discussion and neoplasia. RIEDEL'S STRUTH

This uncominon malady furnishes another example of localised peculiar fibrosis. Unfortunately there does not appear to be any concise inforration on exactly what constitutes the disease nor is there agreement on its nature, aetiology, or natural history. There are, nevertheless, sufficient premises to consider it a separate disease entity but with the qualification that it is still imperfectly defined.

In 1896 Riedel described a form of chronic inflammation of the thyroid gland which transforned it into a bulky tumour composed of dense fibrous and sclerotic tissue. The entire gland was involved and converted into a mass of alnost iron-hard consistency, and so generally adherent that extirpation was difficult or impossible. He made no mention of extensive lymphocytic infiltration nor the formation of 
seminal follicles in the fland. He further observed that the disease did not behave like carcinoma, for which it was commonly mistaken, and that it did not respond to appropriate therapy for the common specific chronic inflawations. Since this initial communcation the definition has been widened by some observers, and narrowed by others with the result that misunderstandings have been caused. The characterisation of rare diseases is always a matter of difficulty for the same reason that ex pede Herculanem is an unsound principle for a physician. Not a few rare conditions have been described on very small series, e.g. the uncommon nervous disorders, endocrine gland dysfunction syndromes, and etc., and it is inevitable that errors are made. Nindful of the succinct injunction of Dr. Ferguson-Smith, - which same he was wont to repeat with enviable precision and rapidity 'The common forns of rare diseases are rarer than the rare forms of cominon diseases!' a cartain latitude in definition is surely justified and this does not reflect criticism on the original observations.

The nature of the disease is unknown but this of course does not preclude copious speculation which is well seen in the numerous titles suggested, sic: Ligneous thyroiditis, woody thyroid, primary chronic inflammation of the thrroid gland, Riedel's thyroiditis, iron-hard tumour of the thyroid gland, and benign granuloma of the thyroid gland, all reflect the varying ideas. Riedel's struma is tactfully noncommittal. Wwing, who suggested the penultimate name in the list, clearly conceived of 'a granulona of neoplastic type', the idea pervades his unquestionably solid contributions to oncology, this name possibly expressed a belief that the disease has tumour-like qualities. The aetiology is also unknown. The relation of this disease to 
Hashimoto's disease is still a vexed question some observers maintain they are distinct e.g. Hellwig, (1938) Boyd, (1945); whilst others affirm that they are simply stages in a single process Shaw \& Smith, (1925). The forner evidence is based on the retention of the same microscopical picture in serial biopsies made over protracted periods and the latter on studies of quantities of material in which gradations from one to the other can be traced. Wost orthodox opinion at present favours the former hypothesis.

Boyd (1945) confesses his own bewilderment at the 'mixed' types one meets in actual practice. In this connection it is quite probajle that 'burned-out' Graves' disease and myxoedena supply a number of these equivocal cases.

The natural history of the lesion is elusive, because so frequently it is fully established when the first examination is made. It is debatable whether studies of large series shewing progressive degrees of fibrosis are admissible as evidence of the process particularly in a structure like the thyroid gland. It has been suggested, De Courcy (1943), that the lesion is prinarily a peri-adenitis but the morbid anatomy points rather to an augmentation of existing fibrous tissue in the stroma of the gland as well. This proceeds centripitally bearing down on the acinar tissue and at the same time reducing its blood supply. This mechanical crushing and starvation result in atrophy of the epithelial moiety. At the same time fibrous tissue expands outwards driving contiguous lobules of the gland further apart. In this way absolute increase in size of the gland is attained. The main interest here is the fibrosis which fixes the gland so 
firmly to adjacent structures. This has more than a passing reseiblince to desmoid tumour and is in fact closely parallel. Fixation is probably an essential fature in the disease. In a series of 23 cases collected bo Shair \& Smith (1925) 18 shewed this phenomenon, in the remainder the records were inconplete. In a series observed personally the phenomenon was present in 3 cases out of 4 . The infiltrative tendency of the fibrosis is much the same as in desmoid. POLYSTROSITIS

This term is used to include a group of race conditions in which there is remarkable fibrous thickening and sclerosis of the serous membranes, with a tendency to chronic dropsical effusions into serous sacs. Hany other names have been attached to the diseuse and several to conditions which are regarded as incomplete manifestations of it. The term pervisceritis connotes the same disease in France; in Italy, Concato's disease is identified with the condition and this eponymic title is frequently used in the United Kingdon. Formerly polyormenitis chronica was employed synonymously in parts of Germany. A frequently quoted authority, Kelly (1903), used the name 'multiple serositis'. It is probable, Cappell, (1951) that Picks disease (pericarditische pseudolebercirrhose, 1896) is of the same essential nature.

This multiple nomenclature and the general vagueness of the subject have given rise to extensive searches of the literature to ascertain who was the original sponsor. In the publications available to me many of the early references are to inaccessible sources, chiefly continental in origin. Kelly considered that the first case was reported by van Deen in 1846 but other authorities attribute priority to Lancisi (1728), 
Corvisart (1812), or Chevers (1842).

In the third edition of Corvisart's treatise (1818) there is an account of a case of chronic constrictive pericarditis in a man aged 40 years. The case notes and post mortem findings indicate that this was probably an example of polyserositis. Two other cases of adherent pericardium are described in this section but their identity is dubious.

Chevers described two cases of chronic constrictive pericarditis with ascites, in the first of these (Case Jane.P.) a pleurisy was also present and was doubtfully ascribed to a chronic empyema.

Kelly (1903) published an instructive review of the condition including a full account of a fatal case which he examined himself and 39 cases extracted from the literature. He was the first author to regard Picks disease, chronic constrictive pericarditis, chronic hyperplastic peritonitis \& etc., as being merely different expressions of the same morbid process, and his paper has formed the basis for subsequent work.

Interest in the condition was much stimulated when surgical treatment was found to give considerable relief or even cure in a high proportion of cases. White (1935) related a series of 15 parsonally observed cases, some of whom were successfully treated and Burwell \& Blalock (1938) a further group of 19 cases. The aetiology of polyserositis is not agreed. In Kelly's case careful mirroscopy failed to reveal any evidence of tuberculosis. Four cut of the 39 abstracted cases were considered tuberculous in origin. In inite's series the causation was ascribed to tuberculosis in only 2 cases with certainty. Thite insisted that Picks disease was not related to polyserositis. In Burwell \& Blalock's patients 16 out of the 19 were considered of tuberculous 
origin, 3 due to pyogenic microbes.

The morbid anatomy is variable depending on the extent of the disease. The process is essentially a remarkable hyaline thickening over the affected sites. This is chiefly collagenous whitefibrous tissue with sparse cellular content and of very low vascularity. In some cases organs invested by these laminated deposits shew extension of fibrosis through their capsules suggesting a feeble infiltrative tendency at work. So far as can be understood the process appears to be naturally slowly progressive. While it has been thought that the lesion misht owe its character to some chronic mild irritant this has not been well proved. It appears reasonable to regard it as an example of peculiar fibrosis since it persists in the absence of any identified cause in most cases, and its wayward if slow progress reflects a degree of autonymity.

MYELOFIBROSIS

This is a rare condition in which the haemopoietic elements of the bone marrow are replaced by fibrous tissue. The replacement appears to be a slowly progressive process which ultimetely involves all the marrow previously occupied by active blood-forming tissue. Pari passu extramedullary foci of haematopoietic tissue develop especially in the liver, spleen, and the lymph nodes. Small foci have also been observed in the kidney, pancreas, abdominal fat \& etc.

In common with all rare disease (under 200 cases had been reported in 1946) the characterisation is imperfect and there is some doubt as to whether it really is a morbid entity.

On the otier hand it is reasonable to suggest that myelofibrosis is $/$ a process 
a process which is possibly fundamental in character and recurs as a common factor in certain specific diseases and syndromes.

The phenomenon has been recognised for a long time. Most authorities attribute the initial observations to Henck in 1879. This worker described two cases. One of them was an instance of lymphatic leukaemia in which fibrosis of the bone marrow was found at necropsy. In many respects the feature of myelofibrosis has been overshadowed by the results related to it. Thus after the description of leucoerythroblastic anaemia by Vaughan, (1936), and the conception of 'myelophthisic' anaenia, this blood picture has dominated the subject. While a pure prinary nyelofibrosis certainly exists and does charecteristically produce this anaenia nanj other lesions can bring about the same blood change and nunerically they are overwhelminsly more frequent. nietastatic carcinoma in bone marrow is probably the commonest cause of this; most instances are recorded in cases of mamary, prostatic, and pulmonary carcinorna.

Confusing the issue further are a series of very rare diseases and syndromes. The marble-bone disease of Albers-Schbnberg and Albright's syndrome are the best known examples, and the storage diseases of the reticulo-endothelial syster including Gaucher's disease, Niemann-Pick's disease, and Schuller-Christian's disease nay also give rise to this anaenia.

Unfortunately there is not any authoritative identification of a large number of doubtfully characterised conditions related to these. Albright's original ngame 'osteitis fibrosa disseminata' - which lesion is mainly unilateral - would seem the same as Lichtenstein and Jaffé's 
'polyostotic fibrous dysplasia' in so fur as the bones are concerned. The tem osteosclerosis is sometimes used instead of myelofibrosis. Wettier and 3usk (1937) use it to include osteopetrosis (AlbersJchunberg's disease) and myelofibrosis. Myelosclerosis is apparently employed synonymously aith nyelofibrosis.

Finally there is a chatic group of cases under the headings of 'Ieukaemoid blood picture', aleukaemic myelosis with osteosclerosis (Stevens and Bredeck, 1933), and unusual cases of polycythaenia vera.

This subject leads into that darkness which stultifies the most dilibent efforts to understand it properly. There is however one paper which is most helpful. This is the commuication of Vaughan ard Harisison (1939). Phese authors suggest that the underlying cause is a 'progressive hyperplasia' of multipotential mesenchymal tissues by an unknown stimulus. The leuco-erythroblastic activity they believe nay be partly secondary to the nyelosclerosis and the fibrosis itself may in part be the result of the haemic change. This idea is the reticulosis idea, but quasi-neoplastic fibrosis is surely an equally reasonable nane for the process. NEUROFIBRONATOSIS

This subject invites controversy from the start. in uncomnon disease, yet widely known about, it has evoked violent argument since its initial description. Von Recklinghausen (1882) first emphasized the relation of the lesions to the peripheral nerves and his name is now commonly attached to the condition, the disease was nevertheless well known at least twenty years prior to this, (Hitchoock 1862).

Opinion has backed and veered irresolutely with regard to its 
nature, status, and histogenisis for so long that one wonders how long it is to enjoy its present position.

Some few facts regarding it appear generally accepted. The complex is an entity, this despite the protean morphology it nay present. Thus tie full picture with multiple molluscum fibrosum lesions, pigmentation, plexiform neuroma, hypertrichosis, bone changes, and elephantiasis arabur, represents the entire realisation of the same process as the 'neurofibroma' of skin. The present view is that the disease represents a maldevelopment of the peripheral nervous system, Stout (1949), Willis (1949). For this reason it is proper to term it hamartoma.

The origin of the growtin is less easily settled. It is now considered that it is predominantly neurilemmal with admixture of neurites. These latter are conspicuously absent in most encapsulated neurilemmomata. Stout (1949) laid considerable stress on the specificity of neurilemmal cells being established by tissue culture but curiously enough in this account cultures from neurofibromatosis were not recorded. This author pointed out that malignnant schwannoma may develop on a basis of neurofibromatosis and offered tissue culture evidence to support the contention that these malignant growths were composed of neurilemmal cells. This however is only indirect evidence that neurofibromatosis is neurilemmal in origin, and it is pertinent that neurilemmoma is almost invariably encapsulated and very rarely goes malignant.

The older views which were well expressed by Bwing have much to support them. If cell morphology is reliable than fibroblasts and endothelial cells are certainly present in many neurofibromata and a 
tendency to whorling - as in meningioma is often distinct.

Whether the growth is entirely neurilemmal or partly fibroblastic there is consent that collagen is formed and this is equivalent to acknowledging that fibrosis is a sequel. This fibrosis and simultaneous overgrowth of tumour-like cells whence it is arising may proceed very rapidy. Instances are recorded of relatively sudden pronounced growth of this kind supervening in plexiform neuromata, the process extending up to and even entering the spinal cord, without true sarcomatous trunsforration of the tissue involved. In a similar fashion a localised elephantiasis neuromatosa may spread from an initially restricted site to involve large tracts of tissue. Although neurofibromatosis is a less satisfactory example than others which are cited it is submitted that there is justification for including it since certain of its expressions illustrate tumour-like fibrosis. 
263.

SECTION B.

Eosinophilia, with special reference to

the phenomenon in tumours.

p. $264-270$

Illustrations

Volume III. Fig. 213

Volume IV. p. 30-33. 
EOSINOPHILIA, WITH OBSERVATIONS ON THE PHENONENON IN CYTOMATA

Eosinophile leucocytes are found in the blood and certain fixed tissues. In the circulating blood of normal man these conspicuous cells account for 1-4 per cent of the total leucocytes the aboolute figures being 50-400 per cu. mm. In the fixed tissue of the adult its normal habitat is the bone marrow, but it is also usually found in the stomach, duodenum, caecum and vermiform appendix. In the foetus and neonatal infant it resides in the archemyelon, viz. spleen, liver, thymus gland, pancreas, and less constantly in other sites.

The disparity in morphology between the blood and tissue types is chiefly attributable to the results of the different preparation techniques conventionally adopted, but eosinophile cells peculiar to extra-myeloid sites also exist.

The morphology and tinctorial properties of this cell are well-known. The nature of the oxyphile granules is not fully understood. They are insoluble in fat solvents, weak acids, and alkalis. They are negative to the xanthoproteic reaction with nitric acid, and they contain ionisable iron. This last property was first demonstrated by Barker (1894). Despite the negative reaction with nitric acid they have been considered to be protein products since Jhrlich first suggested that they corresponded to the aleurone granules of ova. (Peyton Rous, 1908, Jolly. 1922). The function of the cell is obscure. As a leucocyte it is unimpressive; it does not respond proportionately to the chemotactic stimuli which excite the neutrophiles, and in fact is characteristically suppressed in the acute inflammatory exudate.

The origin of the eosinophile is not universally agreed. Ehrlich held the view that production was exclusively myeloid and this has been 
upheld by Ringoen (1921) and Biggart (1932). This theory has been widened by the inclusion of an origin from extramedullary myeloid tissue, which locus may be the result of myeloid metaplasia, Lang (1926). The mechanism of origin in this phenomenon has been interpreted differently. Dominici (1909) traced the development of eosinophile leucocytes from lymphocytes and ieidenriech (1910) maintained that this represented a process of phagocjtosis, the ingested particles being broken down erythrocytes. O.H. Bunting (unpublished observations) cume to similar conclusions but considered that plasma cells were the unfed potential eosinophiles. Fallacies attended the early experimental work of this school, and it is now widely abandoned that the granules are derived from effete red cells. On the other hand the transforration of lymphocytes, plasma cells, stem-cells \& etc. to eosinophiles is still supported. Pullinger (1932), Gillman et al (1949). Up till very recently it has been singularly difficult to photograph eosinophiles satisfactorily, and many pathologists are sceptical about the objectivity of drawings. This fact has militated against general acceptance of this theory. All considered it appears probable that myeloid tissue produces most of the eosinophiles but that cytometaplasia also contributes some. It would appear reasonable to anticipate an hyperplasia of the precursors of this cell in the myeloid tissue when eosinophilia is present. This however is difficult to substantiate because narrow is impossible to sample accurately and the augmentation may be very slight, Beattie and Dickson (1943). One corollary of the exclusively myeloid origin theory is that topical eosinophilia must be infiltrative; this postulates the presence of some attracting stimulus resident in the tissue. 
One further theory is mentioned. Gutig (1907), Downey (1914) held that the blood and tissue eosinophiles were different in every way, and should be considered separate cells.. The identity of this tissue eosinophile is distinctive. It is larger than the blood counterpart,. and the granules are less distinct and brownish-pink when stained with eosin. These cells are commonly found in lymph nodes and are almost certainly macrophages.

Eosinophilia in the blood has been extensively studied and the conditions in which it occurs have been well defined, liuir (1941), Thitby and Britton (1942). This has encouraged several generalisations of value, but the phenomenon can be capricious in the face of known causes and idiopathic examples exist. There appears ample evidence that certain exogenous proteins precipitate eosinophilia. Endogenous proteins are possibly also capable of producing the effect. Certain drugs, particularly camphor and nervanol, tend to produce eosinophilia, and less constantly mercury and iodoform are credited with the same property.

Local eosinophilia in the fixed tissues has not received the same amount of study as the more readily examined blood. The phenomenon accompanies many cases of blood eosinophilia but these examples can be set aside. There are conditions in which local eosinophilia may occur $r^{a s}$ an isolated finding, e.g. eosinophilic granuloma, non-specific periappendical granuloma, Musgrove and Docherty (1950); ligneous perityphlitis, Wilson, Dockerty, Waugh and Bargen (1949), and periarteritis nodosa, to mention only a few. It is quite common in gastric ulcers, nasal polyps, and gummata.

In sterile abscesses produced by turpentine eosinophilia may be 
intense, and this has suggested necrosis with endogenous protein release as the exciting cause; a similar idea has been offered to explain the phenomenon in X-ray and radium burns.

Eosinophilia in tumours has not been much studied. Mallory (1913) emphasized that the phenomenon was common in carcinoma of the uterine cervix. Bizgart (1932) recorded local eosinophilia in all of a series of 19 tumours - 14 malignant and 5 benign recovered from a variety of sites. Willis (1947) discussing the local eosinophilia of Hodgkin's disease considered that 'the granulocytes and plasma cells in Hodgkin's appear clearly to be only reactionary elements similar to those seen in many other kinds of tumour'.

It was considered that information on this problem might aid the interpretation of the local eosinophilia of Hodgkin's disease. Material. A series of 500 separate carcinomata encountered consecutively in the Surgical Specimen Routine were examined for local eosinophilia. Since lymphoid tissue is mesodermal comparison with sarcomata would have been more apt but the numbers available were far too small.

The tumours were derived as follows:-

\section{Site}

Integument and its derivatives.

Gastro-intestinal tract.

Genito-urinary system.

Respiratory system.

Miscellaneous (includes lymph nodes).

Total

The bias in favour of tissues and organs amenable to surgery is
Total Number

213.

139.

103.

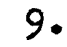

36.

500

/manifest, 
manifest, the series is not representative of cancer incidence in general.

Methods.

The routine sections stained with haematoxylin and eosin were examined first. Where eosinophiles were apparent confirmation was usually made by staining with dilute eosin or by the carbol-chromotrope method. The concentrations encountered were graded as sparse, + , ++ , and +++. The index ++, approximated to the degree of eosinophilia encountered in typical Hodgkin's disease, namely obvious without search. For the purposes of this survey examples exhibiting ++ or +++ were considered topical eosinophilia.

Results.

$\frac{\text { Total Tumours. }}{500 .} \quad \frac{\text { Local Eosinophilia. }}{75 .} \quad$ Rage.

Analysis.

1. Integument and its derivatives.

Site. Total cases. Eosinophilia. Rage.

Skin. Squamous

$\begin{array}{lccr}\begin{array}{l}\text { cell cancer. } \\ \text { Basal cell } \\ \text { cancer. }\end{array} & 38 . & 6 . & 15.8 \\ \begin{array}{l}\text { Malignant } \\ \text { melanoma. }\end{array} & 30 . & 0 . & 0 . \\ \text { Metastatic. } & 12 . & 0 . & 0 . \\ \text { Breast. } & 5 . & 0 . & 0 . \\ & 128 . & 3 . & 2.3\end{array}$

Notes. In 3 cases of squamous cell cancer shewing eosinophilia, genital area skin was affected; the total of epitheliomata in this region was 7 . All the positive breast cases were spheroidal cell tumours, 1 shewed 
Paget's intra-epidermal carcinoma.

2. Gastro-intestinal tract.

\begin{tabular}{|c|c|c|c|}
\hline Site & Total cases. & Eosinophilia. & \%age. \\
\hline $\begin{array}{l}\text { Mouth. } \\
\text { Oesophagus. } \\
\text { Stomach. } \\
\text { Caecum. } \\
\text { Colon. } \\
\text { Rectum. } \\
\text { Vermiform appendix. }\end{array}$ & $\begin{array}{l}23 . \\
7 . \\
24 . \\
37 . \\
20 . \\
2 .\end{array}$ & $\begin{array}{r}1 \\
2 \\
15 \\
4 \\
13 \\
11 \\
1\end{array}$ & $\begin{array}{r}4 \cdot 3 \\
28.3 \\
62.5 \\
100.0 \\
35.1 \\
55.0 \\
50.0\end{array}$ \\
\hline $\begin{array}{l}\text { Bile ducts. } \\
\text { Gall bladder. }\end{array}$ & $\begin{array}{l}2 . \\
3 .\end{array}$ & $\begin{array}{l}0 \\
1\end{array}$ & $\begin{array}{r}0 \\
33.3\end{array}$ \\
\hline $\begin{array}{l}\text { Omentum. } \\
\text { Liver. } \\
\text { Pancreas. }\end{array}$ & $\begin{array}{r}12 . \\
3 . \\
1 .\end{array}$ & $\begin{array}{l}1 \\
0 \\
0\end{array}$ & $\begin{array}{r}8.3 \\
0 \\
0\end{array}$ \\
\hline Parotid gland. & I. & 0 & 0 \\
\hline
\end{tabular}

Notes. The high incidence of eosinophilia in gastric, caecal, colonic and rectal tumours is probably related to the normal presence of eosinophiles at these sites. Little significance attaches to the other relatively high figures since the total numbers are so small.

3. Genito-Urinary system.

\begin{tabular}{lcccc} 
Site & Total cases. & Eosinophilia. & Fage. \\
\cline { 2 - 3 } Kidney. & 10. & 0 & 0 \\
Bladder. & 18. & 7 & 38.8 \\
Urethra. & 2. & 0 & 0 \\
Uterus. & 11. & 2 & 18.2 \\
Cervix. & 23. & 3 & 13.0 \\
Ovary. & 13. & 1 & 7.7 \\
Prostate gland. & 24. & 2 & 8.3 \\
Testis. & 2. & 0 & 0
\end{tabular}

Notes. The figure for bladder cancers is high, most of these growths were papillary carcinomata. The uterine and cervical incidence of eosinophilia is in keeping with general experience. The single positive 
ovarian eosinophilia was in a malignant teratoma from a child.

4. Respiratory system.

Site

Larynx.

Branchial cleft.

Bronchus.
Total cases. Eosinophilia.

0

0

0 page.

0

0

0

Note. The series is too small for reliable results.

5. Miscellaneous.

Site

Total cases.

Eosinophilia.

Qage.

Lymph nodes.

Thyroid gland. 30

4

2

0

6.6

Fascia.

Adrenal (secondary)

1

0

0

1

0

0

0

Note. One of the cases of lymph node metastatic carcinoma was a gastric node, the other was from the scalp.

The limitations of the series preclude authoritative interpretation of eosinophilia in carcinomata generally, but there is evidence that the phenomenon is related to the site of the growth and that it is not so rare as might be expected. It is not related to necrosis but is often accompanied by plasma cell infiltration. 
271.

PART $\quad \nabla$

EXPERIMENTAL

An attempt to reproduce Hodgkin's disease

in rats and mice by trypan blue

poisoning.

p. $272-287$

Illustrations.

Volume III Figs. 214-235.

Volume IV p.89-99 


\section{EXPERIMENTAL WORK ON HODGKIN'S DISEASE}

Foreword. The experimental reproduction of a disease in a suitable laboratory animal integrates knowledge in a unique fashion. The advantages include simplification and control of conditions beyond what is feasible in natural disease, and curtailment of the duration of observations.

Few maladies have resisted the experimental nethod with the success of Hodgkin's disease. It has stultified all attempts. The facts attending this recalcitrance deserve emphasis.

Hodgkin's disease is almost certainly confined to Homo sapiens. Claims of its occurrence in lower animals are rare and its identity in these reports is equivocal. Macllahon (1934), reported a case in a dog. The disease was limited to a single fused cervical node mass on one side; the microscopical picture did not conform to the accepted one closely. Stalker, Schlotthauer, and Feldman (1936), recounted another instance in a dog. The lesions were relatively focal and circumscribed; microscopically they were close to cellular Hodgkin's disease. It is noteworthy that these authors interpreted their findings critically and with caution. Medlar and Sasano, (1937) gave an account of supposed Hodgkin's disease in an elderly fernale rabbit. The photographs of the lesions in this case were not convincing, the fields shewn were very small. Forbus and Davis (1946), reported a reticulo-endothelial system lesion encountered in seven pigs, which they regarded as close to the human disease. This affinity is not borne out by critical analysis of their findings. These reports comprise most of the better substantiated examples, there has perhaps been too sanguine an interpretation of their 
significance by some reviewers. Among the older writings Hodgson (1903); and heFadzean (1903), gave brief references to the disease in horses but the evidence was doubtful.

Transmission of the disease to lower animals has never been successfully accorplished. Attempts have been made since Longcope's pioneer experiments $(1903,1907)$ and many different animals have been used. The general experience is that the grafts die out; if large they may become encapsulated, and if small they are absorbed. Transient reactive hyperplasia of the host lymph nodes has been described in some of the experiments. The universal failure is partly referable to the expected fate of transplants to heterologous species and indicates that an agent capable of inducing the lesion is probably not present in the new tissue.

Tissue culture studies have yielded information about the reticulum cells of the lesion, Grand (1944, 1949), but have contributed little else. Empirical research has naturally a claim, however irrational it may at first appear. Before more particular account is given, one observation is offered.

It is insisted that the production of an ephemeral lesion having some or even tuch microscopical similarity to Hodgkin's disease is a very restricted accomplishment. Failure to appreciate this has been a regrettable shortcoming among certain experimental workers. Any student of the human disease cannot fail to be impressed by the relentless if temporarily hesitant progress which Hodgkin's disease invariably presents. This aspect of its behaviour has never been simulated. It is possible to produce lesions with close morphological 
affinities to the disease by a considerable variety of methods in the common laboratory rodentia. These however are ephemeral, and fail to evolve like the true disease. Examples of this type of reaction are seen in the work of Iiller and Turner (1943), who injected elaborately prepared extracts of urine from patients with leukaemia and Hodgkin's disease into guinea-pigs. In this work the fractions used were one containing hydroxy-acids which appeared to stimulate lymphopoiesis, and another made up chiefly of non-carbinol-acids which had a corresponding action on myeloid tissue. It is pertinent that these extracts were deleterious, the animals seldom survived beyond three to five weeks. Very similar reactions could be induced by extracts of beef. The lesions found were well illustrated, but the fields depicted were very small. Initially promising this line of research appears to have been abandoned.

In 1948 Gillman and Gillman employed parenteral injection of trypan blue in female rats in a study from which they drew analogies between the results of this empirical procedure and those observed in babies whose mothers contracted rubella in early pregnancy. In the course of this work they discovered that tumours of reticulum cell type could be induced by this means in several different organs. In the following year Gillman, Gillman, Gilbert and Spence (1949) produced a comprehensive study of these tumours. In addition they reported changes in lymph nodes of trypan blue treated rats which approximated closely in morphology to Hodgkin's disease and Hodgkin's sarcoma. They interpreted these blastomatoid formations as similar to those in man which Cazal (1942, 1946) regarded as manifestations of 'La reticulose histiomono/cytaire'. 
cytaire'. In brfulness for the Ascheim-Zondek test, (these animals were supp hyperplasia whichweekly to the laboratory by Tuck and Sons).

Emanating from Sc They were distributed as follows:observation - 'ex

the more cynical dictum of a previous and shrewder philosopher "ex

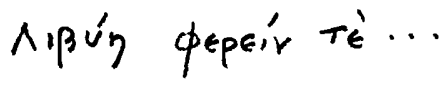

General plan epeír $T \epsilon ́$... ntal work undertaken.

A repetition of the Gillmans' work with trypan blue was attempted. Several modifications were introduced. These included the use of a different brand of trypan blue, its administration to mice as well as rats and alteration in the frequency of injection. A further series of rats were given another vital stain of comparable chemical structure.

As the result of findings established in the preliminary studies, the development of the lesions was followed by serial biopsies in a further series of identically treated rats. The effects of cessation of the trypan blue injections was observed, and attempts were made to transplant the growths obtained.

Material and methods.

\section{Animals.}

Wale albino rats of an inbred laboratory colony of wistar strain were used. The mice were females which had matured beyond their usefulness for the Ascheim-Zondek test, (these animals were supplied weekly to the laboratory by Tuck and Sons).

They were distributed as follows:- 


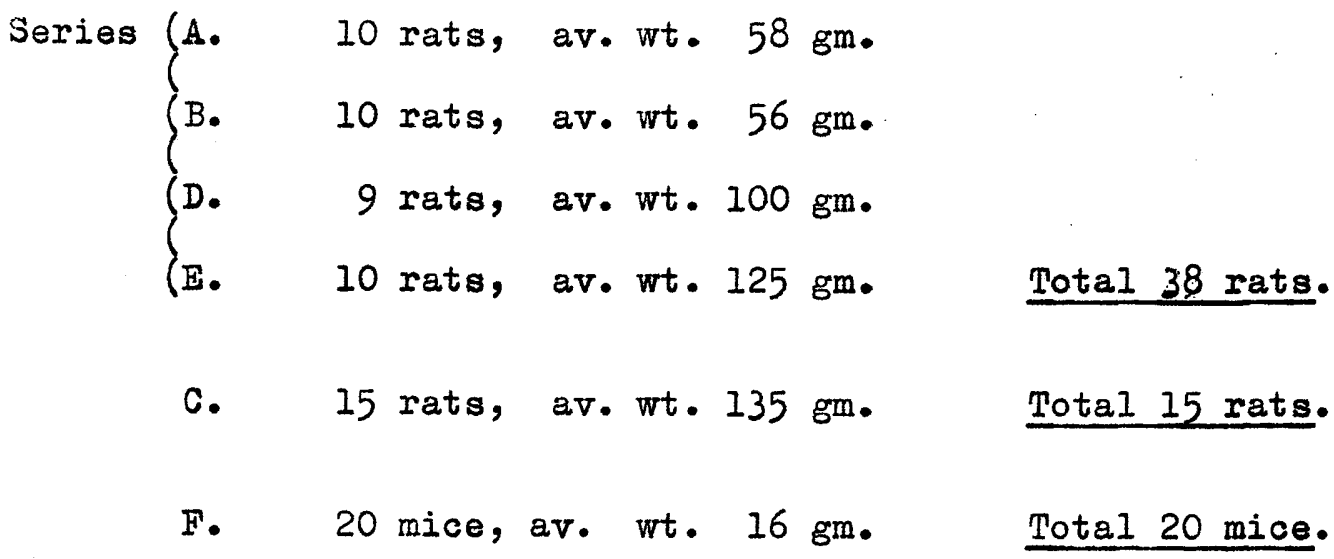

$\begin{array}{ll}\text { Controls. } & 10 \text { rats, av. wt. } 58 \mathrm{gm} \cdot \\ \text { Controls. } & 10 \text { mice, av. wt. } 16 \mathrm{gm} .\end{array}$

Note. A female rath was inadvertently included in Series B. This was overlooked till a litter of eleven was born nine weeks after the experiment started. Nine of the offspring were males and constitute Series D. (The mother was withdrawn from the experiment).

Diet. Rate cake, Rowat Research Institute Diet No.86. Supplemented with bread crusts, occasional cooked bones, and cabbage. Water ad lib. Dyes used.

1. Trypan blue. B.D.H. Batch 647133/471215.

2. Vital new red. B.D.H. Batch $402126 / 471215$.

These were made up freshly in sterile distilled water, $1 \mathrm{gm} / 100 \mathrm{ml}$. Injections.

1. Trypan blue. Series A. and B. $1 \mathrm{ml}$. (= $10 \mathrm{mgm}$. dye $)$ by intra-peritoneal injection every fortnight. Series $D$. and E. the same dosage but given weekly. Series F. $0.1 \mathrm{ml}$. ( $\Rightarrow$ I mgm. dye) by intraperitoneal injection weekly.

2. Vital new red. Series C. $1 \mathrm{ml}$. (= $10 \mathrm{mgm}$. dye) by intraperitoneal injection every fortnight. 
In Series A., B., C., D. and F., the endeavour was to maintain the animals alive as long as possible. They were killed only if they became moribund; or if they survived, to the limit of the experiment.

In Series E. animals were killed at 70,100 and 120 days, after which injections ceased. The remaining seven were subjected to biopsy at 120 days; of these three were killed later, at 140, 160 and 180 days (one was found dead at 167 days). The last three had biopsy repeated at 180 days and were killed at 360 days.

\section{Results.}

Trypan blue, Series A., B., D. and B.

In this group of 38 rats, 5 were lost due to escapes, cannibalism and mistaken identity.

During life the following changes were recorded. After injection of the trjpan blue the animals tended to remain subdued for several $h$ ours. Initially they put on weight more rapidly than the controls but after about two months the position was reversed. Generally by the second injection, whether at 14 days or 7 days interval, general staining of the tissues appeared. This coincided precisely with the full description given by Cappell (1929); it did not augment appreciably thereafter. After 2-3 months most of the beasts became emaciated and weak; they sat hunched up and shivering. In many the abdomen became swollen, and their movements ataxic. Anaemia of the order $3 \times 10^{6}$ erythrocytes per cu.rm. was observed. Terminally they sickened rapidly, diarrhoea was common.

At death the common findings were:-

1. Ascites. The fluid was faintly dye tinged and had a peculiar foul 
metallic odour. It was occasionally turbid and infected. 2. Liver. The organ was usually considerably enlarged (average for series A., B. and D. 32.3 gm., average for controls $12.5 \mathrm{gm}$ ) It was dark water-chocolate colour, the surface was sometimes very faintly stippled but hardly granular. Pumour-like nodules 1-10 mm. were found in 18 cases ( 54 per cent). These varied in colour from pale sky-blue to deep royal blue. They were scattered indiscriminately through the liver substance. Central necrosis was commonly visible in the nodules. 3. Spleen. Inlargement of relatively moderate degree was common. (Average for series A., B. and D. $4.6 \mathrm{gm}$., average for controls $1.3 \mathrm{gm}$. ) Thuour-like nodules were never encountered.

4. Lymph nodes. Apart from pale blue staining these structures did not shew morbid changes.

5. Bone marrow. This was uniformly pale slaty-grey to dull greyishblue. Rarely it was faintly gelatinous.

Intercurrent àisease. Sarcoptic infestation was encountered sporadically. This was treated with 'tetmosol' (Tetra-ethylthiueram monosulphide) with satisfactory results. Several animals were found to be lousy at necropsy. Rat bronchiectasis (Passey, Leese, \& Knox, 1936) was a troublesone complication which forced the abandonment of the original series (not recorded here). In the present records this lesion also appeared but not to a serious degree, the controls shewed a rather lower incidence of it. Microscopical examination.

Liver. There was a remarkable focal proliferation of cells in the portal tracts. In the initial stages this was fairly uniform throughout 
the organ. These formations rapidly assumed the form of sharply demarcated rounded nodules which appeared as buds growing from an axial stem of portal tract tissue. Their origin appeared to be from pericytes in the adventitiae of the vascular radicles. The component cells conformed to a single type with the following characteristics. They measured 9-15 $\mu$ diameter, rarely nore, and were typically spheroidal or polygonal, but wedge-shaped or spindle-shaped when closely packed. The cytoplasm was non-granular, tending to be refractile, and oxyphile. Inclusions e.g. dye, haemosiderin, or debris, were common. The nuclei were trachychromatic and roughly oval. Detail was difficult to discern due to their density but they resembled shrunken twisted skeins. Nitoses were rare. Reticulin formation by these cells was the rule, at least in mature deposits. The formations usually increased with age but individual deposits outstripped others. Central cystic change and necrosis were common. In a small proportion of cases demarcation became poor, the growth rate increased and the deposits becane tunourlike. The progression of the lesion was capricious in cases followed by biopsy in Series $\Xi$. The cessation of dosage at $120 \mathrm{mgm}$. in seven of these animals was followed by advance to the tumour-like picture in two animals. In the remainder the appearance remained static or regressed.

Spleen. Enlargement was related only to general reactive hyperplastic change and engorgement or oedema. Haemosiderosis in the red pulp was seen occasionally. Tumour-like focal aggregates of macrophages were not encountered. (In all the spleens fields microscopically similar to Hodgkin's disease could be found; these were seen where septal connective tissue and megakaryocytes were in propinquity. The 
resemblance is illusory, it did not inature at all). Iymph nodes. In several cases the axillary nodes shewed partial replacement of lymphocytes by plasma cells and mast cells. Lesions comparable with the hepatic ones were never seen. An isolated finding in one node was diffuse fibrosis of the cortex. Bone marrow. This was not consistently exarined. There sections were taken it was comion to encounter scattered macrophages in the tissue, many contained dye.

Perivascular proliferation of cells like those in the liver lesions was constantly observed in the lungs, kidneys and omenta.

In all 12 attempts were made to transplant the tumour. Saline suspensions were made with aseptic precautions and injected intraperitoneally into adult male rate. Despite much care infections usually resulted, and eight of the animals died within three weeks. In the remaining four no trace of the inoculum was found at necropsy. Series F. Mice receiving trypan blue.

Staining developed within a few days of the first injection and was exactly comparable to that observed in rats. On the other hand their condition remained normal. Animals were killed at 70, 100, 120, 170, $180,200,220,250$ and 300 (2 animals) days. The remainder survived in excellent health and were killed at 360 days. None were lost from the series and none died spontaneously.

At death faint general staining of the tissues was seen. The lymph nodes were readily found due to their faint blue colour, and were generally much easier to recover than the nodes in the rats. Ascites was not recorded and the livers shewed only a dark water-chocolate 
colour. Splenomegaly was absent.

Nicroscopical examination.

Liver. Circumscribed nodular hyperplastic aggregates of macrophagelike cells were observed in three animals (170 and 360(2) days). They were scanty and small in each case. In all animals after 70 days exposure to dye small haenopoietic foci were seen; most were localised in the sinuses, and a few were in portal tracts. In all the Kuppfer cells were prominent due to dye content. Haemosiderin was very scanty in these cells.

Spleen. Haemosiderosis of moderate degree was common in the red pulp, but was not consistently related to the time of exposure. Lymph nodes. Sinus catarrh was seen occasionally. Kidneys. In all cases numerous dye granules were found in the first convoluted tubules of individual nephra. Focal interstitial or perivascular lesions were not encountered.

Lungs. Scanty perivascular and peribronchial deposits of small densely staining nuclei were seen. Dye and haemosiderin-laden macrophages were common in the alveoli.

Series C. Rats receiving vital new red.

Unlike trypan blue this dye did not appear toxic to rats in the dose used. All the animals remained outwardly healthy throughout the experiment, and none died spontaneously. Staining developed within three days of the first intra-peritoneal injection but partly on account of its colour it was difficult to detect unless controls were examined at the same time.

Six animals were killed after 27 fortnightly injections and six after 28 injections. One animal was accidentally killed in the first week of 
the experiment, and two escaped, after almost a year.

At death very faint pink staining of the tissues was appreciable. The only conspicuous abnormality, which was seen in 7 animals, was enlargement of the juxta-caecal mesenteric lymph nodes. These were swollen and grey, and section revealed gelatinous material apparently occupying cysts in their substance. Ascites was not observed. Hepatomegaly and splenomegaly were absent, and the tâches laiteuses were not obvious. The average liver weight was $9.5 \mathrm{gm}$. , controls $12.5 \mathrm{gm} . ;$ average spleen weight $0.9 \mathrm{gm}$. , controls $1.3 \mathrm{gm}$. Microscopical examination.

Liver. These were alnost entirely normal. In two livers minute and scanty circumscribed aggregates of macrophage like cells were noted. The Kuppfer cells did not shew any increase.

Spleen. In four moderate haemosiderosis in the red pulp was noted, otherwise the glands were normal.

Lymph nodes. The peripheral nodes shewed sinus catarrh inconstantly. The changes in the caecal nodes were confirmed to be cystic change initiated in the lymphatic sinuses. The cellular conformation of these nodes remained normal.

Lungs. Rare aggregates of small macrophage-like cells were encountered in most.

Kidneys. Granules of dye were always seen in the tubule cells but rarely were they numerous.

Comment on findings, and discussion.

The most inpressive finding is that when rats are injected with relatively small doses of the vital stain trypan blue at fortnightly 
intervals over a period of four to five months tumour-like nodules of distinctive structure develop in the liver in a considerable proportion of the animals ( 15 out of $23 ; 65 \%$ ).

Progressive anaenia, emaciation, and weakness also result. Injection of the same individual doses with twice the frequency induces the same changes more rapidly.

In mice subjected to the same treatment these tumour-like changes in the liver are not produced to any extent, but anaemia may develop.

The growths produced appear to be constituted by a single celltype which morphologically is a macrophage.

Rats given vital new red under the same conditions do not shew changes beyond inconstant haemosiderosis in the spleen.

In these experiments distinctive lymph node lesions were not observed in any of the animals and the 'Hodgkin-like' picture reported by previous workers was not reproduced. Occasionally slight alteration of lymph node cytology was encountered, plasma cells and mast-cells appearing to replace lymphocytes. Interpretation of the hepatic lesion.

It is considered that it would be erroneous to designate the cellular growtis obtained by chronic trypan blue poisoning as tumours, without qualification. Pathologists whose experience is chiefly derived from human morbid anatomy are well aware that to call a growth tumour 'is an award of considerable magnitude. In experimental cancer research, which is now virtually a separate subject, a less critical employment of the term neoplasm can be detected. In lower animals the sensitivity to neoplastic disease is nearly always much lower than in 


\section{4.}

man, and constitutional disturbances e.g. illness, cachexia and anaemia are not so prominent. The anomalous brisk activity and good general health of 'cancer mice', which animals may have enormous tumours, is remarkable. These growths whilst legally cancer, are hardly the same in a moral sense. At microscopical level many experimental tumours present an indefinable appearance of artefact, at least to an observer familiar with human tumours.

In the present study the following features support the interpretation of the lesions as turnour. The growths present uniformity of the proliferating cell-type. There is compuratively little pleiomorphism, certainly inuch less than is characteristic of a typical granuloma. This cell-type is atypical although it approximates closely in most respects to an histiocyte or macrophage. The possible affinities to erythroblast are mentioned but have not been specifically studied. In a proportion of cases more active proliferation is exhibited and simultaneously there is loss of demarcation of the growth which can be regarded as infiltration. There is in addition the phenomenon of progressive growth after removal of the presumed genetic/ stimulus. Hinor features supporting the view include the tendency to necrosis and degenerative change.

On the other hand most of the growths are sharply circumscribed and apparently indolent. Progression either with the continued exhibition of the dye or after its cessation is not invariable, some regression being cowmon. The growths are confined to the liver and even where they appear malignant locally there is failure to metastasize. Finally attempts to transplant the tumours to other rats failed con- 
sistently. This last phenomenon of serial transmission is characteristically a property of tumour, but the practical performance of the test is less certain than its theoretical possibility.

The property of producing reticulin neither reinforces nor detracts from the tumour hypothesis. The elaboration of reticulin by macrophages (histiocytes) is well authenticated, Curran, (195I); Nicol and Abou-zikry, (I953).

In summary the growths are experimental tumours of characteristically low malignancy. They appear peculiar to the liver and are without close counterpart in human neoplastic disease. Morphologically they are sarcomata.

Assuming this view to be correct their genesis requires explanation. It might be held that repeated parenteral injection of a vital dye acting as a stimulant to the reticulo-endothelial system cells might result ultinately in neoplastic change. This has little to commend it. Generalised tumour production throughout the system was not found and the similar colloidal dye vital new red failed to cause similar growths. The total dye given was so small - generally under $0.5 \mathrm{gm}$, that in the latter case there was not even hyperplasia of the spleen. It is reasonable to infer that trypan blue may be carcinogenic in rats.

This dye is not generally credited with carcinogenic properties but this does not reflect final disposal of the possibility. The recent work of Simpson, (1952) and Warshall, (1953) following the Gillmans' studies, confirms that the dye evokes experimental tumours in rats. Boyland, (1952) has emphasisized that in the present provisional list of chemical carcinogens powerful ones are chiefly considered, but that it is being learned that many less spectacularly potent agents also exist. The 
effective dose of powerful carcinogens under optimum and standard conditions is very small. It has been demonstrated that as little as $0.2 \mathrm{mgm}$. of methylcholanthrene is capable of inducing skin papillomata in mice, Hieger (1953). Here trypan blue appeared effective in doses of about $120 \mathrm{mgm}$. In biological assay work disparities of this order viz. I:600 are not exceptional, and this further credits the dye as a carcinogen under the present conditions of test. The restriction of the effect to one tissue site is a common phenomenon encountered in cancer research.

It is beyond the scope of the work to investigate the chemistry of this action. Sorne azo-dyes e.g. Butter Yellow, have been shewn to be carcinogenic and this property is ascribed to the presence of substituent methyl radicles in the amino-azo-benzene nucleus. o-tolidine, the basis of trypan blue contains two methyl radicles but is reported to be devoid of carcinogenic activity, Jimpson (1952).

Two additional factors deserve mention. In general terms rats are readily susceptible to experimental sarcoma, Pullinger (1953), which has been induced by a variety of procedures. In contrast the mouse is refractory although it is typically susceptible to carcinoma.

This neoplastic propensity of rat connective tissue may even be evoked by culture and sub-culture of normal rat fibroblasts without the intervention of any recognised external factor. This almost incredible fact modifies ideas concerning the role of carcinogens in sarcomata of this animal and suggests that even the weakest carcinogens might be unexpectedly potent in this species.

Also it has long been known that the route of administration may /determine 
determine the action of a carcinogen. Superficial application is more likely to lead to carcinoma and parenteral injection to sarcoma.

\section{Summary.}

The prolonged administration of trypan blue (B.D.H.) in $10 \mathrm{mgm}$. doses at fortniütly or weekly intervals by the intra-peritoneal route induces a characteristic cellular proliferation in the hepatic portal tracts of rats. The type-cell of these lesions is a macrophage and the lesion is considered to be an experimental tumour.

The procedure also produces chronic poisoning and anaemia. Mice are refractory to this treatment and rats treated in the same method with vital new red do not shew similar changes.

In my hands the method has failed to produce lesions which simulate Hodgkin's disease. 


\section{SUEILARY AND CONCLUSTONS}

In Part I an atteupt was nade to offer a brief and reasonably critical account of the inherent peculiarities of the structure and of the diseases of the reticulo-endothelial system. Particular emphasis was accorded to works which have served to integrate the concept of lymphoid tissue sarcoma. Thile it is doubtful whether knowledge is yet adequate enough to endorse this idea universally, it has the advantage of being a practical generalisation which simplifies the subject. The view that the morbid process, primary reticulosis, was covered by the generic lymphoid tissue sarcoma was also supported.

In Part II some account was griven of the lymphatics and lymphoid tissue. In this outline attention was drawn to the mysterious and bewildering problems inseparable from the system. The structure of lymph nodes was given with observations on their development, involution, and possible neogenesis in adult life. From these studies it emerged that the full complenent of lymin nodes in the locus examined was probably attained in adolescence or early adult life, and that fat replacenent was the usual mode of atrophy. Attention was also drawn to the rarity of fibrosis in physiological nodes, except where it was the result of blood vascular hyaline change.

In Part III Hodgkin's disease was studied. In the introduction of this part of the work the historical aspect of the malady was recorded, with, it is hoped, advertisement of interesting and possibly less wellknown facts about it. This was followed by a critical consideration on the nature of the disease and its morbid anatomy, the latter being 
illustrated in part by analyses of the cases coming to necropsy at Glasgow Royal Infirnary over a period of fifty years. In this part also were the findings of a large series of biopsy specimens. Here endeavours were made to shew the microscopical variations in morphology in the lesion, and to demonstrate the affinities of other Iymphoid tissue sarconata with the disease. Vithin the resources available the generic lymphoid tissue sarcoma vas established, and links between the better recognised variants were presented with a reasonable degree of conviction. In the necropsy series a detailed study of thirteen cases of Hodgkin's disease or reticulum cell sarcona was offered. In these it was shewn that the favourite locus was lymphoid tissue, that complete systenatisation was rare, and that metamorphosis to a more tumour-like lesion was common.

In Part IV two components of the Hodgkin's disease complex were studied in relation to general pathology. The view that fibrosis, an essential and inherent peculiarity of the Hodgkin's disease lesion, was represented in certain other morbid states was submitted. This was illustrated by brief accounts of some diseases where quasi-neoplastic features are shewn by connective tissue. Eosinophilia in tumours was also made the subject of investignation and revealed that the phenomenon, while possibly not so rare as night be expected, was not nearly so common as in Eodgrin's disease. Some evidence was found for the cytometaplastic origin of eosinophiles in Hodgkin's disease, but possibly due to the restriction to histological as opposed to cytological methods, the results were not highly conclusive.

In Part $V$ an experimental attempt to reproduce the disease by 
chronic trypan blue poisoning of rats and mice proved unsuccessful, although interesting results followed.

The main contention in this thesis has been that Hodgkin's disease is a neoplasm. Perhaps the following may influence the reader more convincingly than I have been able to do by so much work. The reasons for human beliefs depend chiefly upon Authority, Intuition, and Scientific llethod. The last two have been exploited as far as I have been able; the forenost remains. is a junior student I saw a case of Hodgkin's disease first in the wards of the Late Professor Archibald Harrington, at Glasgon Royal Infirmary. I was chagrined at the doubt cast on its nature in the discussion which followed the demonstration; at twenty, one is very intolerant of obscure aetiology! On my return home I imprudently assailed my Father with the question at the dinner table, where even renal oedema was taboo. He was exceedingly angry. There was a dreadful silence, and then he relented - 'of course it is tumour, - but mind to whom you say that!' Nothing more was said. I submit that this terse pronouncement has been my most precious axiom, with deepest respect and affection. 


\section{ACKNO, VLEDGINENTS}

This work was carried out during the tenure of a Dr. Foulis Memorial Scholarship in Pathology. The cost of the experimental studies and most of the photography and drawings was met by this grant. I thank the Trustees very sincerely for this award. It is my regret that the Trustees are anonymous because it is difficult to convey sincerity with conviction to an impersonal body, and to explain how much these funds have ameliorated the problem of surprisingly expensive research.

I am indebted to a great many people for gifts of material, for the loan of slides and for notes of cases. In particular I thank my Mother, Dr. W. Shaw Dunn, Li.J., D.Sc. for the gift of ny Father's collection of Hodgkin's disease slides which provided the first nucleus of this work.

I thank Professor G.L. liontgomery, li.J., my former Chief, and Professor T. Symington, H.D., for their sustained personal interest and encouragement.

I also thank the Physicians and Surgeons of Glasgow Royal Infirmary for their invariable generous and patient courtesy. In particular, Dr. Alex. Brown, H.D., F.R.C.P., and Mr. William Reid, F.R.C.S.; I received great help also from Dr. Worrison and his staff of the Radiotherapy Department.

Wiy own colleagues in the Department of Pathology at Glasgow Royal Infirmary, who must have come to regard this protracted work as the veritable Reticulum of Penelope, have been unstintingly generous in shewing me material and drawing my attention to cases. It is a very sincere pleasure to thank Dr. Alice Marshall, Dr. Alastair Currie, Dr. Robert Curran, Dr. Robert Patrick, Dr. William Crane, Dr. Bruce Voodger and Dr. Peter Pullar. 
From outside Fospitals I have also received very generous aid. In particular from Professor Alan C. Lendrum, Dundee Royal Infirmary; Dr. John C. Dick, H.D., Stobhill Hospital; Dr. Robert IlacAndrew, Law Hospital; and Dr. James P.A. Halcrow, Hairmyres Hospital.

The staff of the Library of the University of Glasgow have given me most kindly assistance, and have on many occasions obtained for me rare or comparatively inaccessible texts.

I am obliged to Wr. Donald F. Hay, F.I.I.I.T., chief technician at Glasgow Royal Infirmary, and to lifr. P.J. Flliott, Hember of the Royal Photographical Society, for instruction and advice on photographic methods. Mrs. M.G. Brown, a Senior Typist at Law Hospital is due my gratitude for her painstaking work in transferring my difficult left-handed script into perfect type.

Finally I thank my wife, Dr. llaida Shaw Dunn, for her incredible patience throughout the prolonged period of this study and the small members of the family for whom 'Daddy's Hodgkin' was virtually Pandora's box. 


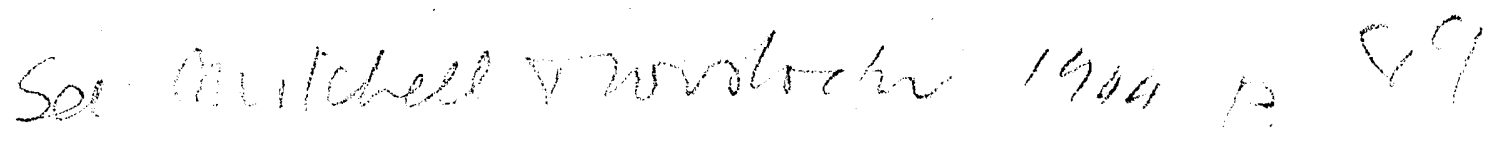

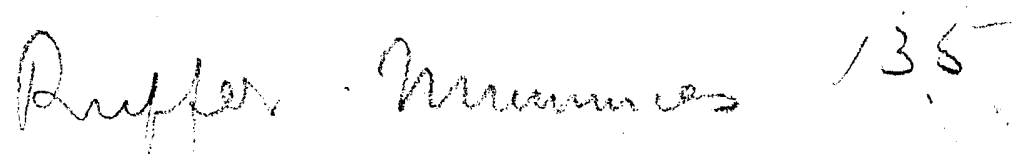

thurecon, $\forall>0$ Zever Symmers (95).

otever 1913. 'She:

b t 1952 


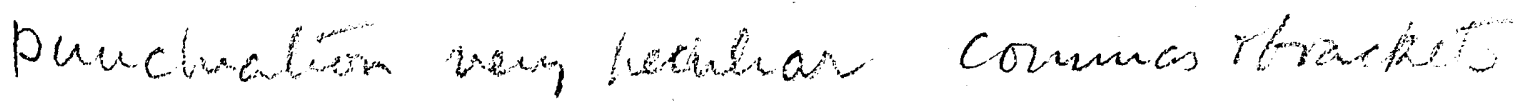

$P 17 .\left\{\begin{array}{l}\text { antonymis }=\text { antomomy } \\ 26\end{array}\right.$
20 sycophantic.

cell-nest $=$ cell nest cohnteim $y$ offee $=y$

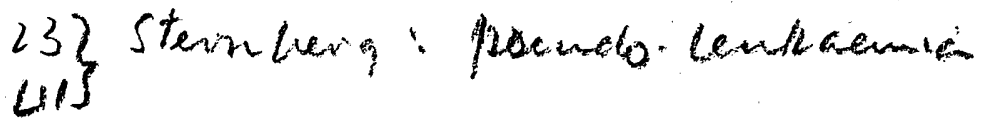

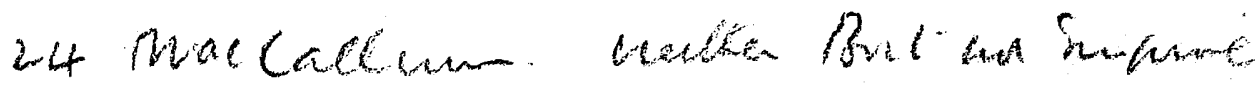
38. Holoma

49 idented to melented

68-lymuth frope-kein

Barnes

97. innozaliaion

227. atowntic.

Vob torme cosmistares 
VOLUNE II

TABLE OF CONTENTS

A. Biopsy Series Protocols page I

B. Necropsy Series Protocols page 223

References.

page 268 


\section{APPINDIX A.}

\section{BIOPSY SERIES PROTOCOLS}

The pages are numbered to correspond with the serial

number of each biopsy; where the records exceed one page the appropriate serial numbers are retained and followed by the letter 'a'.

\section{Illustrations.}

Volume III Figs. 45-116.

Volume IV p.20-57. 
Urquhart. flard XI. 17th June, 1910. History unknown. Lymph nodes.

Established Hodgkin's disease in which all diagnostic criteria are met. There is appreciable persistence of normal structure in one of the nodes, it is represented by sub-capsular lymphoid tissue and a few survival nodules in the deeper node substance. The capsules are irregularly thickened, especially at the bases of the trabeculae. Concentric re-formation and invasion are absent. Fibrosis is moderate, perivascular lamellation and coralline deposits are the chief expression. The cellular moiety of the new tissue is classical; the proliferating reticulum cells are seen in syncytial form in places, multinucleate giant-cells are numerous, often 2 per H.P. field. Eosinophilia is pronounced. Mitoses average 1 per H.P. field. Necrosis is confined to scattered individual cells. There is faint lobulation of the nodes and the vascularity is definitely low.

Diagnosis. Hodgkin's disease, Typical. 
James Smith. Fard XIX. Dated 24th September, 1910. History unknown. Lymph node from groin. Restained in August, 1940. Not well preserved. Labelled "? Benign stage" (J.S.D.).

There is pronounced fibrosis of the capsule and one large trabecula, small coralline deposits are seen below the capsule. The cellular tissue is curiously uniform, most of the cells are very slightly immature lymphocytes and they are distinctly sparser than normal. A diffuse increase of reticulum cells in present, they are uniformly scattered. Plasma cells are seen but eosinophiles and multinucleate giant-cells are absent. The fibrosis and general simplification are in keeping with Hodgkin's disease but the lack of pleiomorphism etc. are against it. Rate of growth is very slow.

Diagnosis. Indeterminate ? benign Hodgkin's disease. (Further sections might have elucidated this). 
John McNeil. Lymph nodes. Dated 1st December 1910. History unknown. A further specimen of lymph node dated 17 th December 1910 also available.

Established Hodgkin's disease in which all diagnostic criteria are met. The lesion here shews a peculiar but remarkable characteristic, the exodic growth phenorenon. Centrally in the node there is a uniform field of typically pleiomorphic Hodgkin's tissue but this does not quite reach the capsule, a thin rind of small lymphocytes with a few physiological reticulum cells being interposed. This attenuated lymphoid tissue is being driven against the inner aspect of the capsule. A few survival nodes of lymphoid tissue are also notable deeper in the new tissue. The capsule is poorly preserved (artefact) but does not appear much thickened. Trabeculae are not present. Fibrosis is diffuse of synplasmic transformation type; perivascular and coralline fibrosis are slight. Fibrils and fibrillae constitute most of the collagen. Reticulum cells are conspicuously increased and are chiefly of the morbid mononuclear variety, some shew gradations to fibroblast resulting in a subtle medley of both cell types. Typical multinucleate giantcells are scanty but Dorothy Reed mirror-image type are common, lobulated nuclei are also frequent. Eosinophiles are numerous and mostly of blood type. Plasma cells and neutrophils are identified. litoses are few and occasional areas of necrosis are noted.

The second specimen is slightly more muture and the fibrosis is more evident; however, even here the entire node is not involved. Note. A few fat cells are present in this node. This is a rare finding. 
Turner. Fard XIV. Cervical lymph node. Dated 20 th June 1911. History unknown.

Barly established Hodgkin's disease in which most diagnostic criteria are met. Under very low power a faint yet definite coarse segmentation is discernible, the exodic growth phenomenon is apparent as well. Survival follicles and nodules are present. The capsule where present is thickened grossly, true trabeculae are absent. Fibrosis is well established, it is seen in the form of a vaguely whorled mesh in the segments described. Coralline deposits and perivascular lamellation are also present. Hononuclear reticulum cells are relatively scanty, multinucleate cells appearing more common. Distribution of the latter is uniform. Eosinophiles and other granulocytes are rare. Hitoses are few, necrosis is only represented on an individual cell basis.

Diagnosis. Atypical Hodgkin's disease. Eosinophiles are distinctly few and pleiomorphism is less than usual. It might conceivably be confused with lymphosarcoma but not after close scrutiny. 
Firstbrook. Vard XXIX. Axillary lymph nodes. Dated 14th October 1911. History unimown. Recut and stained February 1942.

This is a reticulum cell sarcona of fairly uniform cell typeclose to Gall and Mallony's clasmatocytic stem-cell lymphoma. The cell differentiation direction is wavering in places with bias towards lymphoid cell type. The capsule is not thickened but the concentric re-formation phenomenon is well shewn. Normal lymphoid tissue is totally absent. Fibrosis is present, it is strongly reminiscent of Hodgkin's disease fibrosis too. Diffuse fibrillary and fibril deposits, occasionally assuming the coralline form are notable. The vessels inconstantly shew lamellation. Typical multinucleate giant cells are absent but small forms occur. Posinophiles are absent. There are areas of necrosis with local poljmorph response. Hitoses are very numerous, far beyond the rate seen in Hodgkin's disease.

Diagnosis. Reticulum cell sarcoma, sclerosing in similar fashion to Hodgkin's disease. 
Paterson. Ward XVI. Lymph node. Dated 26th January 1912.

Classical Hodgkin's disease, fairly early. The new tissue is disposed in vague segmental fashion in places and occasional survival aggregates of lymphocytes are present in the interstices.

The capsule is very incomplete due to artefact but thickening is seen locally and also infiltration with the new tissue. Connective tissue swathes of crescentic outline closely simulate trabeculae but the loss of capsule is too extensive to substantiate this. Fibrosis is marked, it is chiefly fibrillary and of the synplasmic transformation variety. Perivascular lamellation is present in addition.

The new tissue is hypocellular due to marked loss of lymphocytes. Reticulum cells are fairly numerous, many being indeterminate reticulum cell/fibroblast types. The giant cells are classical. Fosinophiles are very numerous indeed. The remaining characteristic cells are represented. Mitoses average about 1 per H.P. field and necrosis is absent.

Diagnosis. Hodgkin's disease, Typical. 
Forsyth. Tard XX. Iymph nodes. History uninown. The first sections shew very good typical Hodfkin's disease, the second series from another node present an uncomon variant.

In the former all vestige of normal lymphoid tissue except an occasional aggregate of small lymphocytes has gone. The capsule is not preserved unfortunately, but new trabeculae enclosing the Hodgkin's disease segments are discernible, this change recalls the results in sclerosing Brill's disease. Fibrosis is pronounced and all varieties are exhibited in some degree. The reticulum cell proliferation is well shewn, the cells are chiefly of the morbid mononuclear type. The siant cells are typical. Eosinophiles are extraordinarily numerous; in addition small mononuclear cells with lymphocyte nuclei and eosinophile ground-glass cytoplasn are seen. In many cases actual eosinophile granules are present in these and a lymphocytic origin of sone of the eosinophiles seems very likely. Plasna cells are conmon but polymorphs are rare. Mitoses are numerically above average but there is no necrosis. In the second set multinucleate siant cells of Ianghan's type are the commonest giant cell. Up to 60 nuclei vere counted in one cell. There is not any evidence of tuberculosis, e.g. follicles, caseation etc.

An interesting feature is seen in these specimens regarding the relation of reticulin to collagen. The correlation of the silver impregnations with the routine sections is poor, they appear quite unrelated.

Diagnosis. 1. Hodgkin's disease, Typical.

2. Hodgkin's disease, Atypical, Langhan's giant cells. 
McIntyre. O.C.C. History unknown.

The specimen is a lymph node shewing late Hodgkin's disease.

There is not any persistence of normal structure and the capsule is incomplete.

Fibrosis dominates the picture; centrally there is a mass of almost avascular and acellular coralline collagen which peripherally merges with a mantle of active Hodgkin's tissue, the latter is imperfect being traversed by trabeculae. The new tissue is characteristic except for the paucity of eosinophiles but this is common in mature lesions. Mitoses are very few. The appearances support the idea that the lesion starts centrally and drives out. The lesion is subtle in the preparation but the column behind the furled colours is a unit of the same army.

Diagnosis. Hodgkin's disease, Typical late. 
Brazill. R.H.S.C. Dispensary. Four Lymph nodes. History unknown.

In one of the nodes, the smallest, there is some surviving lymphoid tissue; follicles and sinus are represented. This persistent tissue is limited to the extreme periphery of the node. The node capsules exhibit irregular thickeninğ and hyalinisation. Concentric reformation and invasion are absent. Trabeculae are poorly developed and consist of blunt fibrotic wedges. Fibrosis is present but fairly early, it is chiefly in fibrils and fibrillae related to the mesh. Perivascular deposits and coralline formation are little in evidence. The reticulum cell increase is characteristic and there is copious production of morbid mononuclear types. Typical Hodgkin giant-cells are present in about the usual number and there is vague topical aggregation of them locally. In one section a typical Langhan's giant-cell is seen apparently encircling an Hodgkin's giant-cell. This is extremely interesting because it suggests that the cells are quite distinct - a mutual antipathy existing between them. The Iunghan's cell can reasonably claim to be altruistic in function and although one swallow does not make a summer it is a tiny evidence in favour of the neoplastic character of the Hodgkin cell. (This remarkable ocourrence has not been seen again). Eosinophilia is typical and the remaining characteristic cells are present. Here the polymorphs are definitely related to focal necroses. Cellular activity is of the normal order. The exodic growth phenomenon is very well shewn. Diagnosis: Hodgkin's disease, Typical. 
T.K.D. Regent. Cervical and Axillary lymph nodes. Dated lst June 1912, 21st June 1912 and August 1912. History unknown.

Seven lymph nodes are avialable for study, and several striking findings are notable. In one node fully established typical Hodgkin's disease is present and yet perfectly normal physiologically active lymph node tissue containing follicles with pale centres and sinuses is still present in the same gland. Under very low power a vague frontier between the two tissues is just visible giving an impression that the new tissue is driving into and overwhelming the old. Under ordinary low power demarcation becomes guesswork. The exodic growth phenomenon is seldom so emphatically demonstrated and a further fact emerges - the only reaction of the normal tissue is a general reactive hyperplasia, not a 'Iymphoid hyperplasia' which is usually postulated as the incipient change. In five other nodes the typical Hodgkin's disease picture is completely characteristic, all criteria are fully satisfied. In the remaining node the multinucleate giant cells are unusually numerous giving the appearance of Harthin's Hodgkin's sarcoma but eosinophiles are still very copious. This variation in picture is seldom able to be confirmed in specimens from the living since diagnostic resections are linited in extent. The phenomenon is however amply demonstrated in full necropsy studies.

Diagnosis. Hodgkin's disease, Typical. 
McFarlane. Tard XX. Two lymph nodes, incomplete. History unknown.

There is not any persistence of normal lymphoid tissue in either specimen. The capsules shew pronounced fibrous thickening and hyalinisation. The fibrosis is advanced and obscures evidence of concentric re-formation, the trabeculae are particularly massive. A coarse net is formed which segments the node. Perivascular lamellation is the most obvious display of collagen formation, the other forms including coralline deposits are difficult to recognise. The reticulum cell proliferation is chiefly productive of morbid mononuclear types and Hodgkin's giant-cells are few. Bosinophilia tends to be focal. It is pertinent that these cells are mainly located in areas where lymphocytes persist. Plasma cells are more numerous than usual and transitions between these and lymphocytes are common. Judged by the number of mitoses growth is slow. The growth in irregular large islets is still retained despite the maturity of the lesion.

Diagnosis• Hodgkin's disease, Typical, late. 
Agnew. W.R. Dispensary. Dated 10th August 1912. Four lymph nodes. History unknown.

These specinens are poorly preserved, attempts to restain one of the slides met with indifferent results. Where the capsules are preserved there is uniform thickening and hyalinisation. The trabeculae are thickened and in their imaediate vicinity the appearances of typical Hodgkin's disease are seen. Beyond these limits the nodes are replaced by a uniform small round cell tumour which corresponds to mixed reticulum cell/Iymphosarcoma type. There is total absence of eosinophiles in the homogeneous tumour portion and mitoses are numerous. The exodic growth phenomenon is particularly well illustrated in one node, the peripheral mantle of crushed lymphoid tissue containing germinal follicles which have been flattened by pressure.

Diagnosis. Wixed reticulum cell and lymphocyte series sarcoma, corresponding to intermediate cell type of Ehrlich and Gerber (1935); with fields adjacent to the trabeculae morphologically identical with Hodgkin's disease. 
R.B.C. Dispensary. Dated 12th August 1912. Three lymph nodes. History unknown.

In each specimen there are fairly numerous survival follicles of lymphoid tissue, most contain normal active pale centres. Their situation is not limited to the periphery of the nodes. Capsular thickening is apparent in only one gland. The trabeculae are very poorly developed. Fibrosis is extensive, perivascular lamellation is seen but most of the collagen is present as a diffuse fibril and fibrillar mesh with scar-like areas of unusual intensity. Reticulum cells are numerous, norbid mononuclear examples are about average in number but the majority resemble epithelioid types. Typical Hodgkin giant-cells are present but there are more which correspond closely to Langhan's type. A form with a complete ring of peripheral nuclei is common. Eosinophiles are present in adequate numbers but neutrophiles are few. The lesion appears curiously inactive. Ifitoses are hard to find.

Diagnosis. Atypical Hodgkin's disease, Early but established. Abnormal multinucleate giänt-cells (Langhan's type). Bears a faint resemblance to endothelial tuberculosis. Origin in the loose lymphatic tissue probable. 
Ward XV. August 1912. Five lymph nodes. History unknown.

This is a very interesting set. In several of the nodes there is a rind of persisting normal lymphoid tissue including germinal follicles with pale centres and fragments of marginal sinus. The capsules of all specimens are thickened. In one mode the growth pressure of the new tissue has caused massive infarction. Nearly all the Hodgkin's tissue is dead, except for a thin sub-capsular rind in which some normal (reactive) lymphoid tissue is identified. In the largest node new trabeculae are forming giving a coarse follicular pattern superficially like Brili's diseaee but the segmentation is less regular. Fibrosis is chiefly seen as perivascular lamellae and capsular thickening but incipient diffuse fibril formation is in progress. The reticulum cells are unusually numerous and although most are of morbid mononuclear type small 2-3 nuceolated giant-cells are comnon. Typical Hodgkin giantcells are present. Iosinophilia is diffuse and heavy, other granulocytes are rare. Plasma cells are very scanty. Mitoses are about average.

Diagnosis. Hodgkin's disease, rather more cellular than usual. Some affinity to Brill's disease but regard as typical. 
Allan. XXXIV. Single section of lymph node. History unknown.

There is not any remaining normal tissue. The capsule is grossly thickened and hyalinised. Coarse segmentation by pseudo-trabeculae is pronounced. Fibrosis is chiefly by perivascular accretion and is gross. Coralline deposits are particularly marked. The reticulum cell proliferation is unusually florid, most approach the macrophage type as regards nucleus but are syncytial. Typical Hodgkin giant-cells are present but the majority are intermediate between this variety and Langhan's type. They are grotesquely angular and irregular, recalling the foreign body giant cell. Eosinophiles and plasma cells are very scanty. Neutrophiles are absent. Mitoses are rare but occasional areas of necrosis are notable.

Diagnosis. Atypical Hodgkin's disease, late. Pleiomorphism limited. No eosinophilia. Inclining towards fibrifying reticulum cell sarcoma. 
Turtle. C.C.C. Dated 6th November 1912. Five lymph nodes. History unknown.

Rare survival follicles and islets persist. Elsewhere there is a very wide replacement of the node tissue by a rather homogeneous cellular Hodgkin's tissue. Capsular thickening and hyaline change are present and some concentric re-formation is detected. The new tissue despite its uniform appearance low power meets all criteria for its recognition, eosinophilia is unusually marked. The fibrosis is patchy and mostly of the coralline deposit variety. Mitoses are absent.

Diagnosis. Hodgkin's disease, accept as typical but rather uniform and cellular. 
Lee. VI. February 1913. A single lymph node. History unknown.

A few fragments of the marginal sinus tissue persist, the littoral cells are laden with haemosiderin. The capsule is grossly thickened and hyaline change is advanced. Concentric re-formation is notable, several crescentic swathes of new fibrous tissue have formed. The few trabeculae present are thickened by fibrotic accretion. Perivascular lamellation is pronounced but fibrosis in the lymphoid tissue is limited to small scar-like areas of coralline type. The reticulum cell proliferation is about usual, but large lymphocytes of immature variety still predominate. Typical Hodgkin giant-cells are present but the mirror image type is much commoner. Eosinophilia is mild, necrosis and mitotic figures are scanty.

Diagnosis. Hodgkin's disease, typical but rather less pleiomorphic than usual. 
Inglis. XXXIV. Dated February 1913. Single lymph node. History unknown.

This is late Hodgkin's disease. The fibrosis is overwhelming. Definition of the capsule and trabeculae is no longer possible and the new tissue itself is seen in survival islets in interstices of the collagen. In these areas it is typical and easily recognised. The reticulum cells are chiefly of the morbid mononuclear type but Hodgkin giant-cells are present too. Eosinophilia persists and a few neutrophiles are seen also.

Diagnosis. Hodgkin's disease, Typical, late. 
Callaghan. XX. Dated 13th June 1913. Three lymph nodes. History unknown.

This is primarily a mixed lympho-reticulo sarcoma (intermediate type of Ehrlich \& Gerber (1935)). There is not any survival of normal lymphoid tissue. The capsules are thickened but also infiltrated and over-run. Concentric re-formation is present. The tumour is growing in coarse ill defined nodules 1-2 mm. dianeter which are defined by swathes of connective tissue. Fibrosis is also notable in among the tumour cells giving the descriptive 'alveolar sarcoma' picture. Hodgkin's giant-cells are present but very sparse. Eosinophilia is absent and plasma cells are not seen. Mitoses are numerous, 2-3 per H.P. field; necrosis is confined to individual cells.

Diagnosis. Intermediate type reticulum cell sarcoma, Fibrifying. Some features of Hodgkin's disease, oocasional fields would pass for it. 
A.T. Dated September 1919. Cervical lymph node. History unknown.

This is nearest to follicular lymphoma but fibrosis is taking place in it. The false follicles are irregular in size and shape. Their pale centres present an unusually pleiomorphic cell picture. Nany fields indeed would pass for Hodgkin's disease. The extra-follicular tissue is even closer to the latter in morphology. Fibrosis is present round many follicles, sometimes in their centre, and perivascular lamellation is marked. Most of the proliferating reticulum cells are of the morbid mononuclear type but scanty typical Hodgkin's giant-cells are visible as well. Bosinophiles, plasma cells and neutrophiles are very sparse. Mitotic activity is well above Hodgkin level.

Diagnosis. Brill's disease, sclerosing; very close to Hodgkin's disease. 
Identity unknown beyond M.R.I. 27:243. History unknown. Several sections of four different lymph nodes. The appearances are the same in each.

This is an interesting example of a rare variant of Hodgkin's disease. Normal lymphoid tissue is absent. The capsules show slight but definite fibrous thickening of hyaline character. The exodic gronth. phenomenon is displayed well and in a few places the new tissue is being driven into the substance of the capsule. Fibrosis is chiefly expressed in the formation of extensive fin fibrils, with occasional spidery scars. Perivascular deposit is seen round some vessels but this localisation is inconstant. Coralline deposits of collagen are absent. The reticulum cell proliferation is meagre, morbid mononuclear types are identified but are few. liultinucleate giant cells are very numerous and the great majority are of Langhan's trpe, the typical Hodgkin cells being hard to find. Bosinophilia is pronounced, but other granulocytes and plasma cells are few. Mitoses are rare and necrosis is absent. The commonest cell present is the small lymphocyte and it appears normally mature.

Diagnosis. Atypical Hodgkin's disease, Langhan's type multinucleate giant cells. Some reduction of pleiomorphism. 
Identity. 30:200. (Path. Lab; University of Glasgow Label.) History unknown, believed to be from a patient in Manchester Royal Infirmary. A large slide presenting sections of five apparently different lymph nodes.

This is of particular interest in two respects. Normal lymphoid tissue is identified in nodes shewing established Hodgkin's disease, and metamorphosis to Hodgkin's sarcoma (Warthin's reticulo-endothelioblastoma) is seen in three of the nodes.

One node, $V$, which is adjacent to IV, shews only reactive change with much macrophage production. There is haemosiderin storage in some of these cells. Node III shews late Hodgkin's disease, the only irregularity being a low eosinophil content. Node I shews Hodgkin's disease and Hodgkin's sarcoma, between which transition is clearly discernible. Nodes II and IV shew almost pure Hodgkin's sarcoma which is becoming uniform. In this case the sarcomatous transformation is producing a tumour of unquestionably malignant character which morphologically is a reticulum cell sarcoma of the variety clasmatocytic stem cell lymphoma of Gall and Mallony. The eosinophils have almost disappeared from these lesions. The growth is diffuse in the pulp end the sinus tissue appears remarkably intact in some places. (This case is more fully described under Hodgkin's sarcoma q.v•)

Diagnosis. Hodgkin's disease, Typical. Hodgkin's sarcoma. (Reticulo-endeothelioblastoma of Warthin), Transition between the two appearances is convinoing. 
Campbell. Ref. M.R. 1066:33. Dated November 1933. History unknown. A single lymph node $1.5 \times 1 \mathrm{~cm}$., from the axilla.

This is an interesting variety of atypical Hodgkin's disease which possibly forms a link between the benign Hodgkin's disease of Harrison and Hodgkin's disease, it is undoubtedly nearer to the true disease. There is persistence of normal (reactive) lymphoid tissue at the subcapsular periphery and a distinct exodic growth phenomenon is discernible. The capsule is irregularly thickened and attenuated, it is not invaded. Trabecular lobulation is not apparent. Fibrosis is intense at one pole of the node and is mostly of coralline variety though perivascular reticulum cells are present and a few typical Hodgkin giant cells are seen. Eosinophils are sparse and so are plasma cells and neutrophils. Mitotic figures are rare and there is not any necrosis. Normal mature small lymphocytes are very copious except in the fibrosed area.

Diagnosis. Atypical Hodgkin's disease. Very few eosinophils, rather a focal lesion. ? Link with benign Hodgkin's disease of Harrison. 
Mrs. Margaret Wright aet 59. Ref. G.R.I. 1336:33. 3rd October 1933. Following trauma two years ago patient noticed masses on her head. 14 months ago the post-auricular chain of lymph nodes began to enlarge, and eventually others at the base of the neck enlarged as well.

\section{Cervical lymph node.}

This is late typical Hodgkin's disease. The lesion is particularly fibrous and it is not possible to identify the capsule with certainty. The fibrosis is mainly in compact swathes of fibrils which segment the node in irregular fashion. Perivascular lamellation is also seen. The cellular areas of the lesion are located in these collagenous casemates. The reticulum cell increase is slight, but morbid mononuclear and Hodgkin giant cells are identified. Eosinophilia persists but is restricted to the more cellular portions. Plasma cells and neutrophils are scanty. Mitotic figures are very rare, necrosis is shewn only by occasional individual cells.

Diagnosis. Hodgkin's disease, Typical, late. 
Watson. Ref. G.R.I. 1409:35. History unknown.

Inguinal lymph node.

This is an example of reticulum cell sarcoma which exhibits certain features characteristic of Hodgkin's disease.

The bulk of the node is occupied by tumour, at one pole normal reactive lymphoid tissue is seen being driven against the inner aspect of the capsule. On the lymphoid tissue side of the line of contact of tumour and normal tissue, a crescent of fibrous tissue is forming. The node capsule is thickened and hyaline and, significantly, it is not invaded. The tumour is a stem cell lymphoma type of which the nuclei shew a definite notching of their membranes which are pachychromatic. Aggregates of tumour cell nuclei which simulate Hodgkin giant cells are common but no cytoplasmic component is present. Mitotic activity is high, 3 per H.P. field. Fibrosis exists in the growth both as a coarse mesh and a fine filigree; and in some areas coralline deposits are present. A reticulum impregnation supports the interpretation of sarcoma and not that of anaplastic carcinoma. High power scrutiny reveals many fields which would pass for Hodgkin's disease.

Diagnosis. Reticulum cell sarcoma. Shews affinities to Hodgkin's disease viz;- failure to invade the capsule, Hodgkin type of fibrosis, focal Hodgkin morphology. 
Unknown. Ref. G.R.I. 1425:35. History unknown. An enlarged inguinal lymph node.

It is difficult to decide what is the most appropriate category for this lesion. Morphologically it is a fibrifying reticulum cell sarcona in which an unusually perfect follicular pattern is present. The cellular deposits are mostly spheroidal or deformed spheroidal and correspond very closely with the false follicles of follicular lymphoma. These are set in a matrix of dense collagenous fibrous tissue which is made up of compacted coralline variety elements. In some areas mixtures of cellular and fibrous purts exist which are alnost indistinguishable from Hodgkin's disease, even to the presence of Hodgkin giant cells. The growth is not slow growing, mitoses are common; but at the same time the casemates of collagen seen impenetrable. Massive necrosis presumably due to infarction is seen at one part. Wile perifollicular fibrosis is common in late follicular Iymphoma which remains relatively benign, the type of fibrosis seen here is unlike it. Further then such a lesion becomes more malignant the fibrosis is scanty as a rule and the follicular pattern fades.

Diagnosis. Reticulum cell sarcoma, presenting an unusual pattern of fibrosis. Related morphologically to Brill's disease and Hodgkin's disease. 
Micclintock. Ref. A.T.:36. Decenber 1936. (Private patient of the late Professor Archibald Young).

This is an example of Brill's disease in a lymph node. The lesion is of considerable standing and fibrosis is pronounced. The capsule is not preserved or no longer distinguishable. Fibrous shells have formed round the giant follicles and are as much as 100 micra thick in places. Perivascular lamellation fibrosis is marked and scattered coralline deposits are frequent. The tumour is apparently of slow growth, mitoses are not frequent. Deep in the node towards one pole an infarct of sorne considerable age is present. It measures $1 \mathrm{~cm}$. diameter, (specimen $3 \times 1.5 \mathrm{~cm}$.

Diagnosis. Brill's disease. Late, remaining tolerably benign. Fibrosis pronounced. Some affinities to Hodgkin's disease. 
Allan. Patient under Dr. Hugh liorton. December 1936. A cervical lymph node.

This is an example of Brill's disease in which the follicles are small and the follicle cells more lymphoid than reticular in their affinities. The lesion must be regarded as malignant; there are transgression of the capsule, deposits outwith the capsular breaches which reproduce the tumour pattern, and infiltration of the fat. Definite, but unimpressive, fibrosis is seen round some follicles. This is not apparently related to the node reticulum being compressed but to small blood vessels which are undergoing hyalinisation. Further fibrosis is seen round larger blood vessels and also in the pulp (follicles).

Diagnosis. Brill's disease, undergoing malignant change to small cell lymphosarcoma. Tendency to sclerose slight, but present. 
Ref. Ayr County Hospital. 3:37. Holograph (J.S.D.) "? plump cellular stage" on label. A small lymph node $(11 \times 3 \mathrm{~mm})$. Site unknornn.

Small lymph nodes are nearly always inadequate for reliable diagnosis of Hodgkin's disease. This example is more informative than most but the assessment is provisional.

There are areas of normal tissue remaining, these consist of small deposits of loose lymphatic tissue round the hilar intrustion and portions of the medullary lymph sinuses. Elsewhere the pulp and follicles are replaced except for tiny survival islets of lymphocytes. The capsule is definitely thickened and there is a perceptible increase of connective tissue round arterioles in the node substance. The new tissue is composed of a uniform crowd of slightly abnormal reticulum cell nuclei whose cytoplasmic component is not discernible. The nuclei are larger than normal, oval, and tending to become vesicular; oxyphilic nucleoli are common. They approach the morbid mononuclear cell of Hodgkin's disease, but most are not large enough to qualify for this characterization, a very few may be considered typical, but Hodgkin giant cells are not seen at all. Eosinophils are present but sparse. Plasma cells and neutrophils are very scanty. The reticulum cells present many mitoses.

Diagnosis. Indeterminate. Possibly cellular Hodgkin's disease. Possibly reticulum cell sarcoma. 
Cannon. Ref. Greenock. 10/37. A lymph node from the posterior triangle of the neck of a boy aged twelve years.

The node is a small one $(10 \times 4 \mathrm{~mm}$ ) and has been sectioned at four levels.

At one level the appearances are within normal considering the patient's age. A further level shews only one morbid area and the remaining two are diffusely affected.

The capsule is of normal thickness. The exodic growth phenomenon is evident and the normal architecture of the node is disappearing. In its place ill-defined lobules approx. $1 \mathrm{~mm}$. diameter are appearing. These are composed predominantly of normal lymphocytes but scattered morbid mononuclear reticulum cells and typical Hodgkin giant cells are present. Incipient fibrosis is present, it is seen mainly alongside the trabeculae and as diffuse fibrillae. Eosinophils are absent and so are plasma cells and neutrophils.

Diagnosis. Hodgkin's disease, early. Pre-eosinophilia stage.

Note. This decision is arrived at after many exaninations at intervals over several years; it is admittedy partly intuitive. The tissue has the capriciously disordered look of Hodgkin's disease. 
Hind. Ref. H.R. 369:37. 13th April 1937. A case of Hodgkin's disease previously confirmed by biopsy in thich recurrence appeared related to insufficient X-ray therapy.

The specimen consists of two small nodes with adjacent areolar tissue and an incomplete larger node. The intact nodes shew general retention of their architecture with unusually marked sinus hyperplasia of the solid type. The pulp shews the enhanced heterogeneity of cells characteristic of reactive change but occasionally large tumour-iike nuclei of reticulum cell type are appearing. The damaged node shews a picture which may be regarded as extremely cellular Hodgkin's disease or reticulum cell sarcoma. Here the capsule is of normal thickness where it is preserved, but it is being over-run by the tumour. Fibrosis is present, all varieties except coralline deposits are seen, but the amount is very small. The reticulum cell proliferation is extraordinarily profuse and many of the cells are typical morbid mononuclears. In addition Hodgkin giant cells are present in large numbers; some giant cells conform closely to Langhan's cells in the spatial arrangement of their nuclei but the resemblance is otherwise superficial. Posinophils are present, but rather below the concentration typical of Hodgkin's disease. Plasma cells are present and neutrophils are notable in focal areas. Mitoses, including spectacularly abnormal ones are common, up to 7 per H.P. field. Necrosis is absent.

Diagnosis. Atypical Hodgkin's disease, very cellular, tumour-like, or reticulum cell sarcoma. (The retention of eosinophilia is anomalous). 
Ref. M.R. 835:37. August 1937. History unknown.

This is an example of either slightly atypical highly cellular Hodgkin's disease or reticulum cell sarcoma. The capsule is slichtly damaged but is thickened slightly elsewhere. It is over-run at several points by the growth. The new tissue is arrenged in ill-defined follicles which are unequal in size and often mutually distorted. Fibrosis is slight, it is visible round blood vessels and as tenuous deposits of fibrillary and fibril type. The predominant cell type is a morbid mononuclear reticulum cell anong which many typical Hodgikin giant cells are scattered. Fosinophils are distinctly numerous and plasma cells are also common. Neutrophils in small aggregates and scattered diffusely are frequent. The mitotic count is very high, as many as 20 being visible in some H.P. fields. Necrosis is limited to occasional individual cells. It is really equivocal whether this is more deserving of one or other of the alternative designations suggested. If microscopical morbid. anatony means anything this lesion is among the most convincing examples of the essential identity of the two processes.

Diagnosis. Atypical Hodgkin's disease, Cellular, or reticulum cell sarcoma. 
Ref.M.R. 1046:37. 20th October 1937. History unknown.

This is an example of atypical Hodgkin's disease in which eosinophilia is lacking and in which the usual capricious pleomorphism is obscured by the persistence of large numbers of small lymphocyte series cells. The capsule is imperfectly preserved due to artefact, occasional relics of the marginal sinus are recognisable. Concentric re-formation of this envelope is well shown at one pole. The new tissue is irregularly follicular in arrangement with a distinct pseudo-lobulation. Fibrosis is well established, perivascular lamellation, fibrillary and coralline varieties are all seen. Morbid mononuclear reticulum cells are present but not very numerous. Wost of the reticulum cell nuclei are normal in appearance. Hodgkin giant cells are present and several shew central aggregates of their nuclei, recalling the Touton giant cell. Eosinophils are not seen. Plasma cells and neutrophils are scanty. Mitotic figures are rare. Almost mature small lymphocytes are very numerous and obscure the pathognomonic picture in places.

Diagnosis. Atypical Hodgkin's disease. Absence of eosinophils. Diminution of pleomorphism due to numerous small lymphocytes, link with Benign Hodgkin's disease. 
John Ritchie. Aet 21 years. Ref. M.R. 1184:37. Enlargement of cervical lymph nodes of six months duration.

This is early Hodgkin's disease in which the new tissue is seen only in focal areas of the pulp of the node. Much of the anatomical cortical tissue is normal and follicles with pale centres are numerous. The cells of the last locality are not visibly contributing to the new tissue at all.

Fibrosis is already present in all forms except coralline deposits. Morbid mononuclear reticulum cells are conspicuous and there are fairly numerous Hodgkin giant cells. Eosinophilia is marked, but plasma cells and neutrophils are very few. Mitosis is seen but is infrequent.

Diagnosis. Hodgkin's disease, Typical. Unusually early, clearly originating in the pulp. 
Isabella Donaldson. M.R. 142/38.

The specimen is an incomplete enlarged lymph node $(27 \times 15 \mathrm{~mm}$. It is an example of pleiomorphic reticulum cell sarcoma with considereble affinity to Hodgkin's disease. The whole tissue is morbid. The capsule is grossly thickened, up to $1 \mathrm{~mm}$. in places and abstricted deposits of the tumour are embedded in it. Several densely fibrous trabeculae of great width are present. Fibrosis is chiefly expressed in the above forms but some perivascular deposit is also present. The tumour is composed of large morbid reticulum cells with discrete cytoplasmic bodies. Synplasmic growth is visible in small areas. The nuclei are trachychromatic, assume bizarre forms, and frequently exhibit oxyphile nucleoli. Hodgkin giant cells are identified, they are rather below the usual size. Eosinophilia is absent. Mitoses are not numerous - many apparent figures prove to be pyknotic fragments. Massive necrosis is present in several areas, at one part forming a crescent $6 \times 2 \mathrm{~mm}$. Deposits of tumour are present in lymphatic and blood vessels.

Diagnosis. Reticulum cell sarcoma, pleiomorphic. Affinities to Hodgkin's disease (fibrosis, cell forms). 
Ref. M.R. 346:38. March 1938. History unknown.

This is a follicular lymphoma in which the false follicles are small and composed of small deeply staining cells, recalling the solid follicles of normal lymphoid tissue. The capsule is imperfectly preserved but appears to be infiltrated. Fibrosis is present; the component round arterioles is surprisingly large, and coralline deposits are notable in a few areas, apparently being laid down in the follicles. Reticulum cell proliferation is evident, morbid mononuclear types are identified and a very few Hodgkin giant cells are visible. Eosinophilia is absent. The mitotic activity is about 2 per H.P. field.

Diagnosis. Brill's disease. Perivascular and coralline fibrosis present, scanty morbid reticulum cells of Hodgkin's disease discernible. 
Campbel1. Ward 8. Ref. M.R. 1441:38. November 1938. An axillary lymph node.

This is a lymph node which has been partly atrophic before the lesion developed. There is a formation of large follicles at the periphery of the convex border but elsewhere a physiological sinus hyperplasia is the chief feature.

Normal physiologically active lymphoid tissue is present in the concave side of the node. The sinuses are tightly packed with normal littoral cells many of which contain erythrocytes. The capsule is thickened but chiefly on the convexity of the node, which is a nornal finding in involuting nodes. There is moderate fibrosis, this is seen in the trabeculae and as coralline deposits. Almost all the latter can be accounted for by vascular fibrosis, i.e. it is not integral to the lesion. The large follicles are not uniform in size or outline, their pale centres are often composite nodules of cells including reticulum cells, lymphocytes and haemosiderin-laden macrophages. Their cellular heterogeneity is in contrast to the pale centres of the false follicles in Brill's disease. Small Hodgkin giant cells are present in very small numbers and an occasional eosinophil is noted. Plasma cells are common. The mitotic activity of the reticulum cells is low, below 1 per H.P. field.

Diagnosis. Indeterminate. ?? Early (focal in node) Brill's disease. Regarded as probably reactive. 
(Man). Aet 2l'years. Ref.M.R. 147:B9. Femoral triangle lymph node. $22 \times 15 \mathrm{~mm}$.

This is an example of follicular lymphoma which is growing as a large circumscribed nodule in the lymph node and shews pronounced topical eosinophilia and perivascular fibrosis.

A crescentic mantle of crushed normal (reactive) lymphoid tissue can be made out round most of the tumour nodule. The capsule is normal.

In the tumour nodule many false follicles of varying size and shape have developed. Most are of the solid variety the predominant cell being the srnall lymphocyte. Norbid reticulum cells are present but are few. A very few Hodgkin giant cells are seen. Eosinophils are distinctly numerous, comparable with an average case of established Hodgkin's disease. Slight fibrosis is seen, it is mainly perivascular with a small fibrillary moiety. Mitotic activity is moderately high, up to 4 mitoses per H.P. field.

Diagnosis. Brill's disease. Focal in lymph node. Affinity to Hodgkin's disease. (İosinophilia, slight fibrosis). 
Unknown. Ref. M.R. 288:39.

This is lymphadenoid tissue probably lymph node almost entirely replaced by reticulum cell sarcoma.

At one point of the periphery nerve tissue is over-run by the tumour. There is fibrosis which segments the gro⿰t⿱亠䒑 into alvoolar sarcoma, in addition perivascular and fine fibrillary components are seen. The tumour corresponds to the clasmatocyte stem cell lymphoma of Gall and Mallory, occasional Hodgkin giant cells are seen but their cytoplasm is small in amount. Eosinophils are practically absent. Plasma cells and cells of acute inflammation are common. Hitoses are very numerous.

Diagnosis. Reticulum cell sarcoma. Fibrosis component reminiscent of Hodgkin's disease. 
Unknown. Ref. M.R. $368: 39$.

This is Hodgkin's disease of highly cellular uniform type, it is distinctly tumour-like in appearance.

The capsule shows local thickenings, chiefly at the bases of trabeculae, elsewhere it is thin. The exodic growth phenomenon is well shewn but the peripheral mantle of lynphoid tissue is in process of being taken up in the tumour process. Fibrosis is present but in an unusual form. A delicate fibril mesh of open type has formed and is joined by very small dibrillae from the tumour cells. These latter are very uniformly dispersed and the majority are small. Morbid mononuclear and Hodgkin giant cells are identified in the mesh but most of the cells are slightly immature lymphocytes and their precursors. Eosinophils are present but not numerous. Plasma cells are noted but neutrophils are absent. Unequivocal mitotic figures are not numerous, average 2 per H.P. field; but densely staining nuclei are common. Necrosis is not present.

Diagnosis. Atypical Hodgkin's disease, very cellular, distinctly turnour-like. 
Unknown. Ref. iI.R. 382:39.

This specimen is an example of reticulum cell sarcoma which shews mingled features of lymphosarcoma and true reticulum cell tumour. All vestige of normal structure in the affected node is lost. Where it is preserved the capsule shows marked fibrous thickening, but it is over-run and destroyed in most of the specimen. Fibrosis is present in the tumour but is relatively slight. Occasional perivascular lamellation and a little diffuse fibril formation are seen, several sharply demarcated areas of coralline deposit are noted. The predominant cell has the nucleus of a large fininature lymphocyte which is rather pale staining. The cytoplasm is very indistinct. These cells are closely set in sheets but interspersed uniformly throughout them are, typical morbid mononuclear reticulum cells, Dorothy Reed mirror-image cells, and rare Hodgkin giant cells. Occasional cytoplasmic inclusions are visible in these cells possibly reflecting a macrophage activity. Eosinophilia is absent. Mitoses are numerous but necrosis is not present. Diagnosis. Reticulum cell sarcoma, intermediate type. 
Unknown. Ref. M.R. 402:39.

This is a singularly fine example of early typical Hodgkin's disease. The new tissue appears in the form of indistinct nodules $0.5-2 \mathrm{~mm}$. diameter which occupy about two thirds of the section. The remaining tissue is normal reactive lymphoid tissue, it is situated between the new nodules and at the sub-capsular periphery. The exodic growth phenomenon is well shewn and it is apparent that where the surviving lymphoid tissue is being crushed, the sinus tissue promptly disappears. In the field of the lesion fibrosis of all varieties is detected, in particular crescentic swathes formed by compaction of fibrils. The reticulum cell changes are typical in every way and Hodgkin giant cells are already numerous. Eosinophilia is pronounced. The mitoses vary locally but between one and two are observed in most high power fields.

Diagnosis. Hodgkin's disease, Typical. Barly, still focal in the lymph node. 
Unknown. Ref. IH.R. 410:39.

This node which is incomplete is entirely replaced by tumour. Low power examination conveys an impression of almost complete homogeneity but this is dispelled by high power study. The growth is a reticulum cell sarcoma which is shewing little tendency to sclerose. The cells are free spheroidal morbid reticulum cells in the looser parts of the tumour but their cytoplasmic outlines are not discernible in other areas. Cells corresponding to the large morbid mononuclear reticulum cell of Hodgkin's disease are very comnon and several almost typical Hodgkin giant cells are identified. The only detracting feature of the last nared is their small size. Fosinophils are absent though plasma cells are present. Mitotic figures are very numerous, often over 20 being visible in a high power field. Superficially the lesion approximates closely to cellular Hodgkin's disease but the very inconspicuous fibrosis and lack of eosinophils removes it from this category.

Diagnosis. Reticulum cell sarcoma. Affinities to cellular Hodgkin's disease. 
Unknown. Ref. M.R. $1184: 39$.

This specimen is remarkable. All the criteria for the diagnosis of Hodgkin's disease can be met, yet the picture is a quite astonishing parody of the typical disease.

The lymph node shews a fairly uniform pronounced fibrosis of the capsule and trabeculae. Diffuse fibril and fibrillary deposits, although tenuous, are present. Coralline deposits are absent, but perivascular lamellation is striking in some cases. The reticulum cell increase is almost entirely given over to the formation of large morbid mononuclear and Hodgkin giant cells. The former nearly all exhibit the "owl-eye" type of nucleus due to their pronounced nucleoli. Eosinophilia is emphatic and tends to be localised in the chinks between the large cell foci and the cells are set in among small lymphocytes. Plasma cells and neutrophils are scanty but recoverable. Mitotic figures are present in about average numbers; necrosis is limited to individual cells.

Diagnosis. Atypical Hodgkin's disease. Unusual type, overgrow th of morbid reticulum cells but with retention of typical complex. 
Unknown. Ref. M.R. 380:40. A cervical lymph node.

This specimen is a lymphoid tissue tumour which does not conform to any orthodox category. It is nearest to a follicular lymphora and has possibly evolved from this growth.

The capsule is thickened appreciably but is infiltrated and over-run in many places. Concentric re-formation by crescentic swathes of naturing fibrous tissue is notable. Trabeculae are represented, they shew irregular thickening and fuse with perifollicular fibrous tissue deep in the node. Fibrosis is expressed mainly in this fashion, but some perivascular lamellation and occasional coralline deposits are visible. The false follicles exhibit striking disparity in size and demarcation, nany large ones appear compound due to subdivision, the nodules being delineated by surviving small lymphocytes. In places the pulp shews little follicular organisation and here morbid mononuclear reticulum cells are common. The pleiomorphism is not very pronounced and Hodgkin giant cells are rare. Eosinophilia is absent.

Diagnosis. Brill's disease, (nearest), sclerosing. Affinity to Hodgkin's disease evident. 
Unknown. Ref. M.R. 469:40.

This is a follicular lymphoma with distinct affinity to lymphosarcoma. The capsule of the node is considerably attenuated and is over-run in the greater part of its extent. The tumour is composed of very numerous small solid type follicles which are chiefly composed of young lymphocytes, reticulum cells being unusually scanty. Fibrosis is very little in evidence, small fibrillary deposits are discernible in the remains of the pulp between contiguous follicles. Eosinophilia is absent. Reticulin inpregnation confirms the follicular disposal of the tissue and so far as can be gauged there is little if any increase.

Diagnosis. Brill's disease, malignant. Affinity to small-cell lymphosarcoma. 
Unknown. Ref. M.R. 927:40.

This is Hodgkin's disease of an unusual type. The lesion is less pleiomorphic than ordinarily and the reticulum cell proliferation is productive of very numerous macrophage type cells.

The specimen is a lymph node which is incompletely involved in the disease process. One pole shews well preserved lymphoid tissue in which the sinuses are packed with large macrophages. The node capsule is indifferently, preserved but markedly thickened where present. The exodic grouth phencrenon is well shewr. Fibrosis is pronounced. Perivascular and coralline deposits are present but not marked; the chief expression is symplasnic transformation, with the production of a uniform fine filigree. The reticulum cell proliferation already noted above does include infrequent norbid mononuclear cell types and very rare Hodgkin giant cells. Eosinophils are absent. The srowth rate is apparently very slow.

Diagnosis. Atypical Hodgkin's disease. Differentiation of reticulum cells to macrophage type. No eosinophilia. Rather uniform and tumour-like. 
Unknown. Ref. I. R. 1087:40.

This specimen is small, in fact fragmentary. It is described as tumour from the iliac fossa. It is apparently part of a lymph node which had previously been atrophic with fat replacenent.

The lesion is probably a sclerosing reticulum cell sarcoma with morphological affinities to Hodgkin's disease but the specimen is inadequate for proper diagnosis. The reticulum cells are pleiomorphto and include nany Fodgkin forrs, eosinophils are pressent but scanty.

Diagnosis. Speciraen inadequate. (Probably reticulum cell saroona, some affinities to Hodgkin's disease). 
Unknown. Ref. A.C.H. $14 / 41$.

The specimen consists of two small abdominal lymph nodes replaced by reticulum cell sarcoma of intermediate (mixed) type with affinities to Hodgkin's disease.

Under very low power a distinct follicular arrangement is discernible. The tumour nodules are imperfectly outlined by swathes of fibrous tissue. The capsule is becoming thickened and is over-run at one point with new growth in the perinodal fat. Fibrosis is chiefly expressed as above, the centres of the nodules are almost purely nuclear. The type-cell has a large pluwp fairly pachychromatic nucleus with chromatin granules, but many typical morbid mononuclear reticulum cells are visible on close examination. Rare Hodgkin giant cells without typical cytoplasm are present. Eosinophils are present in sufficient numbers to be found easily. Mitoses are numerous up to 6 or 10 being countable in high power fields. Necrosis is not seen.

Diagnosis. Reticulun cell sarcoma, sclerosing in parts. Intermediate cell type. Slight eosinophilia. Slight affinity tó Hodgkin's disease. 
LicCluskey. Ref. I.R. 540:41 and 999:41.

History. This patient had two previous biopsies on account of swollen lymph nodes in the axilla. The first is untraced, the second two months previously established a diagnosis of reticulo-sarcona, the term being a generic usuage. The third specimen M.R. 540:4l consists of four small axillary nodes and the scar of the previous biopsy. The fourth biopsy is a nodule removed from the abdominal wall several months later.

One of the axillary nodes in hyperilastic and seen alone could not be regarded as neoplastic, incipient abnornality is restricted to loss of sinus tissue and slight immaturity of the lymphocytes. The remainder are replaced by active Iymphoid tissue tumour which is characteristically homogeneous. The capsules are of normal thickness, hyaline and at several places are over-run by tumour cells. Invasion of adjacent fat is conspicuous. The type-cell stains almost as deeply basophil as an immature lymphocyte but is larger and has often the misshapen yet distinct nuclear membrane of a morbid reticulum cell. Cytoplasm is impossible to define in most of these cells. Typical morbid mononuclear reticulum cells are present, but Hodgkin giant cells are not. (Foreign body giant cells with inclusion are numerous in the scar). Occasional eosinophils are seen but not plasra cells. Mitoses are numerous and necrosis, especially pyknosis, is pronounced. There is a conspicuous lack of fibrosis, a little fibril formation is seen but that is all. In the abdominal deposit isolated fragments of skeletal muscle simulate coralline fibrosis.

\section{Diagnosis. Lymphoid tissue sarcoma. Intermediate type (Iympho} Reticulo-sarcoma), Lymphoid tendency preponderating. 
Ref. II.R. 609:4I.

History. Unknown beyond the fact that the specimen is a cervical lymph node which had been enlarged (solitary) for about a year.

Superficially the appearances are typicalfollicular lymphoma. Scrutiny reveals that some of the peripheral follicles have normal cells in their pale centres and are surrounded by thin imperfect zones of normal small lymphocytes. The false follicles display the usual low power appearance of homogeneity

The capsule is greatly thickened, being over $1 \mathrm{~mm}$. deep in places, it is densely hyaline but tumour cells are insinuated into chinks in it. The exodic growth phenomenon and concentric re-formation of capsule are evident. True trabeculae are scanty but new swathes of connective tissue are conspicuous between the follicles. The fibrosis is unusually copious in this case; perivascular lamellation, fibrils, and even coralline deposits are seen. The tumour cell type tends to have a reticulum cell type of nucleus but it is small. A very few small atjpical Hodgkin giant cells are present. Eosinophils, plasma cells, and neutrophils are inconspicuous. Growth rate is slow and necrosis is not a feature.

Diagnosis. Brill's disease, sclerosing. Shews definity affinity to Hodgkin's disease. 
America. Ref.1096:41.
(i) Tonsil.
(ii) Recurrence in Uvula two years later (reference relates to second specinen.

The specimen of palatine tonsil shews eurly neoplastic change in the pulp lymphoid tissue. The growth is a uniform cellular tumour of low mitotic activity. The type cell has a reticulum cell nucleus but the cytopliasn stains similarly to plasma cell cytoplasm. Fibre formation is little in evidence. Numerous agparently normil follicles of normal-sized small lymphocytes are scattered through the growth. In some of these follicles small pale centres are visible. Polymorphs are present in the crypts but in much the same quantity as in normal tonsil.

In the recurrence specimen the malignancy of the tumour is enhanced, and fibrosis is more in evidence. Mitoses are numerous and the previous uniformity of cell type is being lost. Occasional multinucleate giant cells are identified which are smaller than typical Hodgkin giant cells but otherwise closely similar.

Diagnosis. Reticulum cell sarcoma, sclerosing slightly. Beconing pleiomorphic. 
Case of W. Rolland. Ref. Bolton R.I. 4893:38. Three cervical lymph nodes.

The specimens are unfortunately not good, fixation and cutting are poor. The lesion present is atypical Hodgkin's disease which has a tumour-like hornogeneity and peculiar giant cells.

Traces of surviving nornal lymphoid tissue can be identified, they include fragments of marginal sinus tissue and peripheral follicles of solid type. The capsule shews only doubtful thickening. Trabeculae are not represented. Fibrosis is curiously patchy; perivascular lamellation and capillary halinisation are notable. Diffuse fibril, fibrillary, and coralline deposits are also present. The reticulum cell proliferation has not the usual capricious heterogeneity and the commonest cell-type present is lymphoblastic in type. Morbid mononuclear reticulum cells are present, and typical Hodgkin giant cells can be seen. Small multinucleate giant cells which are nearer to the foreign body variety than to Langhan's type are conspicuous, in some cases these are huddled in little clusters round coralline collagen deposits. Eosinophilia is present but is of a low order. Other cells are scanty. Mitoses are identified with difficulty in the preparations.

Diagnosis. Atypical Hodgkin's disease. Peculiar giant cells. Thumour like. Eosinophils few. 
Case of Cleminson." Ref. 213:38. Cervical lymph node. History unknown beyond the fact that this patient was recorded to be alive and well three years later. Provisionally labelled by J.S.D. as "? reticulosis, with uniform small cells, fibrosis".

The specimen is an enlarged incomplete lymph node. There preserved, the capsule is uniformly hyalinised and just appreciably thickened. Several crescentic bands of hyaline connective tissue are visible which segment the node imperfectly into nodules $2-3 \mathrm{~mm}$. diameter. Low power the lymphoid tissue appears replaced by a uniform small cell growth in which a few, small, widely dispersed germinal centres survive. These have tiny irregular pale centres, 7-10 cells across, in which the component cells have pleiomorphic nuclei but few or no mitotic figures. The peripheral lymphocytes are typical small lymphocytes. In comparison the type-cell nucleus of the diffuse growth is larger and leptochromatic - a less mature lymphocyte. In addition to these cells slightly enlarged reticulum cell nuclei are apparent but typical Hodgkin giant cells are not present in any of the material available. A few eosinophils are seen near the fibrous septa, other cells are conspicuously absent. The groith rate is low in spite of high cellularity, there is seldom more than one mitotic figure in high power fields. (Many blood capillary vessels with much swollen endothelial cell nuclei present a similarity to giant cells but their true nature is clear on scrutiny).

Diagnosis. Benign Hodgkin's disease. 
Mr. D. Presented initially with lymph node enlargenent in the neck stated to have been present for ten years. Biopsy was performed 2lst Way 1936 ref. P.S. 87:36. A further microscopical examination ref. P.S. 5:37 was made 7 th January 1937 after irradiation. The patient was reported alive and well in December 1938.

The first specimen is a lymph node in which the capsule is slightly thickened and densely hyaline except at a few sites where its composite lamellae are split by infiltrating lymphocytes.' This invasion is possibly artefact and is distinctly limited. Survival of normal tissue is minimal and takes a curious form; there is a thin but defenite perithelial cuff of normal small lymphocytes round most of the small blood vessels deep in the node. These serve to emphasize an otherwise very indistinct false follicle arrangement of the tumour tissue. The tissue is composed of mutually identical cells which correspond to rather young lymphocytes, even high power the purity of the cell type is remarkable. Fibrosis is present; for the greater part it consists of scar-like deposits of the coralline variety which are very similar to those observed in Hodgkin's disease. A small contribution of fibrils and perivascular lamellae make up the remainder. A very few morbid mononuclear cells are present but there are not any giant cells. Bosinophils, plasme cells and neutrophils are exceedingly rare. Mitoses are scanty and necrosis is not seen. The second specimen shews what is virtually an infarcted node, there is a large central area of coagulative necrosis. Adjacent to this is a young connective tissue mantle with bizarre reticulum cell or fibroblast nuclei and moderate eosinophilia. Curiously, the arterioles do 
not shew ahy endarteritis.

Diagnosis. Iymphoid tissue sarcoma, relatively benign indeterminate category. Related to Brill's disease. Related to benign Hodgkin's disease. 
Duguid. Ref. 5064:42.

The specinen represents part of a large fused lymphoid tissue mass. Except at one edge where fragments of the original capsule persist all vestige of normal structure has gone. The capsule is grossly thickened and hyaline, and it is continuous with a very thick fibrous mesh in which are inserted numerous small nodules of typical Hodgkin tissue 1-4 mm. diameter. Miost of the fibrosis takes the form of mature swathes of compacted fibres, either fibrillary or coralline deposit is evident. The islets of cellular tissue are composed of young lymphocytes, morbid reticulum cells including giant cells, eosinophils, and fibroblasts. Transitions between reticulum cells and fibroblasts are common. The eosinophilia is unusually copious considering this is a late stage. Necrosis with many pyknotic polymorphs is noted in many foci. Plasma cells are rather remarkably confined to the least mature (innermost zone) connective tissue fringing the follicles. In a slide stained specifically for eosinophils there is very little evidence of transformation of plasma cells to granular series. Ifitoses average 1 per high power field. The vascularity of the lesion is low; vessels of arteriole size are rare.

Diagnosis. Hodgkin's disease, Typical, late, shewing very striking follicular pattern. 
Andrew Young. Aet 18 years. Ref. Stobhill P.42:834.

History. A painless swelling appeared in the left side of the neck about five months ago. An initial clinical diagnosis of tuberculous lymphadenitis was abandoned since the tumour increased steadily in size. The patient has no other complaints.

This is a cervical lymph node shewing an unusual form of atypical Hodgkin's disease. The reticulum cell proliferation tends to be focal and eosinophils are few. A small adjacent node included in the specimen is still physiological. There is some persistence of normal lymphoid tissue in the larger (morbid) node. This appears as small crushed. crescent shaped deposits being driven against the capsule. The exodic growth phenomenon is well illustrated. The habit of growth is peculiar, all elements of the Hodgkin's disease complex are discernible but the reticulum cell proliferation is focal, large masses of morbid mononuclear and Hodgkin giant cells forming irregular islets and strands. The capsule is thickened and hyaline, and concentric reformation is visible. It is not infiltrated.

Fibrosis is seen in all forms except coralline deposits. The reticulum cell proliferation appears excessive due to the fact that the cells are not uniformly disserninated. In places they are symplasmic. Eosinophils are present but in small numbers. Plasma cells and neutrophils are present but meagre. Mitoses average I per H.P. field. Necrosis is not a feature.

Diagnosis. Atypical Hodgkin's disease. Eosinophils few. Reticulum cell proliferation is tending to be focal.

Note. This patient died in 1951. Full necropsy was carried out see special P.M. series, E. $35: 51$. 
Unknown. Serial biopsy specimen. Ref. 768:42, and 938:42.

Both specimens shew the same features. The lesion does not conform to any of the commoner variants of lymphoid tissue tumour but is nearest to follicular lymphoma.

Fibrosis is a well developed change. The capsules are considerably thickened and the trabeculae are similarly affected. In addition perivascular lamellation is notable in the trabecular vessels. Fibrils and fibrillae are present in the pulp in relation to macrophage foci and near the edges of follicles. Follicles of small size and irregular shape are present throughout the nodes, they are mainly of the solid variety and. the centres are composed of active reticulurn cells differentiating to lymphoid type, mutual compression of follicles is seen in some areas. The pulp contains many aberrant cells of morbid mononuclear reticulum celltype. Rare Hodgkin giant cells are identified. Eosinophilia is absent. In both nodes large focal areas of necrosis are present and some disintegrated polymorphonuclear leucocytes are visible at their periphery. In some areas aggregates of macrophages are seen, many of these cells are degenerate but between the surviving ones a fine collagen mesh is discernible.

Diagnosis. Lymphoid tissue tumour, indeterminate, nearest to Brill's disease. Docal necrosis, fibrosis and abnormal proliferation of cells in the node pulp. 
Unknown. Skin. Identity. Ref. 863:42.

This specimien consists of skin and subcutaneous tissue. In the later coalescent deposits of Hodgkin's tissue are present together with physiological granulation tissue and purulent abscess. The Hodgkin's tissue contains numerous evenly dispersed morbid mononuclear reticulum cells and a small number of typical Hodgkin giant cells. Iymphocytes and eosinophils are copious, many of the latter are mononuclear and of lymphocytoid type. The granulation tissue contains fairly numerous iron pigment-laden histiocytes. These cells are conspicuously different from any in the tumour proper, the nuclei are typically small and eccentrically placed. Fibrosis is difficult to assess due to pre-existing collagen in the site, but a fine fibrillary mesh is discernible in the tumour and is continuously related to morbid reticulum cell processes which taper off into fibrillae. The morbid process is readily recognisable as Hodgkin's disease despite its superficial uniformity. The abscesses noted shew central necrosis and abundant polymorphs, capillaries are little in evidence. Localisation of the lesion is sharp.

Diagnosis. Hodgkin's disease, of skin. (Mfycosis fungoides, a variety). Atypical fibrosis. Pseudo-suppuration. 
Unknown. Identity ref. 1132:42.

The specimen consists of four lymph nodes, two moderately enlarged, the others of normal size. All shew uniform but slight thickening of the capsules, in one case two adnate nodes shew mutual destruction of their capsules at the region of contact. In most areas there is a very thin rind of compressed but otherwise apparently normal lymphoid tissue including rare fragments of sinus tissue. This exodic growth manifestation is disappearing in places. Trabeculae are few, those present shew incipient accretion of collagen fibres along their course but these are not yet compacted. Fibrosis is less than usual in typical Hodgkin's disease; perivascular lamellation, diffuse fibril deposition, and symplasmic transformation are the main expressions of it. Coralline deposits are not seen. The reticulum cell increase is well above the usual and appears much at the expense of lymphocytes. Iymphocytoid differentiation is common, but typical morbid mononuclear type reticulum cells are more numerous. Hodgkin giant cells are present but tend to be small with few nuclei. Eosinophils are numerous, unexpectedly so for this cellular type of lesion. Plasma cells and neutrophils are also commoner than usual. Witoses are up to 4 per H.P. field. Necrosis, except for occasional individual cells, is not a feature.

Diagnosis. Hodgkin's disease, tumour-like type. 
Cameron. Ref. 154:43.

The specimen is a lymph node entirely replaced by reticulum cell sarcoma. There is not any vestige of normal structure except for portions of the original capsule. This tunic is thickened and hyaline where it persists and concentric re-formation is noted. Elsewhere it is over-run and destroyed by the tumour cells which infiltrate out into the adjacent areolar tissue. True trabeculae are not represented and the new collagen of the tumour is still diffuse. Perivascular lamellation is not a feature, the fibrosis is chiefly expressed by diffuse fibre and fibril formation which imparts an indistinct alveolar pattern to the tumour. Coralline deposits are common and occasionally appear to be condensations of the ground fibres. The type cell of the growth is a reticulum cell with a rather small nucleus, superficially it resembles a lymphocyte series cell but high power scrutiny negatives this. Nucleoli are common and typical morbid mononuclear reticulum cells are identified readily. Hodgkin giant cells are present but forms with more than three nuclei are rare. Eosinophilia is absent and plasma cells and neutrophils are inconspicuous. The growth rate is high judged by Karyokinetic figures which average about 4 per high power field.

Diagnosis. Reticulum cell sarcoma, sclerosing. Fields of Hodgkin's disease are present (less topical eosinophilia). 
Ref. R.M. Cervical lymph nodes.

This is atypical Hodgkin's disease which is very tumour-like in appearance and is beginning to conform to Warthin's Hodgkin's sarcoma, type II (Reticulo-endothelioblastoma). Normal lymphoid tissue does not persist except for small islets at the edge of the nodes. The capsule is markedly thickened and extremely hyaline. There is much fibrosis in the node. Most of it assumes the form of irregular crescentic swathes and small irregular stellate scars; under low power an interlacing of the fibres, reminiscent of fibro-sarcoma, is appreciablo. Perivascular deposit is common too, but coralline formations are scanty. The reticulum cell increase is unusually florid and both morbid mononuclear and Hodgkin giant cells are particularly numerous. Eosinophilia is present but rather below average intensity. Mitotic activity is very difficult to assess, there are so many aberrant hyperchromic nuclei present; unequirocal examples of Karyokinesis are rare. Several small necrotic foci are present.

Diagnosis. Atypical Hodgkin's disease, Tumour-like. Close to Hodgkin's sarcoma (Warthin's type 2), but eosinophilia persists. 
Ref. G.R.I. 390:43.

This is an example of soft cellular rather tumour-like Hodgkin's disease. Polymorphonuclear leucocytes are profusely scattered through the new tissue. Small poorly demarcated islets of lymphoid tissue and fragments of marginal sinus survive at some points of the periphery. The capsule is thickened but is still cellular, trabeculae are not represented. Fibrosis is slight, all varieties are encountered but most is fibrillary. It forms a fine filigree and is a good example of symplasmic transformation. Almost all the fibres stain red with van Gieson's stain. Reticulum cell proliferation is pronounced and lymphocytes are very much repressed. Rost of these reticulum cells are small and uniform though typical morbid mononuclear and Hodgkin giant cells are present. Fosinophilia is marked despite the large number of neutrophils present. Small necrotic patches are frequent. Under very low power the exodic growth phenomenon is appreciable at some places.

Diagnosis. Atypical Hodgkin's disease. Highly cellular, tumourlike. Fibrosis mild (? inflamed). 
Marion Carlyle. S.O.P.D. Mr. Hutchinson. Ref. G.R.I. 596:43. Cervical lymph node enlargement of several months duration. 21st April, 1943.

This is typical Hodgkin's disease, the lesion is fully established; the new connective tissue renders the cellular part of the node folliculoid. There is not any persistence of normal lymphoid tissue. The capsule is grossly thickened and hyaline with numerous trabeculae running from it to cut the node into a mosaic. In addition to this coarse fibrosis, perivascular deposits and fibril formation in the cellular islets are common. Coralline formation apparently the result of compaction are also found. Reticulum cell proliferation is pronounced in many of the islets with copious production of morbid mononuclear and Hodgkin giant cells. The latter are frequently necrotic and they are small. Eosinophils are numerous, often focal, and mononuclear forms are common. Plasma cells and neutrophils arepresent in about the usual concentration. Mitotic figures are difficult to identify with certainty but are probably few. Several minute fibrinoid necrotic foci are present. Diagnosis. Hodgkin's disease, Typical. 
Ref. G.R.I. 1168:43. A large single lymph node $4 \mathrm{~cm}$. diameter.

This is classical Hodgkin's disease at a moderately early stage. The entire node is morbid, the capsule is fairly evenly thickened being approximately $I \mathrm{~mm}$. Wide but trabeculae are hardly represented. Incipient concentric re-formation of the capsule is indicated but the true capsule is devoid of any infiltration. Fibrosis of all varieties is encountered; perivascular lamellation is present throughout and swathes of new fibrous tissue are being compacted into bands which are forming the new tissue into nodules. Fine fibrillar filigrees are formed in the more cellular areas and some coralline fibrosis is observed. The reticulum cell proliferation is general and besides the differentiation (or reversion) to morbid mononuclear and Hodgkin giant cells there is easily traced transformation to fibroblast and epithelioid type cells. A few unusual giant cells are noted, their nuclei shew a horse-shoe arrangement but are otherwise unlike Langhan's cells. Eosinophilia is marked, plasma cells and neutrophils are present in small numbers. Mitoses average $I$ per H.P. Pield; necrosis is limited to individual cells.

Diagnosis. Hodgkin's disease, Typical. (Moderately early). 
Ref. Ayr C.H. P.C.32.

This is a good example of typical Hodgkin's disease, the eosinophilia is unusual in its intensity.

Several survival follicles with pale centres and survival islets are present, they are situated relatively deep in the node. They appear remarkably incongruous being surrounded by typical Hodgkin's tissue. The capsule is grossly thickened and hyalinised. New connective tissue trabeculae of very irregular size and course are seen. All forms of fibrosis are present in quantity, the change is coarse. The reticulum cell proliferation presents in the usual degree; fibroblastic transformation is well shewn besides the characteristic morbid mononuclear and Hodgkin giant cells. The latter are typical but necrosis is common in them. The eosinophilia is a most impressive element in this case. Some high power fields seem almost totally composed of these cells. Plasma cells and neutrophils are present but in the usual quantity. Mitoses number 1-2 per H.P. field. Focal necrosis is common, the demarcation of these areas is curiously sharp.

Diagnosis. Hodgkin's disease, Typical. (Eosinophilia very pronounced). 
Nurse Forbes. Aet 22. Ref. G.R.I. 1947:43. History of painless swelling of left cervical lymph nodes of unknown duration. Node resected December 1943. Received X-ray therapy after transfer to Medical House; Known to be dead (1950). Necropsy not performed.

This is atypical Hodgkin's disease of tumour-like type. Eosinophils are almost absent and the growth is relatively homogeneous with a preponderance of small cells. The capsule is grossly thickened and islets of the new tissue are set in it, this phenomenon appears the result of concentric re-formation of the capsule with engulfment of the cellular parts, rather than transgression of the capsule. Fibrosis of other types is much less in evidence. The reticulum cell proliferation is productive of young lymphocyte type cells, the typical mononuclear and Hodgkin giant cells being overshadowed. Eosinophils are very rare, but plasma cells and neutrophils are above average in number. Mitoses are common, up to 4 per H.P. field. Necrosis is confined to individual pathological cells.

While areas of typical Hodgkin's disease are easily found the greater part of the tissue is not orthodox.

Diagnosis. Atypical Hodgkin's disease. Tumour-like type, rather small uniform cells. No eosinophilia. 
Leighton. Ward 26. Mr. Swanson. Ref. G.R.I. 1887:44. Axillary lymph nodes.

This is an example of atypical Hodgkin's disease of an unusual and somewhat treacherous character. The true nature of the change is obscured by the development of epithelioid cells which occur in irregular patches. High power scrutiny dispels all doubt of the true pathology.

The exodic growth phenomenon is present but is indistinct. The peripheral rind of $\dot{t}$ issue is hardy normal but contains many more small lymphocytes than the new tissue. The capsule shews local thickening and concentric reformation is discernible. Fibrosis of all varieties is identified but it is small in amount and difficult to find without special staining. The reticulum cell proliferation is expressed in the usual way with perfectly typical morbid mononuclear and Hodgkin giant cells but epithelioid cells are much more conspicuous. They are disposed in irregular patches without any tendencies like 'endothelial tuberculosis'. Eosinophilia is moderate, plasma cells and neutrophils are very scanty. Mitoses are numerous for Hodgkin's disease, 2-3 being visible in certain H.P. Pields; two minute areas of necrosis are seen.

Diagnosis. Atypical Hodgkin's disease. Epithelioid cell islets present. Fibrosis slight. 
Jean Lornie. Ward 4. Dr. David Smith. Ref. G.R.I. 600:44.

This is early Hodgkin's disease. Most of the capsule is lost by artefact and it is not possible to gauge whether thickening is present. Fibrosis is already fairly pronounced all forms being encountered but coralline deposit is very scanty. The reticulum cell hyperplasia is only moderate. Most of the cells produced appear to be imrature lymphooytes but the pathognomonic ones are also present. Eosinophilia is confirmed; the staining leaves much to be desired but their number is probably high. Plasma cells are scanty but neutrophils are scattered diffusely through the tissue. Mitoses are numerous, 4-5 per H.P. field. Necrosis is limited to individual cells.

(Very little material is available from this case, the spare blocks having been used for class purposes).

Diagnosis. Hodgkin's disease, Typical, Barly. 
Unknown. Ref. G.R.I. 586:45.

This is a good example of early, established Hodgkin's disease which is not involving the whole node and demonstrates the exodic growth phenomenon. The new tissue occupies the central part of the node and is growing just like a metastatic cancer nodule and driving a rind of reactive lymphoid tissue up against the capsule. The capsule is not thickened but the marginal sinus is obliterated, at the margin of the tumour tissue there is a fibrous layer forming, an example of early concentric re-formation of the capsule. Fibrosis of all varieties is already evident. The reticulum cell increase is of the usual order and productive of typical morbid mononuclear and Hodgkin giant cells. Eosinophils are numerous. Plasma cells and neutrophils are little in evidence. Mitoses average about 1-2 per H.P. field. Necrosis is not seen.

Diagnosis. Hodgkin's disease, early established. Partial involvement of node. 
Maloolm Ross. Aet 17 years. A joiner. Ref. G.R.I. 2671:48. Swelling in the left side of the neck of several months duration.

This is early Hodgkin's disease. There is remarkable survival of physiological lymphoid tissue. Follicles, both solid and with pale centres, are present. The latter are restricted to the periphery of the node. Fragments of sinus tissue are also discernible. The pale centre reticulum cells do not appear to contribute to the new tissue which in this case is convincingly restricted to the pulp (functional medulla of Robb-Smith).

The capsule shews irregular thickening, most of it being seen at the base of small wedge-like trabeculae. The exodic growth phenomenon is well demonstrated and concentric re-formation of the capsule is striking. An inner false capsule round the outer margin of the new tissue is distinctly visible. Fibrosis is well established, all forms are identified but the coralline variety is minimal. Reticulum cell proliferation is conspicuous and morbid mononuclear types are very numerous. Typical Hodgkin giant cells are common. Several Langhan's multinucleate giant cells are present and appear directly related to foci of necrosis. Eosinophils are very numerous, several large foci of several hundred are seen. Mitoses number approximately 2 per H.P. Pield. Necrotic foci are common, they are chiefly at the expanding edge of the lesion. (See also Serial 76).

Diagnosis. Hodgkin's disease, Typical. Early with retention of focal character of growth. 
Mrs. Margaret Leonard. Aet 29. Ref. G.R.I. 2146:47. Noticed swelling in left armpit one month previously. Clinical examination revealed enlarged nodes in the neck.

Specimen consists of four large smooth nodes, the largest $4 \mathrm{~cm}$. diameter.

This is early Hodgkin's disease. The coarsely follicular organisation of the new tissue is definite and the exodic growth phenomenon together with concentric re-formation of the eapsule is well shewn. The tumour is atypical, small cells being predominant and eosinophilia slight.

There is patchy survival of lymphoid tissue and sinus tissue at the periphery of some of the nodes. The capsule is distinctly thickened, this is uniform but only moderate in degree. Fibrosis of all varieties is encountered but is slight overall. The reticulum cell proliferation is less prominent than usual due to the high number of small and slightly immature lymphocytes. Morbid mononuclear and Hodgkin giant cells are identified but they are scanty. Eosinophils require a patient search, they are present but distinctly rare. Mitoses are few, less than one can be found consistently in high power fields. Necrosis is absent.

Diagnosis. Atypical Hodgkin's disease. Small cells predominate, no eosinophilia, fibrosis slight. Some affinities to small cell lymphosarcoma.

Note:- Patient died 2nd December 1950, P.M. 332:50. G.R.I. q.V. 
Thomas Finnigan. Aet 74 years. G.R.I. 2387:47. Enlarged axillary lymph nodes for doubtful period, probably months. Also has tumour over frontal bone and in chest wall. Specimen of axillary node sent. See also serial 135 .

This is a poorly differentiated reticulum cell sarcoma which exhibits some affinity to Hodgkin's disease. The entire node is neoplastic, the capsule being extensively destroyed and the tumour invading the surrounding fat. The growth assumes the general form of alveolar sarcoma, groups of cells occupying the spaces of a plexiform fibrous tissue mesh. The cell type is spheroidal with indistinct cytoplasm of pale amphophilic staining reaction and morbid reticulum cell nuclei of various shapes and sizes. The cells are discrete in some areas, and fused to form plasmodial masses in others. Typical Hodgkin giant cells are present. Eosinophilia is absent and plasma cells are not apparent. Polymorphs are notable round small foci of necrosis which are seen. Perivascular fibrosis is seen round the arterioles present in the tumour. The activity of the growth is high, 4-5 mitotic figures can be found in most high power fields.

Diagnosis. Reticulum cell sarcoma, poorly differentiated. Affinities to Hodgkin's disease. (Hodgkin giant cells, fibrosis). 
Thomas Hume. Aet 32 years. Ref. G.R.I. 444:48. Painless enlargement of lymph nodes in the posterior triangle of the neck. Duration uncertain. Specimen. Cervical lymph node.

This is an example of moderately early Hodgkin's disease in which the origin of some of the integral fibrosis is well seen.

The node capsule is of normal thickness, it is intact and concentric re-formation is not evident. Several ill defined pre-existing lymphoid follicles are discernible. Fibrosis is slight but well beyond the amount seen in normal nodes. Perivascular lamellation and fine fibrillary deposits are the chief expression. The latter is traceable to the cytoplasm of reticulum cells which have assumed an epithelioid appearance. While the nuclei of these cells is close to those of proliferating fibroblasts the cytoplasm is rarely spindle-shaped. These fibrifying reticulum cells are the commonest of this scion but morbid mononuclear and Hodgkin giant cells are plenteous. A few of the latter shew horse-shoe arrangement of their nuclei. Eosinophilia is conspicuous but the other cells, plasma cells and neutrophils are scanty. Mitoses average 1 per H.P. field, necrosis is absent.

Diagnosis. Hodgkin's disease, Typical, early, established. Diffuse involvement of almost whole node structure. 
Isobel Topping. Let 36 years. Under Dr. David Smith. Ref.G.R.I. 110:49. Complaint of malaise and swelling of the right side of the neck for five weeks. Right tonsil enlarged. Firm discrete glands in the right posterior triangle of the neck. The peripheral blood is normal. (This patient later developed Hodgkin's disease in the breast, see Serial 81. (Ref. G.R.I. 2583:50) Known to be dead, 1952, necropsy not performed.

This is early Hodgkin's disease. The entire node appears affected but small lymphocytes are still copious, these particular cells are just appreciably less mature than normal ones. The capsule already shews hyaline thickening and fibrosis of all types is present. Perivascular deposit is the chief expression of the change. The reticulum cells shew a very uniform increase, many morbid mononuclear forms are present but in general they are small. Typical large ones and Hodgkin giant cells are however present. Eosinophilia is less conspicuous than usual and tends to be focal. Plasma cells and neutrophils are scanty. Necrosis is limited to occasional giant cells. (Note, the peculiar veins of Ehrich are packed with normal small lymphocytes).

Diagnosis. Hodgkin's disease, Typical, early, established. 
Malcolm Ross. Aet 17 years. "Ref. G.R.I. 2939849. Previously diagnosed Hodgkin's disease (vide Serial 71). Clintoal relapse following $X-r a y$ therapy and exhibition of nitrogen mustard.

The specimen is just reoognisable as a lymphoid struoture. The capsule is greatly thickened by lamellae of collagen but nuolel of fibroblast and fibrocytescan still be made out. At one point a portion of the marginal sinus persists. Areas of the oharacteristio new tissue are recognisable but certain changes are noteworthy. Plasma oello, frequently binucleate, are common, and the fibrosing prooess is florid. Eosinophils are absent but occasional polymorphs are seen. Numerous pyknotic nuclei are scattered widely through the tisene, these appoar to be chiefly lymphocytic.

Enhancement of fibrosis, loss of eosinophilla and abundanoe of plame cells appear to be the chief festures.

Diagnosis. Hodgkin's disease, post-irradiation. 
Mrs. Margaret McCulloch. Aet 64 years. Ref. G.R.I. 2237:49, 2913:49. Complaint of sudden severe swelling of all limbs which persisted for four weeks and then subsided. Enlarged cervical nodes for past week. Exceedingly ill; the peripheral blood is normal. The biopsies are of cervical and epitrochlear nodes and were taken at an interval of six weeks. The patient died six weeks after the second operation, necropsy was not obtained.

The microscopical appearances are the same in both specimens apart from slight increase in the fibrous tissue of the capsule in the second node. This is cellular rapidly growing Hodgkin's disease which clinically merits the term acute Hodgkin's disease. Persistence of normal structure is seen, in the first node several lymphoid follicles persist and in the second much of the marginal sinus remains but the lumen contains abnormal cells.

Capsular thickening is seen only in the second specimen. This barrier is not invaded but small islets of the new tissue, with many plasma cells, are enclosed in the substance. Fibrosis is relatively slight; perivascular lamellation is certainly present in the few vessels of size present, but diffuse fibril and fibrillary deposits are the chief form. Very early coralline deposit is shewn in process of forming and the node of origin is beautifully disclosed. A focus of morbid reticulum cells which have assumed an epithelioid morphology are beginning to fibrose, the process being a direct transformation of their cytoplasm into collagen, this commences as a condensation of their peripheral cytoplasm 
into fibrils which by mutual accretion form the familiar tortuous cylinder. The reticulum cell proliferation is pronounced and many typical morbid mononuclear forms are present. Hodgkin giant cells are present, they tend to be rather small. A few giant-cells shew similarity to Langhan's type but their nuclei are not characteristic. Hosinophils though present are scanty, similarly the plasma cells and neutrophils are few. Hitoses average 4-5 per H.P. field.

Diagnosis. Atypical Hodgkin's disease. Soft cellular tumour-like variety. Rapidly fatal. 
Wrs. Caroline Cameron. Aet 42 years. Ref. 2444:49. Patient was admitted for investigation of suspected intestinal obstruction. Laparotomy was carried out 26th October 1949 and an inoperable retrom peritoneal tumour was found. This specimen consists of a portiom of this growth. The patient died three days later. An incomplete necropsy was performed but, there is not any record available. (From outside hospital, without a morbid anatomist).

The specimen shews a limiting cellular membrane at one dige wich is serosa, beneath this a zone of fatty areolar tissue overlies an ingigling cellular tumour which is widely necrotic.

The growth is morphologically a pleiomorphic reticulum cell sareom in which morbid mononuclear type and Hodgkin giant cells are presemt. Eosinophils are very numerous; plasma cells and neutrophils are copiouss near the necrotic foci. Mitotic activity is considerable. Pibrosis new reticulin formation are confirmed by special staining.

The special interest in this case lies in the remarkable similarty of certain areas of the tumour to Hodgkin's disease. Morphologioanly it is a link between the two, its lymphoid origin is probable in sections fragments of lymph node are visible. (The neoropsy sections shew deposits of the same tumour in the bowel).

Diagnosis. Reticulum cell sarcoma, pleiomorphie, Bosinophilia present. 
British Soldier. Aet 23 years. Ref. I.N. 20:50. The specimen is a cervical lymph node received from Capt. Cowan, R.A.M.C., Cowglen Military Hospital. Complaint of swelling in the neck, left side, of uncertain duration. The general health of the patient is excellent.

The capsule shews irregular thickening in places. Where it is still normal there is surviving sinus tissue and a thin rind of subjacent lymphoid tissue. These normal tissues are hyperplastio but still in physiological degree.. The exodic growth phenomenon of the tumour is particularly well shewn, and capsular re-formation is seen, sometimes outflanking survival islets of non-neoplastic lymphoid tissue. The preexisting trabeculae are thickened and new swathes of connective tisgue are forming. Fibrosis is absolutely typical; perivascular lamellation, fibrils, fibrillae, and corelline deposits are all seen. The reticulum cells are conspicuously increased and the majority are pleiomorphic morbid mononuclear types. Hodgkin giant cells are prominent. Topical eosinophilia is marked; plasma cells and neutrophils are also seen. On an average two mitoses are seen in high power fields. Minute foci of necrosis are notable. (This is amongst the most classical examples of the disease at an established yet early stage that I have seen).

Diagnosis. Hodgkin's disease, Typical, early, established. 
Hugh McLachlan. Aet 51 years. Ref. G.R.I. 351:50. 958:50. History. Patient has noticed glandular swellings in his right elbow region and the right axilla for three years. Anaemia is present. (The first biopsy was mis-diagnosed as reactive). At the second operation the axilla was cleared entirely, with apparent benefit. He reported back in July 1950, but did not return. He died 22nd December 1950, at home, necropsy was not obtained.

This is an example of an unusual variant of Hodgkin's disease. It corresponds most nearly to Ziegler's Type II, but does not conform exactly.

The capsule and trabeculae are thickened sufficiently to arrest attention. The lymphoid tissue of the node is rendered into small psoudo- follicles in most areas, by incipient perivascular fibrosis of the peculiar veins of Erich. The tissue thus arranged looks superficially normal but close scrutiny reveals quite numerous pathological reticulum cells and rare Hodgkin giant cells. The lymphocyte is still by far the commonest cell present but it is an immature type with a large nucleus which is relatively leptochromatic. Fibrosis of fibril and fibrillary type is discernible, especially in the second specimen, but it is not obtrusive. Eosinophils are absent from both. Plasma cells are present in larger numbers than is usual in the disease. Mitoses are very infrequent, many fields being devoid of them.

Diagnosis. Atypical Hodgkin's disease. No eosinophils. False follicles, affinity to lymphoma folliculare. 
Isobel Topping. Aet 38 years. Ref. G.R.I.W.S. 2583:50.

History. This patient came under the care of Mr. Stevenson in July 1950 on account of a localised swelling in the breast. This tumour was resected and sent for microscopical examination. At this time the previous history (See Serial 75) was unknown.

The specimen consisted of a firm fibro-fatty mass rather larger than a walnut.

Breast tissue of normal appearance is readily identified in parts of the specimen. The normal connective tissue of the gland, both the primitive gland field variety and the general stromal type present usual appearances. Several small and one large ( $1 \mathrm{~cm}$. diameter) deposit of typical Hodgkin's disease are present; there is not any evidence that these have arisen in ectopic lymph nodes. The tumour shews well developed fibrosis which is coarse and hyaline, and differs sharply from the native formations. The cellular component of the lesion is quite characteristic and is some areas typical Hodgkin giant cells are very numerous. Eosinophilia is slight. A distinct irregular follicular growth is notable. In places surviving epithelial structures, acini and ducts, are over-run by the growth.

Diagnosis. Hodgkin's disease, Typical. Unusual site - mammary gland. Presumed autochthonous development in ectopic lymphoid tissue. 
Hugh Gray. Aet 54 years. A signalman. Ref. G.R.I. W.S. 3776:50. History. Patient complained of a swelling in the left inguinal region which had originated four years previously. This increased in size rapidly during the last two months. Aspiration was attempted in November 1949. The present specimen was resected 26th November 1950.

The specimen is presumed to be a part of an enlarged lymph node but vestiges of original structure are absent. This is an example of reticulum cell sarcoma corresponding exactly with the clasmatocytic stem cell lymphoma. The type cell is a morbid mononuclear reticulum cell; many shew acidophile nucleoli. In all areas the cells are mutually separated; several typical Hodgkin giant cells are present. Fibrosis is seen chiefly as perivascular lamellae and scar-like areas of compacted fibrils. In addition a fine filigree of stout reticulin fibres is demonstrable. Eosinophils are very few and a few polymorphonuclear leucocytes are seen near necrotic areas. The latter are massive infarcts. Mitoses are very numerous, about 20 per high power field.

Diagnosis. Reticulum cell sarcoma. (Clasmatocytic stem cell Iymphoma). Shews distant affinities to Hodgkin's disease.

Note. This patient died a few weeks later from pulmonary embolism, and necropsy (G.R.I. P.M.304:50) revealed restricted spread of the disease, in the abdomen and thorax. Deposits were confirmed in the liver and in abdominal nodes by microscopy. 
Bernard Duffy. Aet 35 years. A lebourer. Ref. G.R.I. 763:50 932: 50 . History. This patient was unable to offer any account of his illness, his cerebration was very slow. The presenting symptoms were enlargement of the axillary lymph nodes and jaundice. The initial biopsy, (763), was from the left axilla, the second, (932), from the portal fissure.

This is an example of classical Hodgkin's disease which was originally missed. The error was made because the first sections shewed a lymph node in which fibrosis was the only morbid feature of note. Further sections revealed the true character of the lesion. This case emphasizes the paramount importance of appreciating that fibrosis of obscure cause in lymph nodes is a most sinister finding. In both specimens surviving lymphoid tissue, including follicles, pulp, and sinus tissue, persist. The exodic growth phenomenon however is not displayed, the growth of this lesion is relatively indolent. The capsules shew irregular fibrous thickening and trabeculae are visible. Fibrosis of all varieties is encountered but by far the greatest portion is coralline. Morbid mononuclear cells, Dorothy Reed mirror-immage cells, and Hodgkin giant cells are all present. Eosinophils are present, but slightly below the usual number. Even in the cellular areas of the lesion small Iymphocytes are conspicuously few.

Diagnosis. Hodgkin's disease, Typical, late. 
Catherine Quearns. Aet 16 years. A Winder. Ref. G.R.I. 3904:50. History. Patient complained of a small lump present behind the left ear which was present two months. During the past week another swelling has started below her chin. Pediculosis capitis was present. After biopsy the wound healed rapidly and the patient was dismissed with instructions to return in three months. She has never returned to date (November 1954).

This is a slightly enlarged lymph node shewing pronounced reactive change in which epithelioid cell change is seen throughout the pulp. In some areas the appearances very closely simulate Hodgkin's disease but the reacting cells are distinctly small and the general architecture of the node is retained. Fibrosis is present but is not of Hodgkin's disease conformation.

There is general retention of normal architecture, this is difficult to appreciate with the ordinary low power but is well seen with lower magnification (X 30). The germinal follicles are irregular in outline with occasional abstriction of fragments of the pale centre tissue. The pulp is mottled due to numerous small aggregates of epithelioid cells and rare individual large reticulum cells. The capsule is thickened and the fibres are hyaline; in most parts a fairly uniform infiltration of small lymphocytes and plasma cells is present. Fibrosis is seen in the trabecular vessels, and round other vessels in the node. Very fine fibrillae of collagen are present in the cytoplasm of many of the epithelioid cell collections. The reticulum cells encountered have been 


$$
84 a .
$$

noted above, very few shew hyperchromatic or distorted nuclei. Rare mirror-image cells are seen but again they appear physiological. Hodgkin giant cells are not present. Eosinophils and polymorphs are present in small numbers. Mitoses are chiefly seen in the pale centres but several are also noted in the pulp.

Diagnosis. Chronic inflammatory reaction, non-specific. Isolated and aggregated epithelioid cells in pulp. Very few true morbid reticulum cells. Simulates Hodgkin's disease. 
George W. Henderson. Aet 40 years. A fireman. Ref. G.R.I. 3673:50. History. Painless swelling in right side of neck of three months duration, latterly swelling has appeared in the right axilla. it operation two masses of cervical lymph nodes were removed one weighing $70 \mathrm{gm}$. and the other $15 \mathrm{gm}$.

This is a very interesting lymphoid tissue tumour with a peculiar morphology. Features of Hodgkin's disease and large cell lymphosarcoma are intermingled giving what may be described as "lymphoblastic Hodgkin's disease". There is very little remaining normal lymphadenoid structure, survival islets of small lymphocytes constitute the sole evidence. The capsule is irregularly thickened and hyaline, slight concentric reformation is evident and invasion is present. Trabeculae are few but partake of the capsular thickening. Fibrosis is present but slight. Patches of diffuse fibrils are seen together with very delicate sinuous fibrillae and some typical coralline deposits are seen. The majority of the cells are mutually similar. They are free cells with round nuclei, folds and wrinkles of the fairly dense nuclear membranes are rare. Chromatin content is variable but usually large, in the forms of indistinct blobs. Amphophilic nucleoli are very frequent. The cytoplasm is oxyphile, very finely granular, and concentric round the nuclei; angular deformity due to apposition is common. Typical large morbid mononuclear reticulum cells are scattered through these cells and typical Hodgkin giant cells are present in quantity, they tend to occur in ill defined foci. Eosinophils and neutrophils are very rare. Some iron pigment is notable.

Diagnosis. Large cell lymphosarcoma with some features of Hodgkin's disease, or Atypical Hodgkin's disease, "Lymphoblastic". 
Thomas Gartland. Aet 46 years. A labourer. Ref.G.R.I.W.S. I/2966:50. History. The patient presented with a swelling in the left parotid gland region which was provisionally diagnosed as a salivary adenoma.

The specimen is a small lymph node in the depths of which an intrusion of connective tissue containing salivary gland duct and gland acini is present. This node was reported as shewing the appearances of early Hodgkin's disease but I find myself unable to agree.

The lymph node is small, $14 \times 7 \times 5 \mathrm{~mm}$. There is considerable persistence of the normal structure but it is masked by the hyperplasia which is present. The follicles can still be made out, they are large and some are diffuse at the periphery. Sinus tissue is well preserved in many parts. The capsule is not thickened. One trabecula bearing salivary gland elements is seen. There is some fibrosis, chiefly paratrabecular, in the form of diffuse fibrils. There is a reticulum cell hyperplasia, mainly in the pale centres of the follicles but also in the pulp. Most of the cell nuclei are small, although a few merit the term morbid mononuclear type. Rare binucleate forms are seen but no true Hodgkin giant cells. There is not any eosinophilia. Plasma cells are frequent and a few scattered neutrophils are observed. Hitoses are numerous locally - 4 or 5 per H.P. field.

Diagnosis. Reactive hyperplasia. ? Lympho-reticular medullary reticulosis of Robb-Smith.

Note. This patient is alive and well (November 1953). X-ray therapy has been given in several occasions. In the most recent survey available small nodes are reported to be palpable in the axillae; the spleen is not enlarged. 
Catherine Prentice. Aet 38 years. Ref. G.R.I. W.S. 3731:50. History. This patient complained of an axillary tumour four years ago. She was treated in Prof. J.A.G. Burton's wards and underwent an operation at this time. Thorough search of the records in the pathology department has failed to discover any reference to examination of a specimen from the patient. About two weeks previous to the present specimen a biopsy was performed but the material was so extensively necrotic that diagnosis was impossible (3451:50). The present specimen is a grossly enlarged axillary node.

This tumour is regarded as either Hodgkin's disease of highly cellular tumour-like type or a fibrifying reticulum cell sarcoma in which eosinophilia is present. A feature of unusual interest is the inclusion in the tumour tissue of a small physiologically reactive lymph node. This node is being invaded by the neoplasm at one pole. The capsule of the neoplastic node is of normal thickness but hyaline. Concentric re-formation attempts are evident but in many places invasion has occurred. Thick trabeculae are seen. Fibrosis is present, it is relatively uniform. Perivascular lamellae are common, and the fibrils present are compacted to form a coarse even mesh, the lacunae of which are filled with a delicate filigree of fibrillae. Several coralline deposits are present. The type-cell is a morbid mononuclear reticulum cell which corresponds with those of Hodgkin's disease. Small Hodgkin giant cells are common. Eosinophils are as numerous as in typical Hodgkin's disease. Mitoses are very frequent as many as 20 being present in some H.P. fields. Necrosis is chiefly individual cell type. 
$87 a$.

Diagnosis. Atypical Hodgkin's disease, highly cellular tumourlike type, or reticulum cell sarcoma with eosinophilia. Intimately related to normal (reactive) lymph node. 
Thomas McGuiness. Aet 77 years. A pensioner. Ref. G.R.I. W.S. 3770:50. History. Vague; for some time patient has noted the appearance of hard bony lumps on the long bones. His present complaint is of a painful swelling on the front of the left leg. This was diagnosed as a bone cyst and material curetted from it was sent for examination.

This is an indeterninate tumour which has affinities to reticulum cell sarcoma, myeloma of plasma cell type and Ewings endothelioma of bone. The tumour appears very homogeneous under low power examination but high power scrutiny dispels this impression. The type-cell is superficially like a large plasma cell but the nucleus is less chromatic and oxyphile nucleoli are present. A fine reticulin mesh is present round many individual cells and groups of cells. Actual collagen is negligible. In addition typical morbid mononuclear reticulum cells and rare binucleate hyperchromatic cells are seen. Mitoses are few.

Diagnosis. Reticulum cell sarcoma, nearest to myeloma of atypical plasma cell variety.

Note. Further examination revealed 'cysts' in both femora, tibiae and left ulna but none in the skull. Bence-Jones protein was reported and then denied. Patient was traced till mid-December 1950 when he was very ill. 
Mrs. Edwards. Aet 46 years. An housewife. Ref. G.R.I.W.S. B.4079:50. History. Patient has had periods of fever over the last three months latterly associated with enlargement of the right cervical lymph nodes. This was attributed to a decayed tooth but leukopoenia (4,500 per c.mm.) prompted the physician to revise the diagnosis.

This specimen is a cervical lymph node shewing an exceptionally good example of classical established Hodgkin's disease.

Several lymphoid follicles at the periphery of the node survive, they have pale centres. The capsule is markedly thickened and hyaline all round the node. Concentric re-formation is well shewn and at one level a portion of the new tissue is seen in it. The exodic growth phenomenon is still discernible. Trabeculae are poorly developed. The blood vascularity is high and well seen because of congestion. The fibrosis is pathognomonic, all varieties are seen. The reticulum cell hyperplasia is characteristically protean and irregular. Iarge numbers of morbid mononuclear types are present together with numerous typical Hodgkin giant cells. Eosinophilia is present and many plasma cells can be found. Neutrophils are discernible throughout the node and are more concentrated round small focal necroses. Mitotic activity averages 2 per H.P. field. Diagnosis. Hodgkin's disease, Typical. Whole node not implicated. 
William A. Britton. Aet ? (elderly). Ref. G.R.I. W.S. 3875:50. History. The patient presented with a painless swelling in the left groin which had developed over the last few months.

This is a fibrifying reticulum cell sarcoma with pleiomorphic cells which has possibly arisen from a follicular lymphoma. Parts (microscopically) recall Eodgkin's disease. A further point of interest is the incomplete involvement of the node in the tumour process.

One pole of the node consists of physiologically reactive lymphoid tissue and a narrow rind of similar tissue is seen in places elsewhere, the exodic growth phenomenon being well shewn. The tumour has an irregular follicular structure the false follicles being imperfectly separated by swathes of fibrous tissue. The capsule is thickened and some concentric re-formation is evident. Fibrosis is expressed in all forms in marked degree. The cells of the tumour are a mixture of small pleiomorphic hyperchromic morbid reticulum cells and cells shewing lymphoid features. Ill-formed giant cells are noted occasionally. Eosinophils are absent, even with Dominici's stain. Plasma cells and polymorphonuclear leucocytes are scanty. Mitoses are numerous but necrosis is limited to occasional individual cells.

Diagnosis. Reticulum cell sarcoma, intermediate type, sclerosing. Fibrosis of Hodgkin's disease type. Ill-defined follicular pattern. ? Arising on Brill's disease. 
Andrew Cooney. Aet 10 years. A scholar. Ref. G.R.I. T.S. 3191:50. History. Complaint of swellings on both sides of the neck which have been present for six months. Examination revealed enlarged, discrete, firm mobile lymph nodes which were painless.

This is an example of moderately early typical Hodgkin's disease. The characteristic node of growth is well shewn. In general the morbid cells of the lesion are just appreciably smaller than usual.

Several lymphoid follicles persist even in the anatomical medulla; several shew pale centres with normal cells. A peripheral mantle of hyperplastic lymphoid tissue is seen in one node, the exodic growth phenomenon is well shewn. The capsule is thickened but this is mainly restricted to the base of the trabeculae. The new tissue in the node is arranged in coarse irregular follicles mutually isolated by collagen deposits. Fibrosis is not yet marked but all varieties are encountered. The reticulum cell proliferation is productive of many small cells although the characteristic ones are present. The Hodgkin giant cells are small and often occur in small groups. Eosinophils are present but require special stain to demonstrate them well. Plasma cells and neutrophils are noted in small numbers. Mitoses are very few though many equivocal forms exist. Focal necrosis is seen but the areas are very small.

Diagnosis. Hodgkin's disease, Typical. Incomplete involvement of node. Exodic growth phenomenon well shewn. Follicular growth. 
'Plump cellular stage, eos good'. Ref. unknown. Specimen received from Dr. W. Shaw Dunn. It has been reatained. History unknown beyond the fact that the patient was a boy and is now dead.

This is an example of typical Hodgkin's disease in which the complex of the lesion is dissociated, revealing the components in simpler fashion than usual. The capsule shews irregular hyaline thickening which is marked in places, and the trabeculae are also much fibrosed. The exodic growth phenomenon is seen in one segment, where a rind of physiological lymphoid tissue persists. Concentric remormation is not seen. Fibrosis of all types except coralline deposit is seen. Small short lengths of fibrinoid are also present, these may represent collagenous degeneration. The reticulum cell proliferation is pronounced and several different varieties are being produced. Morbid mononuclear cells are present in abundance. Epithelioid cells of large size with smaller nuclei are also seen, these have rather angular acidophile cytoplasm in which minute fibrillae can be detected. These cells tend to occur in islets and are continuous with sinus littoral cells in some cases. Typical Hodgkin giant cells are present in the usual numbers. Eosinophils are very copious, nearly all are binucleate, and conform to the blood type. Plasma cells are scanty. Polymorphonuclear leucocytes are copious, they form definite micro-abscesses, and are related to necrotic foci. liitoses are numerous, 2-3 per H.P. field.

Diagnosis. Hodgkin's disease, Mypical. Slight follicular arrangement of new tissue. Incomplete involvement of node. 
Matthew Dickie. Aet 14 years. A scholar. Ref. L.338:48. History. Complaint of painless lumpy swelling in the left side of the neck of unknown duration. This patient was traced till January 1951. Since diagnosis he has had Hurchison fever episodes approximately every three months. Blood transfusion has been given latterly with apparent benefit.

This is an example of Hodgkin's sarcoma of the variety Warthin (1931) terms type 2 or 'reticulo-endothelioblastoma'. The specimen consists of four nodes. In some there is remarkable survival of cortical lymphoid follicles in certain areas. The capsules shew very irregular fibrous thickening, in places this is intense, in others attenuation is seen. Concentric re-formation is appreciable. Fibrosis of all types is seen, the perivascular component is constant and the fibrillar deposit is chiefly seen in ' $Y$ '-shaped strands. The reticulum cell increase is pronounced and productive of large ill-demarcated rounded areas of morbid mononuclear and Hodgkin giant cells. In addition some epithelioid cell types are being formed. Fosinophils are very scanty. Mitoses are few, below 1 per H.P. field.

Diagnosis. Hodgkin's sarcoma (Tarthin's type II). Hodgkin giant cells and allied cells proliferating to the exclusion of others, regression of eosinophilia. 
John Shepherd. Aet 25 years. A lecturer. Ref. G.R.I. W.S. 4477:50. History. Slightly enlarged painless lymph nodes have been present for the last 18 months in the right inguinal region. Small nodes are palpable in the left groin and also in the left axilla. This specimen consisting of inguinal nodes was originally reported as an 'early low grade type of Hodgkin's disease'.

These nodes are not sufficiently enlarged to be counted abnormal in size for a man of this age. The capsules are irregularly thickened and hyaline. There is almost universal retention of normal architecture in all three, two can only be regarded as physiologically active. The third, the largest shews several diffuse areas of pulp hyperplasia. Here fibrosis is seen round several vessels and as swathes of diffuse fibrils but the connective tissue is continuous with the hilar intrusion.

Reticulum cell hyperplasia is present in the pale centres and pulp, in the latter situation pathological forms are seen including typical morbid mononuclears. Eosinophils are more numerous than in normal (non-alimentary) nodes, but the increase is only convincing with sections stained by Dominici's method. Plasma cells and neutrophils are few. Mitoses are notable in the pale centres and hyperplastic pulp.

Diagnosis. Intense reactive hyperplasia? Early neoplastic change? Regard as indeterminate.

Note. This patient did not receive irradiation, the Paul-Bunnell reaction was negative. Three years later he is alive and in good health with complete regression of glandular swelling. 
Wrs. Annie Welsh. Aet 59. A housewife. Ref. G.R.I. W.S. 374:51. History. The patient attended surgical dispensary complaining of a small firm nodule which had been present two or three months on the left temple. This was diagnosed clinically as a sebaceous cyst and was resected.

The specimen is a lymph node. Superficially the appearances suggest metastatic carcinoma simplex but prolonged scrutiny does not support this diagnosis. The capsule is much thickened and pre-existing and new trabeculae cut the node into a mosaic. The tissue in the mesh consists in most cases of a curious central aggregate of pale staining epithelioid cells which are morphologically morbid mononuclear reticulum cells. (It is my experience however that metastatic malignant melanoma and occasional carcinomata can produce cells which very closely mimic these cells). Mitoses are present, but they are definitely less than might reasonably be expected if this is cancer, multinucleate forms are present but are few. Surrounding these pale cell masses are zones of highly reactive Iymphoid tissue. Fibrosis is present in other forms besides the mesh. Coralline deposits, perivascular lamellae and diffuse fibrillae are all identified. In some of the pale cell areas argyrophile fibrillae are seen. Eosinophils are present in the lymphoid tissue in numbers well above normal. Focal necrosis is seen in some of the pale cell areas.

Diagnosis. Indeterminate. It may conceivably be reactive. It may represent Ewing's trabecular type of lymph node endothelioma. It may be metastatic carcinoma or melanoma. It may be a dissociated type of. Hodgkin's disease. 
Note. A primary tumour has not been discovered to date, November 1954. Patient has reported at regular intervals and has always appeared weIl. 
Alan Wilson. Aet 23 years. 1 discharged soldier. Ref. G.R.I.W.S. 439:51. History. This patient came under observation on account of a large swelling in his left armpit of several months duration. During his sojourn in the medical wards he was also found to have an osteoporosis of the skull and the Wassermann reaction was strongly positive.

This is Hodgkin's disease of cellular type. The affected nodes are very large, $5 \times 4 \times 3 \mathrm{~cm}$. etc. In the mass a whole node has been infarcted. Survival follicles are seen in most nodes. The exodic growth phenomenon is very well displayed in several of the sections, but concentric re-formation of the capsule is absent; the advancing edge of the new tissue is bordered directly by reactive lymphoid tissue. The capsules are thickened and hyaline, trabecular thickening is also marked. Fibrosis of all varieties is confirmed but the process is less conspicuous than usual. The reticulum cell proliferation is quite obvious and productive of morbid mononuclear types and typical Hodgkịn giant cells. In addition small islets of macrophage type cells are seen. Eosinophils are numerous, many have single spheroidal lymphocytoid nuclei. Plasma cells are present, they tend to be in small focal collections rather than diffusely scattered. Mitoses are below average in number.

Diagnosis. Hodgkin's disease, fairly cellular type, accopt as typical. Faint follicular pattern discernible. Incomplete involvement of nodes. 
Peter J. Williamson. Aet 40. A marine engineer. Ref.G.R.I.W.S.(C) 1593:51. History: Since return from South Africa in March 1951 patient has exhibited the Murchison phenomenon. This was attributed to malaria presumed to have been transmitted in liberia at a port of call. Response to treatment was unsatisfactory. Enlarged nodes were found in the neck. The Paul-Bunnell reaction was negative and two specimens of blood taken for culture were sterile.

This is early Hodgkin's disease in which there is incomplete involvement of the nodes and striking increase of reticulum cells with corresponding reduction of other cells. The Hodgkin tissue is focal in two parts of one node, a central zone of normal lymphoid tissue separating them. The exodic growth phenomenon is apparent. The capsule is wider than normal but the collagen lamellae are still separate and not impacted. Concentric re-formation is not a feature. Trabeculae are hardly. represented. Fibrosis is incipient; perivascular lamellation is inconspicuous, diffuse fibrils are scanty, and fibrillae are the chief manifestation. Coralline deposits are not observed. Reticulum cell hyperplasia is marked, it is chiefly productive of a small morbid mononuclear lymphocytoid type of reticulum cell but typical Hodgkin cells are easily found among them. Giant cells are common. The reduction of lymphocytes is pronounced and those present are immature, with big nuclei. Eosinophils are present but not numerous. Plasma cells are frequent, particularly in the marginal zone. Neutrophils are scattered through the new tissue and some are collected in small foci. Mitoses average 3-4 per H.P. field. (Vascularity of the nodes is high, traumatic haemorrhage is seen). 
Diagnosis. Atypical Hodgkin's disease, tumour-like. Fibrosis slight and subtle. Undue reticulum cell proliferation. Affinity to reticulum cell sarcoma. 
John Larkins. Aet 24. Under Dr. Hacewen. Ref. G.R.I.W.S. 553:09 575:09. History. Chronic glandular enlargement in the neck and axillae together with general weakness and anaemia. The cervical swelling commenced 16 months ago on the left side.

The lesion present is typical Hodgkin's disease. The specimen consists of five different nodes, the process is at much the same stage in each. Considering the blocks are 43 years old preservation is very good. The eosinophils are often disrupted but are quire recognisable.

The capsules shew pronounced hyaline fibrosis, at some points very small islets of the new tissue are incorporated in them. There is a well marked exodic gronth phenomenon in three of the nodes and surviving (peripheral) lymphoid tissue is discernible. Fibrosis is pronounced; the change is shewn in all the standard forms, perivascular lamellae are particularly well shewn. The reticulum cell proliferation is productive of morbid mononuclear and Hodgkin giant cells. These cells tend to be aggregated in masses. The eosinophilia is characteristic. (The imperfect preservation of these cells is already noted). Plasma cells and neutrophils are numerous, the latter are nore numerous in the several necrotic areas which are present. lifitoses are difficult to identify with certainty due to imperfect preservation. Note - several giant-cells of Langhan's type are present.

Diagnosis. Hodgkin's disease, Typical. Incomplete involvement of nodes. Inconspicuous atypical feature, Langhan's giant cells. 
Wirs. Mary MoGhee. Aet 22 years. A housewife. Ref. G.R.I.W.S. 1925:42. History. Nine weeks ago patient noticed sweliing in the right side of the root of the neck. Dyspnoea present. Four weeks ago a similar swelling developed on the left side.

The specimen is a cervical lymph node, it has been examined at four levels. There is not any survival of the original lymphoid tissue. The capsule is grossly thickened and hyalinised, concentric re-formation is well illustrated. The trabeculae, which are probably new formations, are very stout but do not penetrate the node regularly nor deeply. Fibrosis is well advanced, all forms are displayed and the perivascular component is the most obvious. The reticulum cell proliferation is of the usual order with the production of typical morbid mononuclear and Hodgkin giant cells. Eosinophils are copious and many lymphocytoid types can be found. Plasma cells are identified but are scanty. Neutrophils are numerous throughout the cellular part of the lesion, many are pyknotic especially those in an infarct which is present. Mitoses are of the usual order 1-2 per H.P. field.

Diagnosis. Hodgkin's disease, Typical. Late, sclerosing markedly• 
James Shearer. Aet 48 years. An engineer. Ref. G.R.I.T.S. 1806:42. History. Patient developed an intractable pruritus. Examination revealed enlarged lymph nodes in the left posterior triangle of the neck.

This is an example of tumour-like Hodgkin's disease. The cellular pleiomorphism is less arresting than usual and the typical capricious heterogeneity is lacking. In the main small cells predominate. The exodic growth phenonenon is well shewn, a rind of physiologically reactive lymphoid tissue is seen at the periphery and concentric reformation of the capsule is seen at points. The true capsule is normal except where the new tissue actually reaches it, here thickening is present. Invasion is confirmed at two points. Fibrosis is observed in all forms except coralline deposit, it is relatively slight. The reticulum cell proliferation is productive of very numerous small cells which morphologically are intermediate between the typical reticulum cell of Hodgkin's disease and an early lymphocyte. They are almost 'normal' reticulum cells but are excessive in number. Typical morbid forms and scanty Hodgkin giant cells are present. Eosinophils are very copious and include many lymphocytoid forms. Neutrophils are scanty and plasma cells are also difficult to find. Necrosis is limited to occasional individual cells. Nitotic activity is of the usual order.

Diagnosis. Atypical Hodgkin's disease, tumour-like type. Homogeneous growth, reduced fibrosis, invasion of capsule. Uniform small reticulum cells. 
William Cummings. Let 66. Ref. G.R.I.W.S. 1460:42.

History. Recurrence of cervical lymph node enlargement. Previous lymphadenopathy which was irradiated. There is not any record of a biopsy having been taken.

This is an inguinal lymph node shewing established typical Hodgkin's disease. The lesion does not involve the whole node; in parts it is more cellular than usual. The cellular components of a cresoentic marginal portion of the node are normal; there is intense lymphatio oedema of the sinuses in it. The demarcation of the tumour tissue is sharp at some points but almost imperceptible at others.

The capsule is much thickened and hyalinisation is proceeding, the exodic growth phenomenon is perfectly shewn and concentric re-formation is evident. Deposits of the new tissue are seen in the capsule but are probably isolated by the last-named process. The trabeculae are extensively fibrous. Fibrosis of all types except for coralline deposits is well shewn. The reticulum cell proliferation is marked locally, large morbid mononuclear types being most numerous. Typical Hodgkin giant-cells are numerous. Two Langhan's giant cells are seen. Eosinophils are copious and plasma cells are frequent too, neutrophils are chiefly limited to the necrotic foci. Mitotic figures are present in normal numbers.

Diagnosis. Hodgkin's disease, Regarded as typical, Slightly·more cellular than usual, but only locally, incomplete involvement of node. 
Webster. Ref. G.R.I. W.S. 188:43. History unknown.

This is an example of a tumour-like variety of atypical Hodgkin's disease. The lesion is uniformly cellular, fibrosis is sparse and eosinophils are absent. There is not any survival of normal lymph node structure. The capsule is incomplete in the specimen but is thickened where it is preserved. Local concentric re-formation is detected. Fibrosis is present but is slight; most of the collagen is fibrillary and derived by symplasmic transformation. The other forms are meagre. Reticulum cell increase is general and productive of many large morbid mononuclear cells with some epithelioid and lymphoid types. Hodgkin giant-cells are present in about the usual numbers. Eosinophils are absent and plasma cells are hard to find. Neutrophils are unusually numerous, scattered diffusely, and in areas of early necrosis. Mitoses which are unequivocal are few.

Diagnosis. Atypical Hodgkin's disease. Tumour-like homogeneity• No eosinophils. Fibrosis slight. 
Robert Morrison. Aet 34. Ref. G.R.I. W.S. 1436:43. History. Swelling in the left side of the neck of four months' duration.

This is highly cellular tumour-like Hodgkin's disease which might reasonably be regarded as malignant from the histology. It would be equally correct to term the lesion pleiomorphic reticulum cell sarcoma.

The capsule is of normal thickness but attenuated in places; at one point it is over-mun by the new tissue and the latter is growing in the peri-nodal areolar tissue. Throughout the node small survival follicles of solid type are discernible, the exodic growth phenomenon is seen. Trabeculae are not present. Fibrosis is slight; perivascular lamellation is seen round several capsular vessels, fibrils and fibrillae are scanty although reticulin fibrillae are much in evidence, the coralline form is absent. Reticulum cell increase is pronounced and general. Host of the cells are small but have hyperchromatic nuclei. Typical morbid mononuclear cells are copious. Multinucleate giant-cells are present but the cell outlines are indistinct giving an impression of smallness. Eosinophils are present but in small numbers, plasma cells are likewise low. Necrotic polymorphs and small lymphocytes are numerous. The mitotic figures are unusually frequent 5-7 being seen in many H.P. fields. Necrosis is limited to individual cells.

Diagnosis. Atypical Hodgkin's disease, highly cellular tumour-like type. Transgression of capsule, fibrosis slight, eosinophils low. 
James Hyslop. Ref. G.R.I.W.S. 1844:43. History unknown.

The material available is unfortunately not in good condition, it is traumatized and badly fixed.

The lesion corresponds most nearly to follicular lymphoma but is shewing mild eosinophilia and fibrosis of Hodgkin type. The capsule is possibly slightly thickened since where it is preserved the collagen is hyaline. It is not possible to pronounce upon the presence of infiltration.

The new tissue is disposed in false follicles some of which are incompletely outlined by compacted collagen fibres. Fibrosis is expressed as above; in several areas coralline deposit is notable. The other forms are doubtfully present. The type-cell is a reticulum cell shewing definite lymphoid type nuclei, individual morbid mononuclear types are identified and several Hodgkin giant-cells can be made out. Eosinophils are present in small foci, most are of mononuclear lymphocytoid variety. Plasma cells and neutrophils are very few. Mitoses are very scanty. Necrosis is limited to individual cells.

Diagnosis. Brill's disease, nearest. Some sclerosis, mild topical eosinophilia, affinities to Hodgkin's disease. 
James McLaughlan. Ref. G.R.I. T.S. 635:44.

This is moderately early established Hodgkin's disease; the remarkable feature is that the affected node is small, $6 \times 5 \times 5 \mathrm{~mm}$, and provides an exception to the general rule that large nodes are more likely to furnish reliable diagnosis.

Inconspicuous fragments of marginal sinus tissue and peripheral lymphoid tissue persist but the remains are insufficient to illustrate the exodic growth phenomenon. The capsule is uniformly thickened being fully $0.6 \mathrm{~mm}$. wide. It is hyaline and infiltration is not seen.

The new tissue is very faintly folliculoid in arrangement, a pattern just detectable with very low power (XIO). Fibrosis is expressed in all forms but perivascular lamellation is the conspicuous one. The proliferated reticulum cells are highly pleiomorphic, ranging from cells identical with fibroblasts to typical Hodgkin giant-cells. Eosinophils are present in characteristic numbers, and there is a diffuse scattering of noutrophils; plasma cells are hard to find. Mitoses average under 2 per H.P. field. Incipient infarction is seen at one pole.

Diagnosis. Hodgkin's disease, typical. 
lirs. Jean bic Intyre. Ref. G.R.I. i.S. (c) $46: 45$.

This is an example of Hodgkin's disease at a late stage. Features of interest include the persistence of eosinophilia and relative overgrowth of the morbid reticulum cells in the surviving foci of the cellular parts of the lesion.

The capsule and trabeculae are very prominent and intensely sclerosed. This collagen formation is irregularly cribriform, and the speces are occupied by the cellular tissue. All forms of fibrosis are encountered. The reticulum cell increase is particularly striking in foci, several high power fields approximating to mixed cell sarcoma. Large morbid mononuclear types predominate but there are plenty of characteristic Hodgkin giant-cells as well. Eosinophilia is intense especially at the margins of the cellular foci. Witoses are still present in about the usual numbers. Neutrophils are copious and tend to be focal. Necrosis is confined to individual cells.

Diagnosis. Hodgkin's disease, typical, late. Overgrowth of large morbid reticulum cells locally. Persistence of eosinophilia. Affinities to Hodgkin's sarcoma (Warthin's type II). 
Margaret Hall. Ref. G.R.I. W.S. 222:45.

This is typical Hodgkin's disease which is involving part of the lymph node only, a crescentic marginal portion of original structure remains. The lesion is characterised by very numerous giant cells.

The capsule shews pronounced local thickening but this change is absent opposite the crushed norral lymphoid tissue. Concentric re-formation is absent. The demarcation of the new tissue is abrupt and an artificial cleft divides it from the normal for some distance. This phenomenon emphasizes the focal character of the disease. Fibrosis is expressed in all forms, but the coralline deposits still appear feathery in places. Trabecular thickening is gross. The reticulum cell proliferation is profuse with many aberrant forms including numerous misshapen Hodgkin giant-cells. Eosinophils are copious, their distribution is very even throughout the lesion. Plasma cells are scanty but many neutrophils ean be seen. Mitoses average 2-3 per H.P. field. Necrosis is limited to individual cells. The sharp demarcation of normal and neoplastic tissue permits easy comparison of the lymphocytes in both formations; those in the tumour are distinctly larger with slightly less deeply staining nuclei.

Diagnosis, Hodgkin's disease, typical, (large cells more numerous than usual). Partial involvement of node well shewn. 
Robert Kelso. Aet 69 years. A ploughman. Ref. G.R.I. W.S. 262345 . History. Patient presented with painless glandular masses in both groins.

The material is poorly preserved. The capsule is widely avulsed and tears are present. This is Brill's disease wìch is sclerosing. All normal structure is replaced by mutually contiguous large false follicles. Some are poorly outlined by para-vascular collagen and in the remaining pulp interstices diffuse fibril forration is seen. The type cell is the usual internediate reticulum cell/lymphocyte precursor seen in the disease. A very few typical morbid mononuclear retioulum cells are present and one (!) Hodgkin giant-cell. There is striking absence of all granular series leucocytes and also of plasma cells. Mitoses are numerous $2-3$ per H.P. field. By silver impregnation definite increase of perifollicular reticulin is apparent.

Diagnosis. Brill's disease; sclerosing in places. 
Wrs. J. Holden. Aet 55. Housewife. Ref. G.R.I. W.S. $302: 45$ (i) 561:45 (ii) History. Patient complained of a swelling under the angle of the jaw on the right side. This recurred after removal and irradiation. Patient died 4th June, 1945. (P.M. 144/45. G.R.I.)

(i) This is an incomplete lymph node from the sub-maxillary region, small portions of salivary gland are present. There is not any persistence of normal structure. The tissue is entirely given over to reticulum cell sarcoma of clasmatocytic stem cell type. The tumour shows a very faint follicular pattern in places, the capsule where preserved is transgressed and is being destroyed. Fibrosis is visible but only as slender fibrillae. The total collagen is small.

The tumour cells are rather small morbid mononuclear cells with typical amphophilic spheroidal nucleoli in their quasi-vesicular nuclei. The nuclei are 12-16 p. diameter. A few large types and giant-cells are to be found. Granulocytes are absent and plasma cells are very few. Mitoses are very frequent often 10 are visible in H.P. fields. (ii) The material is similar in all respects to (i). Diagnosis. Reticulum cell sarcoma, clasmatocytic stem cell lymphoma. Hany individual cells identical with those seen in Hodgkin's disease, fibrosis slight, faint follicular pattern. 
Hugh Montgomery. Ref. G.R.I. W.S. $564: 45$.

This is a cervical lymph node shewing typical Hodgkin's disease, the eosinophilia is just within the limits of acceptability. A feature of interest is the uniform immaturity of the lymphocytes, direct comparison with normal small lymphocytes is afforded by the presence of survival islets. Capsular thickening is irregular but gross where it obtains. Concentric re-formation is visible. Fibrosis is most extensive, the trabeculae are large and coarse, and all other forms are encountered. The origin of coralline deposits from compacted fibrils is clearly indicated here. Reticulum cell increase is pronounced and many grotesque forms are seen, necrosis of these cells is common. Typical Hodgkin giant-cells are numerous. The eosinophilia is less than usual, neutrophils are little in evidence. Mitoses are rare - many H.P. fields are devoid of them. There is not any focal necrosis.

Diagnosis. Hodgkin's disease, typical. (Eosinophils low). Fibrosis very characteristic. 
John Walsh. Ref. G.R.I. W.S. 801:45.

This is uniform cellular tumour-like Hodgkin's disease. The node is incomplete due to artefact but all the tissue present is implicated. The capsule exhibits uniform thickening of moderate degree, true trabeculae are absent. Fibrosis is chiefly in the form of perivascular accretion, the other forms are tenuous and slight. The reticulum cell increase is relatively slight and the cells are dispersed through a matrix of numerous immature small lymphocytes. In addition to the distinctive morbid mononuclear and Hodgkin giant-cells there are many small aggregates of epithelioid cells, consisting in most cases of 10-15 cells. Their cytoplasm is undergoing collagenous transformation to give coralline deposits. Eosinophilia is of the usual order but the cells tend to be focal. Plasma cells and other granulocytes are scanty. Mitoses are meagre and so far as cells can be identified in this biological phase they are in small cells probably lymphocyte precursors. Necrosis is seen in occasional morbid reticulum cells.

Blood vascularity of this lesion is high.

Diagnosis. Hodgkin's disease, almost typical. Uniform cellular lesions with tumour-like appearance. Fibrosis subtle. 
Gavin Struthers. Ref. G.R.I. W.S. 1596:45.

This is Hodgkin's disease of the soft cellular variety and it is shewing certain tumour-like features. In a large thick walled lymphatic in the adjacent areolar tissue the cells of Hodgkin's disease are visible; the only missing element is true connective tissue, the cells are set in an acidophile granular fibrin-like matrix.

The capsule is intact, thickening is present but it is within the wider limits permissable in inguinal nodes. Deposits of the new tissue are seen in the hilar intrusion portion of the capsule, but they appear to be isolation phenomena rather than infiltrative. Fibrosis is slight; the only manifestation which is easily obvious is perivascular lamellation, but scrutiny reveals fine fibrillary production in the pulp. The reticulum cell proliferation is more florid than normally seen in Hodgkin's disease, the typical morbid mononuclear and Hodgkin giant-cells are present in quantity. Eosinophilia is marked, some are of the lymphocytoid type. Plasma cells are absent but scattered polymorphs are seen. Mitoses are rather above average.

Diagnosis. Atypical Hodgkin's disease. Soft cellular variety with little fibrosis. Tumour-like homogeneity. 
George Bell. Aet 38 years. A moulder. Bef. G.R.I. W.S. 1702:45. History. Complaint of pain in the left loin and groin, present for several weeks. Examination revealed enlarged nodes in the left groin.

This lesion is a peculiar mixture of Hodgkin's disease and Brill's disease. Both processes are clearly recognisable and separable in different parts of the nodes.

The capsules have been widely avulsed by artefact but where present they shew hyaline thickening. Concentric re-formation is discernible but normal lymphoid tissue is absent. A fairly uniform giant false follicle pattern with the characteristic shrinkage clefts is seen in most of the tissue but some areas reflect typical Hodgkin pattern. Fibrosis of all types is seen, it is chiefly exhibited by the Hodgkin lesion; at one area deep in the node a uniform sheet of proliferating fibroblastic reticulum cells is present. Elsewhere the distinctive morbid reticulum cells of both lesions are encountered. Typical Hodgkin giant-cells are present and tend to be focal. Eosinophils are present in adequate numbers in the Hodgkin areas. The mitoses are numerous. The lymphocytes throughout are immature in type.

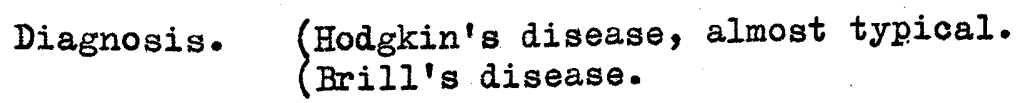

Representation is about equal. 
Mrs. Betty Andison. Aet 66 years. A housewife. Ref. G.R.I.W.S. 1732:45. History. This patient exhibited the Nurchison phenomenon and complained of pruritus of the back. She was very ill and weak and a swelling was found in the neck.

The specimen is a cervical lymph node, shewing late typical Hodgkin's disease.

There is not any survival of original node structure. The node capsule as received is only moderately thickened, it is probably incomplete. Hyalinisation is almost total. All forms of fibrosis are pronounced, particularly coralline deposits. Reticulum cells are relatively few and many appear almost physiological. The typical. varieties including Hodgkin giant-cells are nevertheless present. Eosinophils are lower than in typical young lesions but plasma cells are more numerous. Mitoses are very infrequent. The lesion as a whole has an indolent burnt-out appearance but as usual some small active-looking foci can be found.

Diagnosis. Hodgkin's disease, typical, late. 
Thomas Grant. Ref. G.R.I. W.S. 1775:45. History. Swelling in the posterior triangle of the neck.

This is an example of established Hodgkin's disease which is typical but shews a few minor deviations.

The entire node is involved. The new tissue is arranged in an irregular folliculoid manner in a coarse fibrous mesh. The capsule is very greatly thickened and extensively hyaline. Fibrosis of all forms is encountered but fibrillary deposit is much less conspicuous than the grosser manifestations. The reticulum cell proliferation is florid with the production of numerous intermediate cell-types in addition to the morbid mononuclear and Hodgkin giant cells. Several atypical giant-cells closely similar to foreign body tjpe are noted. Eosinophilia is extreme, densely packed masses of these cells are seen in many parts. Plasma cells and neutrophils are scanty. Mitoses average 1 per H.P. field. Necrosis is absent, but haemosiderin is seen in small amount in minute foci.

Diagnosis. Hodgkin's disease, typical. Eosinophilia intense, rather homogeneous growth in parts, rare atypical giant-cells. 
Wrs. Mary ifcAllister. Bef. G.R.I. 1868:45.

This is a good example of fully established typical Hodgkin's disease with extensive fibrosis and yet only involving part of the node.

A crescentic portion of physiologically reactive lymphoid tissue is present at the periphery of one side. In addition a survival follicle with a pale centre is located deep in the node. The capsule is very markedly thickened and hyalinised, except where it covers normal tissue. Fibrosis in all forms is seen, the perivascular deposits are huge. The reticulum cell proliferation is characteristic and productive of typical morbid cells. Hodgkin giant-cells are numerous and tend to be grouped. Eosinophilia is pronounced, and plasma cells and neutrophils are found with ease. Mitoses average 1 per H.P. field. Necrosis is absent.

Diagnosis. Hodgkin's disease, typical. Incomplete involvement of node despite maturity of the lesion. 
Robert Law. Aet 34. Ref. G.R.I. 289:46. History. Swelling in the neck. (Note: Hodgkin's disease was diagnosed clinically in 1943, biopsy was not performed).

This is Hodgkin's disease which is typical except for the presence of many focal aggregates of epithelioid cells. The entire node is diseased. The capsule is widely lost by artefact, in the surviving fragment thickening is not appreciable. Fibrosis is relatively scanty but all varieties are seen. The reticulum cell increase is notable, many small scattered islets of epithelioid cell types are being produced and collagen fibril is being produced in these foci. The characteriatic morbid mononuclear and Hodgkin giant-cells are present, the latter are distinctly scanty. Eosinophils are also rather lower than usual. Plasma cells and neutrophils are rare. The mitoses average 1 per H.P. field. Focal necrosis is absent. A notable feature is the comparative immaturity of the lymphocytes in the lesion.

Diagnosis. Slightly atypical Hodgkin's disease. Many foci of epithelioid cells. Eosinophils low. The lesion has a dissociated appearance.

Note. Thispatient died in G.R.I. See special P.M. series. Ref. G.R.I. 193:50. 
Effie MeKellar. Ref. G.R.I. 896:46.

The appearances seen in this example of Hodgkin's disease are rare. The lesion is apparently confined to the pulp tissue of the node; many lymphoid follicles, both solid and with pale centres are dispersed through the gland. The intervening tissue is a tumour-like homogeneous Hodgkin tissue which under low power seems curiously uniform. Sinus tissue is not recognisable.

Local thickening of the capsule is seen. Fibrosis is present in all forms but a coarse uniform mesh of fibrils is the chief manifestation. In places these form pseudo-trabeculae. The reticulum cell proliferation is profuse with a high proportion of morbid forms. The Hodgkin giantcells are rather small. Eosinophilia is somewhat less than characteristic. Plasma cells are scanty and neutrophils are very rare. Mitoses are of average frequency. Focal necrosis is absent.

Diagnosis. Atypical Hodgkin's disease. Tumour-like, confined to the node pulp, remarkable survival of lymphoid follicles. 
Fred Higgins. Aet 20 years. Ref. G.R.I. 965:46. Hiatory. A swelling has been present in the neck for eight months, it is painless. Note. This patient died at home 15th October 1948. X-ray and nitrogen mustard had very little effect.

This is an example of rapidly growing cellular Hodgkin's disease. Fibrosis is minimal in most areas. Although highly cellular, the capricious pleiomorphism is not lost.

The entire node is morbid. Capsular thickening is seen and concentrie re-formation is visible at several places. The trabeculae are much thickened and often infiltrated with eosinophils. Blsewhere fibrosis is slight and restricted to slight perivascular lamellation, together with a delicate fibril filigree. The reticulum cell proliferation is productive of very numerous cells, a large number of which look like fibroblasts. The morbid mononuclear and Hodgkin giant-cells are typical. Eosinophilia is pronounced, but the other cells are scanty. Mitoses are much more numerous than usual, 5-10 per H.P. field, there is much local variation. Focal necrosis is absent.

Diagnosis. Atypical Hodgkin's disease. Highly cellular, rapidly growing type. 
William Morrison. Ref. G.R.I. 1227:46.

This is late typical Hodgkin's disease. The node does not retain any normal structure. Capsular thickening and hyalinisation are very marked. The new tissue is irregularly folliculoid due to pseudotrabeculae. Fibrosis of all forms is seen, fibrillar deposit is slight compared with the gross manifestation of the other forms. The reticulum cell moiety of the lesion evidences the characteristic pleiomorphism and the typical morbid cells are present. Eosinophils are remarkably numerous, many lymphocytoid forms are present. Plasma cells and neutrophils are present in small numbers. Mitoses average 1 per H.P. field. Necrosis is linited to individual cells.

Diagnosis. Hodgkin's disease, typical, late. Note - retention of rarked eosinophilia. 
Urs. Watkins. Ref. Q.R.I. 1520846.

This is Hodgkin's disoase, while probably falrly oarly, it is present throuzhout the node. There are focal aggregates of eptholiold cells which are producing fibres. The oapsule is of normel thickness and is hyaline. Prabeculae are poorly represented. Fibrosis is present in all forms but is relatively slight. Fibre production is present in the epithelioid cell foci which represent the majority of the proliferated reticulum cells. Large morbid mononuclear and Hodgkin giant-cells are present but are widely scattered; many of the epithelioid cells have 'owl-eye' nuclei with prominent oxyphile nucleoli. The eosinophilia is moderate, many lymphocytoid varieties are visible. Plasma cells and neutrophils are scanty. Mitoses are of the usual order. Neorosis affects occasional individual cells.

Diagnosis. Hodgkin's disease, almost typical. Production of epithelioid cells in small islets, fibrosis is seen in these foci. 
Nurse Mary McLeach. Aet 24 years. Ref. G.R.I. 1602:46. History. Painless swelling in the right side of the neck for ten months. Hote. This patient received X-ray therapy with transient benefit and died early in 1949.

The lesion is moderately early but established Hodgkin's disease. The node is incompletely involved. There is a crescentic marginal rind of normal lymphoid tissue and the exodic growth phenomenon is well shewn. The non-neoplastic tissue is infiltrated with eosinophils in some places. The capsule appears normal, re-formation is just appreciable. The new tissue shews an irregular nodular habit due to gross thickening of the trabeculae. Fibrosis is well established, all forms are encountered but the coralline variety is minimal. The formation of collagen fibrillae and fibrils from morbid reticulum cells is very well illustrated. The reticulum cell proliferation is florid with production of the characteristic morbid mononuclear and Hodgkin giant-cells. Eosinophilia is pronounced, it is very diffuse. Plasma cells and lymphocytes are numerous; a few widely scattered neutrophils are present. Mitoses average 2 per H.P. field. Necrosis is absent.

Diagnosis. Hodgkin's disease, typical. Incomplete involvement of node. 
Thomas Conlie. Ref. G.R.I. 1652:46.

This is an inguinal lymph node the seat of lymphosarcoma. The tumour cell-type is an immature small lymphocyte which is better differentiated than the large cell lymphosarcoma (Iymphoblastic type of Robb-Smith).

An ill-defined rind of possible normal lymphoid tissue is seen marginally against the inner aspect of the capsule. The nuclear size of the lymphocytes is smaller here and the cells are compacted. The exodic growth phenomenon can be detected but the process is much less distinct than in many cases of Hodgkin's disease. The capsule is attenuated and infiltration right through it is visible at several points.

Reticulum cell nuclei are readily found on high power examination, typical morbid mononuclear cells and one typical Hodgkin giant-cell: are present. The trabeculae are appreciably thickened; fibrosis is limited to several striking examples of perivascular deposit and small coralline formations. Haemosiderin is notable in some of the perivascular lamellae. Eosinophils are present but require much search to locate. Very few plasma cells and neutrophils are discovered. Mitoses are not numerous, and are difficult to identify with certainty because of frequent pyknosis and karyorhexis.

Diagnosis. Small cell lymphosarcoma. Slight Hodgkin-like fibrosis and rare Hodgkin type reticulum cells. 
William McGowan. Aet 60 years. Ref. G.R.I. 1861:46. History. Patient came under the care of Professor Burton. Laparotomy was performed for suspected abdominal tumour, enlarged lymph nodes were discovered.

The specimen is a mesenteric lymph node. This is Hodgkin's disease of fibroblastic type which is now relatively inactive. The picture is one of extensive collagenous replacement of the node, the Hodgkin's tissue surviving in small nodules and clefts in the collagen. All trace of normal structure has gone. The capsule is thin but hyaline. Fibrosis is very diffuse and amorphous masses of collagen are seen as well as coralline deposits. The reticulum cell proliferation has apparently been chiefly productive of fibroblastic cells. Morbid mononuclear and Hodgkin giant-cells are sparse. Several Langhan's type giant-cells are present. Eosinophilia is absent - very few are found, but plasma cells with deeply oxyphile cytoplasm are frequent. Other granulocytes are virtually absent. Mitoses are not seen.

Diagnosis. Atypical Hodgkin's disease, mature diffuse fibroblastic type. Low eosinophils, a few Langhan's giant-cells. 
Peter Campbell. Ward 34. Mr. Mowat. Ref. G.R.I. 2110:46. History. Patient diagnosed as retro-peritoneal tumour. Node recovered at laparotomy.

This is a lymph node from the edge of the retroperitoneal tumour. The node still shews evidence of atrophic fat replacement. Survival islets of lymphocytes are present. The capsule is of normal thickness, it is invaded at several points and tumour deposit is identified in blood and lymph vessels. Fibrosis of all types can be recognised but it is distinctly slight. The tumour is composed of morbid reticulum cells among which 'owl-eye' nucleated types and Hodgkin giant-cells of small size predominate. Eosinophilia is absent. Plasma cells and neutrophils are scanty. Mitoses average 3-4 per H.P. field.

Diagnosis. Hodgkin's sarcoma (Warthin's type II reticulo-endothelioblastoma). Sclerosis slight。 
Alex. Fainagh. Ward 35. Wr. Mowat. Ref. G.R.I. 2117:46.

This is a cervical lymph node shewing typical fairly cellular Hodgkin's disease.

The entire node is involved in the disease. The exodic growth phenomenon and concentric re-formation of the capsule are not seen. The new tissue exhibits a poorly defined folliculoid habit. New young imperfectly collagenised swathes of connective tissue are leaving the capsule at irregular intervals. Wost of the fibrosis present is perivascular, diffuse fibril formation is slight; similarly fibrillae are obscure but present. Coralline deposits are not seen. The reticulum cell increase is readily apparent and many morbid forms are being produced. Morbid mononuclear types are plenteous but typical Hodgkin giant-cells are sparse. Eiosinophilia is marked. Plasma cells are notable also and several giant forms are present. Neutrophils are sparse. Mitotic figures average 1 per H.P. field. Necrosis is slight and restricted to individual cells. Haemosiderin deposit is identified. The lymphocytes are decidedly immature.

Diagnosis. Hodgkin's disease, typical. 
Nrs. Nary Rae. Aet 67 years. Ref. G.R.I. $31: 47$ and 139:47. History. Patient presented with a painless swelling in the right side of the face. The initial biopsy was reported as sinus hyperplasia probably inflammatory, the second established the diagnosis of Hodgkin's disease. The patient died 6 th January 1948.

Unfortunately both specimens are damaged and the material is difficult to study.

The earlier one reveals one very definite morbid feature - marked thickening of the capsule. Where preservation is reasonably good a certain loss of normal architecture is seen, together with the appearance of scanty large morbid mononuclear reticulum cells in the pulp. Othermise non-specific change is all that can be reported. In the second specimen fibrosis is apparent as fibrils, fibrillae, and perivascular lamellae. Reticulum cell proliferation is easily confirmed and typical Hodgkin giant-cells are present. Wosinophils are few, plasma cells and. neutrophils are little in evidence, the last named are seen in the vicinity of small haemorrhage in the new tissue. The lymphocytes are large immature types. The new tissue has a homogeneous tumour-like appearance.

Diagnosis. Initially indeterminate but probably significant. Second specimen - Atypical Hodgkin's disease. Few eosinophils. Tumourlike. Some affinities to lymphosarcoma. 
lirs. Agnes falker. Aet 63 years. A housewife. Ref. G.R.I. 71:47. History. Patient had a small painless swelling in the left side of the neck which had been increasing in size for a year. She was treated with irradiation but responded badly and died January 1949.

This is cellular atypical Hodgkin's disease involving a cervical node. The entire node is diseased. Capsular thickening and hyalinisation are marked, there are deposits of the cells of the new tissue in lymphatics. Concentric re-formation of the capsule is seen in places. The trabeculae are much thickened but do not penetrate deeply. Fibrosis in the node substance is relatively slight. Perivascular lamellation, fibril, and fibrillary deposits are all sparse. The reticulum cell increase is florid, most approach a macrophage in appearance and some of these cells occur in small plasmodia. Large morbid mononuclear type and typical Hodgkin giant-cells are conspicuous. Tosinophils are all but absent, plasma cells and neutrophils are not diffused through the new tissue. Several massive infarct-like areas of necrosis are ringed with neutrophils giving an abscess picture. Mitoses are numerous, they average 3-4 per H.P. field.

Diagnosis. Atypical Hodgkin's disease. Highly cellular, rapidly growing. Little fibrosis, no eosinophils. Affinities to Hodgkin's sarcoma or reticulum cell sarcoma. 
James Calderwood. Aet 38. A collier. Ref. G.R.I. 143:47. History. Intermittent diarrhoea and constipation for 15 months. Has lost four stone in weight and is anaemic. Laparotomy revealed enlargement of the abdominal lymph nodes.

The specimen is a mesenteric node $5 \mathrm{~mm}$. diameter. It is completely replaced by rather homogeneous Hodgkin's disease tissue.

The node is enclosed in a slightly thickened capsule. At one pole a crescentic area of normal follicles, sinuses, and cords persists. The exodic growth phenomenon is well seen. The new tissue is coarsely folliculoid. Fibrosis is chiefly expressed by diffuse fibril formation; perivascular and fibrillary components are slight, and the coralline form is not seen. The reticulum cell increase is pronounced with a striking reduction of small lymphocytes. Liost have small hyperchromic nuclei but large morbid mononuclear cells are present. Typical Hodgkin giant-cells are conspicuous because most of them are dead. Eosinophils are rather low in number, lymphocytoid types are notable. Plasma cells are rare but polymorphs are common especially near necrotic foci, of which there are several. Witoses average 3-4 per H.P. field.

Diagnosis. Hodgkin's disease, probably typical. Fibrosis and eosinophils low, but this is common in mesenteric nodes. Pleiomorphism less than usual. 
James Iyttle. Aet 63. Ref.G.R.I. 241:47. History. A swelling in the right side of the neck for $2 \frac{1}{2}$ years; glandular masses found in both sides of the neck. (Iraced up till 9th November 1949 when he was dyspnoeic and had a psoriasiform lesion of the skin).

This is cellular Hodgkin's disease which almost completely involves the node.

The capsule is norral where it is preserved. Immediately beneath it the marginal sinus is intact at several points, it is filled with normal looking macrophages. (This is an unusual finding; sinus tissue is lost earlier than follicular or cord components as a rule). The exodic growth phenomenon is not appreciable. Fibrosis is slight, all forms except coralline are discernible. The reticulum cell proliferation is marked and there is production of many macrophage type cells which are often aggregated into islets. The morbid mononuclear and Hodgkin giant-cells are well represented. Eosinophils are not numerous. Plasma cells and neutrophils too are meagre. Necrosis is limited to occasional individual cells. Mitotic figures average 2 per H.P. field. Diagnosis. Atypical Hodgkin's disease, cellular type. Fibrosis slight, eosinophils low. Does not qualify for tumour-like. 
Richard laxwell. Aet 31 years. Ref. G.R.I. 446:47.

History. Sustained a blow on the left testicle about eight weeks ago, the related groin lymph nodes swelled up and then the nodes in the opposite side enlarged. Further nodes enlarged in the left side of the neck and left axilla. All remained rather soft. After diagnosis X-ray therapy was given with a poor response, after progressive deterioration he died 6th October 1947. Total duration eight months.

This is tumour-like Hodgkin's disease in a cervical lymph node. At the periphery of the node at one level examined 13 survival nodules are present, several have pale centres but the component cells of those are not entirely normal. The exodic growth phenomenon is no longer apparent. The new tissue is very uniform and diffuse without any indication of nodular arrangement. The capsule of the node is thin, in some parts it is even attenuated but actual invasion is absent. The few trabeculae present are small but appreciably thickened. Fibrosis is slight on the whole. Perivascular lamellation is seen, fibrils and fibrillae are also apparent. Coralline deposits are lacking.

The reticulum cell increase is very pronounced, many of the nuclei are of epithelioid or fibroblastic type. Numerous morbid mononuclear types and nany small but otherwise typical Hodgkin giant-cells are easily found. Bosinophils are extremely numerous often presenting in smal I abscess-like foci. Plasma cells are present in moderate numbers. Neutrophils are distinctly scarce. Liitoses average 1-2 per H.P. field. The lymphocytes in the lesion are imnature with larger paler staining nuclei than usual.

Diagnosis. Atypical Hodgkin's disease, tumour-like. Fibrosis slight, very uniform type of growth. 
Betty Flannigan. Aet 14 years. A schoolgirl. Ref. G.R.I. 458:47. History. In February 1947 she felt sick and complained of pain in the left shoulder. She also drew attention to a small lump in the left supraclavicular fossa. Examination revealed much deep lymph node involvement. She received X-ray therapy but died $2 \frac{1}{2}$ years after diagnosis (29th September 1949).

This is a cervical lymph node shewing late typical Hodgkin's disease. No vestige of normal structure remains. Capsular and trabecular fibrosis are very pronounced. The cellular part of the new tissue is distributed in follicular fashion through the interstices of the coarse fibrous mesh formed from the capsule and trabeculae. Fibrosis in all forms is met. The reticulum cell proliferation is of the usual order and the characteristic morbid forms are readily identified. Posinophils are still numerous and are comonly concentrated in the fibrous tissue, some lymphocytoid types are noted. Plasma cells and neutrophils are easy to find, the latter are frequent round the multiple small necrotic foci present. Mitoses average 1 per H.P. field. A small amount of haemosiderin is present in the node.

Diagnosis. Hodgkin's disease, typical, late. Retention of eosinophils. 
Mrs. Catherine Jamieson. Aet 31 years. A housewife. Ref. G.R.I. 1201:47 July, 1860:47 October. History. Swelling in the neck and axilla of several months duration. Peripheral blood normal. The first biopsy was reported as inflamatory lymphadenitis.

The appearances are very similar in both specimens but invasion is pronounced in the second.

The is reticulum cell sarcoma arising from a follicular lymphoma, the original pattern is being lost. Normal lymphadenoid structure is not present. The capsule is of normal thickness where it is preserved but it is widely over-run and destroyed. Fibrosis is present; new connective tissue is discernible as swathes of fibrils, which are occasionally compacted round many of the false follicles. In addition there is some diffuse fibril formation and fibrillae are appearing in the non-follicular parts of the tumour. The reticulin impregnation reinforces this interpretation. The tumour is composed of intermediate reticulum/lymphocyte cells, the majority tending to the reticulum cell nucleus prototype with wrinkled membranes. Large mononuclear forms and small multinucleate giant cells are present but sparse. Eosinophils can be found but are certainly scanty. Plasma cells and neutrophils are occasional. Mitoses are numerous on the whole but there is much topical variation, some H.P. fields shew as many as 6. Necrosis is limited to odd individual cells.

Diagnosis. Reticulum cell sarcoma. Arising from follicular lymphoma, sclerosing in parts, chiefly where follicles are disappearing. Fields can be found which mimic Hodgkin's disease. 
Mrs. Illary Hean. Aet 62. A housewife. Ref. G.R.I. 2157:47. History. For the past year has had a lump of variable size in the left axilla. Till recently had palpable glands in the neck on the same side. The present specimen is from a mass of very large nodes, total weight $186 \mathrm{gm}$, removed from the axilla.

This is Hodgkin's disease which is typical in all respects except that eosinophils are almost absent. The lesion is universal, normal structures are all lost. The new tissue is coarsely and irregularly nodular, the cellular parts resting in the spaces of the fibrous grid. Where it is preserved the capsule is grossiy thickened, sorne fusion with adjacent nodes is perceptible. Hyalinisation is not complete, in the trabeculae, plump fibroblastic cells a re still copious. Fibrosis of all types is encountered. The reticulum cell proliferation is florid, in addition to morbid mononuclear and Hodgkin giant-cells there are many cells with characters midway between epithelioid cells and fibroblasts. Eosinophils are only discernible after much search. Plasma cells are numerous, quite frequently in small islets. Scanty neutrophils are diffuse through the tissue. Mitoses average 1 per H.P. field. Necrosis of focal type is not in evidence.

Diagnosis. Atypical Hodgkin's disease. No eosinophilia, otherwise typical. 
Thomas Finnigan. Aet 75 years. Retired. Ref. G.R.I. 157:48. History. See also Serial 73. The present specimen consists of two nodules removed from the scalp.

There is not any evidence that these deposits are growing in lymphadenoid tissue. The tumour presents similar appearances to those observed in the previous material but sclerosis is much better developed. The affinities to Hodgkin's disease are remote but the tumour is reminiscent of cellular fibrosarcoma in many parts.

Note. This specimen should properly belong to Serial 73; the identity of the second biopsy was not realised at the time due to the patient's name having been mis-spelt..

Diagnosis. Reticulum cell sarcoma, sclerosing. Pleiomorphism of nuclei diminishing• 
William Barr. Aet 65 years. Retired. Ref. G.R.I. 180:48. History. Swelling of the left groin of six weeks known duration. The spleen was enlarged and soft. Ascites was present and the patient was very ill. The specimen is a large inguinal node $5 \times 4 \times 2 \mathrm{~cm}$.

This is an example of large cell lymphosarcoma in which fibrosis is relatively conspicuous. There is not any survival of normal structure. The tumour is growing in a curious folliculoid fashion like the overlapping lobules of cod-roe. The lobules are oval and massive, approx. $1.5 \times 2.5 \mathrm{~mm}$. The capsule is appreciably thickened and the fibrous augmentation is even better displayed by the trabeculae. Infiltration of the capsule by tumour cells is prominent at most parts. Fibrosis in the tumour is chiefly perivascular and in the form of fibrils; for a tumour of this nature it is well above the amount conceded by orthodox descriptions. The type-cell of the neoplasm is a reticulum cell shewing early lymphoid differentiation. The nuclei are spheroidal, moderately leptochromatic and vesicular. The cytoplasm is usually distinct but plasmodia are common in focal areas. Eosinophils, plasma cells and neutrophils are absent. Mitoses average 3-4 per H.P. field. Necrosis is not a feature.

Diagnosis. Large cell lymphosarcoma. Folliculoid pattern discernible. Sclerosing slightly. 
James Beaton. Aet 24 years. Ref. G.R.I. 319:48. History. Enlarged painless lymph nodes have been present in the left side of the neck for four months.

This is a cervical lymph node which I believe to be an example of Benign Hodgkin's disease (Harrison 1952). It was originally reported as early Hodgkin's disease.

The capsule and trabeculae are greatly thickened and hyaline, this constitutes the main alteration visible - it is easily seen on naked eye examination of the section. The lymphoid tissue is distorted in a subtle fashion; solid follicles and ones with pale centres are present especially peripherally, but they are not normally disposed. Centrally the pulp has overgrown and exodic growth is seen. Fine fibrosis is not present but coralline deposits are numerous. Reticulum cell proliferation is seen in the pale centres of follicles and also in the pulp but in the latter site it is inconspicuous. Scanty large mononuclear forms and two Hodgkin giant-cells are identified in one section. Eosinophils, plasma cells, and neutrophils are conspicuously absent. Hitoses are very scanty indeed. Necrosis is absent.

Diagnosis. Benign Hodgkin's disease. Capsular, trabecular, and coralline fibrosis. Rare Hodgkin giant-cells, slow growth. 
John Kelly. Aet 25 years. A driver. Ref. G.R.I. 1215:48. History. Patient has had a swelling in the left side of the neck for several months, its size has been static and it is painless. It was diagnosed clinicaliy as a sebaceous cyst and resected.

This is early active Hodgkin's disease in a cervical lynph node. Under low power poorly demarcated follicular aggregates of lymphocytes can be seen. High power examination dispels this impression.of surviving normal tissue. The lymphocytes are immature with large leptochromatic nuclei. Set in among them in multiple irregular islets are areas of cells simulating pale centre tissue.' Some of these reticulum cells have large morbid nuclei. The exodic growth phenomenon is not apparent and concentric refformation of the capsule is absent. The capsule is of normal thickness but hyalinised. Fibrosis is seen in increased stoutness of the trabeculae and as fibrils, fibrillae and coraline deposits. Perivascular formation of collagen is lacking. (There is an absence of sizeable vessels). The reticulum cell proliferation outwith the folliculoid formation noted above is marked. Scanty epithelioid cells are seen, but numerous morbid mononuclear types and Hodgkin giant-cells are identified. The latter are small but otherwise typical. Dosinophils are numerous and are diffuse. Plasma cells are absent - an unusual event. Polymorphs are likewise scanty. Píloses average 2 per H.P. fiela. Mecrosis is absent.

Diagnosis. Hodgkin's disease, typical, early, active. ? early disorganisation of follicle tissue of node. Primary origin in node pulp is likely. Affinities to reticulum cell sarcona. 
Mrs. Isabella Beaumont. Aet 64 years. A housewife. Ref. G.R.I. 1493:48. History. A painless swelling in the right side of the neck for seven months.

It is difficult to decide which is the most appropriate category for this example of lymph node tumour. The capsule of the node is incorpletely preserved but the remnants are thin. Trabeculae are hardy represented. Portions of marginal sinus and several peripheral follicles are identified; in addition fragments of trajectorial sinus tissue survive. The bulk of the node however is devoid of normal architecture and composed predoninantly of small cells. This diminution in size is particularly noticeable in the srail lymphocytes which conform to the type seen characteristically in some lymphosarcomata. Fibrosis i.e. actual collagen formation, is absent, but reticulin is definitely increased as a fine pervading filigree. Scattered through the small lymphocyte population many small morbid mononuclear and multinucleate giant-cells are discerned. Poorly demarcated paler staining areas visible low powrer are seen to be made up of small angular cells with ground-glass oxyphile cytoplasm and lymphocytoid nuclei. Eosinophils are almost absent, plasma cells are scanty. Neutrophils are doubtfully present. Mitoses are very infrequent most examples turn out to be pyknotic fragments.

Diagnosis. Indeterminate. (reported accordingly). Possibly lymphosarcoma, ? leukaemic. Possibly atypical Hodgkin's disease (early). 
Maurice Aherne. Ref. G.R.I. 2256:48.

History. Reputed case of proven Hodgkin's disease. Initial biopsy specimens have not been traced. Onset believed January 1946. Treated repeatedly with $X$-ray therapy, latterly with no response. Received nitrogen mustard course. Died lst September 1949 of Hodgkin's disease.

This is tumour-like Hodgkin's disease in an axillary lymph node. The node is totally diseased. The capsule shews thickening and islets of new tissue are present in it. Trabeculae and swathes of new connective tissue cut the cellular components into irregular nodules. In the nodules the lesion is curiously regular and uniform with indistinct whorled arrangement. Fibrosis is roderately early in maturity and present in all forms except coralline deposit. Reticulum cell proliferation is universal and mostly productive of a small fibroblastic type of cell. The characteristic morbid mononuclear cells and Hodgkin giant-cells are present in rather small numbers. Eosinophils are scanty but neutrophils, often as micro-abscesses are very numerous. Plasma cells are scanty, most are located at the periphery of the node. Mitoses are few, below 1 per H.P. field. Many infarct-like areas of necrosis are notable both peripheral and central.

Diagnosis. Atypical Hodgkin's disease - tumour-like. Uniform with whorling, no eosinophils. Areas very like fibro-sarcome, ? late diffuse fibroblastic type of growth. 
Mrs. Agnes Tannahill. Let 47 years. A housewife. Ref. G.R.I. 729:46, 2611:48. History. Swelling of the lymph nodes in the neck, axillae, and groins of several months duration. The initial specimen is a cervical lymph node, the second a node recovered from the vicinity of the sac of a femoral hernia which had been present seven or eight years.

This is a good illustration of the instability of follicular lymphoma (Brill's disease).

The first specimen shews considerable fibrous thickening of the node capsule. The tumour shews typical false follicles which are better seen peripherally, the pattern is indistinct centrally. There is considerable pleiomorphism of the reticulum cells both in the false follicles and central pulp. Large morbid mononuclear and Hodgkin giant-cells are readily identified, but integral fibrosis is absent and there is no eosinophilia.

In the second specimen there is almost total loss of follicular pattern. Fibrosis of all varieties is present, fibrils being the chief form. The capsule is widely destroyed and over-run by the tumour cells. The cellular pleiomorphism is enhanced a little and the cell nuclei are larger, there are proportionately more lymphocyte series cells of primitive type. Hodgkin giant-cells are present in very small numbers and are distinctly small. Eosinophils are absent, plasma cells are very meagre. Mitoses average 4-5 per H.P. field. Necrosis is not a feature. Tumour deposits are confirmed in the lumen of an artery and permeation of the wall is present.

Diagnosis. Pleiomorphic reticulum cell sarcoma. Arising from Brili's disease, sclerosing. 
Susan Ball. Aet 37 years. A housewife. Ref. G.R.I. 281:49. History. Painless slowly growing swelling in the left side of the neck, present for the past two years. Diagnosed provisionally as tuberculous lymphadenitis.

The specimen consists of several pieces of cervical lymph node which are fused. All are neoplastic except one, the normal node retains its architecture and cell structure and is almost entirely encircled by tumour. This latter phenomenon is of much interest as it supports the view that systematisation is not necessarily a feature of tumours of the reticuloendothelial system.

The neoplastic nodes shew reticulum cell sarcoma of undifferentiated type presenting certain morphological affinities to Hodgkin's disease. The capsules where shewn are thick and hyaline but are extensively transgressed at many points. Concentric re-formation is notable. A faint nodular habit of growth is exhibited by the tumour. Fibrosis of all forms is seen but it is moderate. The tumour cells are pleiomorphic morbid reticulum cells shewing little differentiation, free cells are present but most form imperfect plasmodia. Large morbid mononuclear types are comrnon but Hodgkin giant-cells are scanty. Bosinophils are absent and plasma cells are very meagre. Neutrophils are hard to identify due to pyknosis. Litoses are very numerous 8-10 per H.P. field.

Diagnosis. Reticulum cell sarcoma, sclerosing in places. Affinities to Hodgkin's disease close. A physiologically reactive lymph node is enclosed in the tumour. 
Thomas Scott. Aet 55. A fitter. Ref. 372:49. History. Scattered discrete painless enlarged lymph nodes in the neck, axillae, and groins of nine wontins duration. Cough is present whd gustric sustoms are 5roublesome.

The specimen is a cervical lymph node shewing atypical Hodgkin's disease in which small cells predominate and eosinophils are absent. The node is entirely morbid. The habit of growth of the tumour is faintly nodular. The capsule is very narkedly thickened and hyaline, concentric re-formation is present, and at several points there is enclosure of islets of the new tissue in it. In these latter sites the tumour does not appear aggressive. Trabeculae, which are probably new fornations partially separate the nodules of the new tissue. Fibrosis of all forms is encountered but are slight as coinpared with the overwhelming sclerosis of the capsule. The reticulum cell proliferation is productive of very numerous small cells but scrutiny reveals large morbid mononuclear types and infrequent Hodgkin giant-cells. Posinophils are absent. Plasma cells and neutrophils are conspicuously numerous, well above averarg for a pure Hodgkin lesion, it seems likely that inflammation is responsible as the necrotic foci are not very numerous nor large. Mitoses average 2 per H.P. field. The lymphocytes include many immature forms.

Diagnosis. Atypical Hodgkin's disease. Rather uniform, small cells. No eosinophils. ? Infected. 
Lirs. Mary Hunter. Aet 58 years. A housewife. Ref. G.R.I. 1425:49. History. Painless swellings in the neck of three months duration.

The material is poor, the node collapsed on removal. This is a small cell lymphosarcoma. The capsule is indifferently preserved due to being over-run by tumour cells and traunatized. The exodic growth phenomenon is displayed. Fibrosis is minimal, several small scar like areas of compacted fibrils are the only manifestation of note. Nearly all the cells are definitely of the lymphocyte series and small nuclei preponderate. The few large nuclei found are blood vascular endothelial. Eosinophils are absent and plasma cells are sparse. (Haemorrhage due to trauma is seen). Witoses are difficult to find due to pyknosis, many of the necrotic nuclei assume the characteristic lymphocyte form of bivalve type.

Diagnosis. Small cell lymphosarcoma. Affinities doubtful. (Poor specimen). 
William Thomson. Aet 30 years. A railwayman. Ref. G.R.I. 1629:49. History. Referred to out-patients dispensary on account of persistent cervical adenitis. The specimen is a cervical lymph node.

This is Brill's disease in which the follicular pattern is being lost or is poorly developed. Invasive features are notable, and some fibrosis is present.

The entire node is morbid. There is subtle mimicry of the normal architecture by the pseudo-follicles which are mostly of the solid variety, and composed of cells which are closely similar to those of the pulp. Shriniage clefts are observed in many cases. The capsule is of normal thickness, it is transgressed at several points with spread of the tumour cells into the adjacent areolar tissue. Fibrosis is just appreciable, minute representatives of all varieties are found. The type cell of the growth is an inmature lymphocyte. Rare morbid mononuclear forms are to be seen but there are not any giant-cells. Fosinophils and other granulocytes are absent, plasma cells are very scanty. Hitoses average less than 1 per H.P. field. Necrosis is not a feature.

Diagnosis. Brill's disease, regard as malignant. Follicular pattern less distinct than usual. Fibrosis slight but definite. 
John Ferguson. Aet 56 years. Artificer. Ref. G.R.I. 1966:49 16th August 1949, 2677:49 2nd November 1949. History. Generalised moderate lymph node enlargement present for one month. Morbilliform eruption present over the skin. The first biopsy is a cervical node, the second is from the axilla.

This is an example of Iymphoid tissue sarcoma in which the appearances are not appropriate to any of the common categories, the microscopical appearances did not alter very much in the observed course.

The first specirnen is a small node $10 \times 8 \times 6 \mathrm{~mm}$. There is a rind of survival tissue beneath the capsule which is fairly sharply distinguishable from a homogeneous tumour-like tissue occupying the centre. The exodic growth phenomenon is very well illustrated. In this node the capsule is normal in thickness and is not invaded. In the central new tissue fibrosis is present. Perivascular lamellae, fibrils in swathes, and fibrillae are seen. There is reticulum cell proliferation with the production of a uniform mixture of small morbid mononuclear cells and immature lymphocytes. Eosinophils are present in small numbers. Rare small giant-cells are visible. Mitoses are few.

In the second specimen, which is several centimetres across, the capsule is thicker and infiltrated by tumour, normal lymphoid relics have disappeared. The growth is similar to the previous stage except that growth is much quicker. In most areas the lymphoid propensities are greater but in a few the appearances are very close to cellular Hodgkin's disease. Necrosis is not evident. 
Diagnosis. 1. Indeterminate, ? intermediate Iymphoid tissue sarcoma.

$$
\text { 2. Indeterminate. Lymphoid tissue sarcoma }
$$

(malignant) probably nearest to lymphosarcoma but has close affinity to cellular Hodgkin's disease. 
Lirs. Jemima Campbell. Aet 56 years. A housewife. Ref. G.R.I. 1993:49 August 1949, 3093:49 December 1949. P.M. 41:50 January 1950. History. Vague abdominal pain of three months duration. Palpable mass present in the epigastrium; provisionally diagnosed as mesenteric sarcoma. The patient died of Iymphosarcoma 30th January 1950.

The first specimen is a mesenteric lymph node, the second is from the axilla. This is lymphosarcoma in which a false follicle pattern is visible in the earlier biopsy but is much less distinct in the later specimen. There is not any persistence of normal structure in either node. The capsules are thin, attenuated in places, and over-run by small lymphocytes. Fibrosis in the tumour is minimal but is slightly better seen in the later node, it is in the form of diffuse fibrillae. The type cell is an immature lymphocyte with a large moderately deep-staining stippled nucleus, however many morbid mononuclear types are visible intermingled in the second specimen. Several small multinucleate giant-cells are also present in 2. Eosinophils are absent and the other cells, plasma cells etc. are rare. Hitoses average 4-6 per H.P. field. Necrosis of individual cells is noted.

Diagnosis. Large cell lymphosarcoma. Possibly arising from follicular lymphoma. Apparently becoming more primitive (rise in proportion of reticulum cell types). 
Jack Lockhart. Aet 18 years. Ref. G.R.I. 2094:49. History. Swelling of the cervical lymph nodes of several months duration.

This is a perplexing case of lymph node enlargement of indeterminate character. The specimen is a large lymph node $(2.5 \times 2 \times 1.5 \mathrm{~cm}$.) from which the capsule has been widely avulsed. The original diagnosis of Hodgkin's disease is unquestionably erroneous. The appearances are more reflective of inflammatory reaction and hyperplasia than tumour but such gross enlargement is anomalous. The patient is alive and well (4 $\frac{1}{2}$ years later). Only a small fragment of capsule persists. The node is beset with indiscriminately scattered hyperplastic germinal follicles. In many the outline of the pale centre tissue is distorted but does not shew any tendency to separate from the peripheral lymphocytes. The pulp shews irregular local over-growth which drivesthe follicular tissue aside in places. Several cases of marked perivascular fibrosis, and small irregular scar-like fibrous areas are also visible in the pulp. Several micro-abscesses are present most in the pulp but some apparently in follicles.

In the follicular tissue the pale centres are crowded with rapidly proliferating reticulum cells and tingible Korper are copious. The cells are chiefly physiological in size but some are morbidy large with rare binucleate forms. In the pulp similar reticulum cell overgrowth is seen, here morbid forms are commoner but tend to be smaller than typical examples. True Hodgkin giant-cells are not identified. Eosinophils are very scanty but plasma cells abound. Neutrophils are present in the suppurative foci, pyknosis is common. 
Diagnosis. Indeterminate, regarded as probably inflammatory. Himics Hodgkin's disease, but insufficient pleiomorphism, absence of diffuse fibril collagen, and retention of normal structure preclude acceptance of original diagnosis. 
Mrs. M. MoDade. Aet 41 years. A housewife. Ref. G.R.I. 2319:49. History. A painless swelling has been present in the left axilla for the paist six years. This was provisionally diagnosed as a cyst and was resected, it measured $4.5 \mathrm{~cm}$. diameter. The peripheral blood is normal.

The specimen is a greatly enlarged lymph node shewing lymphosarcoma with unusual features. An ill-defined rind of reactive physiological lymphoid tissue without follicles is present at the subcapsular periphery. The exodic growth phenomenon is well shewn. Deep to this reactive tissue is a uniform lymphocytic tumour which does not shew any follicular pattern. The node capsule is moderately thickened but is infiltrated with tumour cells at many points. Fibrosis is present in unusual amount for growths of this kind. Perivascular lamellation is marked, fibrils and fibrillae are also notable. Small coralline deposits are seen round several of the larger vessels. The type cell of the tumour is a small lymphocyte which is almost mature. Large morid mononuclear reticulum cells are scattered sparsely but evenly through the growth and small islets of epithelioid cells are also found. Several typical Hodgkin giant-cells are present. Bosinophils and neutrophils are absent but a few plasma cells are visible. iffitoses average 2 per H.P. field. Necrosis is not seen.

Diagnosis. Small cell lymphosarcoma. Some affinities to Hodgkin's disease viz. fibrosis, capsule and integral; morbid mononuclear and rare Hodgkin giant-cells. 
Angus Sinclair. Aet 57 years. A joiner. Ref. G.R.I. 2376:49. History. Has suffered from malaise and lassitude for the last four months. Swellings have been present in the neck. The blood shews chronic lymphatic leukaemia.

This is small cell lymphosarcoma in a cervical node. The blood vessels and lymphatics of the adjacent areolar tissue are packed with small lymphocytes. The only surviving normal structure is part of the marginal sinus. (This is an unusual finding). The node capsule is thick and hyaline; infiltration is equivocal. Fibrosis is very slight, a few collagen fibrils are denonstrable round the vessels by special method. There is not any increase above normal. The type-cell of the tumour is an immature small lymphocyte, the nucleus of which is fainter staining than mature small lymphocytes. Rare morbid mononuclear cells are identified. Eosinophils and neutrophils are absent, a few plasma cells can be found. Witoses average 2-3 per H.P. field, necrosis is absent.

Diagnosis. Small cell lymphosarcoma, almost pure. 
Patrick Kilgannon. Aet 70 years. Retired. Ref.G.R.I. 2762:49. History. Generalised lymph node enlargernent present for eighteen months. ixamination of the blood revealed chronic lymphatic leukaemia.

The specimen is a small cervical node $9 \times 5 \times 5 \mathrm{~mm}$. shewing lymphosarcoma in which a very indistinct but definite follicular pattern is visible. Very little fibrosis is present.

Norral lymphoid tissue does not survive. The new tissue shews a faint micro-follicular pattern in the deeper part of the node. The capsule is of normal thickness and hyaline, infiltration is confirmed at one point but is not extensive. Fibrosis is very siluht, the deposits are almost certainly hyalinised vessels.

The type-cell is an imnature lymphocyte, intermediate in size between small and large lymphocyte. Rare norbid reticulum cells are present, they are widely scattered. Eosinophils, neutrophils and plasma cells are absent. Hitoses are very few, 1 per 3 H.P. fields. Occasional individual cells are necrotic.

Diagnosis. Lymphosarcoma, pure, with leukaemia. Very faint follicular pattern. 
Rose Bradley. Aet 15 years. Ref. G.R.I. 2773:49. History. Clinical features present sugested appendicitis, operation did not confirm this but the mesenteric nodes appeared enlarged.

This node is inflamed and hịhly reactive, it also represents lymphoid tissue at the zenith of its natural activity. It is possibly an example of the poorly characterised mesenteric lymphadenttis.

The chief justificationsfor its inclusion in the series are the nuclear pleiomorphism of the reticulum cell proliferation in the pulp and the formation of new lymphoid tissue in and outwith the capsule. Scattered eosinophils are present, a constant finding in mesenteric lymph nodes. Plasma cells are more numerous than nornal.

Diagnosis. Physiological reaction and mild non-specific chronic inflammation. 
Isaac Barr. Aet 57 years. A labourer. Ref. 1045:50.

Fistory. Generalised lymph node enlargement present for six weeks.

This is atypical Hodgkin's disease of a peculiar type. The bulk of the new tissue is composed of reticulum cells of epithelioid or fibroblastic type, and fibrosis is chiefly arising by direct diffuse symplasmic transformation. Small areas of typical Hodgkin's disease morphology are identified. Overall the picture is tumour-like. Survival follicles of lymphoid tissue, most with pale centre tissue are present widely scattered through the new tissue; they are not limited to the periphery, this supports the view that the pulp nay be prinariz: involved. The capsule is inperfectly preserved out the pieces remaining are thicisened, infiltration with the naw tissue is present. Trabeculae are not present. Fibrosis is chiefly fibrillar and appears as reticulin at the margins of the epithelioid cells, much is visible with van Gieson's stain and is apparent also in the routine section. The reticulurn cell proliferation is very conspicuous, most of the cells are as noted above. In addition typical morbid mononuclear and Hodgkin giant-cells are numerous. Several Langhan's type giant-cells are found, they are quite indiscriminately scattered. Eosinophils are copious and plasma cells are easily found; these features are probably enhanced by the simplification of the tissue and sparse small lymphocytes. Neutrophils are also fairly numerous and are most concentrated round minute necrotic foci. Hitoses average 1 per H.P. field.

Diaß̧nosis. Atypical Hodgkin's disease, tumour-like. Copious epithelioid/fibroblast cell types. Infrequent Langhan's giant-cells. Fibrosis teruous still. 
Nirs. Eliz. Skillen. Aet 64 years. A Housewife. Ref. G.R.I. L.2071:50. History. Swelling in the right side of the neck which has been present for one year. The patient is a diabetic and is cachectic despite treatment.

The specimen is a cervical lymph node shewing Hodgkin's disease without eosinophils and some tumour-like homogeneity.

There is not any survival of lymphoid tissue. The new tissue shews faintly the expanding nodule habit of growth. Deposits of the tumour are visible in the part of the capsule which is preserved, they appear isolated rather than infiltrative. Thickening of the capsule and trabeculae is pronounced. Fibrosis of all types is encountered and coralline deposit is unusually well evidenced. An interesting anomaly is encountered in the fibrillary manifestation; the fibrillae take the cosin and silver well but stain very faintly with the fuchsin of van Gieson's stain. The reticulum cell proliferation is relatively uniform and the numerous large morbid mononuclear cells are evenly scattered. Typical Hodgkin giant-cells are present in the usual numbers. Eosinophils are very few and other granulocytes are sparse. Plasma cells are scanty. Witoses are not above 1 per H.P. field. Necrosis affects individual cells, chiefly those with large nuclei.

Diagnosis. Atypical Hodgkin's disease. No eosinophils, tend ing towards tumour-like type. 
Urs. Wiclafferty. Aet 43 years. A housewife. Ref. G.R.I. 2158:50. History. A cancer of breast was diagnosed clinically five years ago. Biopsy was not performed. Deep X-ray therapy was administered. A swelling developed in the ipsilateral axilla four weeks ago. The breast and axillary nodes were resected.

This specimen was examined very thoroughly for evidence of tumour, some 20 blocks being taken and all proved negative. The node examined shews a picture which simulates Hodgkin's disease in some respects, sinus tissue is difficult to identify.

There is general retention of the normal architecture of the node, reactive pale centres of irregular outline are present in some of the follicles and in several places there is marked pleiomorphism of the pulp reticulum cells. The capsule is thicker than normal and the trabeculae partake of this change. Diffuse infiltration of these tissues by lymphocytes and plasma cells is notable. Fibrosis in the node tissue is practically restricted to perivascular thickening; the typical lamellation is absent and endarteritic stenosis is common. Rare diffuse fibrils are present in the vicinity of trabeculae. The proliferated reticulum cells assume bizarre forms, but in nearly all the nuclei are noticeably small. Liultinucleate giant-cells are absent. Eosirophils are absent, neutrophils and plasma cells are above normal in number.

Diagnosis. Reactive and chronic inflammatory change. Vascular fibrosis, probably attributable to X-ray therapy. 
John Farrell. Aet 36 years. Ref. G.R.I. 2944:50. History. Painless glandular swellings in the right side of the neck for nine months. The specimen is a cervical lymph node.

This is an interesting example of reticulun cell sarcoma shewing a striking resemblance to Hodgkin's disease.

There is not any survival of normal lymphoid tissue. The capsule is grossly thickened and hyaline, but despite this it is widely infiltrated and transgressed by tumour which is spreading into the adjacent fat. The neoplasm is an alveolar sarcoma and shews the expanding nodule type of growth. Fibrosis is pronounced, all forms are present, but the coralline formation is the most obtrusive. It forms a coarse rather irregular mesh in the interstices of which lie clusters of tumour cells. The type-cell is a free cell conforming to a morbid mononuclear type except that it is rather smaller. The nucleus is more densely staining too, but there are also a fair proportion of typical morbid mononuclear reticulum cells. Small Hodgkin giant-cells are present. Eosinophils are absent and it is difficult to find any plasma cells or neutrophils. Mitoses are very numerous, up to 20 can be seen in H.P. fields. Necrosis is limited to individual cells and is inconspicuous.

Diagnosis. Reticulum cell sarcoma, nearest to "clasmatocytic stem cell lymphoma" but some lymphoid propensities present in the tumour cells. Fibrosis and areas of pleiomorphism very similar to Hodgkin's disease. 
Columba Newcombe. Aet 27 years. Ref. G.R.I. 3579:50. History. Enlarged shotty lymph nodes in the neck and axillae of one months duration. There is not any pyrexia, the peripheral blood is normal, and the Paul-Bunnell reaction is negative.

This is a small cervical node, $8 \times 4 \times 4 \mathrm{~mm}$. There are several areas of interstitial haemorrhage and serous exudate in it. The general architecture remains normal in most areas with retention of lymphoid follicles including many with pale centres. In the deeper part and at some points at the periphery the pulp shews unusual cellular pleiomorphism. The capsule is appreciably thickened but still cellular, at the hilar intrusion, and many small lymphocytes are seen between the lamellae. Fibrosis is present round several large vessels, the largest is apparently the hilar capsule mantle and must be regarded as normal. The trabeculae are also rather more prominent than usual. Scanty fibrils and fibrillae are visible in parts of the pulp. There is considerable reticulum cell increase in the pulp. The cells produced are pleiomorphic but few are sufficiently large or have large enough nuclei to be considered morbid. Small groups of irregularly shaped epithelioid cells are seen at places and scattered singly. Eosinophils are present but not above the usual numbers. Neutrophils and plasma cells are rare. The lymphocytes are normal. Early necrosis is present in the cells in the exudate areas. Mitotic figures are rare except in the pale centre nuclei.

Diagnosis. Indeterminate, considered physiological. Note. Alive and well three years later (27th October 1953). 
Roderick Morrison. Aet 32 years. Ref. Law Hospital 395:51.

History. Swelling in the posterior shoulder region of unknown duration. (It was originally stated that enlarged lymph nodes were not present but this was later revised). The patient responded badly to X-ray therapy and died 30th March 1952.

The material is a biopsy from the tumour of shoulder, it is badly preserved but permits diagnosis. The growth is Hodgkin's disease of tumourlike type. (This appearance is so real that it was in fact reported as reticulum cell sarcoma, however it conforms more closely to Hodgkin's disease).

The tissue is probably a group of small lymph nodes which have thickened fused capsules. Trabeculae are well developed both mature hyalinised bands and swathes of younger tissue. Fibrosis of all forms is encountered but coralline deposits are small. The reticulum cell proliferation is productive of very numerous immature small lymphocytes. These cloak the typical large morbid mononuclear and Hodgkin giant-cells which are readily found on close scrutiny. Eosinophils are few and tend to be focal. Plasma cells are very difficult to identify but this is certainly partly due to the poor preparations. Hitoses are likewise difficult to confirm.

Diagnosis. Atypical Hodgkin's disease, many immature lymphocyte series cells, few eosinophils.

Note. It was later learned that this patient had a severe spastic paraplegia attributed to a deposit in bone at T.7-8 level. 
Moses Norgan. Aet 30 years. A collier. Ref. G.R.I. 506:5l. History. Generalised lymph node enlargement of several months duration.

This is an example of soft cellular Hodgkin's disease shewing tumour-like homogeneity. The eosinophils are much reduced. The node is an axillary one and is large; $20 \times 30 \times 17 \mathrm{~mm}$. Rare follicles and several islets of loose lymphatic tissue persist at the sub-capsular periphery. The exodic growth phenomenon is passing off. The new tissue shews many small focal areas of necrosis or incipient necrosis. The capsule is thin and transgressed in several places. Concentric re-formation is just discernible at some points. Several well developed thick trabeculae are notable, including swathes of fibrils not yet compacted. Fibrosis of all varieties is seen, the greater part of it is in the form of fibrils and fibrillae. The latter are being formed by symplasmic transformation. The reticulum cell overgrowth is marked and lymphocytes are relatively scanty. Nlost of the cells are epithelioid or fibroblast types and their cytoplasmic borders are condensing to form collagen fibrillae. Typical morbid mononuclear and Hodgkin giantcells are copious. Eosinophils are below the usual number but are present in most fields. Plasma cells are scanty but neutrophils are common especially in foci round the necrotic areas. Nitoses are below 1 per H.P. field. Necrosis is focal as already noted.

Diagnosis. Atypical Hodgkin's disease, soft cellular, becoming tumourlike. Fibrosis slight, low eosinophils. Capsule thin and transgressed. 
Robert Shaw. Aet 49 years. Ref. Law Hospital 506:51. History. Complaint of sudden acute colicky pain in the epigastrium. Examination revealed signs of acute intestinal obstruction. Laparotomy was performed and $d$ isclosed a tumour of the colon and enlarged lymph nodes in the abdomen. (The latter were not disturbed).

The tumour is a pleiomorphic reticulum cell sarcoma which appears to have arisen in the lymphoid tissue of the caecal wall. Fibrosis is seen in the neoplasm in the form of strands, some compacted into swathes; fibrils and fibrillae. The type cells are small morbid reticulum cells with hyperchromic nuclei. Large typical ones are present and small Hodgkin giantcells are visible. Eosinophils are present but in small numbers. Plasma cells and neutrophils are absent from the deeper parts of the growth but appear on the ulcerated surface. Nitoses average 2-3 per H.P. field, necrosis is limited to the surface presenting in the lumen. The growth is invading the muscle wall but is not very aggressive.

Diagnosis. Hodgkin's sarcoma. Pleiomorphic reticulum cell sarcom, corresponds closely with Hodgkin's disease in general features but frankly malignant. (Warthin Type II). 
Mrs. Miary Brown. Aet 44 years. A housewife. Ref. G.R.I. 525:51. History. Painless swelling in the right side of the neck for twelve months. The specimen consists of a partly fused mass of large and small lymph nodes. Total size $9 \times 6 \times 6 \mathrm{~cm}$.

This is Hodgkin's disease of soft cellular type which is becoming turnour-like.

There is not any persistence of normal organised lymphoid tissue but small lymphocytes are more numerous at the sub-capsular periphery. The capsule is slightly thickened locally at a few points, in the more tenuous reaches it is infiltrated with the new tissue. Trabeculae are absent. Fibrosis is distinctly light, all forms are encountered except coralline deposits, much is derived by symplasmic transformation. The reticulum cell proliferation is universal and chiefly productive of epithelioid and fibroblastic cells. Both are producing collagen. Typical morbid mononuclear cells and Hodgkin giant-cells are numerous throughout. Eosinophilia is focal, many fields being free of these cells. Plasma cells and neutrophils are common round small necrotic foci. Mitoses average 1-2 per H.P. field.

Diagnosis. Atypical Hodgkin's disease, soft cellular variety with tumour-like homogeneity. Low eosinophilia and tenuous fibrosis. 
Isabella Aitken. Aet 40 years. Ref. Law Hospital 532:51.

History. A lump in the right side of the neck in the supraclavicular region for seven months. An X-ray photograph of the chest taken seven months ago was reported normal. The swelling is painless, the other accessible nodes appear normal.

This is Hodgkin's disease which is typical except that eosinophils are sparse, in all other features the lesion is classical. The example illustrates the principle that while eosinophilia is typical it probably is not an essential feature of the lesion.

Normal lymphoid tissue does not survive. The capsule shews marked fibrous thickening, much of it is still cellular especially on the inner aspect. There is intense sclerosis of pre-existing trabeculae and numerous swathes of new connective tissue have developed. Fibrosis in all forms is pronounced, the coralline deposits are seen to result from fibril compaction and vascular hyalinisation. The new tissue is disposed in irregular nodules. The reticulum cell proliferation is productive of numerous large morbid mononuclear cells and Hodgkin giantcells. The latter are rarely very large. Eosinophils are sparse. Plasma cells are common but neutrophils are rare. Iritoses average 2-3 per H.P. field but the sections are thick. Necrosis is not a feature. Note. A small node $1 \mathrm{~mm}$. diameter adjacent to this large one shews recognisable Hodgkin's disease of the same type.

Diagnosis. Atypical Hodgkin's disease, low eosinophilia the only atypical feature. 
Robert Allan. Aet 46 years. Ref. G.R.I. 660:61. History. Enlarged cervical lymph nodes and nodules in the skin.

This is best described as an intermediate reticulum cell sarcoma possibly emerging from follicular lymphoma. Several nodes are available for study and the appearances are different. In the smaller ones follicular lymphoma is present, the follicles are large, contiguous and beginning to lose their mutual demarcation; in the larger nodes this pattern is disappearing, particularly centrally. The larger nodes probably represent the later stage of the change and are therefore described.

Their capsules are of normal thickness, concentric re-formation is notable between the peripheral pseudo-follicles and the subjacent diffuse growth. Invasion and extension into the adjacent fat is present. The central tissue of the neoplasm shews fields which approximate to small cell lymphosarcoma, to reticulum cell sarcoma, and mixtures of the two. Large morbid mononuclear reticulum cells are found readily but Hodgkin giant-cells are absent. A few Dorothy Reed mirror-image cells are seen. Fibrosis is slight, it is chiefly of diffuse fibril and fibrillary form. The silver impregnation reveals many more pre-collagen fibrillae. Eosinophils, neutrophils, and plasma cells are inconspicuous, the foremost being very sparse. Nitoses are difficult to identify due to widespread pyknosis.

Diagnosis. Retioulum cell sarcoma, intermediate type, sclerosing slightly. Affinities to Hodgkin's disease very close in places. 
James Hogg. Aet 52 years. Ref.G.R.I. 702:51. History. Painless enlargement of the lymph nodes and liver found on clinical examination of the patient who is considered by the dermatologists to be suffering from mycosis fungoides('pre-mycotic' stage).

The specimen is a large axillary lymph node, $30 \times 20 \times 20 \mathrm{~mm}$. The general architecture is preserved but modified. Sinus tissue survives and littoral cell increase is pronounced locally giving a solid packed appearance. Germinal follicles are present both solid types and those with pale centres, in some of the latter the central capillary is hyalinised. The pulp cords are intact but instead of being composed of small lymphocytes nearly all the cells are plasma cells. The capsule and trabeculae are thickened. Fibrosis is seen round vessels and there is some diffuse fibril formation. There is a great increase of reticulum cells in the inter-follicular pulp and haemosiderin is present in the cytoplasm of some. The great majority of these cells have nuclei of physiological size, a few conform to the morbid type, and rare binucleate forms are encountered. Eosinophils are present but are scanty. Mitoses are numerous among the proliferating reticulum cells of the pulp.

Diagnosis. Indeterminate. Reticulum cell proliferation pronounced in areas of the pulp, slight haemosiderosis. Pla sma cells very numerous in capsule and lymph cords deep in the node. Eosinophils present. Regarded as primary, chiefly on account of the size. 
William Fairlie. Aet 51 years. Ref. G.R.I. 1039:51.

History. This patient was operated on for the treatment of varicose veins of the leg. At the operation the groin lymph nodes appeared unduly prominent and three were resected.

The nodes are small, the largest being $10 \times 9 \times 8 \mathrm{~mm}$. The general architecture is retained. The capsules and trabeculae are normal. Iymphoid follicles, some of the them with normal pale centres are present. Fibrosis is present round many of the arterioles of the node and multiple small coralline deposits are seen scattered indiscriminately through the node structure. There is marked proliferation of reticulum cells in ill-defined areas of the pulp, usually in the peripheral inter-follicular loose lymphatic tissue. Thile most of these cells are small and evenly dispersed a few conform to morbid mononuclear type. Eosinophils are distinctly more frequent than in normal nodes. Serial sections reveal that most of the coralline collagen deposits can be accounted for by hyalinisation of blood vessels.

Diagnosis. Physiological reactive change. Small areas which mimic, or rather understudy, Hodgkin's disease are discernible.. The reacting cells are small. 
John Simpson. Aet 35 years. A spring-fitter. Ref. G.R.I. 1862:51. History. A solitary swelling in the axilla present for several months, provisionally diagnosed as a cyst. Naked eye the tumour was considered to be a lipoma. Dimensions $6 \times 3 \mathrm{cms}$, ovoid.

This is an anomalous lymphoid tissue tumour, it bears a superficial resemblance to small cell lymphosarcoma of low malignancy but remarkable fibrosis is present.

The growth is apparently a grossly enlarged lymph node. A few deformed solid lymphoid follicles are discernible at the periphery. Two have imperfect pale centres. The exodic growth phenomenon is present but there is not the reactive change of the peripheral surviving tissue which is usually seen. The capsule is slightly thickened and hyaline, invasion is not seen in the blocks (3) selected for microscopy, concentric re-formation is not seen. Fibrosis is seen in all forms but the most extensive is coralline deposit. It is characteristically similar to the type encountered in Hodgkin's disease. The type-cell of the growth is the small lymphocyte but imnature slightly leptochromatic larger nuclei are comron. Very few morbid mononuclear reticulum cells are seen. True Hodgkin giant-cells are absent. Eosinophils occur in small numbers. Plasma cells are present and neutrophils are seen in tiny pseudo-abscess aggregates. Mitoses are few.

Note. The coralline collagen deposits are traceable to vascular hyalinisation in many cases.

Diaģnosis. Lymphoid tissue sarcoma of low malignancy. Possibly solitary and undisseminated. Sclerosing. Remote affinities to Hodgkin's disease, of benign variety. 
John wilne. Aet 23 years. Ref. G.R.I. 3383:51. History. Swelling of neck present for past 16 months, not increasing in size. (His mother insists swelling was present before this).

The specimen is a large cervical lymph node $50 \times 40 \times 25 \mathrm{~mm}$. of soft elastic consistency. It is difficult to decide the most appropriate category for this tumour. Under hand-lens masnification a distinct follicular pattern is visible, the false follicles being large, up to $3 \mathrm{~mm}$ diameter. At higher magnification this pattern is lost and under the high power objective the tumcur closely resembles cellular Hodgkin's disease. Reticulin compression is not a feature round the false follicles. Several snall lymphoid follicles, some with pale centres can be distinguished at the periphery. The exodic growth phenomenon is demonstrated imperfectly. The capsule is irregularly thickened chiefly at the bäses of trabeculae, it is intact and hyaline. Fibrosis is very tenuous but all forms can be seen. The reticulum cell increase is productive of inmature hymphocytes and large morbid mononuclear cells. Hodgkin giantcells can be identified in many places. Eosinophils are absent. Plasma cells are few and neutrophils are mainly concentrated in young granulation tissue round a large infarct. Mitoses average 1 per H.P. field.

Diagnosis. Iymphoid tissue tumour, indeterminate category• Affinities with lymphosarcoma, Hodgkin's disease and follicular lymphoma. Nearest - Atypical Hodgkin's disease, cellular variety without eosinophils, sclerosis slight. 
Wrs. MoDougall. Let 38 years. A housewife. Ref. G.R.I. 3550:51. thistory. Progressive asthenia for two years. Enlarged nodes not complained of but found on careful clinical examination. The present specimen is a node from the axilla. (Patient died at home six months later).

This is atypical Hodgkin's disease of fibroblastic type at a late stage. Initially this diagnosis was suggested by fibrosis only, many sections were prepared before the characteristic pathognomonic picture was found.

Normal lymphoid structure is totally lost. The capsular thickening is gross but the collagen is still in lamellae. Isolation nodules of the new tissue are enclosed in it, concentric re-formation is pronounced. The node is cut into a very coarse mosaic by enormous trabeculae. Fibrosis in all forms except coralline deposit is confirmed. In the cellular portion typical morbid mononuclear and Hodgkin giant-cells are present but most of the reticulum cell proliferation is expressed in the production of cells very close to typical fibroblasts. Eosinophils are still copious but their distribution in the node is characteristically capricious. Plasma cells are unusually numerous and fairly uniformly dispersed. Neutrophils are rare. Hitoses are very few indeed. Necrosis is not a feature.

Diagnosis. Atypical Hodgkin's disease. Diffuse fibroblastic type. 
Edward O'Farrel. Ref. G.R.I. 5264:51.

History. Progressive asthenia for several months, there is generalised lymphadenopathy but the peripheral blood shews only secondary anaemia.

This is early lymphosarcoma in a cervical lymph node. There is an imperfect peripheral rind of surviving lymphoid tissue; in some localities pale centres are visible in it but the zones of lymphocytes surrounding them are particularly light. The exodic growth phenomenon is well shewn, for a neoplasm of this character. The capsule is of normal thickness but hyaline, infiltration is present at several points. Fibrosis is very slight, perivascular deposits and tenuous diffuse fibrils constitute most of it. The tumour cell is an immature lymphocyte, a free cell with a spheroidal nucleus which is larger and less chromatic than that of a normal small lymphocyte. Morbid mononuclear reticulum cells are very rare, Hodgkin giant-cells are absent. Bosinophils are absent but occasional neutrophils are seen. Plasma cells are scanty. Mitoses average 2-3 per H.P. field. Necrosis is not a feature. Note. Tumour cells are visible in blood vessels and lymphatics. Diagnosis. Iymphosarcoma, small cell type, early• 
John Uhurray. Aet 51 years. A Van-man. Ref. G.R.I. 77:52.

History. Generalised lymphadenopathy with enlargenent of the spleen. The Wassermann reaction was negative. The leucocyte count was 50,000 per cu.mm., the great najority were small lymphocytes. The specimen is an axillary lymph node.

This is lymphosarcoma with leukaemia. The tumour shews a moderately large morbid mononuclear reticulum cell component and fibrosis of the type seen in Hodgkin's disease is present.

The marginal sinus is preserved at some levels; an unusual finding. The exodic growth phenomenon is apparent and the new tissue is disposed in a very faint nodular pattern which is best appreciated naked-eye. The capsule is thickened and hyaline in remarkable degree but invasion with extracapsular deposit is present. Trabeculae are present, they are thickened and partly hyaline. Fibrosis of all types seen in Hodgkin's disease is confirmed even coralline deposits are present in quantity. The type cells of the tumour are 1) an imnature lymphocyte which preponderates, and 2) morbid mononuclear reticulum cells which are however rather small. A very few typical Hodgkin giant-cells are present. Eosinophils are absent and plasma cells are scanty. Neutrophils are totally lacking. Nitoses average 3 per H.P. field; necrosis is limited to individual scattered cells.

Diagnosis. Iymphosarcoma, intermediate cell type. Chronic lymphatic leukaemia present. Affinities to Hodgkin's disease are remarkably close, especially noteworthy are fibrosis and reticulum cell content. 
Mrs. McCall. Aet 22 years. A housewife. Ref. G.R.I. 129:52. History. In the course of a surgical operation for the repair of an inguinal hernia on the right side this node, Cloquet's gland, was removed for good measure.

The node is relatively large but not unduly so considering the patient's age.

This is an example of extreme physiological hyperplasia which under different circumstances might justifiably prompt a diagnosis of tumour at an early stage. There is general retention of the normal architecture pattern but the pale centres of the peripheral gerninal follicles are very large and some follicles are becoming diffuse. In some areas the edges of these merge imperceptibly with the pulp. The capsule is of normal thickness for this site. Trabeculae are poorly represented. Pathological fibrosis is not present - there is some fibrous tissue round the vessels from the hilar intrustion but this is typical of inguinal nodes. There is a marked increase of active reticulum cells both in the pale centres and in the pulp, large morbid mononuclear types are present but none have oxyphile nucleoli. Giant-cells are absent. Eosinophils are very scanty, being well within normal. Plasma cells are increased above the normal number and often occur in small aggregates.

Curiously there is not any real sinus reaction.

Diagnosis. Physiological reaction, marked. Unusual follicular hyperplasia. 
John McGuire. Aet 42 years. Ref. G.R.I. 298:52. History. Complaint of anorexia and loss of weight for the past 14 months. Clinical examination revealed generalised lymph node enlargement of moderate degree; the peripheral blood was normal. The patient received three courses of nitrogen mustard but died six months later (G.R.I. P.M.177:52). The initial biopsy, an axillary node, shews Brill's disease (follicular lymphoma). The necropsy material was poor but revealed total loss of follicular pattern and mutation to large cell lymphosarcoma.

In this specimen a thin mantle of normal small lymphocytes is seen at the periphery, the exodic growth phenomenon being discernible. The capsule is attenuated and split up by infiltrating small lymphocytes the impression gained is that they are being driven through a disintegrating membrane. Concentric re-formation is visible in places. The hew growth is disposed in false follicles of variable size, round these a little collagen is being irregularly deposited. The latter is the only form of fibrosis apparent. The proliferating reticulum cells of the pale centres are mainly shewing lymphoid differentiation but a few large morbid mononuclear types are seen. Hodgkin giant-cells are absent. Eosinophils, plasma cells, and neutrophils are conspicuously absent. In the necropsy specimen some uninvolved lymph nodes are present, e.g. deep cervical. In the remainder examined and in the spleen, the tumour can only be described as diffuse large cell lymphosarcoma. All trace of follicular pattern is obliterated and the type cell is a large primitive lymphoid series one with a leptochromatic vasicular nucleus. 
Diagnosis. Brill's disease, (follicular lymphoma). Transformation to large cell lymphosarcoma. Fatal in six months from initial diagnosis. 
Thomas kicInally. Aet 62 years. A night watchman. Ref. G.R.I. 515:52. History. Hard painless enlargement of the inguinal lymph nodes. The peripheral blood is normal.

This is Brill's disease in an inguinal lymph node. The tumour shews some invasion of the capsule.

There is not any survival of normal lymphoid tissue. The tumour is arranged in numerous large false follicles which are mutually contiguous and confluent in places. The capsule is imperfectly preserved, it appears thicker due to diffuse infiltration and expansion of the fibres by tumour cells. Trabeculae are poorly represented but where seen are unduly thick. Fibrosis of all forms is encountered but the amount is very small; perivascular lamellae account for most. (The collagen content is within normal for an inguinal node). The type cell of the growth is a small reticulum cell shewing lymphoid propensities. The nuclei are rarely spheroidal, most are indented and pachychrornatic. Typical large morbid mononuclear reticulum cells and several small giant-cells are visible in certain fields. Eosinophils are absent. Plasma cells are occasional and neutrophils are restricted to one fragment of marginal sinus. Mitotic activity is high.

Diagnosis. Brill's disease (follicular lymphoma). Regarded as malignant, early loss of pattern. 
William Fulton. Aet 35 years. Ref. Law Hospital 536:52. History. A clinical diagnosis of primary gastric carcinoma was entertained. Surgical exploration revealed an externally normal stomach but enlarged hard lymph nodes in the lesser curvature. The lymph node recovered was originally diagnosed as late Hodgkin's disease but later linitis plastica (diffuse carcinoma) was established as the true diagnosis.

This node is widely replaced by maturing connective tissue and bears a remarkable resemblance to Hodgkin's disease. In this case meticulous examination of sections taken at four different levels revealed a few inconspicuous groups of contiguous epithelial cells and indicated the proper diagnosis. General features of help in this case included lack of hyaline fibrous thickening of the capsule, absence of trabeculae, a suspicious uniformity of the new connective tissue and persistence of sinus tissue. Eosinophils are lacking altogether, an unusual finding in gastric lymph nodes.

Diagnosis. Metastatic carcinoma, mimicking Hodgkin's disease. (True diagnosis confirmed later). 
IIrs. Annie Flanighan. Aet 59 years. A housewife. Ref. G.R.I. 584:52. History. Enlarged cervical lymph nodes of several months duration. In 1948 patient had a thyroidectomy, the gland was reported as hyperplastic. the blood state is not known.

This is indeterminate primary tumour, probably early lymphosarcoma, in a cervical lymph node. The diagnosis is seriously hindered by widespread avulsion of the capsule. There is an imperfect rind of normal hyperplastic lymphoid tissue at the periphery, in a few places small fragments of the marginal sinus can be identified. The exodic growth phenomenon is seen. Centrally the normal tissue is replaced by a very uniform lymphocytic growth. With very low power magnification a vague follicular pattern is discernible at one level. The capsule, where it exists is attenuated except round the inferent vessels. Concentric re-formation is absent. Trabeculae are very poorly represented. Fibrosis is minimal, there is merely hyalinisation of the vessels and these are scanty. The reticulin mesh however is slightly denser than normal. The type cell of the growth is a well formed slightly leptochromatic immature lymphocyte. Morbid mononuclear and rare mirror-image binucleate reticulum cells are evenly scattered through the tumour. All granulocytes and plasma cells are virtually absent. Witoses average 2 per H.P. field. Necrosis is absent.

Diagnosis. Probably early small cell lymphosarcoma. Exodic growth phenomenon present. 
Susan Devlin. Aet 17 years. Ref.G.R.I. E.779:52. History. Patient admitted with exceptionally profuse uterine haemorrhage which required repeated large blood transfusions. General examination revealed enlarged lymph nodes in the neck.

This is a cervical lymph node $2 \times 1 \times 1 \mathrm{~cm}$. which shews very early atypical Hodgkin's disease, the new tissue is almost all confined to the pulp.

There is considerable retention of normal structure; lymphoid follicles, some with pale centres, sinus tissue and parts of the pulp are preserved. The new tissue is patchy and chiefly in the medulla of the node, it reaches the capsule in one level exarnined. The lesion is too early to shew the exodic growth phenomenon but incipient crushing of the cortex is seen at one point. The capsule is definitely thickened and this change affects the few trabeculae present as well. Infiltration is absent. Fibrosis is slight but definitely adequate to cause suspicion. Perivascular deposit, fibrils, and fibrillae are the forms present. Reticulum cell proliferation is manifest in the morbid pulp. The cells produced include perfectly typical morbid mononuclear types, Dorothy Reed mirror-image cells, and rare Hodgkin giant-cells. In addition small aggregates of epithelioid cells are common, some have large hyperchromic nuclei. Eosinophils are present but scanty, many are of lymphocytoid type. Plasma cells and neutrophils are readily found. Mitoses average 1-2 per H.P. field in the new tissue areas. Small focal necroses are common. 
Diagnosis. Atypical Hodgkin's disease, low eosinophils, components rather dissociated. Very early. Only small portion of node is involved. Exodic growth phenomenon appearing.

Note. The patient developed large hard lymph nodes all over the body and the anaemia never remitted. She became progressively asthenic and cachectic and died 26th October 1952. Necropsy was not allowed. 
John Leather. Aet 37 years. A fishmonger. Ref. G.R.I. 1120:52, 1333:52. History. Generalised lymph node enlargement which has been in progress for three months. Abdominal pain has been present for one year, it is epigastric. The initial biopsy was badly mutilated and a repeat was requested, unfortunately an inguinal node was sent.

Both specimens are poor material, they were sent from Helensburgh and inevitably suffered autolysis due to delay.

The second specimen was reported carefully with an account of the changes seen and the diagnosis was left open. The alteration from normal is very slight. The changes giving rise to doubt are -

1. A very slight loss of general architecture with replacement especially in the deeper part of the node by indistinctly follicular lymphoid tissue.

2. A process of re-population of the atrophy fat-cone by lymphoid tissue, indicating proliferative activity.

3. Wany individual cells both of the lymphocyte and reticulum series have unduly large nuclei.

The capsule and trabeculae are fibrous but the site (groin) precludes assessing this accurately. Fibrosis is appreciable round vessels and as fibrils and fibrillae in the pulp. Reticulum cell proliferation is present and though most cells are within normal, a few morbid mononuclear types are seen. Eosinophils are few, neutrophils and plasma cells are very low, too low to regard the lesion as inflammatory. Mitoses average less than 1 per H.P. field. 
Diagnosis. Indeterminate lymphoid tissue hyperplasia, regarded as primary•

Note. This patient died of reticulum cell sarcoma within a year, which diagnosis was confirmed at necropsy (P.M.160:53). A large survey of lymph nodes from all groups shewed reticulum cell sarcoma of intermediate type. Deposits were also confirmed in the liver, spleen and bone marrow. A striking feature of this tumour was the degree of partial implication of the individual nodes. 
Robert Nicholson. Aet 26 years. A Coach Trimmer. Ref. G.R.I. 1528:52. History. Swelling in the midline of the neck of indeterminate duration.

This is an example of probable physiological reactive change associated with marked fibrosis of the node capsule, trabeculae and part of the pulp.

There is general retention of normal active lymph node structure, follicles with pale centres are frequent. The capsule is markedly thickened and hyaline, this change is uhiform. New perinodal lymphoid tissue, plasma cell deposits and etc. are absent from the specimen. The trabeculae are abnormally thick in several instances. Perivascular fibrosis is occasional and fibrils and fibrillae are visible in the pulp in one locality. These latter appear the result of symplasmic transformation. In several follicles the central arterioles shew fibrinoid degeneration, and perivascular macrophages with haemosiderin are seen. The reticulum cells are active in the pale centres and in areas of the pulp, a few large, almost morbid varieties are present. One giant-cell is seen at one level, it conforms to the Hodgkin type but is small. Eosinophils and plasma cells are present but within the range of normality. A few scattered neutrophils are present. Sinus reaction of open type is notable in the marginal sinus.

Diagnosis. Physiological reaction. Fibrosis of capsule marked. Slight fibrosis of pulp. Fibrinoid degeneration present in some vessels. 
Robert Kynaston. Aet 23 years. A labourer. Ref. G.R.I. 2958:52. History. Admitted as a surgical emergency, provisional diagnosis given as '? perforated peptic ulcer'. Considered to be suffering from acute leukaemia. A leucocyte count on admission yielded about 20,000 per cu.mm. The haematologists reported the finding of large mononuclear cells in the peripheral blood, these they considered lymphoblastic. Severe anaemia was present.

The specimen is an axillary lymph node. In general architecture a close mimicry of normal is seen; a peripheral wide zone of normal anatomical cortex exists but underlying this is a homogeneous looking mass of closely packed cells with large deep staining nuclei. The capsule is thick and hyaline, concentric re-formation is apparent, it is enhanced by the striking exodic growth phenomenon present. Well formed trabeculae are seen. Fibrosis of all types is present. liost is vascular (hyalinisation) or peri-vascular. The reticulum cells shew increase with a fair proportion of forras shewing lymphoid tendencies. Typical morbid mononuclear varieties and rare Hodgkin giant-cells are demonstrable. The eosinophils are increased above normal, they appear mature types. Neutrophils are fairly numerous throughout and plasma cells are increased. Note. This patient died four weeks later; before death the haematological diagnosis was revised to acute myeloid leukaemia.

Diagnosis. The original diagnosis, Acute Hodgkin's disease was considered the nearest category, chiefly on account of fibrosis and cellular pleiomorphism. Llay be regarded as lymph node in acute leukaemia. 
Duncan lücLachlan. Aet 45 years. A Railway Guard. Ref. G.R.I. 3227:52. History。 A painless lump appeared in the left side of the neck eight months ago. It has gradually increased in size. It was resected after the provisional diagnosis of lipoma and presented as a fleshy lobulated encapsulated mass $2 \frac{1}{2} \times 1 \frac{1}{2} \times 1$ inches.

This is atypical Hodgkin's disease. The aberrant features include lack of eosinophils and predoninance of sinall cells; it has some affinity to lymphosarcoma.

Normal lymphoid tissue does not survive. The capsule shews very irregular thickening in places but the unaffected stretches are normal. There is doubtful infiltration at several points and concentric re-formation is visible. New trabeculae composed of swathes of immature connective tissue are forming, giving a coarse folliculoid pattern. Fibrosis is present in all forms. The reticulum cell proliferation tends to be focal, it is seen chiefly near the collagen deposits and near the edge. Large morbid mononuclear types and Hodgkin giant-cells are present but less florid than usual. Josinophils are very sparse, small lymphocytoid cells with coarse oxyphile gramules are anong them. Plasma cells and neutrophils are also scanty. Hitoses are very few, less than 1 per H.P. field.

Diagnosis, Atypical Hodgkin's disease. Very low eosinophils. Small lymphocytes very numerous. Slow rate of growth. Affinities small cell lymphosarcoma and ? Benign Hodgkin's disease (not close). 
George illcGaughey. Aet 60 years. Ref. G.R.I. 3359:52. History. Painless enlarged lymph node of neck, duration uncertain.

This is atypical Hodgkin's disease; fibrosis is less than usual, and eosinophils are practically absent.

Normal lymphoid tissue is absent. The capsule shews irregular thickening. Well marked re-formation is present in some sections, invasion is not seen. The trabeculae are thickened and small secondary branches segment the new tissue locally. Fibrosis exists in all forms, perivascular lamellation is the most conspicuous, other forms, especially fibrillary, are scanty. The reticulum cell proliferation is marked but tends to be focal. Large morbid mononuclear cells are common especially at the periphery of the new tissue. Hodgkin giant-cells are few, they are typical but small. Eosinophils are virtually absent. Plasma cells are very few, small lymphocytes are very numerous and appear mature. A few scattered neutrophils are visible. Mitoses average 2 per H.P. field.

Diagnosis. Atypical Hodgkin's disease. No eosinophils, Fibrosis slight. Fairly near to soft cellular variety. 
Joseph Kane. Aet 51. A Janitor. Ref. G.R.I. 3477:52. History. This node was recovered from the lesser omentum at the pyloric end of the stomach. Gastrectomy had been performed for the relief of a benign chronic peptic ulcer.

The lesion is undoubtedly reactive change but it offers an interesting physiological mimicry of Hodgkin's disease. This is partly by virtue of the topical eosinophilia, a very common finding in paraenteronic nodes.

The general architecture is normal. The capsule shews irregular local thicisening and is hyaline. Concentric re-formation is present in marked degree at some parts. Fibrosis adequate to suggest ordinary Hodgkin's disease is lacking, but perivascular deposit is unusual round individual vessels. There is general reticulum cell increase but true morbid varies are absent, here size of the nuclei is the determining factor. Eosinophils are numerous and often focal. The other cells are also fairly common. Sinus tissue is inconspicuous considering this is a gastric node.

Diagnosis. Physiological reaction shewing Hodgkin-like features in mild degree. 
John lieffan. Aet 11 $\frac{3}{4}$ years. Scholar. Ref. G.R.I. 4004:52. History. Nodule present in the occipital region of the neck for the past four weeks, it is painless. Received as '? lymph node'.

This is a cervical lymph node presenting focal epithelioid cell aggregates which shew a tendency to suppurate. In addition there is pronounced capsular thickening and extreme physiological reaction in the pulp.

There is general retention of the normal architecture of the node but sinus tissue is obscure. The pale centres are irregular in outline and some are diffuse. Capsular fibrosis is marked and is being augmented by concentric lamellae from its inner aspect. The trabeculae are increased in size. Fibrosis is seen round a few vessels but is inconstant, there is slight local (sub-capsular) fibril and fibrillary collagen. The reticulurn cells evidence a definite proliferation. This is chiefly expressed in the form of epithelioid cell islets which vaguely suggest tubercles. In some cases micro-abscesses are present centrally. In addition the pale cell and pulp reticulum cells shew increase with fairly numerous morbid mononuclear forms. A few mirror image types are found but giant-cells are absent. Fosinophils are very few. Plasma cells are numerous and many are large.

Acid/alcohol fast bacilli cannot be found in sections appropriately stained.

Diagnosis. Physiological reactive change, possibly tuberculosis. Marked capsular fibrosis. 
Nora Gallacher. Aet 26 years. A bus conductress. Ref. G.R.I.4484:52. History. Swelling of the cervical lymph nodes of one years duration. Recent swelling of the glands in both axillae. Old history of "chest infection". (There is not any active tuberculous disease radiologically).

This is typical Hodgkin's disease in a cervical node. A disrupted rind of normal lymphoid tissue is present below the capsule. The exodic growth phenomenon is present but disappearing. The new tissue is disposed in coarse irregular follicles. The capsule is grossly thickened and hyaline. Concentric re-formation is discernible; there is not any invasion. Fibrosis of all varieties is seen, the perivascular lanellation is remarkable. The reticulum cell proliferation is pronounced, large morbid mononuclear and typical Hodgkin giant-cells are cominon. Lifany of the latter are necrotic. Eosinophilia is intense. Plasma cells are recognisable with difficulty, the neutrophils are likewise hard to identify. Kitoses average 1 per H.P. field. Very small necrotic foci exist.

Diagnosis. Hodgkin's disease, typical. 
Wrs. Annie Kerr. Aet 55 years. A housewife. Ref. G.R.I. 4862:52. fiistory. Dysphagia had been present for six months. A sub-glottal extrinsic swelling was found on examination and there were firm matted lymph nodes present in both posterior triangles of the neck. Oesophagoscopy was negative.

This is an example of late atypical Hodgkin's disease, the growth is tumour-like. The specimen can no longer be identified as lymphadenoid. The cellular deposits of the new tissue are predominantly immature small lymphocyte masses and are set in a coarse hyaline collagenous mesh. Fibrosis of all forms is seen. Fibrils and fiorillae are relatively inconspicuous; the other forms are marked. The reticulum cell proliferation is still active but many of the cells produced are necrotic or going necrotic. Typical morbid mononuclear cells and Hodgkin giantcells are readily found. A few atypical giant-cells like large foreign body giant-cells are visible, they appear to be fusion products of epithelioid cells. Several tiny foci of eosinophils exist but large areas are totally devoid of them. Plasma cells are numerous, especially at the edges of the cellular tissue. Neutrophils are virtually absent. Nitoses are very few.

Diagnosis. Atypical Hodgkin's disease, homogeneous and tumour-like. Very few eosinophils. Mumerous young lymphocytes despite intense fibrosis. 
Annie Forsyth. Aet 62 years. At home. Ref. G.R.I. 4519:52. History. Enlarged lymph nodes in both axillae, duration uncertain.

This is an axillary lymph node shewing lymphosarcoma arising from follicular lymphoma. There is a moderate tendency to sclerose, other minor affinities to Hodgkin's disease are apparent.

The entire node is norbid. The tumour shews a definite follicular pattern which is fairly uniform, the peripheral follicles are larger, beconing diffuse and shew the characteristic artefact shrinkage space round them. The capsule is widely infiltrated by tumour cells but persistent fragnents are thick. Fibrosis of all forms can be found. Most is in the form of compacting fibrils round the false follicles, the other varieties are sparse and inconstant. The type cell of the growth is an imnature (intermediate) lymphocyte but scanty morbid mononuclear reticulum celis and very rare small nultinucleate giant-cells are also present. Eosinophils are present, mostly as scattered groups of 3-6. Plasma cells and neutrophils are rare. litoses are rare, about 1 per 2 H.P. fields. Necrosis is confined to occasional individual cells .

Diagnosis. Small cell lymphosarcoma, ? representing enhancement of malignancy of Brill's disease. Hidespread transgression of capsule, growth in perinodal fat. Slight sclerosis, all forms. Slight affinity to Hodgkin's disease. 
Thomas Quigley. Aet 59 years. Ref.G.R.I. 4910:52. History. (From Alexander Hospital, Coatbridge). A solitary glandular swelling appeared in the right axilla, it was discovered suddenly by the patient when it was already large. The chest is clear radiologically and the general health is good. Two nodes were sent, one $4 \times 3 \times 3 \mathrm{~cm}$. the other I cm. diameter.

Both shew the same alterations. The lesion does not conform to any of the better characterised variants of lymphoid tissue tumour, it shews features of follicular lymphoma and Hodgkin's disease in about equal proportion. A rind of normal highly reactive lymphoid tissue is present in both nodes, it is $0.5 \mathrm{~mm}$. wide. The exodic growth phenomenon is very distinct. The deep tissue is disposed in a definite but irregular follicular pattern. The capsule is not thickened, and it is intact. Light fibrosis is present. Perivascular larellae, fibrils and fibrillae are identified. The reticulum cell proliferation is within the limits of normal but typical morbid mononuclear and true Hodgkin giant-cells are present. A few Langhan's type giant-cells are seen chiefly in groups of epithelioid cells. These latter aggregates are small and irregular, and unlike tuberculous reaction. Eosinophils are few. Plasma cells are very numerous, especially in the normal (reactive) peripheral tissue, and round the ill-defined deep follicles. Witoses are rare. While the pattern is strongly suggestive of chronic irritation the nodes are suspiciously large.

Diagnosis. Lymphoid tissue tumour, relatively benign. Mingled features of Hodgkin's disease and follicular lymphoma present, but insufficiently characteristic to warrant diagnosis. 
Agnes licNiven. Aet 28 years. Ref. Stobhill Hospital 0578:48. History. Asthenia of increasing severity over the last three years. A painful swelling appeared in the left side of the neck recently.

This is a cervical lymph node shewing a highly cellular pleiomorphic Hodgkin's disease picture, the appearances approach Hodgkin's sarcoma (type II of Jarthin) but eosinophils persist, albeit reduced in number. Normal lymphoid tissue is absent. Concentric re-formation of the capsule is well shewn. The cellular part of the new tissue is irregularly folliculoid and set in a coarse collagen mesh. The capsule is grossly thickened and hyaline and permeated with islets of the new tissue. The appearances suggest that the perinodal compensatory lymphoid tissue has become implicated in the disease process. Fibrosis is very marked, all forms are represented but fibrillary deposits are few. The reticulum cell proliferation is florid and almost all are highly pleiomorphic morbid types. Hodgikin giant-cells are very numerous. Rare Touton giant-cells are seen. Eosinophils persist but are scanty. Plasma cells are meagre, neutrophils are frequent including micro-abscesses. Mitoses average 2 per H.P. field in the cellular tissue. (It is likely that sepsis has supervened).

Diagnosis. Hodgkin's disease shewing transition to Hodgkin's sarcoma. Cellularity and pleiomorphism enhanced. Eosinophils reduced.

Note. This patient died in G.R.I. and necropsy was performed. See special P.H. series. 
Mrs. Susan Brisbane. Let 47 years. A housewife. Ref. G.R.I. 98:53. History. Asthenia and tiredness have been present for 15 months. During the past two weeks the left axillary nodes have been enlarged. The leucocyte count was 43,400 per cu.mm, the condition was regarded as subacute myeloid leukaemia.

This is a large axillary lymph node $4 \times 3 \times 3 \mathrm{~cm}$. shewing a remarkable picture of what may be termed acute or atavistic Hodgkin's disease; all the component cells are imnature.

A few survival islets of small lymphocytes persist. Under low power the tumour appears uniform and cursory examination gives the impression of large cell lymphosarcoma. This is dispelled at once on scrutiny. The capsule is thick and transgressed at many points. Re-formation is not evident. Fibrosis is slight, tenuous deposits of all varieties are encountered. The commonest cell present is a spheroidal free cell with a large spheroidal or finely notched nucleus. Oxyphile nucleoli are common and the nucleoplasm is deeper staining than that of typical morbid mononuclear cells. Often the cytoplasm is poorly defined and symplasmic habit obtains. Typical morbid mononuclear and rare Hodgkin giant-cells are identified. Bosinophils of myelocyte type and size are numerous, many lymphocytoid types are also seen. Plasma cells are very scanty. liany necrotic neutrophils are present especially at the sub-capsular periphery. Mitoses average I-2 per H.P. field. Haemosiderin is present in the edge of the tumour.

Diagnosis. Atypical Hodgkin's disease, acute or atavistic type. Conspicuous de-differentiation of component cells. 
Note. This diagnosis was submitted before the blood state was established.

Revised diagnosis. Lymph node in myeloid leukaemia. The patient died two months later (P.M. G.R.I. 97:53). Generalised lymphadenopathy, splenomegaly and hepatomegaly were found; the femoral bone marrow shewed nodular white tissue of similar microscopical appearance. 
James Loudon. Aet 25 years. Ref. G.R.I. 1460:53. History. An abscess developed in the left axilla approximately three months ago, the cause was obscure. It failed to resolve despite antibiotic therapy and a biopsy was taken on 3rd April. This was necrotic. This is a second specimen.

This node shews foci of reticulum cell sarcoma, and areas of typical Hodgkin's disease. The former lesion is a uniform stem-cell type of growth without the pleiomorphism of Hodgkin's sarcoma. Areas of normal lymph node tissue are present with active follicles well represented. Some deep sinuses and parts of the marginal sinus persist. The Hodgkin's disease areas are diffuse in the pulp; the reticulum cell sarcoma areas are better demarcated and some adjoin the former. The capsule is grossly thickened and hyaline, concentric re-formation is not seen but the exodic growth phenomenon is well shewn in places. Invasion is absent. Thick trabeculae are present. Fibrosis is present in all forms except coralline deposits, most is expressed as perivascular lamellation. The reticulum cell sarcoma is composed of large pale cells with indistinct (? symplasma) cytoplasmic outines. The cytoplasm is feebly eosinophil. The nuclei are spheroidal with clear-line nuclear membranes and basophil granular nucleoplasm. Basophil and oxyphile nucleoli are comnon. Eosinophils are scattered through the deposits. In the Hodgkin's disease areas large morbid mononuclear types and Hodgkin giant-cells are present. Eosinophils are commoner in this tissue. Plasma cells and neutrophils are frequent in both growths.

Diagnosis. (Reticulum cell sarcoma, stem-cell type.
(Hodgkin's disease, typical. Co-exist in the same node but transition is difficult to trace. 
Virs. Lala Hoyes. A housewife. Ref.G.R.I. 495:53. History. Swelling in both axillae and groin for six months. Pain in the back and increasing weakness for three weeks.

Three lymph nodes from the groin, two large and one small. The largest measures $2.5 \times 1 \times 1.5 \mathrm{~cm}$.

Two fairly distinct appearances are seen. The larger nodes reveal a soft cellular variety of Hodgkin's disease. These nodes do not contain any persisting normal tissue, the capsules are thin, even attenuated in places and are not much hyalinised. Invasion is not present and concentric re-formation is absent. Mrabeculae are poorly represented. Fibrosis is slight, the chief expression is perivascular accretion, actual lamellation is rare. The vessels thus thickened produce a coarse imperfect segmentation of the new tissue. Diffuse fibrils and fibrillary deposits are present but inconspicuous because lymphoid series cells are still abundant. Occasional coralline deposits are noted. There is a characteristic increase in reticulum cells, nost differentiating to the usual norbia tjpe, but lymphoid differentiation is also seen. Many large mononuclear types and typical multinucleate giant-cells are present. Eosinophils are rare and plasna cells scanty. Neutrophils are also few. Mitoses are scarce, and necrosis is confined to occasional individual cells. The smallest node is normal in structure at one pole; the other part is expanded by a uniform cellular tumour of which the type-cell is the "owl-eye" variety of morbid mononuclear reticulum cell. Survival aggregates of lymphocytes are visible in interstices between the tumour cell masses. Eosinophils are absent and fibrosis is very slight indeed. 
Diagnosis. (1. Hodgkin's disease, atypical in so far as eosinophils are very scanty and fibrosis slight. Soft cellular and tumour-like.

(2. Reticulum cell sarcona.

Note. Patient died 1953. Necropsy was performed, see special P.H. series. 
Alexander Wilkie. Aet 52 years. Grave-digger. Ref. G.R.I. 2129:53. History. Vague illness of several months duration, generalised lymph node enlargement was found on examination. (Since diagnosis incipient chronic lymphatic leukaemia has developed).

This is a cervical lymph node shewing early small cell lymphosarcoma. Small scar-like areas of fibrosis are visible in the tumour. There is a beautifully preserved rind of hyperplastic lymphoid tissue approximately $0.5 \mathrm{~mm}$. wide between the tumour and capsule; it is not entire, the growth reaching the capsule at some levels. The exodic growth phenomenon is seldom better illustrated. The tumour is a very uniform mass of imnature small lymphocytes. The capsule is attenuated, and is transgressed at several places. Trabeculae are absent. Fibrosis is limited to occasional perivascular deposits and small scar-like deposits. The latter are chiefly compacted fibrils. Reticulum cell nuclei including rare large morbid mononuclear forms with big nucleoli are easily found on scrutiny. Granulocytes and plasma cells are absent. Mitoses are numerous, up to 4 per H.P. field. Necrosis is not a feature.

Diagnosis. Small cell lymphosarcoma, incomplete involvement of node. Exodic growth phenomenon marked. Slight fibrosis. 
Francis Moran. Aet 25 years. Ref. O.P. G.R.I. 2348:53. History. Painless enlargement of lymph nodes in the left side of the neck and both axillae. Peripheral blood normal.

The specimen broke up during removal and the capsule became avulsed. Concentric re-formation is not seen. There is not any persistence of normal structure beyond a few survival islets of small lymphocytes. The trabeculae are very poorly represented. Fibrosis is meagre; perivascular lamellation and fibrillar deposits account for most, though coralline deposits are notable in one fragment. Reticulum cell proliferation is fairly uniform and more conspicuous than usual. Most cells of this line are morbid mononuclear types but macrophage types are also well represented. This pleiomorphism is pronounced . Hodgkin giant-cells are numerous, necrosis is common in individual examples. The eosinophils tend to be focal, those localities are in healthy Hodgkin tissue. Plasna cells are very few indeed, the lymphocytes are almost all rather larger than normal small lymphocytes. The growth rate appears about the usual.

Diagnosis. Atypical Hodgkin's disease, early. Cellular, little fibrosis, no plasma cells. 
lirs. E. Drumnond. Aet 80 jears. Cervical lymph node. Ref. G.R.I. 2490:53. History. Angina followed by enlargement of cervical lymph nodes on the left side of the neck. Duration of complaint three weeks.

There is not any survival of normal tissue but a few small aggregates of small lymplocytes are notable at the periphery. The exodic growth phenomenon is almost lost. The node caysule is hyalinised und ruch attenuated; at one point a definite breach is visible. There is much tumour outwith the capsule at all points and it is infiltrating the fat. Fibrosis is early and slight, it is chiefly seen as fibrils and fibrillae, perivascular and coralline deposits are absent. (Senile hyaline degeneration is notable in the blood vessels). The node parenchyma consists of an active intermediate type reticulum cell sarcona. Horbid mononuclear reticulum cells are the predominant cell but lymphocytic differentiation is also seen. Wultinucleate giant-cells acceptable as Hodgkin giant-cells are scattered through the tumour. Tosinophils are absent, other granulocytes are uncomnon, and plasma cells are scanty. Mitotic figures average 10-12 per high power field. Necrosis is beginning in the central portion of the tumour, many of the Karyorhetic nuclei resemble neutrophils but their true character is confirmed by use of the oil immersion lens.

Diagnosis. Reticulum cell sarcoma, intermediate type. Fields very close to Hodgkin's disease are discernible. 
Archibald Scott. Aet 26 years. A collector salesman. Ref. G.R.I. T.5. 3061:53. History. A swelling was first noticed in the right side of the neck 12th July 1953. Painless. The node is from the anterior triangle, others were present.

Survival follicles with pale centres, aggregates of normal lymphocytes and large segments of the marginal lymph sinus persist. The exodic growth phenomenon is shewn, the peripheral mantle consists of equivocally hyperplastic/neoplastic lymphoid tissue. The node capsule is irregularly thickened but still shews many nuclei, invasion is not present. Swathes of delicate young collagen segment the new tissue irregularly. Fibrosis is early, all forms are present. Reticulum cell increase is marked, many of the nuclei are still small and normal looking, but morbid mononuclear varieties are present. Typical multinucleate giant-cells are seen, they are more numerous in focal areas. Eosinophilia is pronounced throughout, but plasma cells are scanty. Many of the lymphocytes present are young forms with big nuclei. Neutrophils are occasional, microabscesses are not seen. Mitoses average rather below $I$ per H.P. field, necrosis is not a feature.

Diagnosis. Hodgkin's disease, typical, early. 
Mrs. Christina Carberry. Aet 51. Housewife. Ref. G.R.I. 2980:53. History. Has experienced pain in the back for several months and during the last month a swelling has appeared in the left side of the neck. Her general health is poor.

This is a cervical lymph node shewing Hodgkin's disease of the soft cellular type. Fibrosis is slight and the lesion is probably early. Several small poorly demarcated deposits of normal lymphoid tissue are seen at the periphery; some are surrounded by the new growth. Fragments of the marginal sinus persist. The new tissue is diffuse, nodulation is not appreciable. The capsule is thickened but hyalinisation is slight as yet. Eosinophils and plasma cells are frequent in between the compacting new lamellae. Trabeculae are not represented. Fibrosis is light; perivascular lamellae, fibrils, and fibrillae are the forms encountered. The reticulum cell overgrowth is marked, typical morbid mononuclear cells and Hodgkin giant-cells are frequent. Neutrophils are commoner than usual, and several micro-abscesses are present. Mitoses average 2 per H.P. field. Necrosis is not a feature.

Diagnosis. Hodgkin's disease, accept as typical; fairly soft cellular variety, sclerosis slight. Incomplete involvement of node. 
John Gray. Ref. G.R.I. 3185:53. History. Swelling in the right groin of two months duration.

Fragments of the marginal sinus and the deeper sinuses persist. Embedded deep in the smaller node are two minute islets of apparently normal lymphoid tissue, the lymphocytes here are distinctly smaller than in the neoplastic part. Very faintly marked follicular growth is apparent especially in the larger node. The capsules are hyalinised and attenuated, at several points they are over-run by tumour lymphocytes with the formation of extra-capsular deposits. Fibrosis is restricted being chiefly perivascular lamellation. Reticulum cells are scanty, most are peripheral and are already shewing lymphopoietic differentiation. Hodgkin giant-cells are not present, nor are there any eosinophils. The type cell of this tumour is an imnature lymphocyte. líitoses are relatively scanty but much variation is seen locally. There are two areas of necrosis in the smaller node and in the vicinity a few neutrophils are seen.

Note. Silver impregnation reveals an unusual amount of reticulin enhancement of medullary mesh especially.

Diagnosis. Small celled lymphosarcoma with slight fibrosis, restricted to the vicinity of blood vessels. 
Natthew Thomson. Ref. G.R.I. W.S. 3221:53. History. Discrete painless enlargement of lymph nodes in the neck of three months duration.

Normal tissue persists in survival islets situated between the illdefined follicles in which the tumour is growing. The capsule shews irregular thickening at the bases of trabeculae. The trabeculae proper are few, and form a scaffolding for new connective tissue swathes which imperfectly delineate the false follicles. Fibrosis is mainly of diffuse fibril and fibrillary character but occasional coralline deposits are seen. The proliferation of morbid reticulum cells is diffuse and unusually homogeneous, many typical mononuclear morbid types are present; Hodgkin giant-cells are present and are aggresated locally. Mosinophils are copious, particularly in the new connective tissue, plasma cells are very scanty. Neutrophils are unusually numerous and there is much focal necrosis. Mitoses do not exceed the usual number.

Diagnosis. Atypical Hodgkin's disease. Soft cellular type, definitely infiltrating capsule. (Tumour-like except for persistence of eosinophilia). 
Wrs. Margaret Hoore. Aet 38. Ref. G.R.I. T.S. 3232:53 and 2736:53. History. Five yeurs previously patient developed a lump in the mid line of the sternum, biogsy was contemplated but not performed. She was treated outside when the lump becine larger and ulcerated. This ulcer subsequently destroyed much of the skin of the breast and chest wall, and she complained of intermittent swellings in the neck. In July 1953 a biopsy of the edge of the ulcer was tiken, ref. 2736:53.

(i) Skin. Due to gross sepsis with abscess formation, diagnosis was difficult. Follicular formations of tissue resembling Hodgkin's tissue were present; fibrosis was seen, but eosinophils were absent. Provisionally I reported this as "mycosis fungoides, probably Hodgkin's disease" but insisted that lymph node biopsy was essential. (ii) Cervical lymph node. The tissue sent was scarred and ragged. Examination of one of the sections revealed it to be atypical Hodgkin's disease at a late stage but included in the mass were two physiologically active lymph nodes.

The capsules of the Hodgkin's tissue nass have lost their identity due to advanced fibrosis, the mass is probably a fusion product. Hyaline change and concentric re-formation are pronounced and the latter, together with thickened pre-existing trabeculae and swathes of new connective tissue cut the glands into segments. The intervening tissue is Hodgkin's tissue in which rultinucleate giant-cells are unusually numerous. Fibrils, fibrillae, and coralline deposits of collagen are present in most areas. In one or two areas histiocytic reticulum cells 
are seen and in a few the sclerosing reticulum cells are so uniform that they recall fibrosarcoma. Eosinophils are present and tend to occur in foci. Plasma cells and neutrophils are present, the latter chiefly related to necrotic foci which are common. Activity is higher than usual, the average mitoses per high power field being 2 .

Diagnosis. Atypical Hodgkin's disease, almost Hodgkin's sarcorna. 
Alexander Laird. Aet 61 jears. A bricklayer's labourer. Ref. G.R.I. T.S. 3281:53. History. Developed a firm painless swelling in the right groin in February 1953, otherwise no complaint. His general health appears good.

The node is entirely neoplastic. The capsule shews a little local thickening but the lamellae are frequently disrupted by infiltration with imnature lymphocytes. The exodic growth phenomenon is present. Trabeculae are poorly developed, they tend to be displaced obliquely. Perivascular fibrosis is appreciable but inconstant, other forms are slight. There are small false follicles in the deeper parts of the node and these shew filamentous collagen between contiguous surfaces. The tumour is predoninantly composed of morbid mononuclear reticulum cells which exhibit much mutual pleiomorphism. Typical Hodgkin giant-cells are present but scanty. Eosinophils are present but require close search. Plasma cells and neutrophils are conspicuously few. The growth rate, judged by mitoses is high, many fields contain 5 or more mitotic figures. Necrosis is limited to individual cells. Deposits of the growth are present in lymphatics. The adjacent fat is invaded.

Diagnosis. Iymphoid tissue sarcoma. Intermediate type. (Reticulo/lymphosarcoma). Possibly originating in Brill's disease?. Possibly proceeding towards reticulum cell sarcoma. 
Archibald Pearson. Aet 30 years. A marine engineer. Identity Ref. G.R.I. J.S. 3478:53. History. Inlarged lymph nodes in the neck of several weeks duration.

All normal structure is lost. A vague follicular pattern is discernible, the chinks between the irregular nodules being filled with lymphatic and blood vessels containing lymphocytes and a few necrotic cells. The capsule where preserved shews gross hyaline thickening. Trabeculae are few but shew fibrous thickening, especially at their bases. Fibrosis is well established. Perivascular deposits are notable and diffuse fibril formations of scar-like distribution are seen. The reticulum cell increase is not marked but characteristic large morbid mononuclear cells are readily found. Hodgkin giant-cells are few. Eosinophils are present but are not numerous. Plasma cells and neutrophils are scanty in most parts. The growth rate is about average 1-2 mitoses per high power field. Necrosis is not present.

Diagnosis. Hodgkin's disease, typical. Early, fibrosing from the start, diffuse growth. 
Dorothy Fox. Aet 27. Ref.G.R.I. 3669:53. History. Swellings have appeared in both sides of the neck and both axillae over the past four weeks. Clinically the size increase is still active.

This is a supraclavicular lymph node shewine the metamorphosis of Hodgkin's disease to Hodgkin's surcoma. A crescentic deposit of normal hyperplastic lymphoid tissue is visible at one segment of the sub-capsular periphery. Follicles, some with pale centres, loose lymphatic tissue, and pieces of sinus survive. In the latter channels morbid reticulum cells are numerous. The exodic growth phenomenon is well shewn. The capsule is grossly thickened and hyaline, some concentric re-formation is discernible. Transgression is not present at the levels eximined. Thuch fibrosis of all forms is seen. New trabeculae are forming from swathes of young connective tissue, and cut the node into segments. The reticulum cell proliferation is unusulily ilorid with the production of copious irregularly pleiomorphic morbid types, Hodgkin giant-cells are very numerous. The latter often occur in agregates. Eosinophils are still present cifiefly as scattered small groups. Plasma cells are common, the neutrophils are copious near the focal necroses which are present. Hitoses average 2 per H.P. field.

Diagnosis. Hodgkin's disease undergoing transition to the variant Hodgkin's sarcoma (II) of Warthin. Eosinophils less obvious. Hodgkin giant-cells very numerous. 
Andrew Shearer. Let 46 years. A tractor driver. Ref. G.R.I. I.S. 4057:53. History. He developed a painless swelling in the left groin one year ago. This has increased in size gradually and he now has hard masses palpable in the left iliac fossa, the right groin, in both sides of the neck, and axillae. General health has remained good.

The specimen is a large axillary node, $3 \times 3 \times 1.5 \mathrm{~cm}$. A thin marginal rind of normal reactive lymphoid tissue is discernible with occasional pieces of sinus tissue. The exodic growth phenomenon is displayed. The capsule is of normal thickness in its greater part but at one locality is increased. In a few places concentric re-formation is detected. The new tissue occupies all the central part of the node, it displays a very faint follicular pattern, the individual false follicles being small, and dense centrally. Fibrosis of all forms is discernible but the amount is small. The commonest cell present is an internediate reticulum cell type, but morbid mononuclear types are present and a few mirror image binuclaate cells can be found. Hodgkin giant-cells are absent. Posinophils and neutrophils are extremely hard to find but some plasma cells are visible. Witoses average 2 per H.P. field.

Diagnosis. Brill's disease, becoming diffuse. Regarded as disseminated. Fields exist which are strongly reminiscent of Hodgkin's disease, especially near the fibrous deposits in the pulp. 
George Fairlie, Het 54 years. A storekeeper. Ref. G.R.I. 4824:53. History. Complaint of sudden pain in the right iliac fossa accompanied by sickness. Examination revealed local swelling. This is a deep pelvic node reinoved at operation.

This is a lymph node shewing active soft cellular Hodgkin's disease which is tumour-like in structure. The identity as lymph node is almost certain, lymphatics with valves are present in the capsule. Meagre crescents of reactive lymphoid tissue including follicles, some with pale centres are present near the inner aspect of the capsule. The exodic growth phenomenon is passing off but in several places the expanding nodules are driving towards the periphery. The new tissue is arranged in an indistinct folliculoid pattern. The capsule is much thickened but hyalinisation is restricted to the outer zone. Concentric re-formation is discernible, infiltration is not. Fibrosis is slight except for the capsular and trabecular components, the latter are composed of compacting fibrils. Fibrillar deposit is universal in the new tissue, the silver impregnation and van Gieson picture are closely similar. Perivascular lamellae and coralline deposit are less marked. The reticulum cell proliferation is extreme. The cells produced are highly pleiomorphic; epithelioid cells, morbid mononuclear types and Hodgkin giant-cells are all present in profusion. Eosinophils are distinctly scanty. Plasma cells are present in the usual numbers, neutrophils are copious round several infarct-like areas of necrosis which are present. Mitoses average 2-3 per H.P. field. 
$204 a$.

Diagnosis. Atypical Hodgkin's disease. Soft cellular tumour-like variety. Eosinophils disappearing. Regarded as transition to morphological variant Hodgkin's sarcoma of Warthin (type II).

Note. In Warch 1954 this patient developed nodules in the skin which shewed reticulum cell sarcoma, eosinophils not present. 
Margaret Galloway. Aet 17 years, A book-keeper. Ref.G.R.I. A.S. 4420:53. History. Patient presented with a glandular swelling in the posterior triangle of the left side of the neck. It was painless and had been present six months. Examination revealed enlargement of the mediastinal nodes on the left side and a moderate neutrophile leucocytosis. Ilsewhere the nodes were normal clinically and the spleen was not palpable. General condition was good.

This is atypical Hodgkin's disease which is apparently a slowly evolving type. The specimen consists of a small physiologically hyperplastic node and a lymphoid mass. The latter is organised as nodules of Hodgkin's tissue set in a coarse fibrous mesh. The nodules are irregular in size I-2 $\mathrm{mm}$. diameter and the mesh up to $1 \mathrm{~mm}$. thick. Capsule is not represented but gross trabecular thickening is seen. Fibrosis is pronounced, it is seen in all forms, especially as compacted diffuse fibrils. Relatively little is seen in the cellular parts of the growth. The reticulum cell proliferation is only moderate and tends to be focal. Morbid mononuclear types and Hodgkin giant-cells are easily identified. Eosinophilia is slight but plasma cells are numerous., The latter occur often in dense foci in the peripheral part of the specimen. Neutrophils are sparse, most are seen in the few small necrotic foci which are present. Mitoses are not seen in many H.P. fields.

Diagnosis. Atypical Hodgkin's disease. Few eosinophils, reticulum cell proliferation focal. Follicular pattern well shewn. Affinities to Benign Hodgkin's disease and Brill's disease. 
John Bibby. Het 45 years. A labourer. Ref. G.R.I. 4467:53. History. Enlarged painless lymph nodes have been present in the neck and both axillae for three months.

This is a large axillary lymph node shewing atypical Hodgkin's disease of a peculiar type. Persistence of normal structure is difficult to assess. Much apparently normal lymphoid tissue is present throughout the node and in one part, fragments of an atrophy fat-cone are preserved. This lymphoid tissue contains many small solid follicles, the arrangement being better indicated in the silver impregnation. The exodic growth phenomenon is absent. The new growth is patchy. Most of the reticulum cell overgrowth is seen in the locus of sinuses, but some is present in patches of the new tissue related to fibrosis deep in the node. Scattered morbid cells can be found in the pulp away from sinus tissue; typical morbid mononuclear and Hodgkin giant-cells are numerous. Transition from littoral cells can be traced. The capsule is much thickened but the inner layers are not yet hyalinised. Concentric re-formation is not present, and infiltration is not appreciable. Fibrosis is slight, most is expressed in perivascular lamellae and swathes of compacting fibrils in new trabeculae. Eosinophils are sparse. Plasma cells and neutrophils are fairly numerous, the latter being common round small necrotic foci which are present. Hitoses average 1 per H.P. field in the lesions.

Diagnosis. Atypical Hodgkin's disease. An unusual type. Eosinophils sparse. Morbid reticulum cells tending to be localised to sinus loci. Possible sinus origin in this case. 
Joseph Hollywood. Aet 23 years. Ref. Law I.P. 811:53. History. Patient has noticed swelling of the lymph nodes of the left side of the neck for six months. The enlargement is painless and there are no other symptoms. The nodes were firm and matted, rendering biopsy difficult.

The specimen is a cervical lymph node $18 \times 14 \times 10 \mathrm{~mm}$. There is a very tenuous rind of hyperplastic lymphoid tissue at one point, it is crescentic and isolated between the true and false capsule, the latter is the result of concentric re-formation. The true capsule is irregularly thickened and becoming hyalinised, definite breaches have been made in it at several places with spread of the turnour into the external tissue. Fibrosis is conspicuous; all forms including direct symplasmic transformation are evident. The silver impregnation and van Gieson stained preparations coircide remarkably closely with the haematoxylin and eosin section. The reticulum cell proliferation is alnost universal and productive of uniform cells. Thile the typical morbid mononuclear and Hodgkin giant-cells are easily found the majority are immature lymphocyte and intermediate types. Eosinophils are absent, plasma cells and neutrophils are rare. Mitotic figures are rare, below 1 per H.P. field. Necrosis is limited to individual cells.

Diagnosis. Atypical Hodgkin's disease, tumour-like, regard as malignant (transgression of capsule etc.) No eosinophilia. Affinities to reticulum cell sarcoma and primitive lymphosarcoma. 
Samuel Bickley. Aat 57. A tailor's presser. Ref. G.R.I. 4583:53. History. Wultiple swellings of the neck, axillae, and groins which have been present for four years. The specimen sent consists of three cervical lymph nodes $15-5 \mathrm{~mm}$. diameter.

This is a relatively pure small cell lymphosarcoma which may have arisen from a follicular lymphoma since a pseudo-follicular pattern is discernible in the deeper parts of the nodes. Persistence of normal structure is doubtful, several reaches of what may be normal (reactive) marginal sinus are present. The exodic growth phenomenon is absent. The node substance is replaced by a mass of immature small lymphocytes which in the deeper parts are arranged in a poorly organised microfollicular pattern. The centres of these follicles are appreciated by being slightly less densely packed. The capsule is generally thin but local thickening is observed at several points. Fibrosis is absent, several vessels shew hyalinisation but distinct perivascular lamellae are absent. The vast majority of cells are immature small lymphocytes. A very few morbid mononuclear reticulum cells are present but typical Hodgkin giant-cells are not found. Bosinophils, plasma cells and neutrophils are absent. Mitoses average 1 per H.P. field, necrosis is absent.

Diagnosis. Srnall cell Iymphosarcoma, pure; some affinities to follicular lymphoma. 
John Young. Aet 58 years. A labourer. Ref. G.R.I. 4643:53. History. Swellings in the neck were noted first five years ago, these increased in size very slowly. On admission gross dropsy, cough and dyspnoea, and generalised lymphadenopathy with splenomegaly were present.

This is of doubtful category, it may be regarded as highly cellular tumour-like Hodgkin's disease and with equal justification as reticulum cell sarcoma. (Hodgkin's sarcoma). The specimen is a cervical lymph node $10 \times 9 \times 8 \mathrm{~mm}$. At several points at the edge crescentic pieces of hyperplastic normal lymphoid tissue survive; they are simple loose lymphatic tissue without follicles. The exodic growth phenomenon is well seen. The new tissue occupies the remainder of the node and is of very uniform diffuse habit - a large single nodule. The capsule shews local thickening but is not hyalinised at these points although it is elsewhere. Concentric re-formation and infiltration are not present. Fibrosis is present in the new tissue in all forms but it is sparse. The reticulum cell proliferation is florid, the great majority of the cells being free with morbid single-lobe nuclei of "owl-eye" type. A few epithelioid cells are present, and Hodgkin giant-cells are very numerous (4 per H.P. field). Eosinophils are present but scanty. The remaining cells are merely occasionally encountered. Witoses average 5 per H.P. field, necrosis is limited to individual cells.

Diagnosis. (Highly cellular tumour-like Hodgkin's disease,
(or reticulum cell sarcoma with Hodgkin's giant-cells.
(Hodgkin's sarcoma).

Note. Patient died 18th November 1953, see Special Necropsy 418:53. G.R.I. 
Mrs. Mair. Ref.G.R.I. 4877:53. History. A painful swelling in the left axilla of two weeks duration.

These are axillary lymph nodes containing nodular foci of reticulum cell sarcoma of stem-cell type with some multinucleate giant cells. These tumour nodules are extensively necrotic centrally. Surrounding them the node tissue shews the changes of typical Hodgkin's disease and it in turn is enveloped in normal reactive lymphoid tissue. Peripherally normal reactive ljomphoid tissue with follicles and occasional remnants of sinus tissue. The Hodgkin's disease areas are diffuse in the pulp adjoining the nodules of reticulum cell sarcoma. The exodic growth phenomenon is shewn by both tumours. The true node capsule is much thickened by lamellae of young connective tissue, hyalinisation is confined to the outermost parts. Concentric reformation is appreciable. Infiltration is not seen. Fibrosis is chiefly fibril and fibrillary, the other forms are seen but are little in amount. The reticulum cell sarcoma is not sclerosing much. In the Hodgkin lesion the reticulum cells conform to the usual morbid types including typical Hodgkin giantcells. Eosinophils are numerous, many are large myelocytic or lymphocytoid types; they are much fewerin the reticulum cell sarcoma. Neutrophils are frequent; most occur in relation to the large necrotic centres of the tumour nodules. Mitoses average 1 per H.P. field in the Hodgkin's lesion and 5-6 per H.P. field in the reticulum cell sarcoma. In the latter the type cells closely approximate to morbid mononuclear types and are free cells. Small giant cells are common. 
Diagnosis. (Hodgkin's disease, typical.
(Reticulum cell sarcoma, stem-cell type, chiefly.

(A form of Hodgkin's sarcoma, note the disproportionate rate of growth of the latter part; the Hodgkin lesion is early since the whole node is not involved). 
Ian Ross MaNab. Aet 19 years. A student. Ref. G.R.I. 5035:53. History. Swelling of the cervical lymph nodes for nine months, recently the axillary nodes have enlarged. Subcutaneous nodes are palpable across the clavicles, progressive dyspnoea and cough are developing.

The specimen is a cervical lymph node shewing Hodgkin's disease which is acceptable as typical although large cells are more numerous than usual. Some highly reactive but probably physiological lymphoid tissue persists. This is seen in a small adjacent node included in the specimen and in a tongue-shaped process in the morbid one. The exodic growth phenomenon is just discernible in a few places. The tumour exhibits a very faint folliculoid habit of growth. The capsule is irregularly thickened, the process is best seen at the base of the trabeculae, hyaline change is in progress. Concentric re-formation is hardly appreciable, there is not any infiltration of the capsule. Fibrosis is seen in all forms. The fibrillary component is very well illustrated. The reticulum cell proliferation is florid. The production of large morbid mononuclear types is above average. Hodgkin giant-cells are numerous, many are small. Eosinophilia is marked, a small proportion of lymphocytoid variety are identified. Plasma cells and neutrophils are scanty. The majority of the lymphociztas are imiuature with large nuclei. Mitoses average $I$ per H.P. field. Necrotic foci are small and scanty.

Diagnosis. Hodgkin's disease, accept as typical; large cells rather above average, verging towards cellular type. 
David Milne. Aet 53 years. Ref. Law 915:53. History. Swelling was first noted in the neck in April this year. Primary tumour was suspected and two biopsies were performed, neither of these has become available for study. The patient developed a further swelling in the right axilla recently. The general health is good.

This is an axillary lymph node shewing typical Hodgkin's disease at a late stage; suppuration is added. There is not any survival of normal structure. The growth is folliculoid, the cellular parts of the lesion being set in a matrix of maturing collagenous connective tissue. The capsule where it can be identified is grossly thickened to $1 \mathrm{~mm}$. or more. Hyalinisation is incomplete and concentric re-formation is poorly shewn. Infiltration is not seen but there is fusion with an adjacent node. Fibrosis in all forms is marked. The reticulum cell proliferation is of the usual order; morbid mononuclear types and typical Hodgkin giant-cells are conspicuous. Eosinophilia is moderate, the cells occur in foci in both the cellular and fibrous components. Neutrophils are very numerous in micro-abscesses which abound, in these areas necrosis is notable. Plasma cells are rare. The lymphocytes are immature in many parts. Mitoses are of the usual order.

Diagnosis. Hodgkin's disease, typical, late. Folliculoid habit well shewn, micro-abscesses common. 
Daniel Bowman. Aet 38 years. A driver. Ref. G.R.I. 5119:53. History. Complaint of glandular swellings in the groins, axillae and neck, present for two months. In addition ulceration has developed round the anus. The tassermann reaction is negative, $\mathrm{X}$-ray of the chest is negative and Frei's test is negative.

This is a cervical lymph node shewing a soft cellular tumour-like atypical Hodgkin's disease. (The skin lesion is tuberculous ulceration in which the microbes were demonstrated).

Normal lymphoid tissue is absent and the exodic growth phenomenon is not seen. A patchy folliculoid organisation of the new tissue is discernible. The capsule is slightly but uniformly thickened. Hyaline change is early. Infiltration is doubtful, two small intra-capsular deposits of lymphoid tissue which are seen are almost certainly physiological. Fibrosis is slight. All forms can be made out except coralline. Wost is still fine argyrophile fibrillary. The reticulum cell proliferation is marked and productive of nany morbid mononuclear cells, Hodgkin giant-cells are meagre. Dosinophils are absent in significant numbers, neutrophils and plasma cells are rare. Necrosis is not a feature and mitoses are of the usual order.

Diagnosis. Atypical Hodgkin's disease. No eosinophilia. Soft highly cellular tumour-like type. 
Villiam Christie. Aet 29 jears. Ref. G.R.I. 5219:53.

History. Glandular swellings have been present in the neck for the past ten weeks. They are painless and are not tender on palpation. No other accessible nodes are enlarged.

The specimen onsists of two cervical lymph nodes shewing identical changes. The lesion is probably nearest to atypical Hodgkin's disease but features of follicular lymphoma and lymphosarcoma are also displayed. Normal architecture and structure are almost entirely lost. The exodic growth phenomenon is not discernible. The node substance is replaced by a more or less well defined follicular fornation in the interstices of which is tissue conforming to that of cellular Hodgkin's disease. In a few of the follicles nornal pale centre cells are present but rost are almost entirely composed of large immature lymphocyte nuclei, rare morbid reticulum cells are seen. The capsule is thickened fairly uniformly, infiltration by lympiocytes is seen, and some lympatics are filled with these cells. Concentric re-fornation is not a feature. Fibrosis is slight. Trabeculae are not represented to any extent. Diffuse fibrils and fibrillae are present but scanty, occasional vessels shew very characteristic perivascular lamellation. Coralline deposit is not seen. The reticulum cell proliferation is notable only in the inter-follicular matrix. Worbid mononuclear and typical Hodgkin giant-cells are numerous. Eosinophils nearly all of blood type are copious in these areas. Plasma cells and neutrophils are noted. Mitoses average less than I per H.P. field. Necrosis is common in the giant cells. 
Diagnosis. Atypical Hodgkin's disease, fibrosis slight. Co-exists with morbid follicular imnature lymphocyte growth. Some affinities to lymphosurcoma. Compare with Serial 203. 
Lirs. Annie Leggate. Aet 73 years. A housewife. Ref. G.R.I. 36:54. History. Presented clinically as a case of carcinoma of the right breast. The mamma was removed surgically. Taked eye a scirrhus was not found, instead numerous larje crimson lymph nodes presented. Inquiry elicited that the patient was treated at home four months previously for glandular fever, diagnosed solely on clinical grounds. (At examination an enlarged node was found in the left axilla). The patient died after operation and necropsy was not obtained.

The absence of cancer was confimed by microscopy. The clinical tumour is caised by a lymphoid tissue tuncur. The turnour does not conforn to those oruinarily recopnised but is morphologically nearest to cellular Hodgkin's disease. All nodes shew the same changes. Myeloid leukaemia is also a possibility. There is total loss of normal structure. The new growth occupies all the substance of the node. The capsule is much thickened by hyaline fibrosis, $1.0-1.5 \mathrm{~mm}$. thick. It is not infiltrated and recalls the appearance of Hodgkin's disease vividly. Fibrosis is otherrise slight, all forms apart from coralline deposit can be found but the perivascular form is not typical. The reticulum cell proliferation is pronounced. Epithelioid cells are common, and next in frequency, morbid mononuclear types. Small Hodgkin giant-cells are identified. Eosinophils, including many lymphocytoid types, are very numerous. Plasma cells are also remarkably comnon. Neutrophils are sparse. Mitoses average 4-6 per H.P. field. Nearly all lymphocytes present are immature forms. Necrosis is rare and confined to individual cells. 
Diagnosis. Iymphoid tissue sarcoma, indeterminate category but nearest to cellular Hodgkin's disease. Eosinophilia pronounced, fibrosis slight, less capsular. Affinities to reticulum cell sarcoma with sone lymphoid propensities. (Similar to nyeloid leukaemia picture).

Note. Periglandular infiltration is seen in the breast, and abnormal white cells are also seen in some blood vessels. The circulating blood was not studied unfortunately and this diagnosis cannot be confirmed. In any event such gross enlargement of lymph nodes is not typical of myeloid leukaemia. 
Ian Kirsop. Aet 25 years. A clerk. Ref. G.R.I. 86:54. History. A painless slowly increasing swelling in the right side of the neck of some months duration. The swelling is slightly fluctuant. Naked-eye the cut surface of the node is a delicate café au lait tint and a faint follicular pattern is discernible in it.

This is typical Hodgkin's disease at an early stage. Persisting normal structures include lymphoid follicles, several with normal pale centres, and survival islets of lymphocytes. These are chiefly disposed peripherally in crescentic areas. The exodic growth phenomenon is well shewn. The new tissue is developing almost exclusively in the pulp of the node and is diffuse. The capsule is hyaline and doubtfuliy thickened, concentric re-formation is not appreciable at one level. Infiltration is absent. Fibrosis is slight. Trabecular thickening is visible and rare perivascular deposit can be found. Fibrils and fibrillae are discernible on close scrutiny especially in the van Gieson stained section and the silver impregnation, symplasmic transformation and coralline deposits are not seen. Reticulum cell proliferation is well shewn. Epithelioid and morbid mononuclear forms are readily found, the latter are highly characteristic. Hodgkin giant-cells are few. Eosinophils are very numerous. Plasma cells and neutrophils are readily found. One typical focus of necrosis is present at one level. Mitoses are few, below I per H.P. field.

Diagnosis. Hodgkin's disease, typical, early. Almost restricted to the pulp. Fibrosis slight, eosinophilia marked. 
Mrs. Catherine Cameron. Aet 30 years. Ref. Dundee R.I. 1186:51. History. Inlarged lymph nodes present in the right inguinal region for 15 months. No other lymph nodes palpable. She had a phlebitis of the right leg in 1950, it lasted a month.

This is part of a large node shewing early typical Hodgkin's disease. The growth is cellular and fibrosis is still slight. Persistent normal structure is identified but is fragmentary, one germinal follicle with a pale centre is seen together with remnants of marginal sinus, at the extreme sub-capsular edge. The exodic growth phenomenon is not convincingly detected. The capsule is still of nornal thickness and cellularity. The new tissue is essentially diffuse in habit although a delicate irregular segmentation is detected with very low power study. Fibrosis is light; new compaction trabeculae of diffuse fibrils are appearing, they have no connection with the capsule. Fibrillae are scanty, likewise perivascular accretions. Symplasmic transformation is in process in patches. Coralline deposits are absent. The reticulum cell proliferation is even and productive of epithelioid, morbid mononuclear and Hodgkin giant-cells. Eosinophils are numerous but other granulocytes and plasma cells are rare. The lymphocytes are slightly immature. Hitoses average 1 per H.P. field, necrosis is absent.

Diagnosis. Hodgkin's disease, typical. Early, fibrosis slight, rather cellular. 
David Hatton. Aet 47 years. Ref. Law Hospital 828:52. History. For the past 15 months asthenia, profuse sweating, and Ioss of weight have become increasingly severe. Severe secondary anaemia was found and enlarged glands were discovered in the right groin.

This is an inguinal lymph node shewing atypical Hodgkin's disease, eosinophils are absent, and necrosis is unusually marked. The growth is distinctly tumour-like in some parts. Further features of interest are the presence of fat tissue in the node substance and evidence of fat atrophy, presumably previous to prinary growth in the gland. Normal lymphoid tissue does not survive, and consequently the exodic growth phenomenon is absent. The new tissue is chiefly irregularly diffuse and micro-folliculoid. The capsule is atrophied to extinction at one pole due to previous fat atrophy. At this point the Hodgkin tissue reaches the fat line directly. Elsewhere thickening is doubtful. Fibrosis is chiefly in fibril and fibrillary form diffusely throughout the new tissue, symplasmic transformation is also seen. Perivascular lamellae and coralline deposits are scanty. The reticulum cell proliferation is productive of many different cell types. Epithelioid and morbid mononuclear varieties with transition forms are commonest, Hodgkin giant-cells are also conspicuous. Eosinophils are virtually absent, and plasma cells and neutrophils are rare. Lymphocytes of mature type are still the predorninant cells in most areas. lhitoses number about 1 per H.P. field, or less. Lassive necrosis of infarct type is marked, in addition individual cell death is very common throughout otherwise viable growth. Diagnosis. Atypical Hodgkin's disease. No eosinophilia. Much necrosis. Diffuse and turnour-like in parts. 
Harie Traynor. Aet 17 years. Ref. Hairmyres Hospital 1735:52. History. Since June of this year the patient has experienced general malaise and presents a painless swelling in the left side of the neck.

These are cervical lymph nodes shewing an uncommon variant of atypical Hodgkin's disease. There is very extensive production of fibroblastic.cells. Small wedges and crescentic areas of reactive lymphoid tissue survive in both nodes. The exodic growth phenomenon is not evident however. The new tissue is disposed in coarsely segmented fashion with much maturing fibrosis round the more cellular nodules. The capsule is still fairly cellular but is grossly thickened, concentric re-formation and infiltration are absent. Fibrosis is pronounced, all forms except symplasmic transformation are visible. The reticulum cell proliferation is very widely given over to cells of fibroblastic characters. Epithelioid cells are rare, morbid mononuclear and Hodgkin cells tend to be aggregated in ill defined foci. Eosinophils are numerous, other granulocytes and plasma cells are cormoner than usual and appear related to necrosis. Mitoses are impossible to assess in this preparation but are probably few. Necrotic foci of characteristic type are seen.

Diagnosis. Atypical Hodgkin's disease. Diffuse fibroblastic type. 
Douglas Law. Aet 23 years. Ref. Eairmyres 804:53. History. Hultiple glandular swellings were discovered in the neck during investigation of bouts of vomiting and jaundice which have developed since February of this year. There is an old history of pulmonary tuberculosis.

This is a cervical lymph node shewing almost typical Hodgkin's disease, the features short of the ideal include very general and conspicuous early fibrosis, and very striking reduction of lymphocytes. Persistence of normal structure is seen. A small peripheral crescent of reactive lymphoid tissue with sinus tissue survives, in addition several lymphoid follicles with pale centres are seen at one point. The exodic growth phenomenon is present but not conspicuous. The tumour is distinctly, if faintly, follicular in habit. Capsular thickening is not impressive possibly due to artefact loss, concentric re-formation is absent. Fibrosis is widespread, diffuse and marked. New filamentous trabeculae are seen springing frorn the capsule. Perivascular deposit is slight. Diffuse fibrils and fibrillae constitute most of the new collagen, the other types are rare. The reticulum cell proliferation is chiefly productive of epithelioid cell types but morbid mononuclear and Hodgkin griant-cells also abound. Bosinophilia is of the usual order. Plasma cells and neutrophils are few. The reduction of small lymphocytes is striking. Witoses average 1 per H.P. field, necrosis is restricted to occasional individual cells.

Diagnosis. Atypical Hodgkin's disease, cellular, diffuse fibroblastic type. Warked lymphocyte reduction. 
Whs. Wargaret Royal. set 72 jears. Housewife. Ref. G.R.I. 1860:53. History. A swelling apjeared in the left side of the neck in llarch 1953 (about two nonths aso). This was provisionally diagnosed as a salivary gland calculus and resected.

This is an almost pure reticulum cell sarcoma. The tumour is fibrosing and in some areas where this is nore in evidence the picture of Hodglin's disease is closely simulated. The specimen is almost certainly a lymph node, a marginal rind of small lymphocytes persists in places but otherwise all vestige of nornal structure is absent. The tumour is very uniform and diffuse in most blocks; in one, scar-like areas of fibrosis and diminished cellularity mimic Eodgkin's disease. The capsule is slightly thickened and hyaline, concentric re-formation is absent and transgression is doubtful. Fibrosis is slight. Wost is in the form of a delicate fibril and fibrillary filigree. Some swuthes of compacting fibrils are seen, perivascular larnellae and corilline deposit are rare. The reticulum cell neoplasia is an almost pure snall norbid mononuclear type, rare multinucleate types are encountered but their cytoplasm is deficient. Eosinophils are very rare, plasma cells and other granulocytes are virtually absent. Hitoses average 5 per H.P. field. Necrosis is fairly pronounced, the foci are poorly demarcated.

Diagnosis. Reticulum cell sarcoma, almost pure single cell type. Some affinities to Hodgkin's disease. 
lirs. Colburn. Aet 45 years. A housewife. Ref. G.R.I. 510:54. History. Swellings have been noticed in the neck for the past three months. Recently those on the left side have become larger and softer. A provisional diagnosis of tuberculous lymphadenitis was made. (After recognition of the true pathology a thorough search was made to discover the primary growth, this has so far eluded detection).

This is an example of metastatic carcinoma of squamous cell type in lymph nodes. In several blocks the sinilarity to Hodgkin's disease is remarkable. The isolated tumour cells mimic morbid reticulum cells to perfection and have evoked a copious diffuse fibroblastic reaction in their vicinity. In addition eosinophil infiltration is marked. There is an indistinct follicular pattern which also recalls the disease. In the remaining blocks, chiefly from the nodes on the left side shew easily recognisable deposits of squamous cell cancer.

Diagnosis. Metastatic squamous cell carcinoma simulating Hodgkin's disease. Primary tumour undiscovered. 
223.

APPENDIX B.

NECROPSY SERIES PROTOCOLS 
224.

\section{NECROPSY SERIES PROTOCOLS}

These are in the same order as in the text, Volume I.

p. 227. The sheets of the individual necropsy

reports are sub-numbered.

Illustrations.

Volume III Figs. 117-205

Volume IV p. 61-87. 
James Raeside, aet 62 years. Ref. \$.16:49.

Anamnesis. The patient, a retired carter was admitted to the Bastern District Hospital, Glasfow, on 25th October 1949. He complained of swelling of the lower limbs and scrotum of about three months duration. Loss of weight, breathlessness, and anorexia were also present. During his stay in hospital numerous investigations were carried out but the results were negative or inconclusive. A tentative diagnosis of neoplasm of lung was considered because his condition was deteriorating rapidiy and lymph nodes became palpable in the axillae. A slight irregular fever persisted throughout his tine in hospital and on 15th November a leucocytosis of 33,200 per cu.mm. was recorded. Blood taken for culture on several occasions was sterile. Death occurred on 3rd December 1949.

\section{Report of Necropsy.}

9 hours post-mortem.

External. Emaciation is present but is partly masked by dropsy.

Reticulo-endothelial system. Iymph nodes. There is generalized lymphadenopathy. The cervical lymph nodes are numerous, slightly enlarged and very firm; at the base of the neck they are partly natted together. The cut surfaces are a prevailing greyish-white with yellowish-white bands running irregularly from the capsules so as to cut the surfaces into a mosaic. In both axillae the nodes are enlarged, hard, and discrete. Then cut they reveal the same appearances as in the cervical group. The mediastinal nodes are grossly enlarged, the right inferior tracheo-bronchial gland measures $5 \times 3 \times 2 \mathrm{~cm}$. They are hard and nodular. The cut surfaces are coarsely mottled grey and white. In the abdomen the nodes along the upper border of the pancreas and alongside the aorta are the most conspicuously enlarged. On section they are seen to have thin tenuous capsules and fleshy homogeneous yellowish-pink gland substance. The nodes of the mesenteric group are unduly numerous but not much larger than normal. In both groins the nodes are large, hard, and closely similar to the axillary group. The spleen is roderately enlarged and firm due to chronic venous congestion. The cut surface is pale crimson and homogeneous except for a few small rounded nodules of yellowish-white tissue deep in the pulp.

Cardiovascular system. A terminal sero-fibrinous pericarditis is present, the heart shews only mild brown atrophy. There is senile ectasia of the aorta.

Respiratory system. Sero-fibrinous effusion is notable in both pleural sacs, (left cavity $1200 \mathrm{cc}$, right cavity $500 \mathrm{cc}$ ) Both lungs are slaty grey, small, and partly collapsed. In the right upper lobe several small ill-defined abscesses are noted. In the right eparterial bronchus the mucosa is nodular but not ulcerated.

Alimentary system. The oesophagus, stomach, and intestines are normal. 
The liver is moderately enlarged and displays the nut-meg pattern of chronic venous congestion. There are several widely scattered deposits of firm white tissue about $I \mathrm{~cm}$. diameter throughout the viscus. They are more angular and less discrete than typical metastatic cancer. The remainder of this system is normal.

Genito-urinary system. A small series of aligned soft yellowish-white nodules $2 \mathrm{~mm}$. in diameter are present in the mucosa of the bladder, near the urethral orifice. The kidneys, ureters and prostate gland appear normal.

Haemopoietic system. The marrow in the ribs is copious, pale red, and contains minute white nodules. In the cervical vertebrae similar appearances are seen. The red marrow of the femur extends down to within $12 \mathrm{~cm}$. of the lower end, a few minute nodules are discernible.

Endocrine system. The thyroid gland is small and fibrous. The single parathyroid gland identified is normal, and the adrenal glands are natural.

Wicroscopical examination:

General description of the lesions encountered.

Late typical Hodgkin's disease with very extensive fibrosis and scanty eosinophils is restricted to certain lymph nodes and small fields of the prostate gland. In nost sites the lesion corresponds to the 'Hodgkin's sarcoma' of Varthin. Transitions between the two pictures, which are regarded as simply different degrees of the same process, are evident. In the majority of the latter appearance the growth presents a poorly demarcated nodular habit. The tissue is made up of Hodgkin giant-cells in profusion, morbid mononuclear reticulum cells, fibroblasts, and fibrocytes. Interspersed between these aggregates is a variable number of small lymphocytes, neutrophils, and plasma cells. Eosinophils are absent. Collagen is generally conspicuous as a filigree of medium and tenuous fibrils. Several exceptions are noted; in the abdominal nodes fibrosis is scanty and the type cell is a morbid mononuclear variety, the appearances being relatively uniform. In a right cervical node a distinct whorling of fibroblastic cells is visible producing an appearance which recalls fibro-sarcoma. None of the sarcomatous lesions appear very rapidly growing. (There was not any antecedent biopsy in this case so that comparison is not possible). Microscopical evidence of tuberculous or other disease is lacking.

Distribution of the lesion. (See diagram).

Both processes are observed in nodes from the cervical, axillary, and inguinal regions. The mediastinal nodes also shew this mixture, and so does one large right pelvic node. In the abdomen all the retroperitoneal nodes examined and the mesenteric nodes shew the sarcomatous Iesion. One node from the portal fissure is exceptional in that the 
ordinary form of the disease co-exists. The bone marrow from one sample of rib shews the sarcomatous lesion, likewise a piece of cervical vertebral body. The femoral marrow is not involved. The picture of Hodgkin's sarcorna is seen in all the spleen and liver lesions examined. The adrenal glands are free of the tumour. Extra-nodal deposits of the sarcomatous form are confirmed in the bladder and prostate gland, the latter also exhibiting fields which would qualify for ordinary Hodgkin's disease. The lungs and kidneys examined by random blocks are free of either lesion.

Diagnosis and Sumnary.

$$
\begin{aligned}
& \text { (Eodgkin's disease. } \\
& \text { (Hodgkin's sarcoma (Warthin type II). }
\end{aligned}
$$

The latter preponderates and appears to have arisen on a basis of the former. This case is interpreted as an instance of metamorphosis of Hodgkin's disease to Hodgkin's sarcoma. The latter is an highly pleiomorphic tumour exhibiting a low degree of malignancy. Anasarca and hydrothorax are notable. Survival of normal lymphoid tissue is slight. 
Agnes McNiven, aet 32 years. Ref. G.R.I. P.in.201:49.

Anamnesis. The patient was fairly well till 1945. At the end of this year she began to experience vague weakness. This hardly amounted to indisposition till early in 1948 when her strength failed and she was obliged to leave work. Her sister died in 1940 at the age of 18 of pulmonary tuberculosis, and she has two brothers at present suffering from the same disease. In view of this it was surmised that she might have the same complaint, but investigation revealed only enlarged lymph nodes in the mediastinum. She was admitted to Stobhill Hospital in May 1948. At this time a painful swelling appeared in the left side of the neck, and in the following month the right side also became affected. A cervical lymph node was removed for microscopical examination 12 th october 1948 and was reported to shew the changes of very active Hodgkin's disease. (Ref. 0578:48. Serial 188). By December of this year both axillae presented swellings and cachexia set in. Deep X-ray therapy was administered with transient benefit; her clinical condition deteriorated steadily and she suffered considerable pain up till death on 5 th July 1949.

Report of Necropsy.

6 hours post-mortem.

External. An extremely cachectic woman, the skin over the chest is brownish-grey and desquamating.

Reticulo-endothelial system. Lymph nodes. The superficial cervical lymph nodes are scanty small and hard, several in the deep groups near the spine are enlarged, firm, and white on section. Wost of the axillary nodes are small and shotty but on both sides a few large indurated ones are present. Nodes could not be found in ei ther antecubital fossa. The paratracheal nodes are enlarged, pale pink, and firm. In the mediastinum there are many large morbid lymph nodes, measuring up to $3 \mathrm{~cm}$. in their long axes. Lost shew central caseation but are otherwise firm and white. The intra-pulmonary nodes at the lung hila are similarly diseased and spread of tumour-like tissue from them extends along the bronchi into the lungs. In the abdomen the mesenteric and retroperitoneal nodes are involved in the disease process. The nodes of the lesser omentum along the lesser curvature of the stomach are enlarged, firm, and pale pink. Small hard white nodules are visible along the mesenteric insertion of the small intestine giving a beaded appearance, to a lesser extent a similar condition is seen in the mesocolon. The nodes at the root of the mesentery appear normal and those along the upper border of the pancreas also seem natural. Below the level of the renal arteries the para-aortic lymph nodes are considerably enlarged and rather fleshy. The cut surfaces are pale pink. The groin lymph nodes are few but several large hard white specinens are present among those found. The popliteal nodes are very small, examples are present on both sides. Spleen. $200 \mathrm{~g} \cdot$ Four discrete white tumour-like nodules sharply outlined by zones of

/congestion 
congestion are present. Their outlines are polygonal rather than round. The pulp is otherwise normal.

Bone marrow. Deposits of the new tissue are doubtfully present in the bodies of the lumbar vertebrae but none are seen in the right femoral marrow, ribs or sternum. The diploe of the skull is normal.

Cardiovascular system. The parietal pericardium is thickened especially at the base being up to $4 \mathrm{~mm}$. thick. It is studded with white tumour nodules 1-2 mm. diameter. The sac contains $250 \mathrm{cc}$. of blood-stained fluid in which fibrin is copious. The heart, $180 \mathrm{~g} \cdot$, shews brown atrophy. The aortic and main arteries are natural being almost devoid of atheroma lesions.

Respiratory system. Nodules of tumour-like white tissue, $1-1.5 \mathrm{~mm}$. diameter, are scattered over the parietal layer of the pleura on both sides. They are particularly numerous over the diaphragm. Approximately $1500 \mathrm{cc}$. of serous fluid occupy the left cavity. Both lungs are small, partly collapsed, and congested. In the right main bronchus an ulcer $1 \mathrm{~cm}$. diameter is encountered overlying a lymph node in the lung substance. A yellowish-grey slough occupies the Iumen of this bronchus.

Alimentary system. The oesophagus is clear of actual invasion but is fixed by the enlarged para-tracheal nodes and the pleural nodules. Small turour-like nodules are present in the peritoneum chiefly the diaphragmatic area; free fluid is slight. A small malignant ulcer is present in the stomach in the lesser curvature near the pylorus. The intestines are norwal internally, peyer's patches and the solitary lymphoid follicles are not at all conspicuous. The liver, $1060 \mathrm{~g} \cdot$, is small and terminally congested. Repeated close slicing discloses several small (under $5 \mathrm{~mm}$.) nodules of the new tissue. The gall bladder and ducts are natural, the pancreas is normal.

Genito-urinary system. Kidneys, left $50 \mathrm{~g}$., right $80 \mathrm{~g} \cdot$; both glands are small but otherwise normal. The left ureter is healthy but the right tube is encircled by enlarged para-aortic (pelvic) lymph nodes.

Dissection reveals actual growth in the ureter wall. The bladder, uterus, and adnexa are natural.

Endocrine system. The hypophysis cerebri is normal. The thyroid gland is small but of normal texture. Both adrenal glands are partly embedded in tumour tissue, dissection reveals small beads of white growth pressing into the cortices but no deep deposits. The thymus gland cannot be satisfactorily identified.

Central nervous system. The meninges are pale but otherwise normal. The brain (1160 g.) is small, pale, and wet, dissection does not reveal disease. 
Microscopical examination:

1. General description of the lesions encountered.

The lesion shews some variation from site to site. In the lymph nodes, the highly cellular pleiornorphic picture with retention of eosinophils seen in the biopsy (Serial 188), is displayed. In the spleen and ureter the picture is closer to the typical disease. In the alimentary and pulmonary deposits tumour-like features are seen. Gradation between the extremes encountered is difficult to follow. This case is considerably corplicated by widespread active fibro-caseous tuberculosis. The latter is present in the lungs, liver and spleen.

\section{Distribution of the lesion.}

The lymph nodes are involved with the exception of the popliteal specimens and one from the mesentery. Tuberculous disease interferes with identification of the process in some mediustinal and para-aortic nodes. Deposits of the new tissue are not identified in the bone marrow of the fernur and sternun, gelatinous degfeneration is marked. The spleen shews the lesion well, many of the fields shew considerable haemosiderin deposit. Tuberculosis co-exists but generally in distinct foci. The spleniculus present does not display the disease. In the four blocks of liver exinined the aisease is not visiole, active and obsolete tuberculosis constitute the nodules seen naked-eye.

Adrenal flands. One was exarnined, the giand is partiy encircled by the new tissue growing in lymph nodes. At one point a wedge of the growth is embedded in the gland but intrinsic deposits are not seen.

The disease is confirned in the duode no-gastric junction (gastric side), ureter, and juxta-intestinal nodules (at insertion of mesentery). Highly cellular foci of the disease are seen in the lungs.

Deposits of the disease are confirmed in the pericardium, pleura, and peritoneum.

Diagnosis and suramary.

Hodgkin's disease shewing transition to the morphological variant, Hodgkin's sarcona. The transition is imperfect and apparently static since alteration from the biopsy is slight. Fibro-caseous tuberculosis co-exists and several normal lymph nodes survive.

The lesion in this case of atypical Hodgkin's disease shews enhanced pleiomorphism and cellularity. Tumour-like propensities exist, but are restricted and shew little alteration after four years. An unusual feature is the location of the disease process in the ureter and serous membranes.

Hydrothorax and sanious effusion of pericardial sac are present. The survival of lymphoid tissue is very slight. 
Robert Law, aet 38 years. Ref. G.R.I. 193:50.

Anamnesis. In 1943 the patient, who was then aged 31 years, developed painless enlarged lymph nodes in the left side of the neck. The history available records that a diagnosis of Hodgkin's disease was made at that time but it is not known whether this was substantiated by microscopy. Biopsy was not performed in this institution till 28th February 1946, Ref. 289:46 Serial 117, with this specimen the diagnosis was confirmed. He He received deep X-ray therapy in Warch 1946, September 1949 and again in February 1950. Subsequently he was treated with nitrogen mustard and blood transfusion.

He was admitted to G.R.I. in mid-ifay 1950, extremely ill. At this time he had severe anaemia and leucopoenia, despite further transfusions his condition deteriorated progressively till death on 10th July 1950.

\section{Report of necropsy.}

2 hours post-mortem.

External. An adult man of slight build. but is masked by dropsy.

Reticulo-endothelial system. 1. Lymph nodes. The superficial and deep cervical nodes are numerous, distinctly enlarged, and firm. Those at the root of the neck and thoracic inlet are the largest, being up to $2 \mathrm{~cm}$. long. All are pale greyish-pink and the cut surfaces are fibrous and wet. In both axillae nodes are scanty, small, and firm; despite close search nodes cannot be recovered from either antecubital fossa. The mediastinal lymph nodes are enlarged, firm, and pale. They are matted together, and are difficult to separate. The inferior tracheo-bronchial nodes are conspicuously enlarged; the right gland measuring $5 \times 3 \times 3 \mathrm{~cm}$. The adjacent areolar tissue is somewhat gelatinous due to oedema. In the abdomen the retro-peritoneal nodes, especially the para-aortic and pelvic groups, are enlarged to the size of broad beans. Wost are pale greyishpink and firm. Those along the upper border of the pancreas and in the lesser omentum also show enlargement, but the small intestinal mesenteric nodes are normal in number and size. In the groins the nodes are small and scar-like. Two small nodes are present in the popliteal fossae, one on each side.

2. Spleen (500 g.) The gland is held to the parietes by light adhesions. It is uniformly enlarged and only moderately firm. In the cut surface small suet-like deposits of new tissue are visible, set in a uniform crimson matrix.

3. Bone-narrow. In the sternum the tissue is widely fatty but several small white nodules are discernible. Tumour deposit is not visible in the lumbar and lower thoracic vertebral bodies. The marrow in the right femur presents a marled red and white pattern throughout, the entire 
cavity being thus occupied. At least part of the paler constituent is fat.

Respiratory system. Recent fibrinous pleurisy is notable on both sides, approximately $500 \mathrm{ml}$. of serous fluid occupy the cavities. Iungs, left $270 \mathrm{~g} \cdot$, right $340 \mathrm{~g} \cdot$ Both organs are partly collapsed, relatively bloodless and mottled greyish-white. In the right lower lobe adjacent to the heart there is an indurated zone of pulmonary tissue, it is in the form of a roughly circular disc $3 \mathrm{~cm}$. diameter and $1 \mathrm{~cm}$. thick. It presents at the pleural surface. The bronchi and trachea are natural.

Alimentary system: Sero-fibrinous ascites (1200 ml.) obtains. Large soft yellow gelatinous clots are present in the fluid. In the stomach a tumour-like growth $3 \times 2.5 \mathrm{~cm}$. is present in the submucosa of the lesser curvature $4 \mathrm{~cm}$. from the pylorus. It closely resembles malignant ulcer but the underlying muscle is freely moveable. Several satellite nodules of white firm tissue surround it. The small and large intestine are not remarkable. Liver (1990 g.) Uniformly enlarged, pale lilac and glaucous. In the cut surface videly scattered suet-like deposits of new tissue are visible. The largest measures $2 \mathrm{~cm}$. diameter. A fine cirrhosis is present. Gall bladder and ducts natural. Pancreas normal.

Cardiovascular system. Pericardium natural. Heart (255 g.) moderately atrophic, there is patchy fibrosis in the posterior wall of the left ventricle. The aorta and main arteries are visibly atheromatous.

Genito-urinary system. Kidneys, left $180 \mathrm{g.,}$, right $170 \mathrm{~g}$. , both glands are pale but otherwise normal. The ureters, bladder and prostate gland appear healthy.

Endocrine syster. The hypophysis cerebri is normal in size and pink. The thymus is atrophied almost to extinction. The thyroid gland is much atrophied the lobes being leaf-like, the parathyroid glands (four recovered) are natural. The adrenal glands and testes are of normal appearance.

Central nervous system. The meninges are normal. The brain (1420 g.) is not remarkable except for pallor.

Microscopical examination:

General description of the lesions encountered.

In most situations the lesion is recognisable as Hodgkin's disease. Fibrosis of hyaline character is conspicuous especially in the capsules and the anatomical medullae of lymph nodes. Coralline deposits and compacted fibres constitute most, but vascular hyalinisation is also present, possibly the result of radiotherapy. Diminution of lymphocytes is notable, when extreme it imparts an unwonted homogeneity to the 
cellular parts of the deposits which then approximate closely to reticulum cell sarcoma. Eosinophils are very scanty. Haemosiderin is present in small amount in most sites.

Compared with the original biopsy the growth is much more tumour-like but the growth rate does not appear high. (See biopsy, Serial 117).

Ividence of tuberculous or other disease is lacking.

Distribution of the lesion. (See diagram).

All the lymph nodes examined shew the lesion except one node from the left popliteal fossa and several from the mesentery of the small intestine. Deposits of the new tissue are present in the femoral bone marrow, which is also the seat of diffuse light fibrous replacement. In the spleen the lesion is nearest to typical, minute foci of necrosis abound, and interstitial fibrosis is extensive. The liver lesions are confirmed, necrosis is common in the bigger deposits and the resemblance to round cell sarcoma is close. The adrenal glands and hypophysis cerebri are free of deposits.

Extra-nodal involvement is confirmed in the stomach. Here the growth is virtually an infiltrating reticulum cell sarcoma. The nakedeye impression of failure to infiltrate is erroneous, the muscle is being actively permeated and destroyed.

The lung lesion is less coherent than apparent naked-eye. It consists of partly coalescent perivascular and peribronchial deposits. The appearances are typical.

Diagnosis and sumnary.

Hodgkin's disease with extreme but incomplete involvement of the lymph nodes. The microscopical picture is altering in various sites towards reticulum cell sarcoma.

The initial lesion was slightly atypical Hodgkin's disease. The presumed duration is seven years, and yet normal lymph nodes are still present. Treated by irradiation and cytotoxic drugs it now presents a close similarity to reticulum cell sarcoma morphologically. Anasarca, hydrothorax and ascites are pronounced. The impression gained from the examination indicates that a very marked reduction of lymphoid tissue has been produced by the disease and by its treatment. 
Anamnesis. Towards the end of 1947 the patient noticed a swelling in the left side of the neck, this was painless and was followed shortly after by sirilar swellings in the left axilla. In November of that year she was admitted and biopsy was performed (Ref. G.R.I. 2146:47, Serial 72). The diagnosis of Hodgkin's disease was established and X-ray therapy was given with a rapid satisfactory response. She remained well till July 1949 when the swellings recurred and a further course of irradiation was given. In June 1950 she was manifestly much weaker and complained of swelling in the epigastrium. This was found to be due to enlargement of the liver. $X$-ray therapy and the exhibition of nitrogen mustard produced regression of this tumour and she remained fairly comfortable till the end of November 1950. She was then re-adritted extremely ill and failed to respond to any therapy. She died 2nd December 1950.

Note. There is a record of a previous operation on the left axilla in 1937. I have been unable to trace whether this was a biopsy. There is not any record in this department of material from this patient at that date.

Report of Necropsy.

External. An adult woman of slight build. but is partly hidden by dropsy.
$5 \frac{1}{2}$ hours post-mortem.

lifarked cachexia is present

Reticulo-endothelial systern. I. Lymph nodes. The cervical nodes are diminished in number, at least in recognisable form, and those identified are sinall and firm. The cut surfaces are pink and white. In both axillae scanty nodes of the same appearance are encountered. Despite careful search nodes camot be found in either antecubital fossa nor in the epitrochiear regions. The nodes at the root of the neck and the paratracheal chains on both sides are augnented beyond the usual number. Several of these nodes are fibrous and pale but the larger ones are only moderately firm and redish-brown. In the mediastinum the nodes are appreciably enlarsed, but probably not increased in number. The pulmonary hilar nodes are normal-looking.

Ihroughout the abdomen the retro-peritoneal and pelvic nodes are small, discrete and firm. Their cut surfaces are moist and pink. The mesenteric groups appear almost normal. In the left groin several large nodes, up to $2 \mathrm{~cm}$. Iong are noted, they are firm and pink. The cut surfaces are homogeneov.s and cream coloured. The remaining nodes in this site and in the right groin are small, firm, and scar-like. Their cut surfaces are rhite. Nodes cannot be found in either popliteal fossa,

2. Spleen $(230 \mathrm{~g} \cdot)$. The gland is tense and firm. Section shews woderate uniform engorgement and a solitary white tumour nodule $1.5 \mathrm{~cm}$. diameter near the hilum. The deposit is angular and surrounded by a deep 
bright crimson zone of congestion 1 nim mide.

3. Bone warrow. We marrow in the sternum and spine is uniformly pale pini, resorption of the cancellous bone is not seen, and tumour nodules are not visible.

In the riut fenur only a small anount of red marrow remains, it is confined to the extremities, most of the space is occupied by fat.

Alimentary system. A small shallow ulcer $6 \times 2$ mo is present on the dorsum of the tongue. The oesophagus is normal. riscites, (4000 nl.), is present, the fluid is faintly opalescent. The stornach and intestines are normal. The liver $(2,500 \mathrm{~g} \cdot)$ is increased in size and weight by virtue of a licge, roughly spheroidal tunour nass, $13 \times 12 \times 10 \mathrm{~cm}$., which occupies much of the right lobe. The growth is creamy-white at the periphery, with occasional red puncta, this zone is about $1 \mathrm{~cm}$. wide and gives way to a pale green very oedematous yet firm centre having the texture of watered silk. Small satellite nodules of suet-like appearance surround it. Further similar nodules are encountered in the left lobe. The gall bladder and ducts are natural. The pancreas is well-preserved, it is nornal.

Respiratory system. The system is normal except for the presence of a solitary nodule of firn pale fawn tissue in the left basal pleura.

Cardiovascular system. Pericardium, normal; the fluid is slightly increased. The heart $(190 \mathrm{~g} \cdot)$ shews brown atrophy. Atheroma is very slight throughout the arterial system.

Genito-urinary system. Kidneys, (Ieft $180 \mathrm{~g} \cdot$, right $155 \mathrm{~g} \cdot$ ). The glands shew only slisht ischaenic scarring. The ureters and bladder are nornal. The uterus shews evidence of a previous parous state but involution is nornal. The left ovary is partly replaced by an hard fibrous tumour nodule 1 cn. diameter, the right gland is natural.

Endocrine system. The thyroid gland is snall but the texture is normal. Both adrenal glands present normal appearances.

Central nervous system. (The head was not opened due to lack of permission).

Wicroscopical examination:

1. General description of the lesions encountered.

The lesions include mature, almost totally hyalinised, deposits of Hodgkin's disease, and cellular tumour corresponding closely to reticulum cell sarcoma. The two growths appear distinct, it is not possible to trace convincing transitions in single specimens but some deposits present intermediate pictures. The sclerosed Hodgkin's disease is confined to 
lymph nodes and the collagenisation is intensified by vascular hyalinisation. In some nodes the identity of the lesion would be equivocal, the persisting nuclei are so sparse. In the sarcomatous lesion the growth is cellular but a degree of dissociation is detected, this is enhanced by the profound reduction of lymphocytes. These necropsy lesions are far more tumour-like than the biopsy specimen.

Ividence of tuberculous disease is absent.

2. Nistribution of the lesions.

Lymph nodes. Obsolete, almost totally collagenised Hodgkin's disease affects an axillary node on the left siae and part of one on the right. A single para-aortic mass of collagen believed to be a node is also present. In the cervical, para-tracheal, mediastinal and groin nodes the lesion is more cellular, in some instances recalling reticulum cell sarcoma. In the cervical group two curious pictures are seen. In one node, left cervical lymph node I, the appearances simulate Hodgkin's disease but fibrosis and disorganisation of node architecture are lacking. In another of this group, node II, a uniform overgrowth of morbid reticulurn cells is seen. This is accentuated by the depletion of lymphocytes. The node architecture is disorganised by fine diffuse fibrosis. In the groin nodes on the left side a uniform tumour composed of morbid mononuclear and multinucleate cells is found, this lesion is sclerosing.

Normal lymph nodes (shewing atrophy) are identified in the left axilla and mesentery. Several presumed nodes were found to be fat or condensed areolar tissue.

The femoral bone marrow is almost aplastic, the tissue is chiefly haemorrhagic fat. Tumour is absent. In the spleen the lesion is identified as rather diffuse reticulum cell sarcoma which retains a background of Hodgkin's disease. Fibrosis is rarked. In the liver the growth is extensively necrotic but where the cellular tissue survives it is composed of morbid reticulum cells with densely hyperchromic nuclei, pleiomorphism is restricted.

The adrenal glands are natural.

Ixtra-nodal deposits of the tumour are identified in the left ovary and in the pleural nodule noted. Rare tumour cells are found also in the connective tissue of the pancreas. The lingual ulcer recorded is a benign non-specific lesion.

Note. In one of the paratracheal nodes an unusual type of multinucleate giant-cell is encountered, viz. a Touton giant-cell.

Diagnosis and summary.

Hodgkin's disease and reticulum cell sarcoma. Thile it is highly /probable 
probable the latter has supervened on the former it is not clearly demonstrated. The virtual disappearance of lymphocytes is notable in the higpatic and groin node lesions as well as in the late Hodgkin's disease affected lymph nodes. This rather supports the view that these lesions are related.

In this case the lymph node lesions as a whole are not so pronounced as usual, this may be the result of therapy. Systematisation is incomplete, but surviving lymphoid tissue is small in anount. Anasarca and ascites are prominent features. 
Andrew Young, aet 27 years. Ref. I.35:51.

Anamnesis. The patient was in good health till early in 1942 when, at the age of 18 he developed a painless swelling in the left side of the neck. This was regarded as tuberculous in character and he was treated for three months in Ruchill Hospital. Later in that year the swelling increased and he was admitted to Stobhill Hospital where biopsy established a diagnosis of Hodgkin's disease, Ref. Stobhill Hospital P42/

834. Serial 57. Deep X-ray therapy was administered immediately afterwards and at intervals till 1945 when further nodes appeared in the left axilla. After a course of X-ray treatment given to destroy these, the patient developed numerous crops of boils which were very troublesome. The disease appeared to remit when the suppurative episodes ceased and he remained tolerably well till 1949 when symptoms recurred. Nitrogen mustard was administered and considerable improvement followed till the following year when the neck glands were grossly swollen. $X-r a y$ treatment was given but the response was poor. He became cachectic and bedridden from the beginning of September 1950, and died 2lst Bebruary 1951.

\section{Report of Necropsy. 6 hours post-mortem.}

External. A tall well-developed man. Vasting and dropsy are evident in the limbs but tine abdomen is full. The skin is a very pale cafe-au-lait tint, several warts are noted on the trunk.

Reticulo-endothelial system. 1. Iymph nodes. The cervical lymph nodes particularly those on the left side are very much increased in number and many are enlarged. They remain discrete in most cases, but the investing areolar tissue is much indurated. These nodes are extremely hard being almost cartilaginous in consistency. Their cut surfaces are white and faintly nodular. Small yellow areas of necrosis are seen in a few of the larger nodes. In the axillae the nodes are small scanty and hard. They are distinctly yellowish-grey. Nodes are not recoverable from either antecubital fossa. The mediastinal nodes are conspicuously numerous and large. They form a coarsely nodular coherent mass 12 X 9 X $8 \mathrm{~cm}$. which encircles the lower half of the trachea. The individual nodes vary much in size. The smallest, the inferior group members measure $2-3 \mathrm{~mm}$. in diameter whereas the largest, those above the lung roots measure $2-5 \mathrm{~cm}$. diameter. They are all extremely hard, white, and nodular. Rarely small necrotic foci are encountered in them. Stibbe's nodes are much enlarged too, they are increased in number and are fused with a tumour-like plaque attached to the basal pericardium. Nodes in both lung hila are also involved by the disease. In the abdomen both the mesenteric and retro-peritoneal nodes are extensively diseased. In the lesser and greater omenta many large hard nodes are encountered. In the mesentery of the small intestine a mass of semi-fused nodes, $20 \times 15$ $X 7 \mathrm{~cm}$, occupies the root of that structure, and minute firm nodules often in small clusters are copious along the insertion of it into the bowel. 


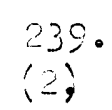

Several of the root nokes are enomous, up to $10 \times 6 \times 4 \mathrm{~cm}$, occasional massive nacrosis is a feature in these giant specimens. The para-aortic group form a roughly fusiform mass $11 \times 7 \times 4 \mathrm{~cm}$, the individual nodes closely resemble those in the mesentery. In both groins scanty small hara white noies ate found, none could be recovened fron the popliteal fossue.

2. Spleen. (450 E.). Uniformly enlared, sinooth and dull plum colour. Repected close slicing and scrutiny fail to reveal any deposits of Hodghin tissue. The appearances are simply those of engorgement.

3. Bone marrow. In the sternum ribs and spine the aarrow is copious homogeneous and rich red, nodules are not visible. In the ferur the marrow is pale yellow and gelatinous throughout, focal lesions are not present.

Cardiovascular system. Pericardiun. Phere is tumour-like invasion of the parietal vall at the base of the heart, nodules of very hard white tissue 2-3 $\mathrm{mm}$. dicneter are visible from the internal surface. The heart $(200 \mathrm{~g} \cdot)$ shews brown atrophy. Atheroma is notably mild in the arterial system.

Respiratory system. Pleurae. Approximately $2000 \mathrm{ml}$. of pale strawcoloured fluid are present in the cavities. On the left side a dense fibrous pleurisy up to $3 \mathrm{~mm}$. thick affects both layers of the membrane. In places fusion, with encystuent of jelatinous pale yellow exudate, is noted. On the right siae similar changes obtain but are less developed and are restricted to the upper lobe area. At the lower end of the trachea intra-nucosal or sumucosil beaded growth of white tumour extends over an area $7 \times 5$ che the deposits shew occasional colasescence to form raised plaques, this growth extends down into the bronchi. Both lungs are partly collapsed. Nodules of hard white tissue are present near the hila of both. Some suppuration is detected in the left organ, apparently due to retention pneumonia. The diaphragm is patchily encrusted with deposits of hard white tissue.

Alimentary system. The oesophagus is nornal. The stonach and intestines are very well preserved and internally they are normal. Peyer's patches are very indistinct. The liver is nornal apart from terminal congestion, repeated close sIicing fails to reveal any tumour deposit. The gall bladder and ducts are natural. The pancreas is normal.

Genito-urinary system. The kidneys (left $160 \mathrm{~g} \cdot$, right $150 \mathrm{~g} \cdot$ ) are normal. The ureters, bladder, prostate gland, and testes are natural.

Fndocrine system. The hypophysis cerebri is normal. The thyroid gland is small but of normal texture, the two parathyroid glands recovered are natural. Both adrenal glands are nornal apart from a curious dull olive and mahogany coloured mottling of the cortices.

Central nervous system. The meninges are healthy, the brain is normal in all respects. 
Microscopical exanination:

I. Generil description of the lesions encountered.

Atypical Hodgkin's disease, corresponding closely to the variety seen in the biopsy, only mature und extensively sclerosed, co-exists with a cellular tumour which is virtually rodgkin's sarcona. Paradoxically transition between the two is imperceptible, they are so very similar. In the "sarcomatous" form the multinucleate giant-cells are very numerous indeed and sclerosis is less evident, the two pictures are simply different degrees of the sime process. An unusual feature is the retention of eosinophils but these cells are nevertheless very scanty.

Suppurative broncho-pneumonia and organising pleurisy are seen in the lungs but evidence of tuberculous disease is absent.

2. Distribution of the lesions. (See diagram).

All the lymph nodes examined sher evidence of the disease in one or both its forms. The cervical, axillary and nediustinal nodes shew chiefly the sarcomatous picture but fields of the densely fibrotic type are encountered in the last two places. In the abdomen the mesenteric and retro-peritoneal nodes reveal almost identical appearances with superior frequency of the surcomatous lesion. In the groin nodes the less cellular sclerosing form predominates.

The samples of femoral bone marron, liver, and spleen are free of the disease in either form. Both adrenal glands are unaffected.

Deposits of the hew tissue dre confirwed in the trachea, both lungs, the diaphragin, und the jleura. Buth kidnejs, the prostate gland and brain are unaffected. A sumple of colon exaninel shews snall normal lymoid pollicles in the subrucosa.

Diagnosis and suminer

Atypical Jodgin'in aisease norphologically close to reticulum cell sarcoma, shewing fuirly complete networphosis to the morphological variant, Eodgkin's sarcora. The lesion, initially typified by unusual ageregation of the proliferating morbid reticulum cells shews enhancement of this phenomenon which together with renarkable production of multinucleate giant-cells gives the picture of Varthin's reticulo-endothelioblastoma. The lesion is not systematised, despite the elapse of nearly nine years. The pulmonary, tracheal, diaphragmatic, and pericardial deposits are probably autochthonous, though it must be conceded that the first named might be embolic in origin.

Oedema is notable in this case. Surviving normal lymphoid tissue is minimal but recognisable in the para-enteronic loci. 
Mirs. Mary Clegg, aet 25 years. Ref. G.R.I. 61:51.

Anamnesis. The patient was well till 18 months ago when she began to feel weak and breathless. These symptoms intensified gradually, and dysphagia with vomitine became troublesome. Pulmonary tuberculosis was suspected and she was treated in Robroyston Hospital for some months, definite evidence of tuberculous infection was not however established. She was admitted to Glasgow Royal Infirmary 13th July 1950. At this time she was pale, sallow, and dropsical. A large pleural effusion, and enlargement of the spleen were discovered, but lymph node enlargement was not a feature. Considerable anaemia and moderate leucocytosis were present. Guinea pig inoculation of pleural fluid failed to incriminate Nyco. tuberculosis as a cause. A diagnosis of "reticulosis, type uncertain" was considered and she received radiotherapy. She was then allowed home. She was re-admitted in October 1950 and spent nearly three months in hospital but her condition slowly deteriorated. On 3Ist January 1951 she was admitted again, manifestly in her last illness. She died 16 th February 1951.

\section{Note. It is established that lymph node biopsy was not performed in this case.}

Report of Necropsy.

External. A very emaciated young adult woman.

24 hours post-mortem.

Reticulo-endothelial system. Lymph nodes. The cervical nodes are small and inconspicuous above the root of the neck. At the thoracic inlet several fleshy, moderately firm, glands are noted, these measure up to $2 \mathrm{~cm}$. in their long axes. Their cut surfaces are pale pink and white. The axillary nodes are few in number on both sides but several large firm specimens exist. On section they are white and coarsely nodular. Nodes cannot be recovered from either antecubital fossa. The para-tracheal chains are prominent, the individual nodes varying from $1-3 \mathrm{~cm}$. diameter. These glands are mutually separable with ease. In the mediastinum the lymph nodes are increased above the usual number and most are appreciably enlarged. On section they appear white except for scattered carbon particles. Stibbe's para-sternal nodes are readily seen due to their enlarsênent.

In the abdomen the retro-peritoneal nodes are numerous and enlarged. The most conspicuous are the para-aortic groups round the coeliac and renal arteries. The glands of the pelvis are very scanty and small. The mesenteric groups are normal in appearance. A solitary small lymph node is found in the left groin but none can be recovered from the right. Both popliteal fossae are similarly devoid of nodes.

Spleen. (190 g•). Three pale nodules are visible from the surface shining through the capsule. Dissection reveals these to be typical suet- 
like masses of Hodgkin tissue approximately $1 \mathrm{~cm}$. diameter.

The bone marrow of the right femur, lumbar and lower thoracic vertebrae, and ribs is pale red and homogeneous. Visible deposits in it are absent.

Respiratory system. $1200 \mathrm{cc}$. of greenish-yellow opalescent fluid lie in the right chest. The right lung is collapsed and covered by a thick fibrin deposit. Section reveals white tumour-like infiltration of the peribronchial tissue which extends out to involve most of the upper lobe. Necrosis is marked in the centres of the coarsely lobular deposits. Small islets of surviving lung tissue are enclosed by the growth and are also visible at the periphery. On both sides the bronchi are normal internally as far as dissection can be carried. The left lung is clear of disease, the only feature of note being a patch of fibrinous pleurisy.

Cardiovascular system. The pericardium is infiltrated in both its layers at the base, the new growth is hard, white, and nodular. Effusion to the extent of $500 \mathrm{cc}$. of faintly turbid fluid is noted. The heart $220 \mathrm{~g}$. shews only atrophy. The aorta is natural, atheroma is minimal.

Genito-urinary system. The kidneys (left $145 \mathrm{~g} \cdot$, right $145 \mathrm{~g} \cdot$ ) are normal. The ureters and bladder are healthy. The uterus and adnexa are natural.

Alimentary system. Ascites (1200 cc.) is present, fibrin is very scanty. The oesophagus, stomach, and intestines shew only post-mortern change. The liver ( $1720 \mathrm{~g} \cdot$ ) is large, a nut-meg pattern is discernible in the cut surface; despite repeated close slicine tumour deposit is not seen. The gall bladder and ducts are nornal. The pancreas is natural.

Central nervous system. Brain ( $1320 \mathrm{~g} \cdot$ ) The organ is normal to nakedeye examination. The meninges are pale but otherwise normal.

Endocrine system. The hypophysis cerebri is small, red, and very soft. The thyroid gland is natural but rather small. An enlarged lymph node is partly embedded in the cortex of the risht adrenal gland, the left gland is normal.

\section{Microscopical examination:}

I. General description of the lesions encountered.

The basic lesion is ageing Hodgkin's disease. This is seen in lymph nodes and the spleen. In these sites fibrosis is pronounced and infarctlike areas of necrosis are present in some cases. Fosinophils are retained in these deposits. In addition transition to the variant here termed Hodgkin's sarcoma is notable. In certain nodes examined both pictures can be seen, in others only the latter is exhibited. (Comparison with antecedent biopsy findings is not applicable). 
There is not any evidence microscopically of co-existent tuberculous or other disease. Preservation of the histological material is unfortunately not very good and this has limited successful photographic illustration.

2. Distribution of the lesions.

Lymph nodes. In the cervical, axillary, mediastinal, and three retroperitoneal nodes the picture is one of mature Hodgkin's disease. one of Stibbe's nodes from the right side Hodgkin's sarcoma is seen in addition. The mesenteric nodes and several retro-peritoneal specimens shew only Hodgkin's sarcoma. Incomplete involvement of nodes is present in two specimens, one from the mesentery and in one para-aortic node. The node from the left groin is normal apart from moderate atrophy.

Only the femoral bone rnarrow was examined, neither lesion is identified in it. In the spleen the form is that of late cellular Hodgkin's disease, necrosis is slight but some haemosiderin deposit is visible. Growth of Hodgkin tissue of moderately fibrous type is confirmed round the right adrenal gland. The gland is closely invested but not penetrated, the new tissue is not present in the gland itself. Fat spaces in the new growth suggest that fatty tissue has been infiltrated in tumour-like fashion. The hypophysis cerebri is very poorly preserved, there is not any evidence of the new tissue in it.

Extra nodal sites shewing the disease include the pericardium and superficial nyocardium. The cardiac muscle is being infiltrated by typical Hodgrin's disease. The right lung lesion is Hodgkin's disease. It assumes the form comnonly seen namely a type of chronic interstitial pneumonia with a central necrotic zone. The disease is not found in any of the blocks of liver exarnined.

Diagnosis and summary.

Hodgkin's disease with metamorphosis to Hodgkin's sarcoma. The transition is displayed fairly convincingly in two sites. The tumourlike propensities of Hodgkin's disease are well shewn by the infiltrative tendencies seen in the heart and peri-adrenal fat. Failure of the disease to systematise is noteworthy. Hydrothorax, pericardial effusion, and ascites are pronounced. There is very severe atrophy of lymphoid tissue but some normal is identified. 
David Hatton, aet 47 Jears. Ref. P.il. 131:52. Law Hospital.

Anamnesis. This patient was first admitted to Law Hospital on 12 th Ausust 1952. He complained of Eeneral weakness, profuse sweating (especialiy at night), loss of weight, and unorexia. These symptoms had been present for one jear. Fe had remittent prexia and severe secondary anaenia. His condition inproved after blood transfusion and he Was transferred to Jtrutholyde Jospital. On 2lst Novernber 1952 he was re-adnitted to Law Hospital where the Wurchison phenomenon was observed during his sojourn. Inlarged limph nodes vere found in the right iliac Possa and groin. Biopsy was perforned (Ref. Iair Hospital 828:52., Serial 218). This shewed Goagrin's disease. His condition deteriorated rapidy, he becane mentally confused and jaundice developed. He died 27 th December 1952.

\section{Report of Necropsy.}

External. An extremely cachectic middle-aged nan. Icterus is present. Healing surgical wounds are present in the right inguinal region, a paramedian abdominal scar is also present.

Reticulo-endothelial system. 1. Lymph nodes. The cervical lymph nodes are normal in number and size but are appreciably firmer than usual. In the left axilla the nodes are enlarged, hard, and numerically increased, the largest measures $2.5 \mathrm{~cm}$. diameter. On the right side they appear almost normal but are firm. The mediastinal and hilar nodes shew moderate anthracosis but are not enlarged nor increased in number. Their consistency is firm. In the abdomen the mesenteric and retro-peritoneal groups are involved in irregular fashion. Of the former those in the lesser omenturn (porta hepatis), the transverse mesocolon, and coeliac group are enlarged. The biggest measure $3 \mathrm{~cm}$. diameter. The nodes in the small intestine mesentery are small and inconspicuous. The paraaortic groups on both sides are considerably enlarged and firm. In the right side of the pelvis nodes are proninent along the right internal iliac vessels. The nodes in the left groin are srall, fairly hard, and scanty, those in the right region are enlarged and firm. Nodes are present in both antecubital fossae and popliteal fossae, they are very small.

2. Spleen. $350 \mathrm{~g}$. The gland is enlarged to over twice the normal size. It is firm and palpably nodular. The cut surface discloses some half-dozen discrete greyish-pink nodules of between 1-7 cm. diameter set in a dark crimson pulp. These tumours are not very hard.

3. Bone marrow. In the sternum the marrow is red and semi-fluid. The marrow of the right femur is greyish-green and gelatinous throughout.

Cardio-vascular system. Pericardium, natural. Heart (230 g.). Brown atrophy is present, the coronary arteries are tortuous but patent to the 
limits of dissection. The main arteries and veins dissected are natural, bile staining of the intima is seen.

Respiratory system. The air passages contain bile stained muco-purulent material, dissection carried down to the snaller bronchi does not disclose any tumour-like lesions. The lungs are snall, confluent bronchopneumonia is seen in both lower lobes. Thumour growth is not visible in either organ nor in the pleurae.

Alimentary systern. The oesophagus is normal. Old adhesions are present in the peritoneal sac, the greater onenturn is inflamed and adherent to the undersurface of the liver. A partial gastrectorny has been performed. The pyloric stump is thickened due to diffuse increase of interstitial tissue, this ceases at the pylorus itself. A pedunculated growth $2 \mathrm{~cm}$. dianeter is present in the posterior wall of the antrum. The intestines shew only putrefactive change. The liver, $1400 \mathrm{~g} .$, is externally normal, repeated close slicing f'ails to reveal any nodules. The gall-bladder and ducts are normal. (The pressure test is positive despite adhesions). The pancreas is much sortened.

Genito-urinary system. The kidneys (left $210 \mathrm{~g}$., right $160 \mathrm{~g} \cdot$ ) are normal. The ureters, bladder, and prostate gland are natural.

Central nervous systen. The meninges are merely bile stained. The brain is normal externally and on dissection.

Endocrine system. (The hypophysis cerebri was over-looked). The thymus gland is atrophied almost to extinction. The thyroid gland is normal; both adrenal glands shew only marked autolysis.

Microscopical examination:

General description of the lesions encountered.

This necropsy was performed two days after death and the preparations are consequently poor. Preservation of structure, i.e. microarchitecture, is reasonably good but the staining properties are badly impaired.

The lesions where present are recognisable as moderately late Hodgkin's disease. Fibrosis and necrosis are extensive and eosinophils cannot be identified. Thereas some latitude of appearance is discernible the growth is much the same wherever it occurs. Comparison with the original biopsy is difficult because of post-mortem change but in some fields the resemblance is close. In a few nodes the loss of lymphocytes is disproportionate and accentuates the impression that reticulum cells are unduly numerous.

Non-specific broncho-pneumonia is present; evidence of tuberculous disea'se is absent. 
Distribution of the lesion.

Lymph nodes. There is striking persistence of non-affected lymph nodes; these are found in the neck, para-tracheal chains, right axilla, mediastinum, mesenteries, and left inguinal regions. In addition the antecubital and popliteal nodes are not diseased. The lesion is identified in the left axilla (four nodes), the para-aortic (retroperitoneal) nodes, a chain along the right iliac vessels and the right inguinal region.

The spleeh is extensively affected, haemosiderosis and necrosis are pronounced. Lesions are ilso confirmed in the liver. The femoral bone marrow shews almost complete gelatinous degeneration.

All other tissues exarnined proved negative. The gastric tumour is a papilioma.

Diagnosis and summary.

Atypical Hodgkin's disease, eosinophils lacking and aplastic anaemia. This case is very interesting because of the restricted distribution of the lesions. It appears probable that the severe unaemia determined death. Oedema is not a feature and lymphoid tissue survival is unisually great. 
Mrs. Catherine Cameron, aet 31 years. Ref. P.W.155:52. G.R.I.

Anamnesis. Warly in 1951 the patient complained of a painless swelling in the inguinal region. On 20 th April 1951 an enlarged inguinal lymph node was removed for microscopical exánination at Stracathro Hospital, Brechin. This was exanined at Dundee Royal Infirmary and Hodgkin's disease was diagnosed, Ref. 1186:51 Dundee Royal Infirmary; Serial 217. Radiotherapy was instituted and ten treatments were given. The patient was pregnant at the time and later gave birth to a normal healthy baby. Thereafter she remained well till ten weeks ago when jaundice, anorexia, nausea, and vomiting supervened. She was admitted to Irvine Central Hospital and her condition improved sufficiently for her to return home after several weeks. Two weeks ago dyspnoea developed suddenly and she became very frightened and distressed. On 17 th liay 1952 she vas admitted to the Gatehouse of Glasgow Royal Infirmary in a dying condition. She lapsed into coma and died 19th lay 1952.

Report of Necropsy.

8 hours post-mortem.

External. A well built adult woman. There is slight anasarca of the lower limbs. Jaundice is not present.

Reticulo-endothelial system. Lymph nodes. The lymph nodes in the neck, axillae, and antecubital fossae are very greatly reduced in number. In the first site a very few were found after prolonged search; they are confined to the thoracic inlet. Nodes could not be found in either antecubital fossa nor in the right axilla. A solitary partly fatreplaced node was recovered from the left axilla.

The mediastinal lymph nodes are numerous and much enlerged. They are discrete, hard, and mite. The largest measures $4 \mathrm{~K} 3 \mathrm{~K} 2 \mathrm{~cm}$. The para-tracheal chains on both sijes are similarly affected but the lesions cease abruptly at the root of the neck. Intra-pulmonary nodes are conspicuous in both Iunes due to their participation in the moroid process.

In the abdowen Iymphadenopathy is considerable. Those in the porta hepatis, the para-pancreatic group and the coeliac group are moderately enlarged and firm. The retro-peritoneal para-aortic group and those along the iliac arteries are hard and white but not much enlarged. One large node $3 \times 2 \times 2 \mathrm{~cm}$. is producing distortion of the inferior vena cava at the level of the right renal artery. Several smaller nodes are pressing on the right ureter below this level. The nodes of both groins are enlarged, hard, and white on section, and on the left side further enlarged nodes are present in the lower angle of Scarpa's triangle. Popliteal nodes cannot be found.

Spleen. (420 g•) The gland is fairly uniformly enlarged but slightly /aistorted 
distorted due to nodules in its substance. The capsule is smooth and free from exudate. The cut surface reveals a pale pink homogeneous pulp in which are set scattered angular white suet-like deposits of Hodgkin tissue. These vary widely in size the largest being $2 \mathrm{~cm}$. in diameter. The hilar lymph nodes are partly embedded in the splenic substance and present the same changes as the other abdoninal nodes. Visible deposits of the new tissue cannot be seen in the liver.

Bone marrow. The red narrow of the sternum appears normal. thoracic and lumbar vertebrae several white nodules are seen. The upper third of the shaft of the right femur is occupied by soft red marrow which is homogeneous, tumour is not found. The narrow of the skull appears nornal, it is very scanty. The nuclei pulposi of the spine are nornal.

Respiratory systeri. The trachea is moderately congested and the lumen is occupied by whitish-yellow mucus and pus. The lower $3 \mathrm{~cm}$. presents nodular tumour-like beading in the mucosa. These nodules are continued downwards into the main bronchi. They are firm, yellowish-white and 2-3 min. diameter. Gross ulceration is not convincingly shewn at any level but some of the larger lesions are stippled with pink. Stenosis of the right main bronchus obtains. The left lung is well aerated and appears normal. The right lung is collapsed and several tumour-like craggy nodules are present below the pleural surface at the periphery. These measure up to 1 cm. diameter. On section the growths are white. A small (unmeasured) effusion of sero-fibrinous character is notable on the right side.

Cardiovascular system. The pericardial sac is distended by approximately 180 cc. of clear yellow fluid. Two small white tumour nodules approximately' $1 \mathrm{~cm}$. diameter are noted in the visceral layer just below the line of reflection from the aorta and pulmonary artery.

Heart (275 g.): The organ is of normal size and configuration. The myocardium is of good quality and the chambers are natural. The aorta and main arteries are of small calibre, intimal change is linited to fatty streaking. Thrombosis is present in both femoral veins, the left vessel is completely occluded, but the right only partially.

Alimentary system. The oesophagus is natural. The peritoneum of the pelvis, especially that in the pouch of Douglas, is beset by numerous small nodules which appear to have developed deep to it and are thrusting forward into the membrane. Exudate is scanty. The stomach and intestines appear entirely healthy; the mesenteric nodes are small and inconspicuous.

The liver ( $1980 \mathrm{~g} \cdot$ ), is large. The shape, consistence, and colour are natural; repeated close slicing reveals only terminal congestion. The gall-bladder and ducts are normal. The pancreas is well preserved and is normal.

Genito-urinary system. The kidneys (left $150 \mathrm{~g} \cdot$, right $170 \mathrm{~g} \cdot$ ) are normal /externally; 
externally; there is some persistence of the foetal lobulation. Section reveals congestion but tumour deposit is absent. Slight hydronephrosis is appreciable on both sides but the pelves and ureters are clear of intrinsic growth. The para-aortic lymph node tumours are the apparent cause. The bladder is small and contracted. Several small aligned nodules are present in the trigone, one srall polypoid outgrowth of f'irm white tissue is seen. The uterus and adnexa are natural.

indocrine system. Pituitary gland. The gland is normal in size and nodules are not visible in tie cut surface. Thyroid gland, small, otherwise natural. Adrenal glands. Both are normal naked-eye.

Central nervous system. The scalp, skull, and meninges are natural. The brain $(1300 \mathrm{~g} \cdot \mathrm{)}$ is nornal.

Wicroscopical examination:

General description of the Iesions encountered.

The form of the lesion is highly cellular tumour-like Hodgkin's disease. Fibrosis is present in most sites, but is chiefly restricted to fibrils and fibrillae; large hyaline masses are rare. In several lesions morbid reticulum cell overgrowth is conspicuous but eosinophils are retained. Compared with the biopsy the growth is much more tumourlike. Microscopical evidence of tuberculosis is not found.

Distribution of the lesion.

Iymph nodes. Lesions are identified in nodes from the mediatinum, abdomen, and both groins. The single left axillary node recovered is unaffected but shews advanced fat atrophy. The disappearance of peripheral nodes including those of the cervical regions is unusually complete. In the bone marrow deposits of the new tissue are small, they are confirmed in the ribs and lumbar vertebrae but absent in the right femoral marrow. In the spleen the lesions are rather more cellulis than usual but otherwise typical. The liver samples studied are normal, there is not even lymphocytic infiltration of the portal tracts. The adrenal glands and hypophysis cerebri were not sectioned. is nucleus pulposus examined shews no evidence of disease.

Ixtra-nodal lesions are confirmed in the trachea, bronchi, lungs, bladder, visceral pericardium, and pelvic peritoneum. In these sites there is destruction of the native tissues by the growth. Ectopic lymphoid tissue foci co-exist with the peritoneal lesions.

The kidneys, thyroid glend, pancreas, breast, and ovaries are normal. Diagnosis and summary.

Cellular Hodgkin's disease shewing enhamement of tumour-like growth 
and properties. The disease ran a relatively short course (known duration 15 months). There is definite failure to systematise throughout the reticulo-endothelial tissue but extra- nodal involvement is quite pronounced.

Anasarca, mild hydrothorax, and hydropericardium are notable, there is moderate survival of lymphoid tissue in para-enteronic sites. 
Iirs. Wala líoyes, aet 41 years. Ref. G.R.I. 119:53.

Anamnesis. The patient was adnitted on 3rd January 1953. She had been attending outpatient dispensary for one year and was being treated for myxoedena. In November 1952 she had begun to feel unwell and a temperature chart tisen at howe shewed a norning elevation of temperature. swout that tine whe noticed swellings in her neck, axillae and groins. In the folloring month she experienced back pains and sciatica. Biopsy was performed (Ref. G.R.I. 495:53, Serial 191). She received a course of nitrogen mustard with little result, and three doses of generalised. radiotherapy with slight regression of the glandular swellings. Femoral thrombosis and dropsy supervened shortly before she died on $23 \mathrm{rd}$ liarch 1953.

\section{Report of Necropsy.}

11 hours post-mortem.

Axternal. A well-built, well-nourished midale-aged woman. The skin of the right thigh is pigmented a delicate brown with darker freckles of the same colour. A small surgical scar in the left groin is similarly pigmented. There is moderate dropsy of both legs.

Reticulo-endothelial system. Lymph nodes. The superficial groups of cervical lymph nodes are small and inconspicuous on both sides, but the deeper groups, especially those along the carotid sheath are enlarged glaucous and fleshy. One node on the left side and lying on the vertebral column at the thoracic inlet measures $5 \times 2 \times 2 \mathrm{~cm}$. On section all these nodes are pale pink, fibrosis is not evident. Nodes are not recoverable from the antecubital fossae. In both axillae several enlaryed soft pinkish-white nodes are present, their number is $f e w$. The retro-sternal nodes of stibbe are markedly increased in number and size and are indistinguishable from the anterior mediastinal groups which also shew a florid increase. In the mediastinum the size of the nodes is modest, most are below $2 \mathrm{cn}$. diameter, besides those nentioned above both paratracheal cinains are conspicuous. The remainder including the hilar nodes are sinall, antriracotic, and apparently clear of tumour. In the abdomen the mesenteric nodes are nearly all normal in number and appearance. The retro-peritoneal groups however, are enlarged, firm, and fleshy. There is a large ovoid tumour mass $24 \times 15 \times 10 \mathrm{~cm}$. occupying the right flank which is apparently of lymjh node origin (retroperitoneal). This is invading contiguous structures including the liver, right kidney, right adrenal gland and the right psoas muscle. There is a solitary enlarged node in the porta hepatis. On both sides the pelvic nodes are enlarged and fleshy. The groin lymph nodes are also increased in size, they are very soft. Lymph nodes are not seen in either popliteal fossa.

Spleen. $210 \mathrm{~g}$. The gland is congested. Repeated close slicing reveals a single white tissue nodule $2 \mathrm{~mm}$. diameter.

Bone marrow. The marrow in the sternum and lower vertebral bodies is 
pink and uniform, the bone is soft due to resorption. In the right femur, red bone marrow is confined to the upper half of the cavity, it is pale and uniform. Fat occupies the rest of the space.

Cardiovascular system. The pericardium shews sone diffuse thickening at the base but tumour nodules are not visible in it. The heart $(250 \mathrm{~g} \cdot)$ shews moderate brown atrophy. itheroma is remarkably slight throughout the arterial system. Recent thrombosis occludes the lower half of the inferior vena cava, where it is encircled by tumour, and both iliac veins and their tributaries are occluded.

Respiratory system. Pleurae. Left, normal. Right, extensive organising fibrinous exudate with loculated encystment of sanious fluid is present. Iungs, left $660 \mathrm{~g}$., right $600 \mathrm{~g}$. An obsolete tuberculous primary complex is present in the left organ. The right lung is partly collapsed, there is palpable induration in the lower lobe. The bronchi and trachea shew engorgement. On the right side the diaphragm is almost $2 \mathrm{~cm}$. thick due to infiltrating white tumour spreading from below, the muscle fibres are visibly separated by the growth.

\begin{abstract}
Alimentary system. A serous ascites (1000 ml.) is present. The oesophagus, stornach and intestines are norinal. The liver (2330 g.) is uniformly enlarged, pallor is marked. Direct infiltration by tumour is seen in the undersurface; apart from this nodules are not present. The gall bladder and ducts are natural. The pancreas appears normal.
\end{abstract}

Genito-urinary system. Kidneys, (left $160 \mathrm{~g} \cdot$, rigint uninown). The left gland appears normal. The upper pole of the right is directly infiltrated $b_{y}$ soft white tumour, the cortex at this point is pale whitish pink and blurred-looking. The ureters and bladder appear normal. The uterus contains a single myoma $3 \mathrm{~cm}$. diameter, the adnexa are normal.

Endocrine systern. The hypophysis cerebri appears normal but is soft. The thyroid gland is uniformly enlarged, whilst retaining its contours it has been subtly replaced by soft pink tumour. It measures $6 \times 5 \times 3 \mathrm{~cm}$. The cut surface shews several well demarcated nodules deep in the growth. Both adrenal slands are much destroyed by tumour, fragments of the right one are found with difficulty.

Central nervous system. The skull and meninges are normal. The brain $1350 \mathrm{~g} \cdot$ is natural.

Microscopical examination:

General description of the lesions encountered.

Preservation of the material is regrettably poor and the illustrations are consequently indifferent. The majority of the deposits are more or less uniform reticulum cell sarcoma in which fibrosis is relatively slight. Reticulin formation is demonstrable. The type cell is a morbid 
mononuclear type similar to that seen in the biopsy specimen, eosinophils are absent. In several sites gross coarse fibrosis typical of late Hodgkin's disease is seen. In several nodes Hodgkin giant-cells abound. By virtue of the biopsy findings this case is interpreted as one of Hodgkin's sarcoma (Tarthin's type II, reticulo-endothelioblastoma) but the neoplastic habit is ennanced to the point where the growth is actively aggressive and destructive. The metanorphosis is more complete than is usually seen, the resultant turnour being more atavistic and approaching the 'stem cell Iyrnphoma' of Gall and lwllory. Thaberculous disease is confirmed in the lungs but the process is obsolete.

Distribution of the lesion.

All the cervical nodes examined shew the tumour, several contain large collagen deposits indicating previous Hodgkin's disease. The axillary nodes are sinilarly affected but one on the right side is only partly replaced. This node shews fat atrophy, the more atrophic portion is free of the growth. Stibbe's nodes and all the mediastinal ones are neoplastic. The retro-peritoneal and pelvic nodes are replaced by tumour, the former group merging with the large mass in the right flank. In both groins the tumour is identified in all but one node from the right side which is unaffected, it shews profound depletion of lymphocytes.

The tumour is present in the fenoral bone merrow and it is extensively necrotic. One sample of anrrow is free of growth and is hypocellular. The single splenic nodule is tumour in which central fibrosis is inarked. Tumour is confirmed in the liver; in blocks taken at random well away from the deposits due to direct extension, the growth is not seen. Both adrenal slands are widely infiltrated, it is not possible to learn whether the growths are intrinsic but naked-eye their investment indicated invasion from vithout. The hypophysis cerebri is free of tumour.

The neoplasn is not present in a nucleus pulposus (Iumbar region) examined nor in samples of omentum. Extra-nodal tunour is confirmed in the thyroid gland which is almost entirely destroyed. The large tumour in the right flank is the sane neoplasm, destruction of skeletal muscle is extensive. Deposits of the growth are seen throughout the right kidney, the left fland is not involved. The diaphrasm, pleura and right lung contain deposits of the tumour. Sections from the brain and optic nerves are normal.

Diagnosis and sumnary.

Hodgkin's disease and Hodgkin's sarcoma, with loss of pleiomorphism, the final picture approaching a pure reticulum cell sarcoma. In this case systemic involvement is a marked feature, the virtual replacement of the thyroid gland is interesting since this coincides with myxoedema 
diagnosed during life. Despite the relatively wide involvement one normal and one partly involved node survive. These findings are highly important since this case may well be regarded as "acute Hodgkin's disease". Cases of this type are generally believed to be truly systematised, (Callender, 1934). Anasarca, hydrothorax and ascites are notable. There is very little survival of normal lymphoid tissue. 
Douglas Law, aet 23 years. Ref. G.R.I. P.I.274:53

inamesis. In liay 1050 the patient was found to have pulmonary tuberculosis, mainly right sided. Collapse therapy was followed by a satisfactory response. Bventually in Cctober 1951 he was declared cured and returned to work. In February 1953 he bad a bout of persistent vomitine, and jaundice developed. He was admitted to Hairnyres Hospital the following month. On exümination multiple glandular swellings were found and biopsy established the diagnosis of Fodgkin's disease (Ref. 804:53 Hairmyres Hospital, Serial 220). He was treated with blood transfusion, nitrogen mustard and trephyline melanine with little benefit. He became progressively worse and was admitted to Glassow Royal Infirinary at the end of June 1953. at this juncture he was extremely ill and despite blood transfusion his condition worsened rapidly till death on 5 th JuIy 1953.

\section{Report of ilecropsy.}

20 hours post-rnortem. Ixternal. A tall cachectic young man; deep orange yellow jaundice is
present. (reight of body $=134 \mathrm{lbs}$. )

Reticulo-endothelial system.

Lymph nodes: The cervical nodes are considerably enlarged and are unduly numerous. The superficial groups in the anterior and posterior triangles are hardest and their increase in size is the least. Their cut surfaces are generally evenly yellow but occasional white areas are seen. The retro-pharyngeal groups are thrown into unwonted prominence because of their gross increase in size, they are softer than the others. They form a columnar mass $10 \times 3 \times 2 \mathrm{~cm}$., nodes beine closely set mutually, but remaining discrete. Individual specimens neasure up to $3 \mathrm{~cm}$. diameter. The cut surfaces are all yellow with occasional petechiae.

The axiliary nodes on both sides are small, hard, and few. Despite much search none are found in either antecubital fossa. is single small subcapsular node is present on the left side. The para-tracheal glands are numerous, enlarged, and rather fleshy. The retro-sternal nodes of stibbe are readily seen due to their increase in size. They are dull red and soft.

The mediastinal lymph nodes are very numerous and moderately enlarged. In most cases they are pinkish-yellow and rather soft. right inferior tracheo-bronchial node is incompletely replaced by pale yellow tumour-like tissue, a rind of dull red congested lymphoid tissue being visible. The pulmonary hilar nodes are small and slate colour on the left side, but are enlarged and firm on the right side.

The mesenteric nodes are small, soft, and generally inconspicuous, except in the lesser omentum and portal fissure, where enlarged fleshy 
nodes are found. The para-pancreatic nodes are small, fleshy, and numerous. The para-dortic and pelvic glands are much enlarged, firm and increased in number. In the groins the glands are small, infrequent, and hard. The popliteal nodes are very small.

The spleen. (1000 $\left.\mathrm{g}_{\bullet}\right)$ is uniformly enlarged and very numerous angular yellowish-white deposits of firm tissue project slightly from the surface. These deposits measure 3-4 mm. in diameter. The cut surface is evenly studded with them; the matrix is dull crimson.

Bone marrow. Uniform red marrow is visible in the bodies of the lumbar and thoracic vertebrae. The right femur contains red marrow in its upper third, below this level there is only fat. Tumour nodules are not seen. The bones of the skull also appear normal.

Cardio-vascular system. The pericardium is natural, approximately $20 \mathrm{cc}$ of bile-stained fluid occupy the sac. The heart $240 \mathrm{~g}$. shews moderate atrophy - Atheroma is virtually absent from the aorta and main branches: dissected.

Respiratory system. Lungs. Left $760 \mathrm{~g}$. Oedema and congestion are notable, small lesions of obsolete tuberculosis are present in the apex. Right $600 \mathrm{~g}$. Partial collapse and fibrous pleurisy are present. Active caseous foci, some with thick fibrous capsules, are seen throughout the upper lobe.

Alimentary system. Localised fibrinous exudate is seen over the viscera, especially the spleen and liver. Several gelatinous fibrin clots lie in the pelvis. Free fluid is small in amount. The stomach and intestines shew only early putrefaction. The liver (2600 $\mathrm{g}^{\circ}$ ) is uniformly enlarged. Scanty whitish-yellow nodules, rarely above $2 \mathrm{~mm}$. diameter are present under the capsule and throughout the viscus. The organ is rich reddishbrown. The gall-bladder is aistended with orange bile, the pressure test is positive. The pancreas is natural apart from bile staining and softening.

Genito-urinary system. Kidneys, left $250 \mathrm{~g}$., right $250 \mathrm{~g}$. Apart from their large size they appear normal. The ureters, bladder, prostate gland, and testes, are normal.

Central nervous system. The brain $1460 \mathrm{~g}$. is nornal and unstained by bile although this pigment colours the meninges and choroid plexuses.

Endocrine system. The hypophysis cerebri is small and dull red. The thyroid gland is atrophic, leaf-like, and bilious. Only one parathyroid gland is found, a small one. The adrenal glands are small but otherwise normal. 
Licroscopical exanination:

General description of the lesions encountered.

All the tumour deposits assume the form of Hodgkin's sarcoma (type II of warthin, reticulo-endothelioblastoma). Horphology conforming to ordinary Hodgkin's disease is not encountered. The growth is a highly pleiomorphic reticulum cell sarcoina with an high content of Hodgkin giantcells, moderate fibrosis, and total absence of eosinophils. Histologically the growth exhibits low malignancy. An interesting feature which is fairly comion in the lyraph nodes is partial involvement of these structures.

Pulmonary tuberculosis is identified and acid/alcohol fast bacilli morphologically identical with myco. tuberculosis are demonstrable in the lesions.

Distribution of the lesion.

Lymph nodes. The lesion is identified in the cervical, right subcapsular, Stibbe's retrosternal chains, the axillary and mediastinal nodes. In the abdomen the retroperitoneal and some of the mesenteric nodes exhibit it. Further lesions of the same character are observed in the left groin nodes. Normal but atrophied nodes are found in the right and left groins and in the right popliteal fossa.

The fenoral bone marrow contains small foci of the growth. In the spleen the tumour is seen together with haemosiderin deposits and caseating necrosis. The liver contains microscopical deposits in the portal tracts. The adrenal glands, hypophysis cerebri, a nucleus pulposus, and the omenta are negative.

The thyroid gland, samples of lungs and skeletal muscle are also free of the growth. The sections of brain and choroid plexus examined are natural.

Diagnosis and summary.

Hodgkin's sarcoina; in this case metamorphosis appears to be complete. There is failure to achieve general systematization. Dropsy is not manifest to any degree in this case and surviving lymphoid tissue is present in quantity. 
Anamnesis. About June 1952 the patient complained of general malaise and a painless swelling in the left side of the neck. She was admitted to Hairmyres Hospital and biopsy of the cervical tumour established the diagnosis of Hodgkin's disease. (Ref. 1735:52. Hairmyres Hospital, Serial 219). A course of X-ray therapy was given, the exposure being local to the neck and thorax. General improvenent with regression of the swelling ensued, but six months ago recurrence developed. Further irradiation failed to influence the disease and she became very weak. This progressed till she was bed-ridden, and she was admitted, dying, to Glasgow Royal Infirmary on 12th November 1953. At this time she had mild anaemia and a large left-sided pleural effusion. A single intravenous injection of nitrogen mustard was given, $5.0 \mathrm{mgm}$., without untoward effect but two days later she passed into the agony and died l6th Novernber 1953.

\section{Report of Necropsy.}

26 hours post-mortem.

External. An extremely enaciated young woman. Where is pallor of the integument but no jaundice. Glandular enlirsements are not palpable. leight of the body, 73 lbs.

Reticulo-endothelial system. 1. Lymph nodes. Superficial lymph nodes accessible to dissection are not present in the neck. A few deep ones are found at the root of the neck. They are small, firm, and ill-defined. The cut surfaces display shaged outlines and a pinisish-white substance. iNodes are also recovered with difficulty and in small numers from the axillae, chiefly on the left siee. Antecubital nodes cannot be found. In the thorax, Stiboe's noles are irregularly enlarged, particularly on the left side. The mediastinal groups are markedly enlarged and hard. llost are white : Itin caseous foci centrally. The paratracheal chains are enlarged as far up as the thoracic inlet. The hilar and intra-pulmonary nodes are grossly enlariged and hard. On the left side several up to $4 \mathrm{~cm}$. diameter are visible. They are continuous uith diffuse massive growth replacing the lung substance of the upper lobe on this side.

The mesenteric nodes are sinall, scanty, and inconspicuous; on section they appear natural. Several retroperitoneal nodes in the para-aortic groups are hard and crager but little enlarged. 'In the pelvis the nodes are small and very few. Both groins have very few nodes, one firm nediumsized one is seen on the right side.

The popliteal fossae are devoid of nodes.

2. Spleen. $60 \mathrm{~g}$. The gland is small, pale, and soft. Repeated close slicing reveals always an homogeneous pale pink surface. Nodules are not present. 
3. Bone marrow. The skull is normal, the diploe is small. There is tunour-like infiltration of the periosteum of the internal aspect of the 3th left rib, the narrow cavity is srall and ary. (Infiltration or fibrosis is visible in the adjacent intercostal muscles but this process is limited in extent). In the sternum and lumbar vertebral bodies the marrow is pale pink, nodules are not visible.

Respiratory systen. The larynx, trachea, and bronchi are natural apart from purulent sputum in the latter. I serous effusion of $1200 \mathrm{cc}$. is present in the left pleural sac. The left lung is collapsed and most of the upper lobe is replaced by hard white or café-au-lait tinted tumour. In addition rounded nodules of similar growth are seen in the lower lobe. The right lung is less collapsed but contains nunerous scattered tumour nodules $0.5-4 \mathrm{~cm}$. dieneter. The picture closely resembles bronchial carcinoma, but oronchial ulceration is not a feature. In the left parietal pleura over the 6th, 7 th and 8 th ribs, and interspaces, small beads of very hard pale crean tissue are seen.

Cardiovascular system. The heart is small and atrophic, dissection was not performed. In the pericardium three tumour noduies $4-5 \mathrm{~cm}$. In their long axes are present in the parietal layer. The aorta is normal and free of atheroma.

Alimentary system. The peritoneum is healthy. The oesophagus, stomach and intestines shew only post-mortem change. The liver $1000 \mathrm{~g}$. is small but normal to naked eye scrutiny, repeated close slicing does not disclose any focal lesions. The gall-bladder and ducts are natural. The pancreas is softened and discoloured.

Genito-urinary system. The kidneys, left $120 \mathrm{~g} .$, right $110 \mathrm{~g}$. are natural, they are clear of tumour. The ureters and blader are nornal, likewise the uterus and adnexa. The breasts are pubertal.

Central nervous system. Brain, $1250 \mathrm{~g}$. Normal.

Endocrine systen. The hypophysis cerebri is small and red. The thyroid gland is sincll but otherwise normal. (Parathyroids, not dissected). The thymus is not recosnisable as a separate structure, it is possibly taken up in the thoracic tumour. The left adrenal gland is partly enclosed by and possibly also invaded by iard white tumour. The right gland appears noriul.

lificroscopical exanination: General description of the lesions encountered.

The great majority of the lesions are recognisable as atypical Hodgkin's disease. It presents very similar morphology to the biopsy but eosinophils are particularly numerous in many sites. It is the diffuse fibroblastic type with swathes and whorls of fibroblastic cells dominating the picture. Hodgkin giant-cells are often distinctly rare, the appearances then recalling fibrosarcoma in general form but 
suspiciously indolent. Focal deposits of the new tissue are quite common in nodes.

In a few sites morbid reticulum cell proliferation is notable but tjpical Hodgkin's sarcoma is absent.

In this case all the specimens taken for rnicroscopy are poorly preserved and the staining properties are badly impaired. Vicroscopical evidence of tuberculosis is lacking̈.

Distribution of the Iesion. Iymph nodes. Lesions are confirned in the cervical, axillary, paratracheal and mediastinal nodes. In the first named group hyaline fibrosis is pronounced. Further lesions are seen in the retroperitoneal and groin nodes but not in the mesenteric ones. These latter are very small.

Bone marrow. The marrow is hypocellular, the sample is free from deposits of the new tissue.

Spleen. Simple atrophy is present, no other changes are visible. The Iiver is free from deposits. The left adrenal gland is enclosed by Hodgkin tissue and at one point there is penetration of the capsule, a zone of necrosis precedes the growing edge of the tumour. Deposits are not found in the gland itself. The systemic involvement is most impressive in the lungs, pericardium and periosteum of the $8 \mathrm{th}$ left rib. In the first named site caseous necrosis abounds but the cellular reaction of tuberculosis is not seen. The kidneys are normal post-mortem specimens.

\section{Diagnosis and summary.}

Atypical Hodgkin's disease; diffuse fibroblastic type. Naked-eye the morbid anatomy recalled neoplasm of lung (see illustration)strongly. There is failure to systematise and the maximurn focus is thoracic. Apart from the lack of evidence of rapid growth the picture is tumour-like. Oedema is a feature in this case, it is chiefly expressed as hydrothorax. Survival of lymphoid tissue is moderate, the spleen being intact. 
John Young", aet 58 years. Ref. P.i. 418:53. G.R.I.

inamesis. The patient was first observed to have a swelling in his neck by his physician; this was five or six years azo. This lump persisted without alteration in size and his general health was good up till 1952. In liby of that year he developed pneunonia and pleurisy and vas treated in Heathfield Hospital, Ayr, for eight weeks. He recovered fairly satisfactorily but a productive coush remained. He again developed pleurisy in June 1953 and was trected in Irvine Central Hospital. for five weeks. Since then progressive tiredness, loss of energy and increasing shortness of breath have developed insidiously. Three months ago an irritating rash of ephemeral duration affected his face and arms. Just prior to admission to Glasyow Royel Infirnary anasarca developed. At this time generalised lymphadenopathy and splenomegaly were discovered. Biopsy (Ref. G.R.I. 4643:53, Serial 209) of a cervical node performed $13 \mathrm{th}$ November 1953 revedied highly cellular tumour-like Hodgkin's disease. The patient aied five aajs later.

\section{Report of Necropsy.}

16 hours post-mortem.

External. Anan of later nidale-age. There is pronounced cachexia Which is extensively concealed by dropsy except in the head. Jaundice is not present. (Veight of body $=103 \mathrm{Ibs}$. )

Reticulo-endothelial system. Lymph nodes. Generalised lymph node enlargement is present. The nodes in the neck, axillae, mediastinum, extra-mesenteric abdominal groups, para-aortic region and groins are increased in number and size, the largest being the size of horse beans. All are rather soft, discrete and fleshy. The cut surfaces occasionally reveal fibrosis but are usually a homogeneous pinkish-grey. The mesenteric glands are not much increased in size. The nodes under the subclavius muscles and Stibbe's para-sternal nodes are prominent due to increase in size. A lymph node, $I \mathrm{~cm}$. diameter, is present in the subcutaneous tissue over the left scapula. Two very small fibrous nodes were found in the antecubital fossae, one on each side. Popliteal nodes could not be found after prolonged search.

Spleen. (230 g.). The viscus is uniformly enlarged. A recent superficial fibrinous exudate is notable on the surface. The viscus is soft and pliable, the cut surface is faintly congested and nultiple small creany white spots are scattered through it, it is equivocal whether they are deposits of the new tissue. (Liver). The portal tracts do not shew any visible deposits.

Bone marrow. The bone of the fernur, sternum, Iumbar spines and skull appears normal, there is little osteoporosis. In the femur red marrow is confined to the upper third. It is clear of visible tumour; in the lower two-thirds gelatinous degeneration is present. The sternal and rib marrow is red and also clear of turnour. 
Thymus gland. The structure cannot be identified separately from the anterior mediastinal lymph nodes.

Respiratory system. The larynx and trachea are natural. There is moderate bronchiectasis of cylindrical type in the tubes to the lower lobes on both sides. The pleurae are thickened and present multiple dense adhesions to the lungs. Approximately 1,000 cc. of serous fluid are present in the thorax. Both lungs are heavy due to oedema and tumour. The upper lobes are congested and dropsical and clear of tuberculous or neoplestic disease. Tumour-like infiltration of greyish-pink colour is visible in the cut surfaces of the lower lobes. It is chiefly parabronchial thoush isolated irregularly outlined nodules about $1-2 \mathrm{~cm}$. are also seen. The large deposits are poorly demarcated. On palpation the affected tissue is cragsy; some necrotic softening is present. The appearances sugsest that the growth has enanated from the mediastinum.

Cardiovascular systen. Pericardium. Several hard white nodules are visible in the inner aspect of the parietal layer at the base. They appear to be totally íbrous. Heart $(300 \mathrm{~g} \bullet)$. The myocardium is of good quality but rather chestnut coloured. The chambers are natural. Witral valve. There is stenosis of button-hole type with rigid calcified margins; the orifice adnits one finger. (The lack of right ventricular enlargement is notable). The remaining valves are natural, slight sclerosis of the cusps being the only feature of note. lioderate atheroma of nodular type affects the coronary arteries. The aorta is atheromatous but the lesions are mild and calcification is slight. Arteries. Hild atheroma is notable with advanced arteriosclerosis. The cerebral vessels show only the latter. Veins. Thrombosis is not present in the large vessels.

Alimentary system. Tongue. The lymphoid tissue at the root is copious, it does not appear involved in tumour. Oesophagus. iNatural. The stomach and intestines do not shew any morbid features of note, in particular tuberculous ulceration is not seen. The liver $(1,350 \mathrm{~g} \cdot)$ is small, truly atrophic and shews terminal congestion. The vascular pattern is well demonstrated in the under surface due to atrophy of parenchyma. Tumour is not encountered on multiple close slicing. The gall-bladder and ducts are natural. Pancreas. Faintly orange-yellow in colour, otherwise normal.

Genito-urinary system. Kidneys (Ieft $150 \mathrm{~g} \cdot$, right $190 \mathrm{~g} \cdot$ ). The left is the seat of renal phthisis with cavitation ard pelvic ulceration. Calcareous change is absent. Deposits of tumour cannot be found. The right gland presents two large depressed scars probably of ischaemic origin; a nodule of firm pearly-white tissue, $6 \mathrm{~mm}$. diameter, is present in the pelvis. The gland is otherwise natural. The ureters are normal and the vesical reaches appear of the usual calibre. The bladder is healthy in appearance. The seminal vesicles contain partly calcified 
caseous debris and both epididymes are tuberculous. Both testes contain small hard white fibrous nodules in their substance.

Central nervous jystem. There is a snall deposit of reddish-white material like old blood clot between the skull and dura at the torcular Herophili. The sinuses are healthy. The leptoneninges are dropsical. The brain $(1,430 \mathrm{~g} \cdot$ ) is normal on dissection. (A few minute cysts are present in the left lateral lobe of the cerebellum near Deiters nucleus).

Indocrine system. The pituitary gland is soft but otherwise normal. The thyroid gland is small and the lobes are leaf-like. Two parathyroid glands were recovered, they appear nomal. The adrenal glands are small but apparently free from disease.

Microscopical exumination:

General description of the lesions encourtered.

Although the material is only 16 hours jost-mortem, the vreservation is indifferent: in miny of the larger lesions ante-morten necrosis is pronounced.

The lesion presents the same appeurance wherever it is found and is identical with the biopsy except where necrosis obtains. is in the nosology of the biopsy, it is equivocal whether it should be regarded as highly cellular tumour-like Hodgkin's disease or as reticulum cell sarcome Josinophils are recognisable in the deposits and therefore the former category is probably nore correct.

There is microscopical evidence of tuberculosis in the Iungs, liver, spleen, epididymis, right renal pelvis and adrenal gland. Acid/alcohol fast bacilli identical with ifco. tuberculosis can be demonstrated only in tile renal lesions.

Distribution of the lesion.

Iymph nodes. The lesion is present in the nodes of the neck, supraclavicular groups, axillae, para-tracheal chains, and mediastinum. The left suprascapular node which presented clinically is also involved. (A corresponding node on the right side does not exist.) The left antecubital fossa node. is involved but the right gland is normal. In the abdomen the retroperitoneal nodes are involved likewise those in the pelvis and both groins. Due to accident the mesenteric nodes escaped examination.

Bone marrow. The lesion is not found in the marrow from the femur, a thoracic, and a lumbar vertebra. Iinute tubercles are present in the femoral marrow.

Spleen. Minute lesions are identified together with caseous 
tubercles. In the liver only the latter are seen and they are rare.

The lesion is not found in the adrenal glands or hypophysis cerebri.

3xtra-nodal deposits are restricted to the lung's and pericardium. Sections of the pancreas, cerebellum, testis, prostate gland, and epididymis are negative. In the kidneys and epididymis active tuberculosis is found. The nodule in the right kidney is a fibroma.

Diagnosis and summary.

Highly cellular Hodgkin's disease with co-existent tuberculosis of acute caseating type. There is widespread but not universal implication of lymphadenoid tissue. Anasarca and hydrothorax are present and there is pronounced reduction of normal lymphoid tissue. 
Mrs. Wargaret Royal, aet 72 years. Ref. P.M. 57:54. G.R.I.

Anamnesis. In lifurch 1953 the patient developed a small swelling in the left side of her neck. This was resected after a provisional diagnosis of salivary calculus had been made. The biopsy shewed reticulum cell sarcoma, almost certainly of lymph node. (Ref. G.R.I. 1820:53, Serial 221). She received a course of radium therapy to the neck and has been attending the radiotherapy department at regular intervals since. In November 1953 she developed a cold with a productive cough and a swelling appeared in the right side of the neck. Since then she developed pneumonia and became bed-ridden. Dyspnoea became severe latterly. died IIth February 1954.

\section{Report of Necropsy.}

$4 \frac{2}{2}$ hours post-mortem.

Ixternal. A well-built somewhat obese elderly woman. Purpuric patches are notable over the limbs: There is oedema of the lower limbs. (Weight of body $=140$ Ibs.)

Reticulo-endothelial system. Iymph nodes. In the neck the superficial lymph nodes are small and inconspicuous. whe few found appear scarred. The deeper nodes, particularly those above the thoracic inlet are considerably enlarged on both sides. They are soft and discrete. The cut surfaces are fleshy and pink with occasional haenorrhagic areas. Fibrosis is not apparent other than in the capsules. The supra-clavicular nodes on both sides are similarly affected. In the left axilla nodes are very scanty. A few nodes shew marked fat atrophy except in the case of one small gland which is white. The right axilla nodes are sirnilar to those at the root of the neck; they are few in number. Nodes were not recovered from either antecubital region. The para-tracheal lymph nodes are normal in number but are individually enlarged up to $2 \mathrm{~cm}$. in their long axes. They are soft, fleshy and pink. The cut surfaces shew extensive haemorrhage. The mediastinal group, particularly those in the anterior compartment, are grossly enlarged, being up to $4 \mathrm{~cm}$. long. These shew anthracosis and considerable necrosis. The hilar and intrapulmonary nodes are considerably enlarged. They present the same features as the former group. The mesenteric nodes shew moderate increase in number and size. Those most affected are seen in the mesentery of the small intestine and in the lesser omentum. The retroperitoneal nodes, especially those about the aorta and its main branches are conspicuously enlarged. The nodes in the pelvis are large and very soft. liany are haemorrhagic. In the groins several large haemorrhagic nodes are present on both sides. A single small node is present in the right popliteal fossa. The left fossa does not contain nodes.

Spleen. (70 g.) The gland is small and atrophic; repeated slicing does not disclose any lesions. 
Bone narrow. There is resorption of cancellous bone in the ribs, sternum and vertebral column. The marrow is pale pink and homogeneous. In the richt femur the upper two-thirds of the shaft is occupied by pale pink marrow, and fat. Nodules are not present.

Respiratory system. Irachea. In the lower third of the posterior aspect of the lumen several white nodules $2-3 \mathrm{~mm}$. in diameter are visible growing in the mucosa. Further nodules are growing down into the bronchi on both sides. The growth is much more marked on the left side. It has brought about extreme stenosis of the left nain bronchus. Iungs (Ieft $370 \mathrm{~g} \cdot$, right $490 \mathrm{~g} \cdot)$. The left lung is almost entirely collapsed. The hilum is converted into a coarsely nodular pink and white tumour-like mass due to the grossly enlarged lymph nodes at this site. Several tumour deposits are present at the periphery of tne organ; these neasure 1-3 cm. in diameter. The rioht lung is fairly normil, apart from increase of nojes at the hilum and a sinele firm nass at the anterior lower margin of the lower lobe. This latter neasures 1 X $2 \mathrm{~cm}$. A serous effusion of approximately $300 \mathrm{ml}$. is present on both sides.

Cardiovascular system. Pericardium. The external layer is adherent to the enlarged glands of the mediastinum, but the merbrane is not infiltrated. Heart $(320 \mathrm{~g} .)^{2}$. Hoderate hypertrophy of the right ventricle is the only morbid feature of note. Both coronary vessels are atheromatous. The aorta shews atheroma, with ulceration and calcification.

Alimentary system. The oesophagus is distorted by the mediastinal growth but is not infiltrated. The stomach is of normal size. The mucosa presents multiple small ulcers scattered throughout it. These have the appearance of malignant ulcers. The duodenum and small bowel are normal. The colon is natural. The liver $(1,060 \mathrm{~g} \cdot)$ is small. Dissection does not reveal any abnormalities. The gall-bladder and ducts are nornal. The pancreas appears slightly scarred in places, but is otherwise natural.

Genito-urinary system. Kidneys (left $150 \mathrm{~g} \cdot$, right $150 \mathrm{~g} \cdot$ ). The only aonormalities visible are several small white poorly denarcated nodules in the cortical tissue of each gland. The deposits are firm and measure I-4 m. diameter. The pelves, ureters and bladder are healthy. The uterus contains a small endometrial polyp. There are several hyaline myomata in the wall. Both ovaries appear natural.

Indocrine systen. The pituitary gland appears normal. The thyroid gland $(78.5)$ shews rather diffuse enlargement of its left lobe. Definite tumour is not identified in it. The single parathyroid gland recovered is normal, but snall. Both adrenal glands are small and not very well preserved; they are otherwise natural.

Central nervous system. The scalp, skull and meninges are natural. The brain $(1,240 \mathrm{~g} \cdot)$ is of normal size. Dissection reveals circumscribed gliosis in the right thalamus. This probably represents an old softening, 
but orange staining is not visible. Recent softening is not visible. The vessels at the base are sclerotic and atheromatous.

Iicroscopical examination:

1. General description of the lesions encountered.

In all sites where tumour is confirmed the type cell is a reticulum cell which shews some degree of lymphoid differentiation. The cytoplasm is feebly oxyphile and indistinct. The nucleus is spheroidal, occasionally crenate or indented und confined by a sharply defined nuclear membrane. The nucleoplasm is not so leptochrometic as in the typical norbid reticulum cell but oxyphile nucleoli are comon. Reticulin and colligen formution are slight in nost examples. In several sites eosinophils abound wind the uppearances upr oxinate closely to cellular Hodghin's disease. Compared with the biopsy the growth is nore lymphoid in cell type.

Microscopical evidence of tuberculosis is lacking, the only other pathological process is a minute chromophobe adenoma in the hypophysis cerebri.

2. Distribution of the lesion.

Lymph nodes. The tumour is confirmed in the right cervical nodes, in one from the left side, in both supraclavicular groups and in the right axillary nodes. Both paratracheal chains, the mediastinal and intrapulmonary nodes all shew the growth. Necrosis in these deep nodes is extensive. All the abdominal nodes taken shew the disease. In both groins tumour is identified and is also present in a single node recovered from the right popliteal fossa. One left cervical node and one left axillary node are free from tumour.

In the blocks taken tumour is not identified in the spleen, lumbar vertebral marrow, nor sternal marrow. Foci are present however in the femoral marrow. The liver is devoid of deposits likewise the adrenal glands and hypophysis cerebri.

Extra-nodal deposits are confirmed in the trachea, bronchi, Iungs, stomach, peritoneum and kidneys.

The thyroid, parathyroids and pancreas are free of tumour.

Diagnosis and sumnary.

Reticulum cell sarcoma. There is evidence of instability of the microscopical picture. Systemic involvement is incomplete, several nodes, the liver and spleen not shewing the lesion.

Anasarca and hydrothorax are present. Iymphoid tissue survival is somewhat greater than might be expected. 
1. Reticulo-endothelial system.

Adami, J.G. 1909. The Principles of Pathology. Vol.1. p.681. London: Hodder \& Stoughton.

Aschoff, L. \& Kiyono, S. 1913. Folio Haematologica. 15. 383. Aschoff, I. 1924. Lectures on Pathology, New York. Ȧstbury, W.T. 1947. Proc. Roy. Soc. B. 134. 303. Belchier, J. 1736. Philosophical Transactions. Vol.39.p.79. Bloom, 7. \& Bartelmez, G.W. 1940. Amer. J. Anat. 67. 21. Bouffard. 1906. Ann. de I'Inst. Past. 20. 539.

Bremner, J.I. \& Weatherford, T.L. 1948. Textbook of Histology. 6th Ed. York. P.A: Blakiston.

Browning, C.H. 1905. J. Path. Bact. 10. 145 .

Burnet, M. \& Fenner, F. 1949. The production of Antibodies. 2nd Ed. Helbourne: Macmillan.

Capell, D.F. 1929. J. Path. Bact. 32. 629.

Cappell, D.F. 1930. J. Path. Bact. 33. 175.

Collins, D.H. 1949. 'Pathology of Articular \& Spinal Disease'. p.255 et seq. London: Arnold \& Co.

Dickson, C. 1908. 'The Bone Harrow'. London.

Drinker, C.K. \& Shaw, L.A. 1921. J. exp. Med. 33. 77.

Duthie, E.S. 1930. J. Path. Bact. 33. 547.

Duval, H. 1900. 'Precis d'histologie'. p.441 et seq. Paris: Masson et cie.

Eberth, R.H. \& Florey, H.N. 1939. Brit. J. exp. Path. 20. 342.

Ecker, A. 1889. The Anatomy of the Frog. Eng. Trans. by Geo.Haslam, M.D. Oxford. Clarendon Press.

Forkner, C.E. 1929. J. exp. Med. 49. 323.

Gaunt, W.E. \& Wright, G.P. 1940. J. infect. Dis. 67. 217. 
Glimstedt, G. 1936. Cited by Andrew \& Andrew 1948. In Amer. J. Anat. Gorer, P.A. 1946. Guy's Hosp. Rep. 95. 7.

Hadfield, G. 1949. Ann. Roy. Coll. Surgeons Ing. 5.89.

Jaffe, H.I. 1927. Arch. Path. 4. 45.

Jones, H.B., Wrobel, C.J., and Lyons, W.R. 1944. J. Clin. Invest. 23. 783 .

Latta, H., Gitlin, D. \& Janeway, C.A. 1951. Arch. Path. 51. 260.

Iison, I. \& Smulders, J. 1948. Nature. 162. 65.

Lowenhaupt, E. 1945. Amer. J. Path. 21.(1). 171.

Hiall, F.P. 1896. John Hopkins Hos. Rep. I. 1896.

Maximow, A.A. 1924. Physiol. Reviews. 4.533.

Maximow, A.A. 1932. In 'Cowdrey's Special Cytology', 2nd Ed. Vol.2. p.601. New York: Hoeber.

Metchnikoff, E. 1893. 'Comparative Pathology of Inflammation'. Translated by F.A. \& B.H. Starling, London.

Metchnikoff, E. 1905. Immunity in Infective Disease. Translated by Francis G. Binnie. (French Ed. 1901). Cambridge: University Press.

Michels, N.A. 1931. Arch. Path. 11. 775.

Moschowitz, E. 1950. Arch. Path. 49. 247.

Muir, R. \& Shaw Dunn, J. 1914. J. Path. Bact. 19. 226.

Neumann. 1879. Berl. KIin. Woch. No.6 Cited by Cohnheim, J. 1889. Lectures on general Pathology. Trans. by Alex. B. McKee. New Sydenham Society, London.

Nicol, T. \& Abou-Zikry. 1953. Brit. med. J. 1.133.

Oakley, C.I., Warrack, G.H., \& Batty, I. 1949. J. Path. Bact. 61. 179. Ranvier, L.A. 1890. C.R. Acad. Sci. Paris. 110. 165. 27 Janvier. Rawson, R.A. 1943. Amer. J. Physiol. 138. 708.

Reeves, D.L., \& Stuck, R.L. 1938. Hedicine. 17. 37.

Renaut. 1907. Arch. d'Anat. Micr. 9. 
Ribbert, A. 1889. Beitr. path. Anat. 6. 185.

Russell, W. 1890. Brit. med. J. ii p.1356.

Sabin, F.R. 1939. J. exp. Hed. 70. 67.

Schittenhelm. 1925. Krankeiten des Blutes, Vol. 2. Berlin. Cited by Piney, A. Recent Advances in Haematology. 2nd Ed. p.7.

Schmitt, F.O., Hall, C.T. \& Jakus, M.A. 1942. J. cell. comp. Physiol. 20. 11 .

Schulemann. 1917. Biochem. Z. 80. 1. Cited by Cappell, D.F. 1929 in J. Prath. Bact. 32. 600 .

Sheldon, J.H. 1935. Haemochromatosis. p.95. London: Oxford University Press.

Woolard, W.H. 1924. J. Anat. 58. 89.

Zsigmondy, R. 1914. Colloids and the UItramicroscope. Translated by Jerome Alexander. p.113 et seq. London: John Viley \& Son.

2. Iymphatics.

Assellio, G. 1628. 'De lactibus'. Ediderunt Alex. Tadimus et Senat Septalius. (Hunterian Museum, University of Glasgow).

Barnes, J.If. \& Trueta, J. 1941. Lancet. i. 623.

Clark, E.R. 1909. Anat. Rec. 3. 183.

Clark, D.R. and Clark, ت.L. 1926. Amer. J. Anat. 38.

Clark, Z.R. and Clark, I.I. 1937. Amer. J. Anat. 60. 253.

Clark, W.I. le Gros. 1945. The tissues of the body. p.219. p.227. 2nd Ed. Oxford: Clarendon Press.

Crandall, L.A., Jr., S.B. Barker, \& Graham, D.G. 1943. Gastro-enterol. 1. 1040 .

Drinker, C.K. 1946. Annals of the New York Academy of Sciences. Vol.46. Art.8. pp. 679-882. pp.807-821. Lymph. $16 \mathrm{Sep}$. 1946. New York.

Ehrich, W.E. 1946. Annals of the New York Academy of Sciences. Vol.46. Art.8. p.823.

Elkes, J.J., Frazer, A.C. and Stewart, H.C. 1938. J. Physiol. 95.68. 
Frazer, A.C. 1946. Physiol. Rev. 26. 103.

Gage, S.H. \& Fish, P.A. 1924-5. Amer. J. Anat. 34. 1.

Herring \& Simpson. 1906. Proc. Roy. Soc. B. 78.

Huntington, G.S. 1914. Amer. J. Anat. 16. 259-316.

Kampmeier, 0.F. 1928. Aner. J. Anat. 40. 413.

Klein, 2. 1875. The Anatorny of the Lymphatic System. Vol.2. Cited by Schafer, I.A. in Quain's Textbook of Microscopic Anatomy, 1912, London. Longmans, Green \& Co.

Leach, i.H. 1938. J. Physiol. 93. 1.

MacCallum, W.G. 1903. Bull. Johns Hopk. Hosp. 14. 394 .

McClure, C.F.T. 1915. Anat. Rec. 9. 563-579.

McClure, C.F.W. 1921. Anat. Rec. 22.

Pullinger, B.D. \& Florey, H.i. 1935. Brit. J. exp. Path. 16. 49.

Rand, H.i. 1950. The Chordates. p.633. Penn. U.S.A. Maple Press.

von Recklinghausen, F. 1870. In 'Manual of Human and Comparative Histology'. Ed. B. Stricker. Vol.1. p.297.

Sabin, F.R. 1902. Amer. J. Anat. 1. 367.

Sabin, F.R. 1911. Anat. Rec. 5 .

Sabin, F.R. 1922. Physiol. Reviews. 2.

Sheehan, H.L. 1931. J. Physiol. 72. 201.

Weed, L.H. 1914. J. med. Res. 31. 51.

3. Lymphoid Tissue.

Bargmann, W. 1951. Histologie und Mikroskopische anatomie des menschen. Band.2. p.242. Stuttgart: Thieme.

Blacklock, J.i.S. 1933. J. Path. Bact. 37. 504.

Bourne, A.G. 1885. Quart. J. micr. Sci.

Bunting, C.H. \& Huston, J. 1921. J. exp. Med. 33. 593.

Clark, Le Gros, W.T. 1945. The tissues of the Body. 2nd Ed. Oxford: The Clarendon Press. 
Corner, I. 1913. Brit. Med. J. 15. 325.

Dominici, H. 1902. In Manuel d'histologie pathologique by V. Cornil, L. Ranvier, A. Brault \& N. Letulle. 3rd Ed. Vol.2. p.581. Paris: Felix Alean.

Downey, H. \& Weidenreich, F. 1912. Arch. f. mikr. Anat. 80. 306. cited by Cowdry, T.V. in 'Special cytology' Vol.2 p.609.

Dukes, C. \& Bussey, H.J.R. 1926. J. Path. Bact. 29. 111.

Ellermann, V. 1923. Folia Haemat. Lpz. 27. 207.

Farris, i.J. 1943. Amer. J. Anat. 72. 223.

Fraser, J.E. 1931. 'A manual of Imbryology'. London: Balliere Tindall \& Cox.

Gall, I.A. \& Stout, H.A. 1940. Amer. J. Path. 16. 433.

Gray, H. 1854. 'On the structures \& Use of the Spleen'. London.

Hwang, J.H.S., Lippincott, S.W. \& Krumbhaar, I.B. 1938. Amer. J. Path. 14. 809.

Hwang, J.H.S. \& Krumbhaar, M.B. 1940. Amer. J. med. Sci. 199. 75.

Krumbhaar, Z.B. 1942. In 'Problems of Ageing'. 2nd Ed. Ch.8. p.139. Edited by T.V. Cowdry. Baltimore: Villiams \& Wilkins.

von Mollendorff, W. 1930. Handbuch der Wikroskopischen anatomie des menschen. VI/I. p.283.

Nakahara, W. \& Murphy, J.B. 1921. Anat. Rec. 22. 107.

Piney, A. 1926. Br. J. Surg. 53. 9.

Pratt, C.I.G. 1931. Lancet. ii. 794.

Reubens-Duval, H. et Fage, H. 1909. C.R. Soc. Biol. Paris. p.802. Williams, R.J. 1939. Amer. J. Path. 15. 377.

Yoffey, J.H. \& Drinker, C.K. 1939 (a). Anat. Rec. 74. 417.

4. Lymph Nodes.

Bayer, K. 1885. Ztschr. f. Heilk. 6. 105.

Bloomfield, A.I. 1915. Arch. intern. Med. 16. 197. 
Bunting, T.I. 1904. J. Anat. \& Physiol. 39. 55.

Bunting, T.L. 1905. J. Anat. \& Physiol. 39. 178.

Conway, E.A. 1937. Anat. Rec. 69. 487.

Denz, F.A. 1947. J. Path. Bact. Vol.59. p.575.

Dorninici, H. et Reubens-Duval, H. 1909. C.R. Soc. Biol. Paris. 18 Dec. Downey, H. \& Weidenreich, F. 1912. Arch. f. mikr. Anat. 80. 306. Downey, H. 1922. Haematologica. 3. 431.

Ehrich, W. 1929. Amer. J. Anat. 63. 347 and 385.

Furata,

Gibbes, H. 1884. Qu. J. micr. Sci. 24. 186.

Gibbes, H. 1893. Amer. J. med. Sci. 106. 316.

Gulland, G.L. 1894. J. Path. Bact. 2. 447.

Hassall, H.i. 1849. Microscopic anatomy of the human Body. Vol.1. 487. Heudorfer, K. 1921. 2. Anat. Entwickl. 61. 365-401.

Jaffe, H.I. \& Richter, 政. 1928. J. exp. Med. 47.

Job, T.T. 1922-23. Amer. J'. Anat. 31. 125.

Jolly, J. 1923. 'Traite Technique d'Hematologie'. Vol.2. p.692. Paris: Maloine et Fils.

Jordan, H.玉. 1934. Anat. Rec. 59. 297.

Latta, J.S. 1922. Anat. Rec. 24. 233.

Reubens-Duval, H. et Fage, H. 1909. C.R. Soc. Biol. Paris. 696. Sanders, A.G. \& Florey, H.W. 1940. Brit. J. exp. Path. 21. 275. Sappey, Ph.C. 1869. Traite d'Anatome Descriptive. 2nd Ed. Tom.2 p.799. Paris: E. Martinet.

Stiles, H.J. 1892. Edinb. med. J.

Sundberg, R.D. 1947. J. Lab. \& Clin. Med. 32. 777.

Taylor, G.W. \& Nathanson, I.T. 1942. 'Lymph Node Metastases'. New York: Oxford University Press. 
Teichmann, L. 1861. Das Sangadersystem, vom anatomischen standpuncte. Leipzig.

Tarthin, A.S. 1901. Amer. J. Anat. 1. 4.

5. Reticulosis.

Bennet, J.H. 1845. Jdinb. med. J. 64. 413.

Cazal, P. 1946. 'La Reticulose Histiomonocytaire'. Paris: Masson.

Craigie, D. 1828. 'Elements of General \& Pathological Anatomy'. Edinburgh: John Stark.

Craigie, D. 1845. Edinb. med. J. 64. 400.

Oberling, C. 1928. Bull. Assoc. Franc. du Cancer. 17. 259.

Robb-Smith, A.H.T. 1938. J. Path. Bact. 67. 457.

Ross, Joan M. 1933. J. Path. Bact. 37. 311.

Scott, R.B. \& Robb-Smith, A.H.T. 1936. St. Barth. Hosp. Rev. 49. 143. Symmers, W. \& Hutcheson, W. 1939. Arch. Path. 27. 562.

6. Iymphoid Tissue Tumours.

Brill, N.E., Baehr, G. \& Rosenthal, N. 1925. J. Amer. med. Ass. 84. 668. Le Count, B.R. 1899. J. exp. Mad. 4. 559.

Cruveilhier, J. 1835-42. Anat. Path. du corps Humain. Tom.2. 34 liv. Custer, R.P. \& Bernhard, W.G. 1948. Amer. J. med. Sci. 216. 625. Dawson, E.K., Innes, J.R.li. \& Earvey, V.F. 1937. Edinb. med. J. 44. 645 . Ewing, J. 1913. J. med. Res. 28. I.

Falconer, E.H. \& Leonard, H.I. 1938. Amer. J. med. Sci. 195. 294. Foot, N.C. 1924. J. med. Res. 44. 417.

Fox, H. \& Farley, D.L. 1924. J. Cancer Res. 8. 162-172.

Gall, E.A. \& Mallory, T.B. 1942. Amer. J. Path. 18. 381.

Giffin, H.Z. \& Hatkins, C.H. 1934. Amer. J. med. Sci. 188. 761.

Hayden, H.C. \& Apfelbach, C.W. 1927. Arch. Path. 4. 743.

Herbut, P.A., Willer, F.R. \& Erf, A.I. 1945. Amer. J. Path. 21. 233. 
Minot, G.R. \& Isaacs, R. 1926. J. Amer. med. Ass. 86. 1185.

J. Amer. med. Ass. 86. 1265.

Muir, R. 1909. In 'A System of Medicine'. Edited by Albutt C. \& Rollerton, H.D. Vol.5. p.811.

MacCarty, W.C. 1930. J. Cancer Res. 14. 394.

Oliver, Jean. 1913. J. Med. Res. 29. 191.

Piney, A. 1926. Arch. Path. \& Labty. lied. 2. 301.

Robb-Smith, A.H.T. 1947. In Recent Advances in Clinical Pathology, idited by S.C. Dyke, p.350, London.

Sugarbaker, I.D. \& Craver, I.F. 1940. J. Amer. med. Ass. 115. Symmers, D. 1948. Arch. Path. 45. 73.

Velpeau, A.A.L.M. 1840-1. Lecons orales de clinique chirurgicale faites a L'Hopital de la Charite. Vol.3. 147. Paris: Balliere.

Warren, S. \& Picena, J.P. 1941. Amer. J. Path. 17. 385. Warthin, A.S. 1931. Ann. Surg. 93. 153. Wells, W.G. \& Llaver, M.B. 1904. Amer. J. med. Sci. 128. 837. 7. Hodgkin's Disease and Hodgkin's Sarcoma.

Anderson, W.A.D. 1948. 'Pathology'. Ch.33. London: Henry Kimpton. Badia, P.D. 1943. Amer. J. Surg. 59. 577.

Baker, C. \& Mann, W.N. 1939. Guy's Hosp. Rep. 89. 83-107.

Baker, C. \& Mann, W.N. 1940. Lancet. 238-1. 23.

Barron, in. 1926. Arch. Path. 2. 659.

Bersack, S.R. 1943. Amer. J. Clin. Path. 13. 253.

Bersack, S.R. 1944. Arch. intern. Hed. 73. 232.

Bright, R. 1838. Guy's Hosp. Rep. 3. 405.

Bristowe. 1853. Trans. Path. Soc. Lond. 4. 178.

Bunting, C.H. \& Yates, J.I. 1913. J. Amer. med. Ass. 61. 1803. 1914. J. Amer. med. Ass. 62. 516.

Burger, R.E. \& Lehman, E.P. 1941. Arch. Surg. 43. 839.

Ciechanowski, S. 1938. Virch. Arch. 305. 206. 
Clark, 1901. Brit. med. J. ii. 701.

Coley, W.S. 1908. Surg. Gyn. Obstet. 6.649.

Cornil. 1865. Arch. gen. de med. Vol.2. 206.

Cowen, R.I. 1949. Urol. \& Cutan. Rev. 53. 521.

Craver, L.F. \& Copeland, M.H. 1934. Arch. Surg. 28. 1063.

Cunningham, W.I. 1915. Amer. J. med. Sci. 150. 868.

Cunningham, H.ت. 1917. Amer. J. med. Sci. 153. 406.

Debré. 1950. Bull. Soc. med. Hop. Paris. Seance de 27 Jan. 1950. 4th Serie. 66th Annee. 76-78.

Delbet, P. 1893. Semaine lied. 13. 430-2.

Delbet. 1895. C.R. Acad. Sci. Paris. No.24.

Dietrich, A. 1896. Beit. KIin. Chir. 16. 377 .

Dunn, J.S. 1939. Personal cominunication.

Dunn, R.I.S. 1951. Glas. med. J. 32-3I.

Falconer, E.H. \& Leonard, M.E. 1949. Ann. Int. Med. 29. 1115.

Fitchett, M.S. \& Weidman, F.D. 1934. Arch. Path. 18. 225.

Fox, A. 1952. Arch. Path. 54. 75.

Fox, H. 1926. Ann. Med. Hist. 8. 370-74.

Fox, H. \& Farley, D.I. 1922. Amer. J. med. Sci. 163. 170.

1923. Amer. J. med. Sci. 166. 313.

Fox, H. \& Farley, D.L. 1923. J. Radiol. 4. 261-267.

Fraser, J. \& Vlekie, E.C. 1933. Edinb. med. J. 40. 455-481.

Gibbons, H.i. 1906. Amer. J. med. Sci. 132. 692.

Goldman, I.B. 1940. J. Amer. med. Ass. 114. 1611.

Goldman, L.B. \& Victor, A.WI. 1945. N.Y. St. J. Med. 45. 1313.

Goldman, E.ষ. 1892. Zbl. allg. Path. Anat. 3. 665.

Goldman, 3.T. 1909. Beitr klin. Chir. 64. 192.

Goormagtigh, N. 1925. C.R. Soc. Biol. 92. 457. 
Gordon, H.H., Gow, A.I. \& Rolleston, H. 1937. British Encyclopoedia of medical Practice. Edited by Sir H. Rolleston. Bt. Vol.6 p.523. Lond on: Butterworth.

Gowers, W.B. 1879. In Reynolds System of liedicine. Ed. Sir J.R. Reynolds. Vol.5. Ch. p.806 et seq.

Grand, C.G. \& Chambers, R. 1949. Cancer Res. 9. 1831.

Greenfield, i.s. 1878. Trans. Path. Soc. Lond. 29. 272.

Greenstein, R.H. \& Conston, A.S. 1949. Lmer. J. med. Sci. 218. 384. Hale-ihite, i. 1924. Guy's Hosp. Rep. 74. 117.

Harrison, C.V. 1952. J. Path: Bact. 64. 513.

Harrison, G.A. 1943. Chemical methods in Clinical Medicine. 2nd Ed. Iondon: Churchill.

Hodgkin, T. 1829. 'A Catalogue of the preparations in the Anatomical museum of Guy's Hospital. London: R. Watts.

Hodgkin, T. 1832. Trans. Hifed. Ohir. Soc. Lond. 17. 68.

Hodgkin, T. 1836-40. 'Lectures on the liforbid Anatomy of the Serous and Hucous Hembranes'. London: Sherwood, Gilbert \& Piper.

Holmes, 0.W. 1859. Elsie Venner. p.168. London: Wm. Paterson\& Co. Hoster, H.A., Dratman, H.B., Craver, L.F. \& Rolnick, H.A. 1948. Cancer Res. 8. numbers 1 \& 2 .

Isaacson, N.H., Spatt, S.T., \& Grayzel, D.M. 1947. Ann. Int. Med. 27. 294.

Jackson, H. (Jr.) Surg. Gynec. \& Obstet. 1937. 64. 465.

Jackson, H, (Jr.) 1939. New Ingl. J. Med. 220. 26.

Jackson, H. (Jr.) and Parker, F. (Jr.) 1947. 'Hodgkin's Disease and allied Disorders'. New York: Oxford University Press.

Jackson, H. \& Parker, F. 1920-47. In Nelson Loose-Leaf medicine. Ed. Talter W. Palmer. Vol.3. p.347. and supp. New York: Thos. Nelson \& Sons.

Jones, C.4. 1946. New Ingl. J. lifed. 234. 65 .

Kamahora, J. \& Terashima, H. 1951. Med. J. Osaka. Univ. 2.4.29. 
278.

Karsner, H.T. 1910. Arch. Int. Med. 6. 175.

Karsner, H.T. 1931. Human Pathology. 3rd Ed. p.304. Philadelphia: Lippincott.

Kofoid, C.A., Boyers, I.M. and Swezy, 0. 1922. J. Amer. med. Ass. 78. 1604 .

Krumbhaar, I.B. 1931. Amer. J. med. Sci. 182. 764.

Krumbhaar, E.B. 1934. Amer. J. med. Sci. 188. 597.

Laser, L. 1951. J. nat. cincer Inst. 12. 259.

L'Esperance, I.S. 1929. J. Immunol. 16. 37.

Lemon, J.S. 1924. Amer. J. med. Sci. 167. 178.

Longcope, T.T. 1903. Bull. Ayer. Clin. Labty.

Longcope, H.T. 1947. Acta med. Scand. Supp. 196. 659.

MacCallum, W.G. 1928. Text-Book of Pathology. 4th Ed. London. p.856. Malpighi, M. 1666. Di Viscerum Structura, Opera Omnia. Vol.2. p.IIl. Bonn.

Narkham, 1853. Trans. Path. Soc. Lond. 4. 177.

Martin, C.F. 1901. J. Med. Res. 6.249.

Medlar, E.H. 1931. Amer. J. Path. 7. 499.

Rifllaret, P., Reilly, J., Bastin, R. et Tournier, P. 1950. Bull. Soc. med. Hop. Paris. 11. 424.

Morpagni, G.B. 1752. Epist. 68.

Mueller, T. 1920-21. J. med. Res. 42. 325.

Murchison, C. 1870. Trans. Path. Soc. Lond. 21. 372.

Murray, G.R. 1909. In 'A Syster of Medicine' Edited by Allbutt, C. \& Rolleston, H.D. Vol.4. (1) p.459.

Naumann, H.N. 1947. Arch. Path. 43. 324 .

Parsons, P.B., Poston, M.A. \& Wise, B. 1939. Amer. J. Path. 15. 634 •

Pavy. 1859. Lancet. 2. 213.

Phillips, R. 1941. Lancet. 240. 480. 
Piney, A. 1925. Med. Press \& Circ. 170. 189.

Potter, E.L. 1935. Arch. Path. 19. 139.

Priesel, A. \& Winkelbauer, A. 1926. Virch. Arch. 262. 749.

Pullinger, B.D. 1932. 'Rose Research on Lymphadenoma'. p.115. Bristol: Wright \& Sons.

Rebuck, J.W. 1947. J. Lab. \& Clin. Med. 32. 660.

Reed, D.Mi. 1902. Johns Hopk. Hosp. Rep. 10. 133.

Robb-Smith, A.T.H. 1947. In 'Recent Adv. in Clinical Pathology'. General Editor S.C. Dyke. London: Churchill.

Robinson, G.I. 1948. Brit. med. J. 2. 822.

Shennan, T. 1927. 'Post mortems and morbid anatomy'. p.353. London: Faber \& Gwyer.

Simmons, C.C. 1903. J. med. Res. 9. 378.

Smith, C.A. 1934. J. Pediat. 4. 12.

Smith, I.C. 1935. Lancet. 2. 874.

Sternberg, C. 1898. Zeit Heilkunde. 19. 21.

Stewart, F.H., \& Doan, C.A. 1931. Ann. Surg. 93. 141.

Symmers, D. 1917. Arch. Int. Med. 19. 990.

Symmers, D. 1924. Amer. J. med. Sci. 167. 157. 313.

Symmers, D. 1944. Arch. Int. Med. 74. 163.

Symmers, D. 1945. J. Amer. med. Ass. 128. 1248.

Symmers, W. St.C. 1953. Personal communication.

Torrey, J.C. 1916. J. med. Res. 34. 65.

Trousseau, A. 1868. Clinique medicale de L'Hotel Dieu de Paris. Vol.3. p.568.

Tullis, J.M. 1949. Amer. J. Path. 25. 829.

Twort, C.C. 1926. Medical Press. 1. 195.

Twort, C.C. 1930. J. Path. Bact. 33. 539. 
Wade, H.i. 1913. J. med. Res. 29. 209.

Wallhauser, A. 1933. Arch. Path. 16. 522.

Nelch, J.E. 1910. Proc. New York Path. Soc. n.s. 10. 161.

White, W.C. \& Proscher, F. 1907. J. Amer. med. Ass. 49. 1115.

Wilks, S. 1856. Guy's. Hosp. Rep. 3. 114.

Wilks, S. 1865. Guy's. Hosp. Rep. 11. 56.

Wilks, S. \& Moxon, i. 1875. 'Lectures on Pathological Anatomy'. 2nd Ed. London: Churchill.

Willis, R.A. 1948. Pathology of Tumours, p.761. Iondon: Butterworth. Wise, N.B. \& Poston, H.A. 1940. J. Amer. med. Ass. 115. 2016. Wray, S. 1943. J. Path. Bact. 55. 75. Wunderlich. 1866. Archiv. fur Heilkunde. 531.

Yamasaki, H. 1904. Ztschr. f. Heilk. 25. 269.

Ziegler, K. 1911. 'Die Hodgkinsche Krankheit'. Jena: Gustav Fischer.

8. Peculiar fibrosis.

Blalock, A., Robinson, C.S., Cunningham, R.S. \& Gray, M.E. 1937. cappele im. 10 i. Arch. Surg. 34. 1049 .

Coventry, H.B., Ghormley, R.K. \& Kernohan, J.i. 1925. J. Bone Jt. Surg. 27. 105. 233. 460.

Durante, G. 1902. In 'Wanuel d'Histologie pathologique' by Cornil, V. \& Ranvier, L. 3rd Ed. 2. 162.

Fairbank, T. 1951. 'An atlas of general affections of the Skeleton'. Edinburgh: D. \& S. Livingstone.

Kellgren, J.H. 1952. Brit. med. J. 1. 1093. 1152.

Kelly, A.0.W. 1903. Amer. J. med. Sci. 125. 116.

Meeker, I.H. 1925. Amer. J. Path. 1. 57.

Murray, M.R. \& Stout, A.P. 1942. Amer. J. Path. 182. 585.

Husgrove, J.T. \& McDonald, J.R. 1948. Arch. Path. 45. 513. 
Macfarlane, J. 1832. Clinical Reports of the Surgical Practice of Glasgow koyal Infirmary. p.63. \& p.66.

Porter, K.R. \& Vanamee, P. 1949. Proc. Soc. exp. Biol. N.Y. 71. 513. Shaw, A.F.B. \& Smith, R.P. 1925. Brit. J. Surg. 13. 93.

Stout, A.P. 1948. Ann. Surg. 127. 706.

Stout, A.P. 1949. 'Tumours of the peripheral nervous system'. In Atlas of Tumour Pathology. Sect.2. fasc.6. Armed Forces Institute of Pathology. U.S. Army. Washington D.C.

Stewart, H.J. \& Mowat, T.B. 1924. Brit. J. Surg. 12. 355.

Teacher, J.H. 1900. 'Catalogue of the Anatomical \& Pathological

Preparations of Dr. Villiam Hunter'. Glasgow: Wrac Lehose.

Vaughan, J.U. \& Harrison, C.V. 1939. J. Path. Bact. 48. 339.

Wood, F.C. \& Krumbhaar, i.B. 1951. In Nelson Loose Leaf lledicine. Vol.4. p. 288 et seq.

9. Eosinophiles.

Barker, I.F. 1894. Johns. Hopk. Hosp. Bull. 5. 93.

Beattie, J.M. \& Dickson, H.I.C. 1943. A textbook of Pathology. 4th Ed. London: Heinemann.

Biggart, J.H. 1932. J. Path. Bact. 35. 799.

Bunting, C.H. 1932. In Cowdry's 'Special Cytology'. 2nd Ed. Vol.2. p.693.

Dominici, H. 1909. Folia Haematologica. 8.97.

Edward, D.S. 1938. Lancet. I. 936.

Friedemann, U. 1934. Brit. med. J. 1. 517.

Howard, W.T. \& Perkins, R.G. 1902. Johns Hopk. Hosp. Rep. 10. 249.

Jaffé, H.I. \& Lichtenstein, I. 1944. Arch. Path. 37.99.

Karsner, H.T. 1931. 'Human Pathology' 3rd Id. p. 185-6. Philadelphia: Lippincott.

Lang, F.J. 1938. In 'Handbook of Haematology' Ed. by H. Howney. Vol.3. p.2103. New York, Paul B. Hoeber, Inc. 
Wusgrove, J.I. \& Dockerty, il.B. 1950. Arch. Path. 50. 427.

Opie, E.L. 1904. (a) Amer. J. med. Sci. 126. 988.

Opie, I.I. 1904. (b) Amer. J. med. Sci. 127. 477.

Ringoen, A.R. 1921. Folia haemat. Ipz. 27. 10.

Symmers, W. St.C. 1950. Arch. Path. 50. 475.

Vaughan, J. 1952. J. Path. Bact. 64. 91.

Weir, I.H. 1903. Amer. J. med. Sci. 125. 74.

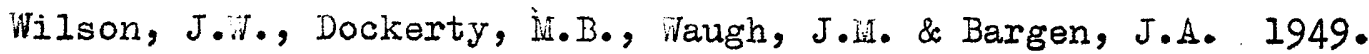
Arch. Surg. 59. 933.

10. Experimental.

Bonser, G.I., Clayson, D.B., \& Jull, J.W. 1951. Lancet. 2. 286. Boyland, E. 1952. Cancer Res. 12. 77.

Cloudman, A. vi. 1939. Amer. J. Cancer. 36. 578.

Cook, J.i. 1943. Chemistry \& Cancer. A lecture to the Royal Inst. of Chemistry of Gt. Britain and Ireland.

Curran, R.C. 1951. Br. J. Exp. Path. 33. 82.

Gillman, J., Iandelstam, T. \& Gilbert, C. 1945. Brit. J. Exp. Path. 26. 67 .

Gillman, J. \& Gillman, T. 1948. S. Afr. J. med. Sci. 13. 11.

Gillman, J., Gillman, T. \& Gilbert, C. 1949. S. Afr. J. med. Sci. 14. 21 .

Hodgson, J.F. 1903. J. Comp. Path. \& Therap. 16. 382.

Hoffman, G.T. \& Rottino, A. 1949. Arch. Path. 48. 230.

Hoffman, G.T. \& Rottina, A. 1950. Blood. 5. 74 .

Marshall, A.H.I. 1953. Acta path. microbiol. Scand. 33. 1.

Miller, F.R. \& Turner, D.I. 1943. Amer. J. med. Sci. 206. 146.

Moore, F.D., Tobin, I.H. \& Aub, J.C. 1943. J. Clin. Invest. 22. 155. MicFadzean, J. 1903. J. Comp. Path. \& Therap. 16. 382. 
Oppenheimer, B.S., Oppenheimer, T.T. \& Stout, A.P. 1948. Proc. Soc. Exper. Biol. \& Med. 67. 33-34.

Passey, R.D., Leese, A. \& Knox, J.C. 1936. J. Path. Bact. 42. 425.

Pearse, A.G., Everson. 1953. Histochemistry, Theoretical and applied. Ch.5. p.81 et seq. London: J. \& J. Churchill Ltd.

Schiller, i. 1937. Amer. J. Cancer. 31. 486.

Simpson, C.L. 1952. Br. J. Exp. Path. 33. 524 .

Smith, R.0. \& Wood, N.B. 1949 (a) J. Exper. Hed. 90. 555.

Smith, R.0. \& Wood, W.B. 1949 (b) J. Exper. Med. 90. 567.

Stewart, H.J. \& Dobson, J.F. 1924. Brit. J. Exper. Path. 5. 65.

Turner, D.I. \& liiller, F.2. 1943. Proc. Soc. Exper. Biol. \& Med. 54. 177 .

Editorial 1953. Lancet. 1. 32.

11. General references.

Butterfield, H. 1949. The origins of modern science. London: Bell. Cheatle, G.I. \& Cutler, M. 1931. Tumours of the breast. Iondon: Ed. Arnold \& Co.

Donaldson, R. 1931. Practical Morbid Histology. 2nd Ed. London: Heinemann.

Ewing, J. 1940. Neoplastic Diseases. 4th Ed. Philadelphia: Saunders. Gross, J. 1950. Ann. H.Y. Acad. Sci. 52. 7. 964.

Mallory, F.B. 1914. The Principles of Pathologic Histology• Philadelphia: Saunders.

Minot. 1905. Amer. J. Anat. 4.

Moodie, R.I. 1923. Paleopathology.

Nicholson, G.it. 1926. The Nature of Tutoour Formation (Erasmus Wilson Lectures 1925) Cambridge: Butterworth.

Osman, A.A. 1937. 'Original papers of Richard Bright on Renal Disease'. Iondon: Ox. Univ. Press.

Sargent, H. 1951. Brit. ned. J. 2. 31 I. 
Senn, N. 1900. The Pathology and Surgical Treatment of Tumours. ch.25. 2nd Id. Philadelphia: i.B. Saunders.

Shaw, A.F.B. 1938. J. Path. Bact. 47. 115.

Thompson, D'A.W. 1942. 'On growth and Form'. 2nd Ed. Cambridge: The University Press.

Trotter, ir. 1930. Brit. Med. J. 2. 129.

White, C. Powell. 1927. The Principles of Pathology. Manchester: The University Press.

12. Technique.

Barrett, A.M. 1944. J. Path. Bact. 56. 133.

Conn, H.J. 1946. Biological Stains. 5th Ed. Geneva, N.Y., U.S.A. Biotech. Publications.

Foot, N.C. \& Henard, M.C. 1927. Arch. Path. 4. 211.

Gatenby, J.B. \& Beams, H.W. 1950. The microtomists vade-mecum (Bolles Lee.) Ilth Ed. Fondon: Churchill.

Gordon, H. \& Sweets, H.H. Jr. 1936. Amer. J. Path. 12. 545.

Little, R.D. 1947. J. Lab. \& Clin. Med. 32. 910.

Iillie, R.D. 1948. Histopathological Technique. Philadelphia \& Toronto: The Blakiston Company.

Mallory, F.B. \& Parker, F. Jr. 1927. Amer. J. Path. 3. 515.

Robb-Smith, A.H.T. 1937. J. Path. Bact. 45. 312.

Stricker, S. 1870. Manual of Human and Comparative Histology. Translated by Henry Power. London: New Sydenham Society.

Waller, A. 1846. Philosophical Magazine. Vol.29. pp. 287 \& 397. Wilder, H.C. 1935. Amer. J. Path. 11. 817.

13. Addenda.

Burrows, H. 1932. Localisation of Disease. London, Balliere Tindall \& Cox.

Drinker, C.K. \& Yoffey, J.W. 1941. Lymphatics, Lymph and Lymphoid Tissue. Cambridge Mass. Harvard Univ. Press.

Mitchell, A.J. \& Wislocki, G.B. 1944. Anat. Rec. 90. 261. 


\section{VOLUME III}

\section{PHOTOGRAPHS.}

1. Mesenchyme.

2. Iymph nodes.

3. Ectopic lymphoid tissue.

4. Hodgkin's disease, Biopsy Series.

5. Hodgkin's sarcoma, Biopsy Series.

6. False Hodgkin's disease.

7. Wixed lesions.

8. Reticulum cell sarcoma.

9. Lymphosarcoma.

10. Brill's disease.

11. Necropsy Series.

12. Peculiar fibrosis.

13. Lymphocytoid eosinophiles.

14. Experimental.

15. Lymph node counts.
Figs. $1-3$

Figs. 4-36

Figs. $37-44$

Figs. $45-85$

Figs. 86, 87

Figs. 88-95

Figs. 96-105

Figs.106-109

Figs.110-113

Figs.114-115

Figs.117-205

Eigs.206-212

Fig. 213

Figs.214-235

Figs.236-246 

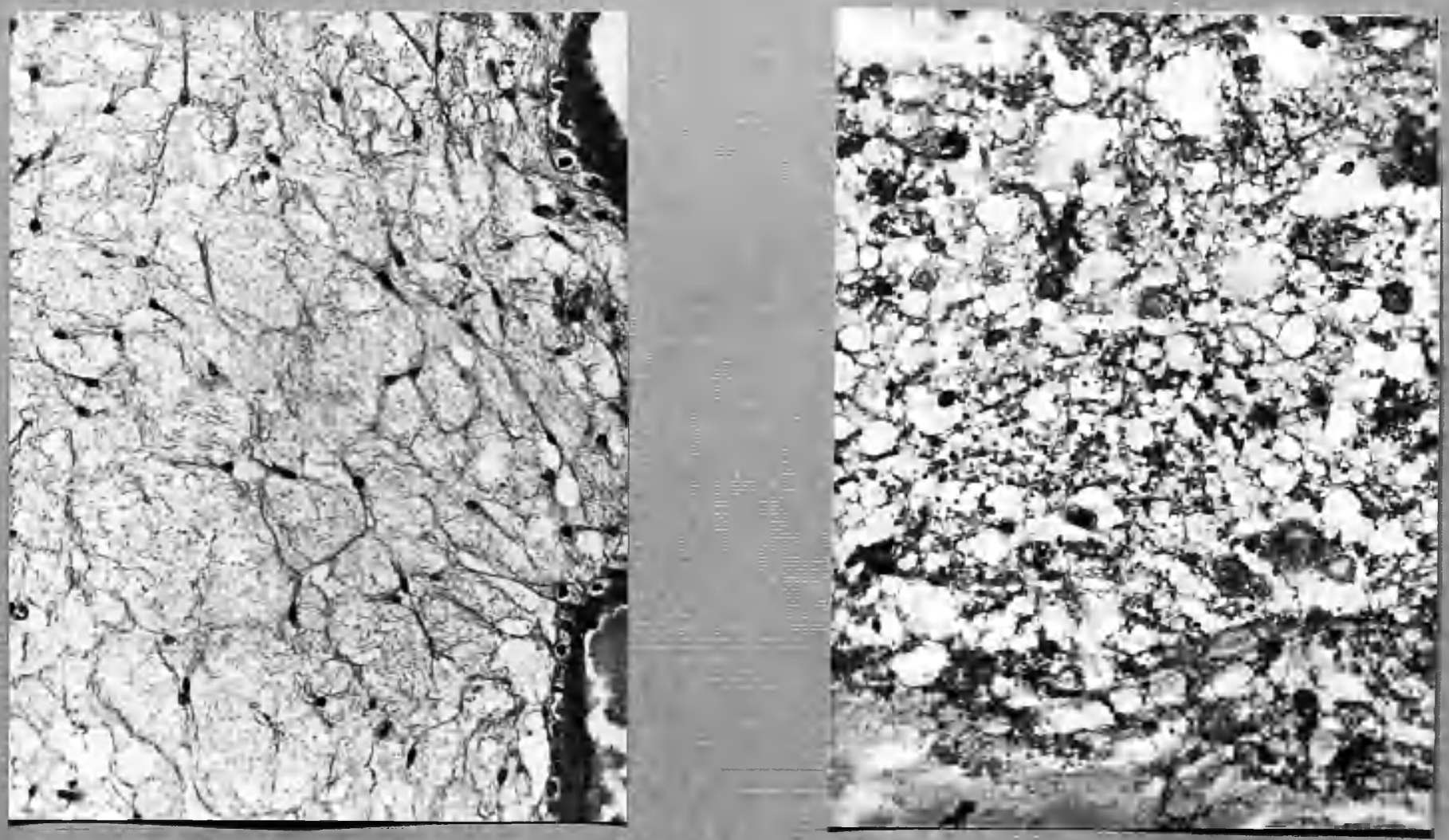

Fig. 1. Garly mesenchyme. Note mutually similar trachychronatic nuclei with stellate cytoplasmic processes. Set in mucoid matrix.

$6 \mathrm{~mm}$ Human embryo. H\&E $\times 200$

Fig. 2. Nucleus pulposus. Iarge widely scattered spheroidal nuclei set in physaliphorous mucoid cytoplasm. The structure does not recall mesenchyme. Girl aet 13. H\&W X 200. 


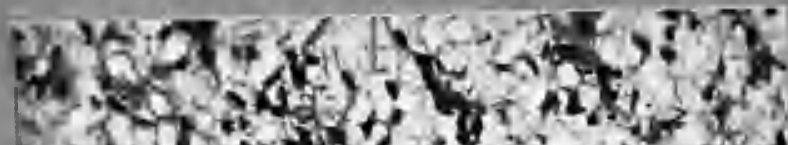

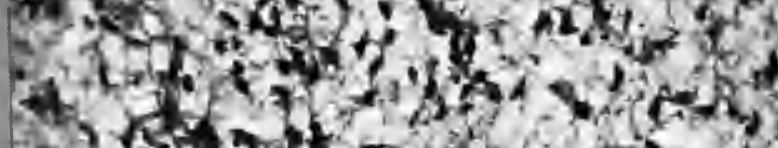

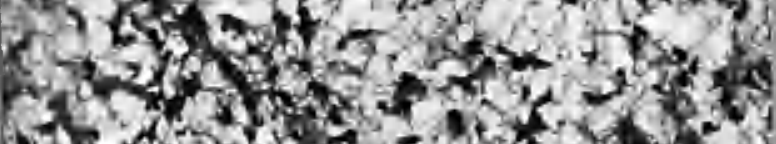

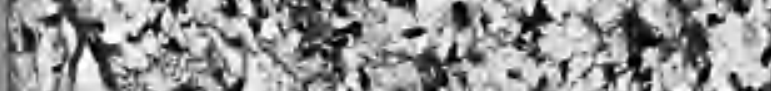

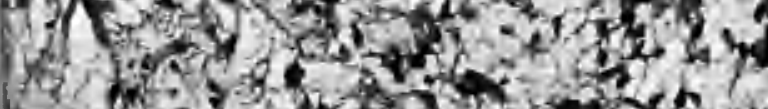

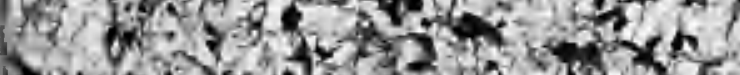

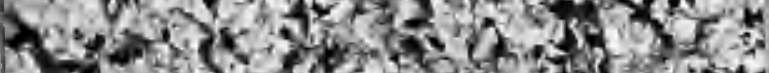

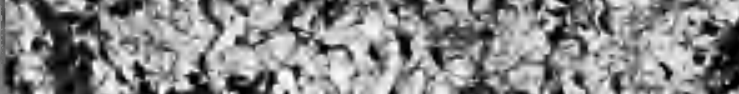

14. 5 .

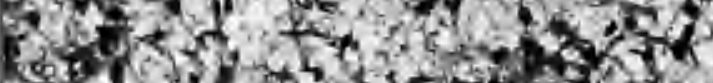

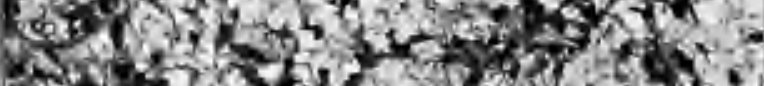

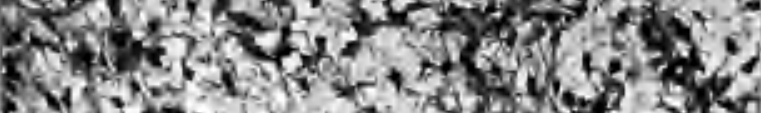

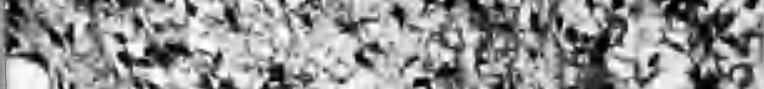

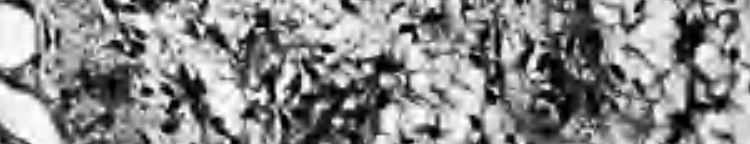

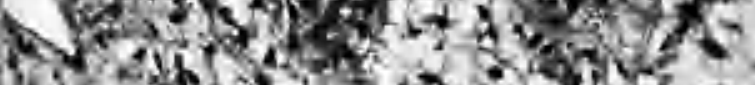

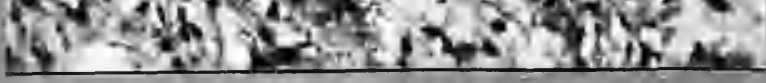

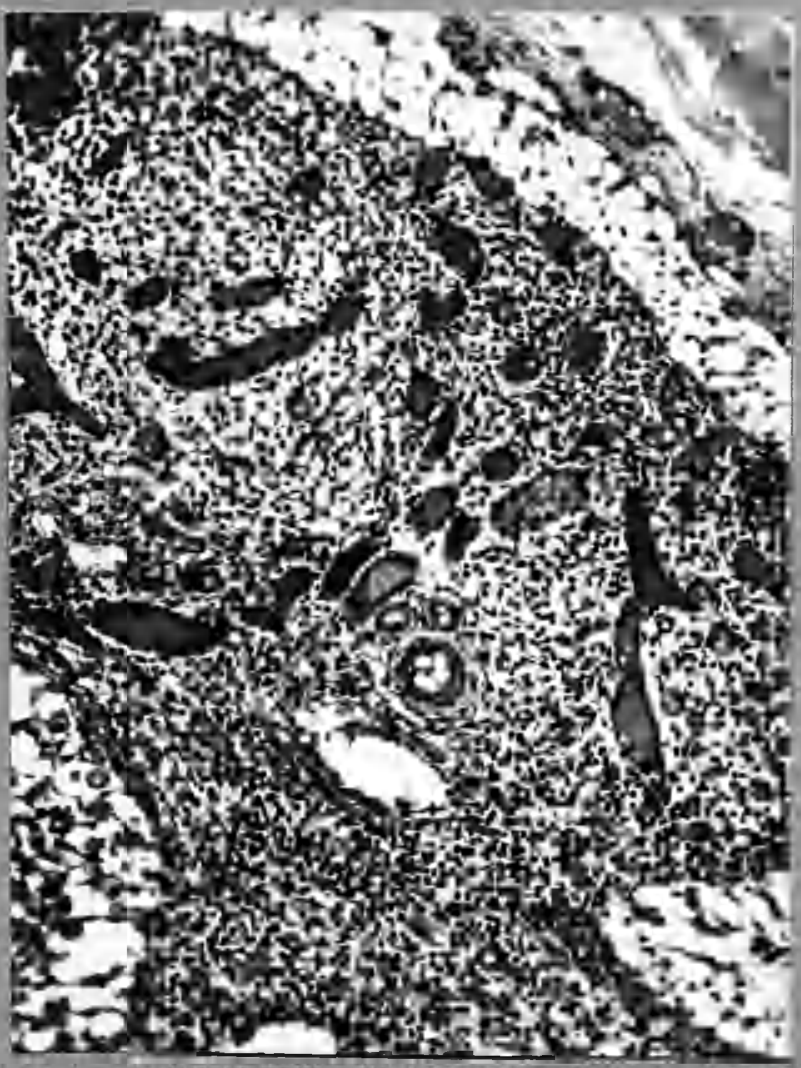

Fig. 4, Axillary lymph node from a midale aged adult, the blood vascularity is prominent as a result of post mortem auto-injection. F.i. 337:49. Hed X 150 . 

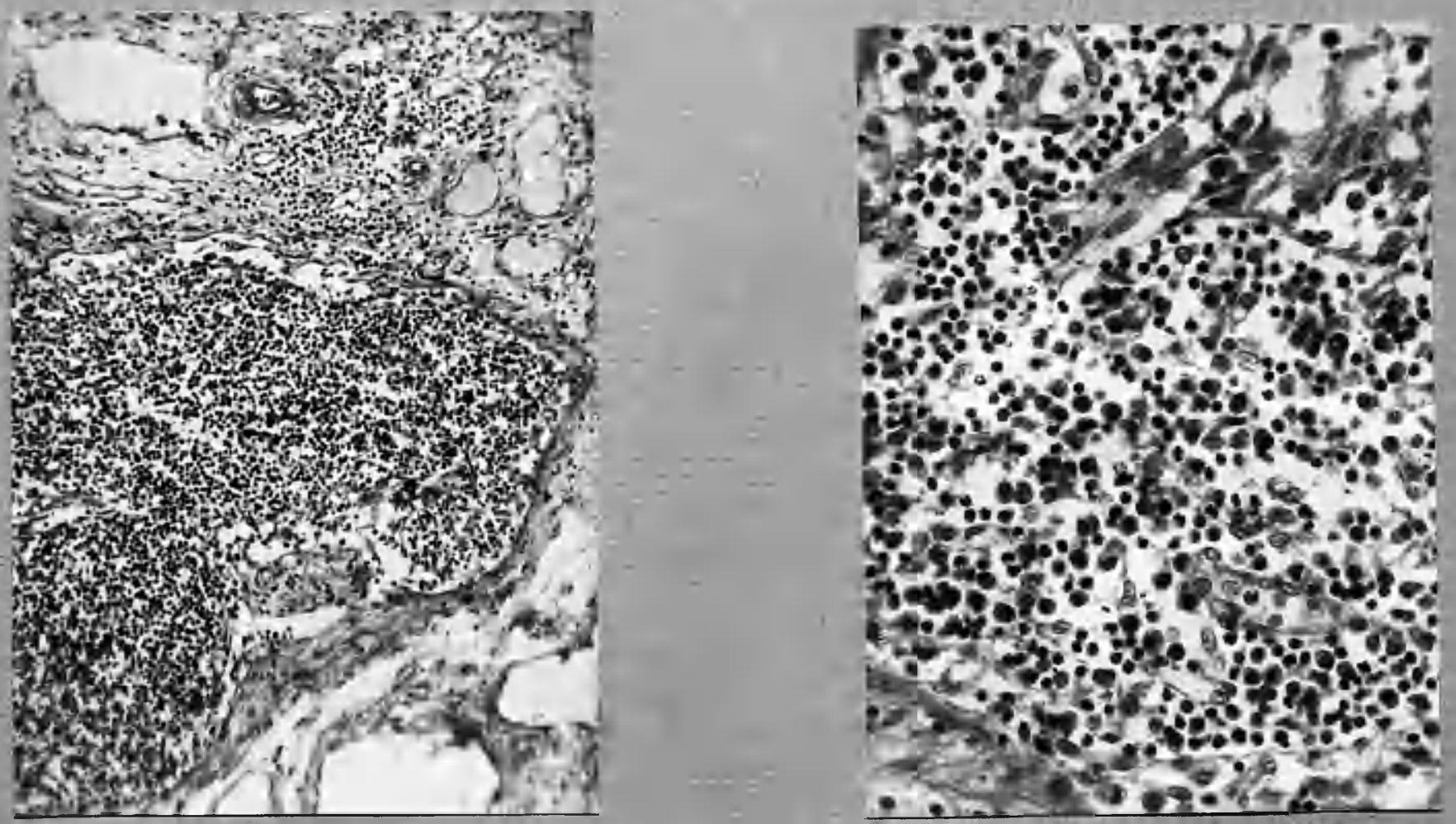
Fig.5
Axillary lympi node, from a wowan aged 83 jedrs. Depopu- lation of silicil limpnocytas is seen but sinus tissue per- sists and is not fibrous. 739. Fic. $\times 90$.

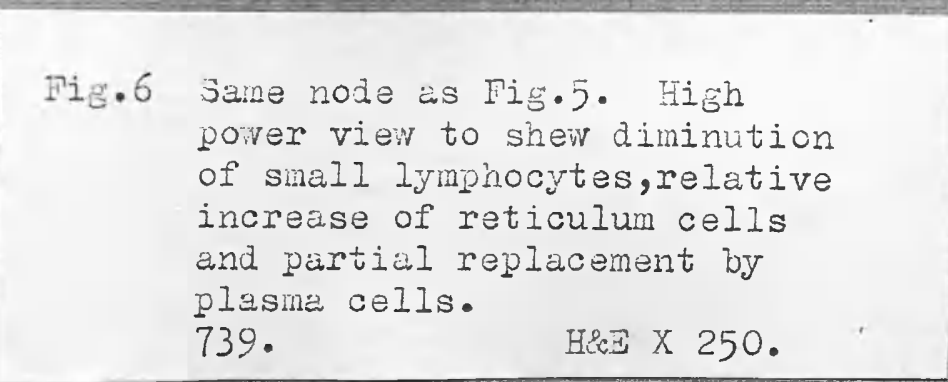




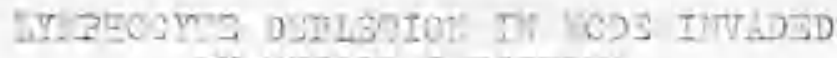 \\ 3V IUJOIP GaRCITOIS}

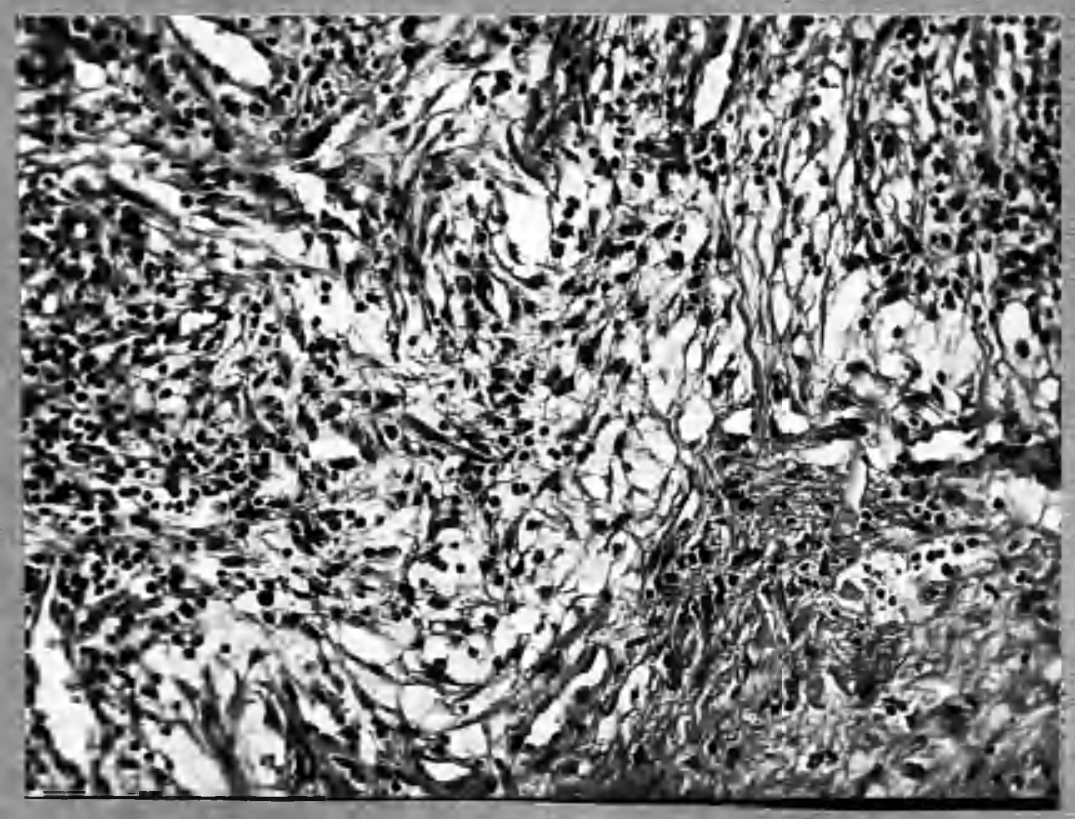

Fig.7 A groin lymph node invaded by mucoid carcinorna. a diffuse Iibroblastic reaction is seen in the marginal sinus. Iymphocyte depletion is already well established. $3408: 52$ H\&政 X 175 . 

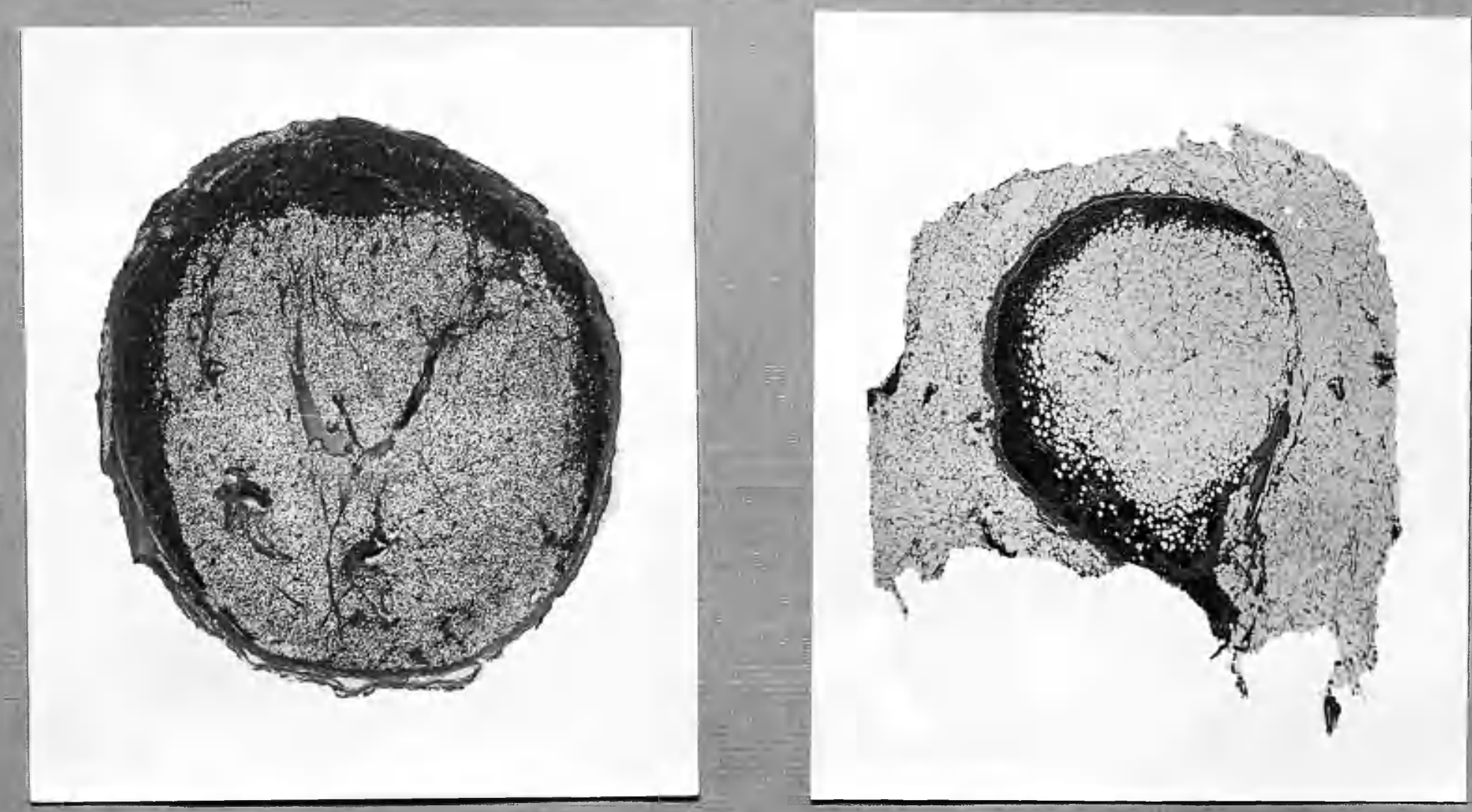

Pig.8 ixillary lymph node shewing moderate excavation by eccentric fat cone. Note capsule is thinned where fat reaches it (below) but does not collapse. Also note fine sprinkling of individual fat cells in lymphoid tissue beyond limit of fat cone. isl1: 50 .
H\&国 X 8 .
Fig.9 Axillary lymph node. Late fat replacement. Note lipomalike uniformity of fat cone and early disappearance of capsule (right). s10:50. 


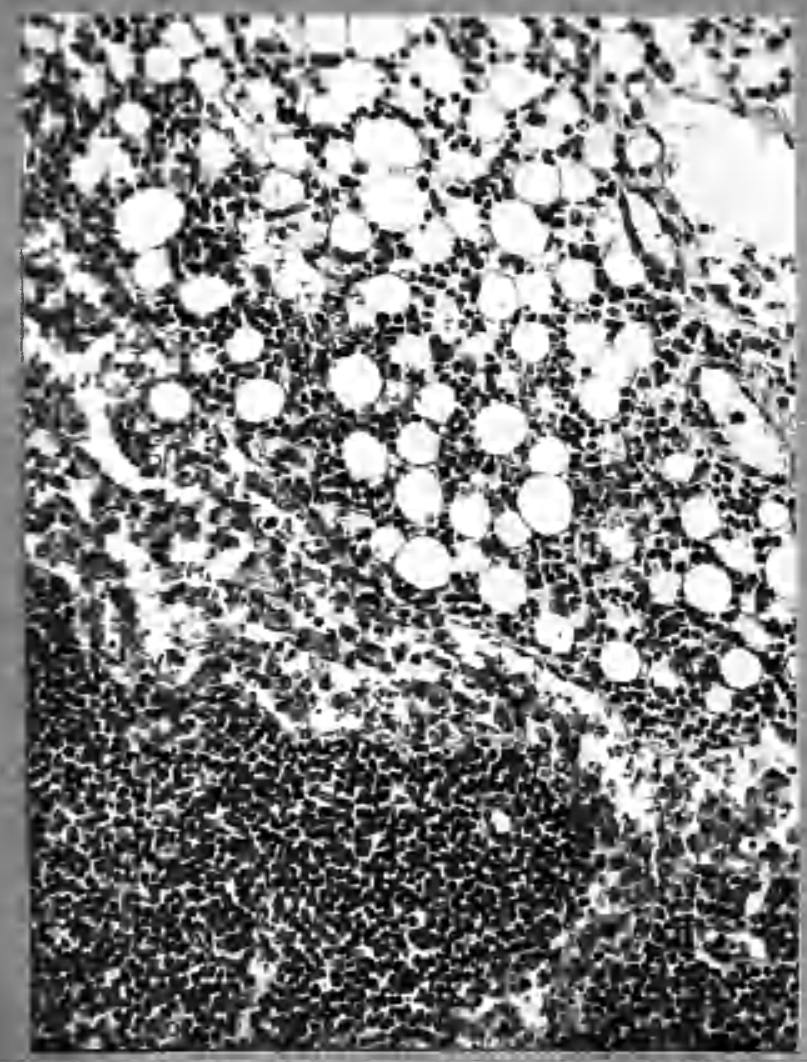

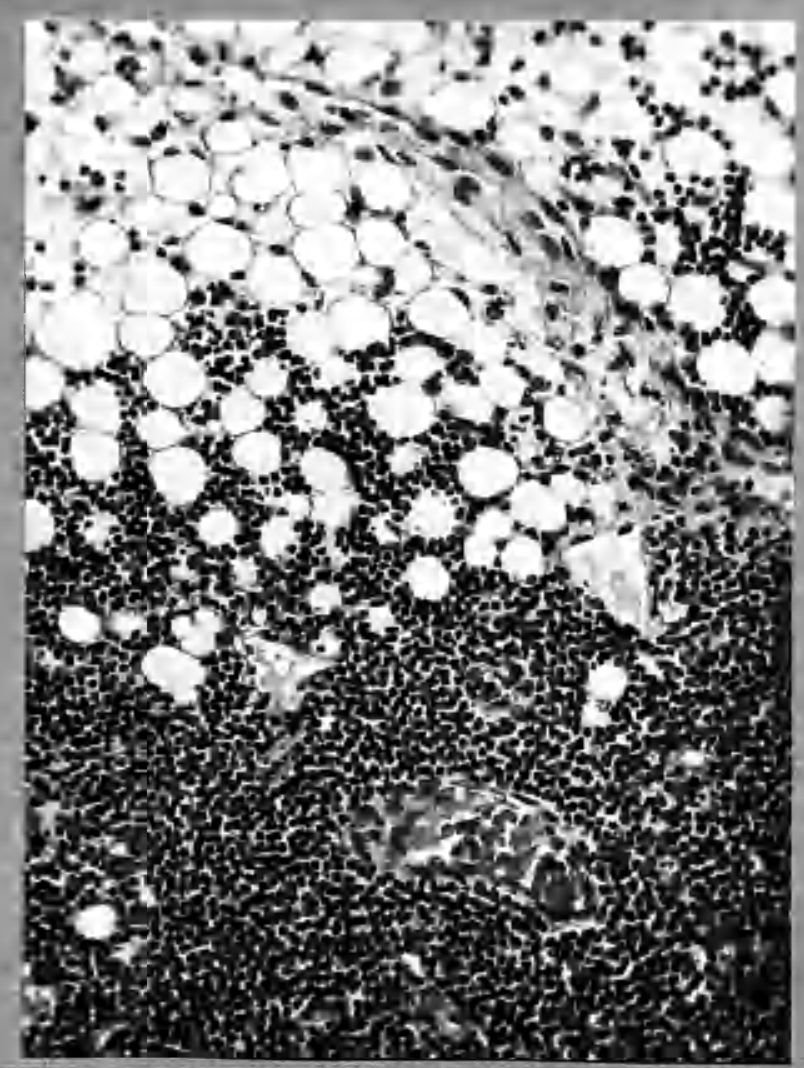

Fig.11 Fat replacement. Wote persistence of sinus tissue whicis is outplanked by irfiltrating fat. 3475:52. Hois \& 200 . 

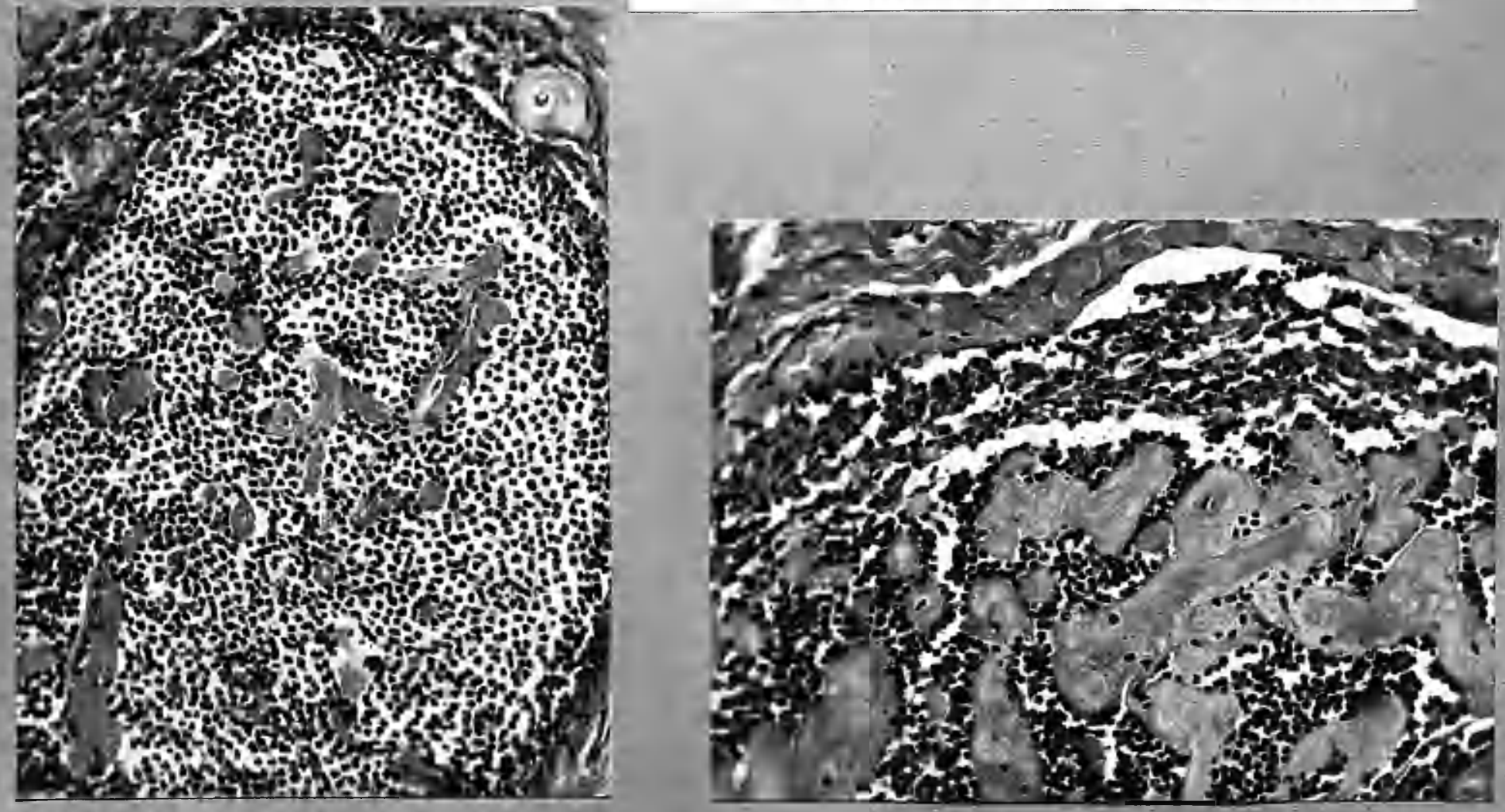

$$
\begin{aligned}
\text { Fig.12 L peripheral solid follicle. } \\
\text { Jurviving sinus tissue is } \\
\text { seen above. Hyalinised cup- } \\
\text { illaries are vell shewn, } \\
\text { nany exhibit stenosed } \\
\text { lunina. The lymphoid tissue } \\
\text { is clear of fibrosis. } \\
\text { lian } 70 \text { jears. Hix } x \text { 250. }
\end{aligned}
$$




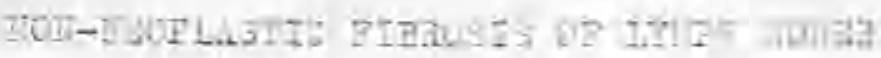
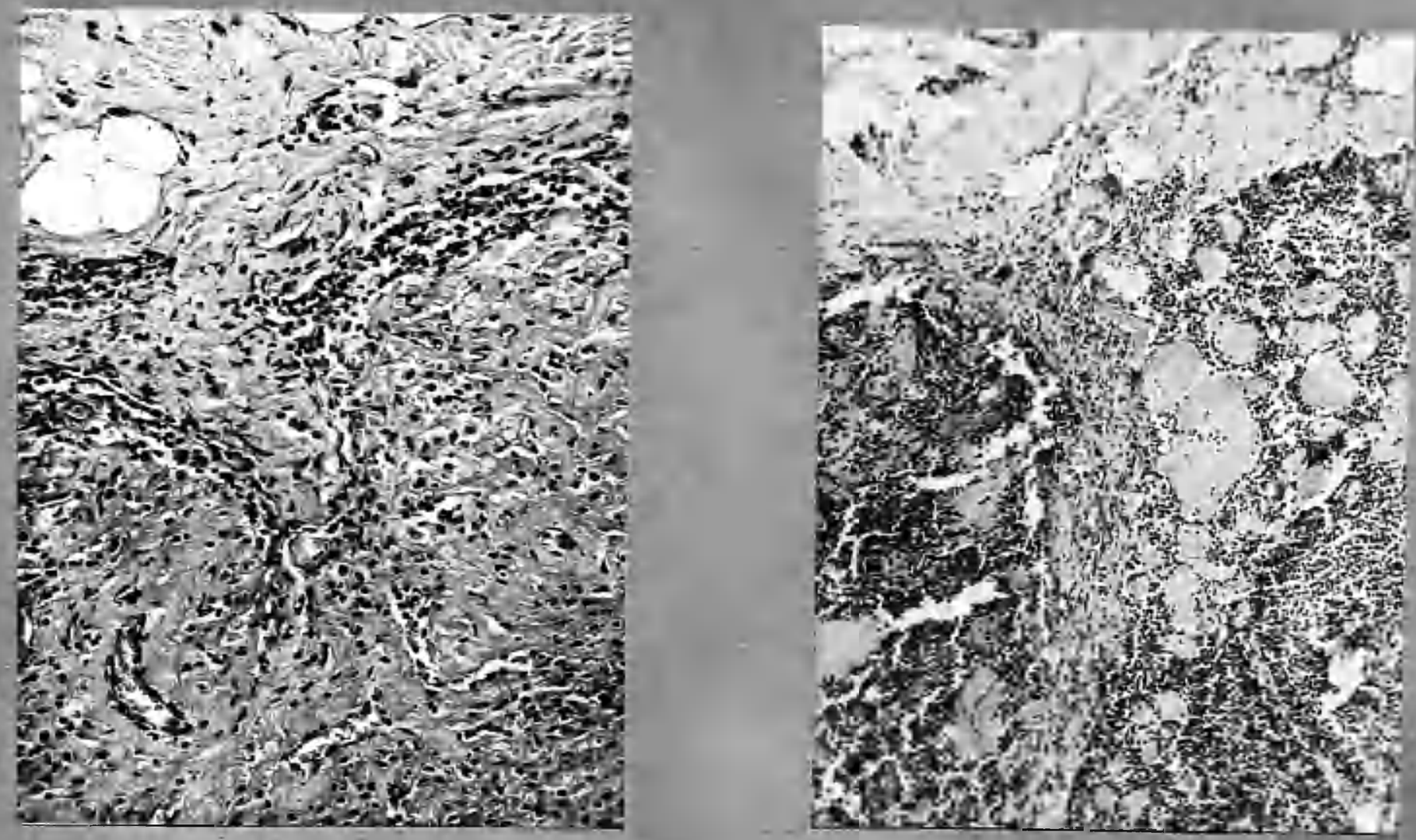

Fig. 14

Ixtreme vascular riorosis.

The line of narginal sinus

can be traced, arrows. The

vessels botton lert are

possible new formations.

roman 73 years. Hav X 250 .

Fig.15 Late vascular fibrosis.

Jurly culcareous change is

present in the collagen, note

the atrophy of the para-

trabeoular sinus tissue.

lonar 75 years. HoE X 100 . 


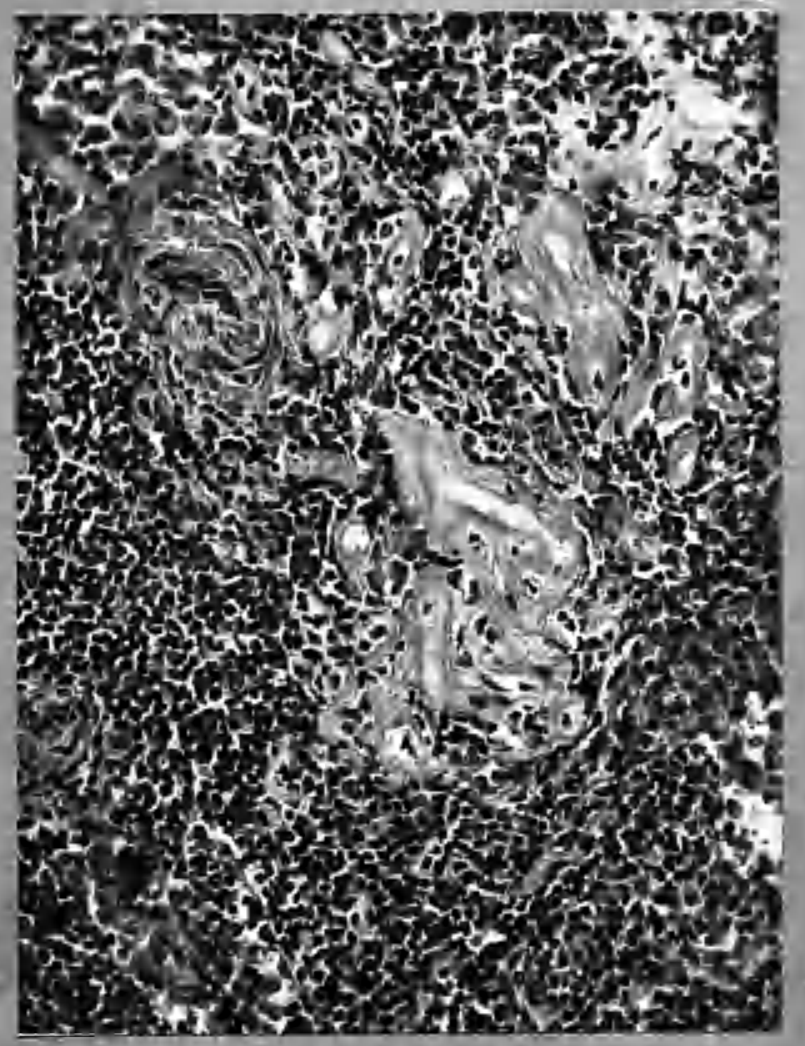

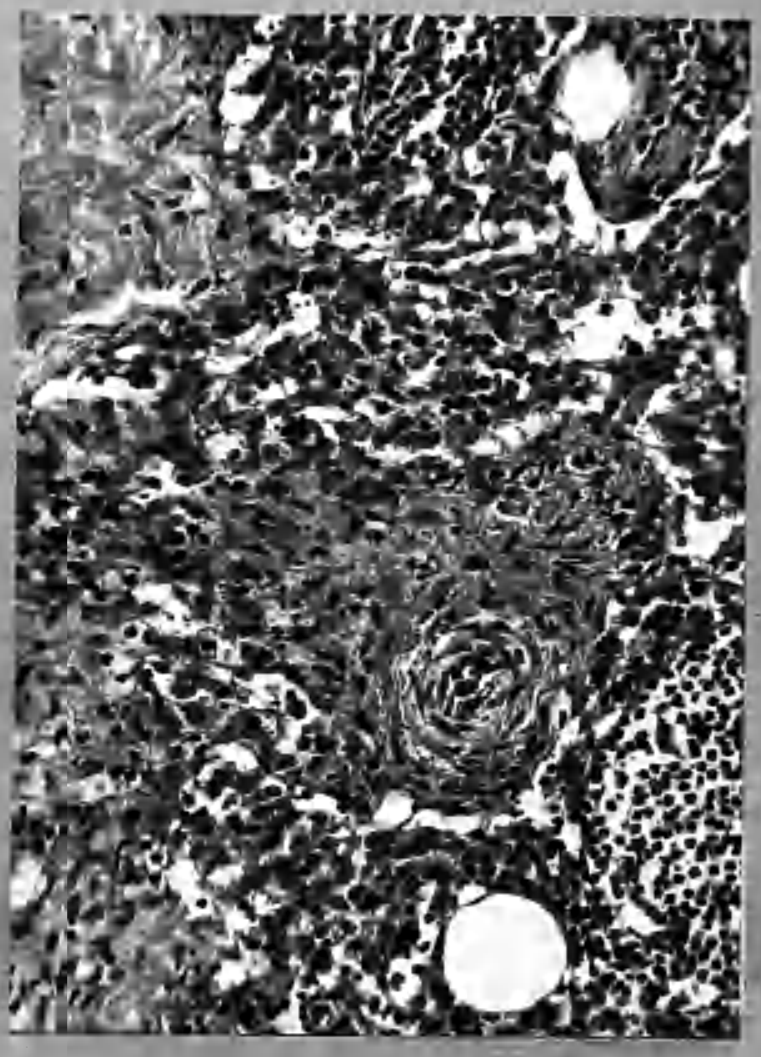

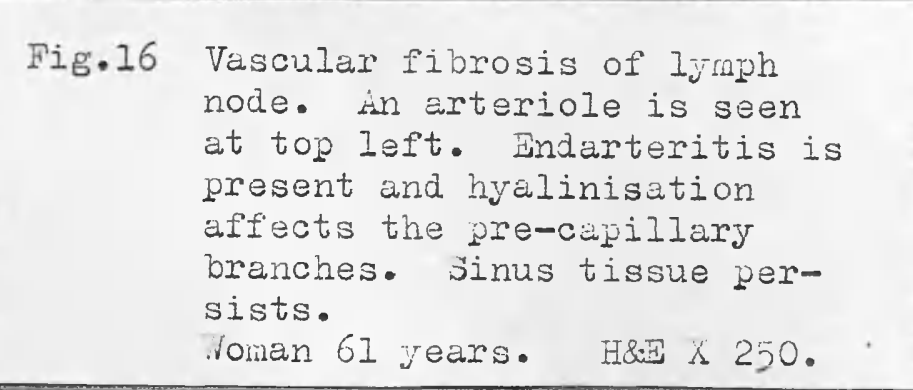

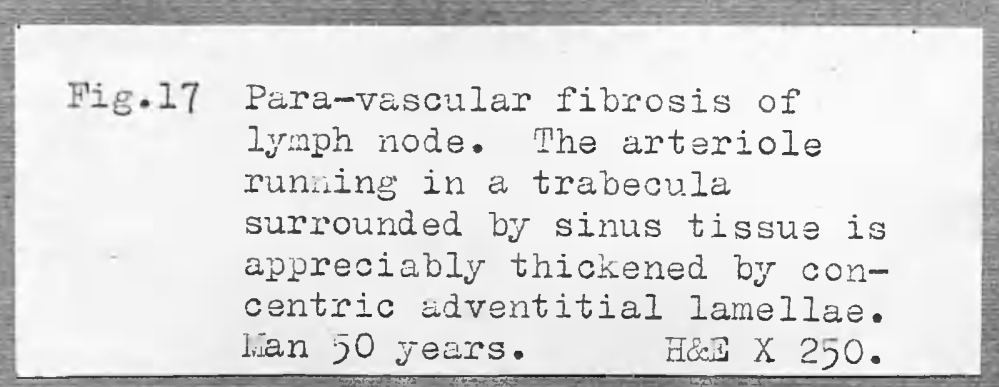




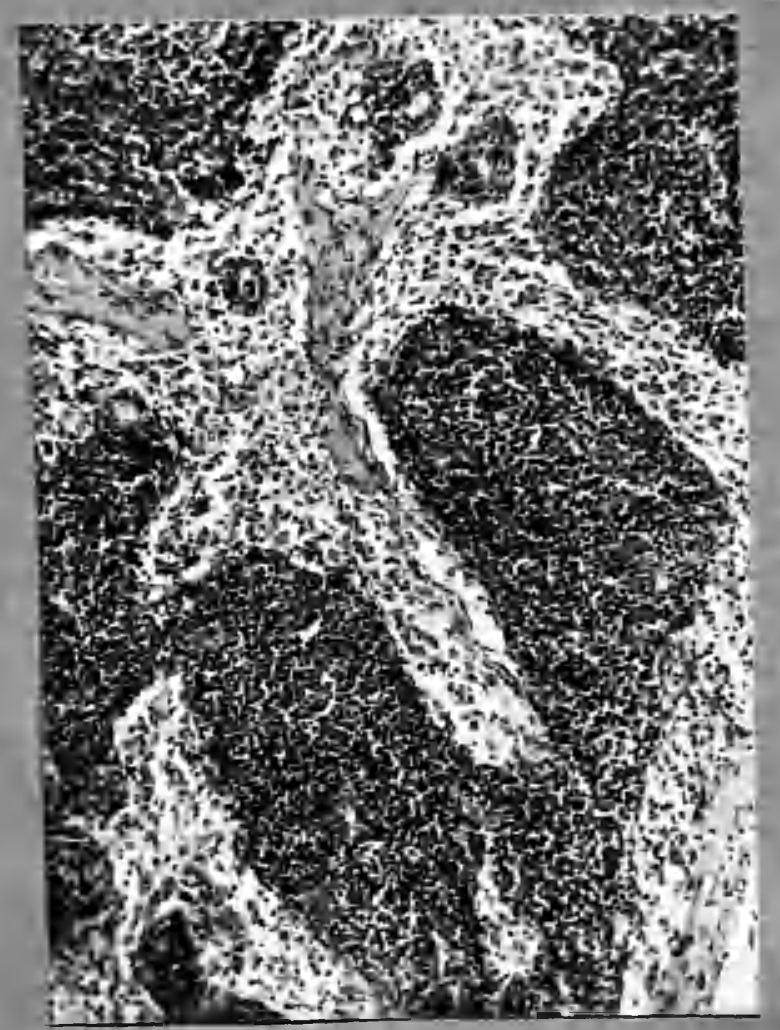

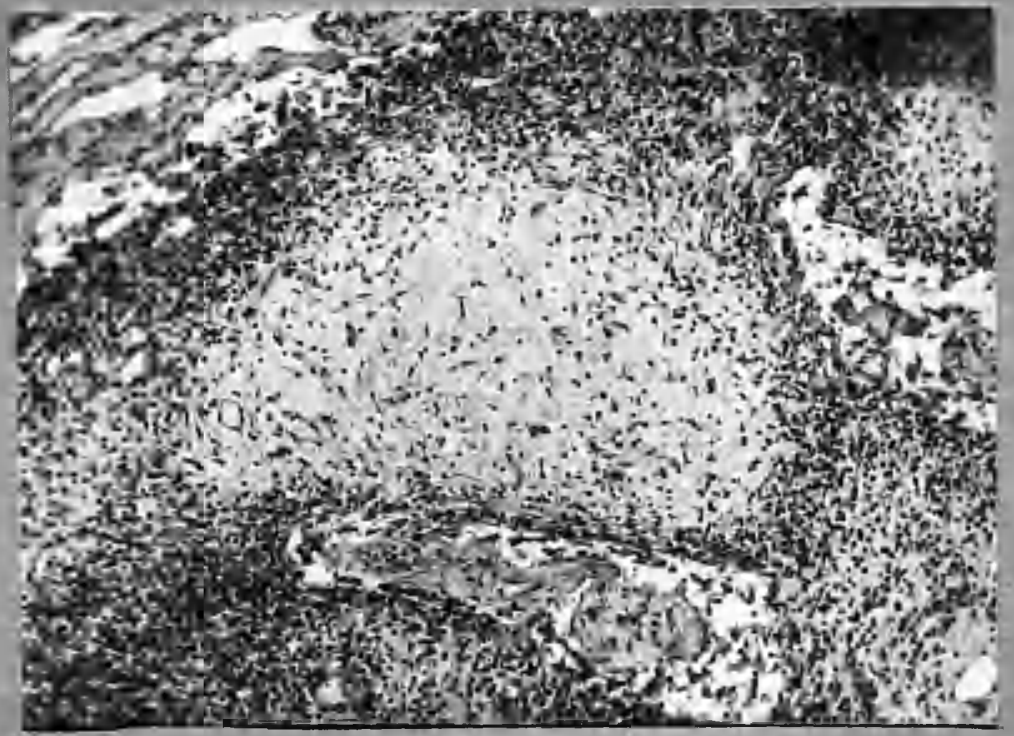

Fig.18 Fara-vascular fitrosis of

ljomph node. Note how a

mantie of sinus tissue per-

sists round the dixial fibrous

tissue.

lian 62 yeurs. Ade $\times 100$.
Fig.19 Lute diffuse fibrosis of the pulp. The deposit resembles scar tissue, note the thin walled vessels in it. (ParaVuscular fíbrosis is seen in the sirus below). This form of tiurosis is very rare. roman 57 years. He: X 100. 

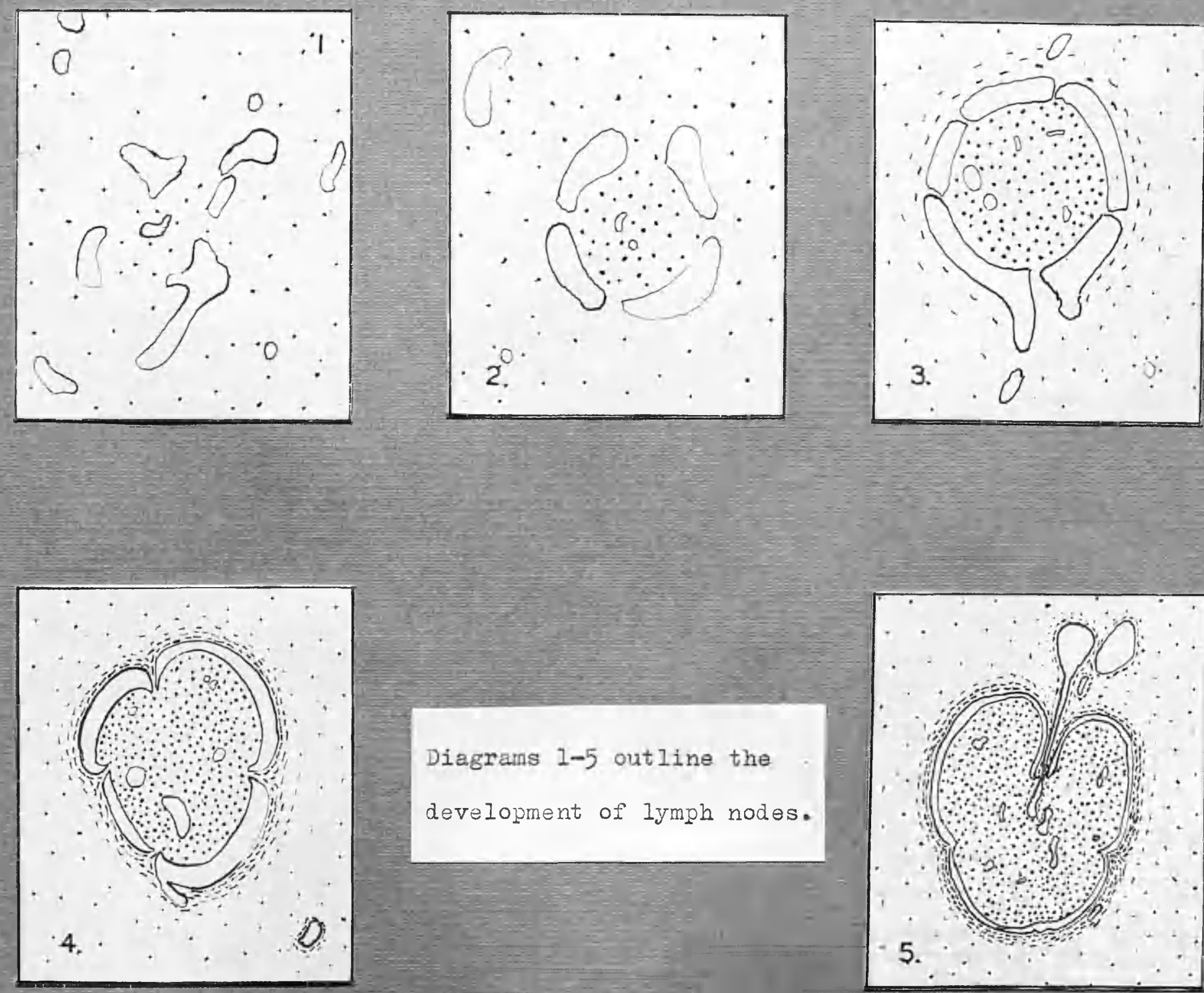

Diagrams $1-5$ outline the development of lymph nodes.

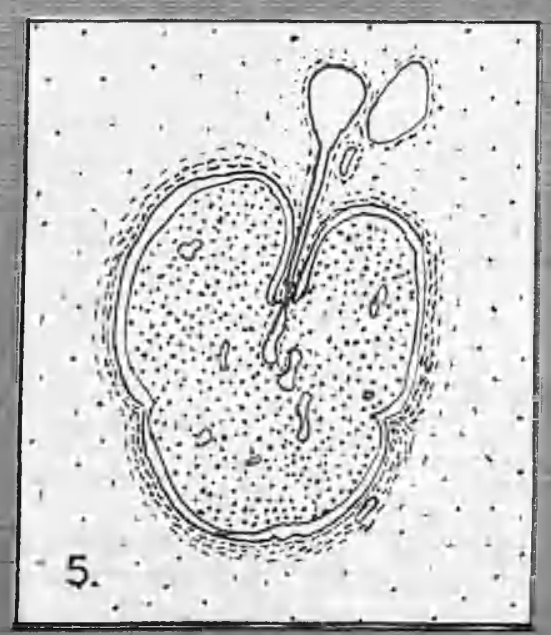



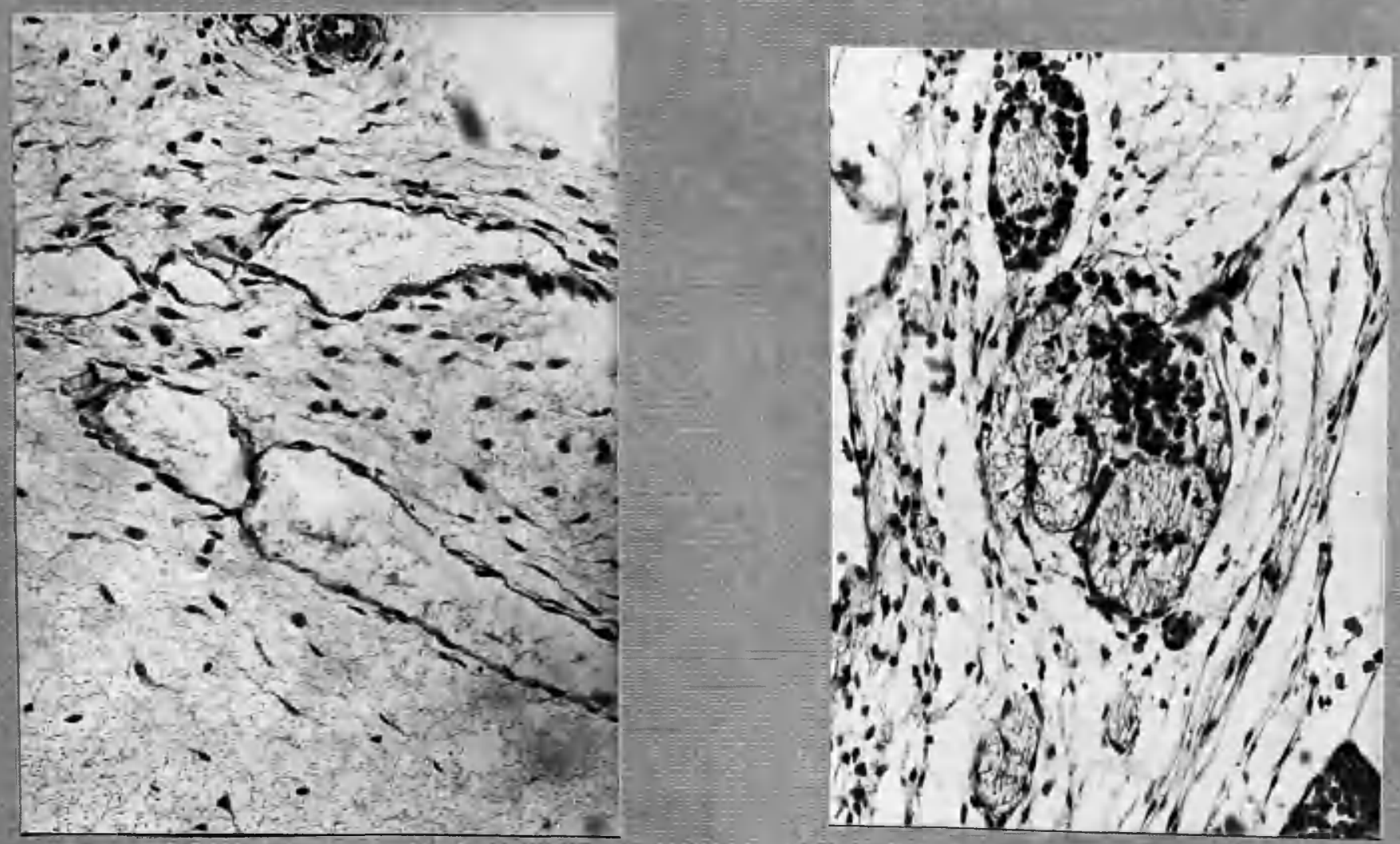

Fig.20 Jarly formation of lymphatic plexus at locus of aeveloping lymph node.

$22 \mathrm{~mm}$ fiunan inbryo $10 \mathrm{z}$ X 200 .

FiE.21 Lymph node anlüge, a snall group of lymphocytes and reticulum cell nuclei are seen in the centre of fusing lymphatic channels. $46 \mathrm{mn}$ Human Inbryo. How X 250. 

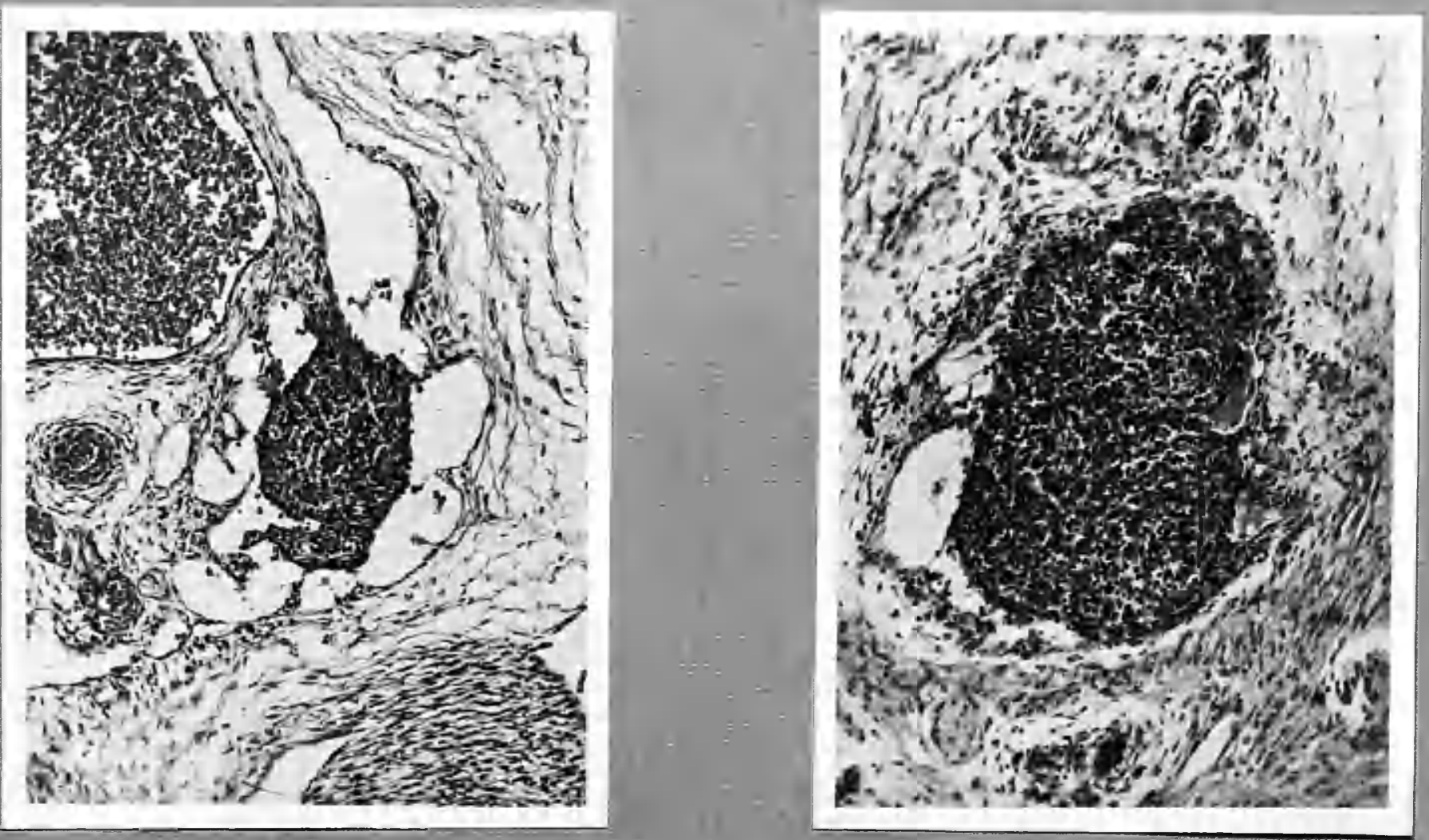

Fig. 22

Lymph node shewing central lymphoid tissue mass and marginal sinus. Note contimuity with lymphatics.

$65 \mathrm{~mm}$ Human Embryo. H\&d X 125.
Fig.23 Lvmph node shewing very faint segmentation of central

Iymphoid mass. The marginal sinus is partly collapsed. At right an early trajectorial sinus is seen.

$78 \mathrm{~mm}$ Human Imbryo. H\& X 125 . 


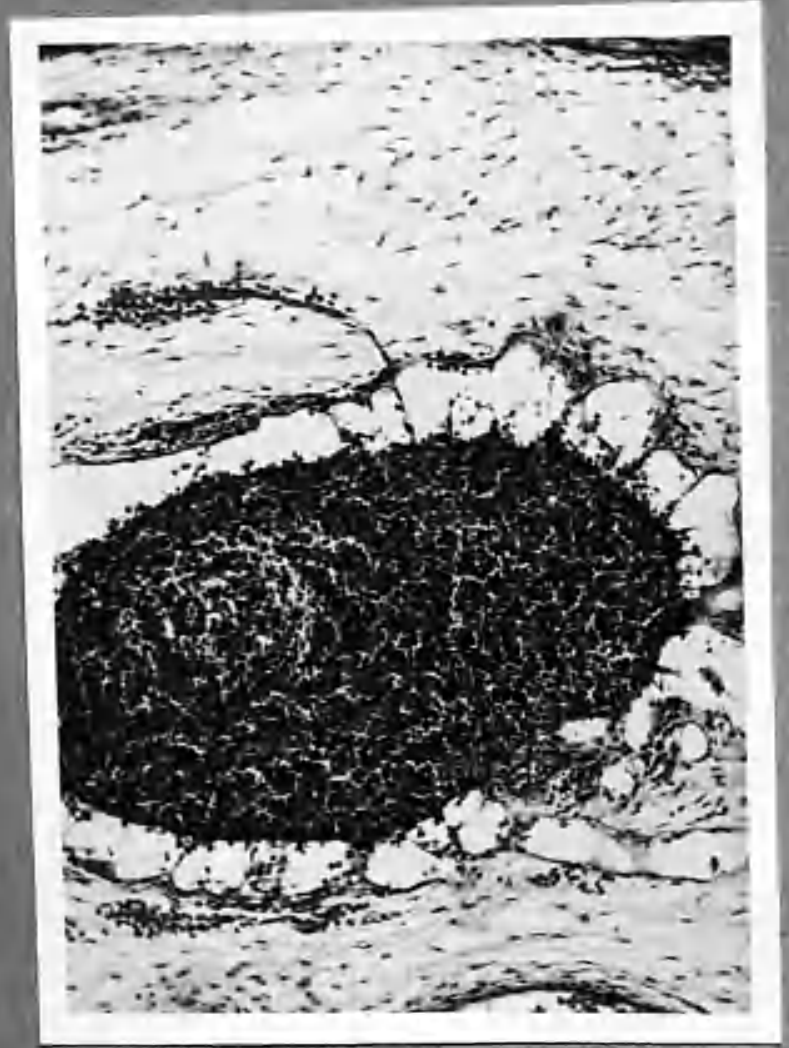

Fig.24 Early foetal lymph node. Sinus structure appearing, also possibly intra-nodal sinuses. Note afferent valved lymphatic at bottom right.

94 mra Euman Foetus. Ex⿱口龰己 X 100.

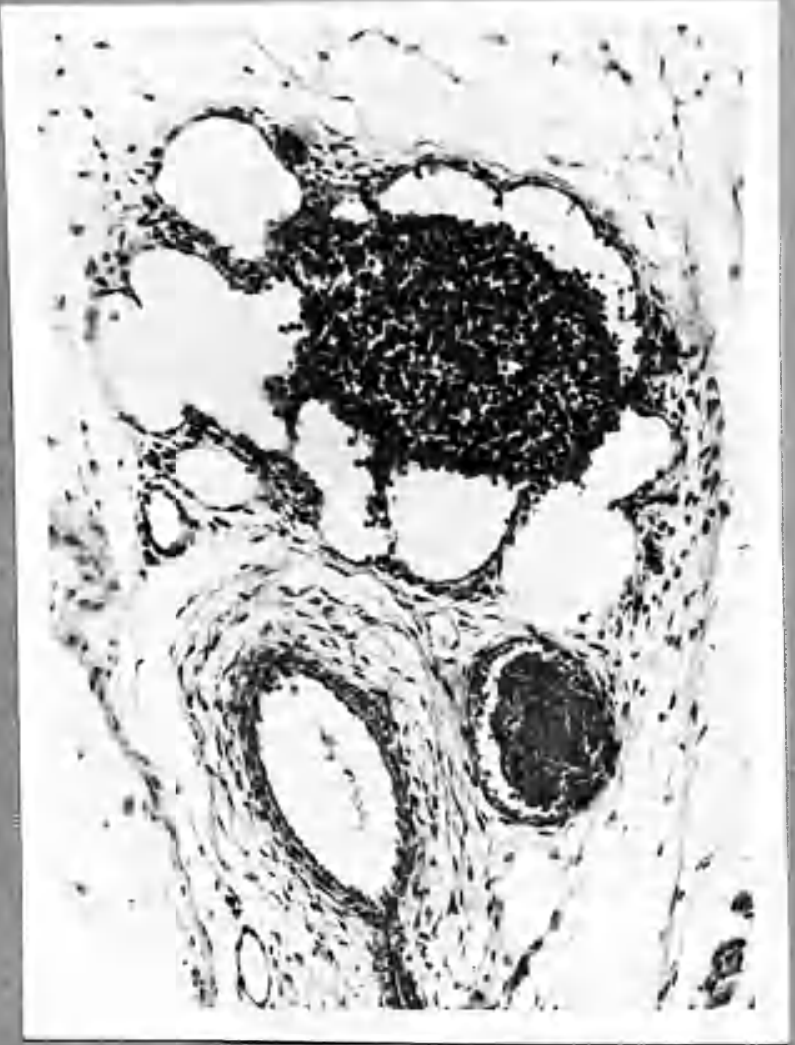

Fig.25 Foetal lymph node shewing approach of definitive blood supply. Small artery and vein. 108 min Human Foetus. Hois X 125. 

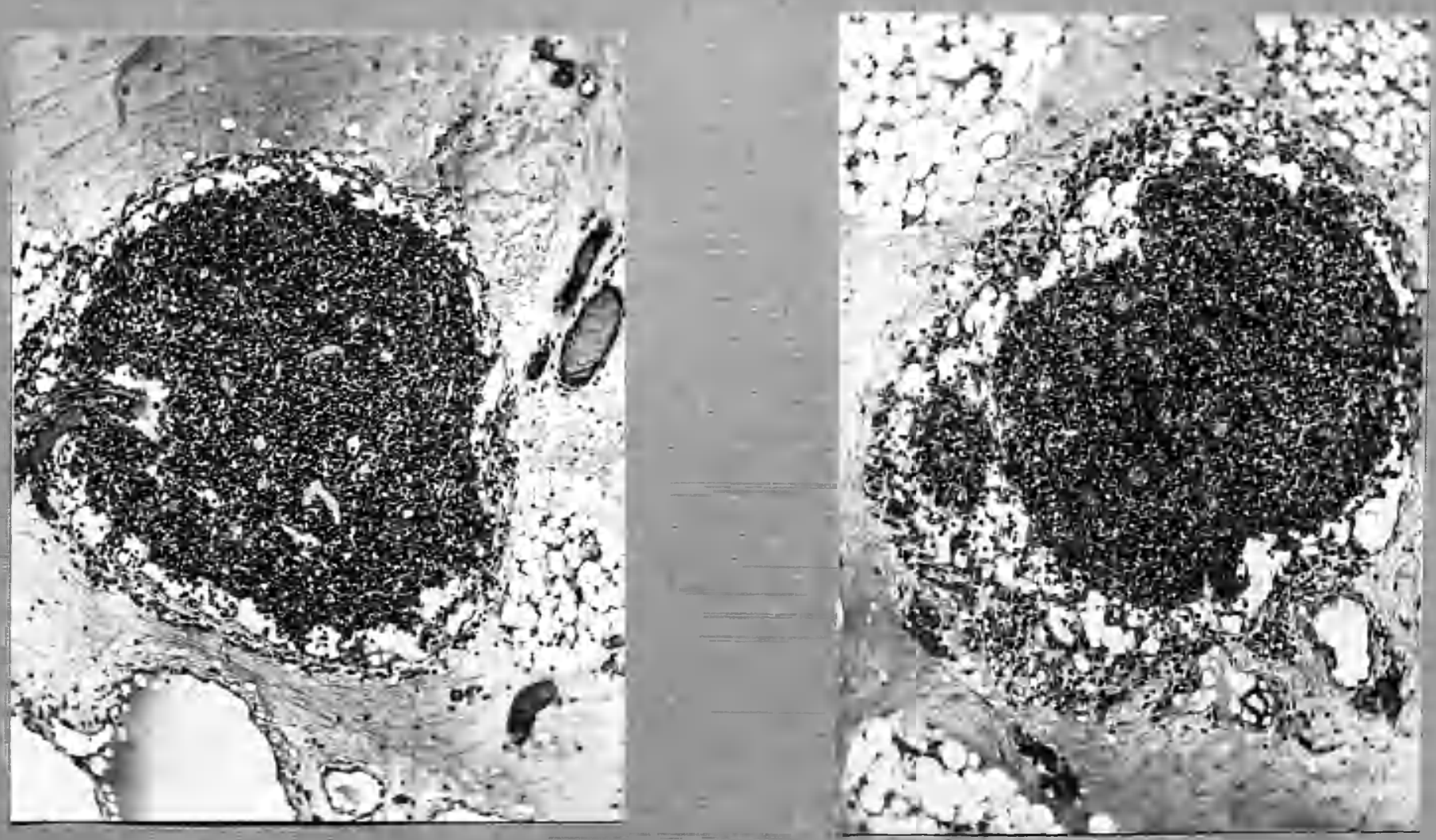

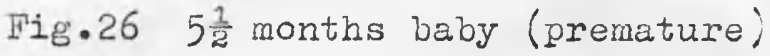
Lymph node. The definitive blood supply is seen entering left and carries a mantle of sinus tissue round it. $5 \frac{1}{2}$ nonths baby. Hß2 $X 90$.
Fig.27 Jarly segmentation of node substance. تlaboration of sinus tissue and early changes in lymphoid tissue density. 6 months baby (premature). 积导 X 90. 


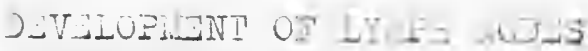
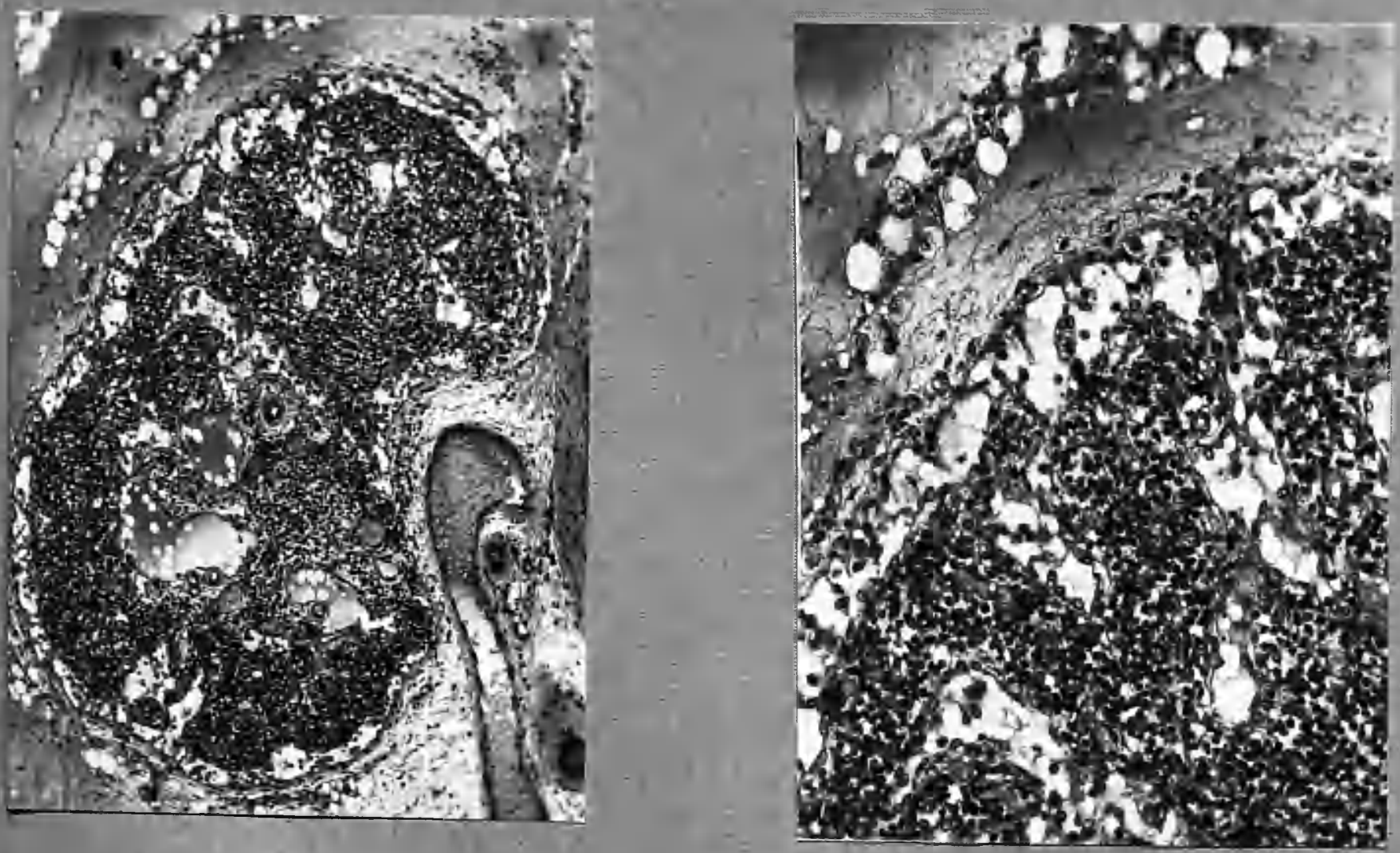

Fig.28 liode at 7 montins intrauterire life. he sinus system is rell developed, and a proper blood supply is present.

7 months baby (premature)

Fig.29. Same node as Tis.28. High porrer to shew detail or developing sinuses. The structure is already chardeteristic.

7 nonths babi (premature). Y $\mathrm{X} 90$ H\&E X 200. 


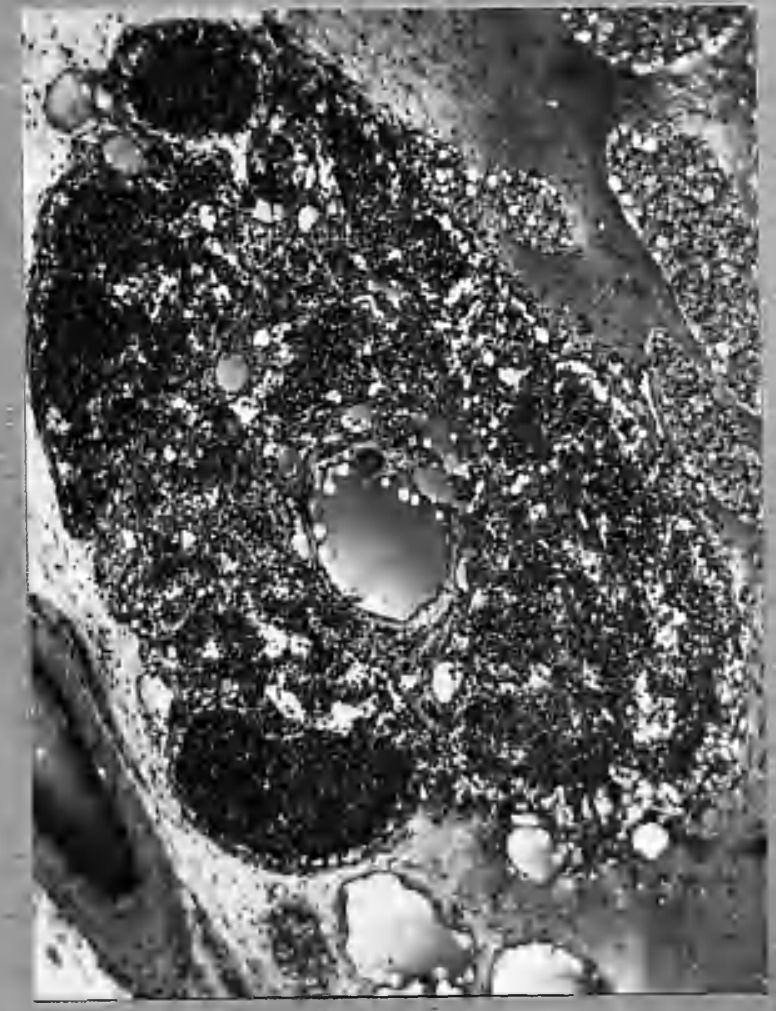

Fig.30 Lymph node at birth. (Axilla). Several solid follicles are now present, and the node is properly forined. Child at tern. Heg X 50 .

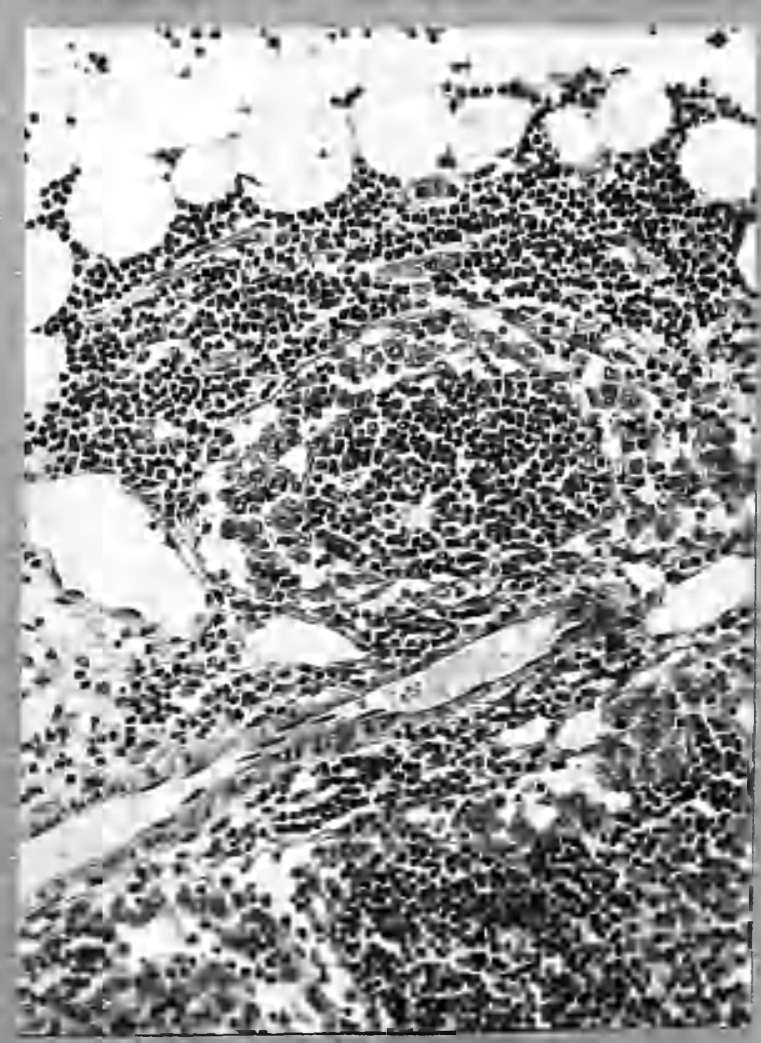

Fíg·3I

Adventitious lymphoid tissue formation outwith the capsule of a node. The vessel tram versing the field obliquely delineates the capsule. sbove it a follicle rith a cuff of new sinus tissue has formed, outwith it is lymohoid tissue in direct contact with the ajjacent areolar tissue.

S.163:49. H8: X 200. 


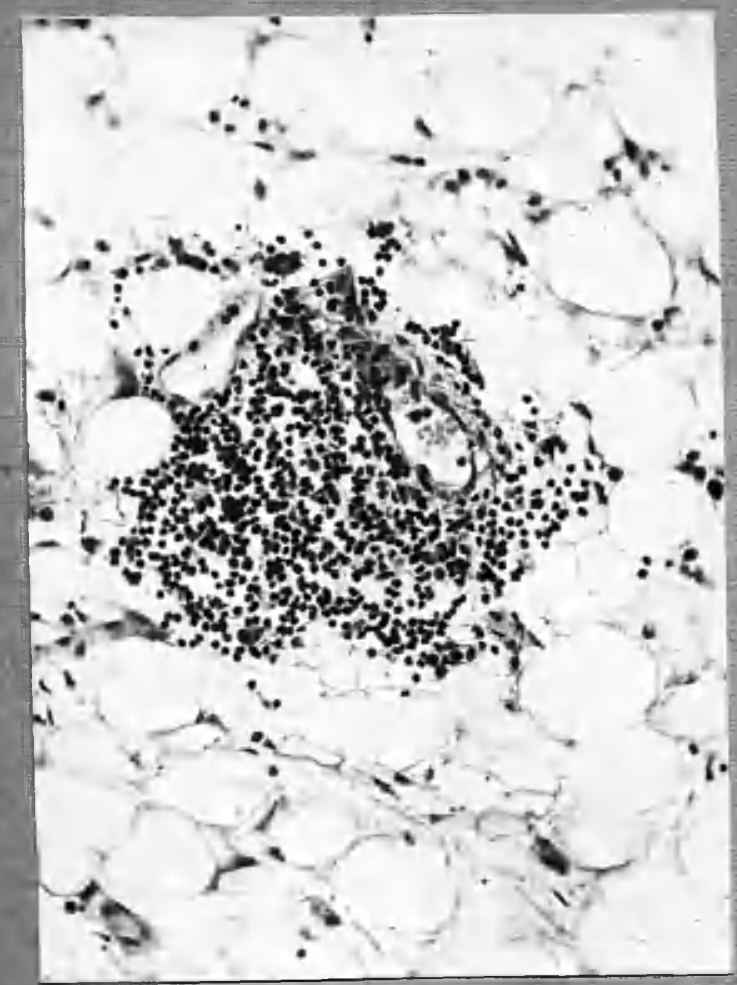

Fig.32 Neogenesis of lymph node in axillary fat. I Iympiatic and capillary are related to focal lymohocytic aggregation. The deposit is not ancapsulated.

s.10:51. प2. $\times 150$.

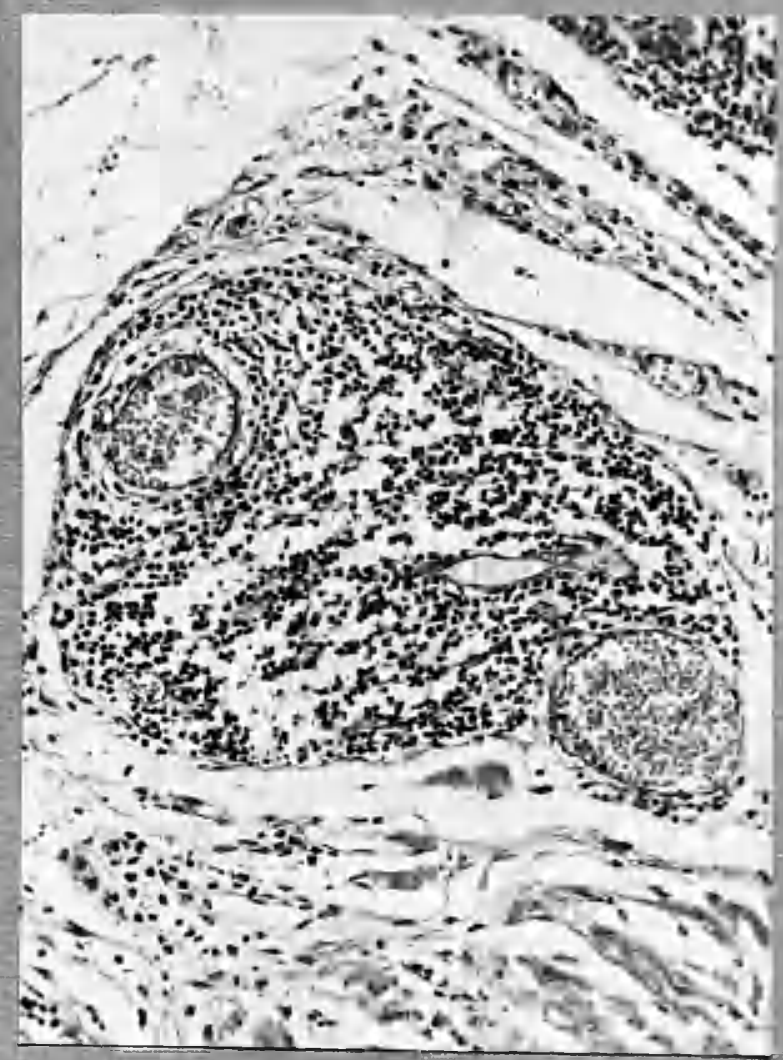

Fig.33 Wewly formed lyrmph node.

Tho sinusoidal capillaries

and a lomphisto are seen in relation to the node which is acquiring a capsule. 3.27:50. HBC X I25. 


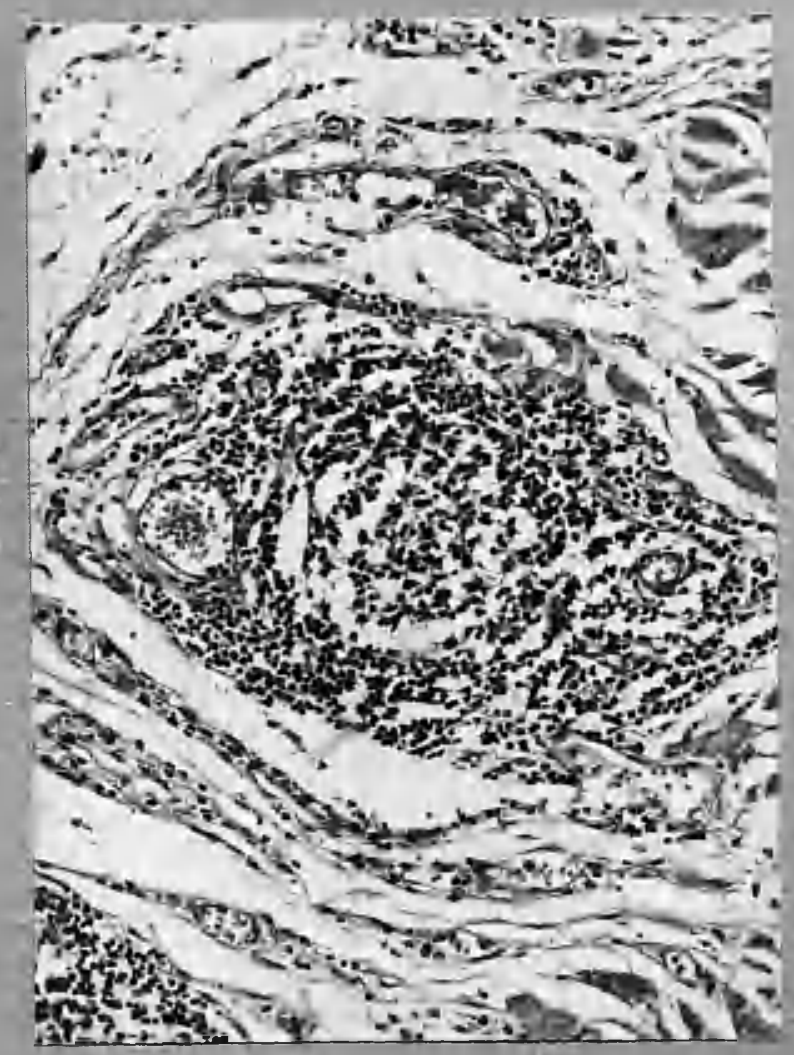

$\operatorname{pig} \cdot 34$
Sane node as Fig.33 at

another level to shew incip-

ient pale centre follicle.

$3.27: 50$.
180 $\therefore$ is 125.
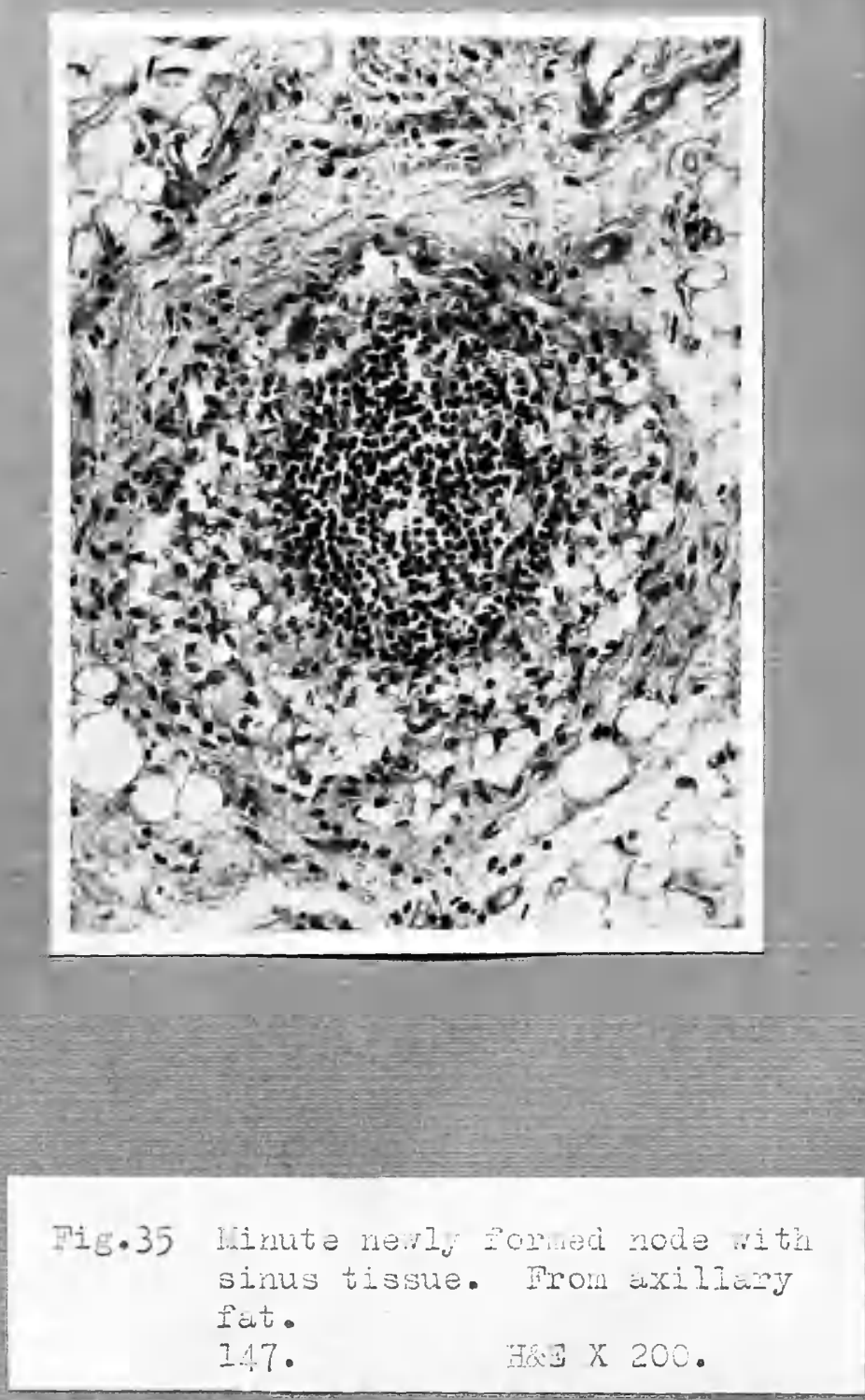


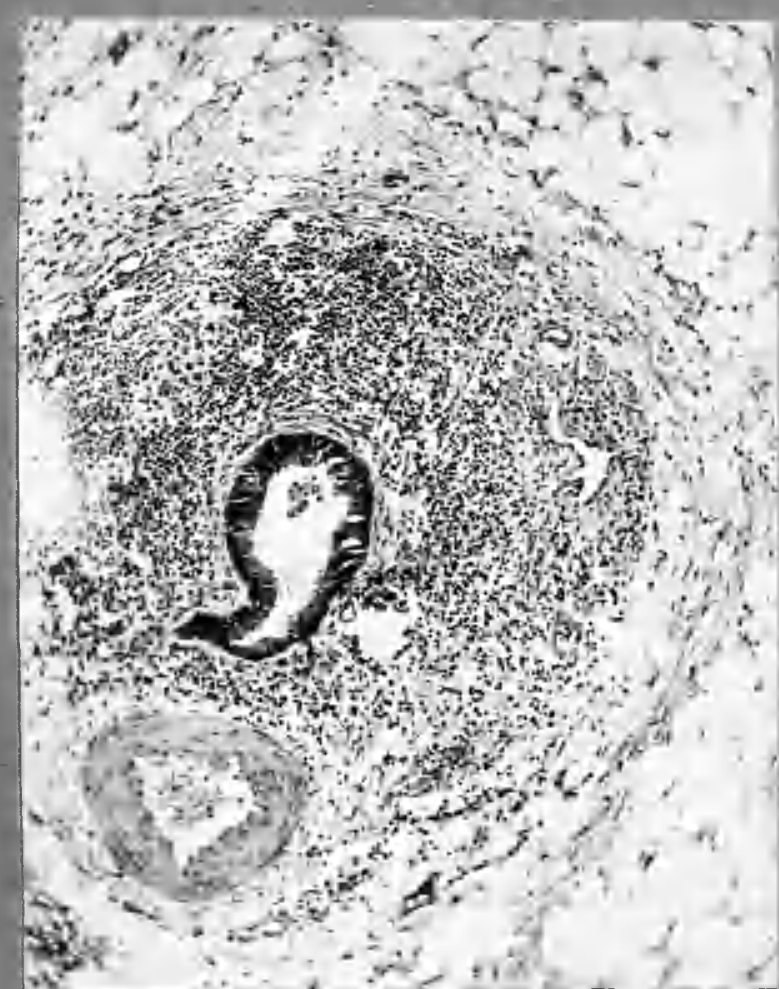

Fig.36 llatastatic cancer in a newly forned node. It is sited in the node in a lymphatic, a true marginal sinus is absent. From mesentery of large bowel.

1462. 발 $\times 65$.

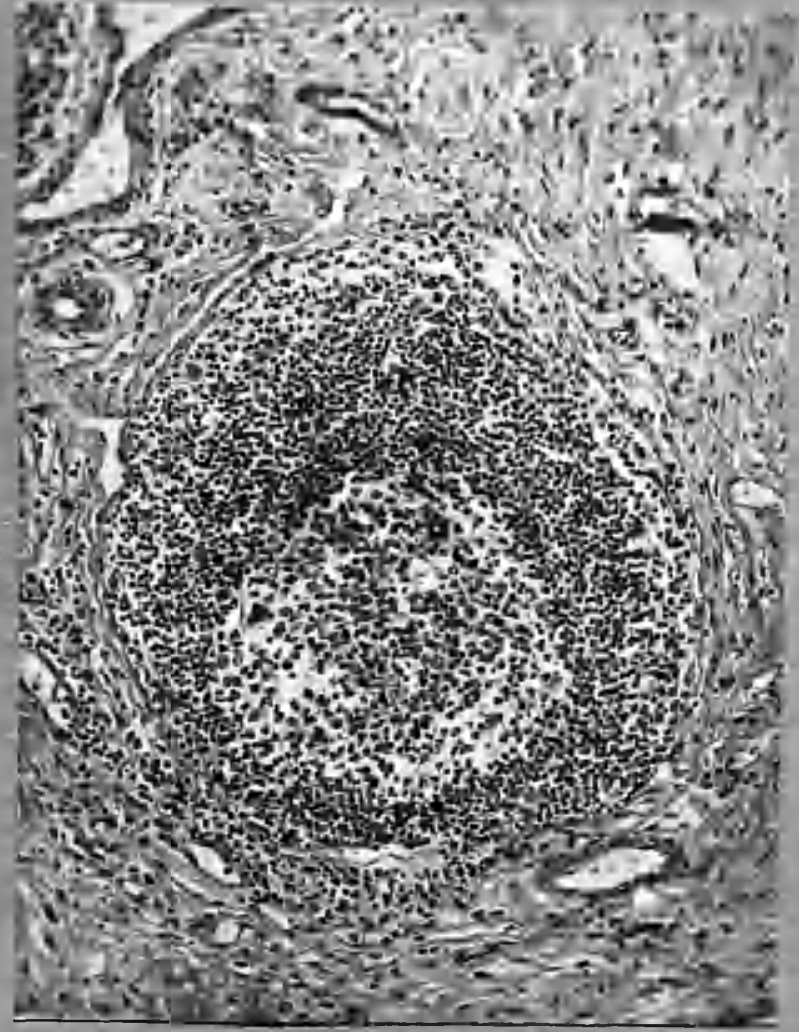

Fig.37 Ectopic lynphoid tissue, prostate land. Note pale centre of new follicle and displacement of blood vessel (right). I. Hic 1150. 

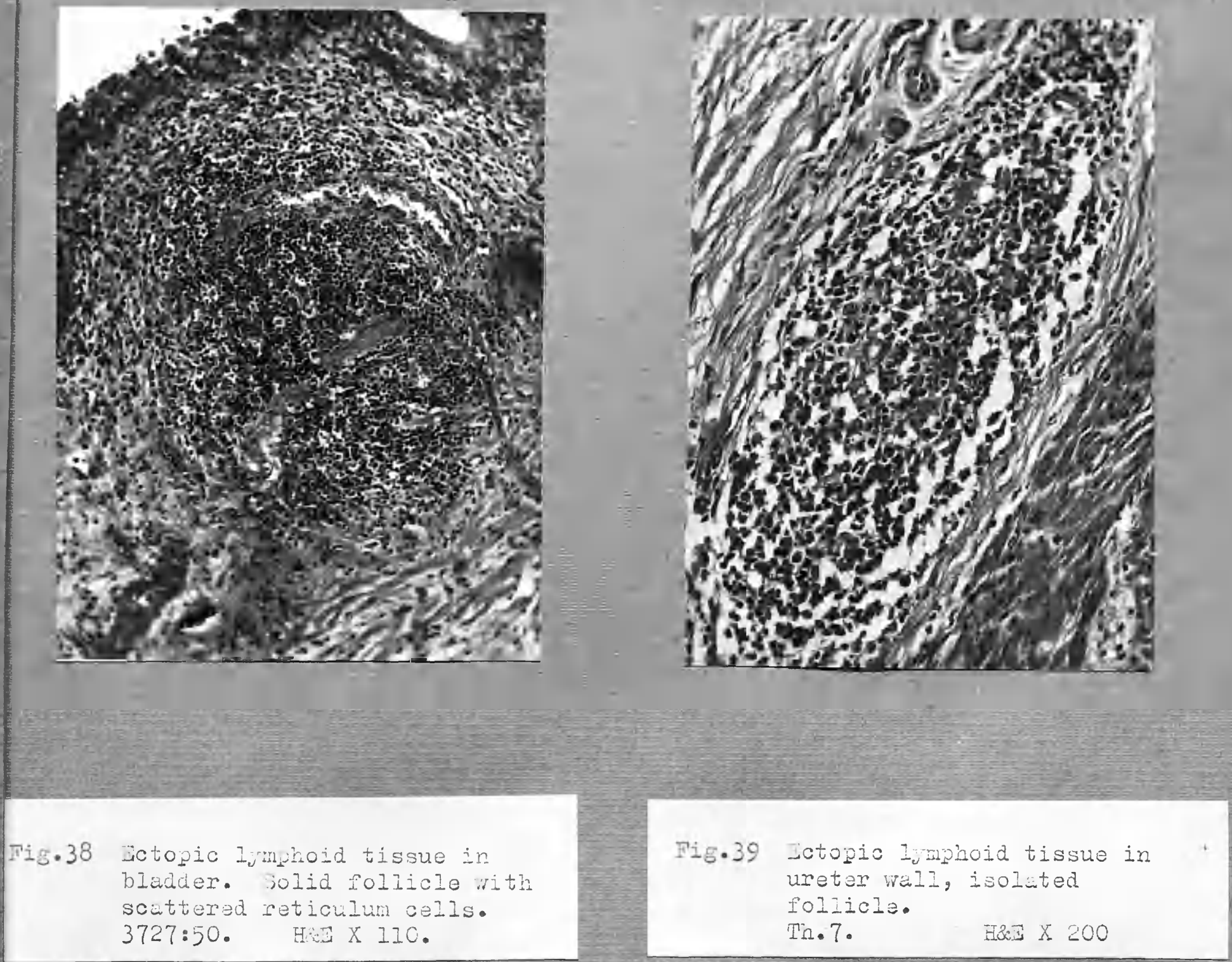


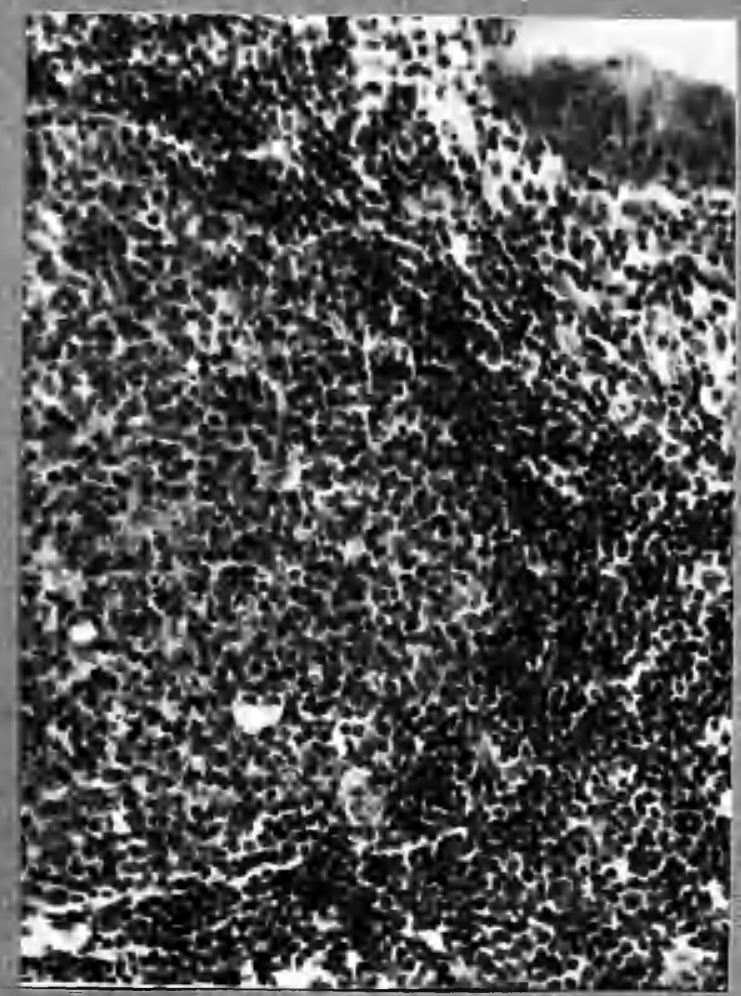

Ris $\cdot 40$
Gctofic Iymphoid tissue in

female breust (chronic

uastitis,

2.182. HXE $\times 250$.

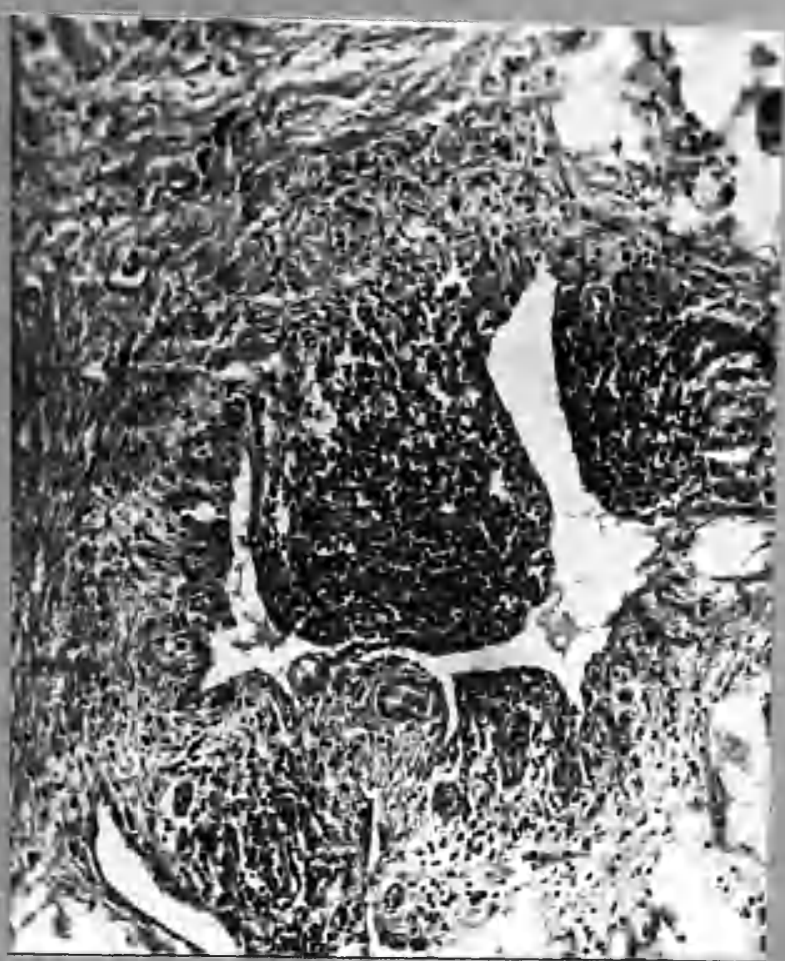

Fis. 41

Jctopic Iraphoid tissue in gal1-olidder. $3787: 50$ 

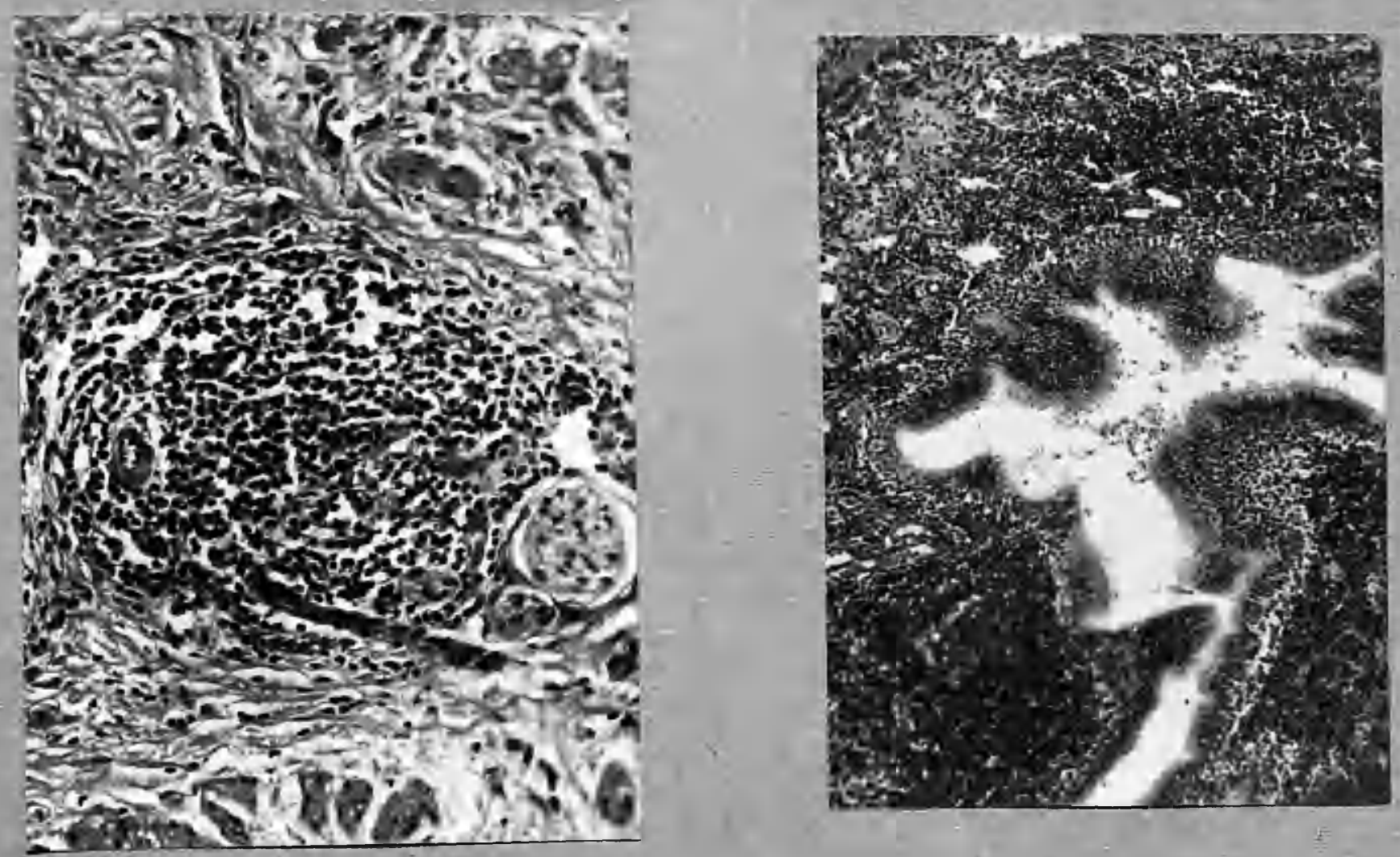

Pig.42 Dctopic lyrighoid tissue in skin (cutis vara) of face. Th. 3. ERe X 250.

Fig. 43 Small bronchus, shewing morbid increase of peri-bronchial lymphoid tissue. Case of bronchiectisis.

Uninom. 파 X 100 . 


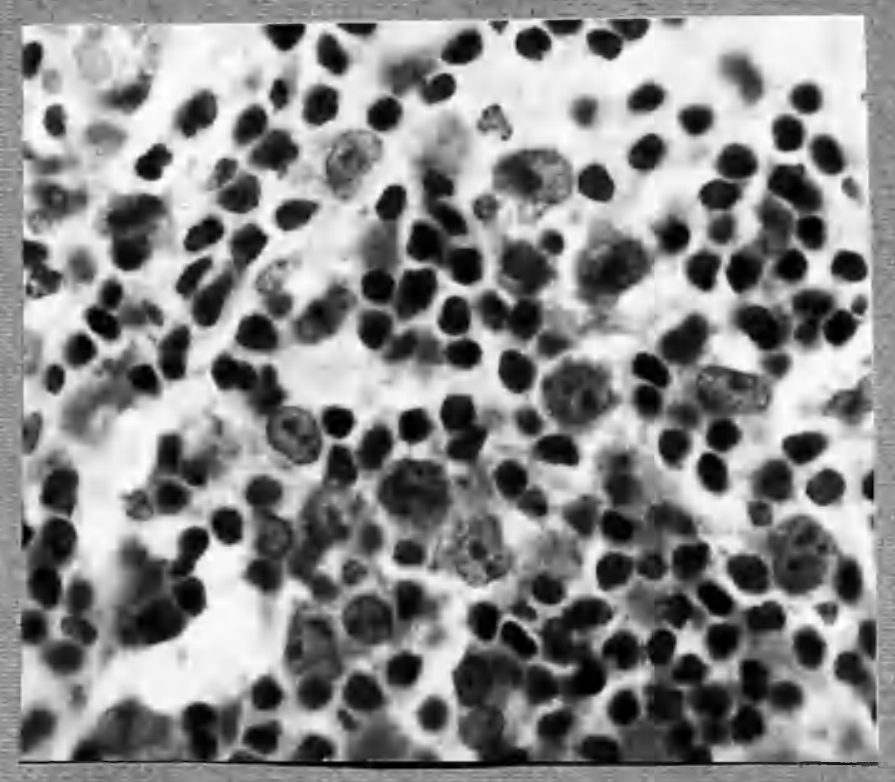

Fis. 44

Eigh porer vien of new

lymprola tissue to shew minute structure. (Blidier). 3727:50. H\&D X 500 . 
HODGKIN'S DIJEAJ. Exodic growth phenomenon.

Pig.45 jerial 70. Typical Gojekin's disemse shering exolic oromin phenomenon, the deniscant crescent is reactive lymphoid tissue, below is the new tissue.

He: $\times 9$.

Pig.46 jerial 40. itypical Lolgkin's disease. ixodic groith phenornenon visible but less pronounced. wisis lesion vas tumour-like. He: $\times 7 \frac{1}{2}$

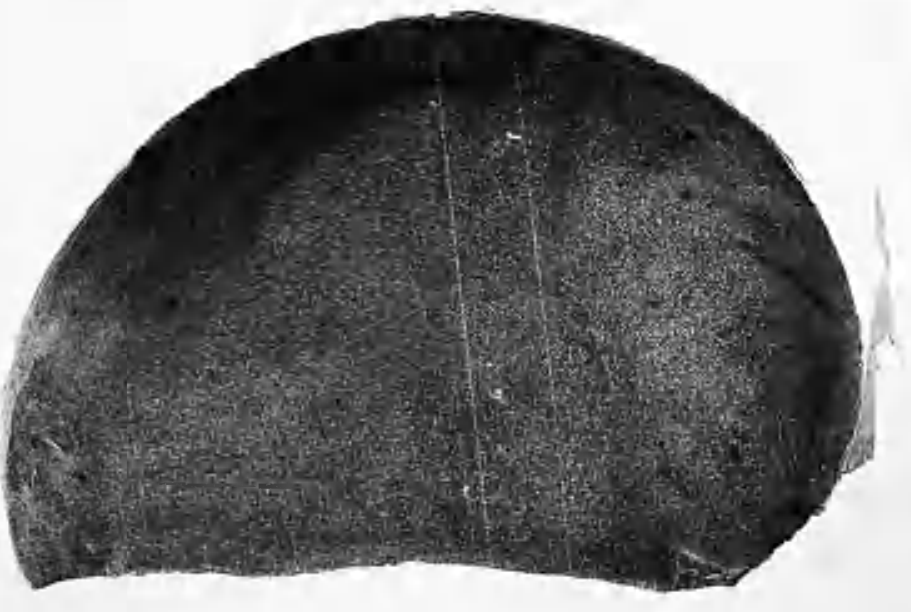


HODGKIN'S DISEASE. Folliculoid habit.

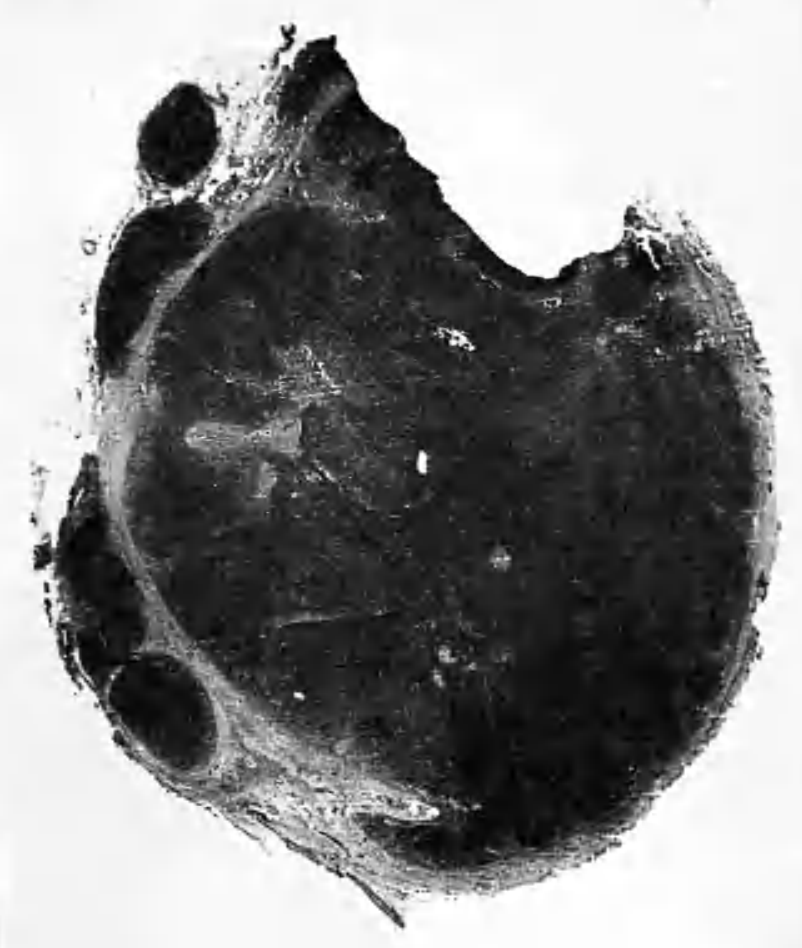

Fig.47 serial 132. Typical Hodgrin's disease. Faintly folliculoid habit is shem. H\&i $\times 7 \frac{1}{2}$.

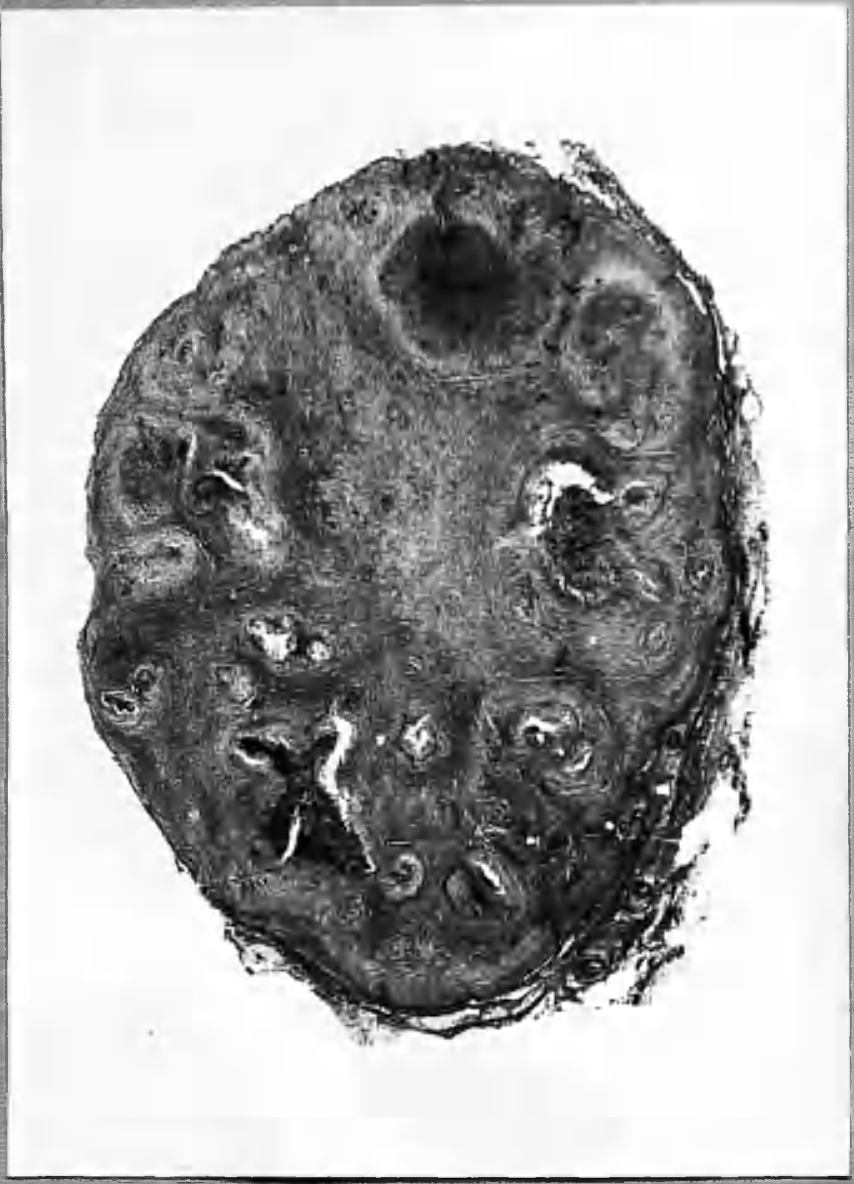

Fig.48 Serial 106. Iute Hodgkin's disecse. Irregular

folliculoid pattern well shewn. 
HODGKIN'S DISAASJ. Folliculoid habit.

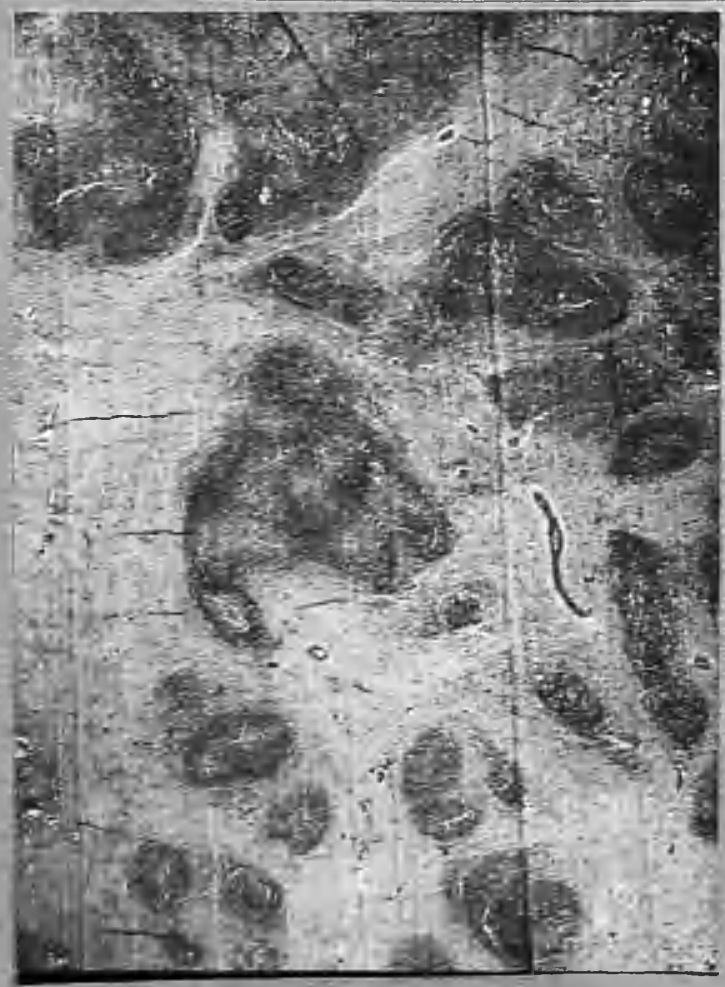

Fig.49 Sterial 56. Inte Fod gin's disease. Snall deposits of active new tissue in a fibrous matrix. Hes $\times 7 \frac{1}{2}$.
HODGKIN'3 JIszu3S, Wicroscopical features-

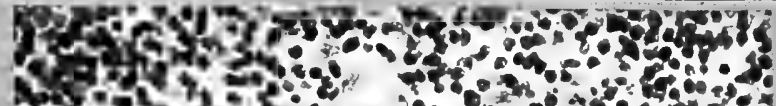
19.27 a

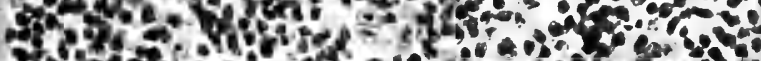

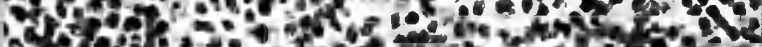

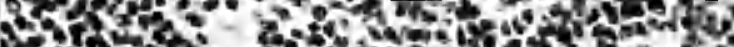

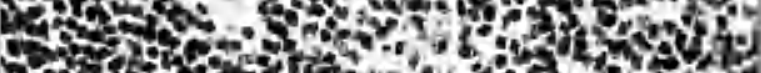
D. \&

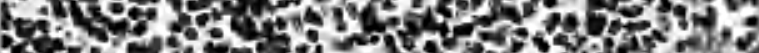

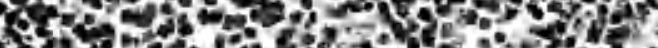

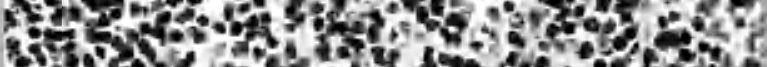

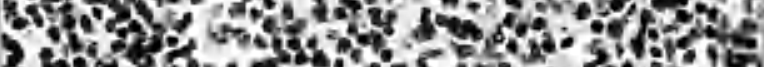

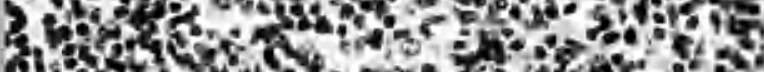

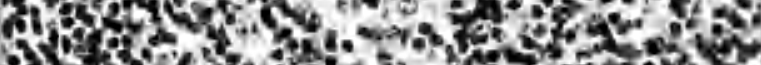

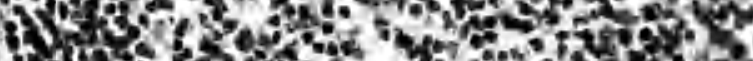

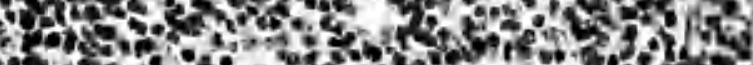

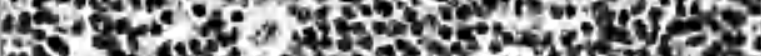

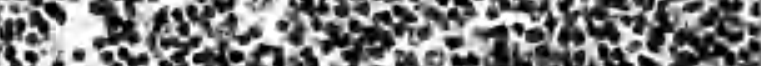

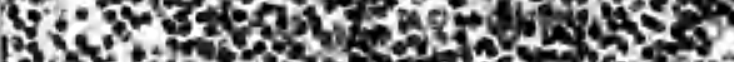
RSt

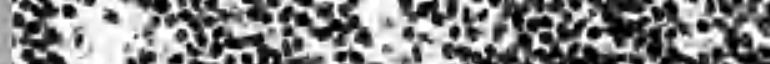

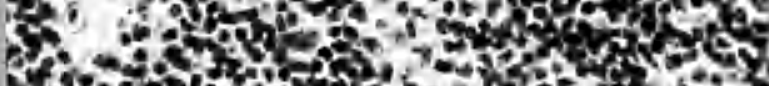

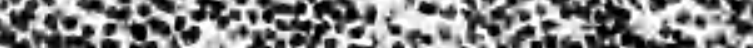

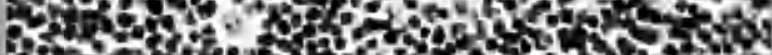

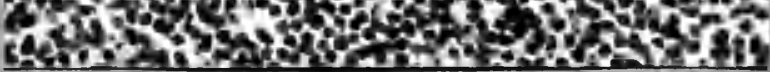

Pig.50 Serial 42. Garly Hodgkin's diseuse. Reticulum cell proliperation with produation of morbid forms. Note the tysical 'orl-eye' muclei. H\&W X 250 . 


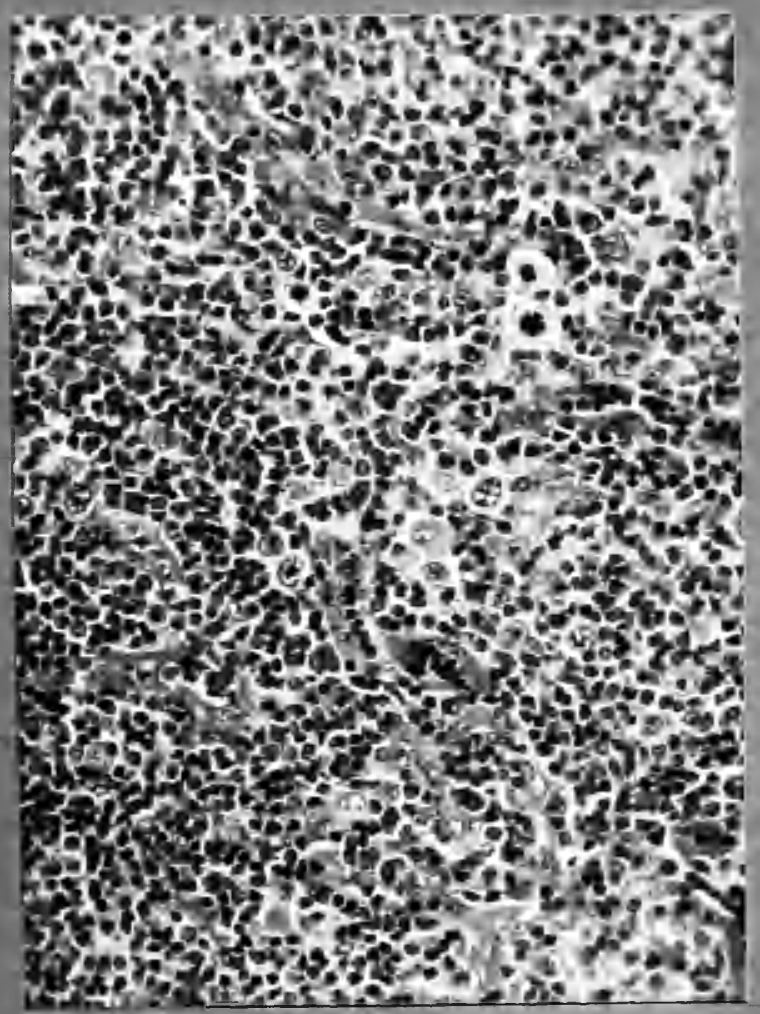

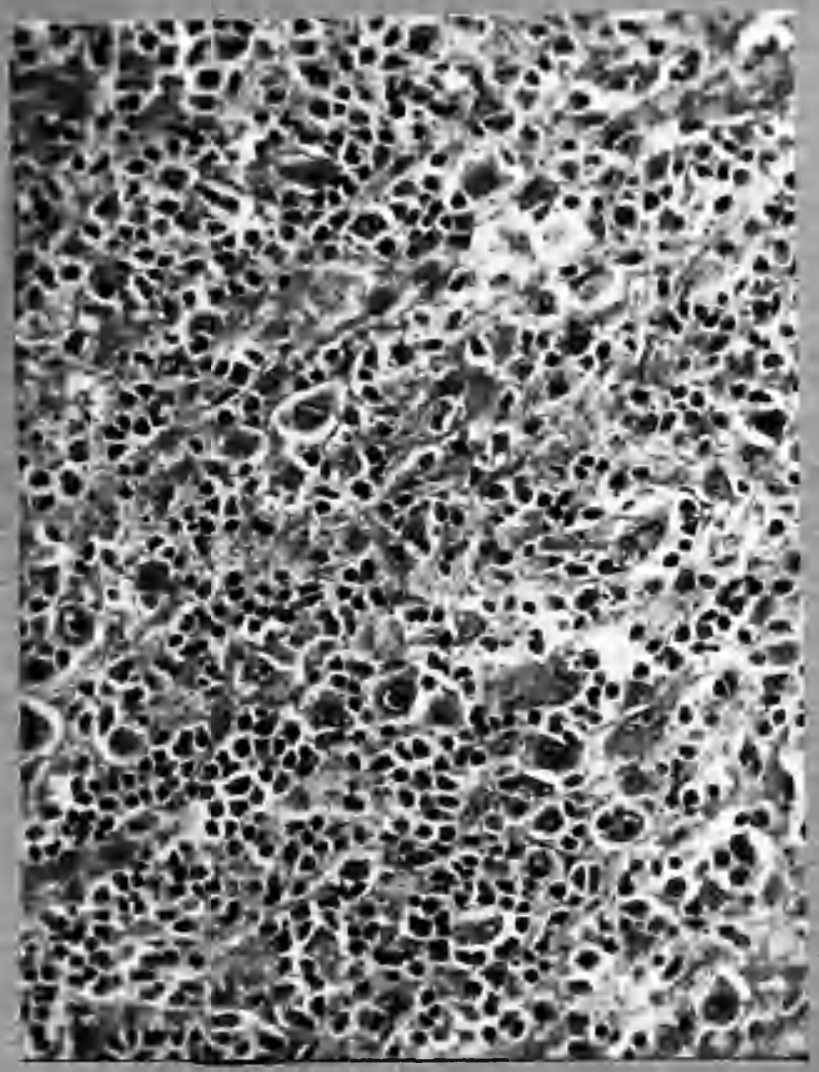

Fig.51 Jarial 89. Jurly lesion. Pleionorphism of reticulum celis eninanced, very sarly ṫiorosis。
Fig.52 Serial 89. Field ahewing eetablished lesion, fibrosis of fibril type is distinct. 

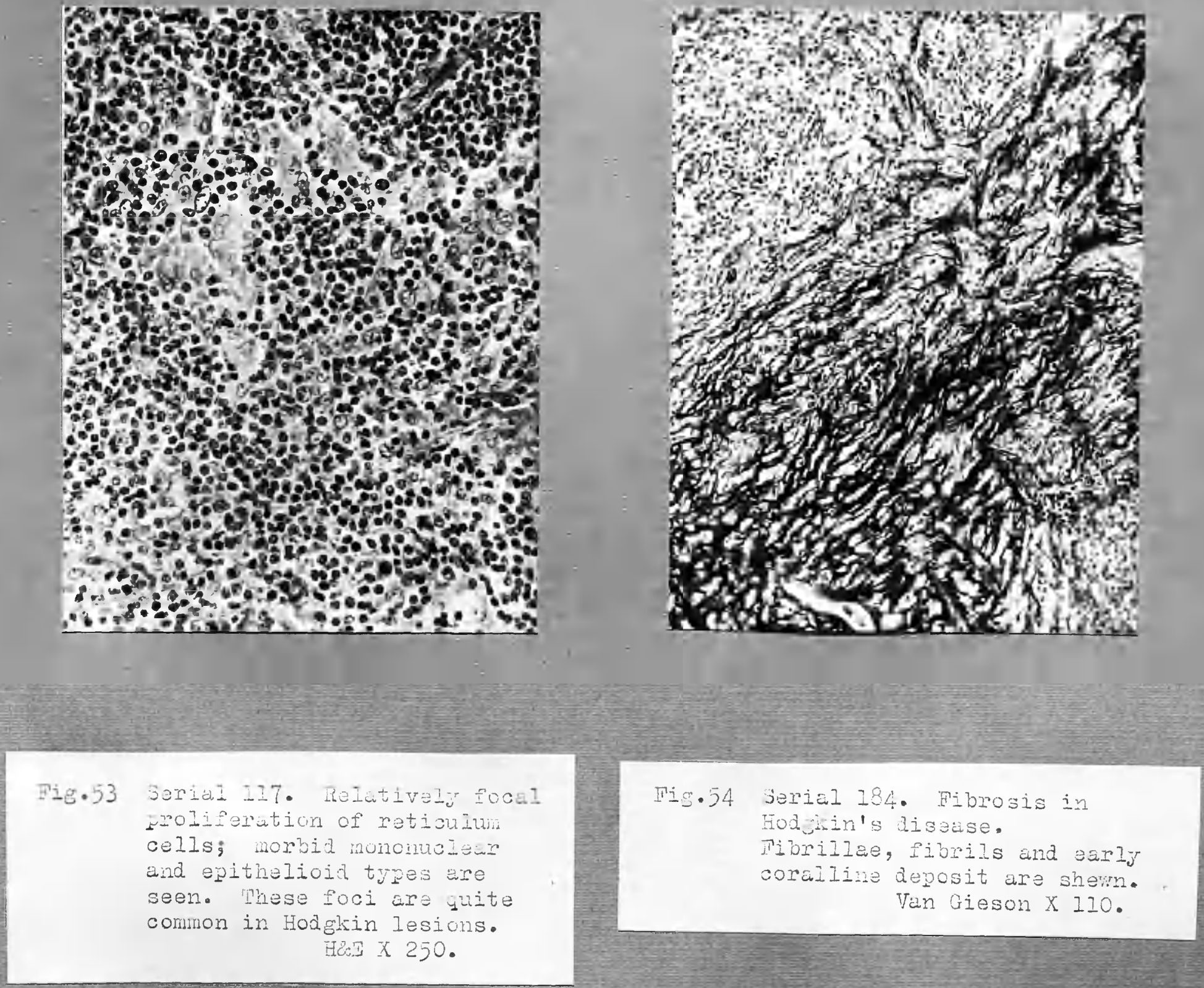

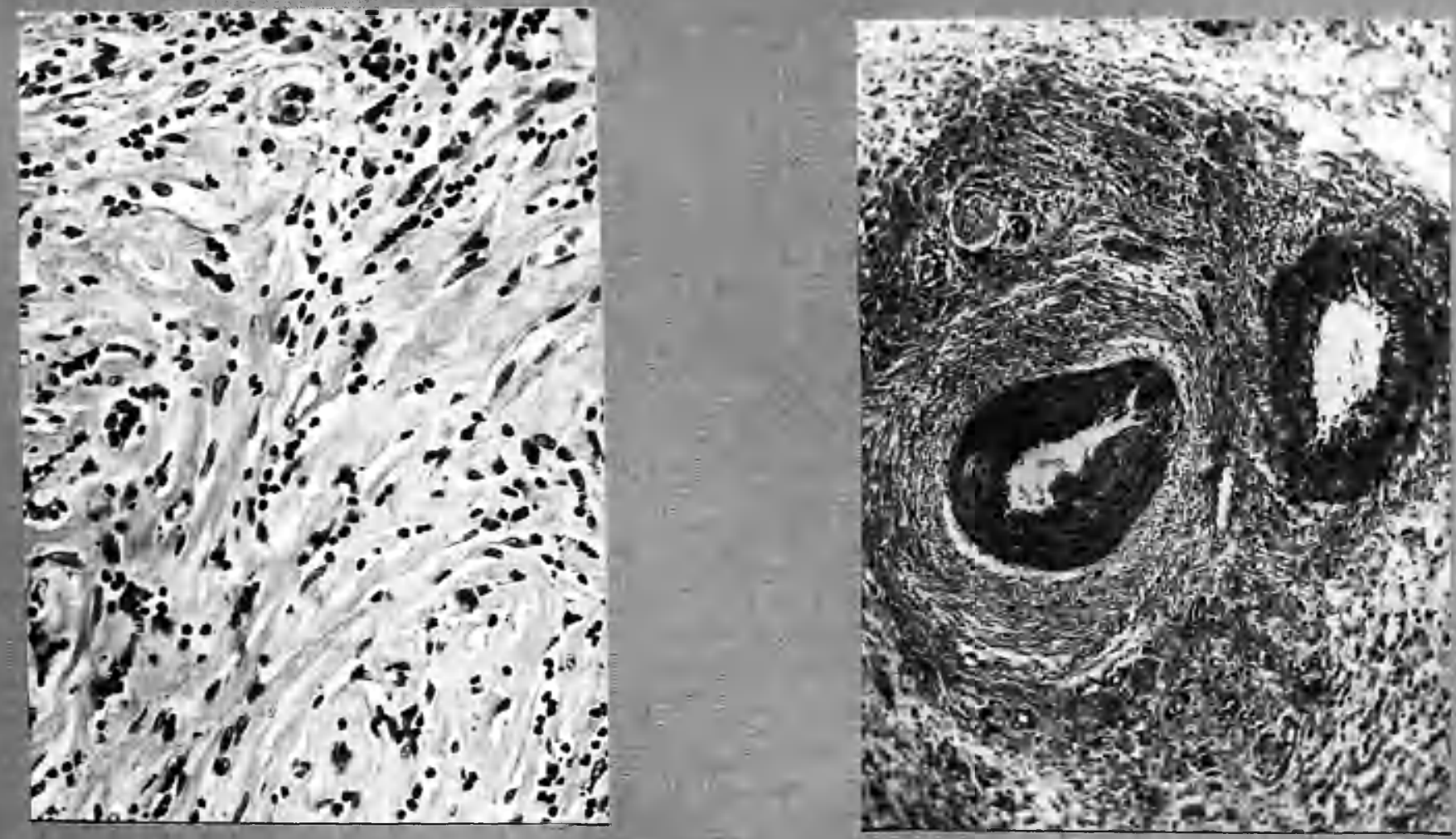

Fis.

Serial 83 (2nd biopsy). late fibrosis in Hod grin's disease.
Fig.56

Serial 184 .

Perivascular

artery and vein. Hos $\times 200$. 

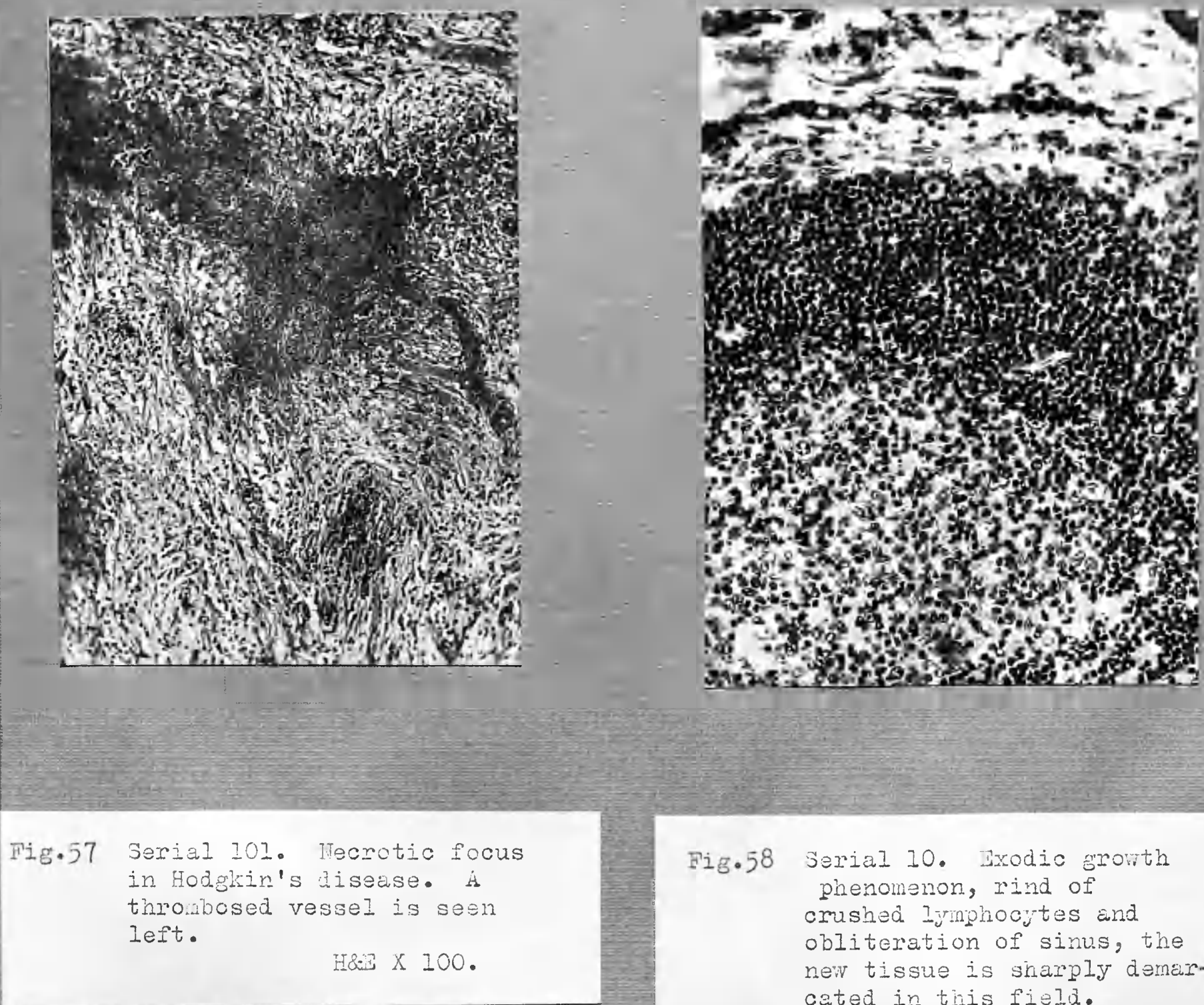

Fig. 58

Serial 10. Ixodic syowth phenomenon, rind of crushed Irnphocytes and obliteration of sinus, the new tissue is sharply demarcated in tinis field. 

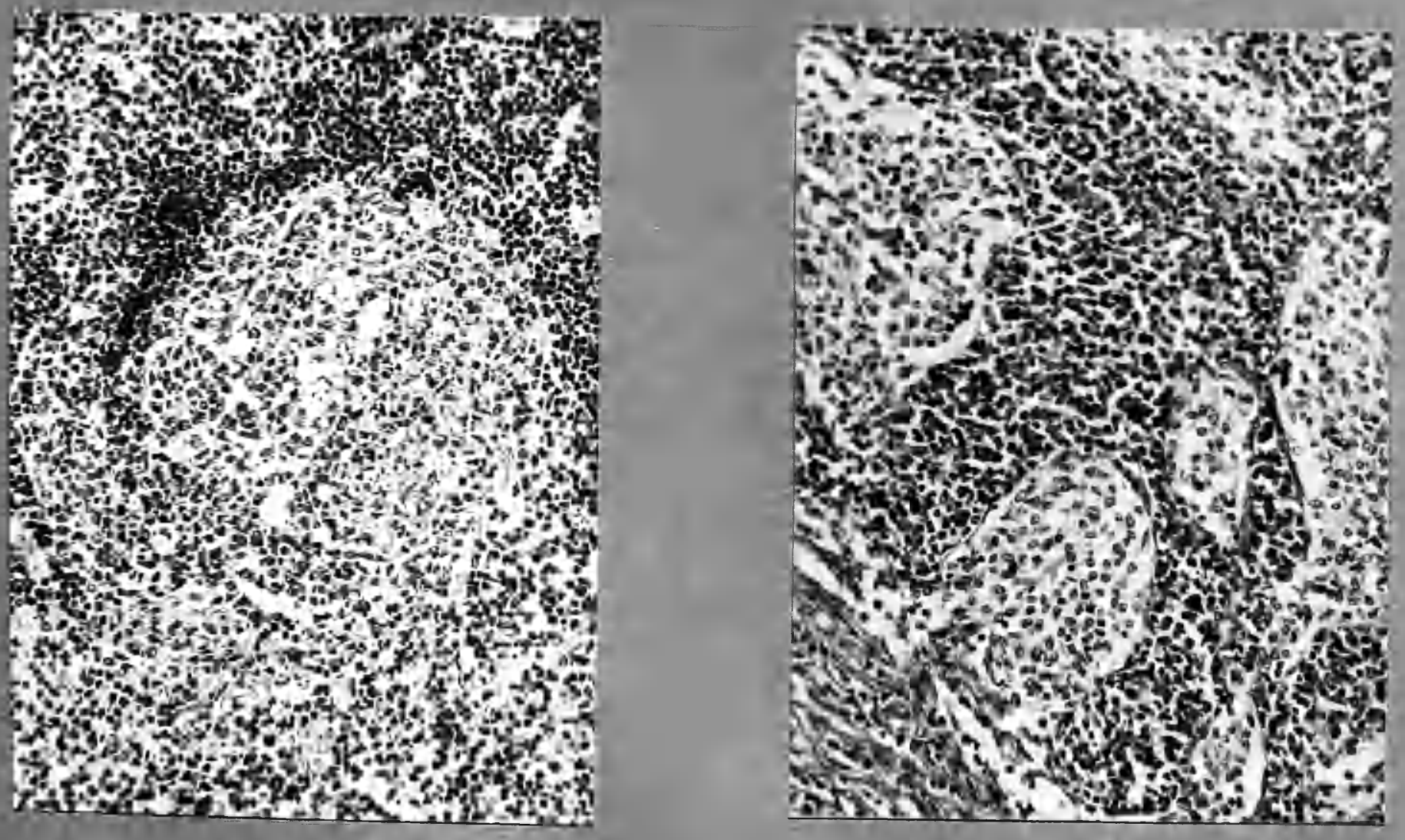

$$
\begin{array}{r}
\text { Fig.59 Serial 89. Surviving } \\
\text { Iymphoid follicle with pale } \\
\text { centie, at peripherjof node } \\
\text { shewing Lodgrin's diseuse. } \\
\text { Has } x 200 .
\end{array}
$$

Fig.60 Serial 199. Normal 1ymph cords and siruses deep in E node shewing Hodgkin's diseuse. 
HODGRT:'S JIJEABE

Paraisturce of nori..l structure.

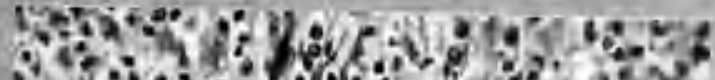
4.

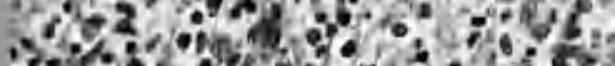

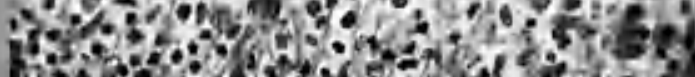

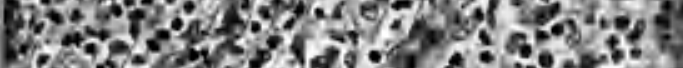

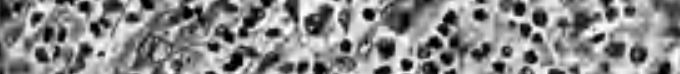

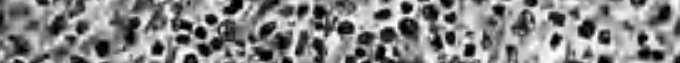

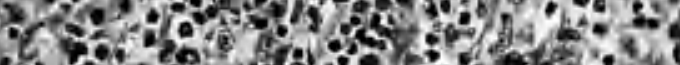

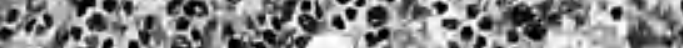
o. kP

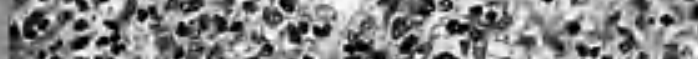

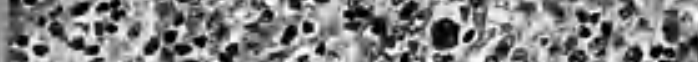

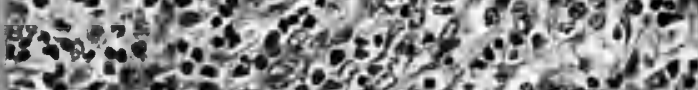
3.1.

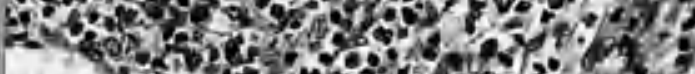

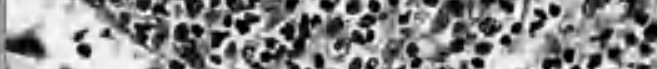

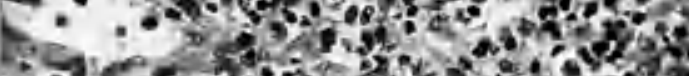

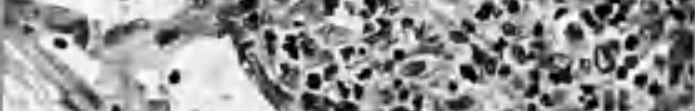

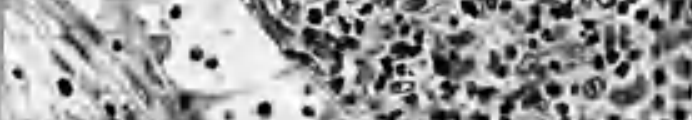
cin

Fig.61 Serial 77. Persistence of narginal sirus in wituical tunour-like Hod firir's discase. This is rare.

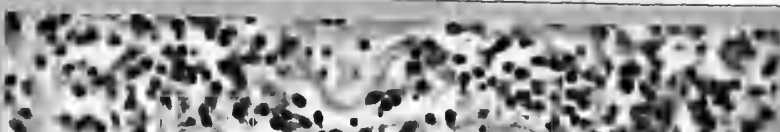

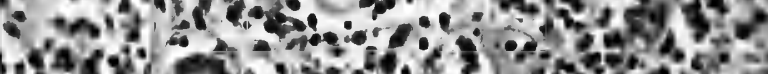
194.

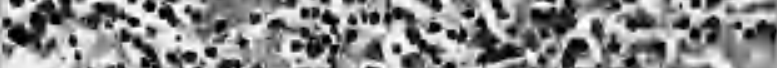

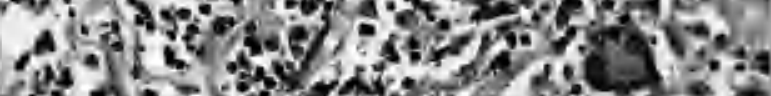

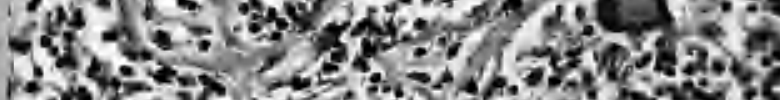

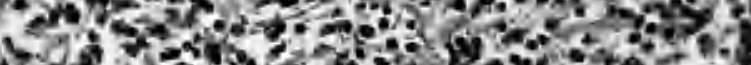

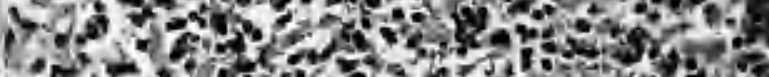

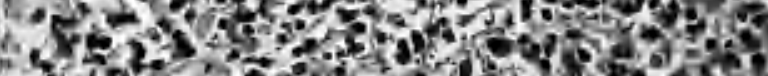

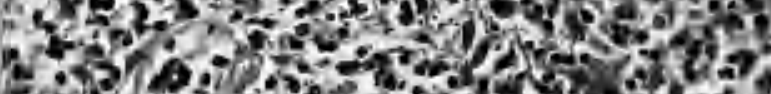

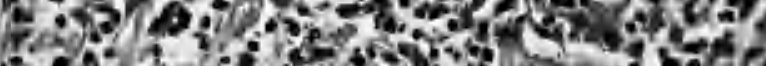

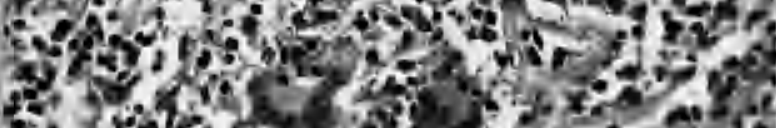

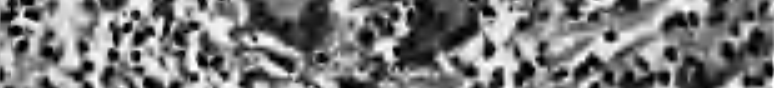
if

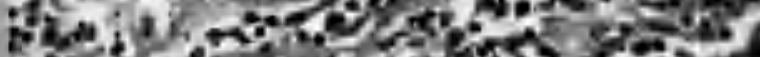

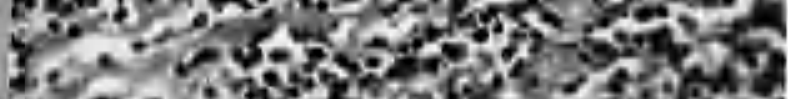

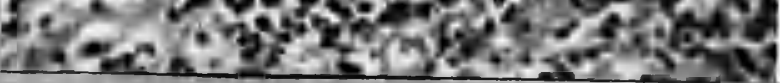

Hise $\times 250$. 


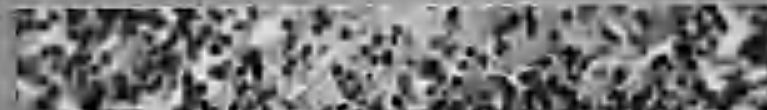

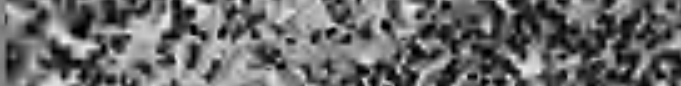

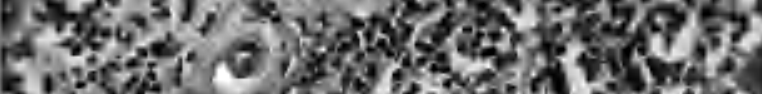

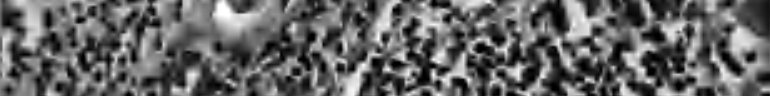
3.

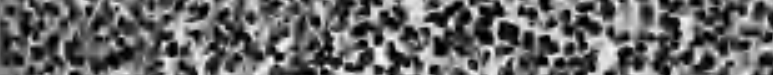

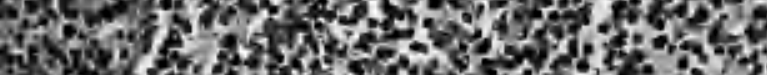

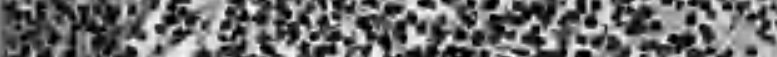
Th

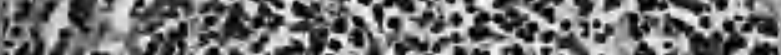

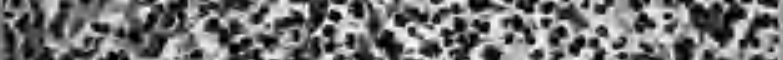

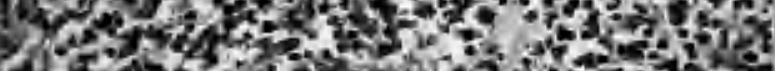

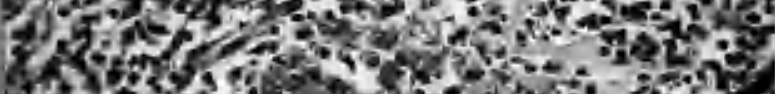

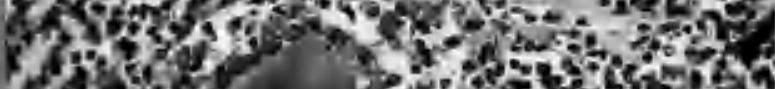

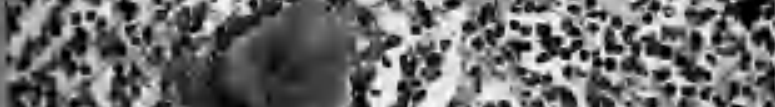

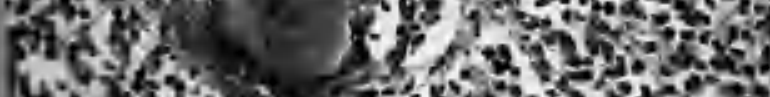

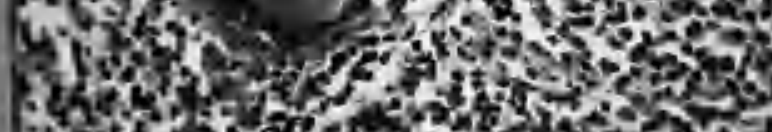

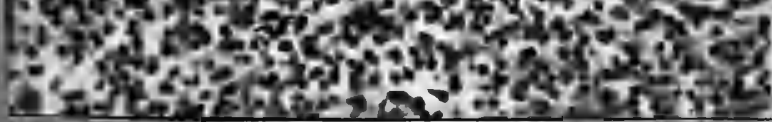

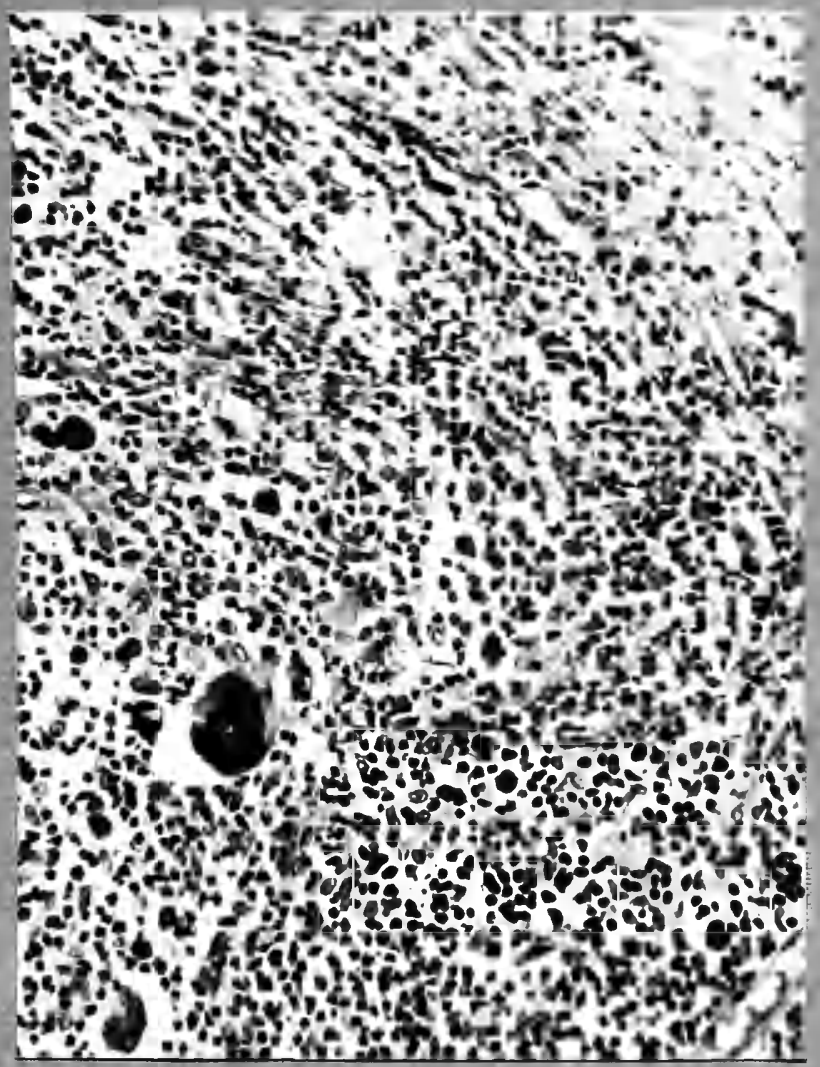

$$
\begin{array}{r}
\text { Fig.64 Jerial 81. Hodgkin's disease } \\
\text { in fentle breust. Mhe new } \\
\text { tissue in a glind field. } \\
\text { Hod X } 200 .
\end{array}
$$




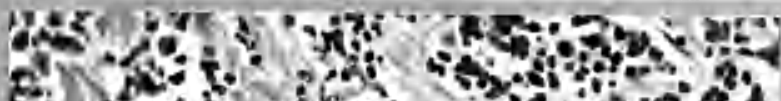

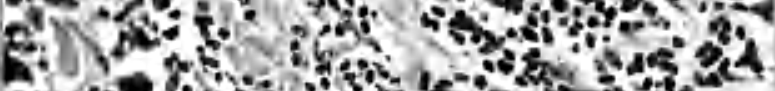

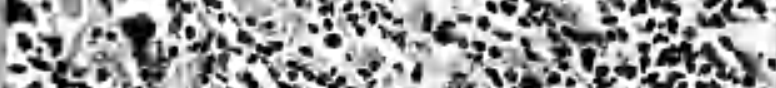
-4.

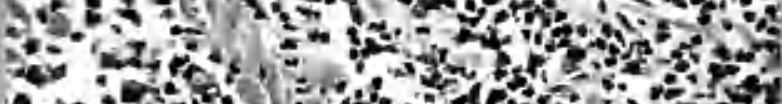

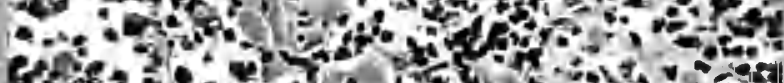

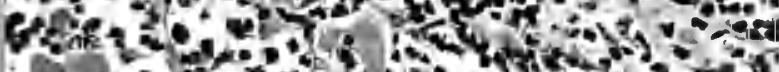

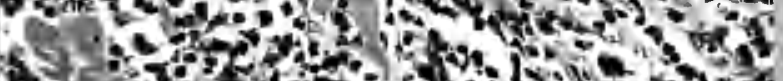

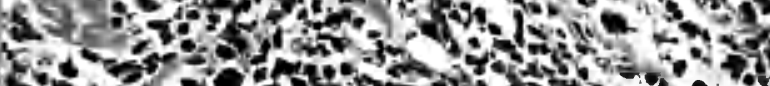

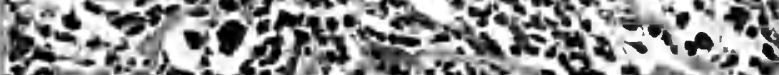

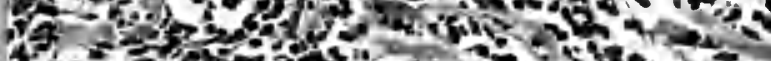

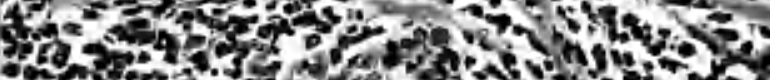

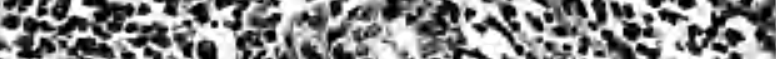

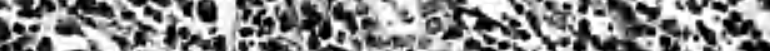

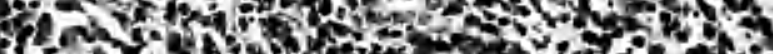

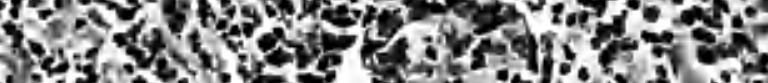

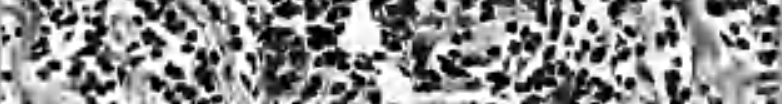

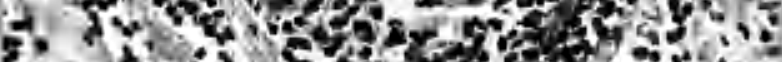

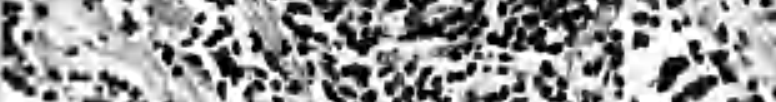

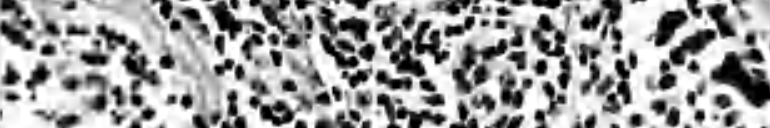
Fops:

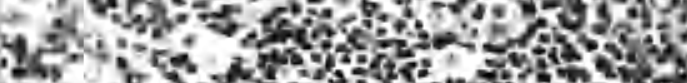

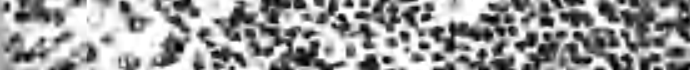

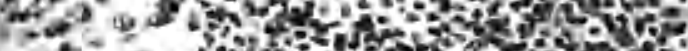

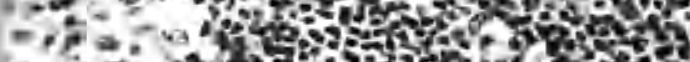

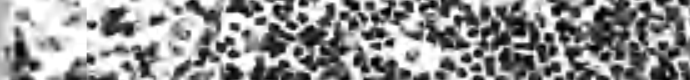

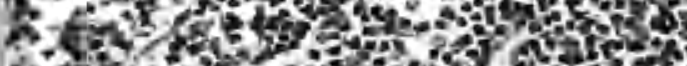

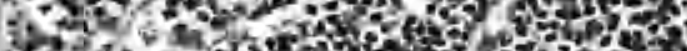

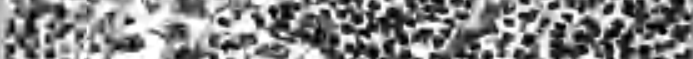

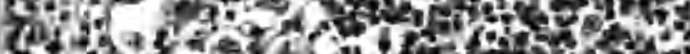

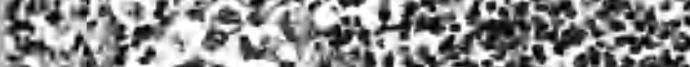

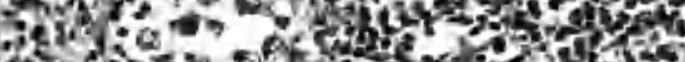

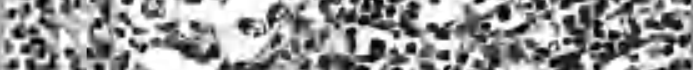
P. Thint

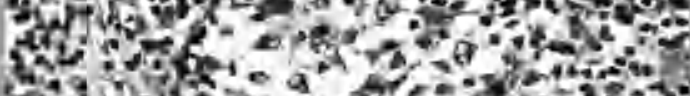
4.M.

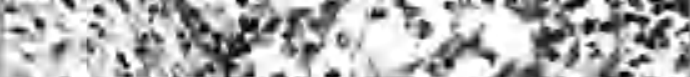

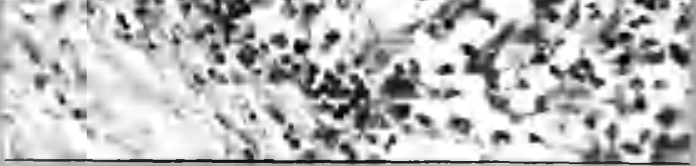

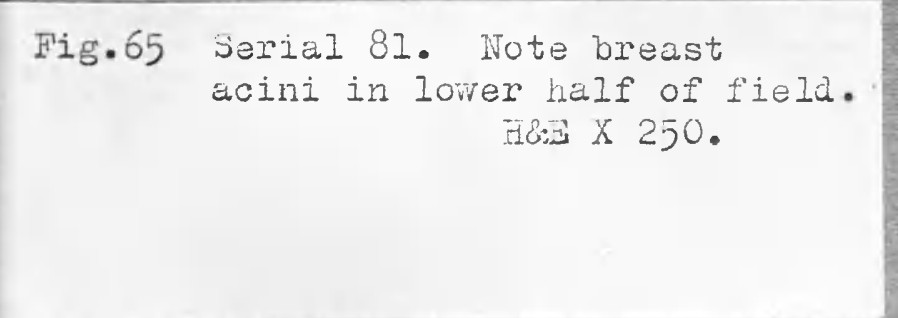

Tig.66 Serial 206. Possible origin of Hodgkin's disease in the sinuses. Irote morbid mononuclesr reticulum calls replacing littoral cells, the pulp is less affected.

Fier X 200. 

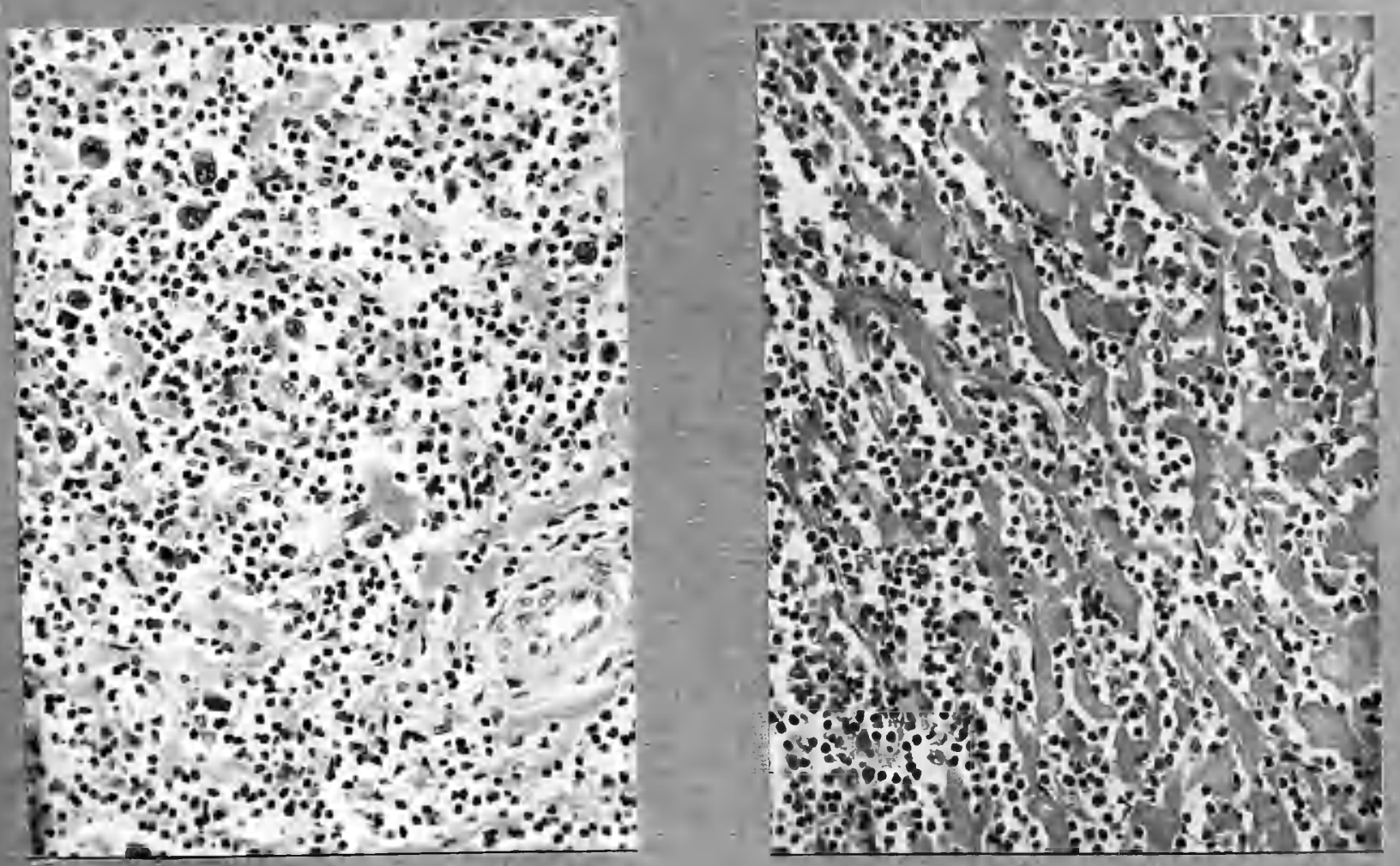

Figs. 67 and 68. Case of apparently isolated Hodgkir's diseuse. G.R.I. P.I. 100/54. See page 185 of text. Small cells predorninete.

H\&S X 250 . 


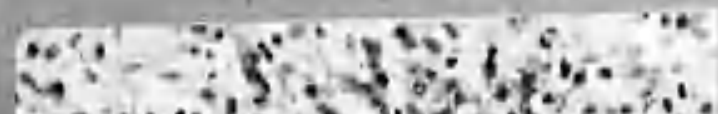

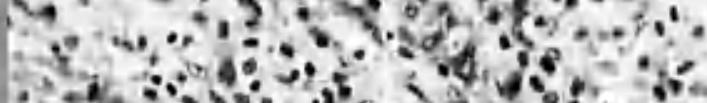

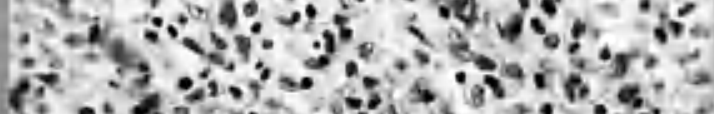

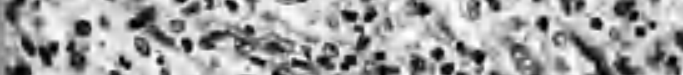

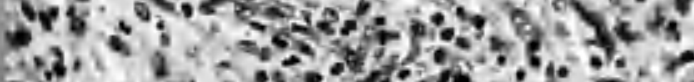

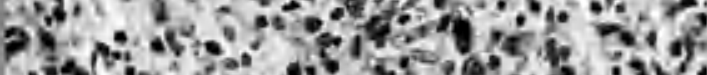

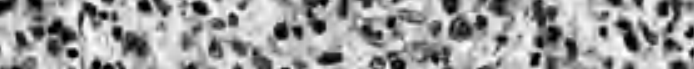

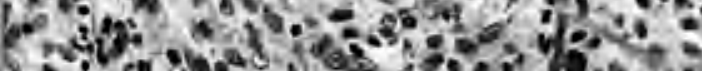

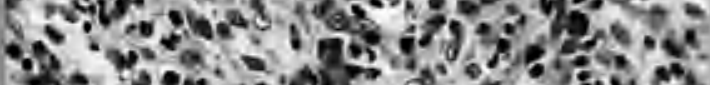

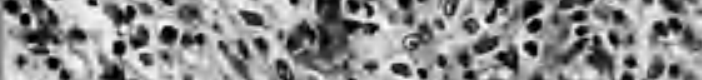

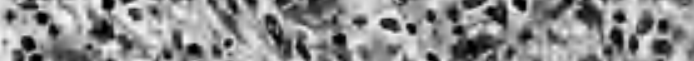

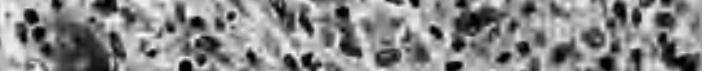

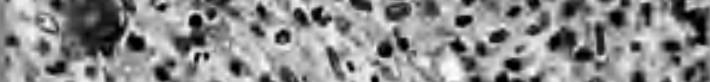
of ${ }^{2}$.

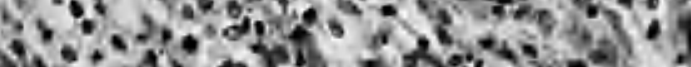

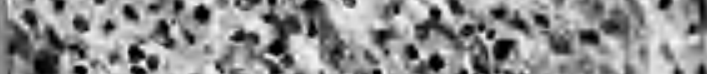

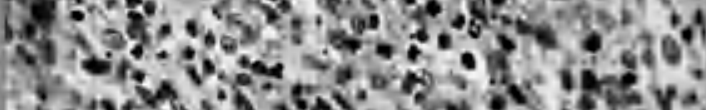

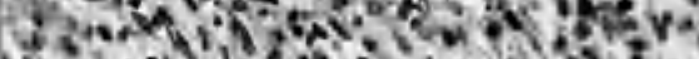

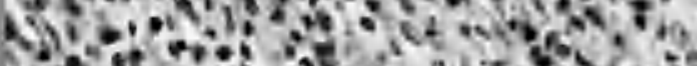

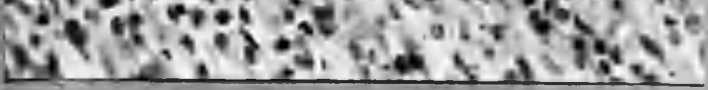

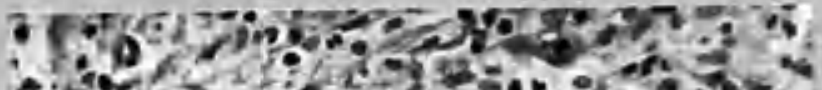

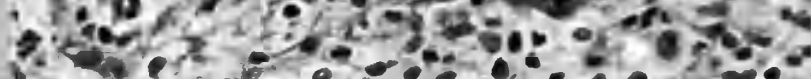

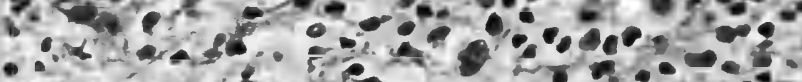

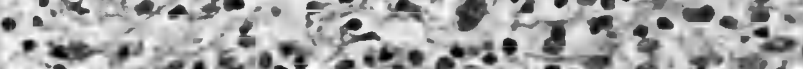
id a

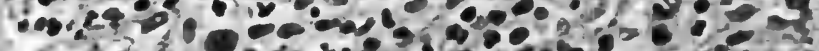

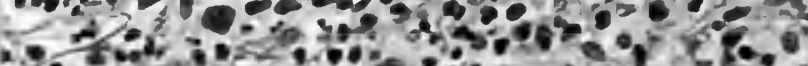

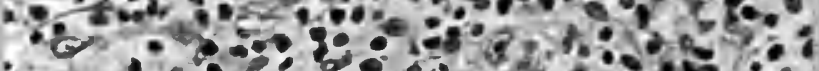
$\because 40$ a

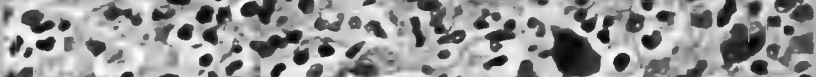

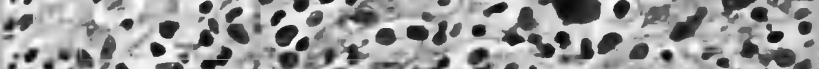
is o t? sion 60 o

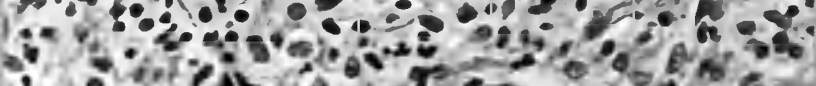

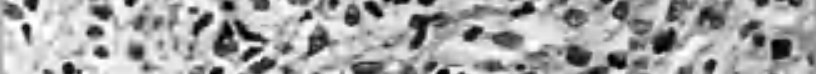

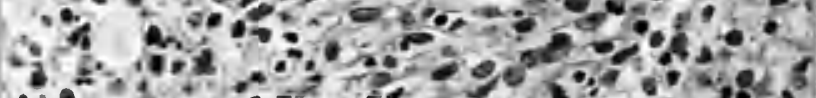
$\because 03,1,0,0$,

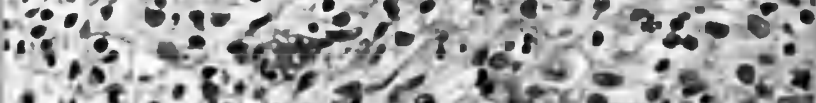
into

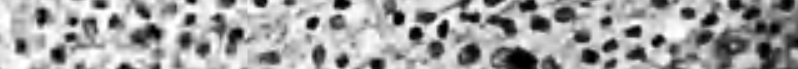

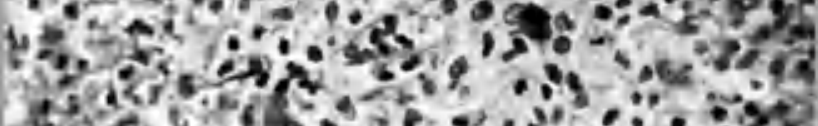

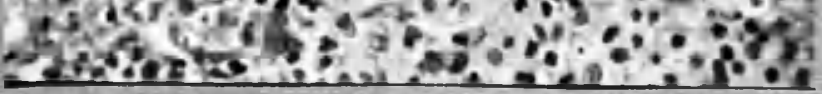

Figs. 69 and 70 .

jerial 133. Hojgkin's disease ill winch egithelioid aelIs are very numerous, symplasmic fibrosis is lignt, it is seen better in Fig. 70 . 


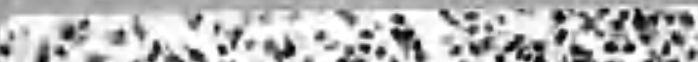

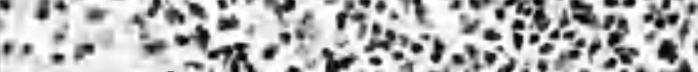

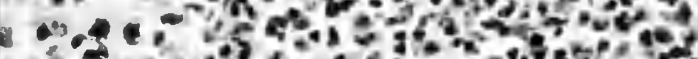

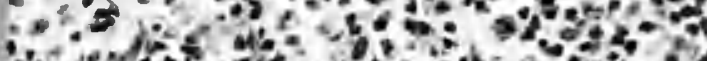

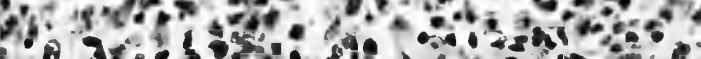

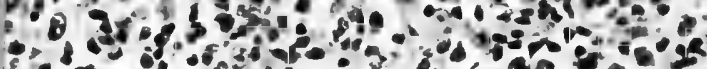

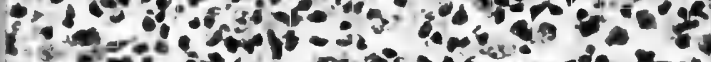

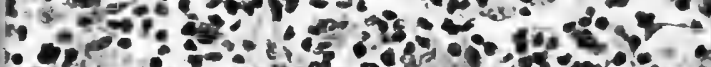

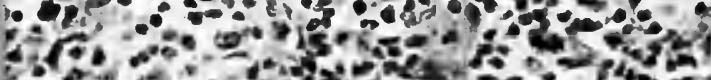

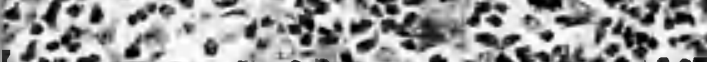

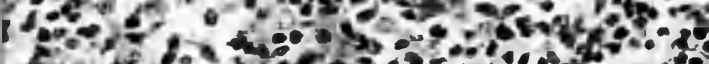

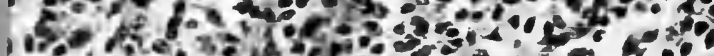

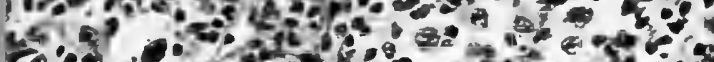

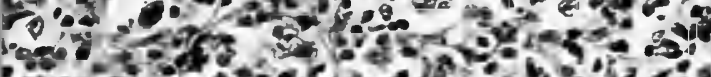

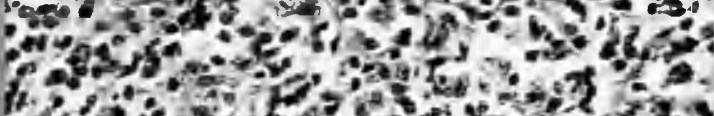
1. 10.90 is

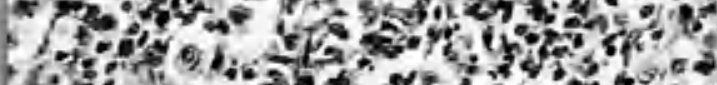
$670^{2}$.

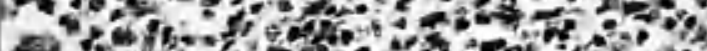

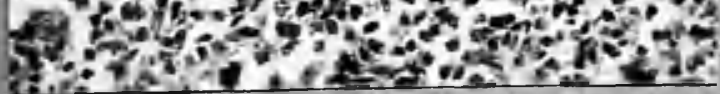

Fig.71 jerial 213. Highy cellular

tunour-inke lodgkin's

disease rith retention of

eosinopnilos. Note relative

unifornity of patuen.

H\&3 X 200.

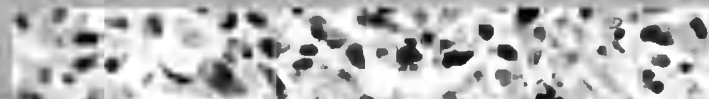

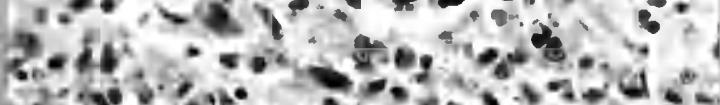

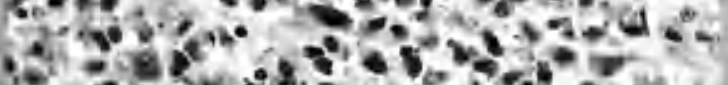

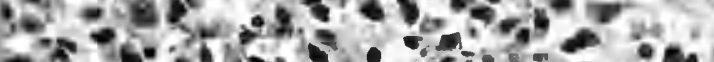

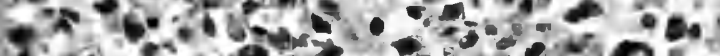

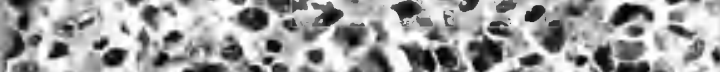

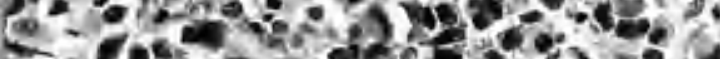

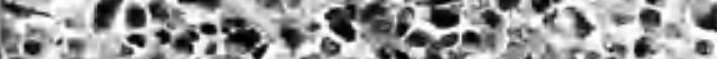

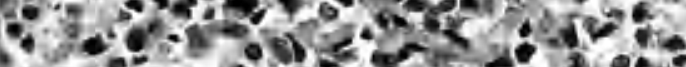

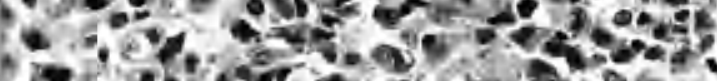
iso. 140 - 10 :

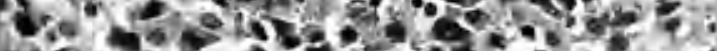

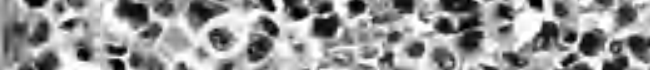

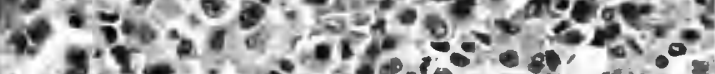

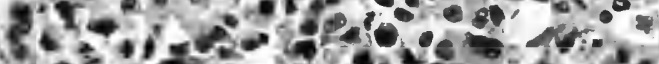
6 7.

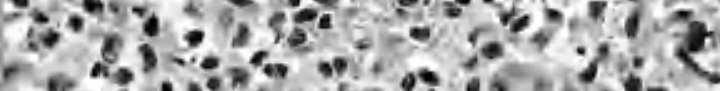

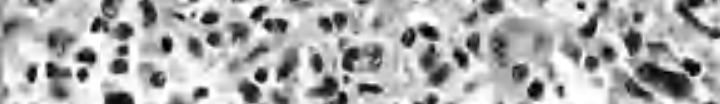

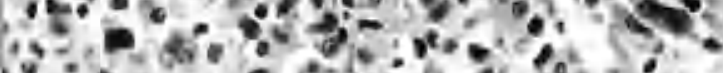

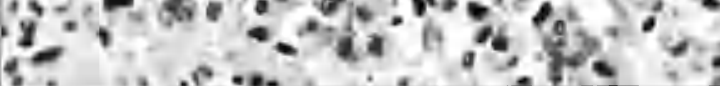

Fig.72 jerial 204. Highly celiular and tunour-like, posinophiles reducea. Fen very larese celis are seen. 


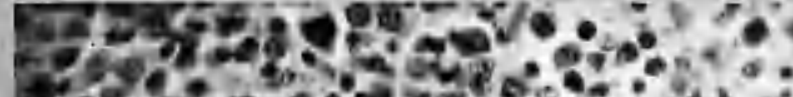

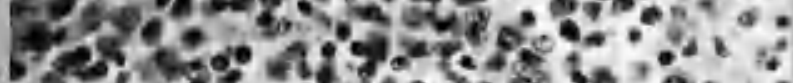

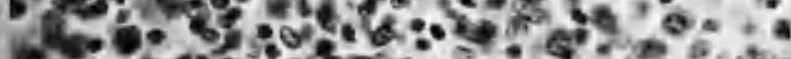

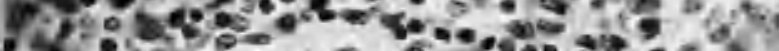

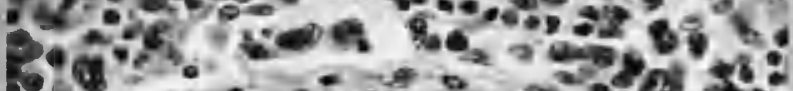

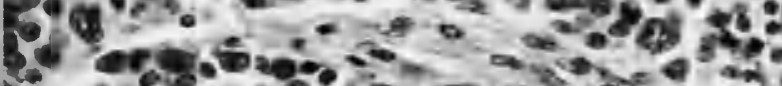

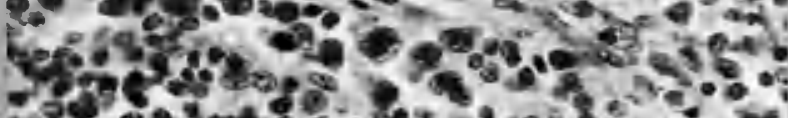

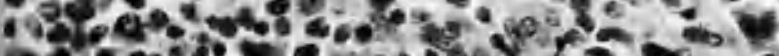

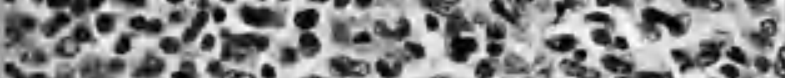

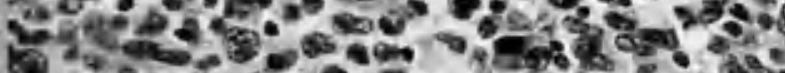

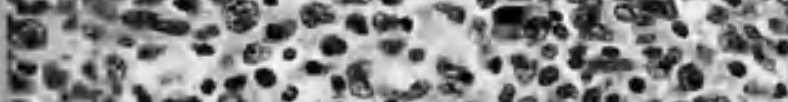

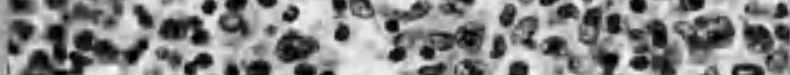

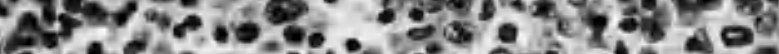

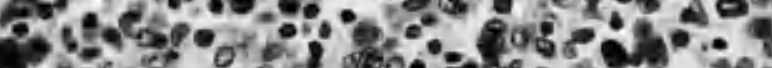

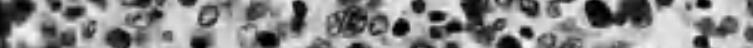

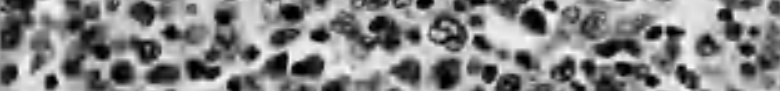

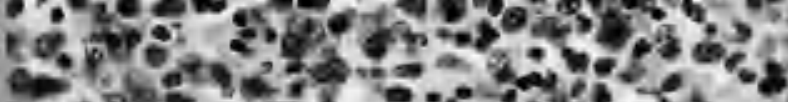

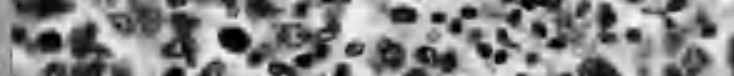
E.c.

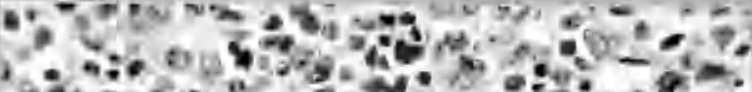

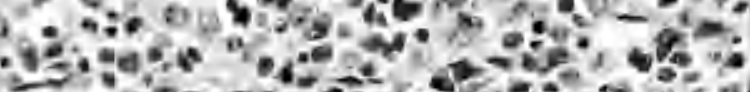
a

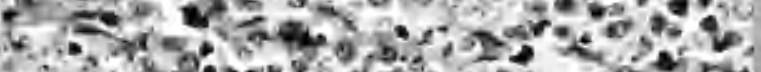

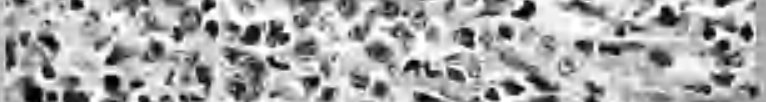

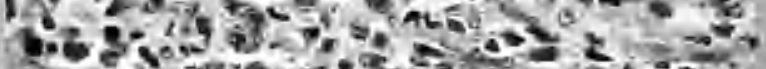
6 s.

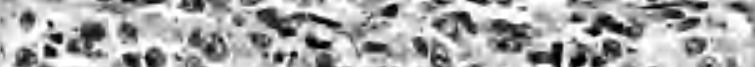

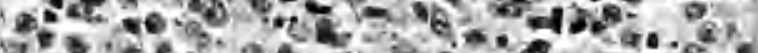

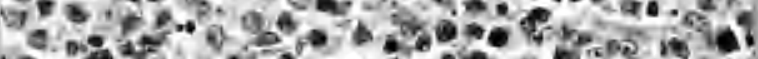

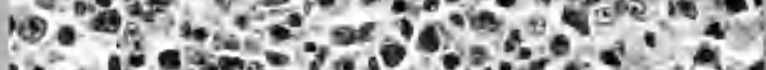

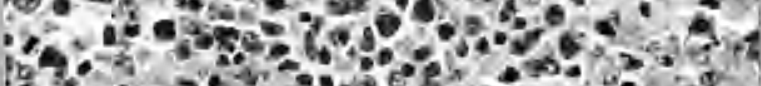

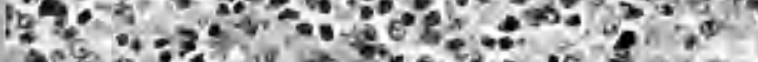

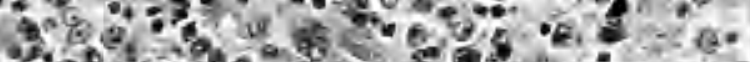

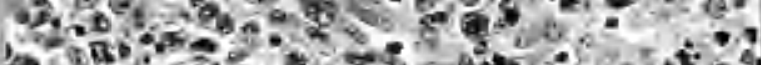

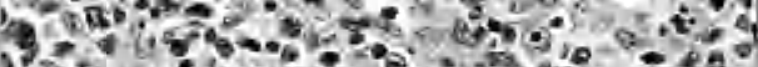

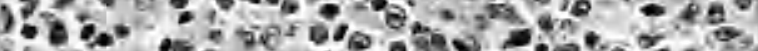

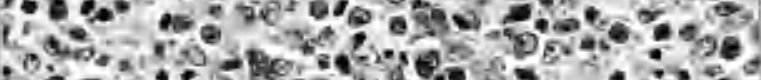

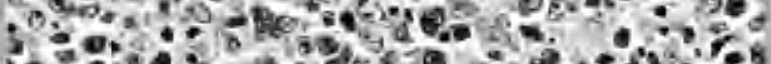

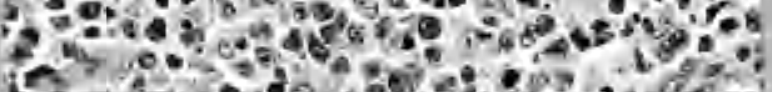

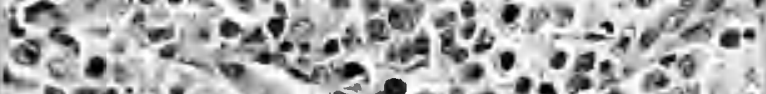

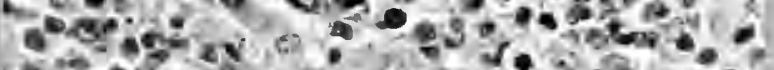

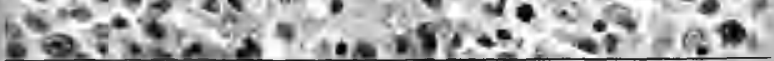

Fig.73 Serial 31. itosology doubtful, either cellular tumourlike Hodgkin's aisesse or reticulum cell sarcona. Losinopkiles present.
Fig.74 Jerial 209. Cellular tumourlike Hoagkin's aisease, or raticulun cell sarcona. Josinophiles are very few. The picture is nearer frank turnour. 


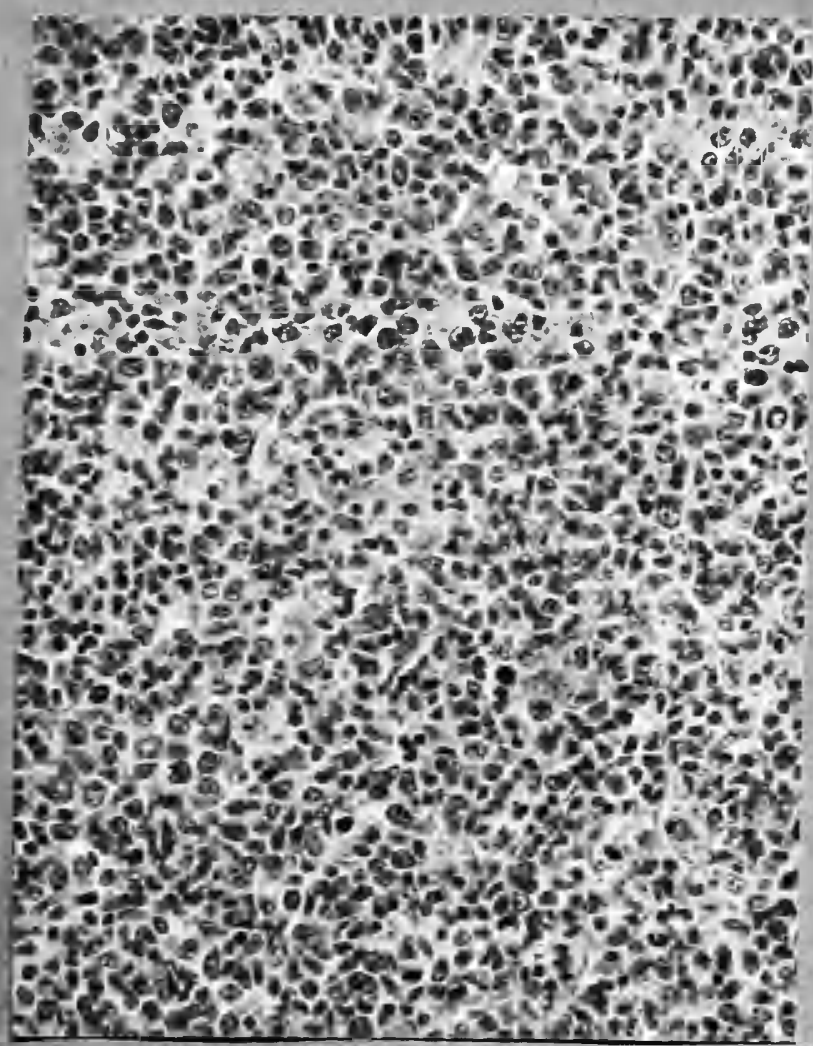

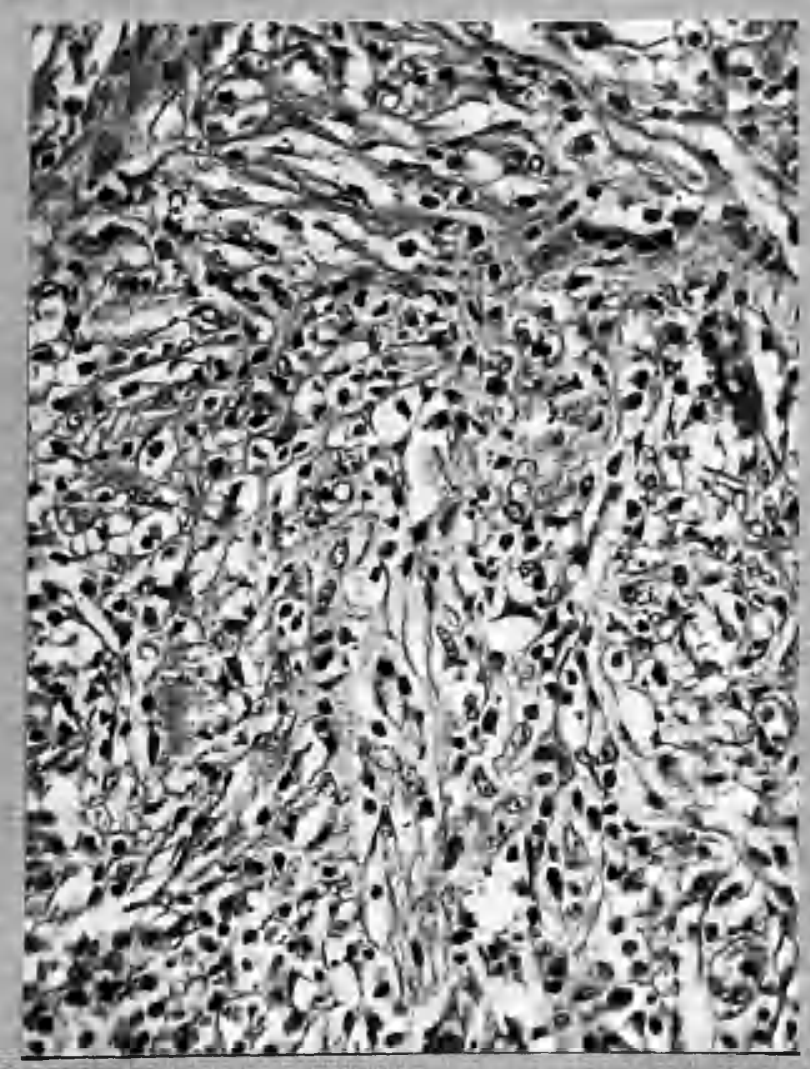

Fig.76 Serial 168. Diffuse fibroblastic Hodgin's disease; pleionorphism linited, lymphocjtes sparse, very uniform tirroughout the node. sarcoma retainins many Hodgkin's disease features. בx $\mathrm{x}$ X 250 . 


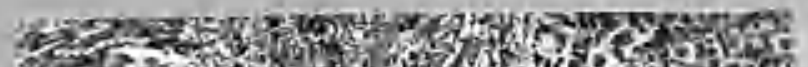

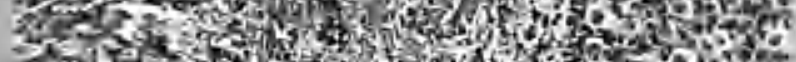

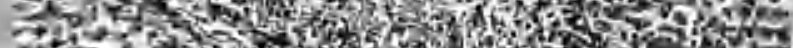

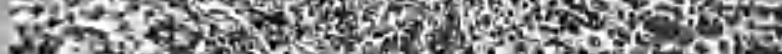
$x$ s.

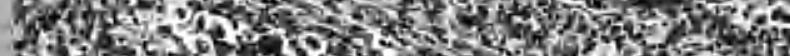

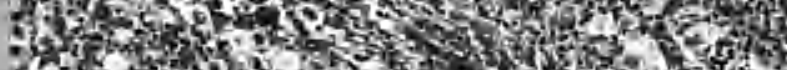

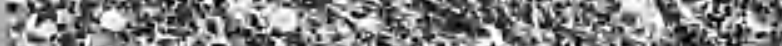

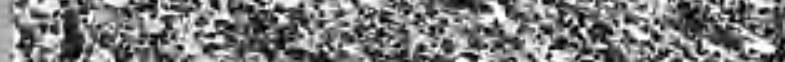

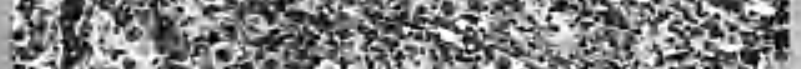

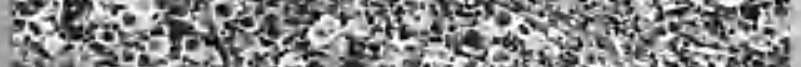

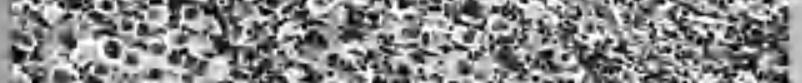
P.

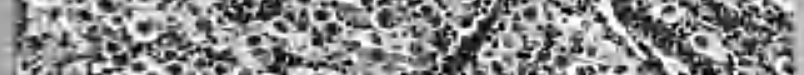

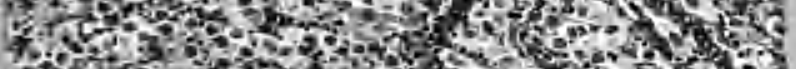

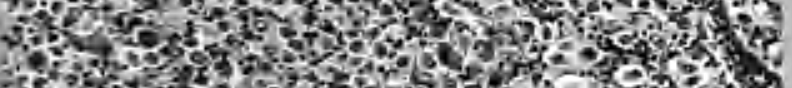

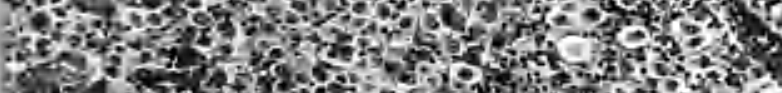

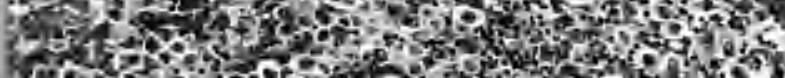

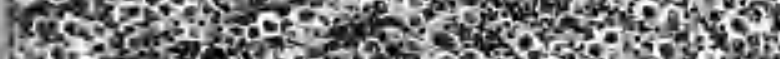

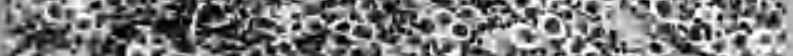

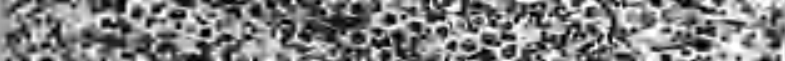
$3060-2$.

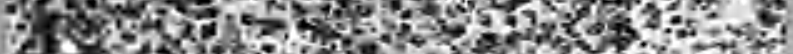

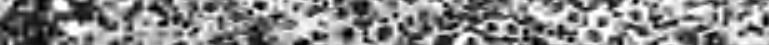

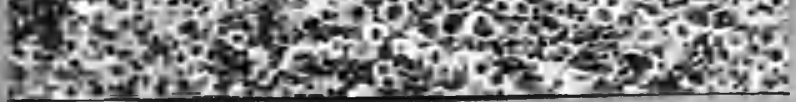

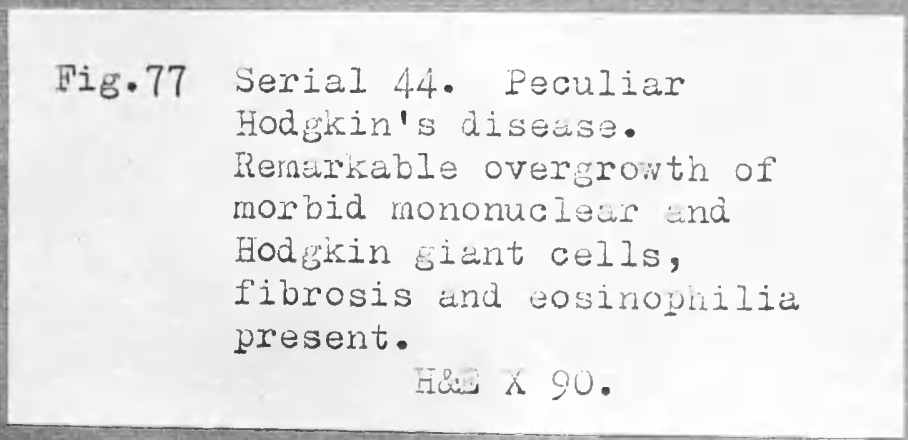

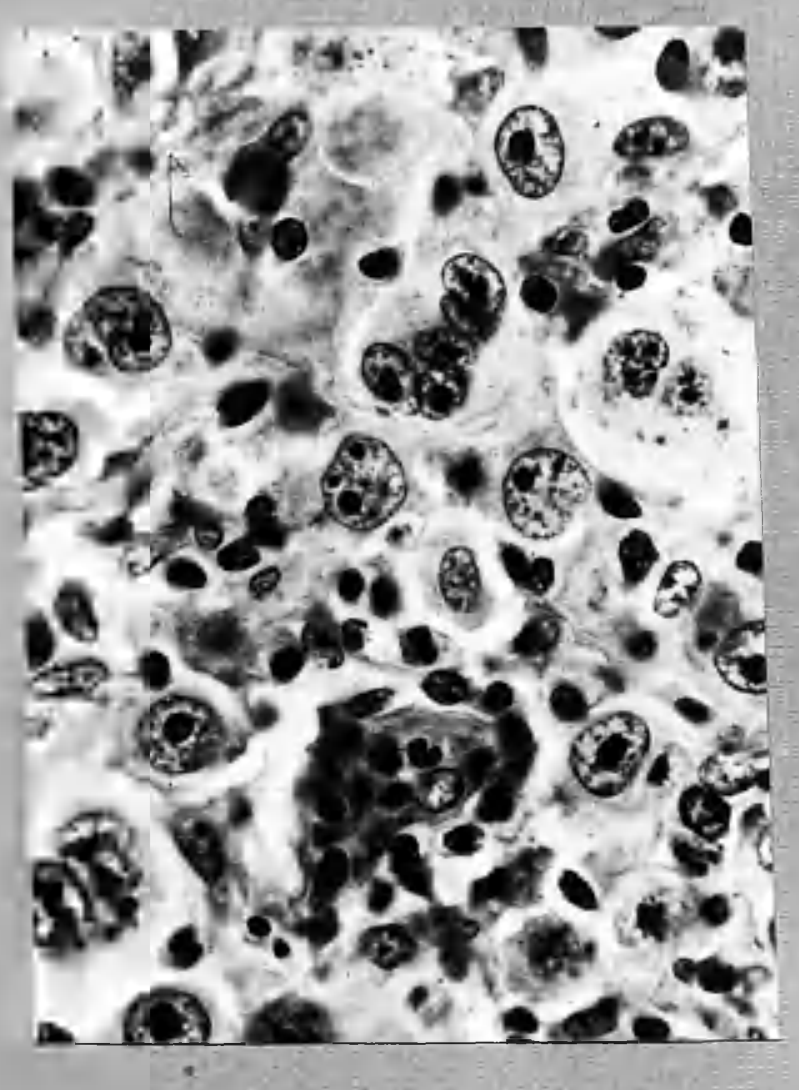

Fig.78 Jerial 4.4. Pecuitar Hodekin's diseuse. Hilgh power view of component cells. Ifei X 600 . 

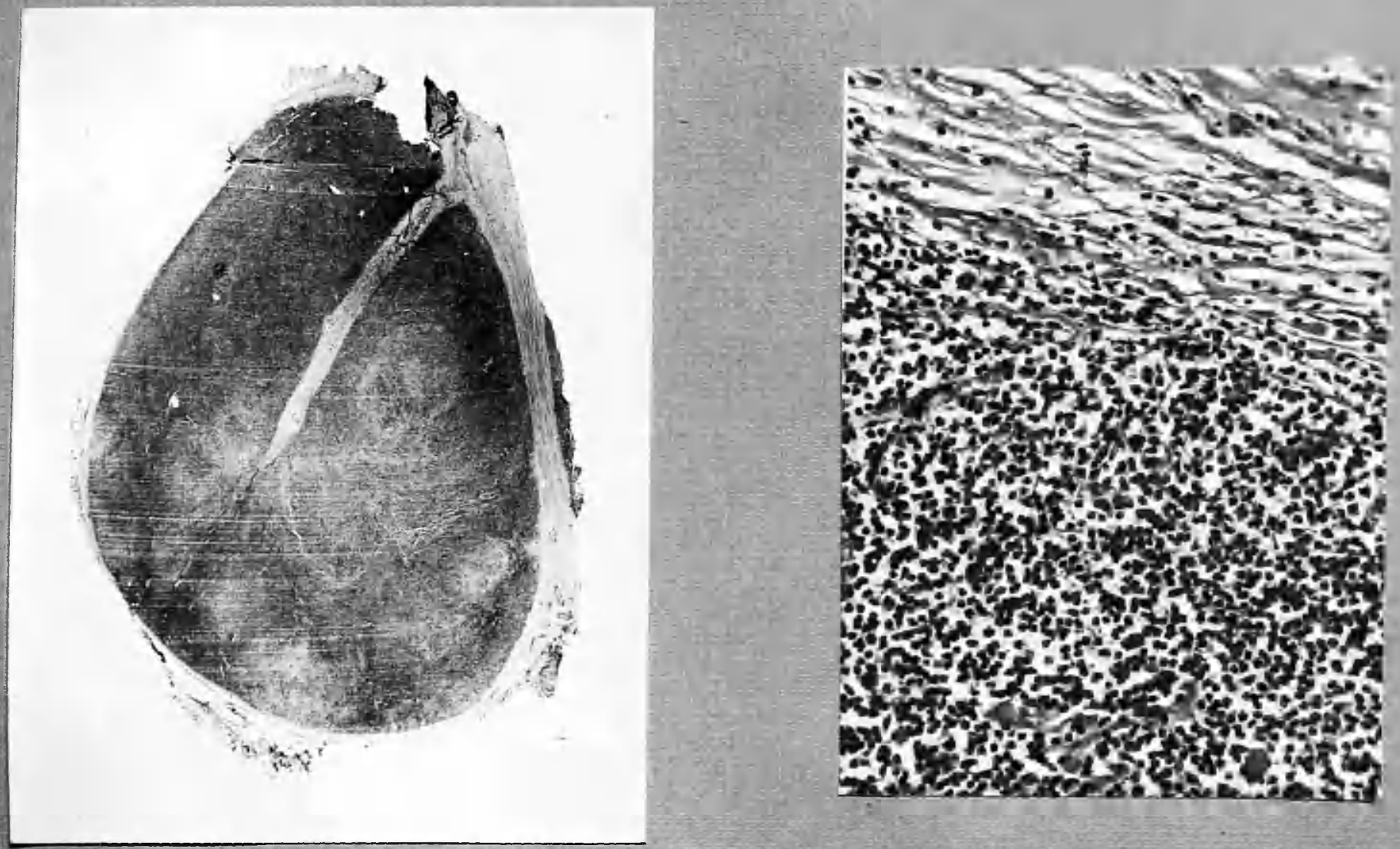

Fig.79 Jerial 2. Benign Hoagkin's disease. ? Pibrosis of capsule and trabecula, faintly follicuioia.

Fis.80 Serial 2. Hich poner of Pig.
79 at capule. Snill lympio-
cytes predominate but
several large cells are
present.
Heid $X 250$.



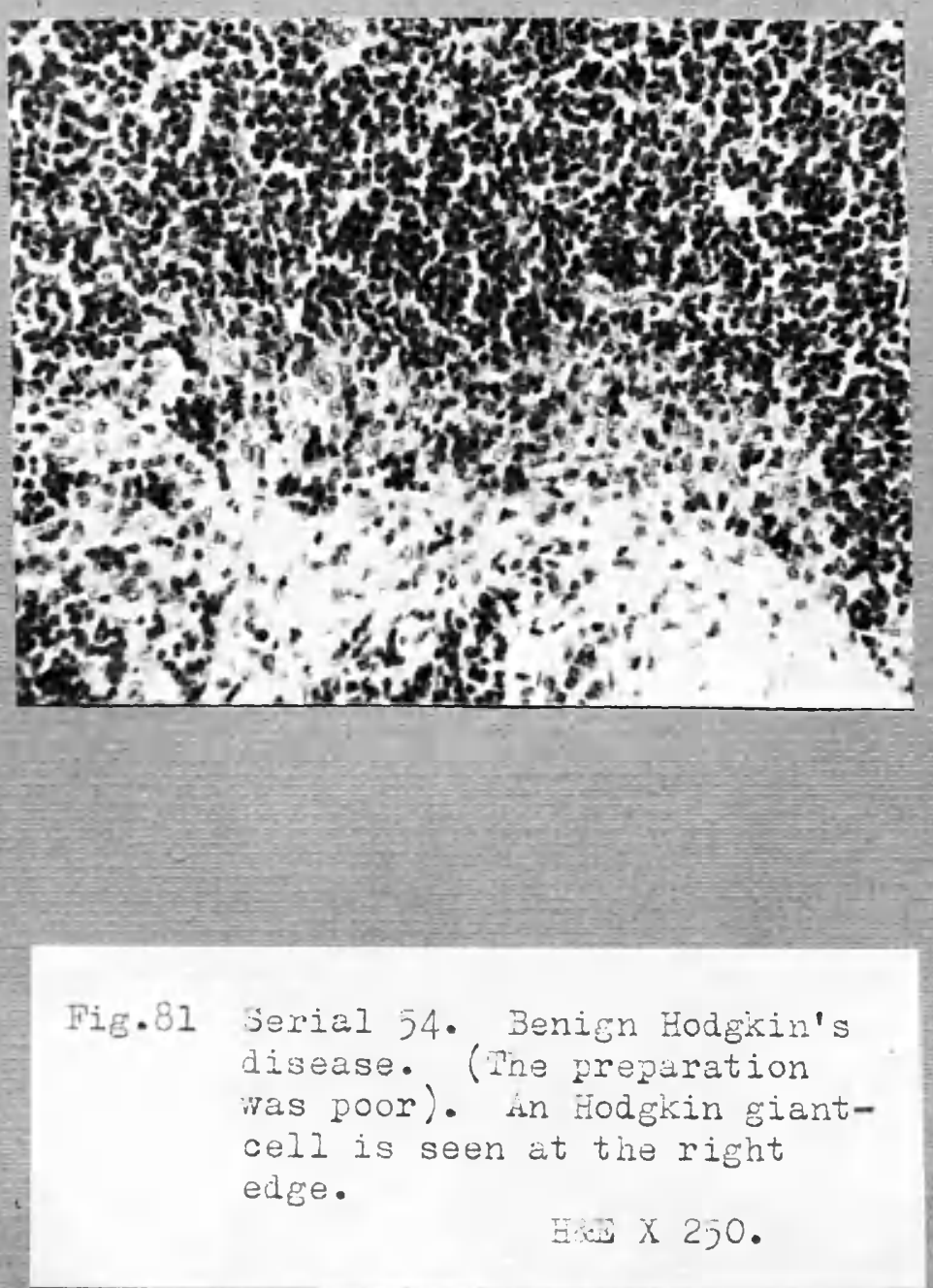

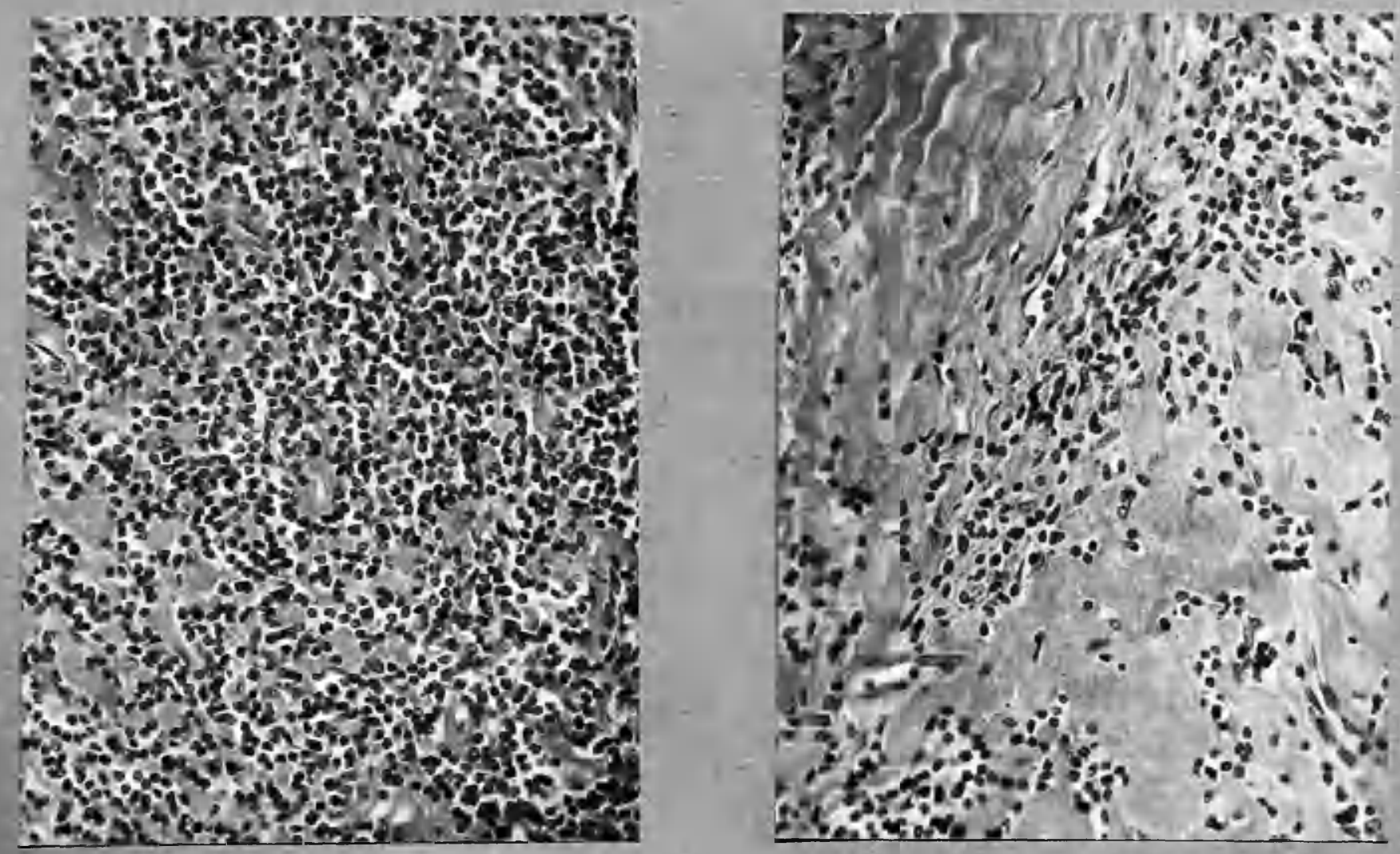

Figs. 82 and 83 .

Serial 23. A Iink lesion between ordinary and benign Hodgkin's disease. Fibrosis pronounced and small cells predorninate.

H3) X 250 

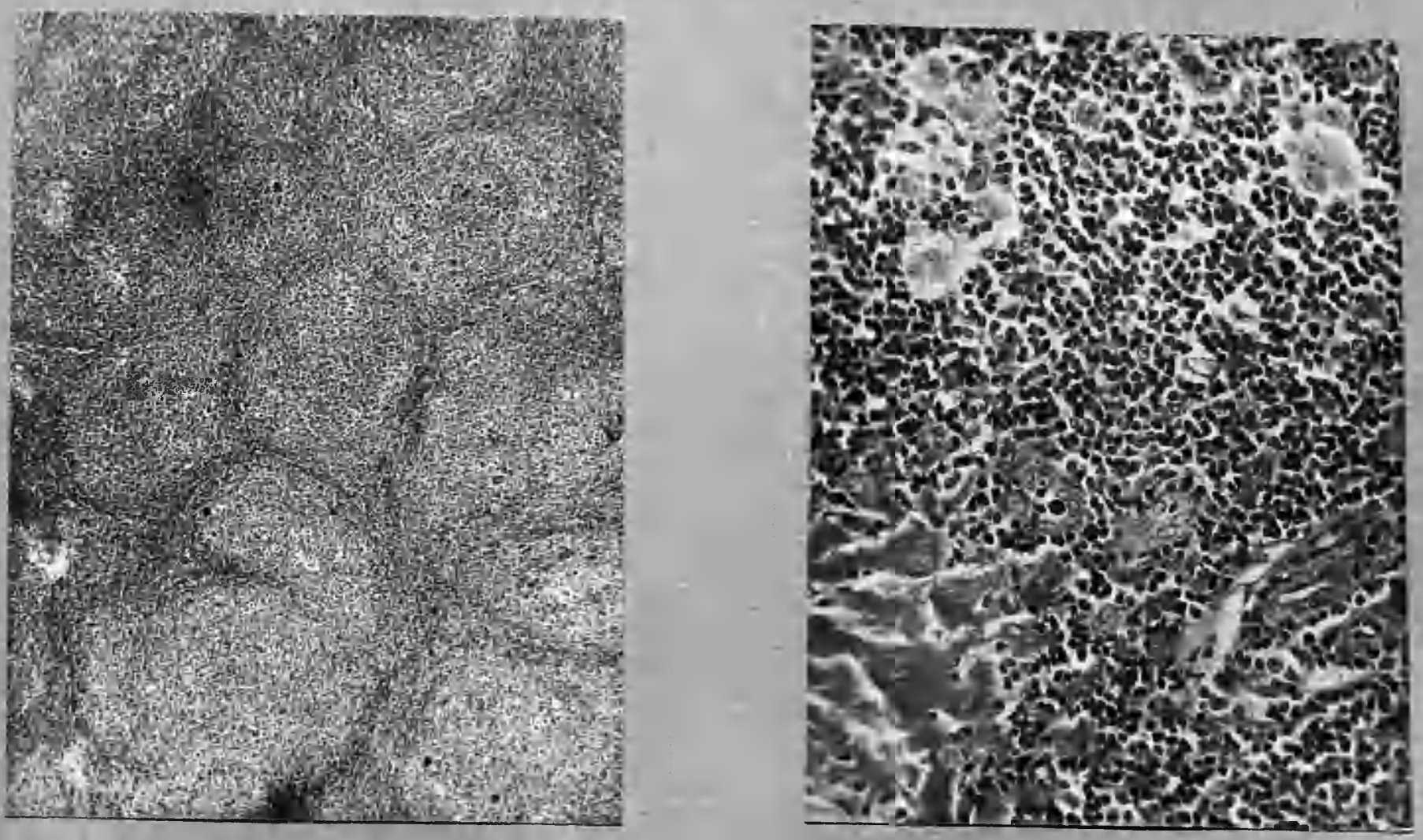

Fig.84 Hodgkin's disecuse with affinity to Brill's disease. Note follicular pattern with giant-cells in the false follicles.
His X 65.

Fig.85 High power view of same lesion as $\mathrm{Fig} \cdot 84$. It is very close to Fodgkin's disease. 1379:54. 


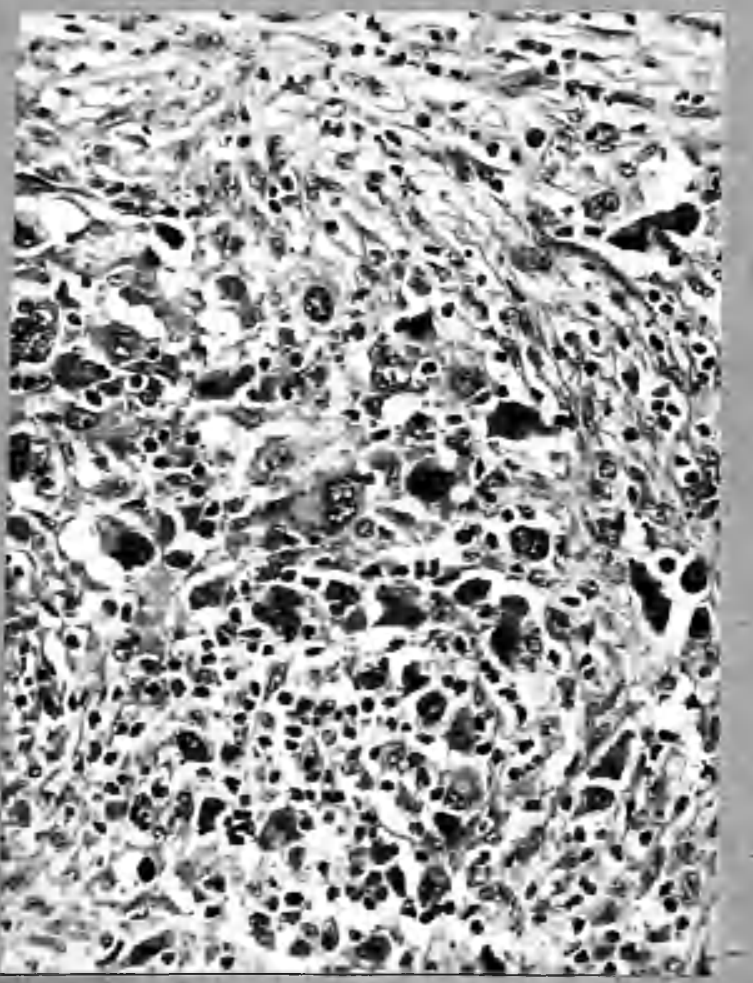

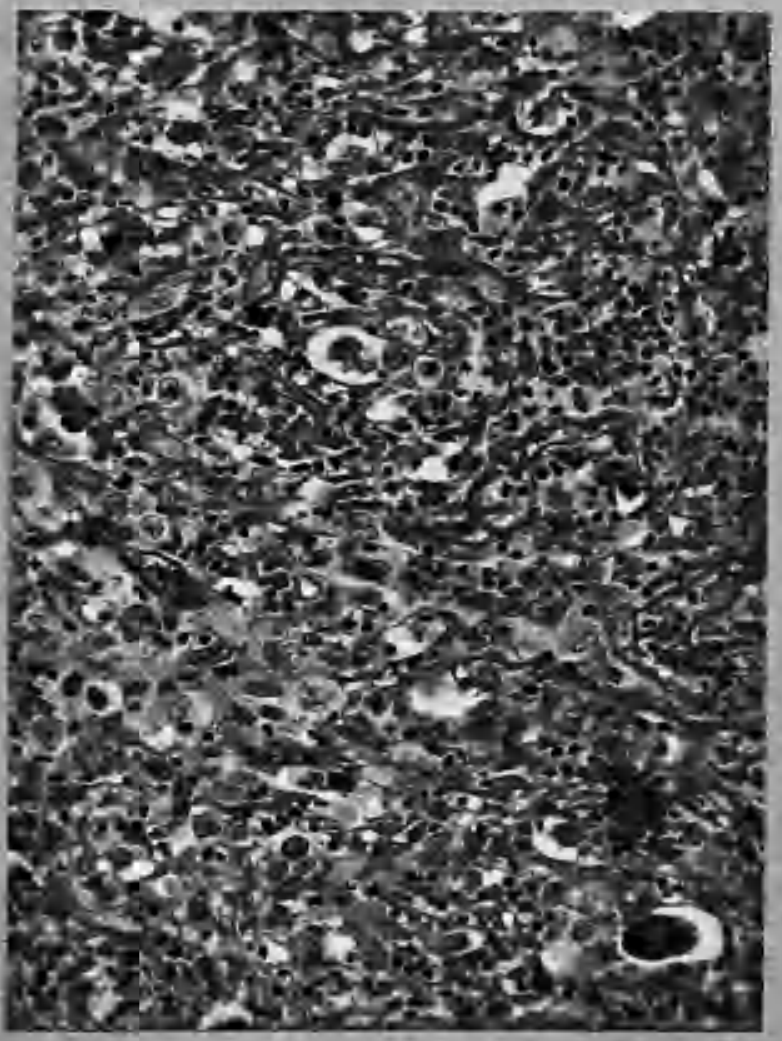

Fig.87 Serial 188. Hodgkin's surcoma (Biopsy). Losinophiles reduced but present, regarded as a transition stage. 


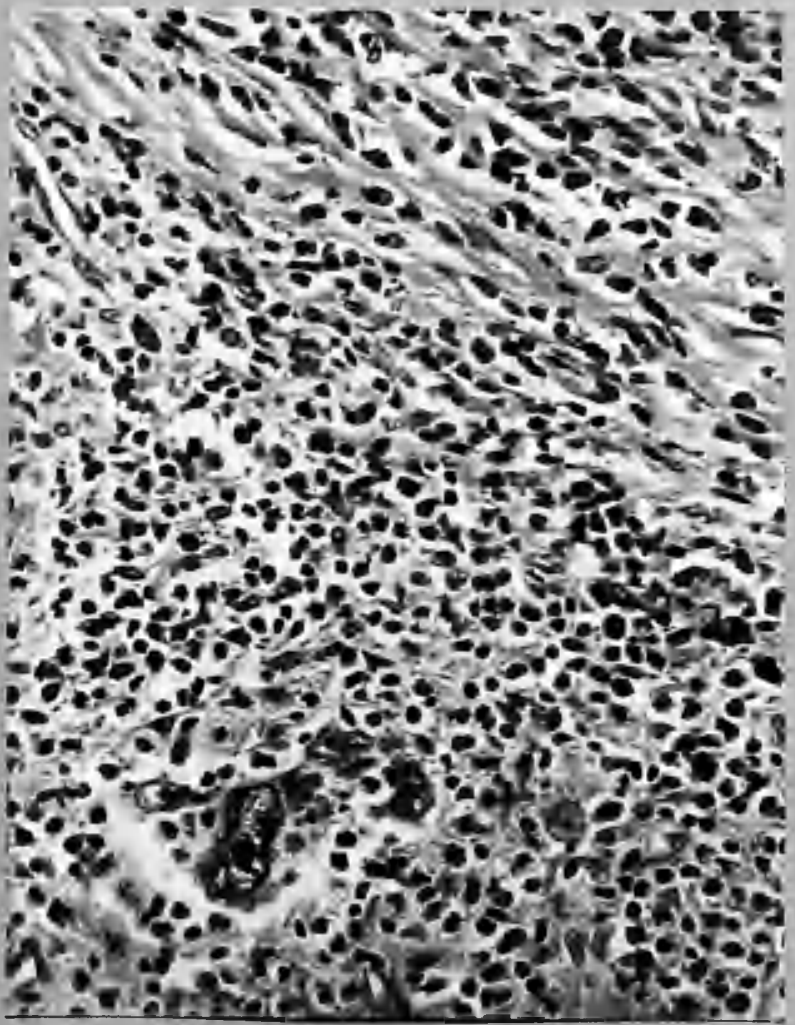

5.5.720.7\%

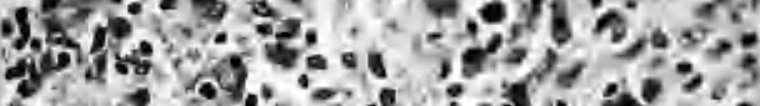

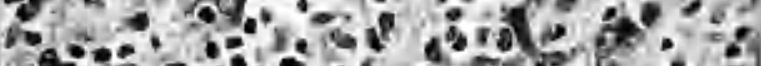

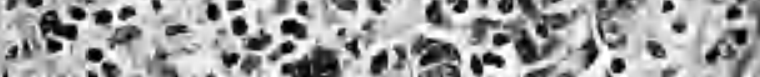
Ha 38 ?

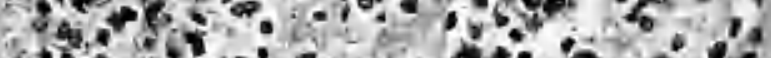

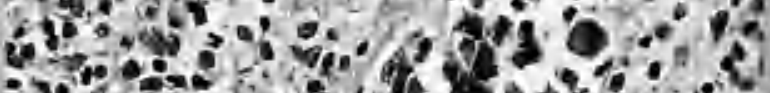

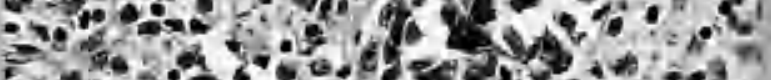
7.

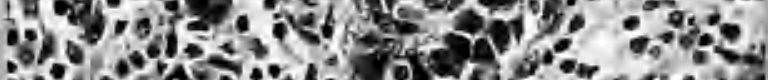

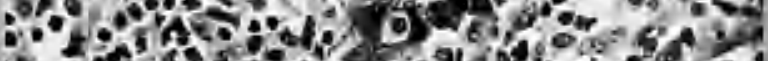

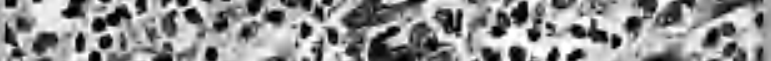

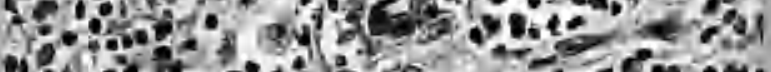

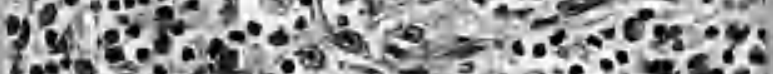

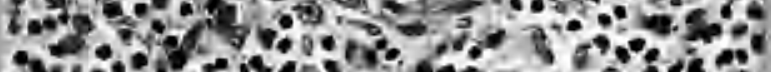

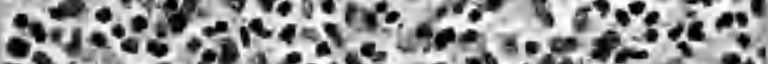

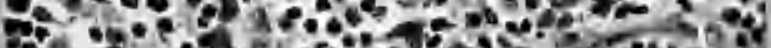

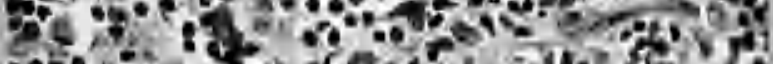

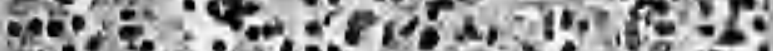

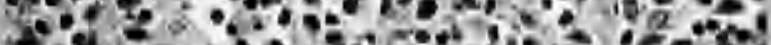

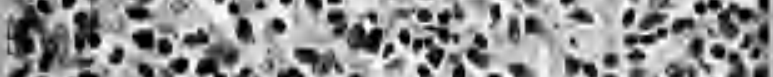

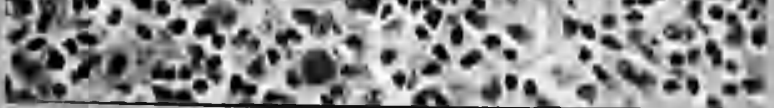

Fíg̈.88 Serial 222. Filse Hodgkin's disease. Difiuse metastatic cancer with eosinopirilia. Cancer giant-celis and diffuse fibrous reaction. H\&E X 250 .
Tig.89. Jerial 222. Palse Hodgkin's disease. Small islet of cancer cells which indicate the true nature of the lesion. Eic: $\times 250$. 

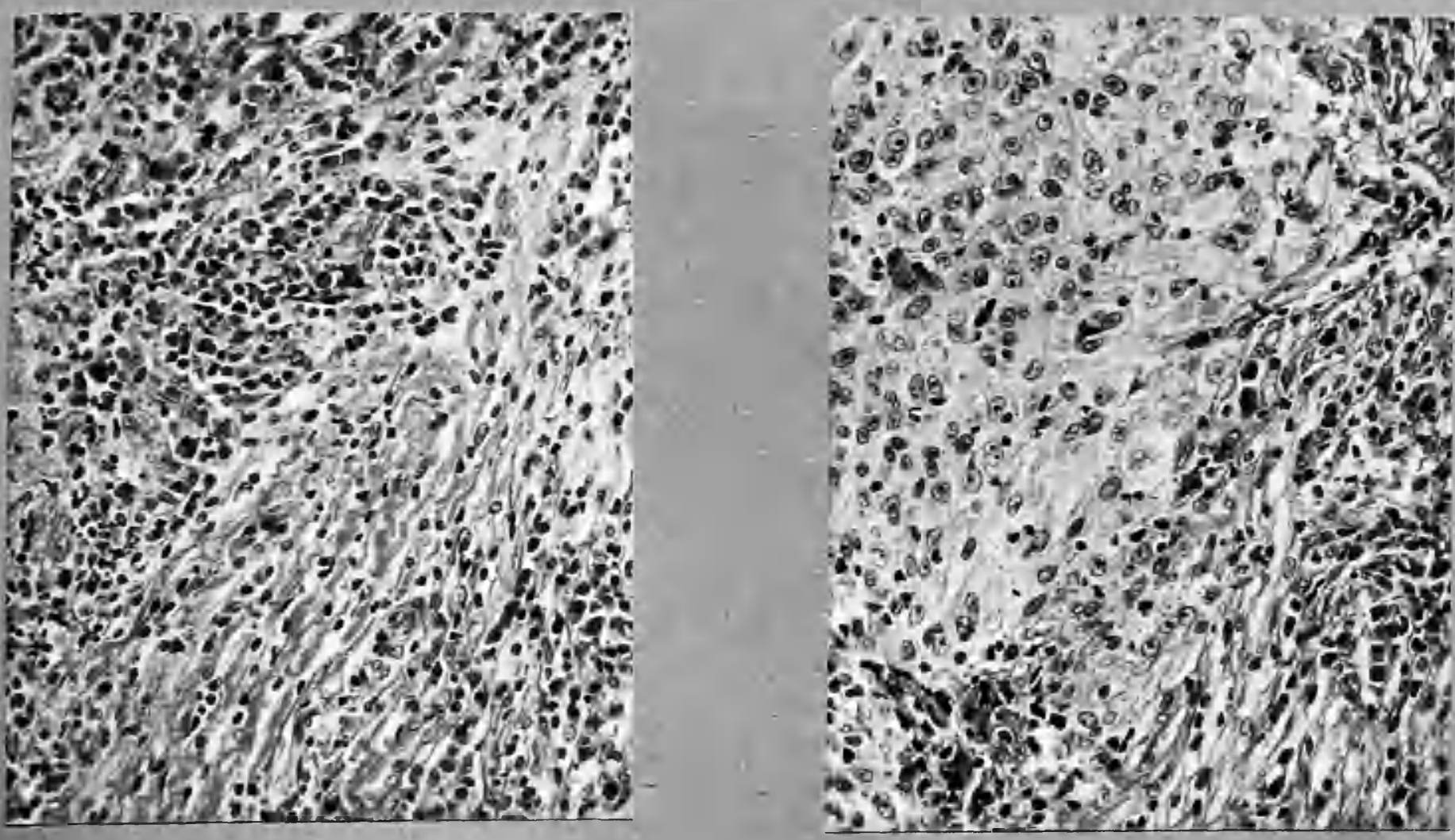

Fig.90 Serial 95. False Hodgkin's disease. Probable cancer metastasis. Diffuse fibrosis limited cellular pleiomorphisn. Hi⿱口龰己 $\times 250$.
Fig.91 Serial 95. False Hodgkin's disease. Plasmodial islet of epithelial cells which mimic morbid reticulum cells closely. 

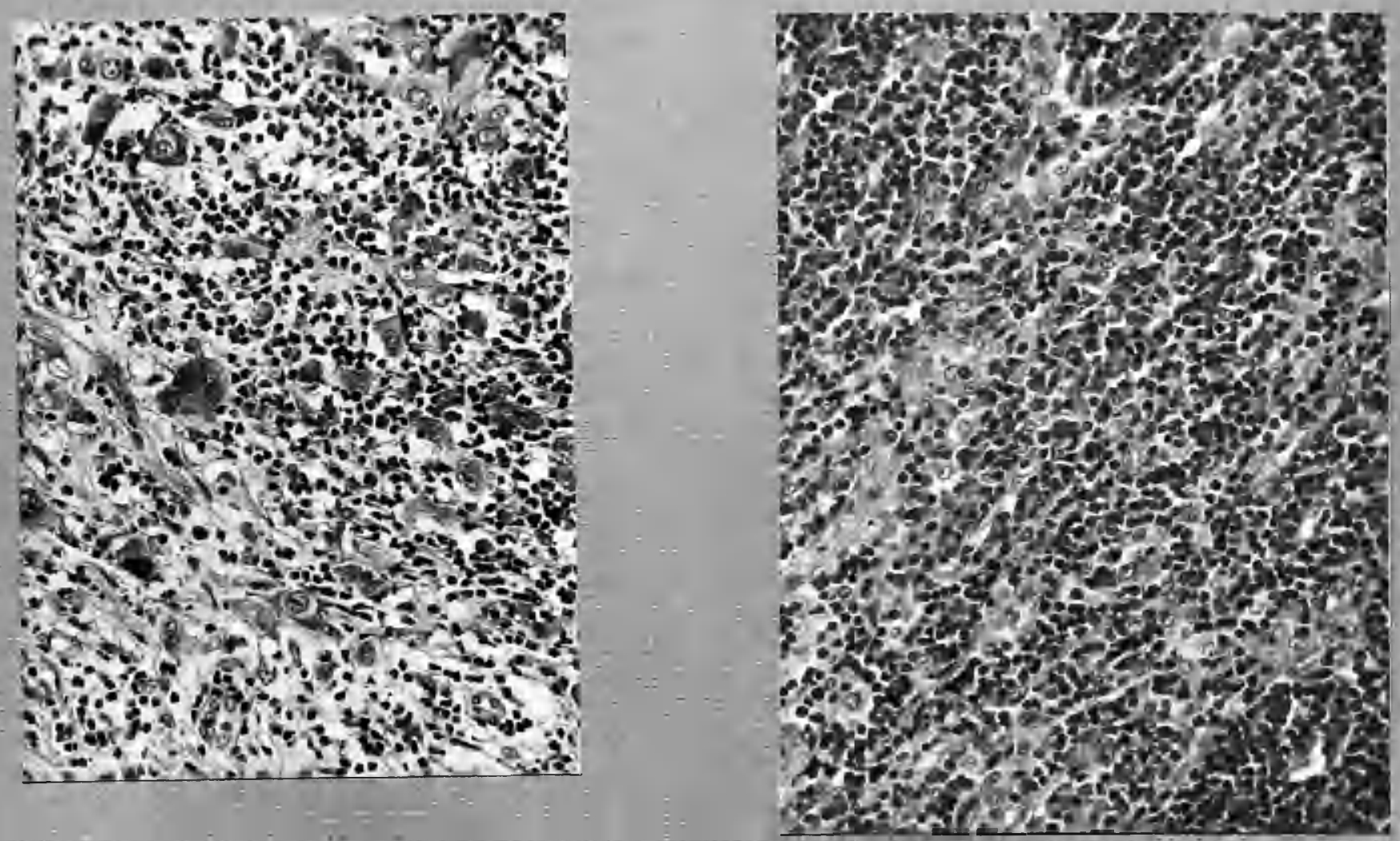

Fig.92 False Hodgkin's disease. Diffuse cancer metastases, from mamary primary (proved). $23: 53$. $\mathrm{H}$ \&政 X 230 .
Fig.93 Serial 86. False Hodgkin's disease. Probably reactive change, diffuse reticulum cell proliferation. Still benign at 3 years. H\&E X 250. 


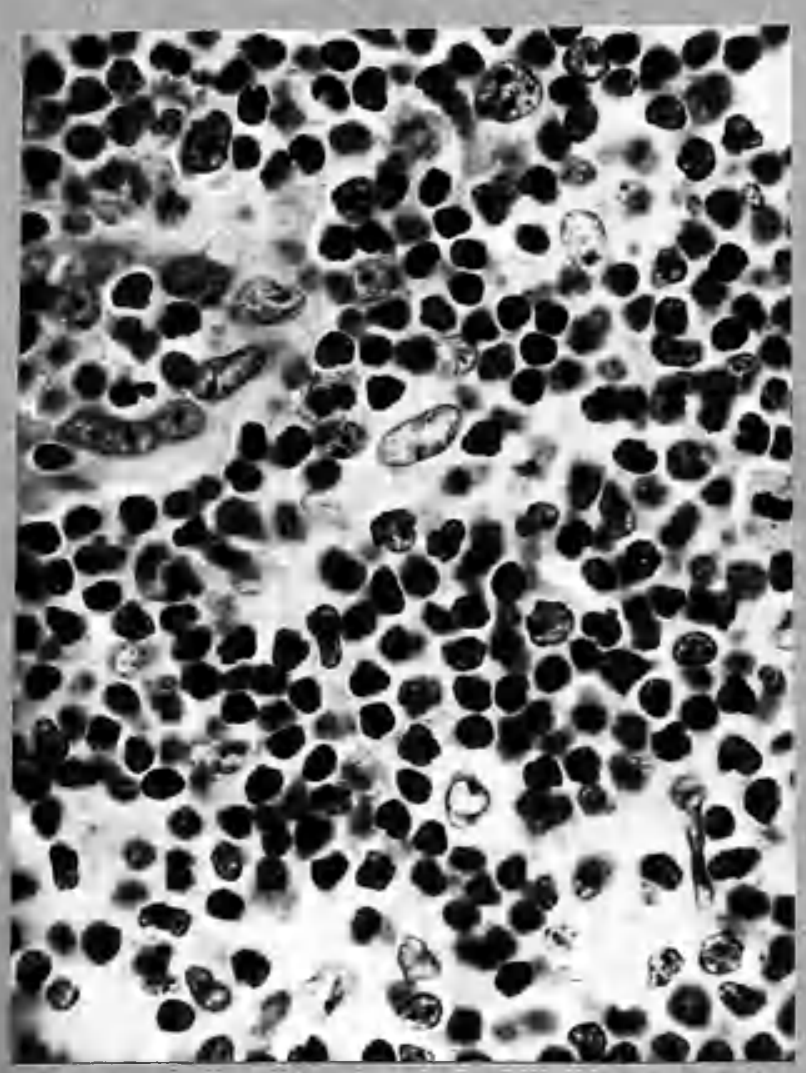

Fig.94 Serial 86. High power view. Note that proliferated reticulum cells are small, even thoueh nucleoli are common.

H8w X 600 .

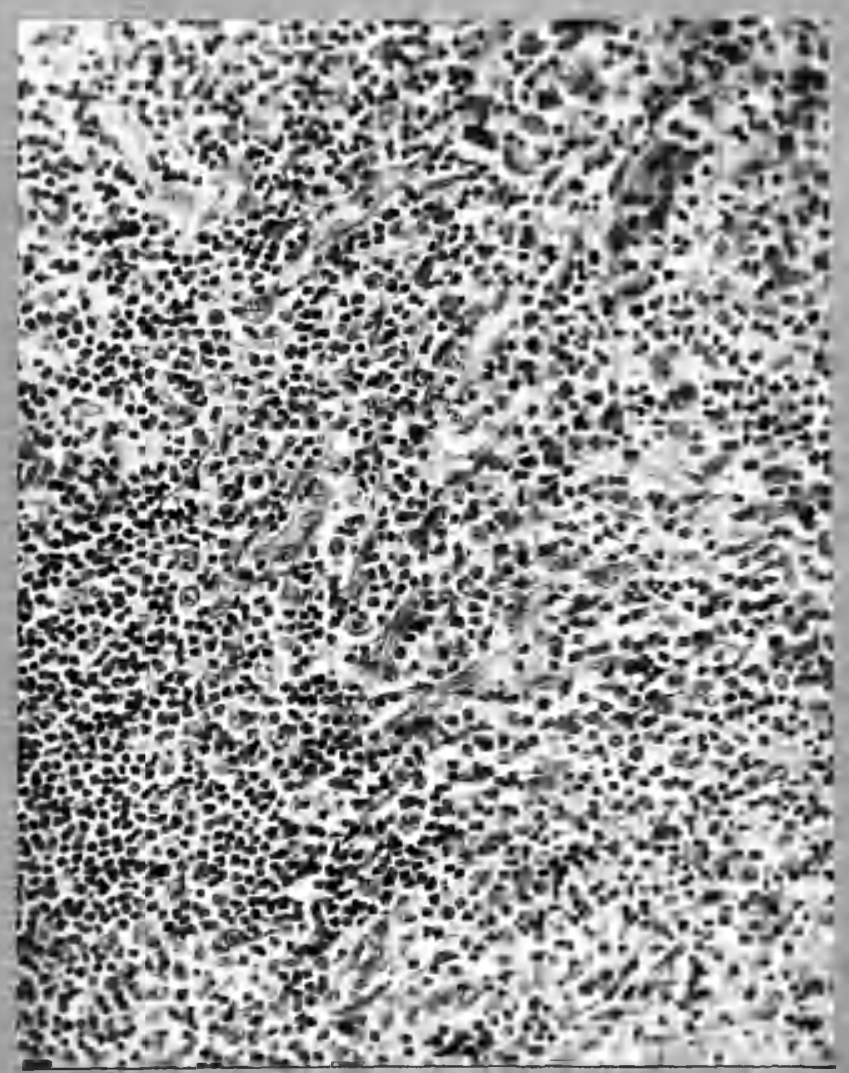

Fig.95 False Hodgkin's disease.
Proven tuberculosis of lymph
node. The diffuse epitheli-
oid cells minic the lesion
closely, but are small.
H\&I X 200.




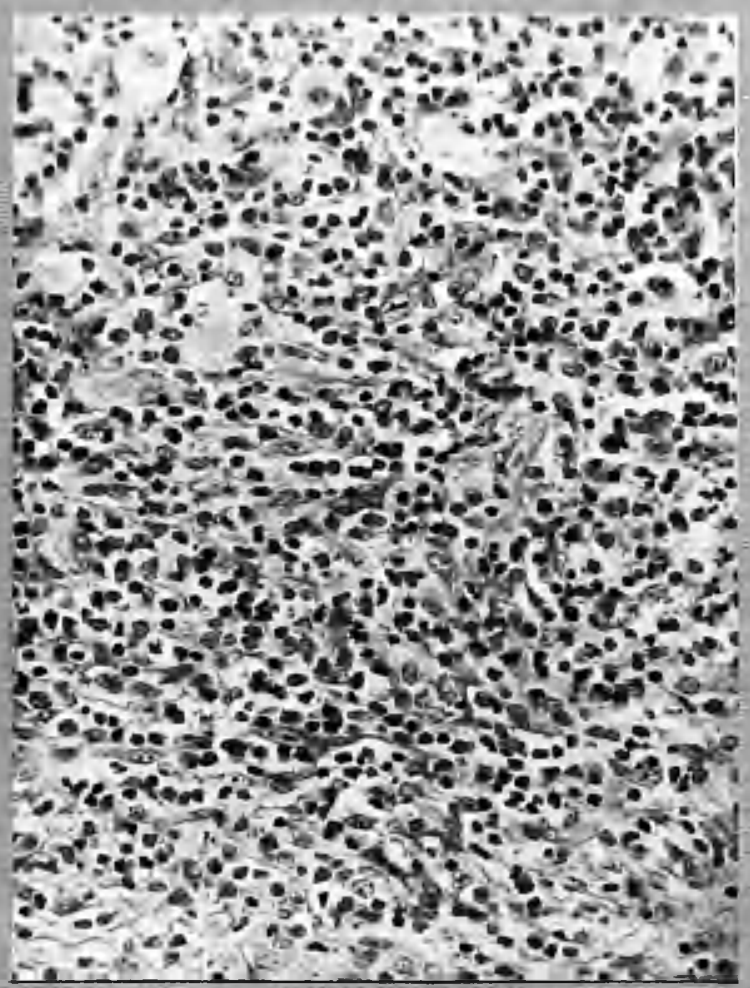

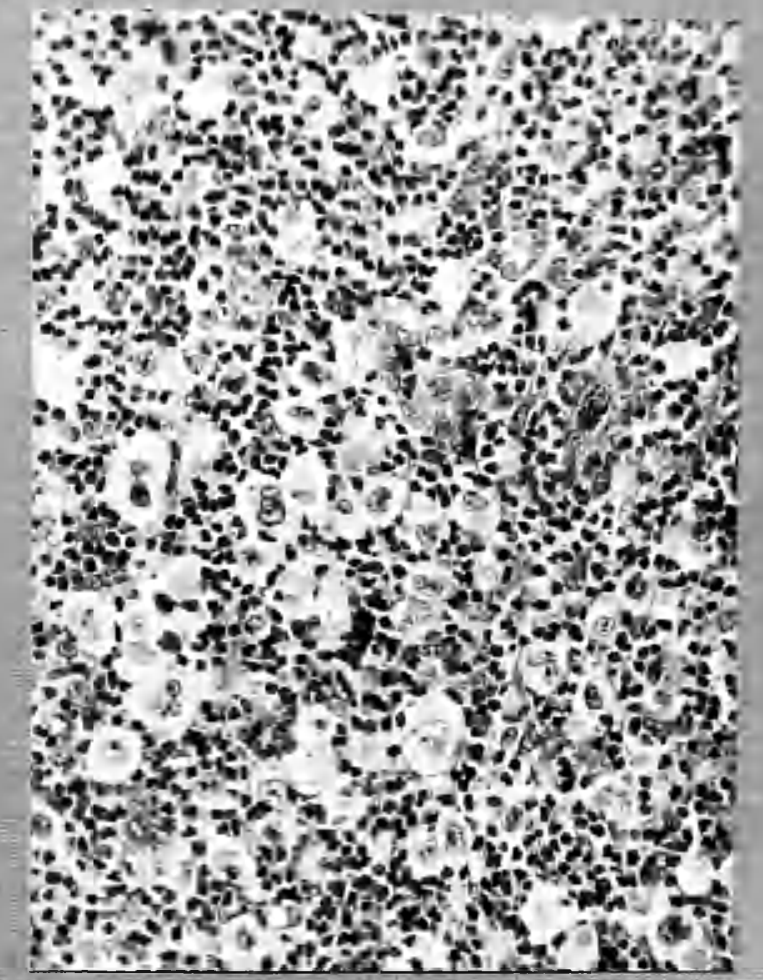

Fig.97 Serial 211. Area 'B'. Hodgkin's sarcoma. Large morbid reticulum cells very numerous. Fig.96. H\&E X 250 . 


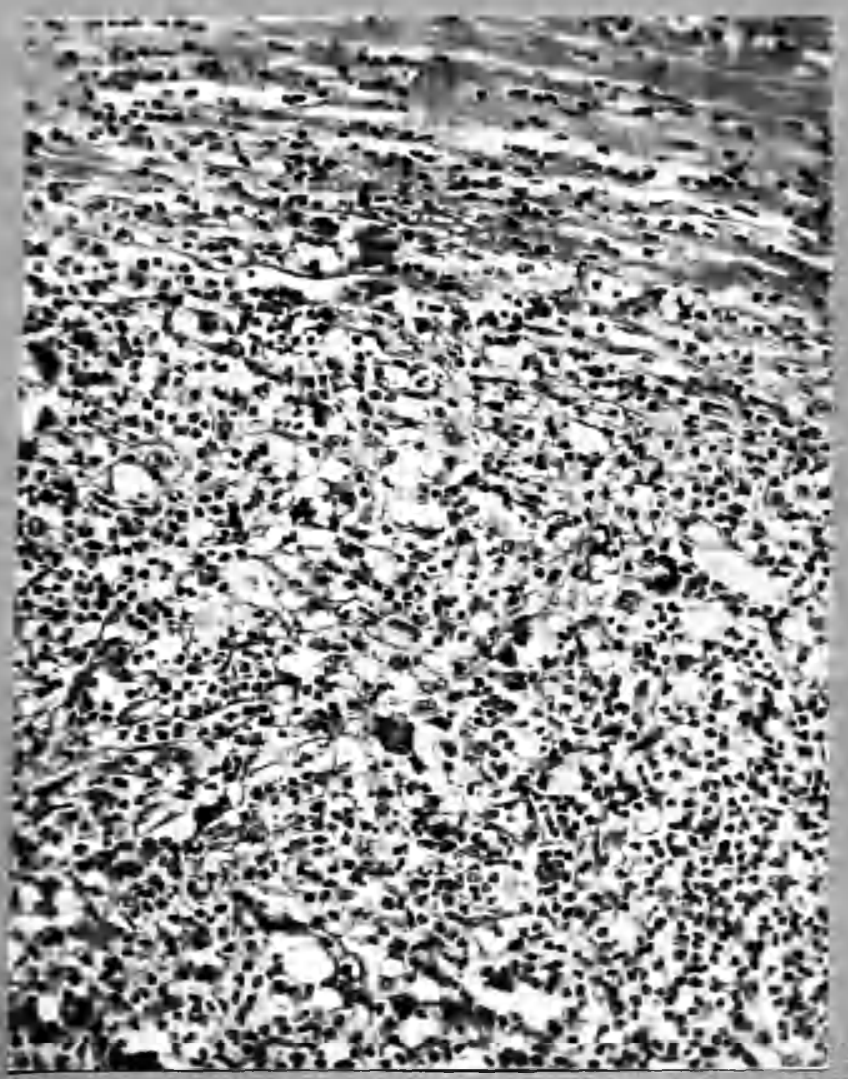

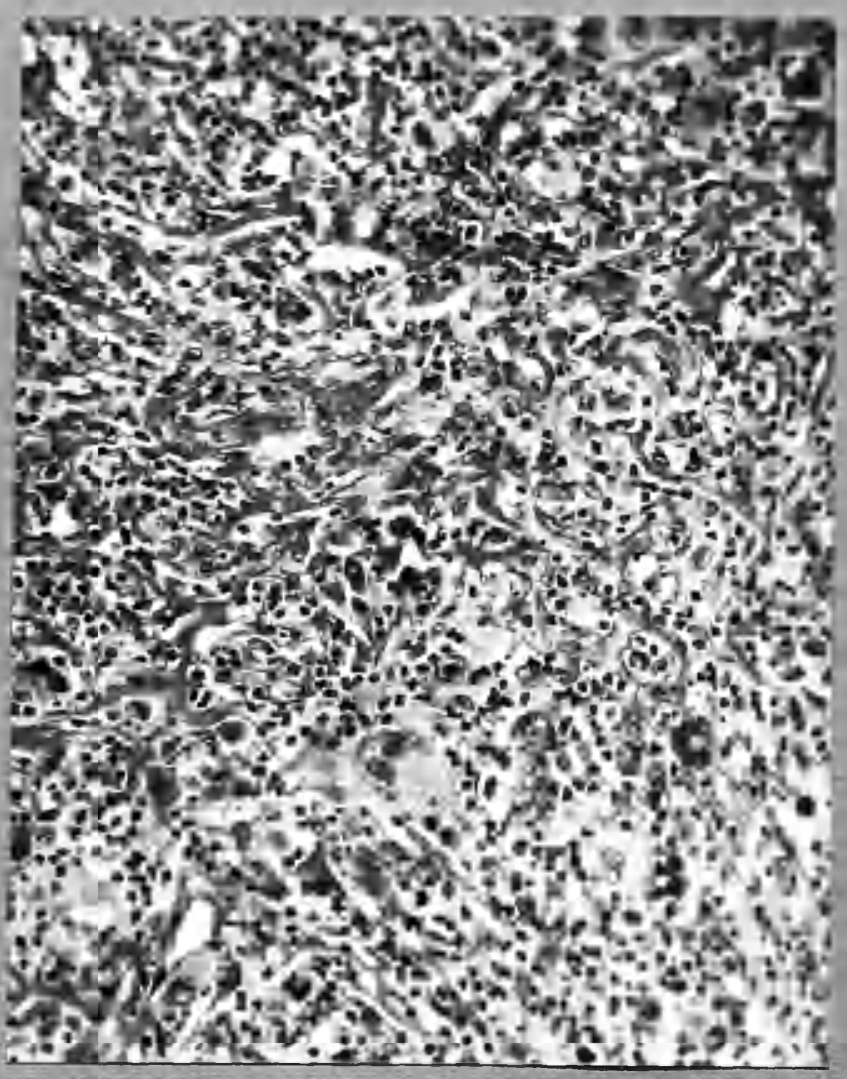

Fig.98 Serial 188. Area 'A' Hodgkin's disease. Fairly typical.
Fig.99 Serial 188. Area 'B'

Transition features between Fig.98 and Fig.100. H\&四 X 200. 
Fig. Serial 188. wrea 'C'. 100 Hodgkin's sarcona.

Hoe X 200.
Fig. Serial 22. Mixed Iesion.

101 Ageing Hodgkin's disease area. H\&\& X 250. 


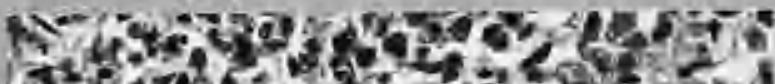
के

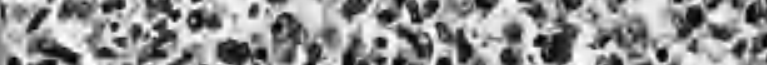

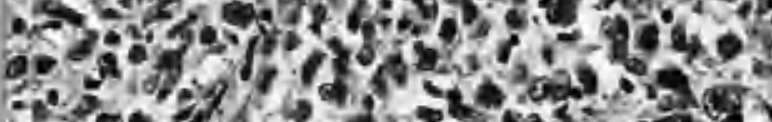

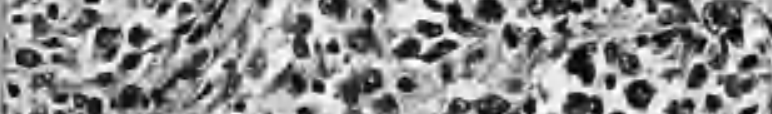

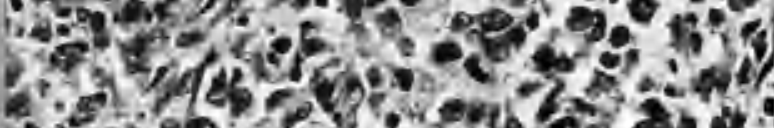

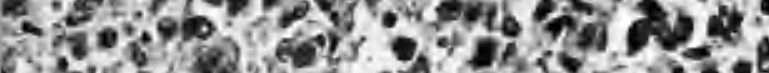

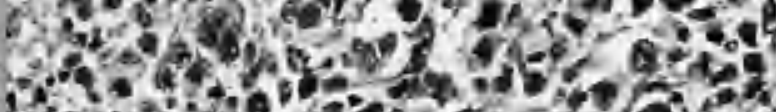

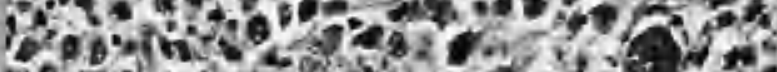

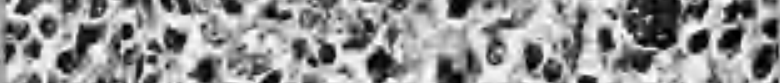

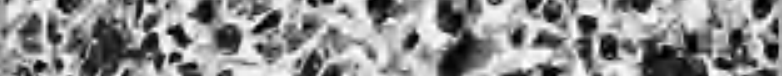

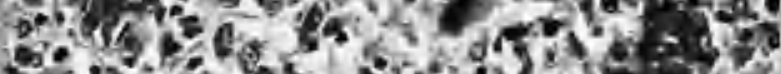
D. ${ }^{\circ}$.

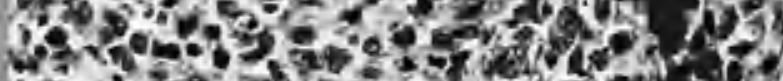

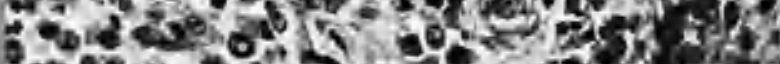

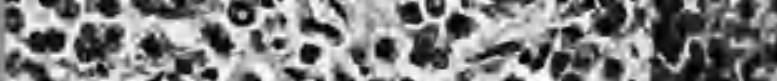

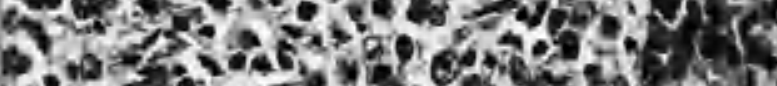

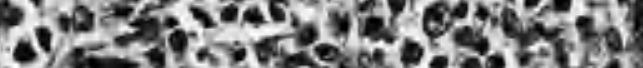

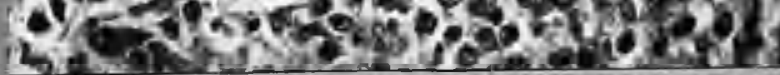

Fig. Serial 22. Lixed lesion.

102. Reticulum cell sarcoma area. How $\times 250$.

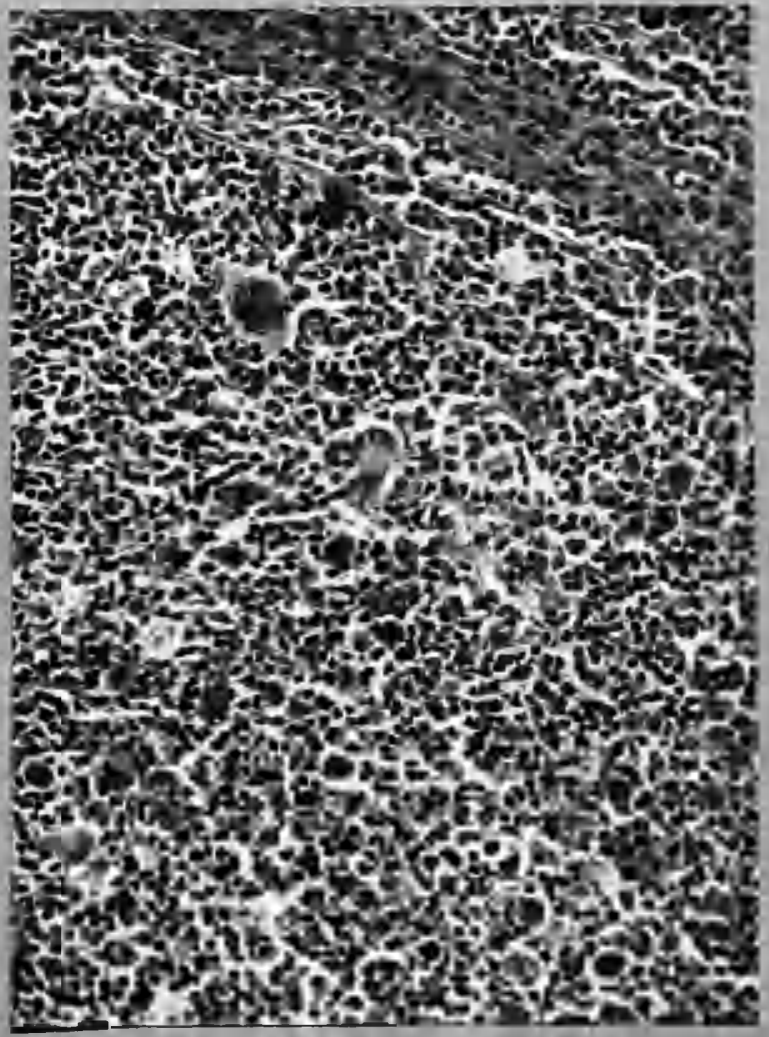

Fig.

Serial 191. Coextant Hodgkin's disease and reticulo- sarcoma in adjacent nodes, same biopsy. Hodgkin's disease node.

H\&C X 200. 
I:

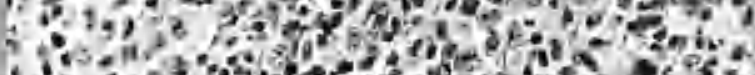
1,6 ;

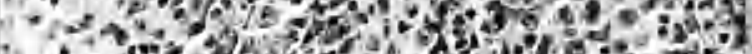
$\therefore$ a

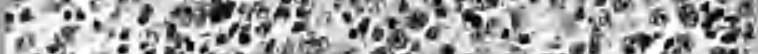

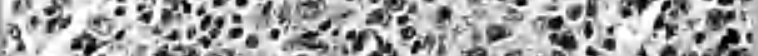

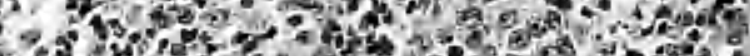

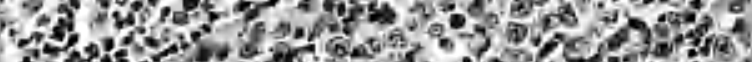

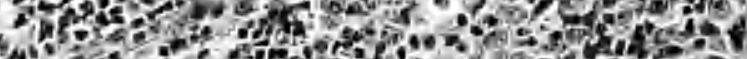

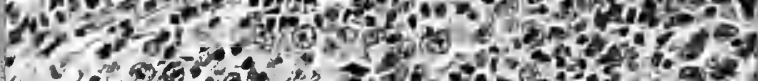

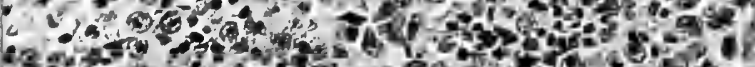
4.

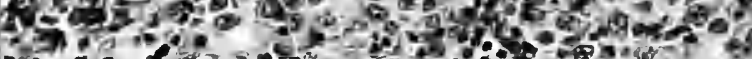

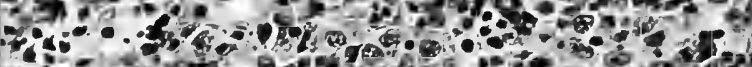
Mar

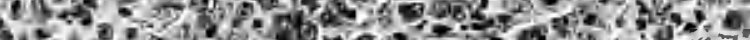

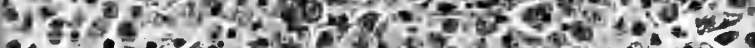

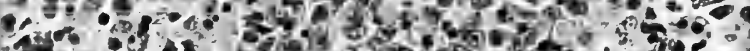

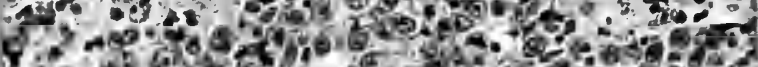

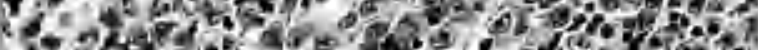

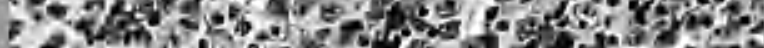
ser.

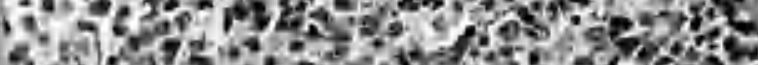

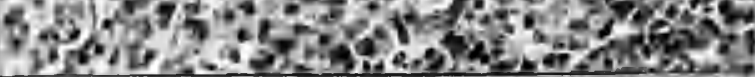

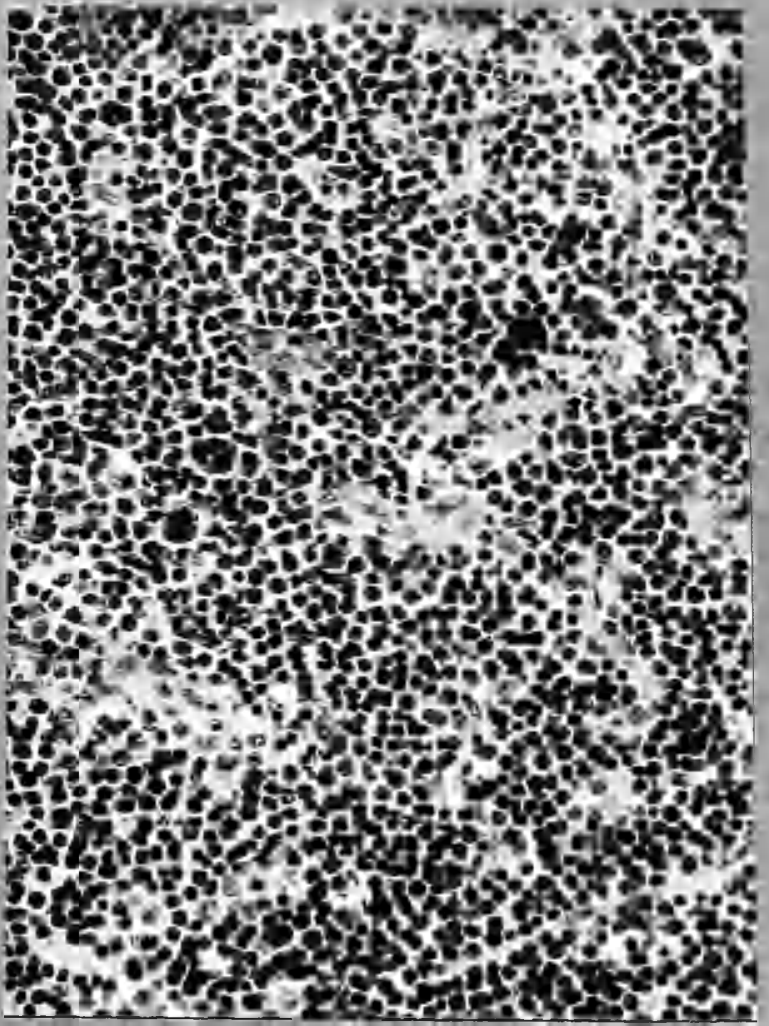

Fig. Serial 191. Reticulum cell

104 sarcoma node. (See also Fig. 103.

H\&E \& 200.

Fig. Serial 85. Curious indeter-

105 minate lesion. ? lymphoblastic Hodgkin's disease. Note very numerous large immature lymphocytes, reticulum cells and giant cells.

H程 X 200。 


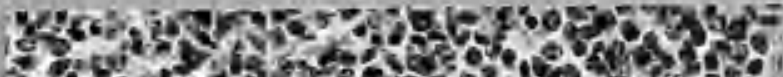

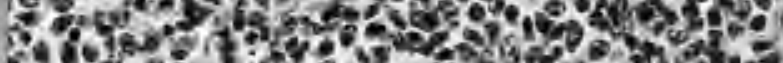

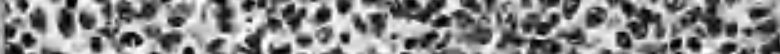

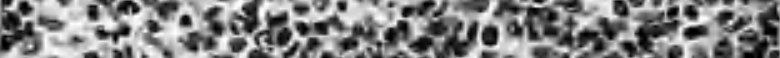

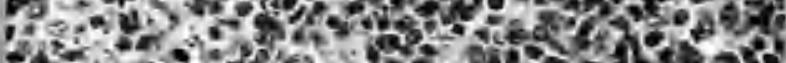

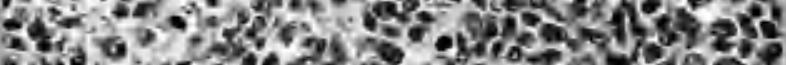

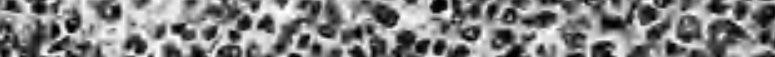

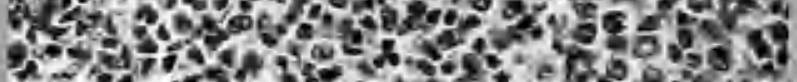

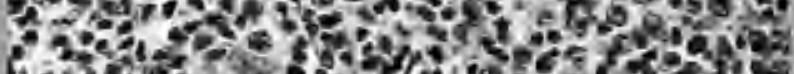

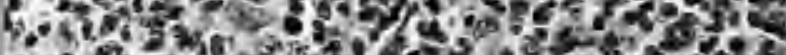

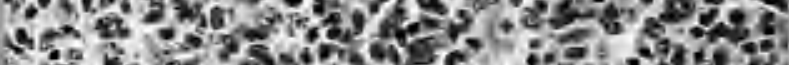

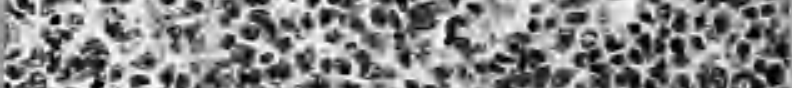

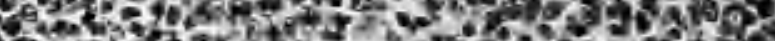

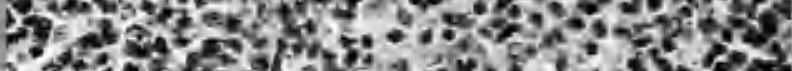

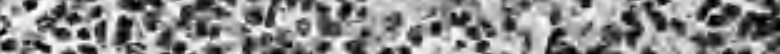

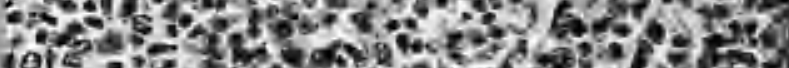

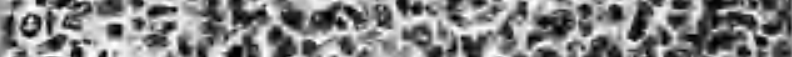

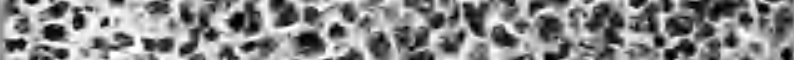

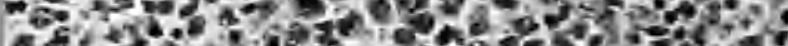

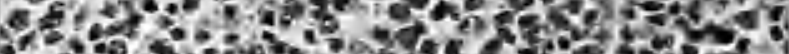

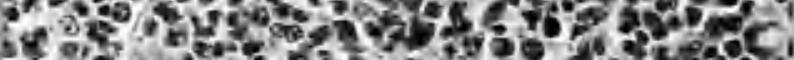

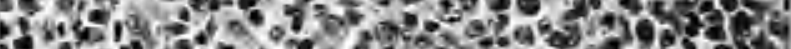

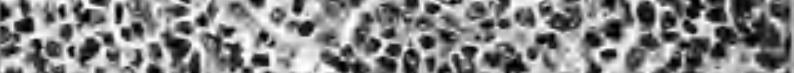

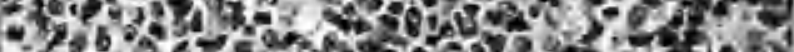

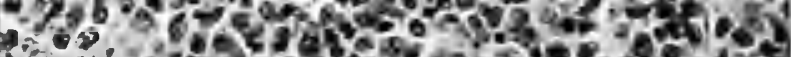

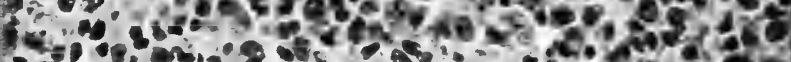

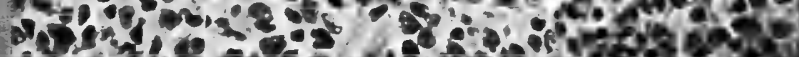
ond is co:

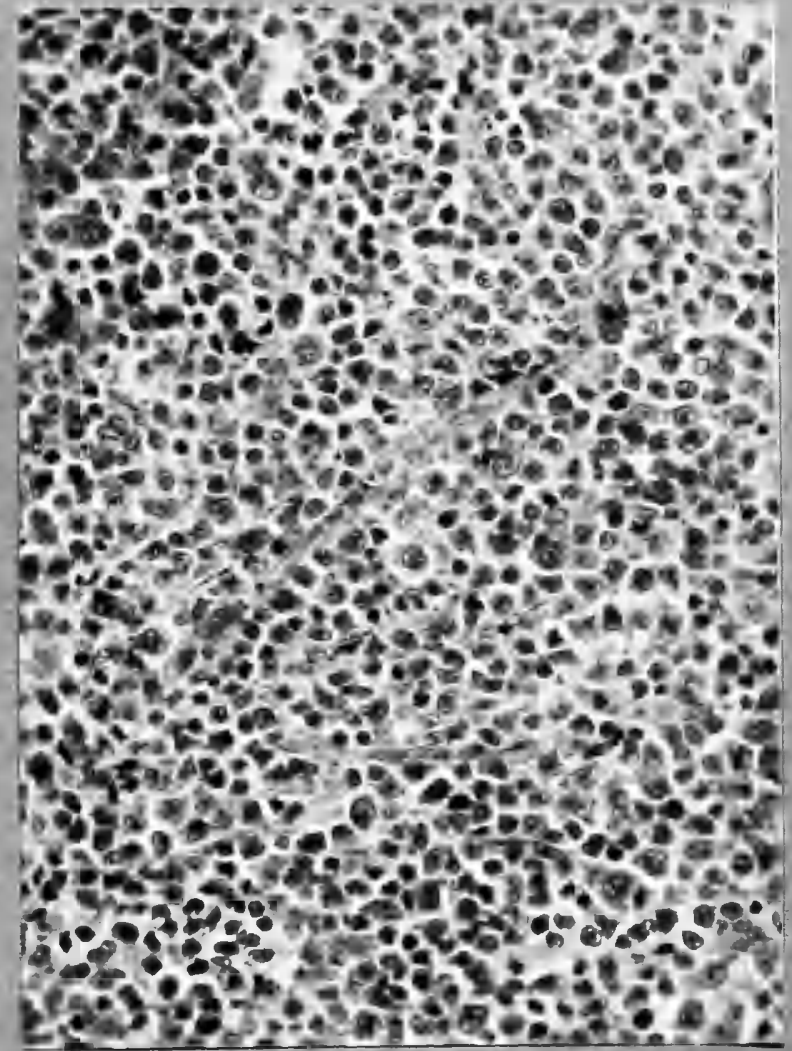

Fig. Serial 82. Reticulurn cell

107 sarcoma, stem-cell type. Fibrosis tenuous. Witoses numerous. 

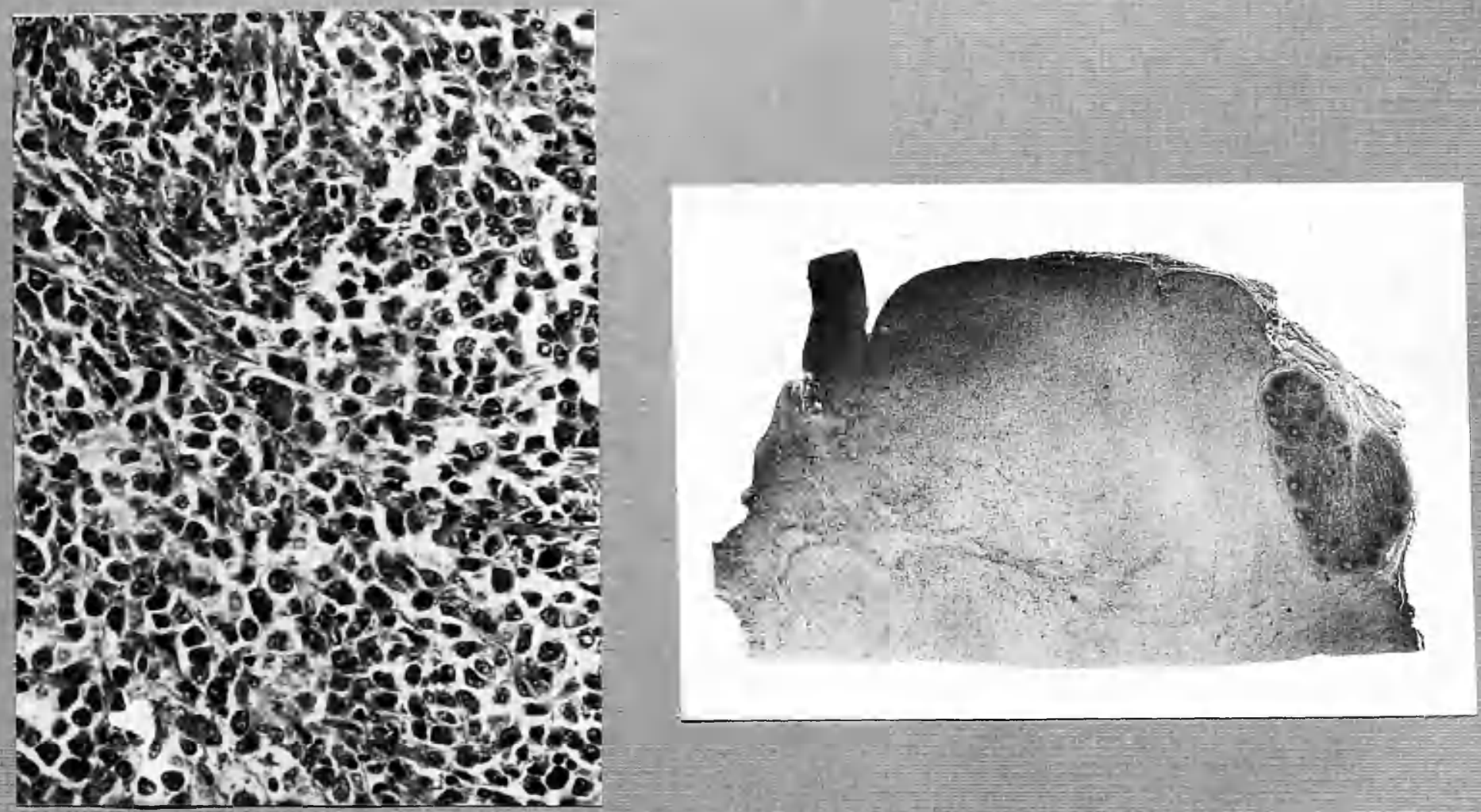

Fig. Serial 35. Reticulum cell

108 sarcoma. Very large morbid mononuclear reticulum cells, some multinucleate; and fibrosis.

H\&Q X 250.

Fig. Serial 12. Beticulum cell

109 sarcoma. Incluied normal lymph node in tine tumour. H'\& $\times 7$. 

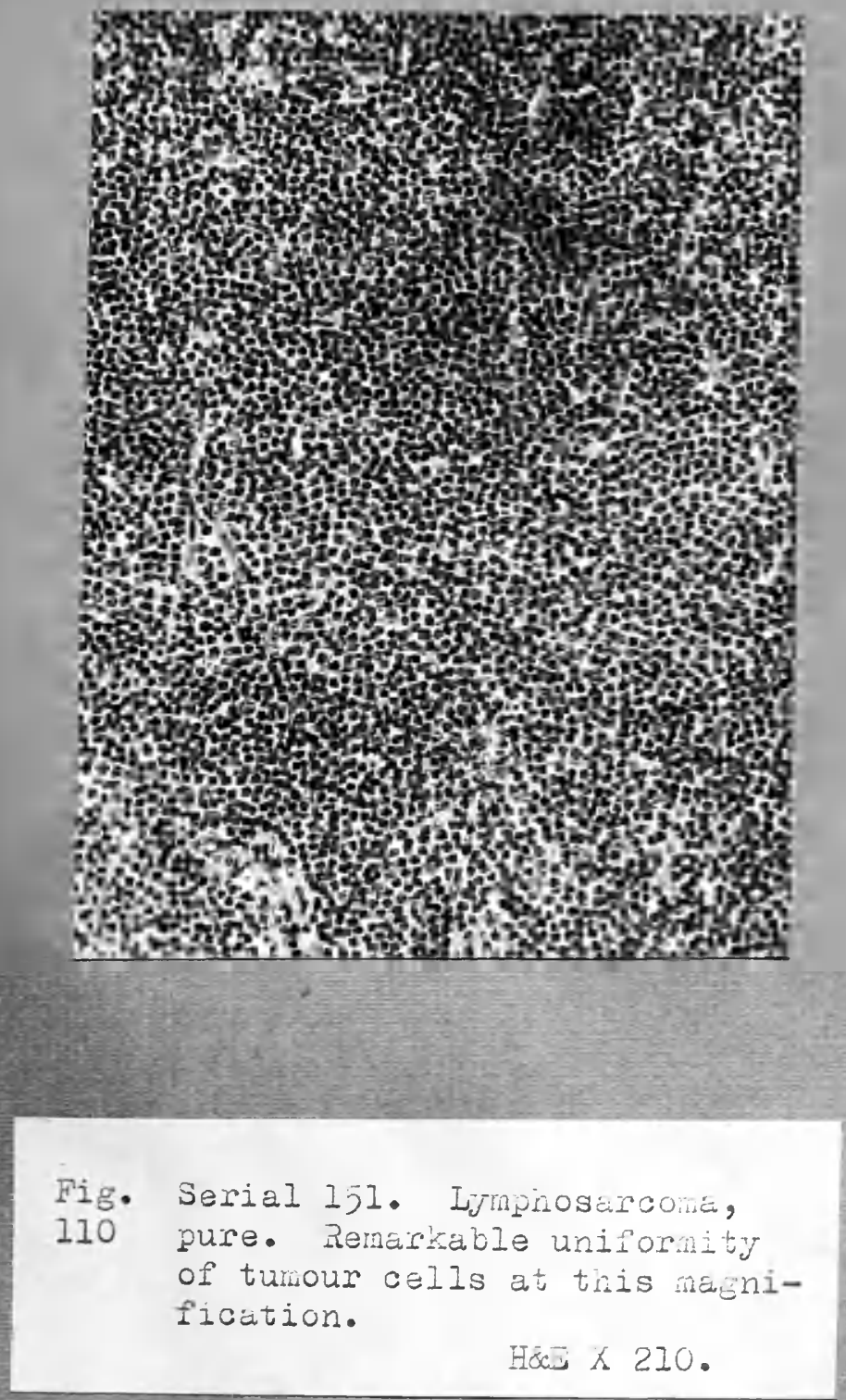
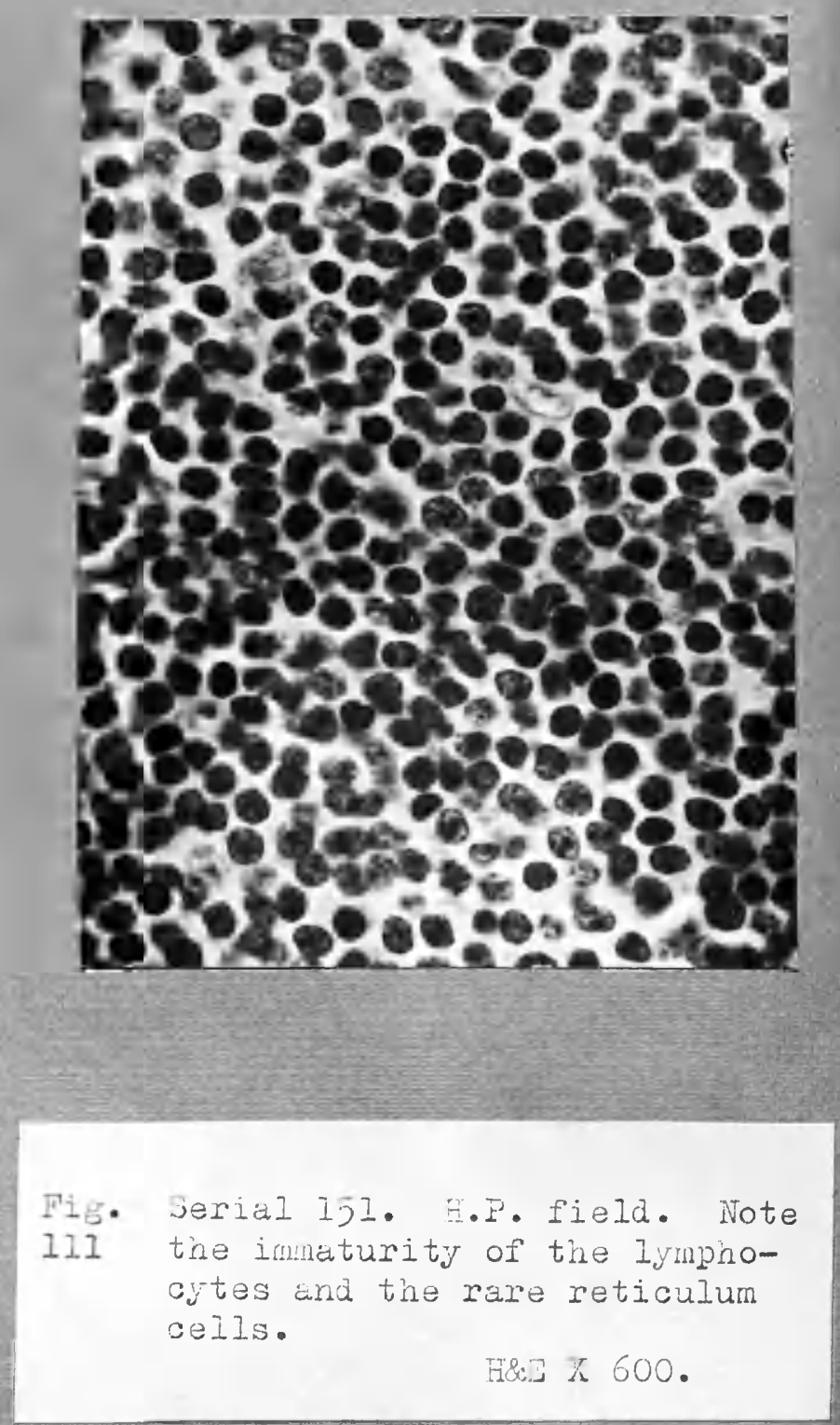

6.t.

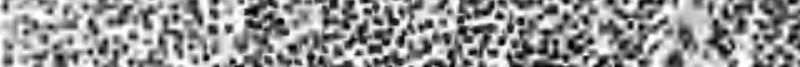

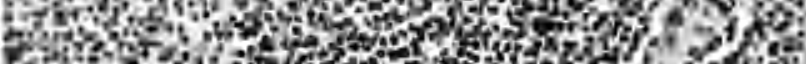

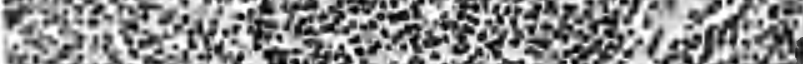

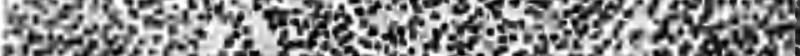

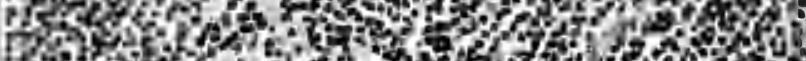

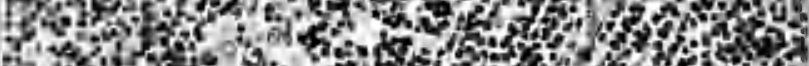

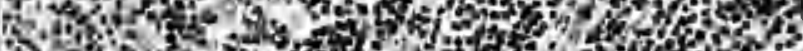

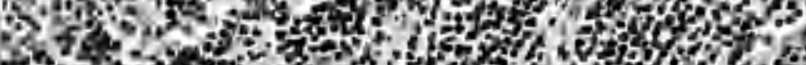

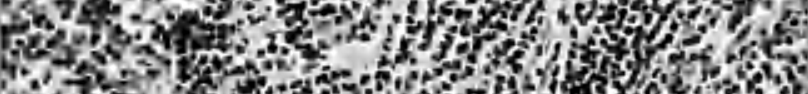

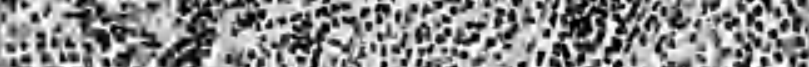
से

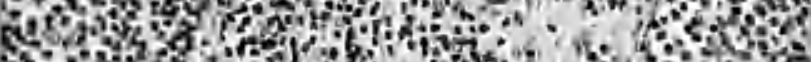

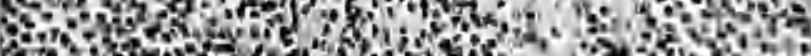

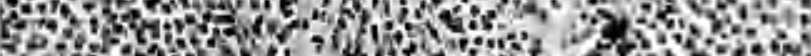

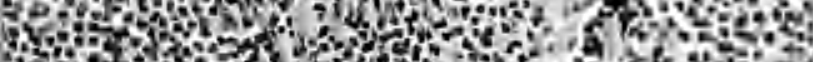

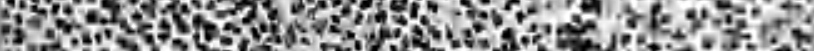

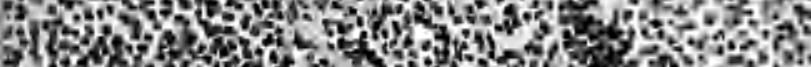

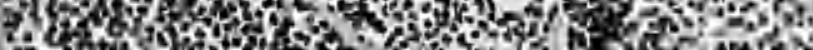

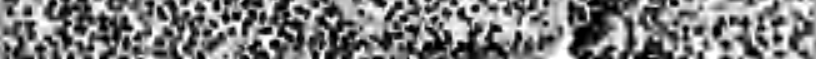

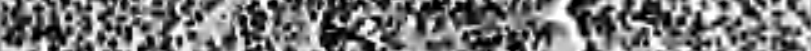

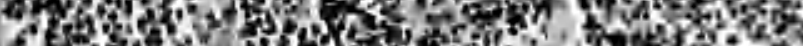

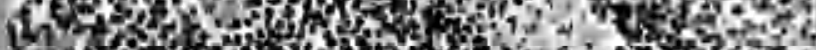

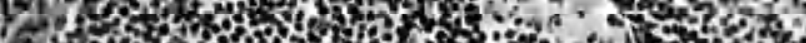
wow

Fig. Serial 192. Lymphosarcoma.

112 Rare norbid reticulum cells and Godgkin type of fibrosis. H\&E X 200.
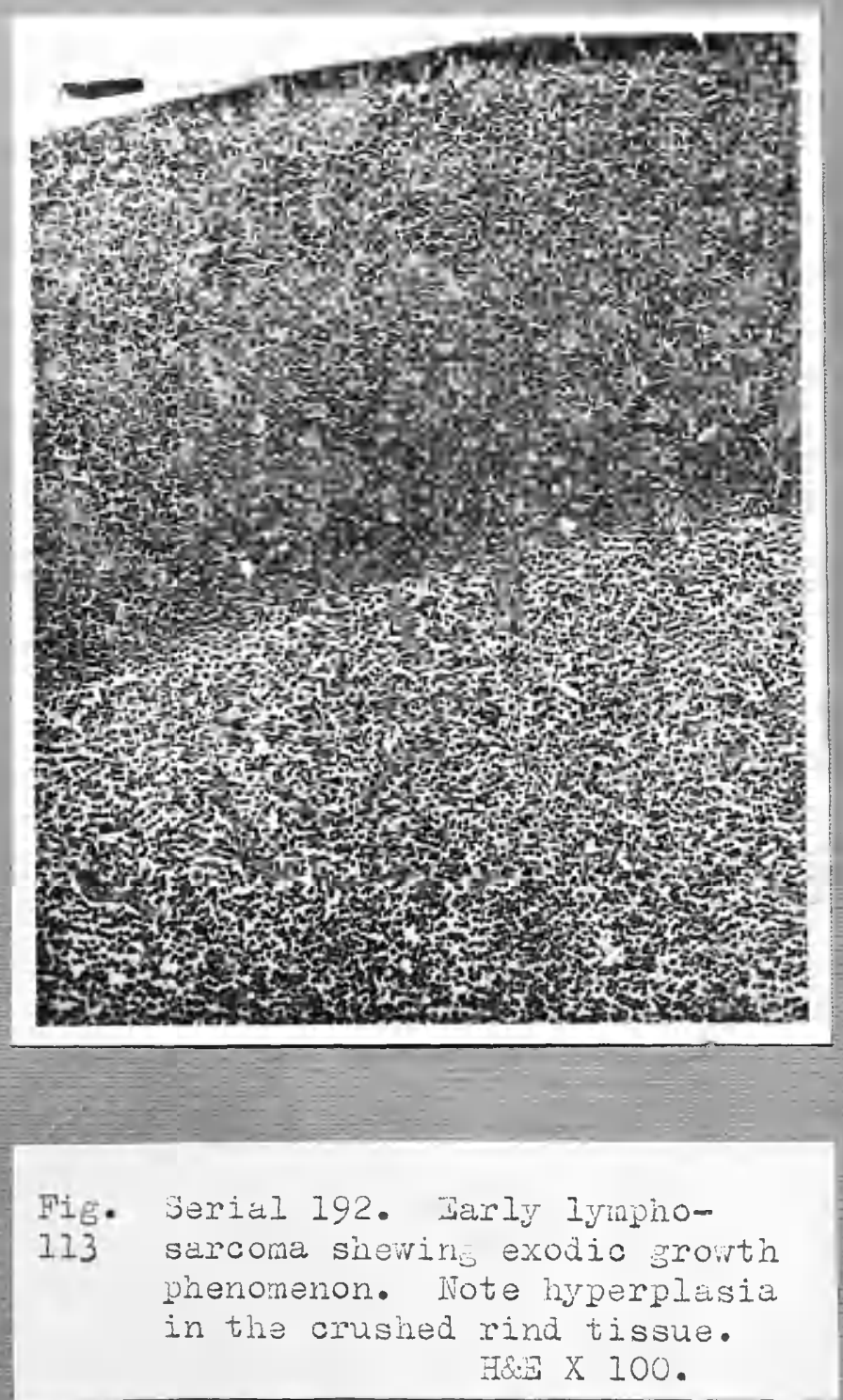

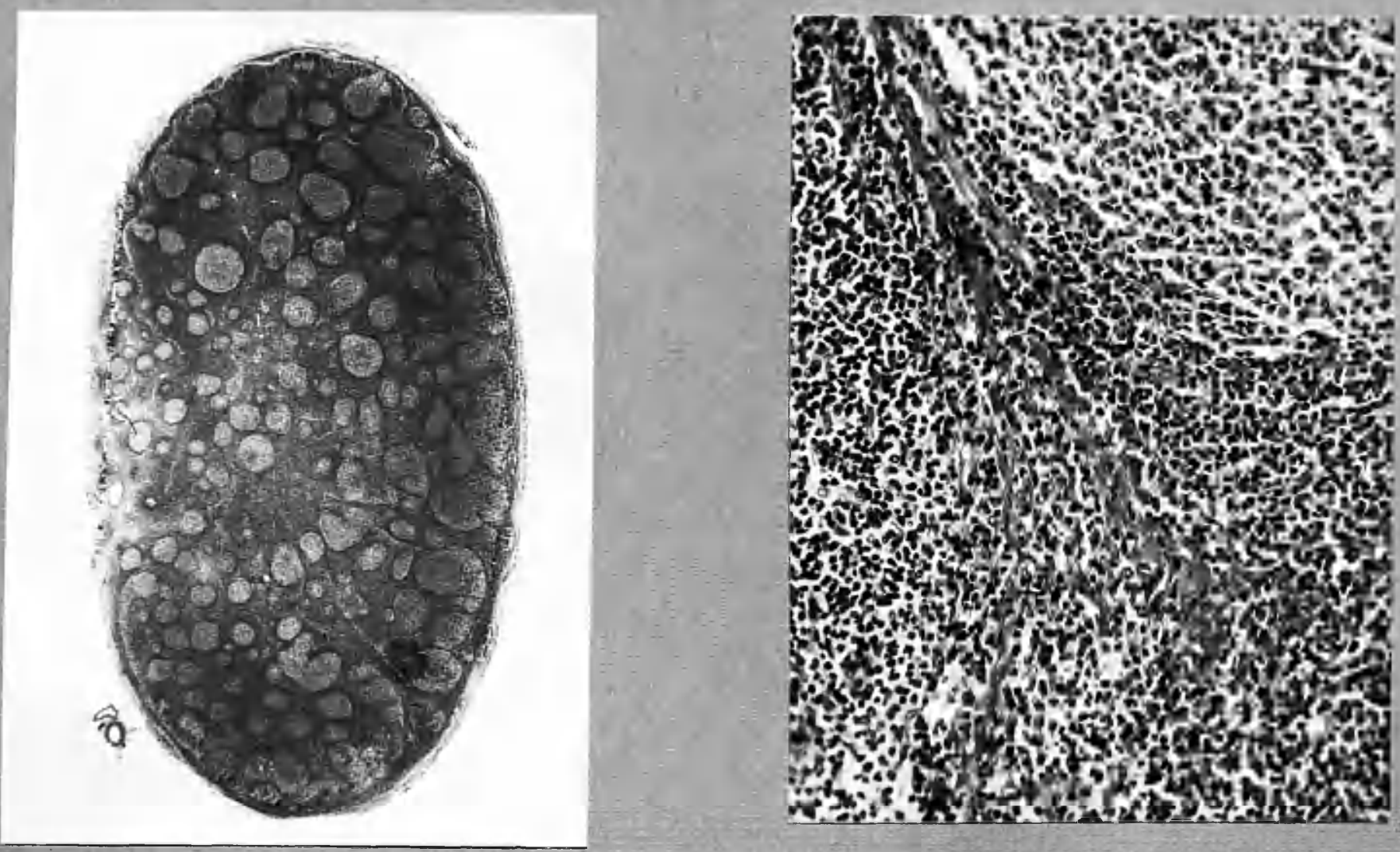

\footnotetext{
Fig. Serial 108. (Re-cut). Brili's 114. disease. False follicles very well seen.

$$
\text { Hoce X } 7 \text {. }
$$
}




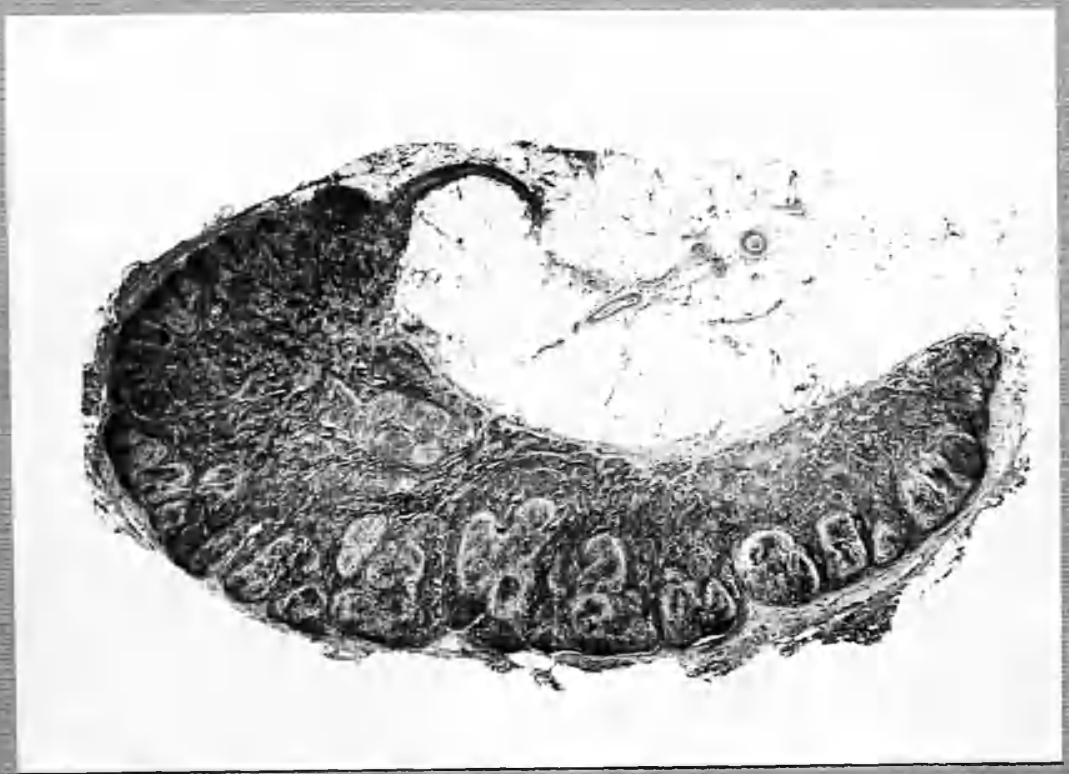

Fig. Serial 37. Indeteruinate

116 lesion, with aflinities to Brill's disease. Whe folicles are very larše. Fide $\times 5$. 


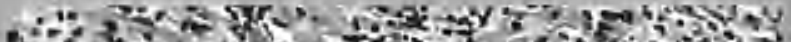

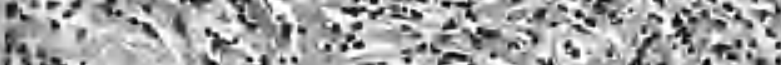

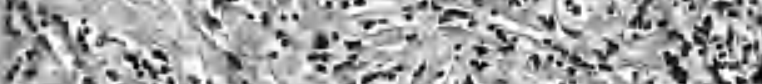

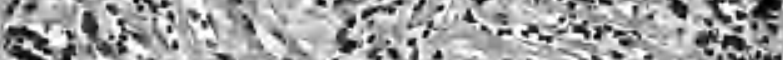

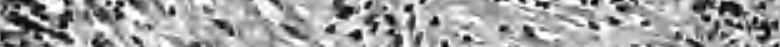
5. 4.

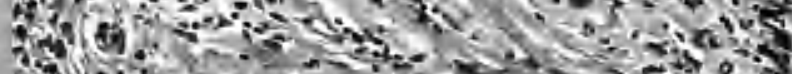

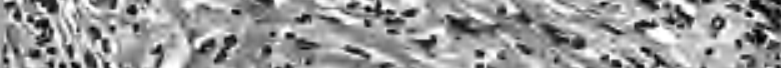

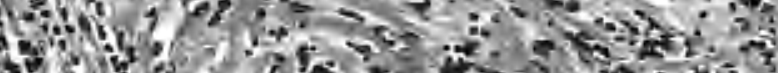

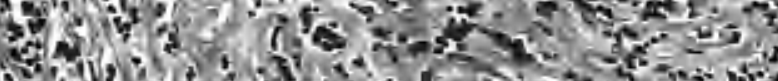

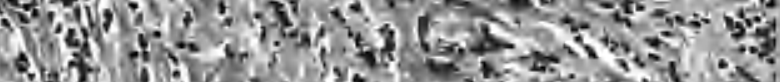

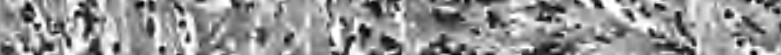
- ${ }^{2}$ in

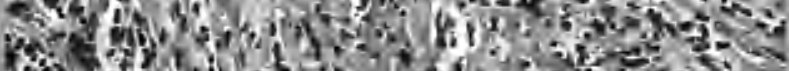

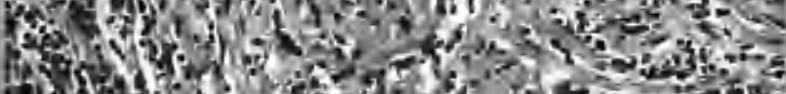

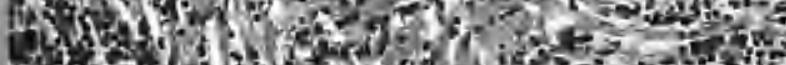

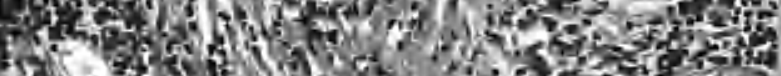

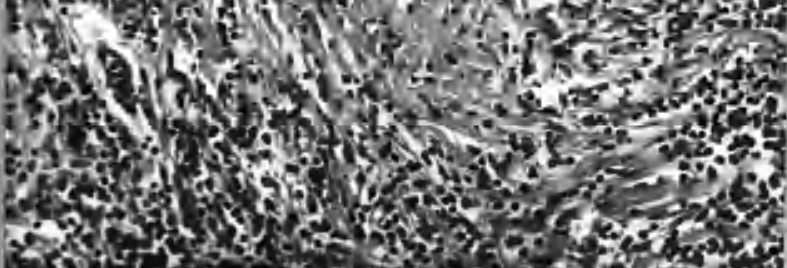

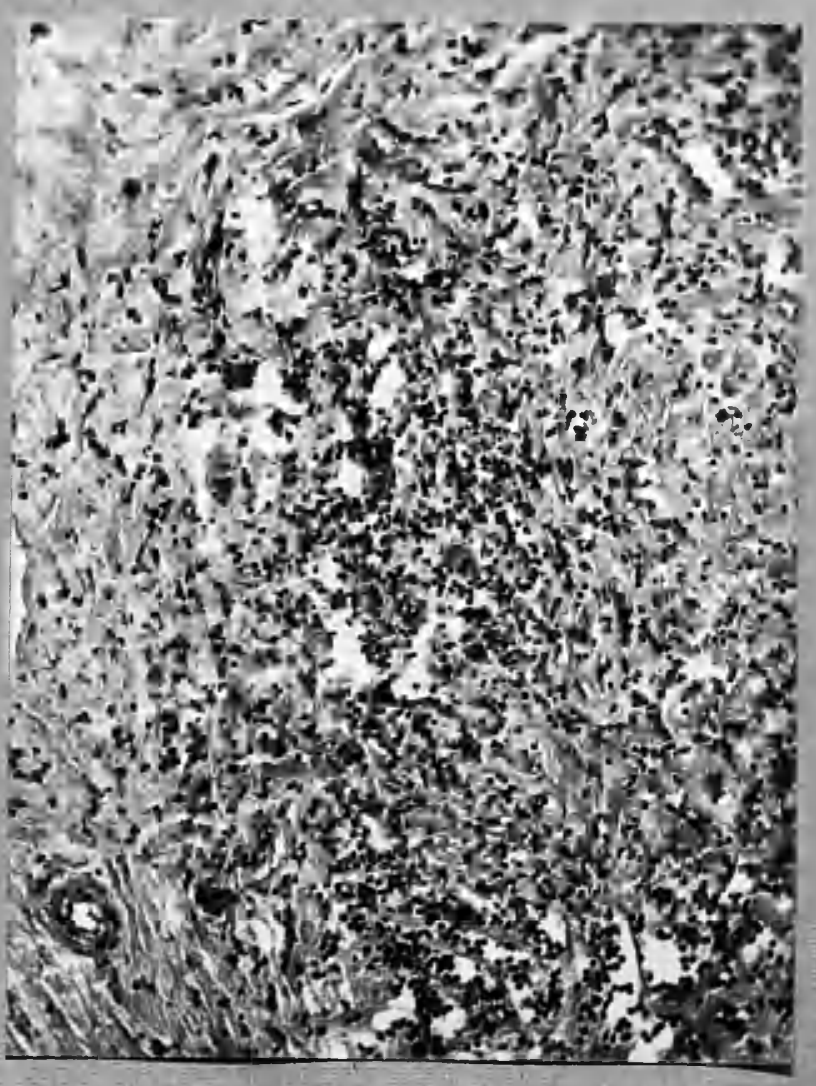

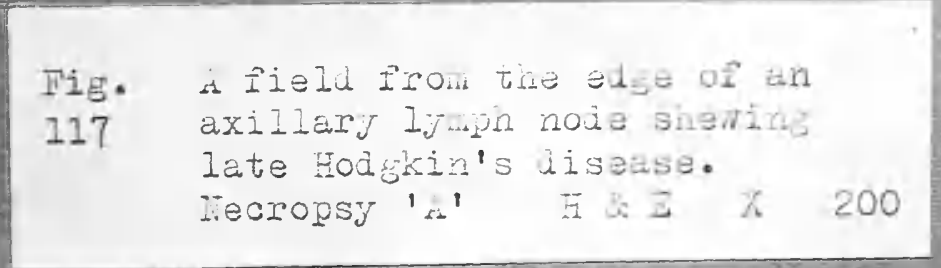

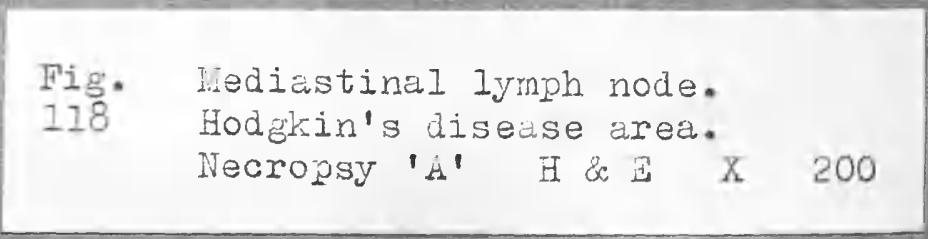




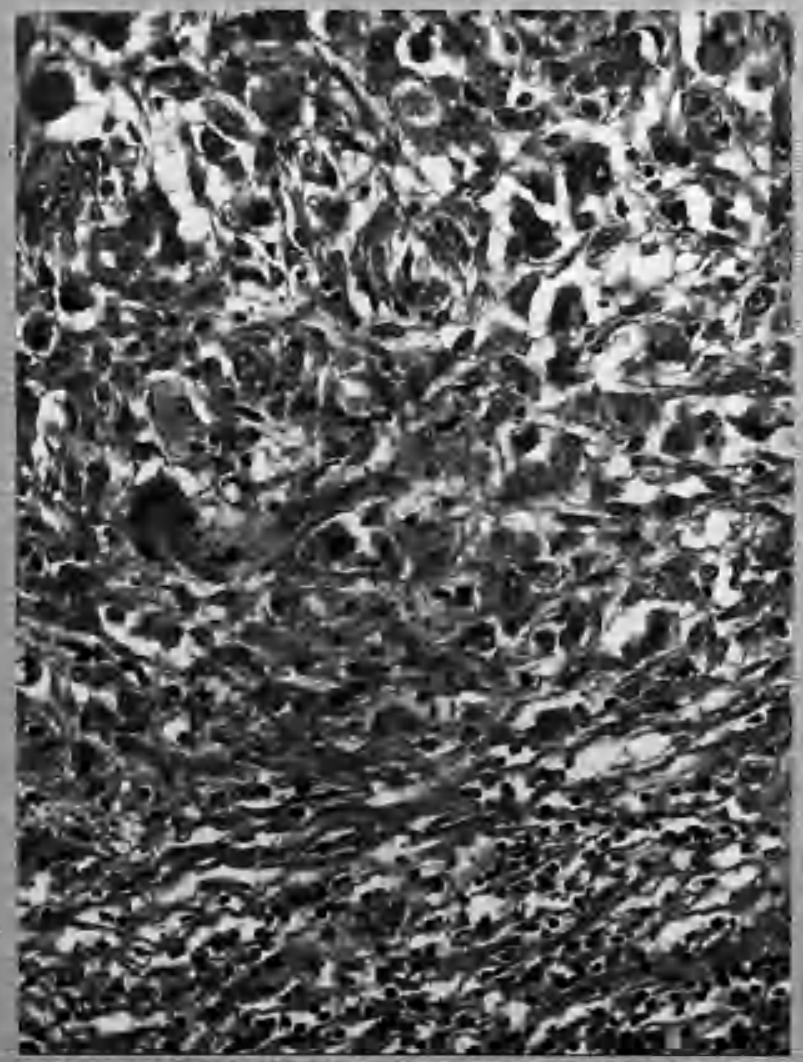

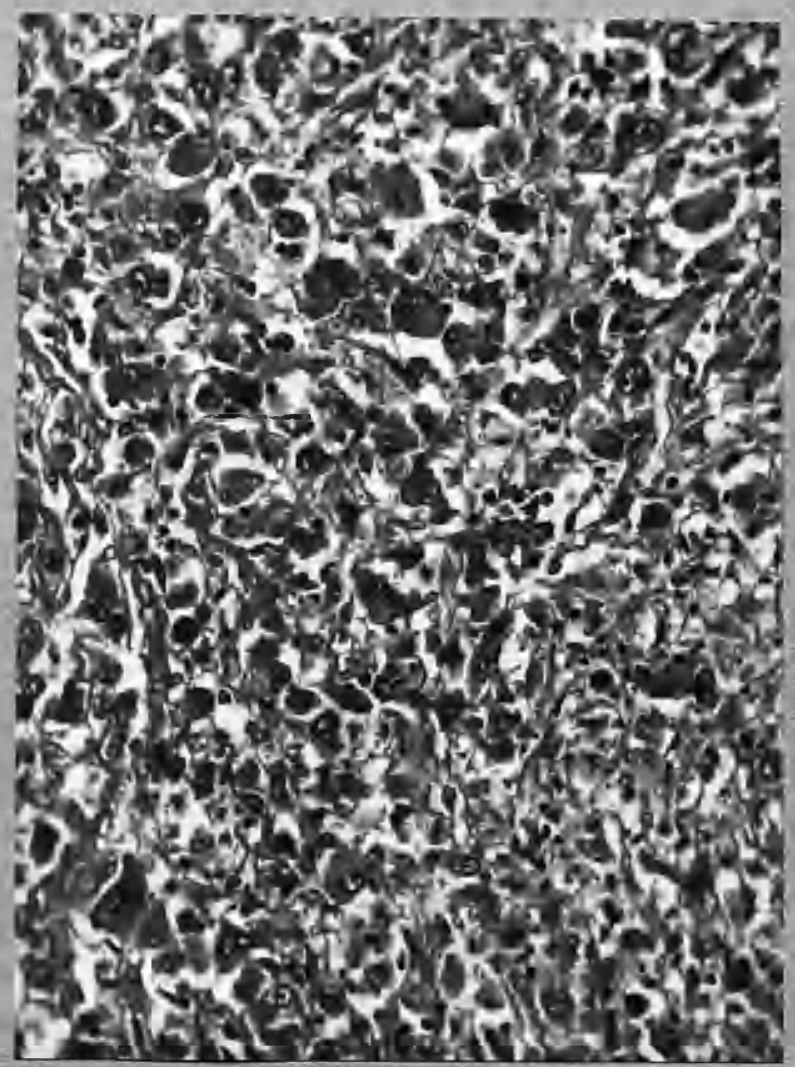

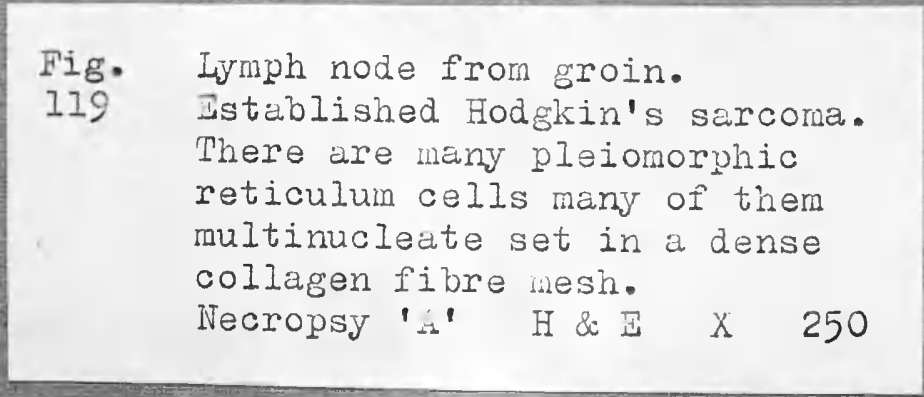

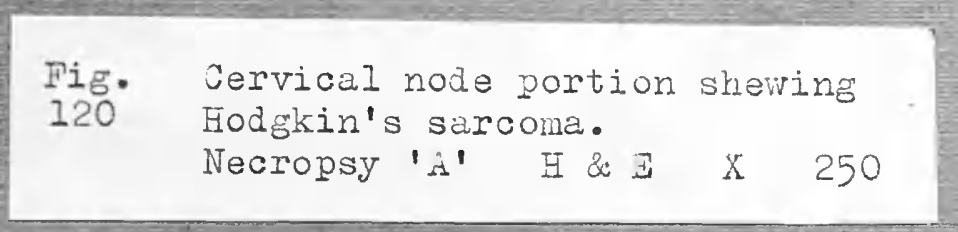




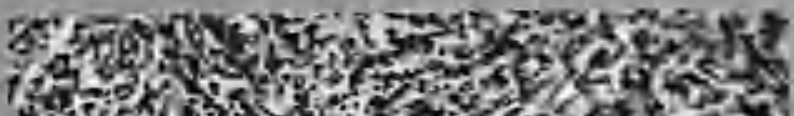

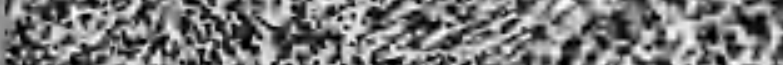

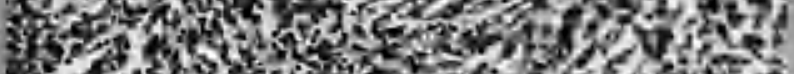

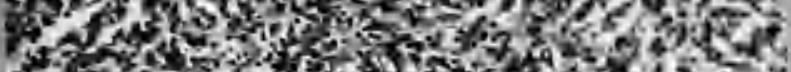

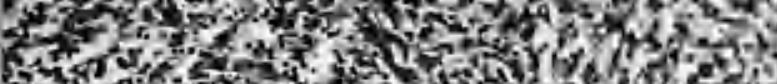

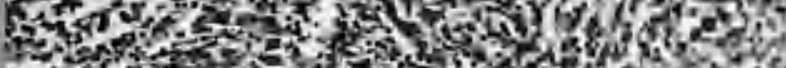

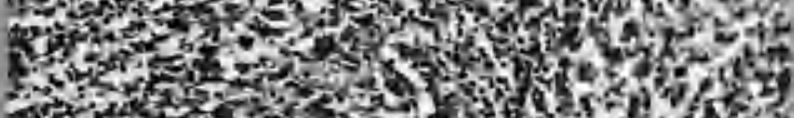

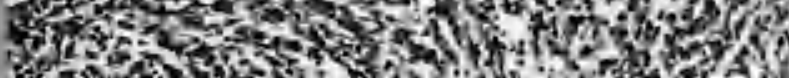

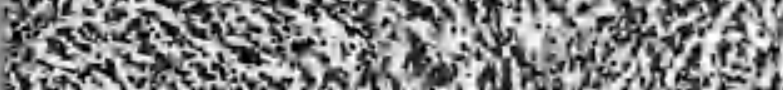

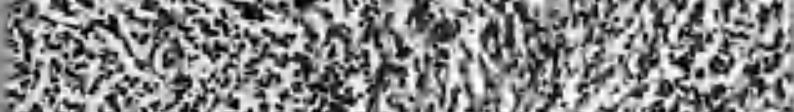

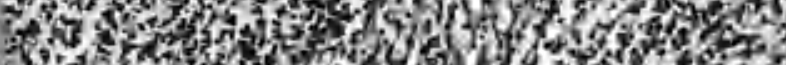

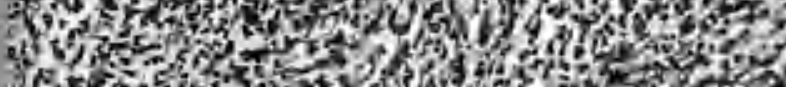

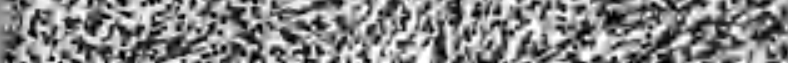

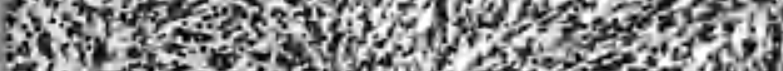
an 130

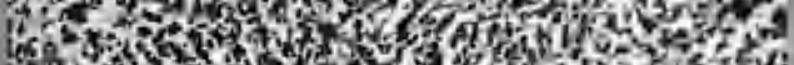

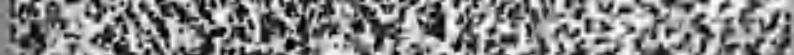

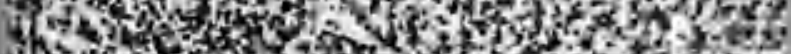
Shith

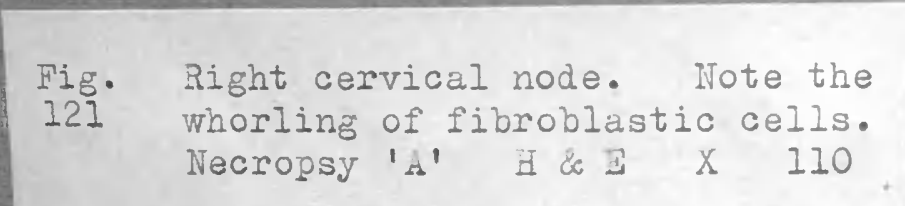

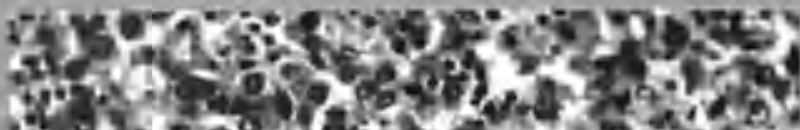

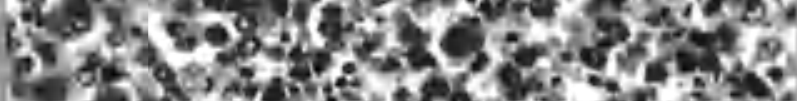

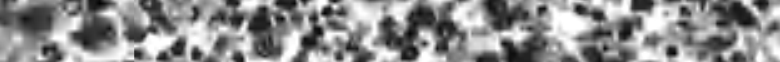

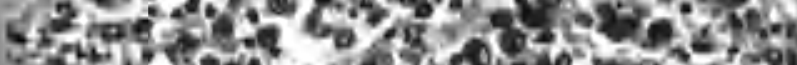

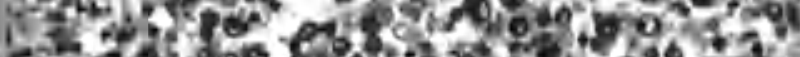

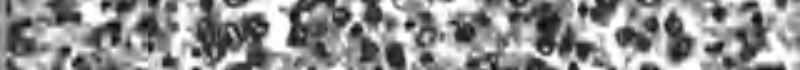

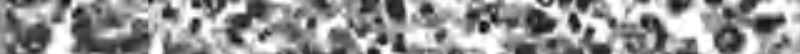

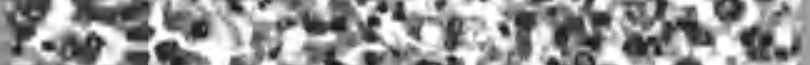

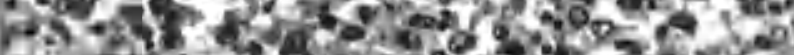

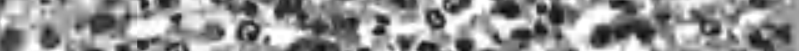

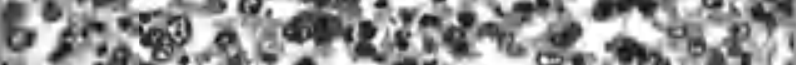

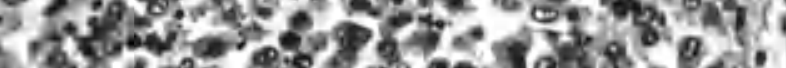

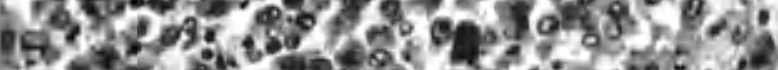

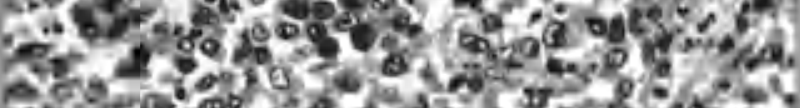

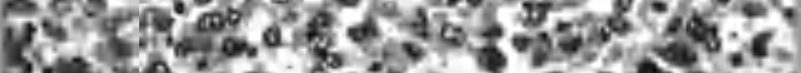

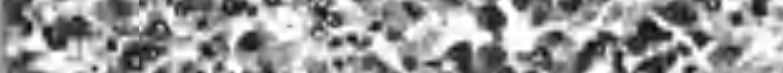

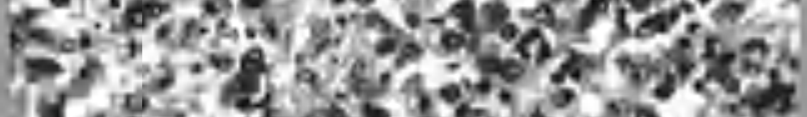

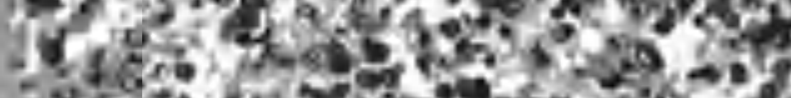

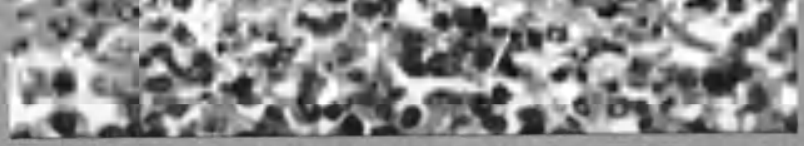

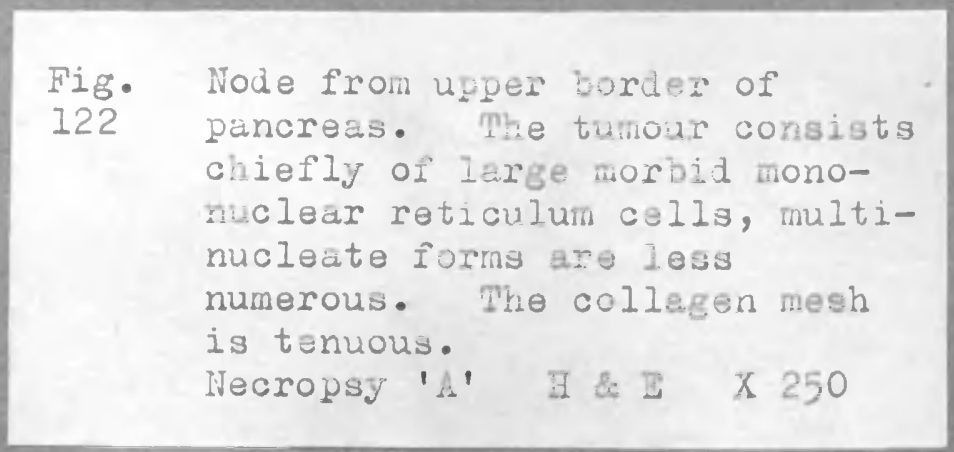



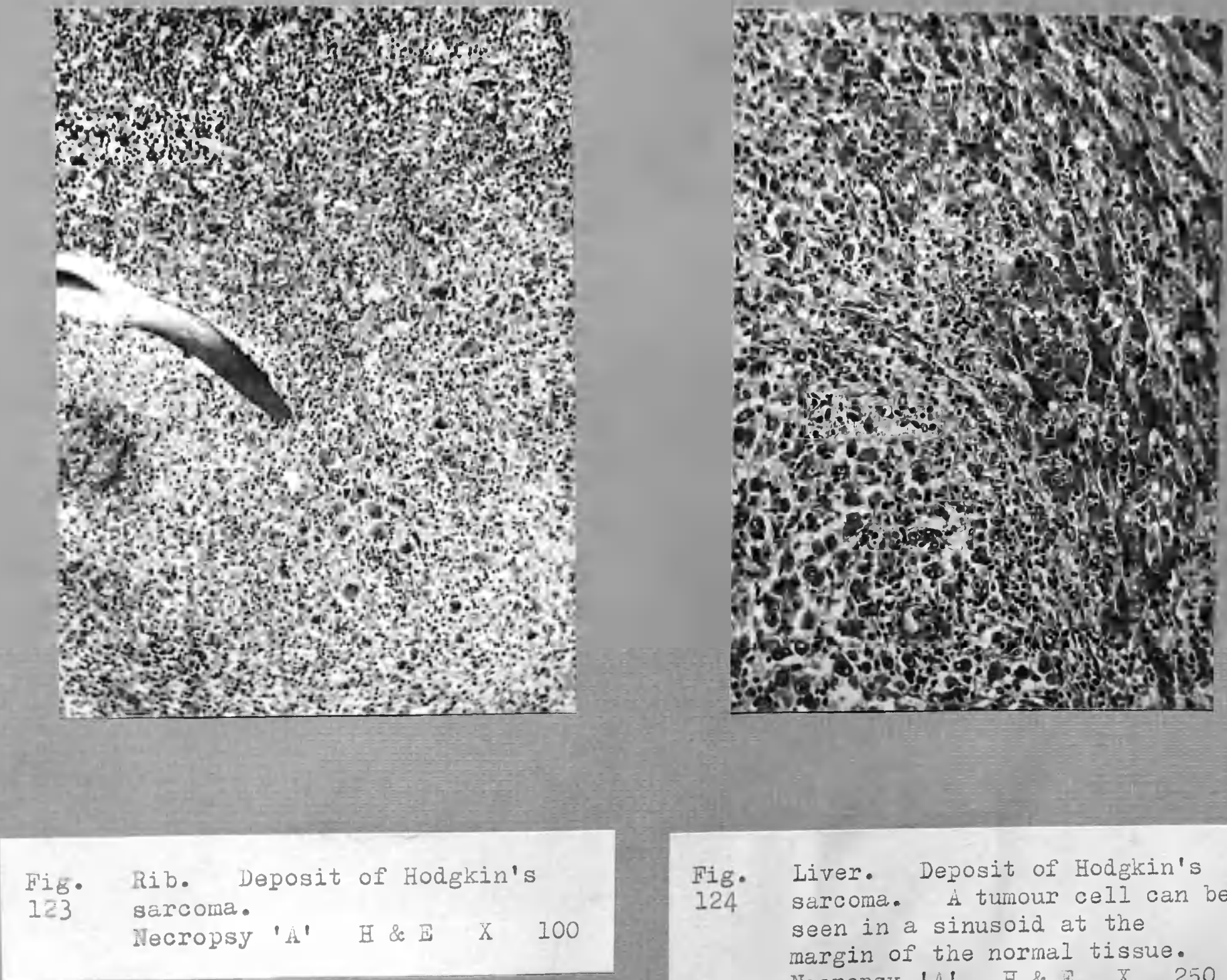

Fig. Liver. Deposit of Hodgkin's
124 sarcoma. A tumour cell can be
seen in a sinusoid at the
margin of the nornal tissue.
Necropsy 'A' $\mathrm{A} \& \mathrm{~A}$ X 250



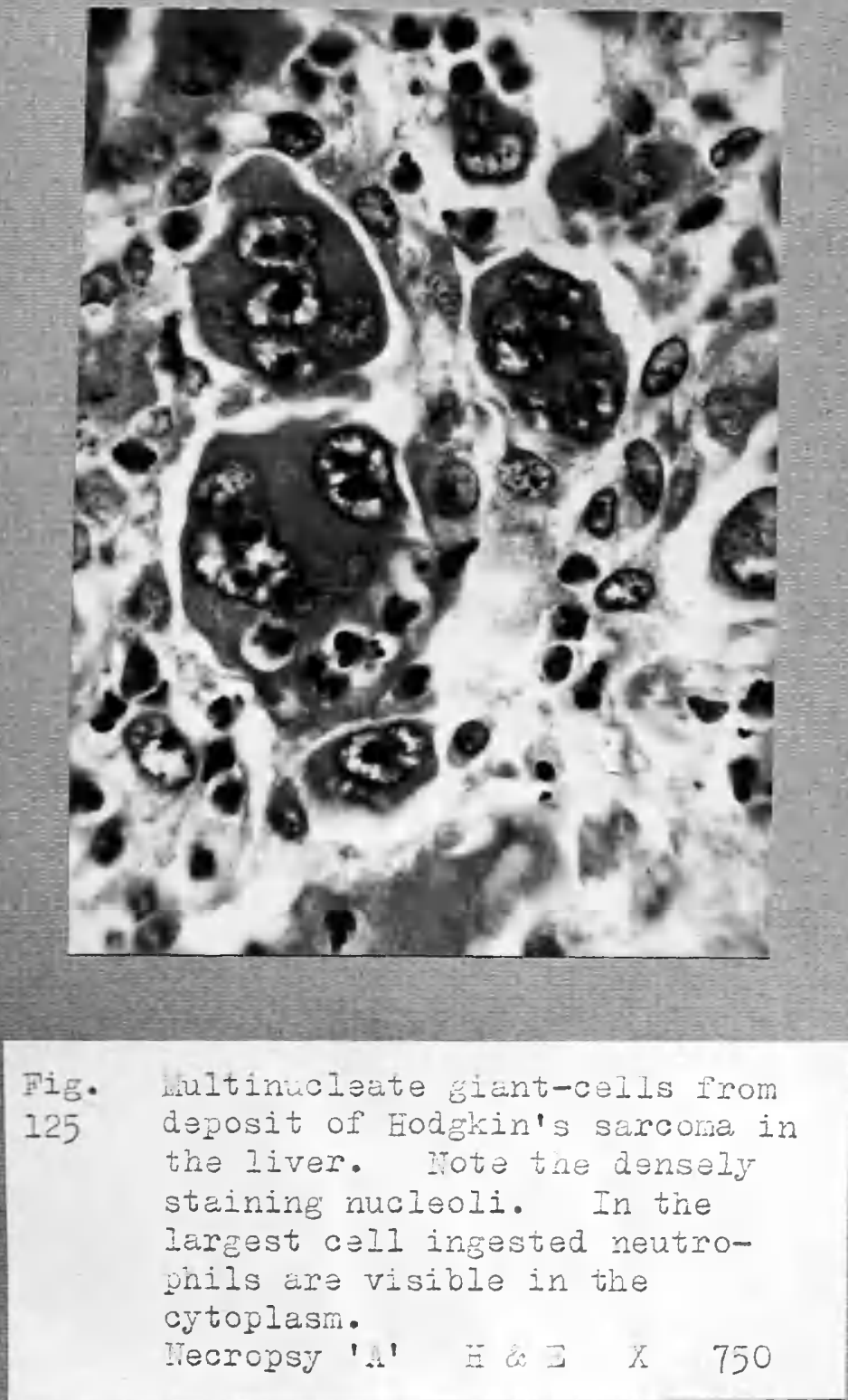

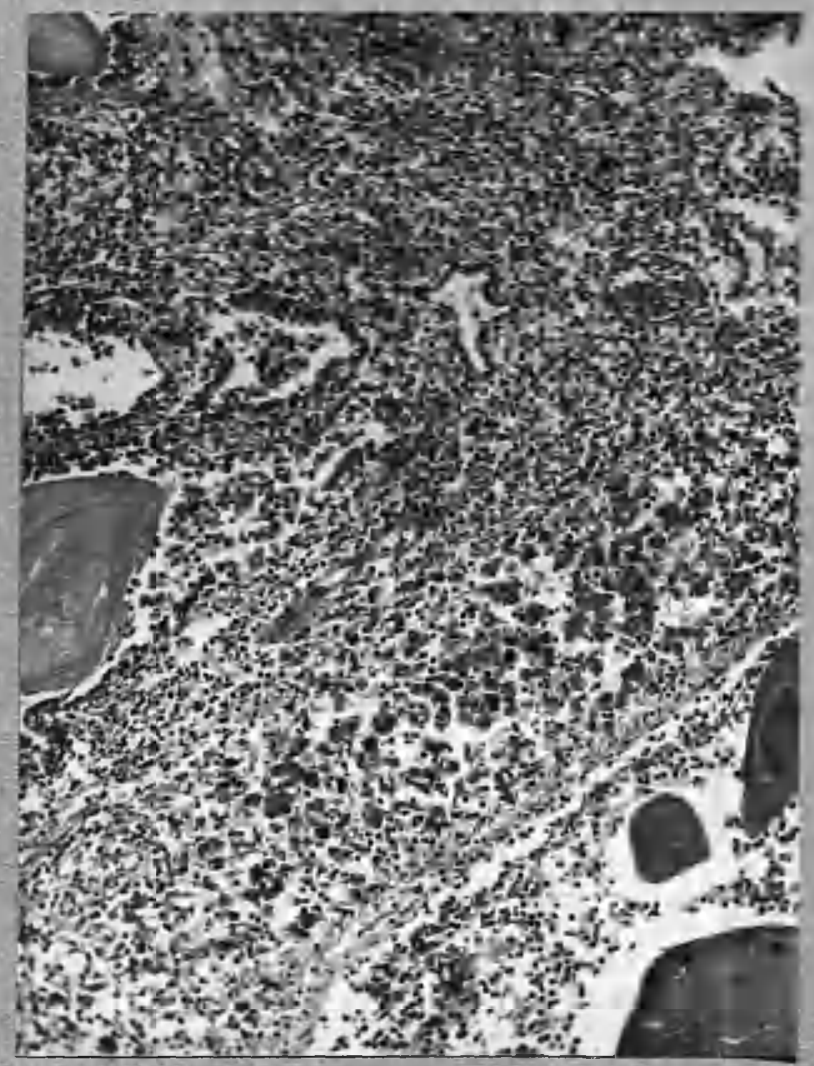

Fig.

Prostate gland, deposit of Hodgkin's sarcoma.

Necropsy ' $A$ ' $\quad \mathrm{H} \& \mathrm{E} X$

110 
Ex: 5 8. thes: Exy 20, Nata

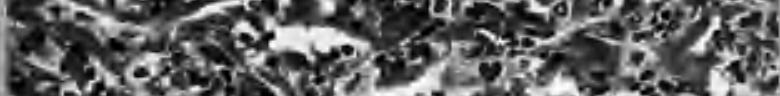

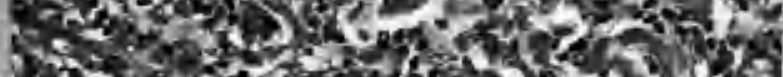

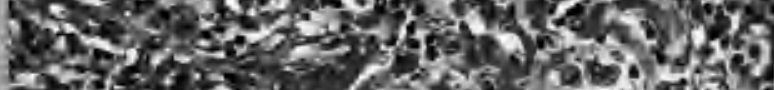

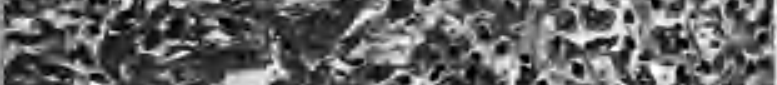

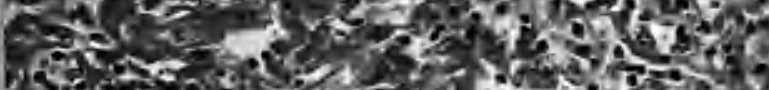

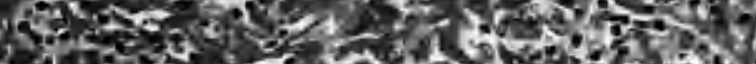

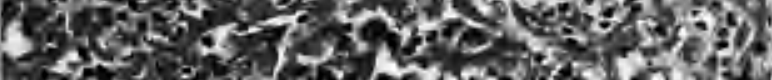

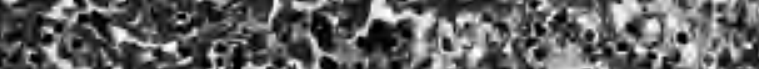

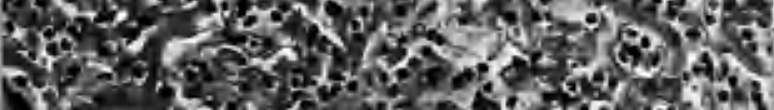

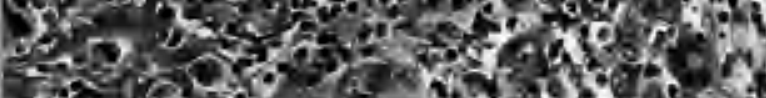

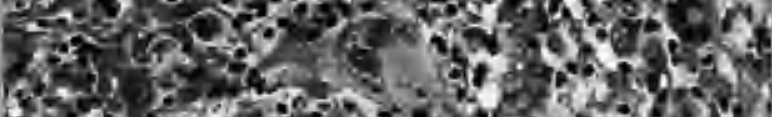

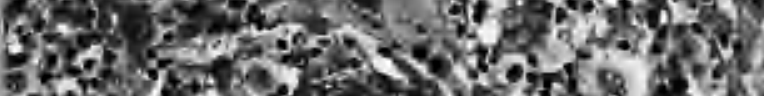

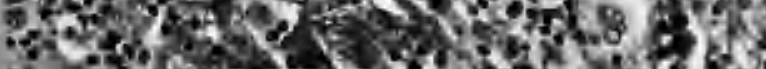

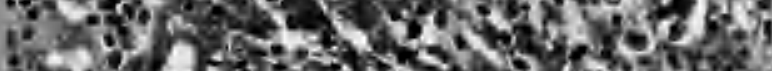

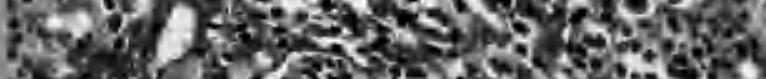

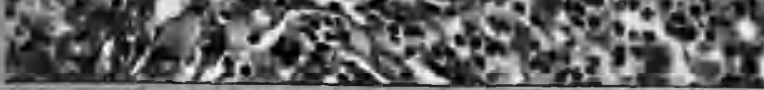

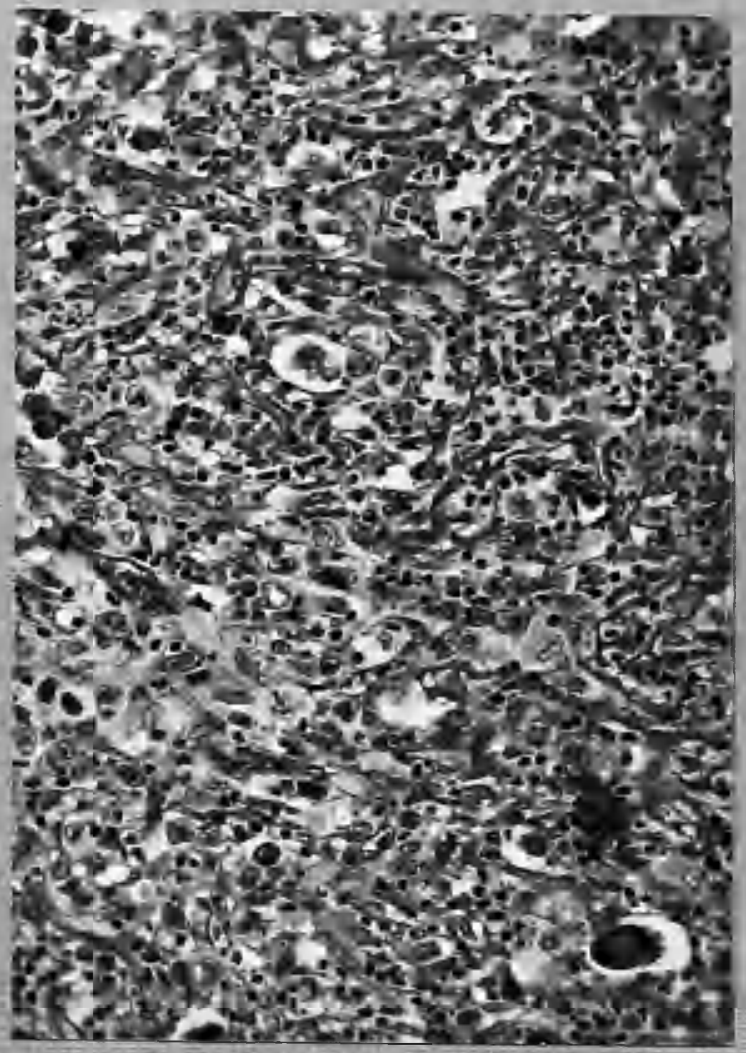

Fis. 127
Biopsy. Highly cellular

pleiomorphic Hodgkin's iisease

shewing weIl developed fibrosis.

Serial 188 I \& $B$ X 200
Fig. Biopsy. From the sume node.

128

overgrowth is unusually florid.

Serial 188

200 


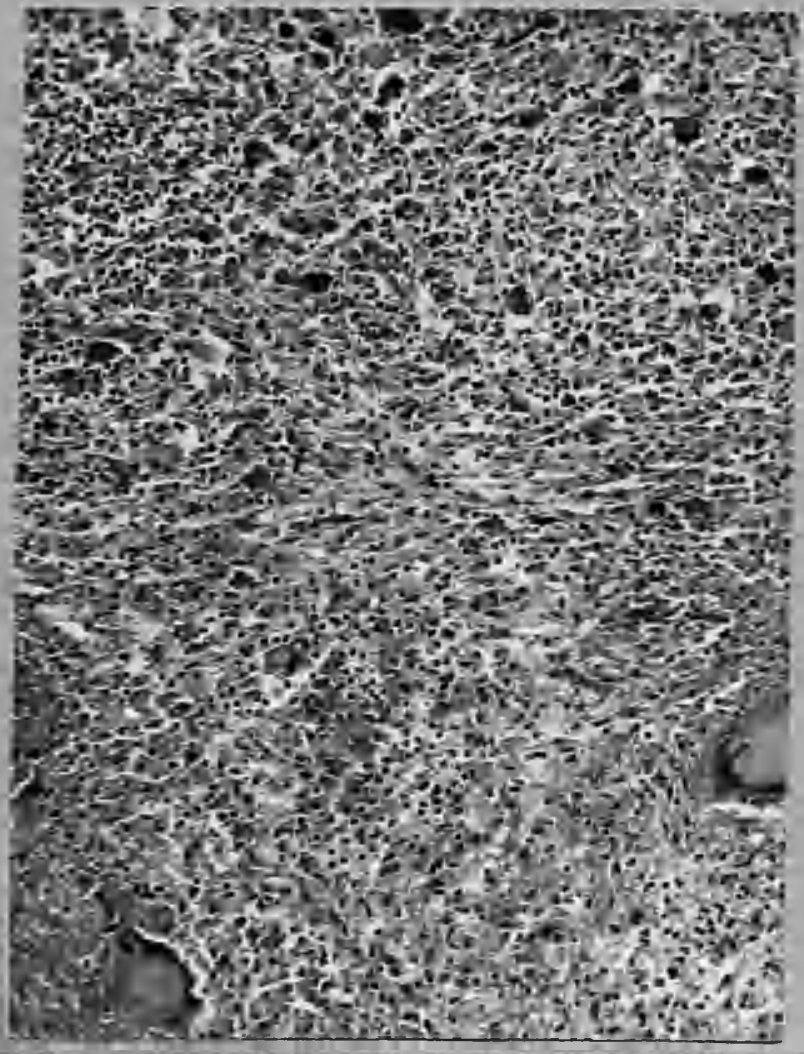

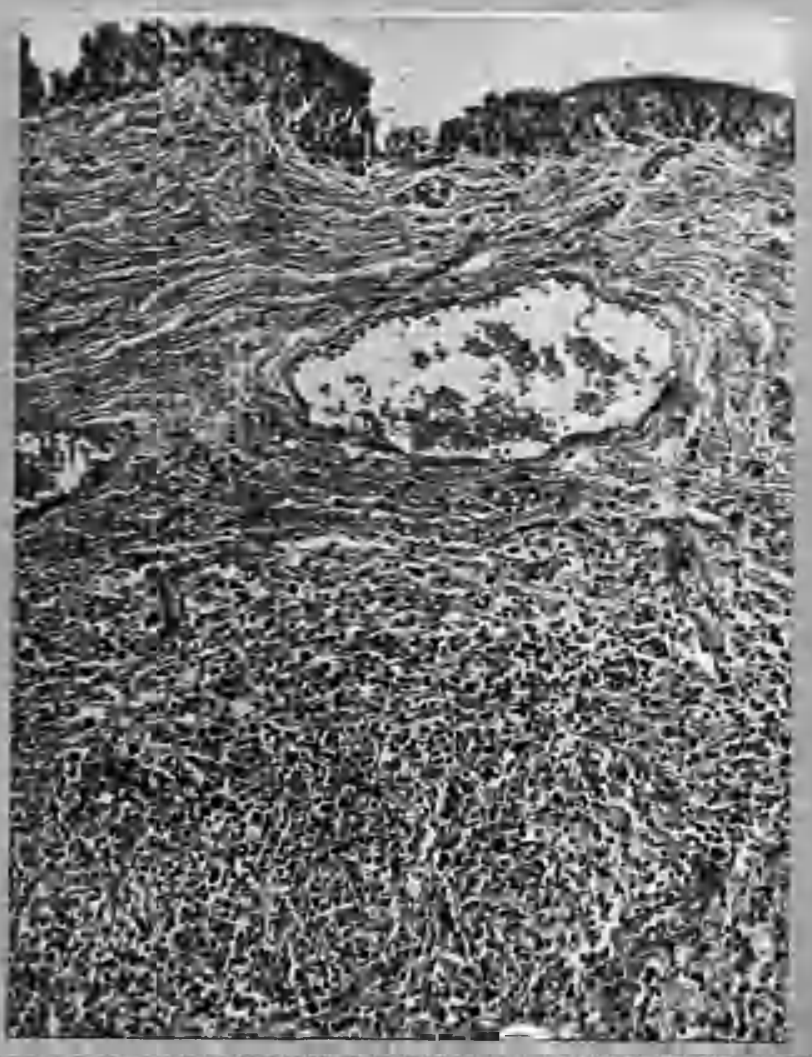

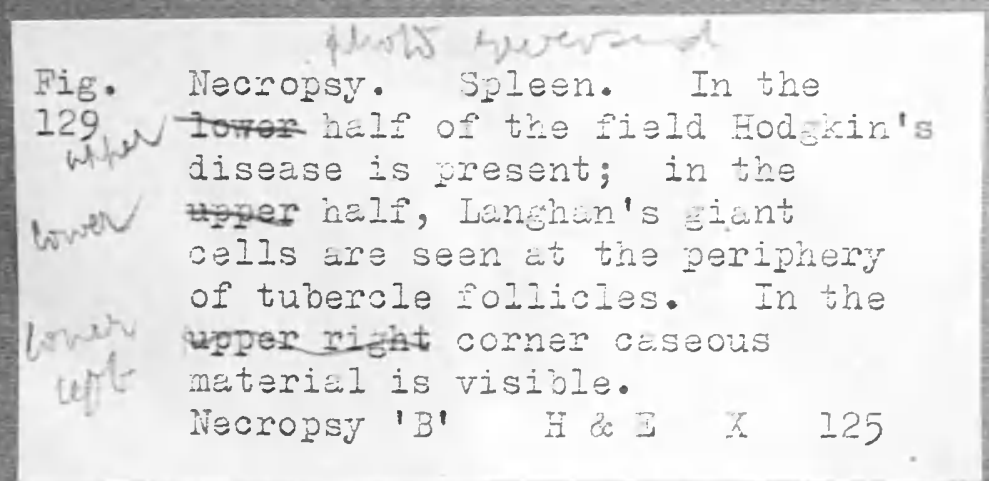

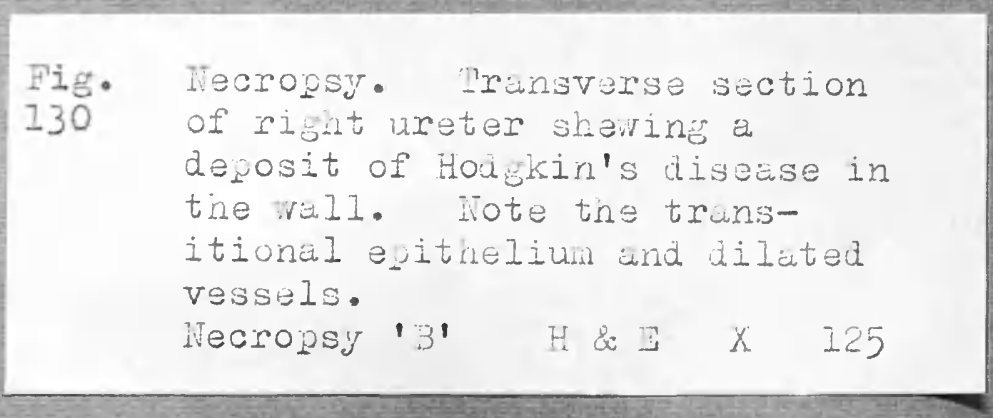




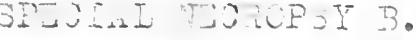

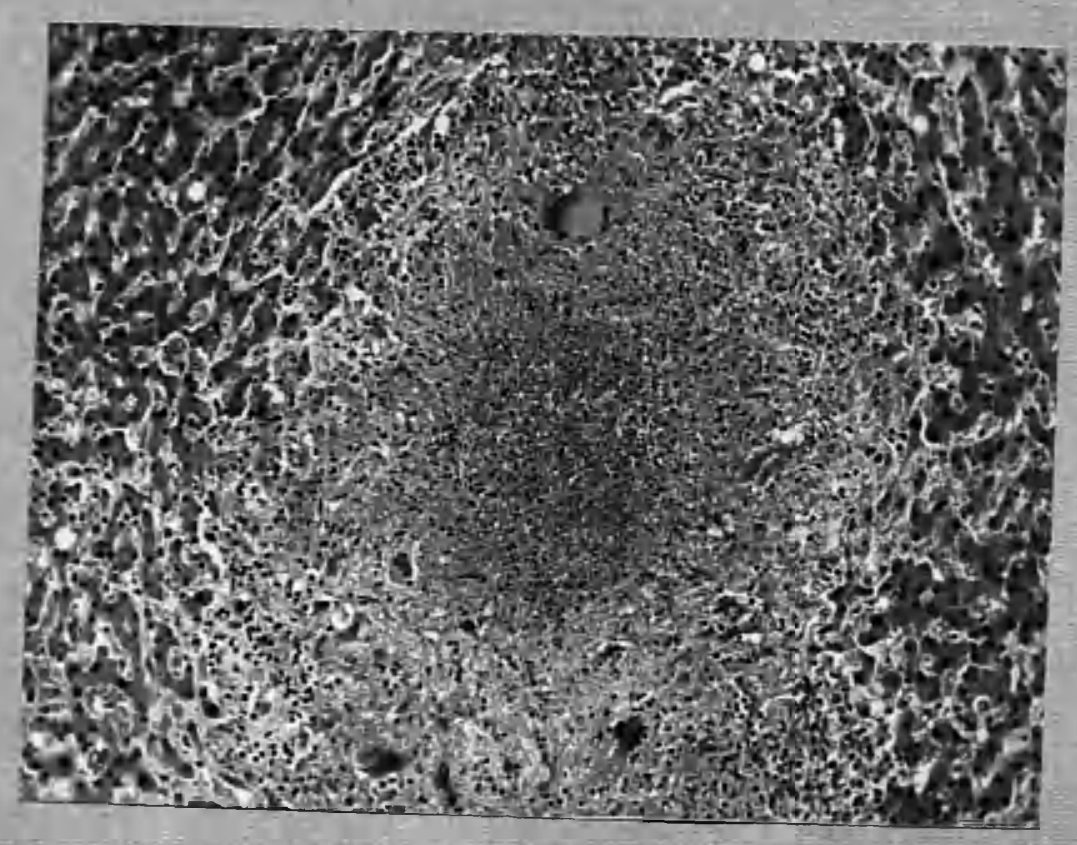

$$
\begin{aligned}
& \text { Fig. Necropsy. Tuberculous follicles } \\
& 133 \text { in the liver. } \\
& \text { Ivecropsy 'B' } \mathrm{B} \text { \& } \mathrm{I} \quad \mathrm{X} \quad 125
\end{aligned}
$$


26.

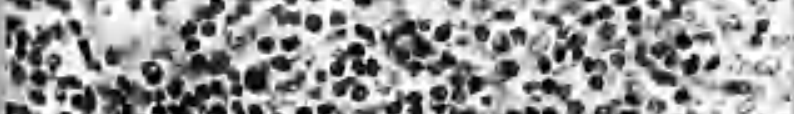
s.

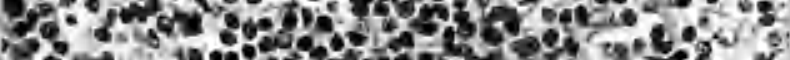

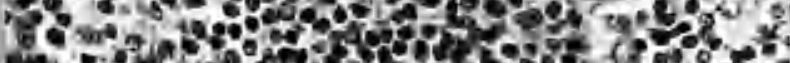

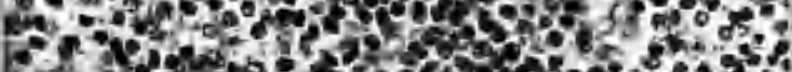

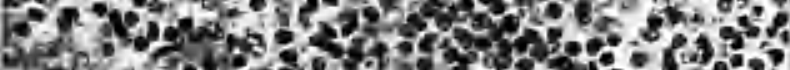

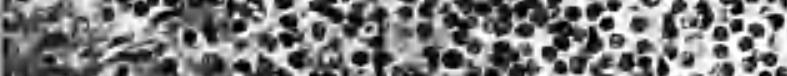

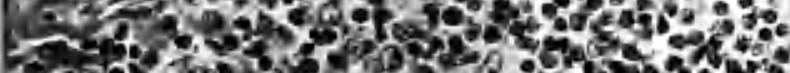

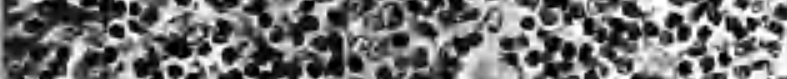

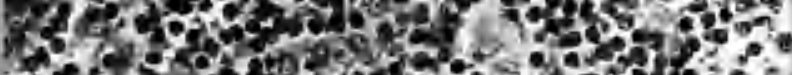

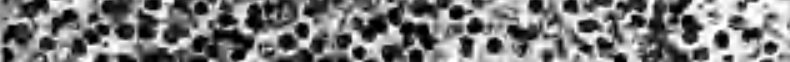

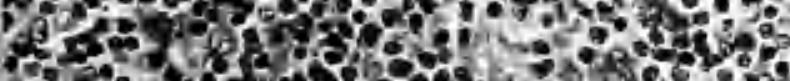

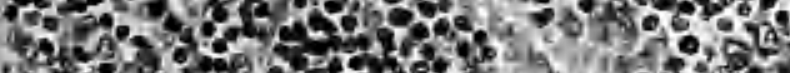

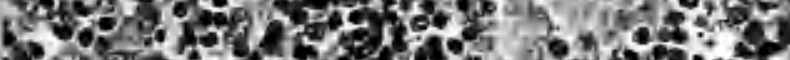

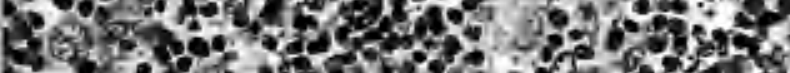

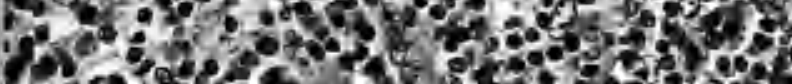

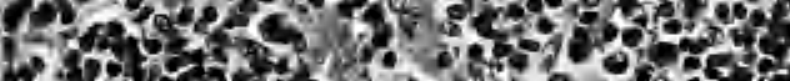

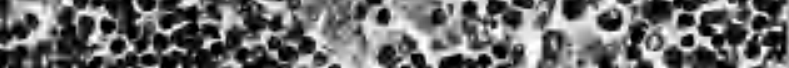

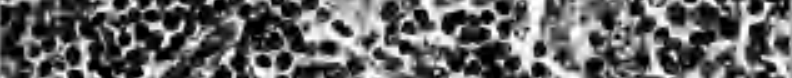

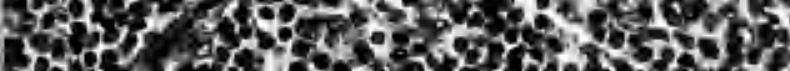

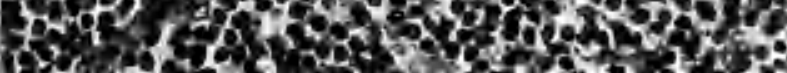
$13920 \mathrm{~m}$.
Fig.
Original biopsy, 1946.
134
Cervical lymph node. The
reticulun cell proliferation is
marked, in adaition to the
usual morbid mononuclear and
Fodgkin giant-cells, epitinelioid
cell islets are visible. The
nuclei of the latter are
relatively feebly stained. Note
the large slightiy innature
lymphocytes.
Serial 117.
H\&:E X 250

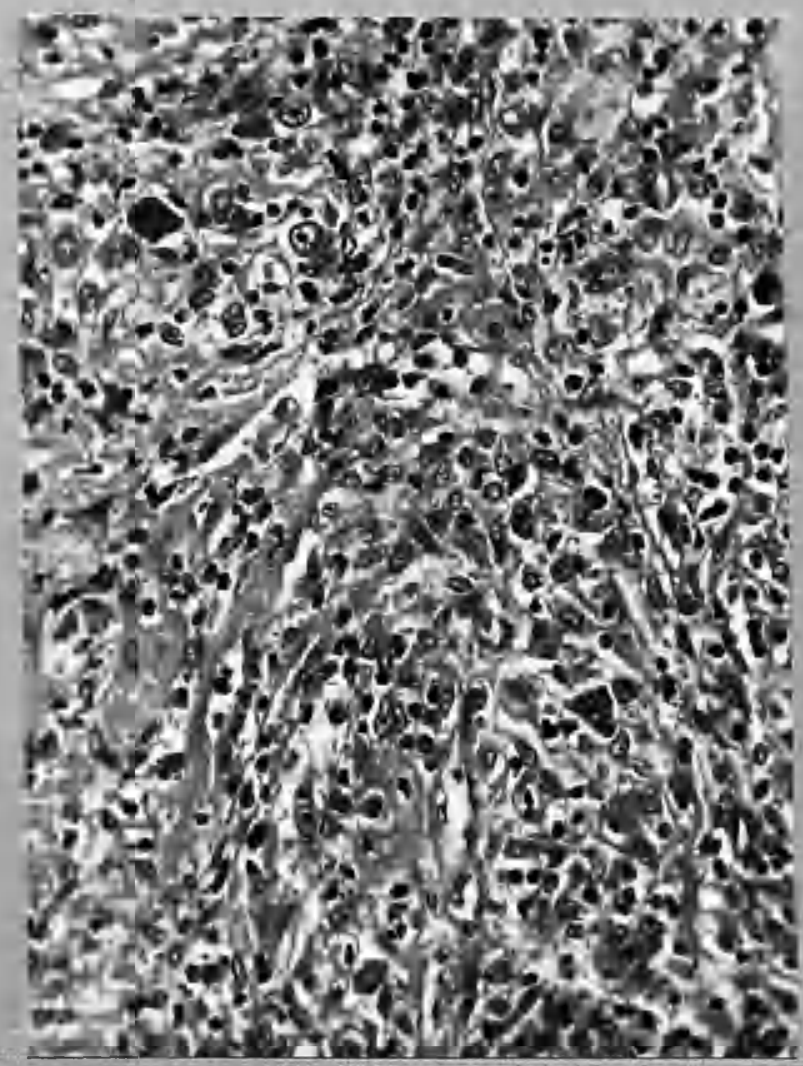

\begin{tabular}{|c|c|}
\hline $\begin{array}{l}\text { Fig. } \\
135\end{array}$ & $\begin{array}{l}\text { Necropsy. Right inferior } \\
\text { tracheo-bronchial node. } \\
\text { Waturing Hodgkin's disease, } \\
\text { lymphocjtes are scanty and } \\
\text { fibrosis is marked. The lesion } \\
\text { is becoming distinctly tumour- } \\
\text { like. }\end{array}$ \\
\hline
\end{tabular}




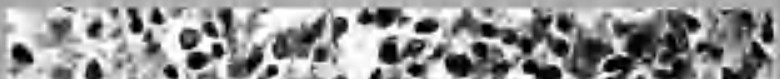

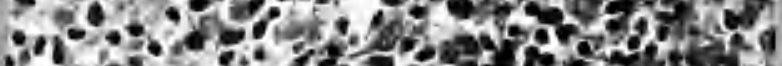

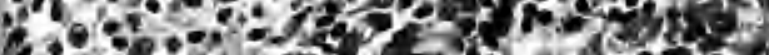

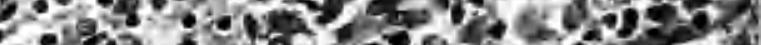

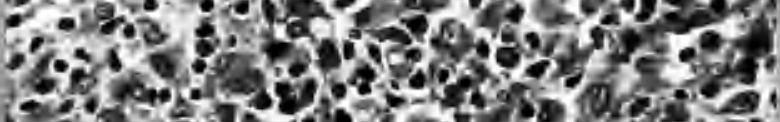

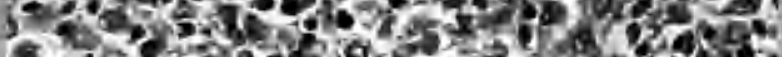

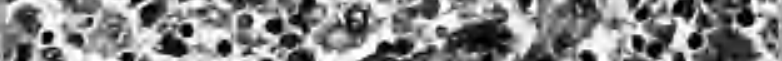

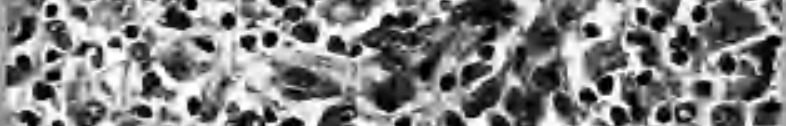

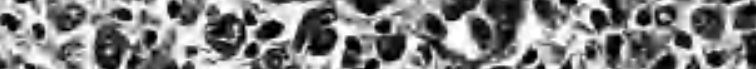

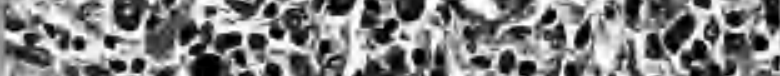

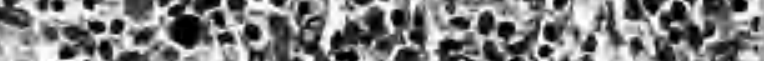

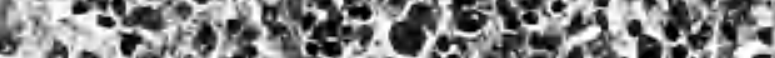

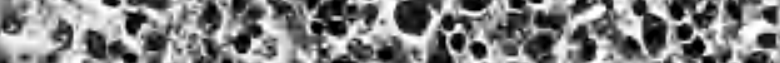

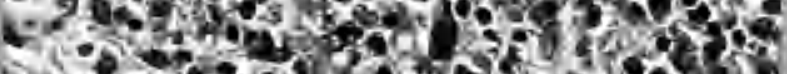

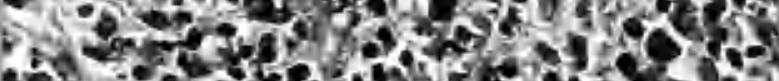

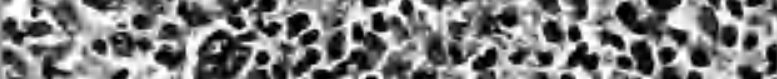

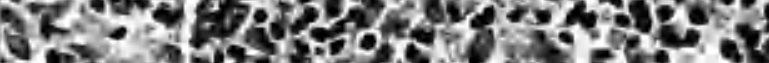
the

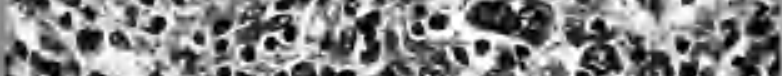

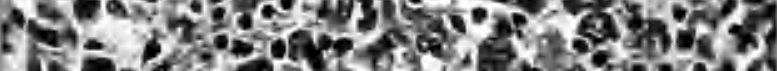

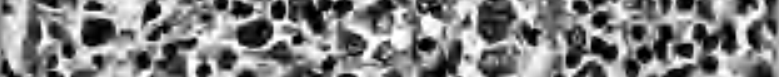

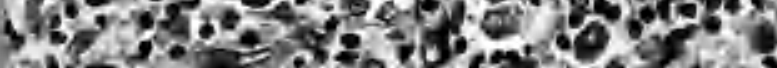

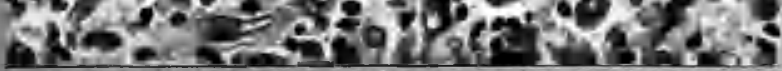
Fig. Necropsy. Juxta-splenic lymph
136 node. Large morbid reticulum cells are much more abundant, the lesion approximates closely to reticulum cell sarcoma. (Hodgkin's sarcora). ITecropsy 'O' Heed X 250

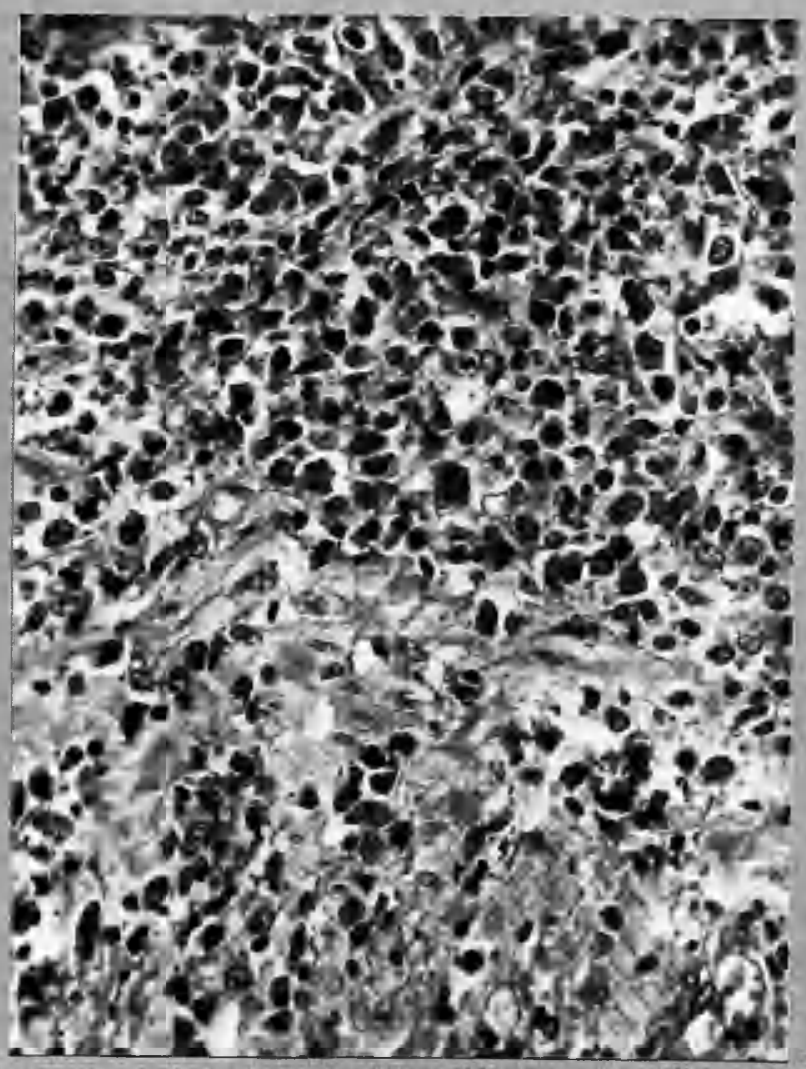

Fig. Necropsy. Wumour of stonach.
137 In this site the tumour is
franky sarcomatous. The celis
are mutualy sinilar but some
nuclear pleiomorisism persists.
In the lower part of the photo-
graph the pale tissue is smootr
muscle being infiltrated and over-
run by the growth.
Necropsy 'o' Hai $X$


450 5 5

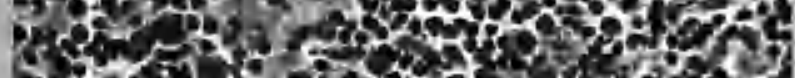

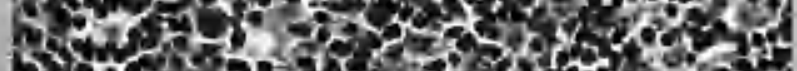

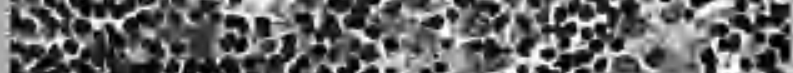

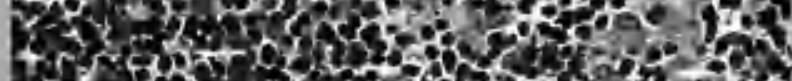

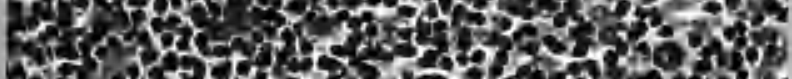

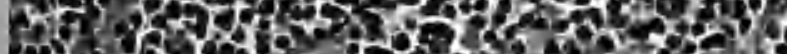

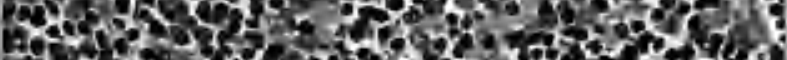

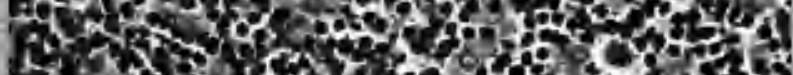

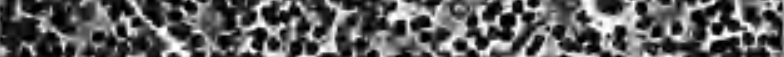

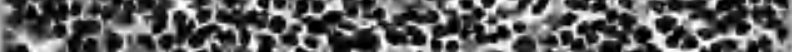

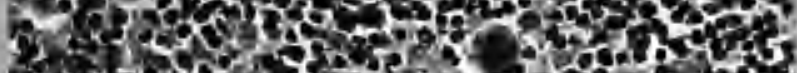

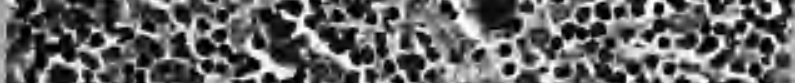
$04 \mathrm{~s}$.

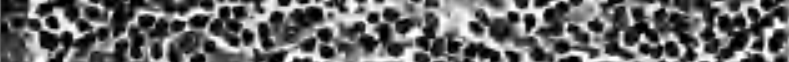

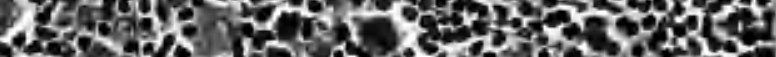

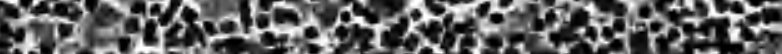

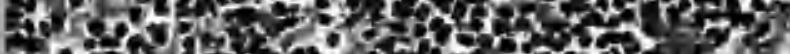

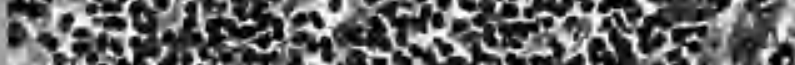
ow 5 H

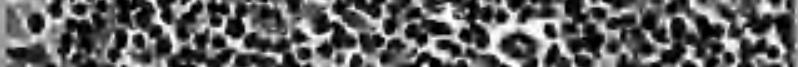

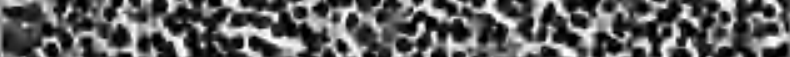

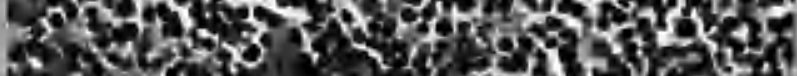

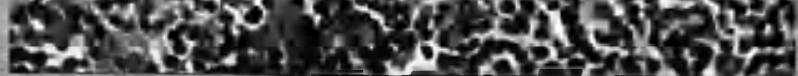
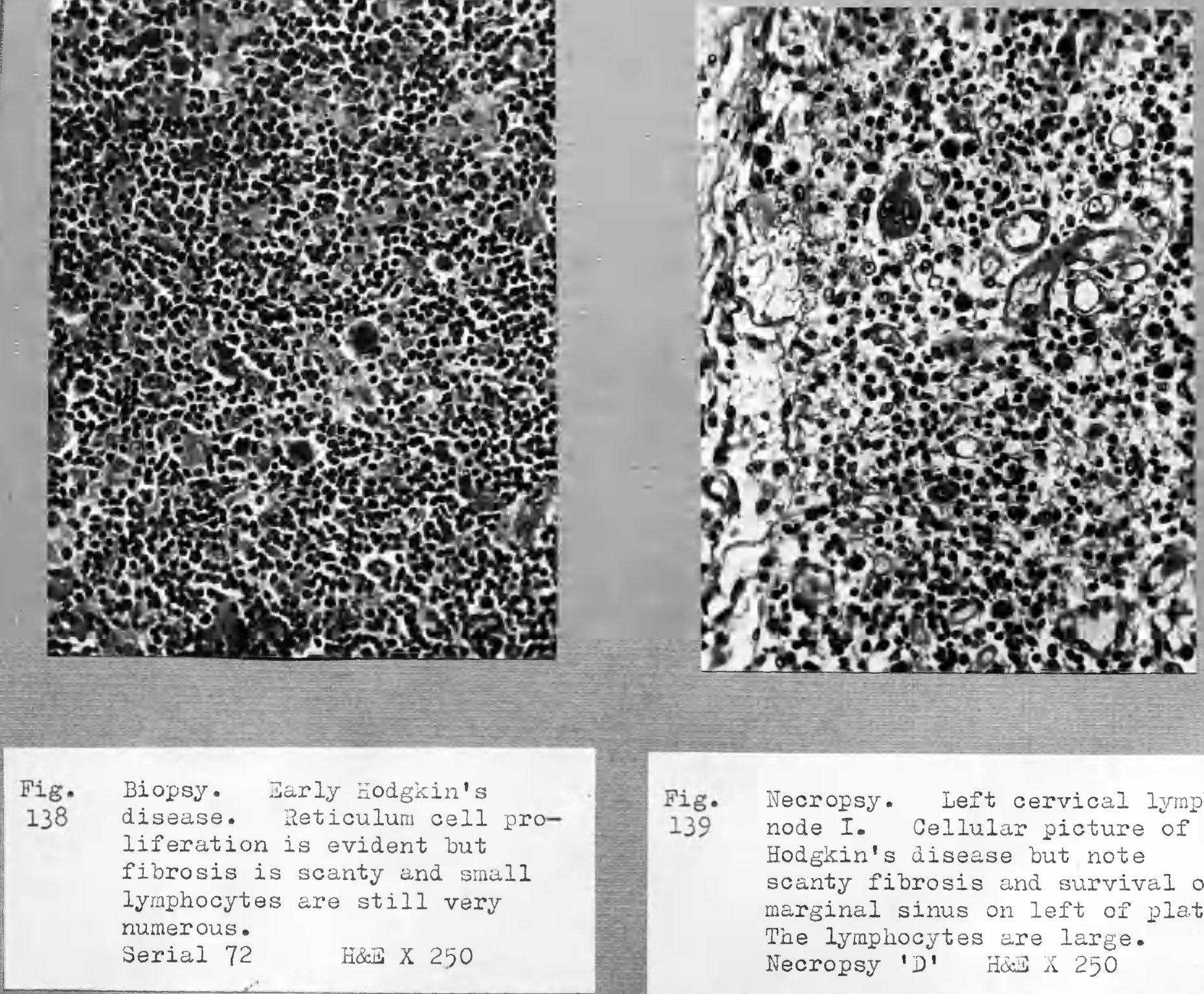

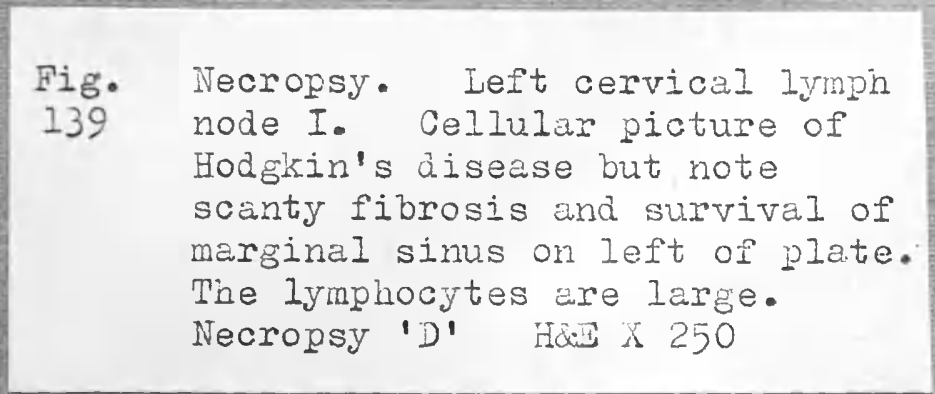




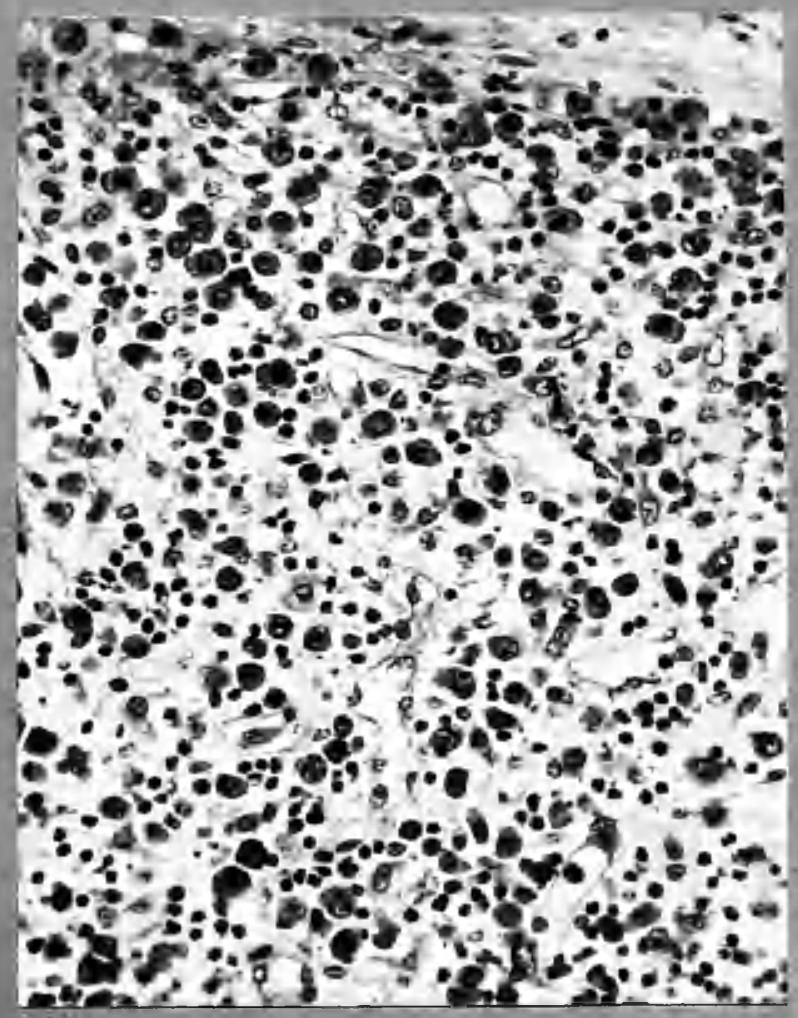

Fig. Necropsy. Left cervical lymph 140 node II. Overgrowth of large morbid mononucelar reticulum cells and merked reduction of lymphocytes. Necropsy ' $D$ ' Hoi $\times 250$
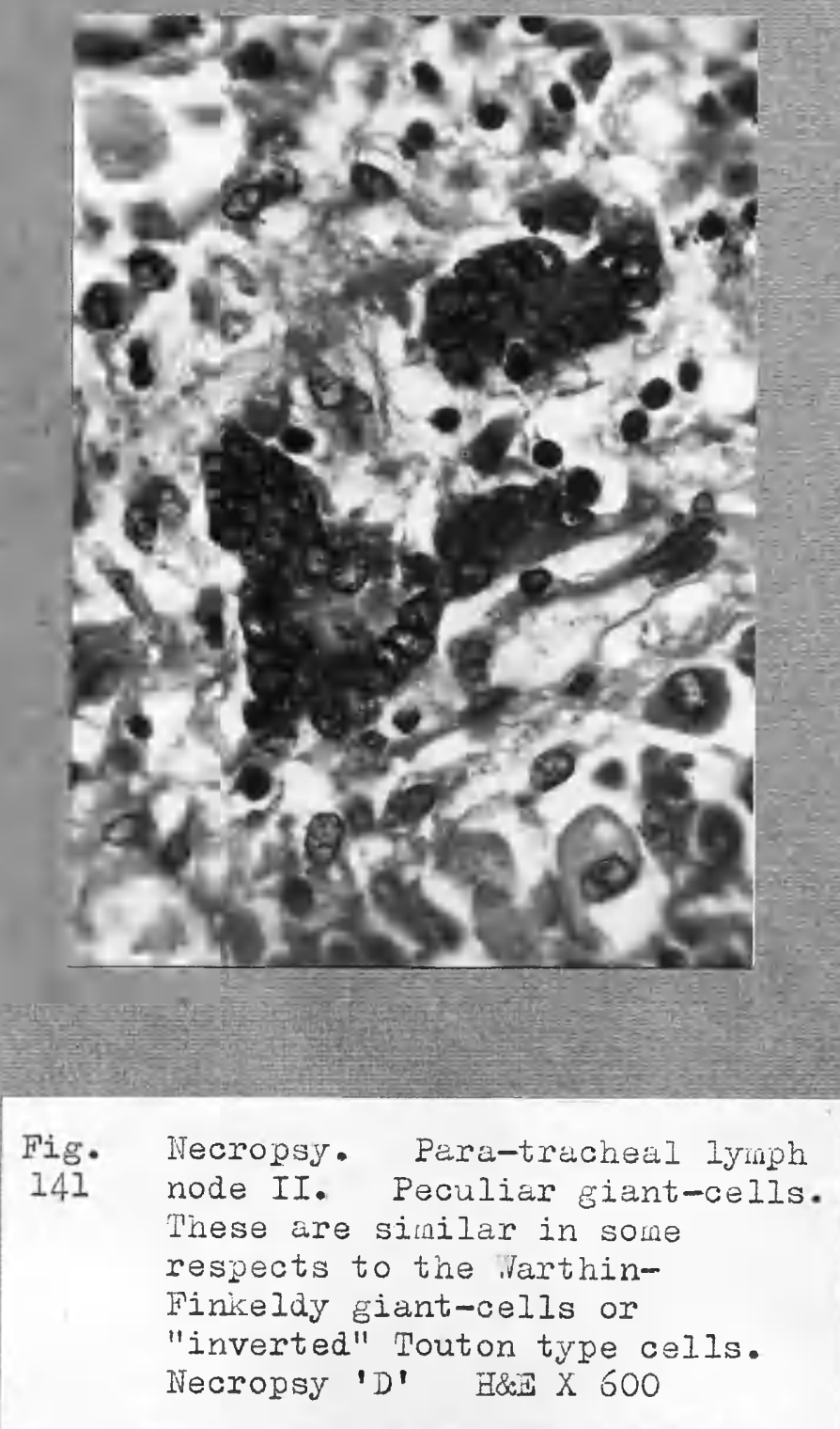


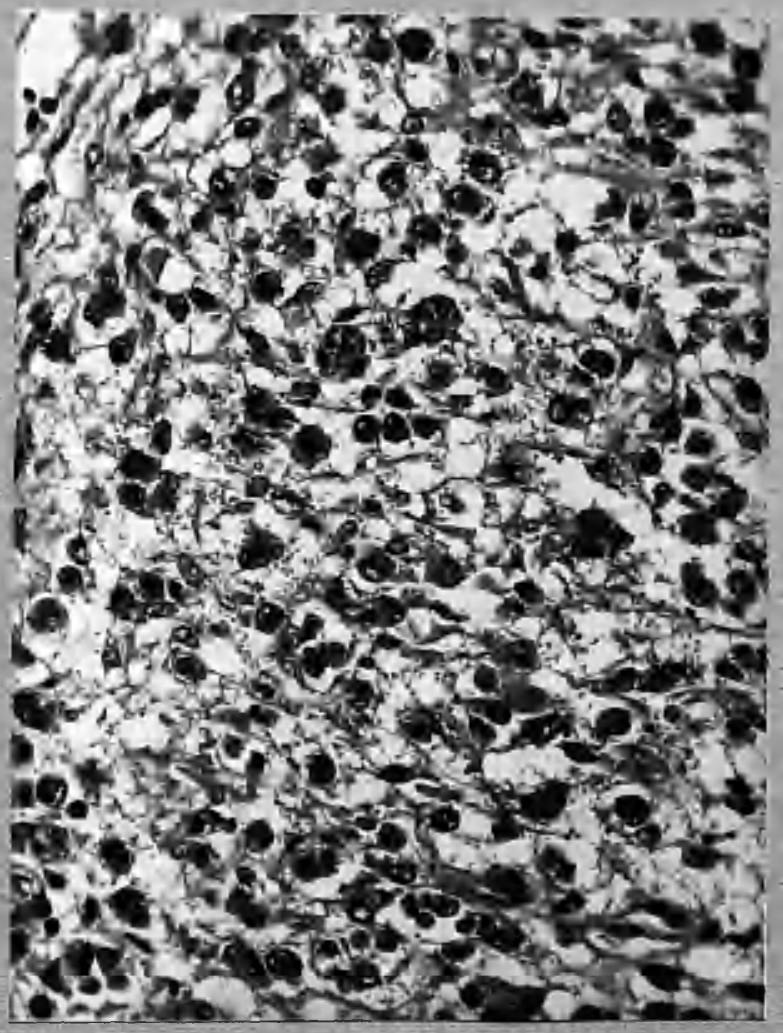

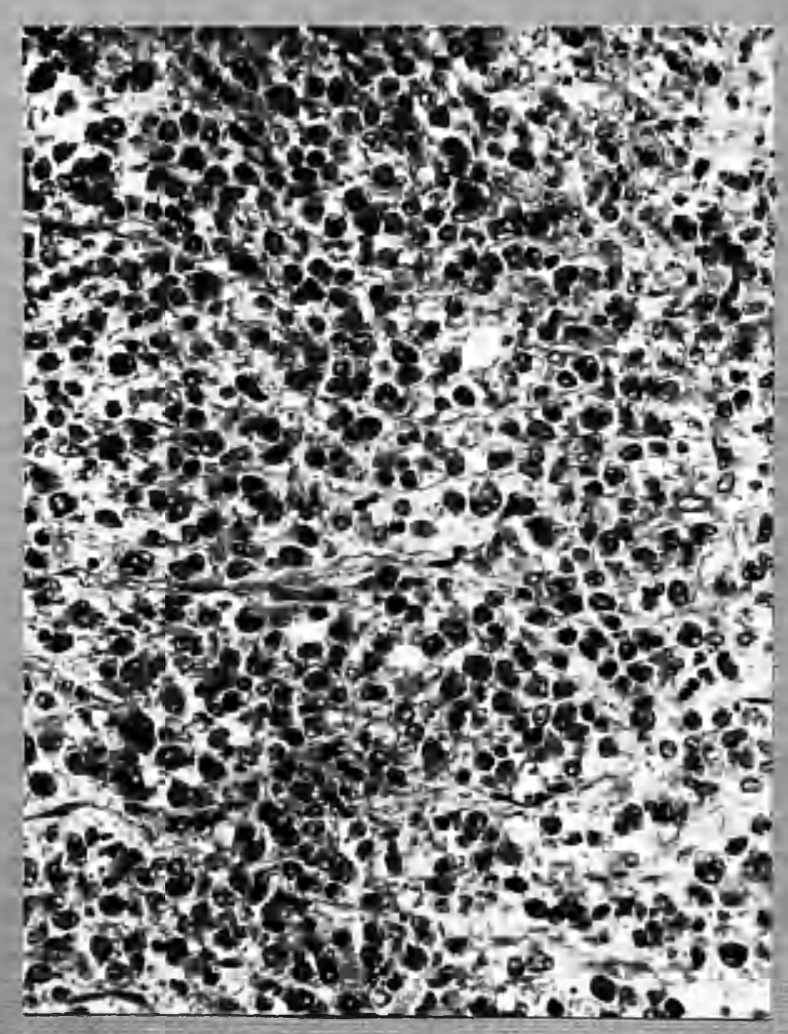

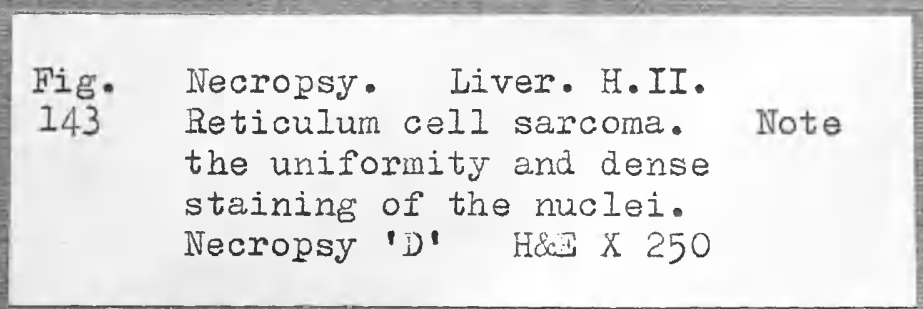




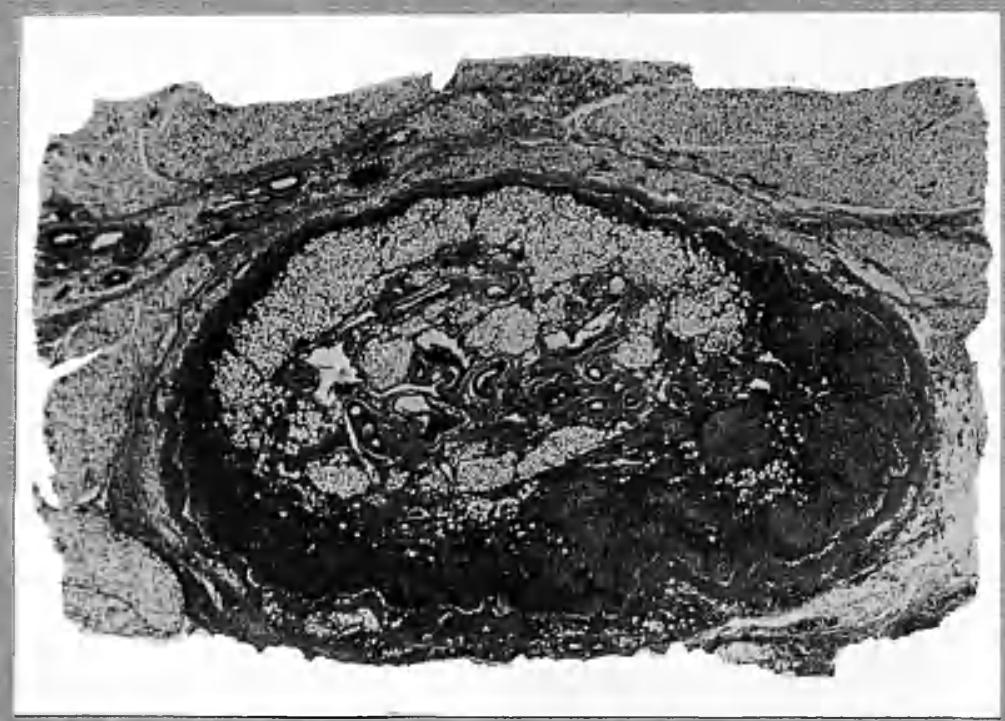

Fig. Necropsy. Left axillary lymph
144 node shewing fat atrophy. Note
the follicles and nornal marginal
sinus.
Necropsy 'j' Hä X IO




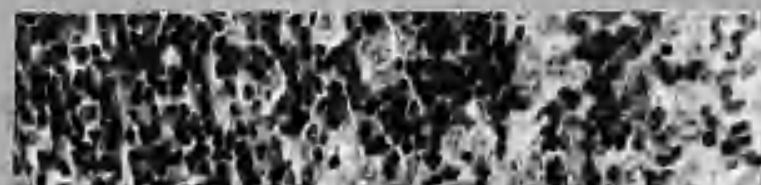

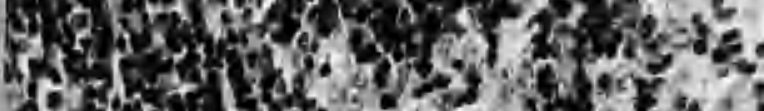

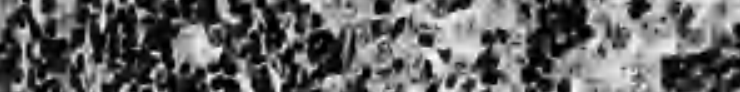
1.

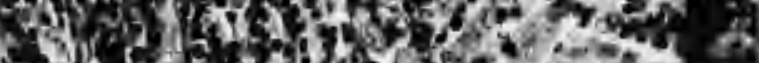

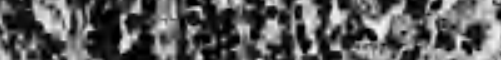

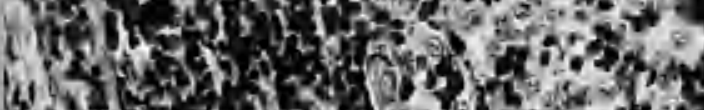

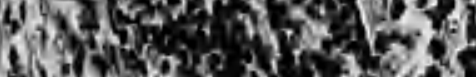

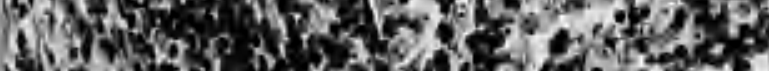

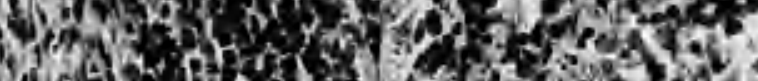

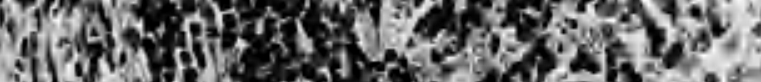
shy

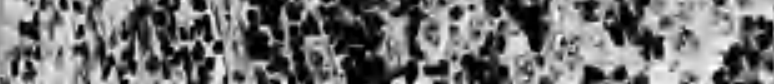

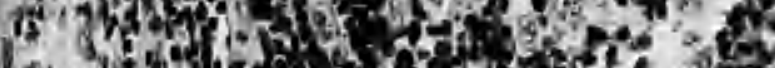
(a) 35 ,

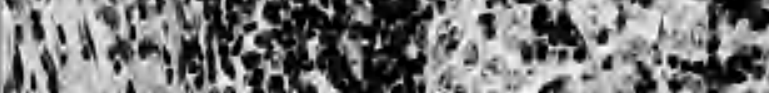

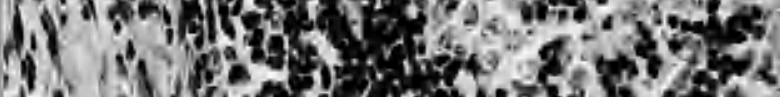

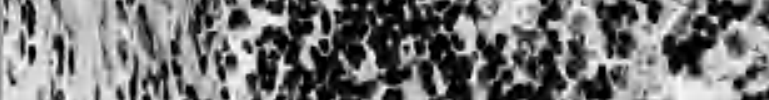
15 ind 1 iv
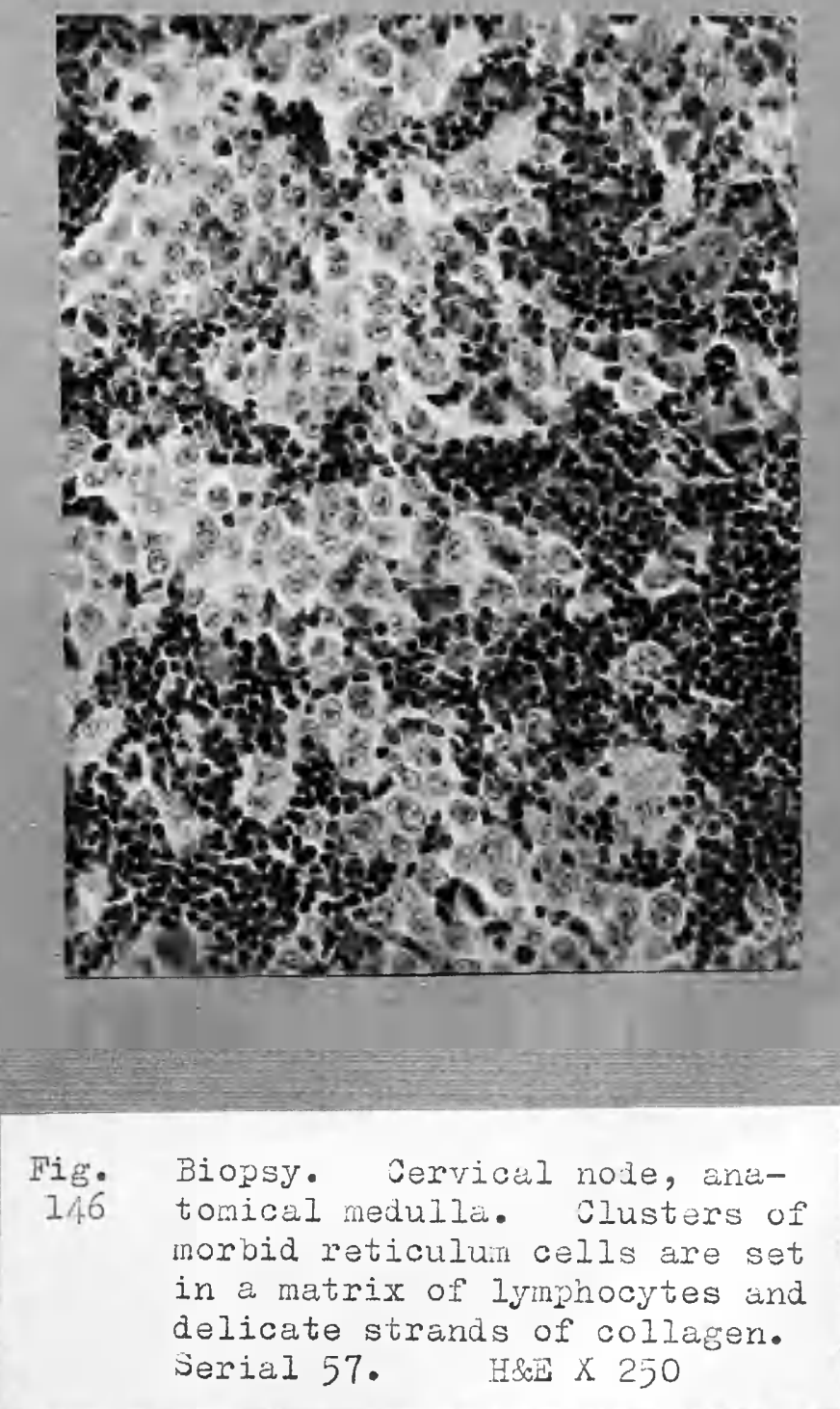

Fig. Biopsy. The edge of the
145 cervical node. On the left
the capsule is visible adjacent
to a zone of crushed lymphoid
tissue. The right side of the
field contains the new growth
with very numerous ageregated
molbid reticulum cells among
which mitoses are freguent.
serial 57 . Hei $x 250$


oriso

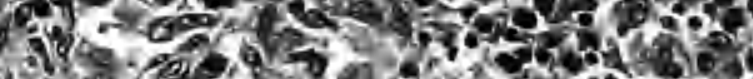

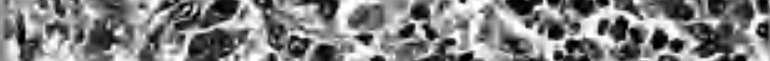

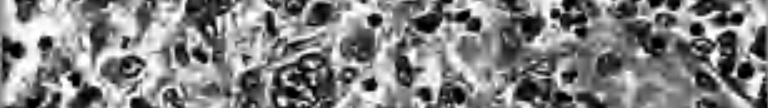

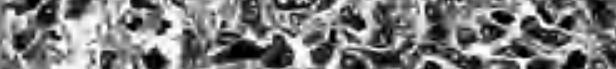
X r

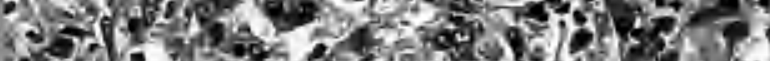

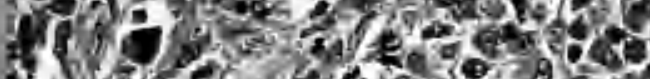

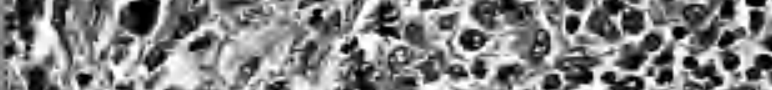

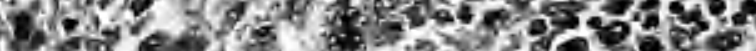

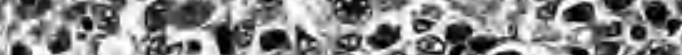

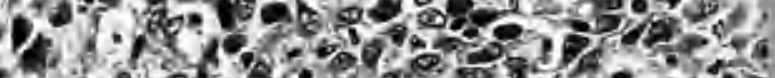

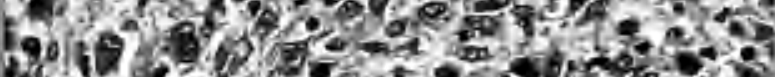

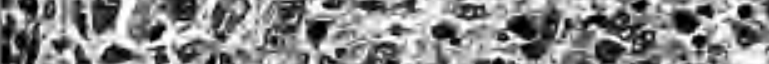

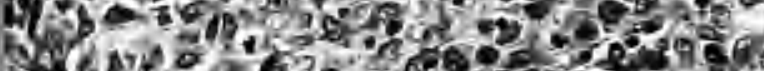

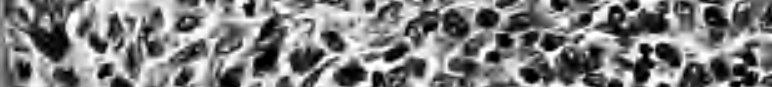

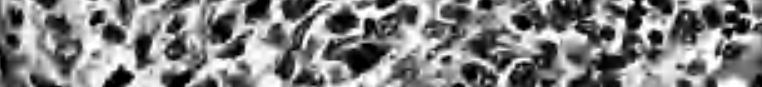

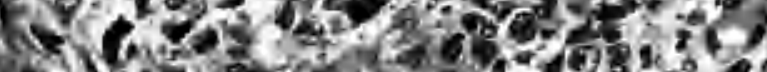

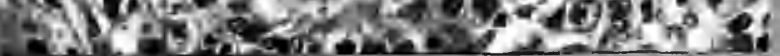

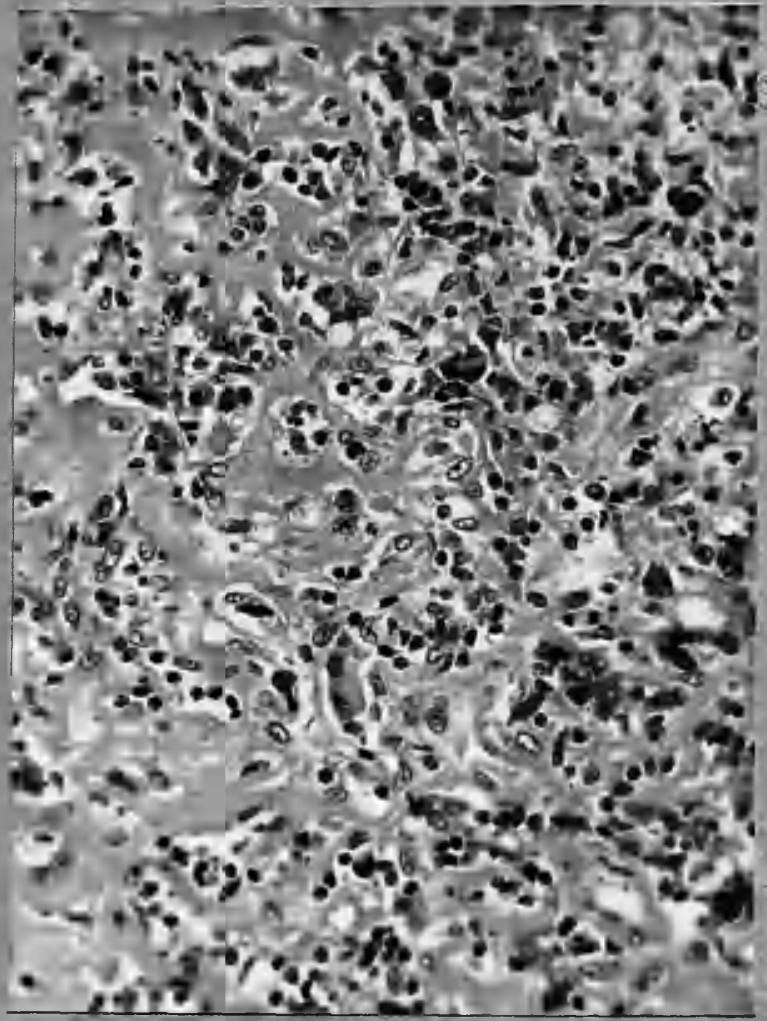

Fig. Necropsy * Para-arotic retro148 peritoneal lymph node.

Sclerosing Hodgkin's disease with numerous morbid reticulum cells. rint of this node presents this appearance. Necropsy ' $\mathrm{A}$ ' H\& X 250 giant-cells. Necropsy ' $\mathrm{H}$ ' HE $\times 250$

Hodgkin's sarcoma. Lymphocytes are very few and eosinophils almost absent. Note the pleiomorphism and numerous Hodgkin 


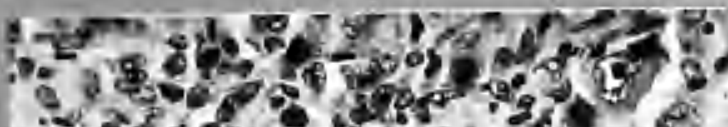

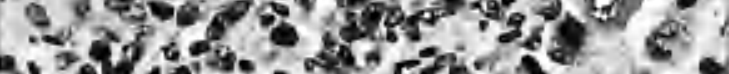

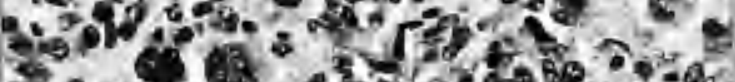

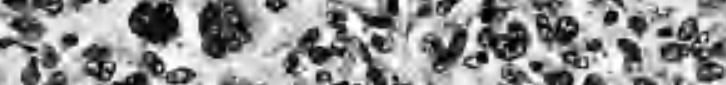

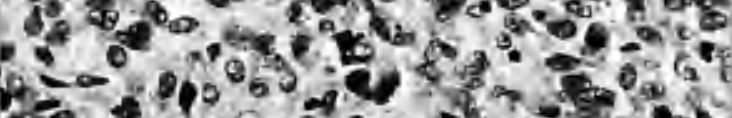

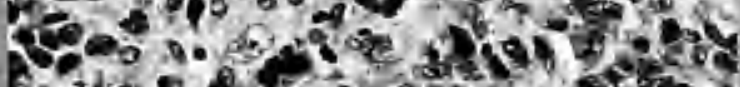

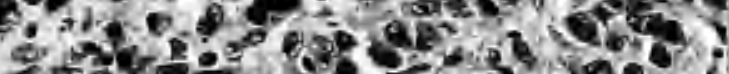

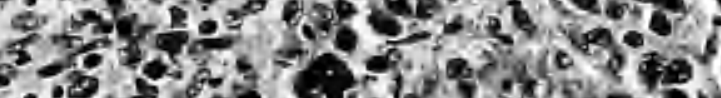

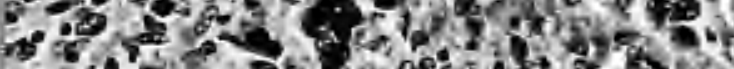

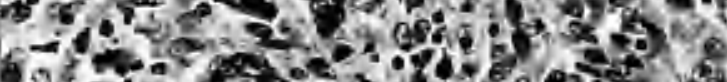
P8.

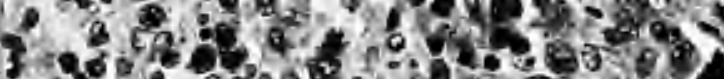
jas a yet as

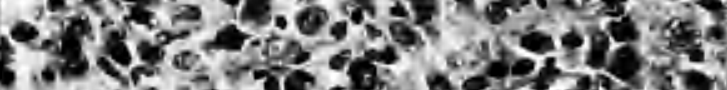

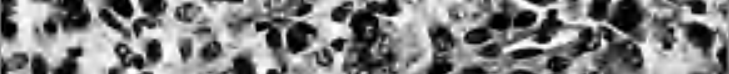

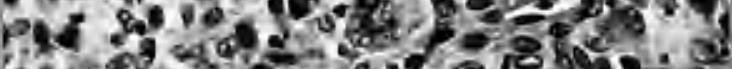

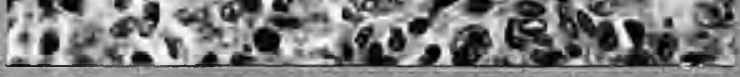

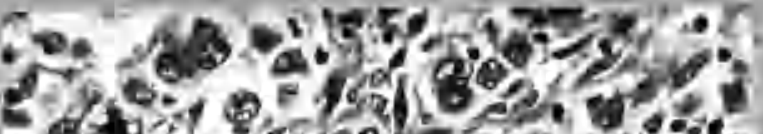

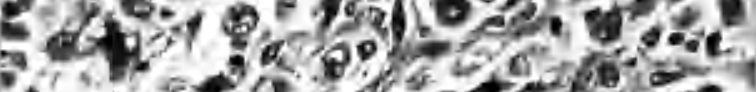

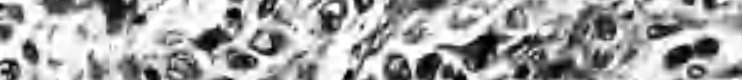

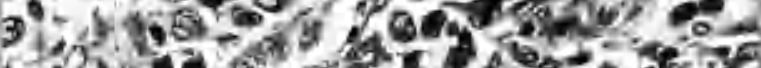

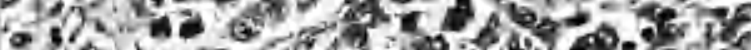

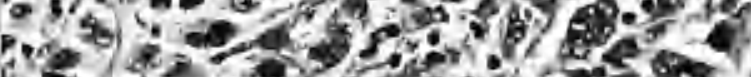

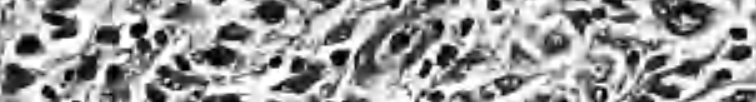

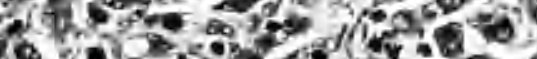

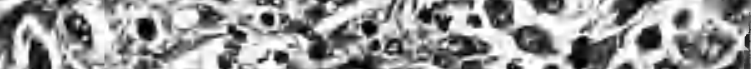

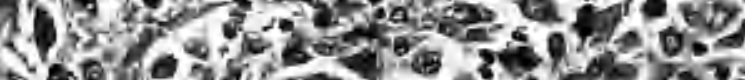

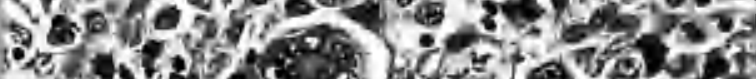

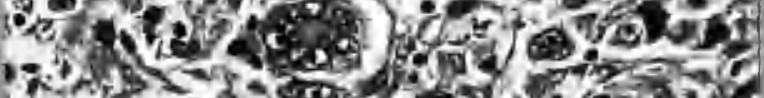
270 . Sorts?

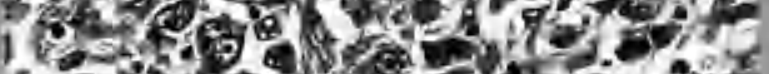

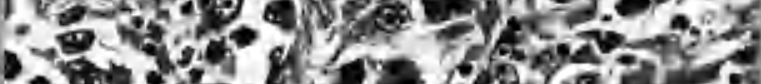

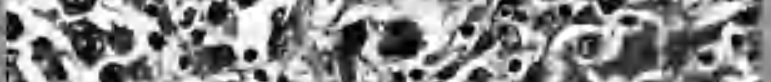
3.

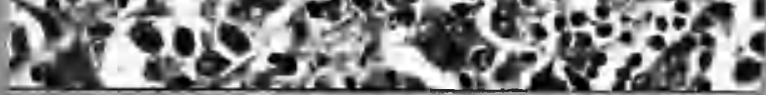

$$
\begin{aligned}
& \text { Fig. Necropsy. Iesentexic lymph } \\
& \text { 149 node 'B'. Iransition stage of } \\
& \text { the lesion. It is equivocal } \\
& \text { whether tins is the ordinary or } \\
& \text { sarcomatous form. } \\
& \text { Necropsy 's Hat } \times 250
\end{aligned}
$$

sig. Necropsy. Cervical lymph node.

150 Hodgkin's sarcoma, sclerosing.

Note the numerous Hodgkin jintcells.

Necropsy ' $\exists$ ' H\&w X 250 


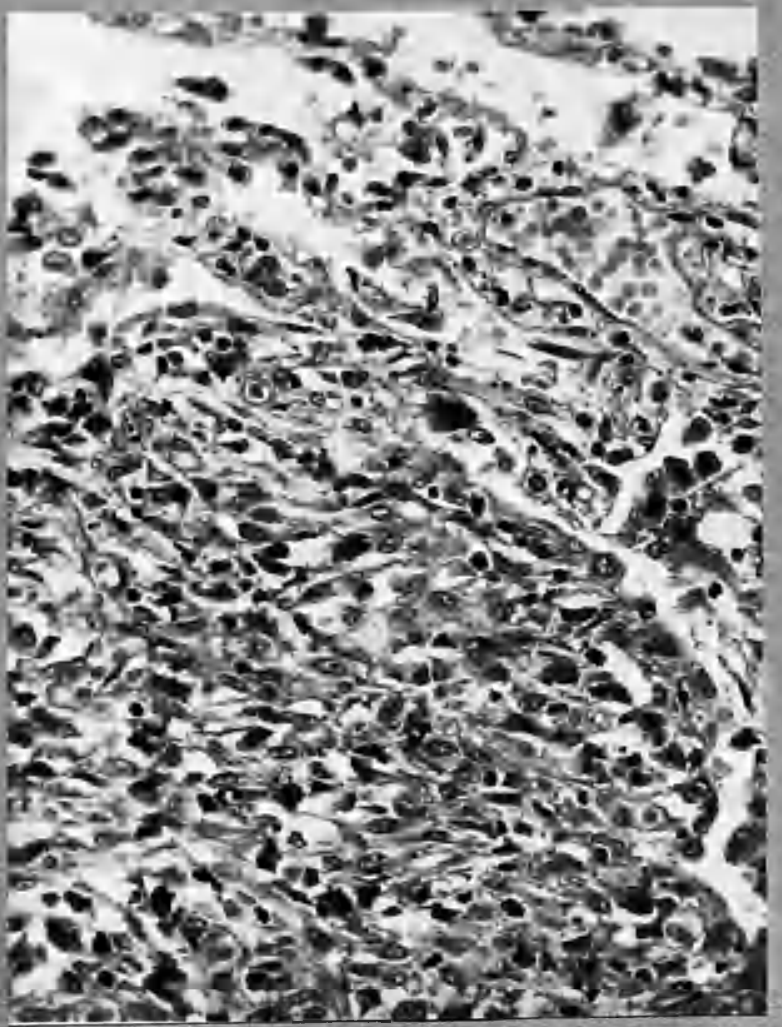

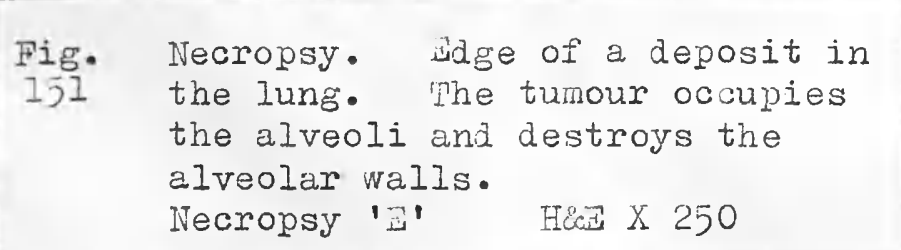

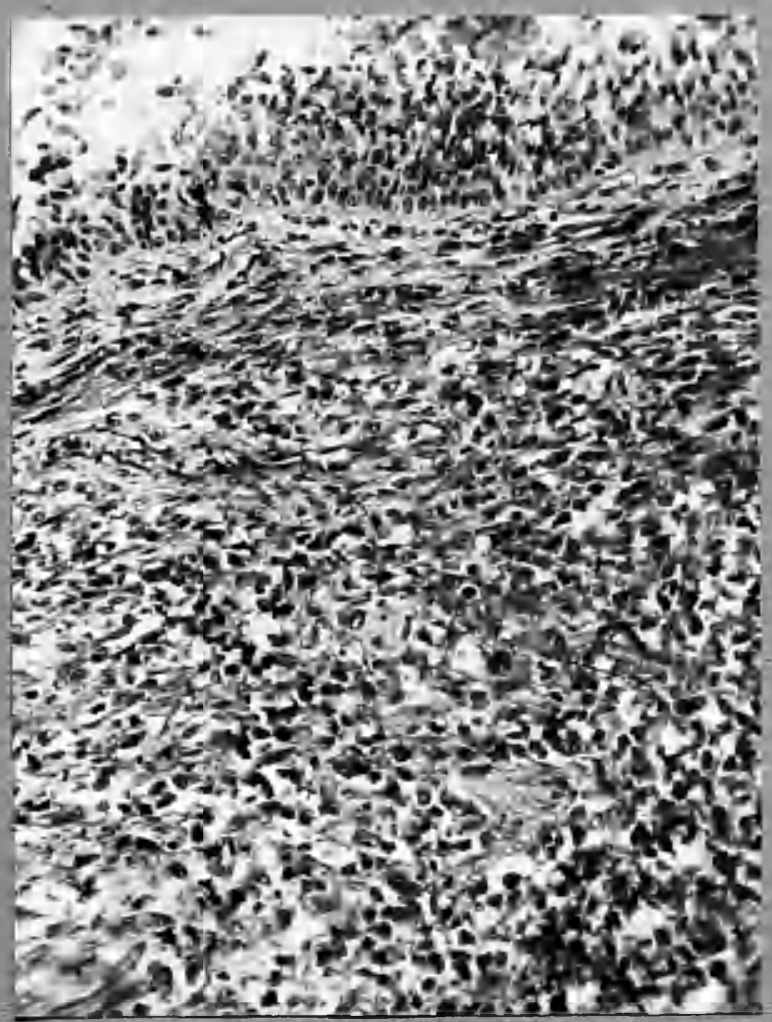

Pig* 152

Necropsy. Trachea. The ciliated columnar epithelial lining is visible in the upper edse of the field. The submucosa is occupied by tumour which has destroyed most of the native tissue. Necropsy 'I' $\mathrm{H} \& \mathrm{E} \mathrm{E} \times 150$ 


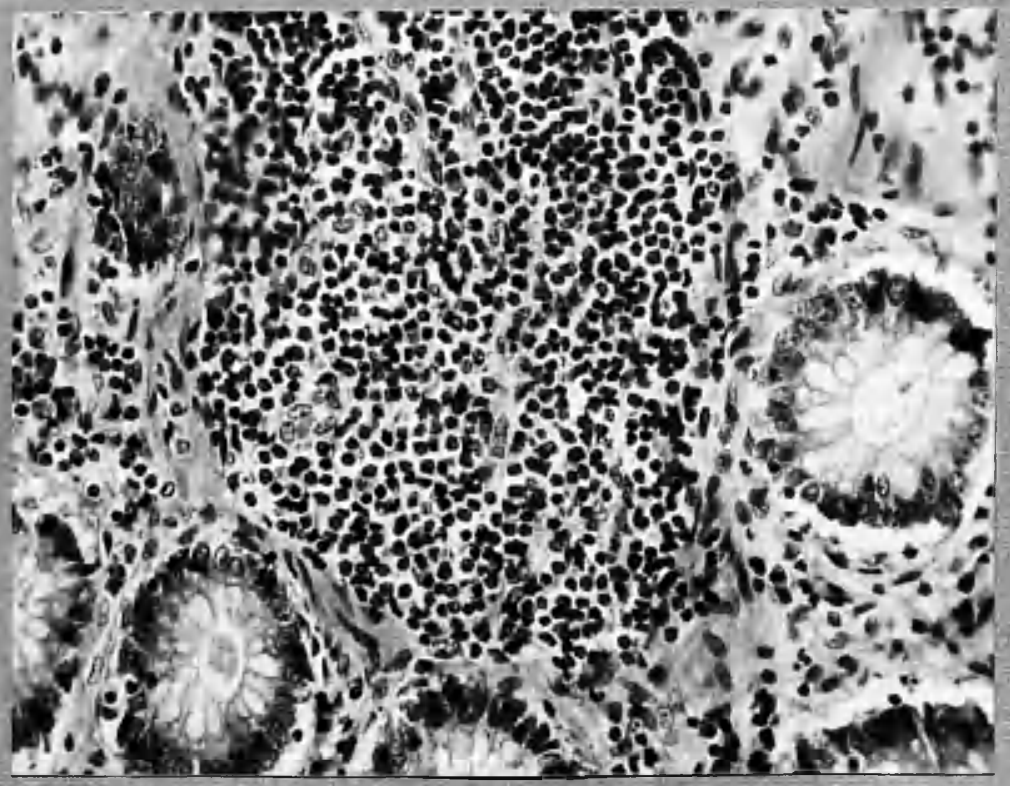

Tig.

Necropsy. Solitary follicles of

153

normal lymphoid tissue in the

colonic submucosa.

Necropsy 'a' IH\& X 250 


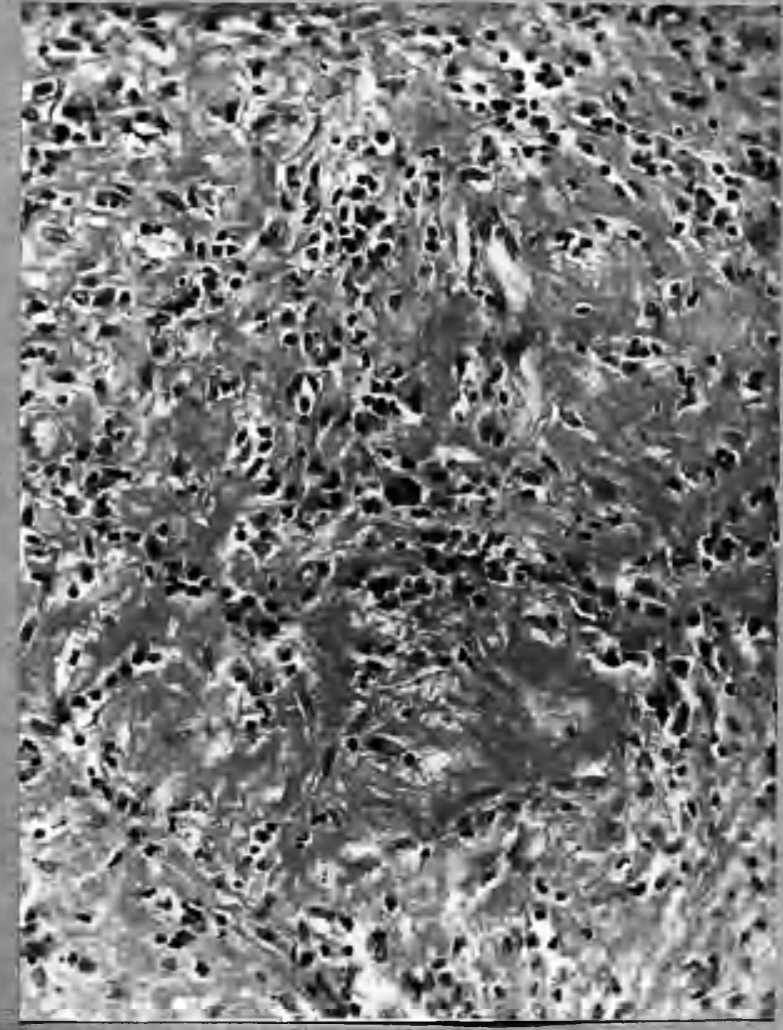

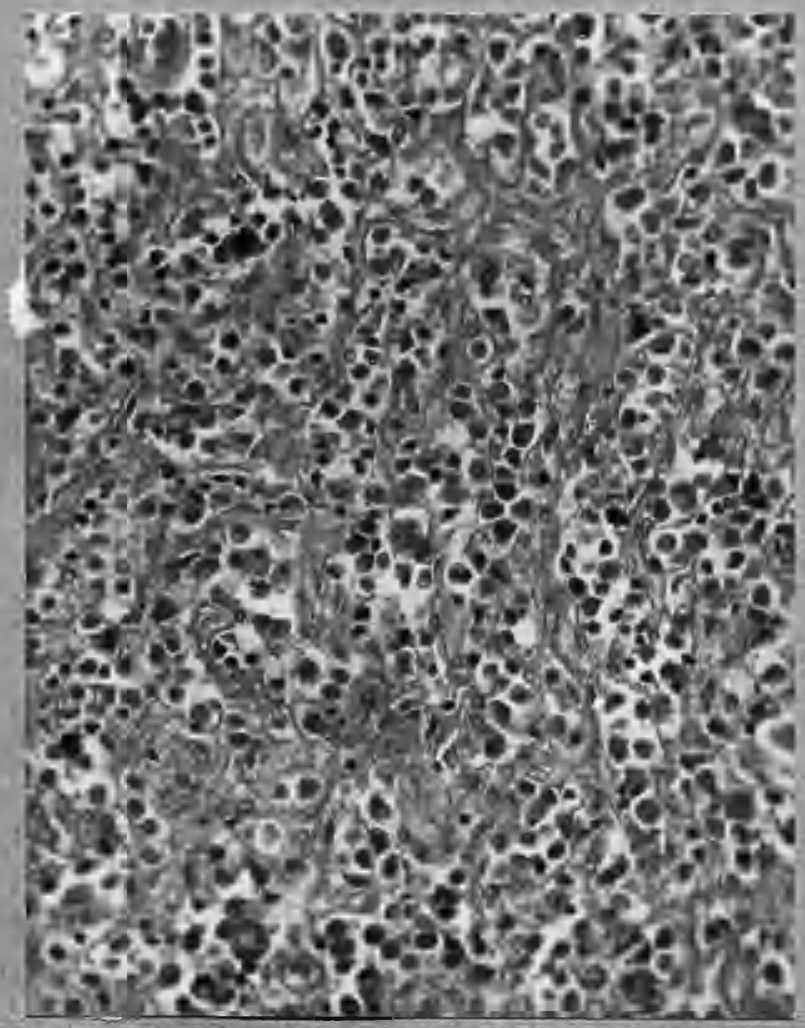

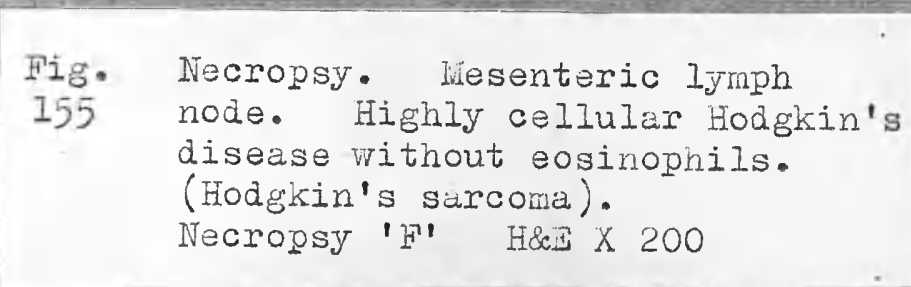




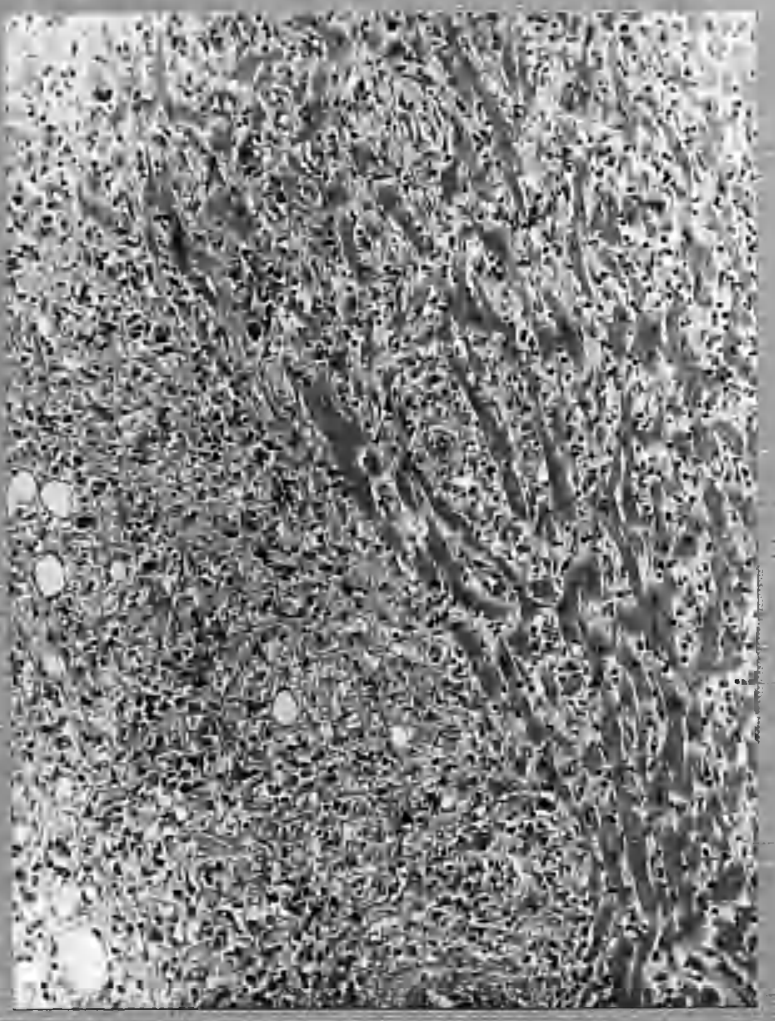

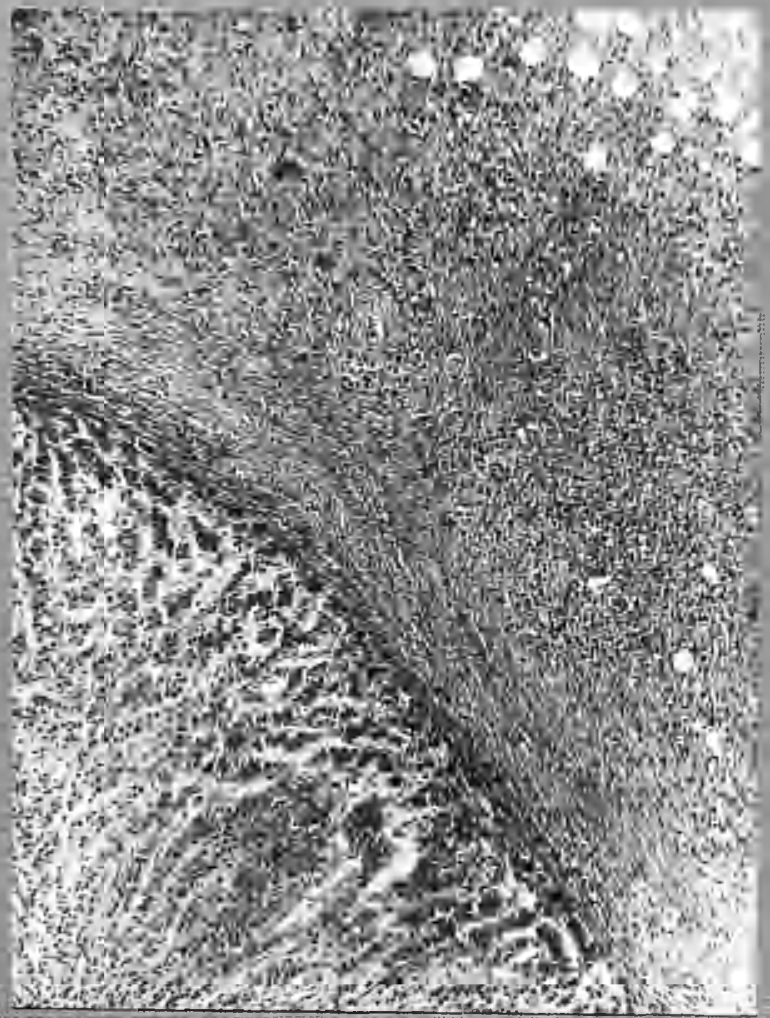

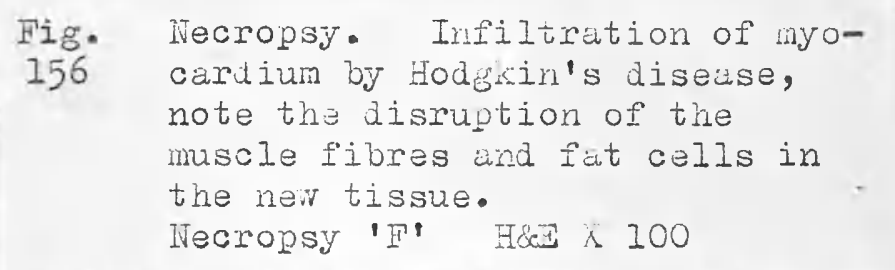

Fig. Necropsy. Infiltration of nyonote the disruption of the mascle fibres and fat cells in ivecropsy 'P' HeE 100

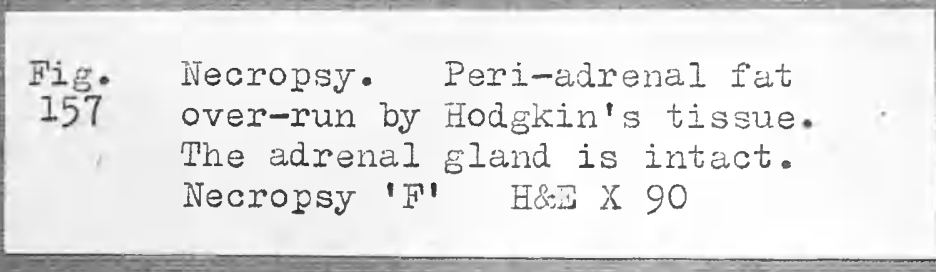




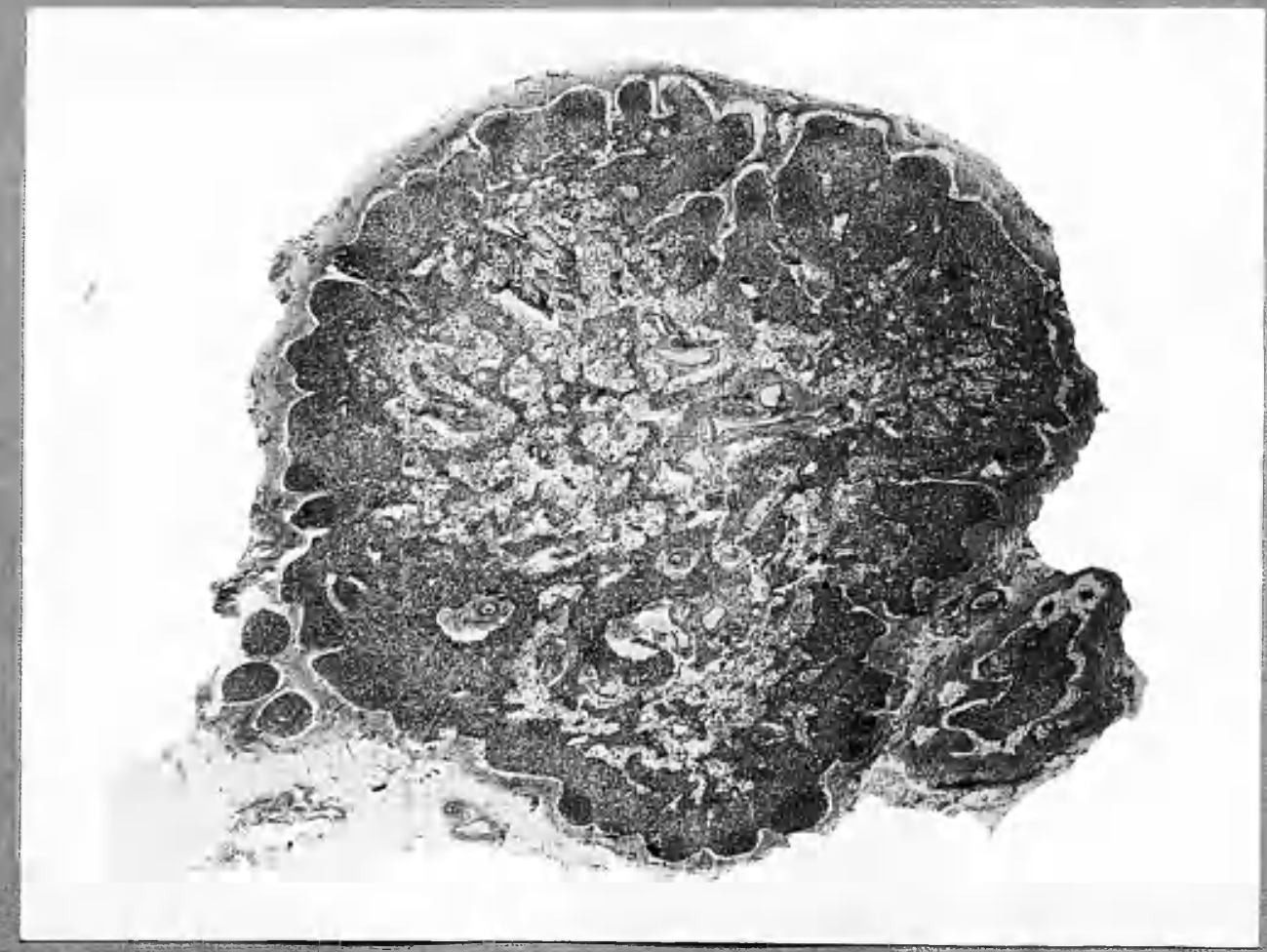

Fig. Necropsy. Inguinal lymph node 158 unaffected by the disease. Necropsy ' $F$ ' H\&Q X 12 
sily

$\rightarrow$ ?

(5).

is 01 - in

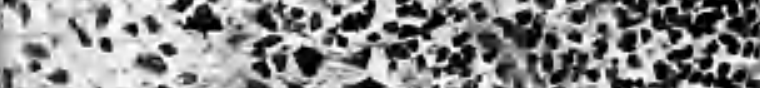

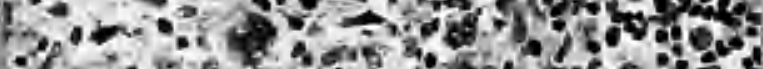

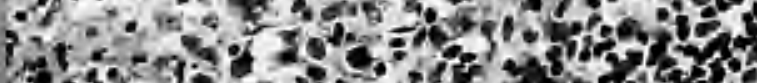

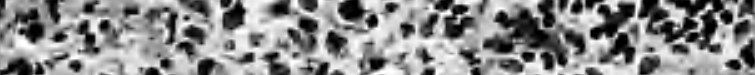

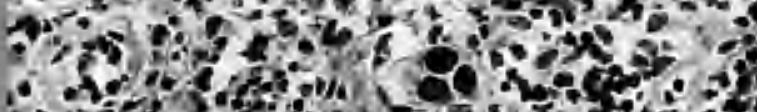

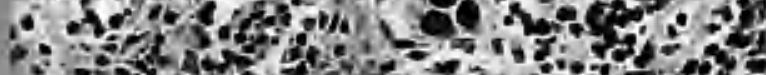

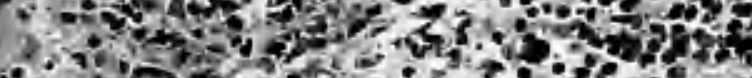

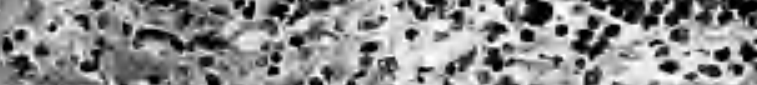

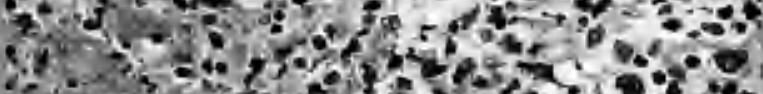

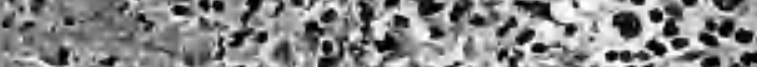

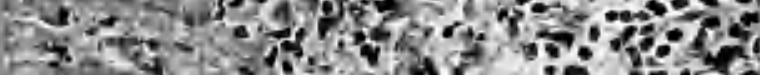

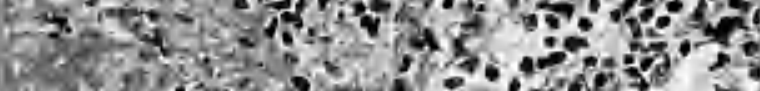

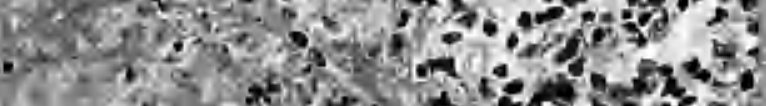

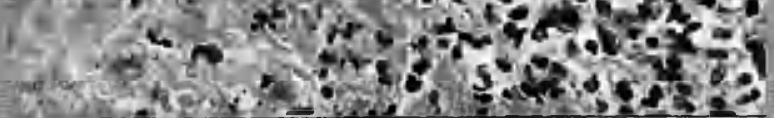
Fig.
Biopsy. Right inguinal node;
159
established Hodgkin's aisease. Note the pleiomorphic reticulum cells, some of which are necrotic. The homogeneous material at pottom left is caseous. Serial 218 $H \& \mathrm{E} \quad 250$

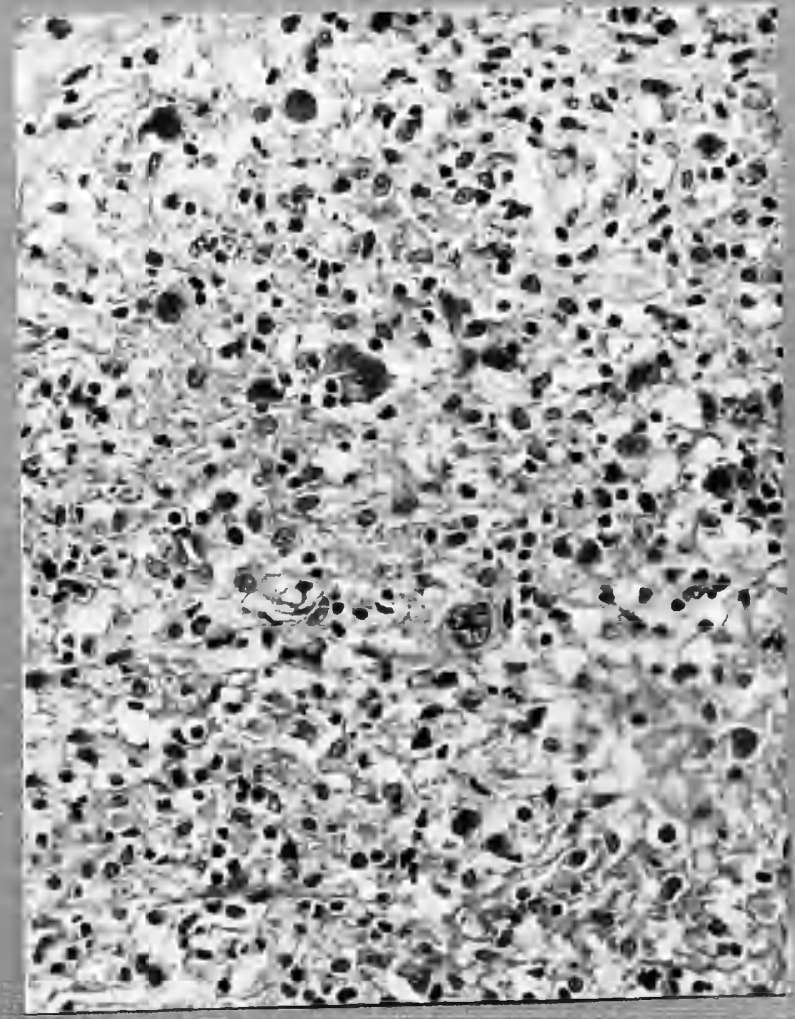

Fig. Necropsy. Right inguinal node;

160 Waturing Hodgkin's disease.

Note fine diffuse fibrosis and paucity of lymphocytes. Necropsy 'G' Hice X 250 


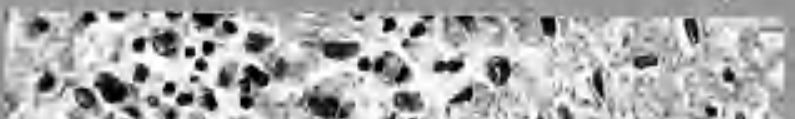

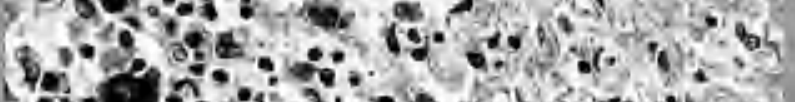

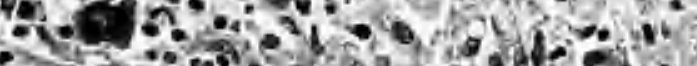

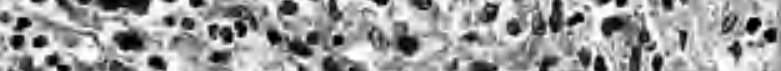

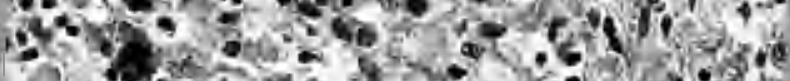

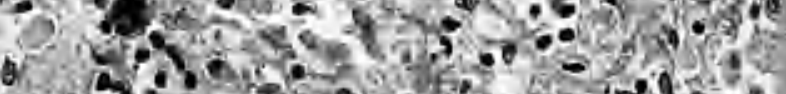

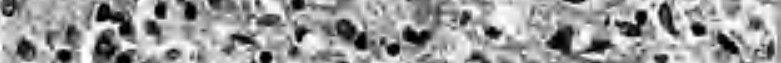

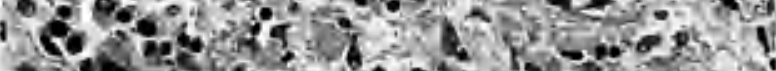

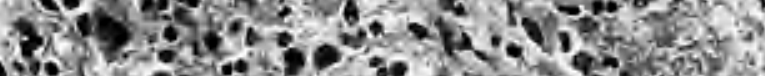

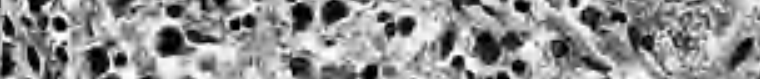

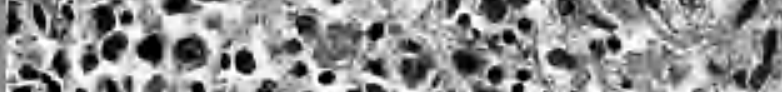
-

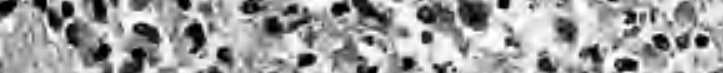

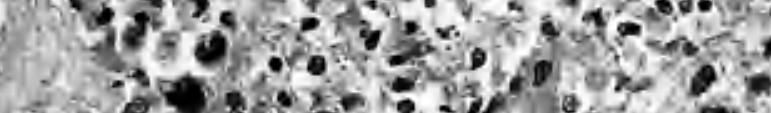

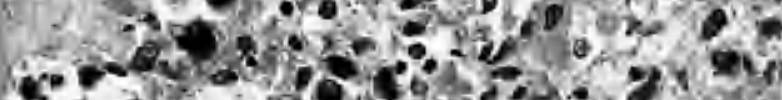
s.

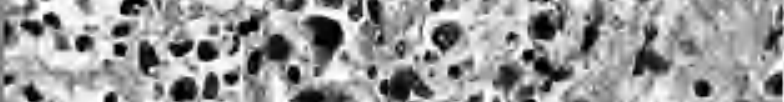

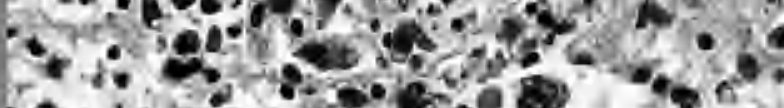

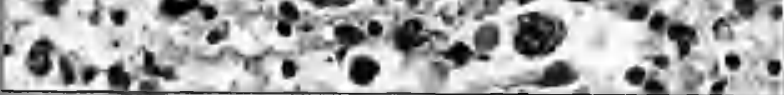

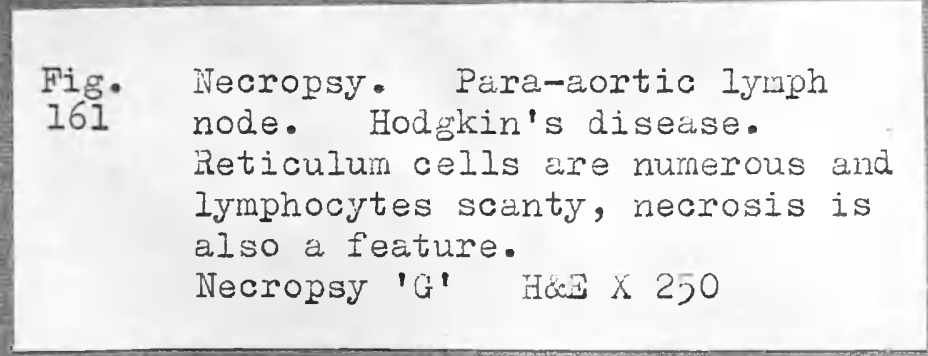

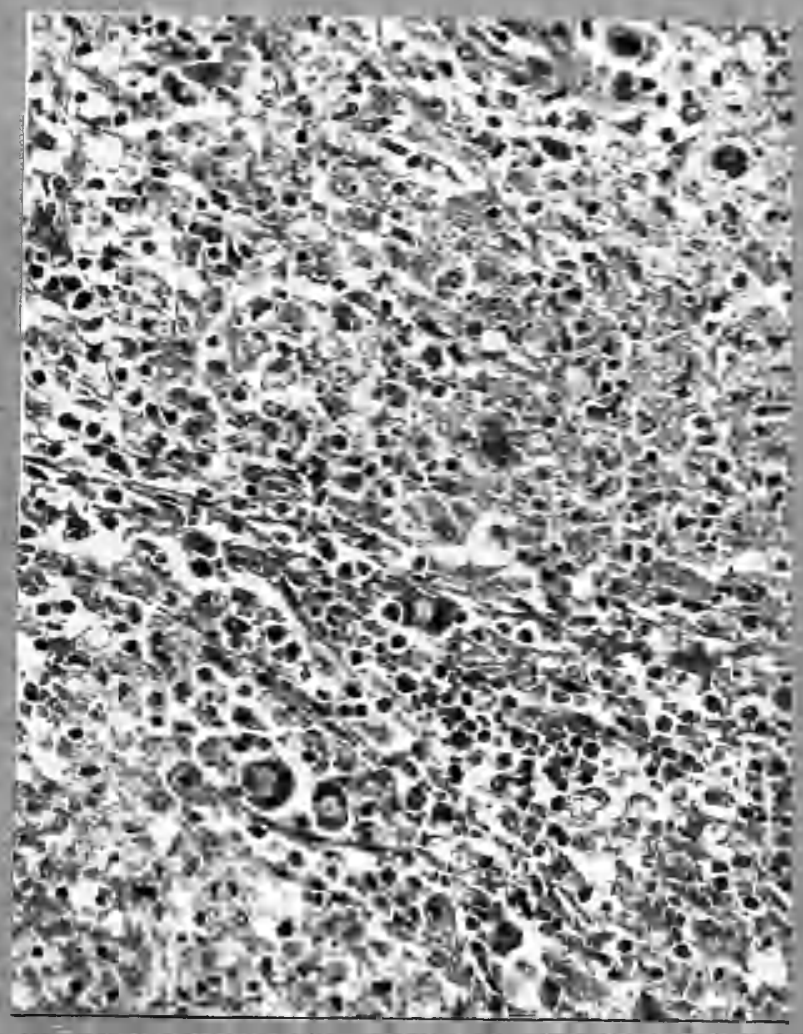

$$
\begin{aligned}
& \text { Fig. Necropsy. Spleen. Hodgkin's } \\
& \text { 162 diseuse. Jxtensive incipient } \\
& \text { necrosis is present. } \\
& \text { Necropsy 'G' H\&Z K } 250
\end{aligned}
$$




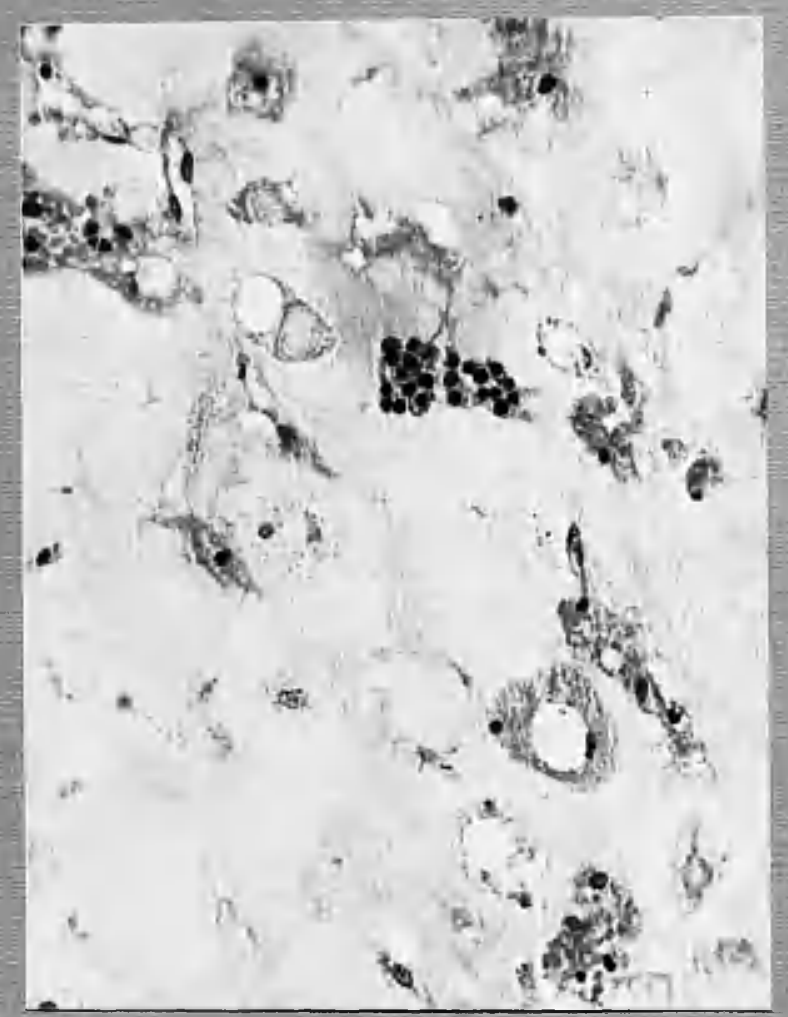

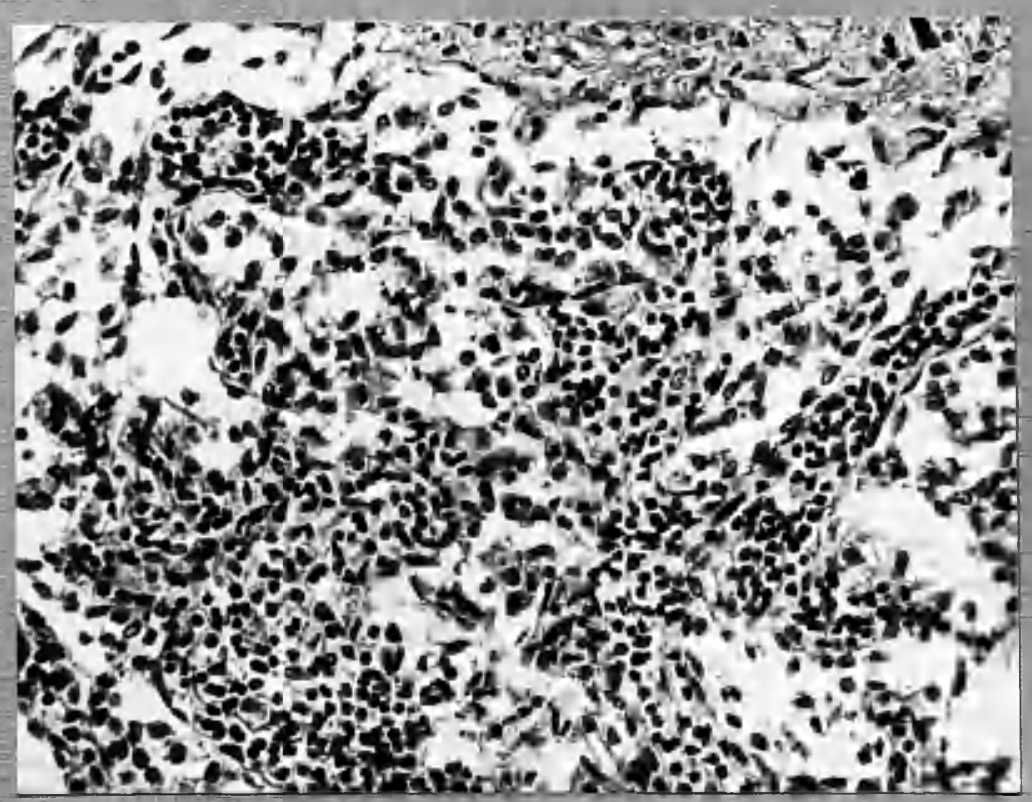

Fig. Necropsy. Wesenteric lymph

164 node. The capsule is at the

top of tine plate. sinus

catarrh of dropsical type is

seen. The Iymphoid tissue is

hypocellular but clear of the

disease.

Necropsy 'G' Hei $\mathrm{X} 250$ to artefact. Necropsy 'G' Hea X 250
Fig. Necropsy. Femoral bone marrow. represents the largest survival islet of haemopoietic tissue in this section. The gelatinous material appears fibrillary due 


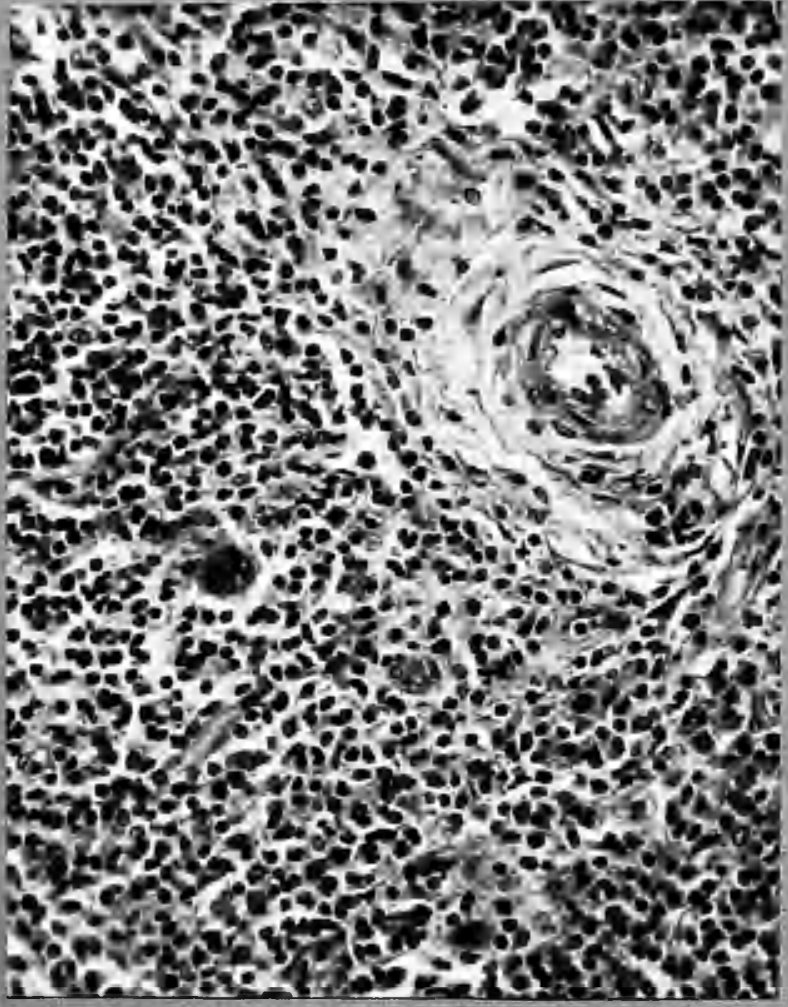

\begin{tabular}{|c|c|}
\hline $\begin{array}{l}\text { Fig. } \\
165\end{array}$ & 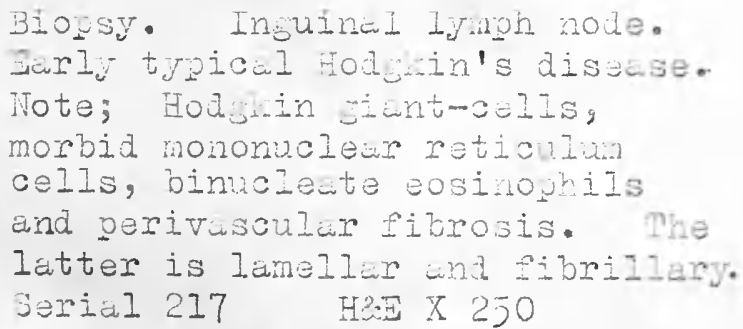 \\
\hline
\end{tabular}
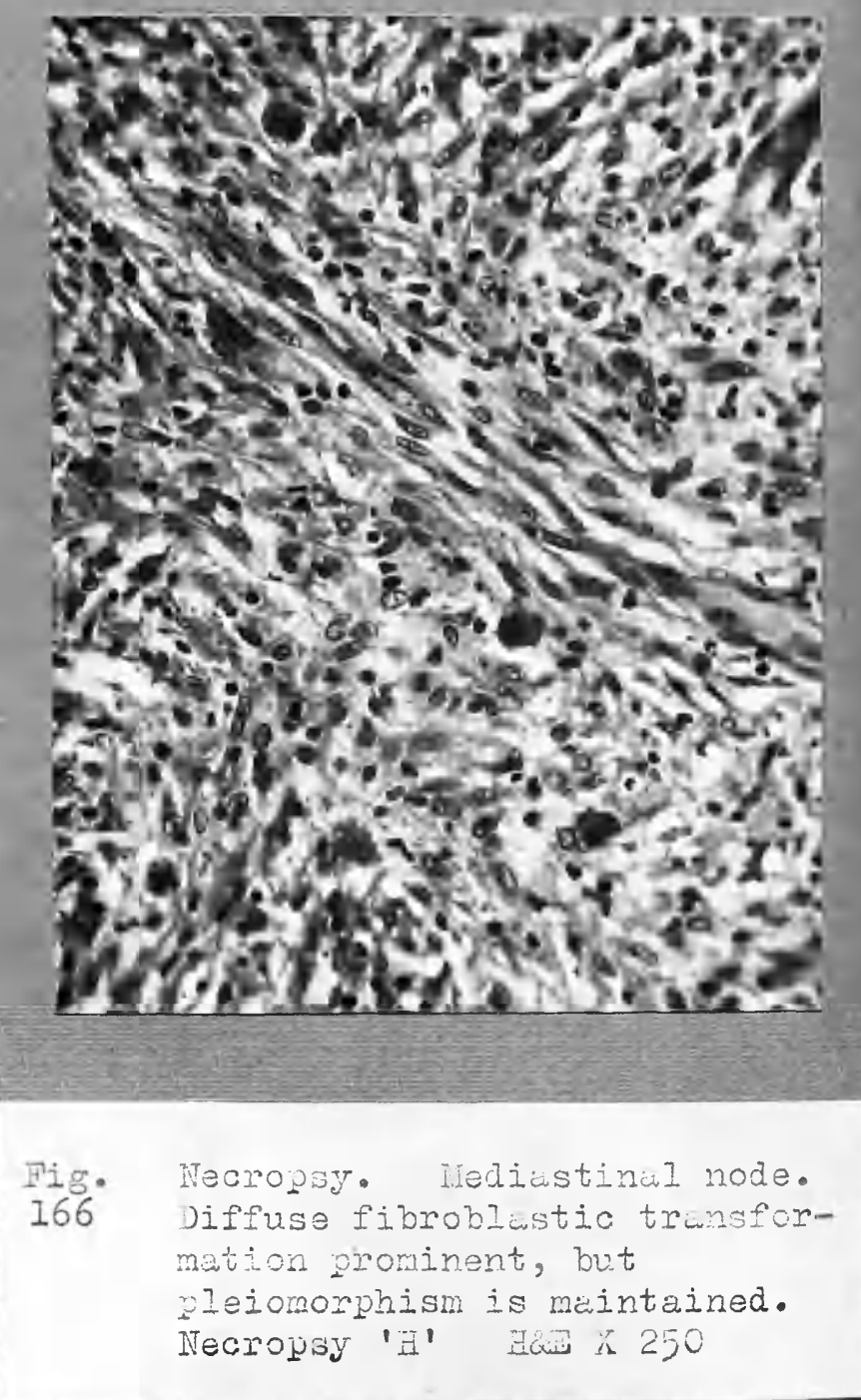


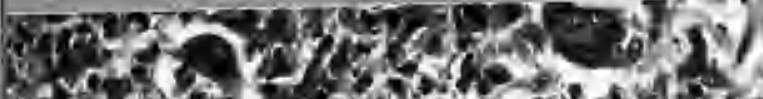

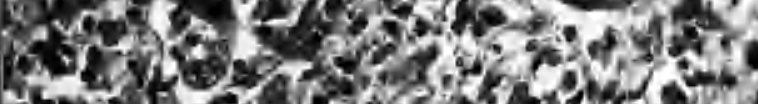

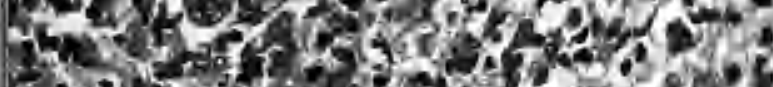

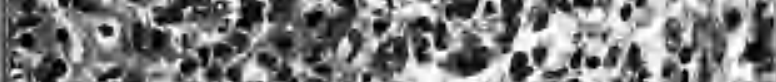

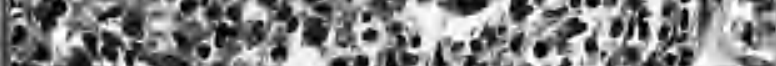

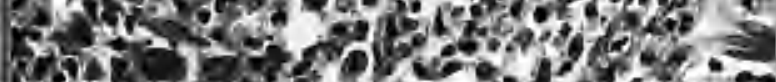

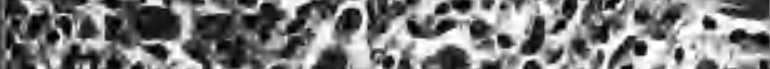

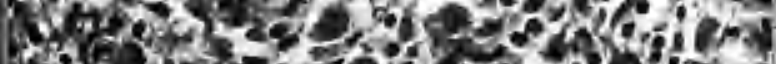

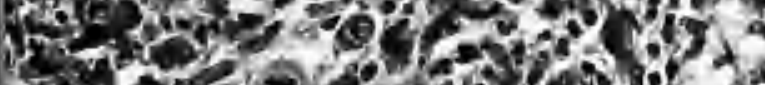
C. 3 -

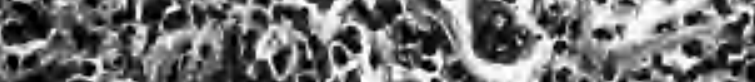

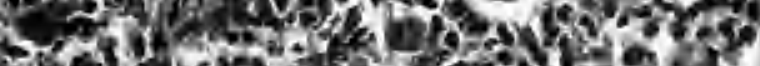

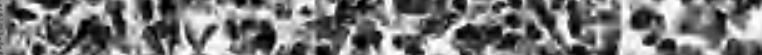

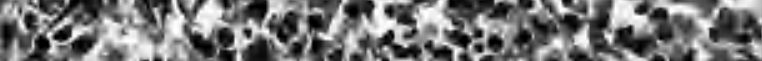

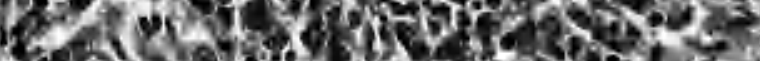

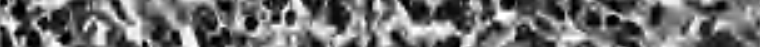

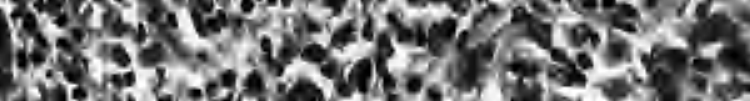

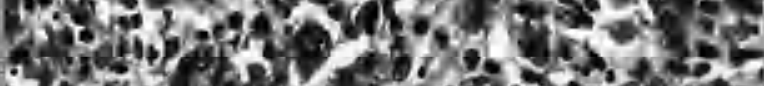

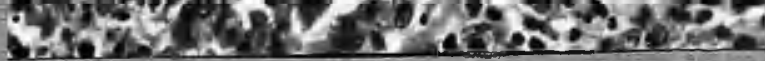

Fig. Necrops. Getropentionas

167 node. पri gly pleionorphic

cellular Hodghin's disease.

Bosinophils wre retuined.

Tecropsy ' ' t'

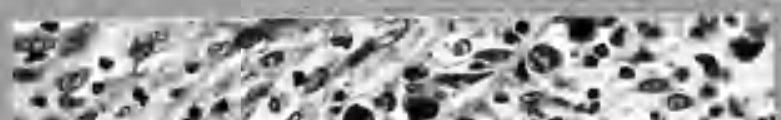

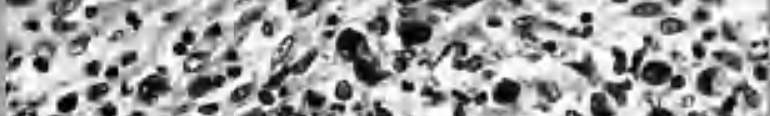

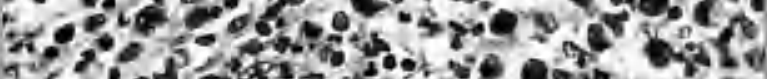

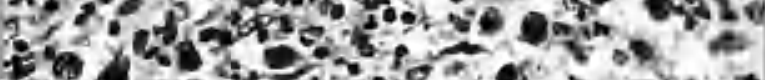

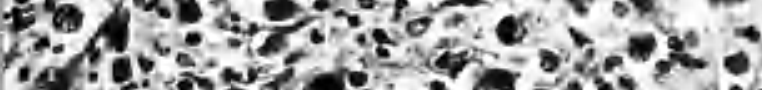
"Af as ins

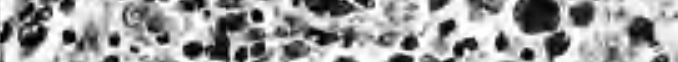

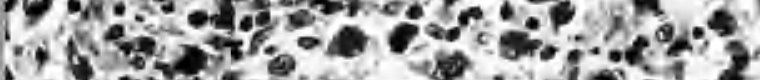

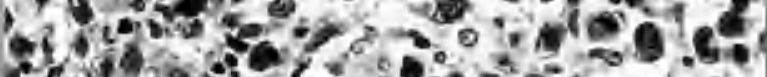

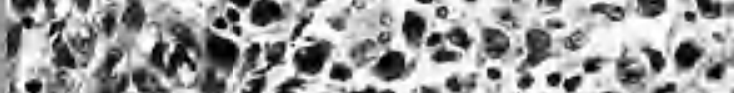

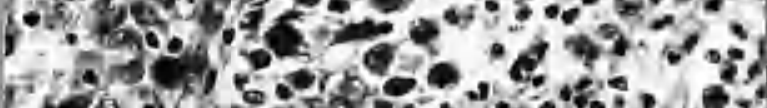

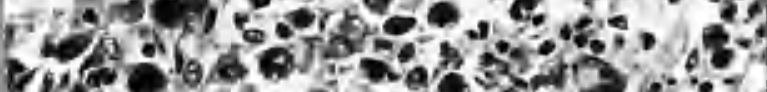

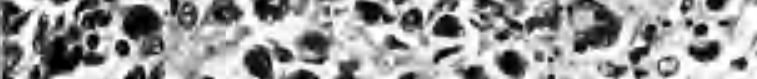

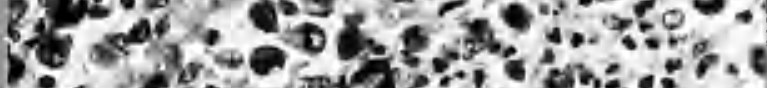

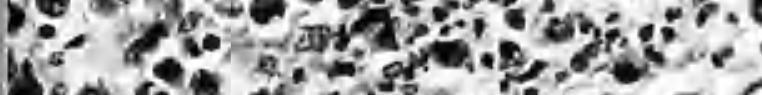

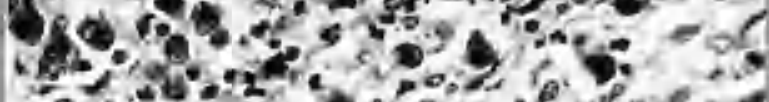

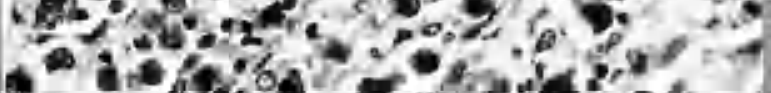

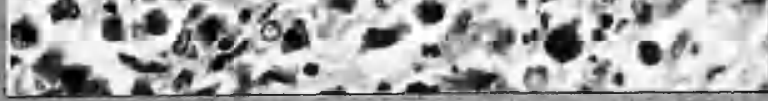

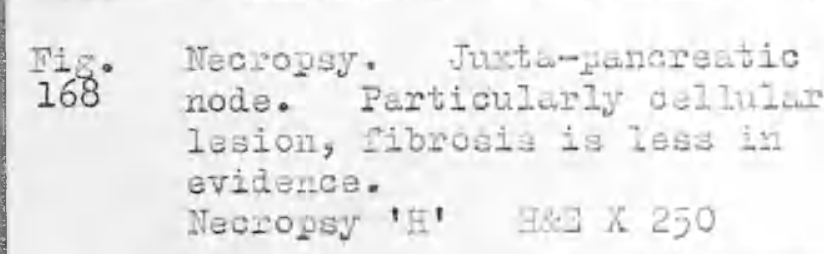




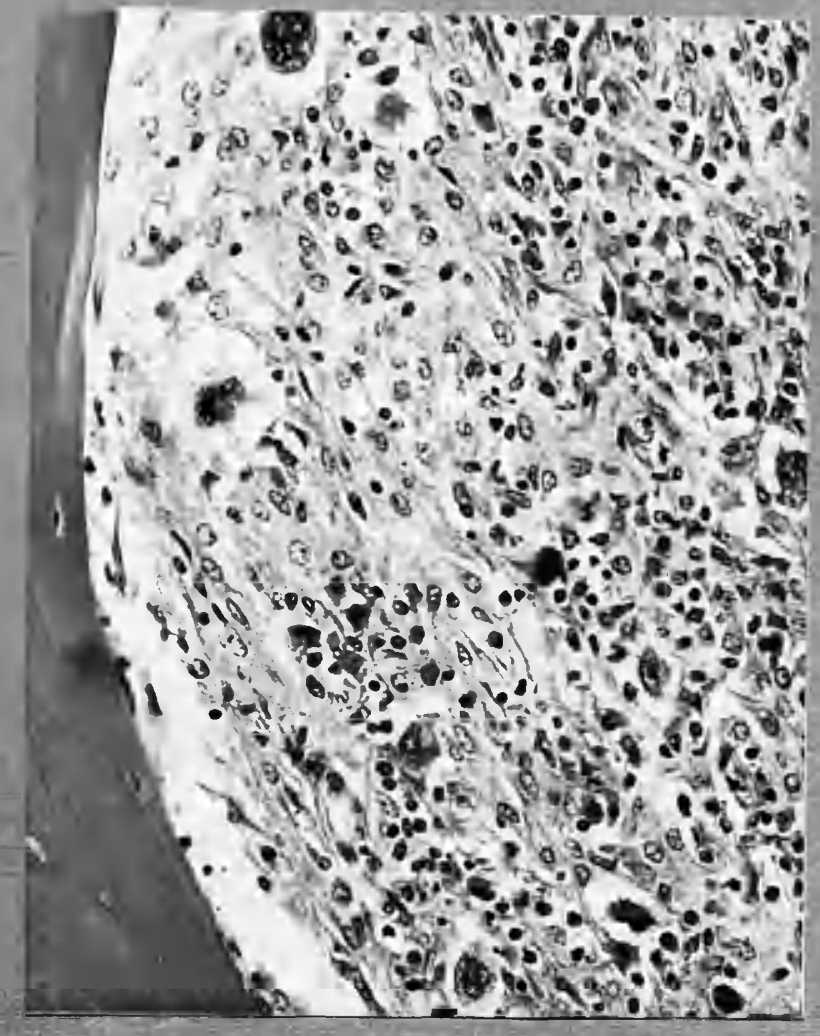

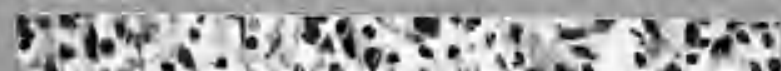

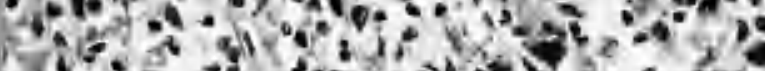

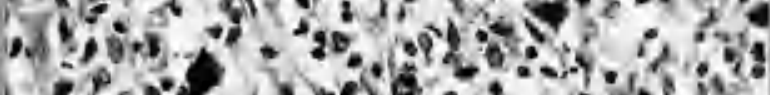

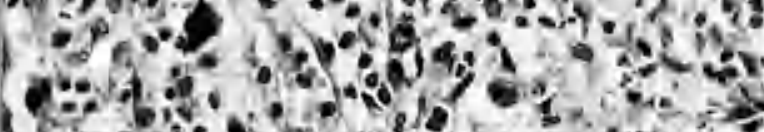

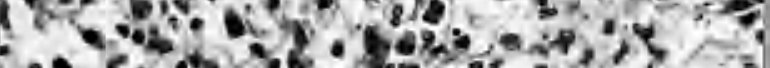

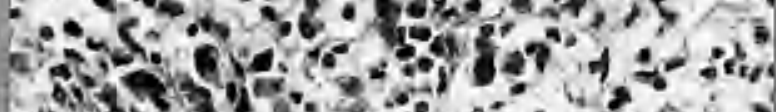

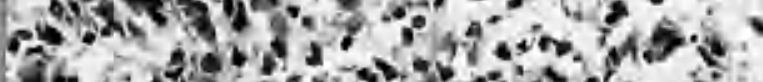

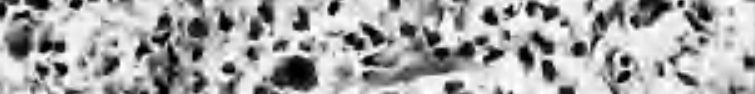

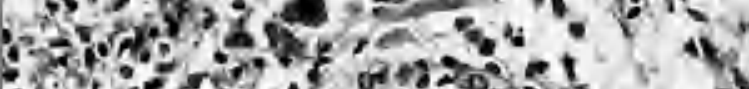

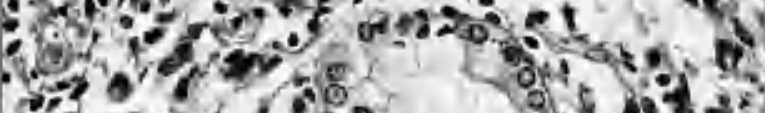

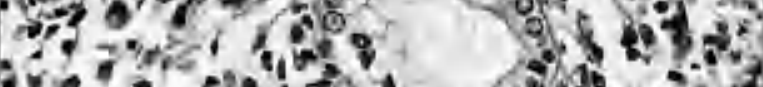

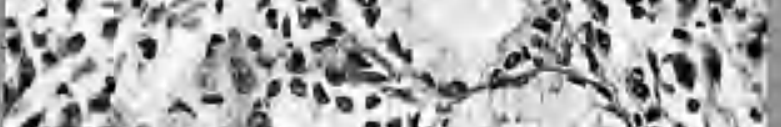
Abs.
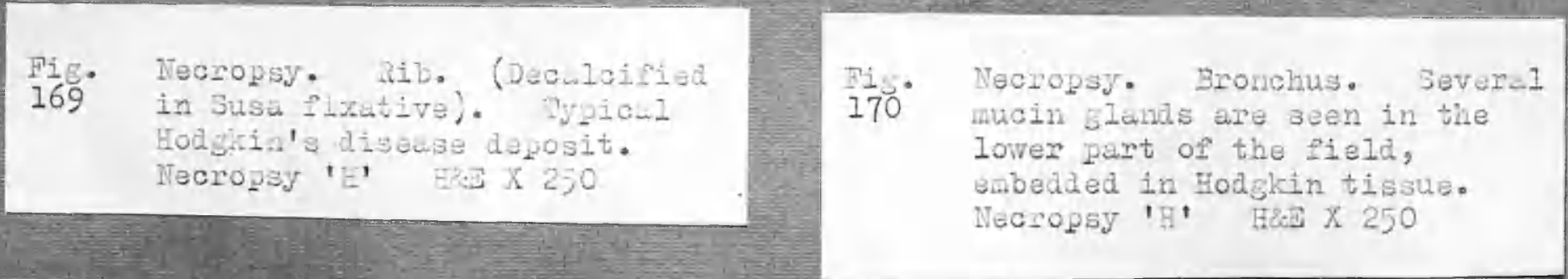

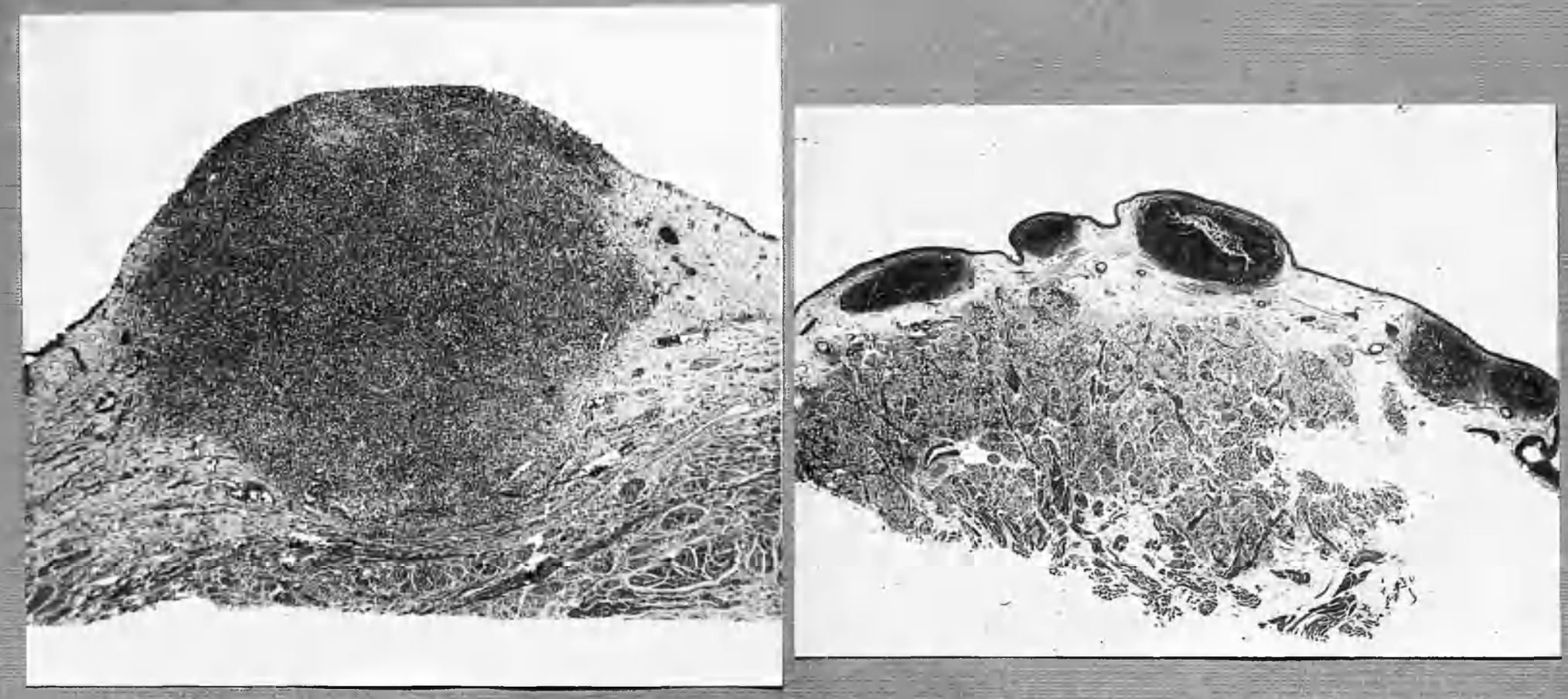

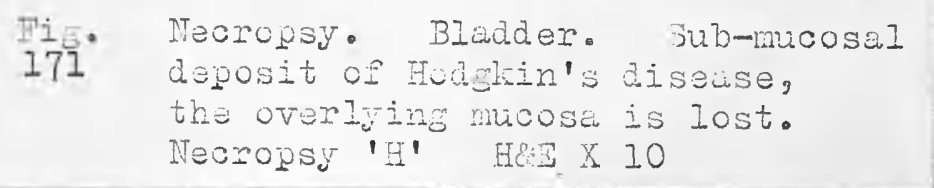

Fig. litecropst. Buse of torgue.

172 Deposits of nontid lymold

tissue. Germinil oentres can just be raide out in two of the foci.

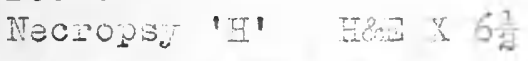




\section{SPECIAL NECPOPSY E.}

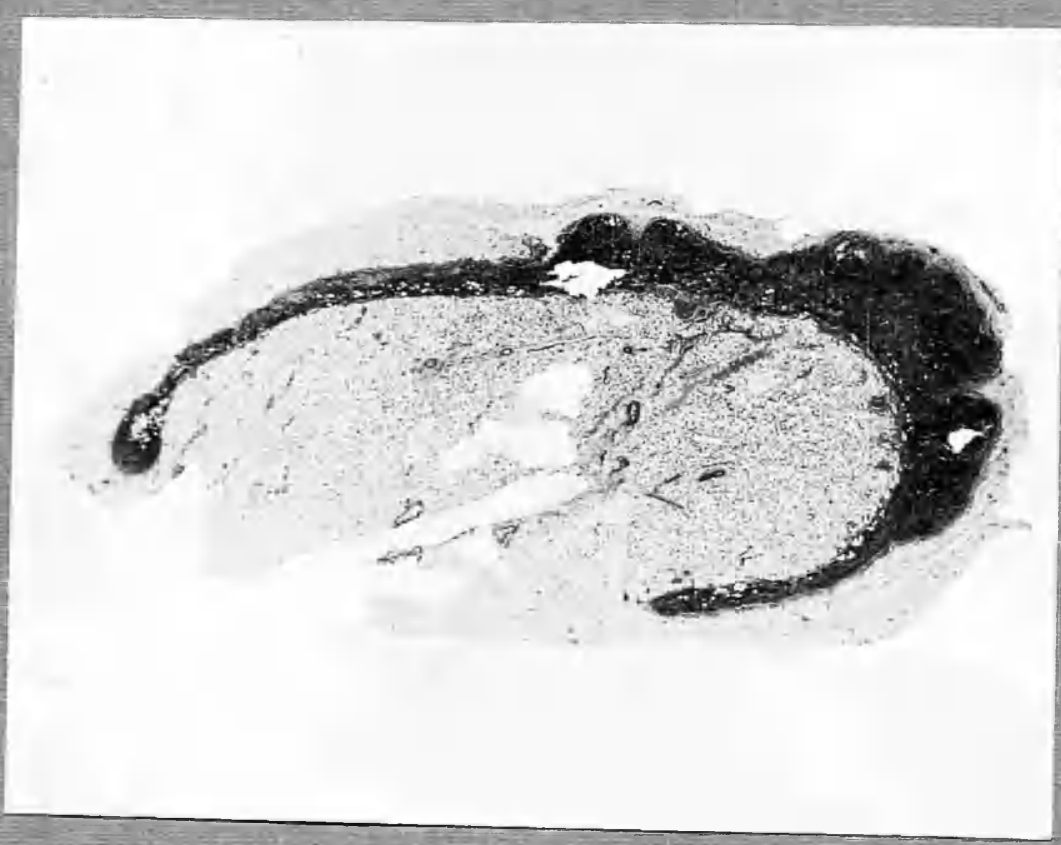

Pig, Nocropey. Ieft axillery lymph 173 hode. Advancel fut atrophy is present. The tissue is otherwise physiological. (The section is imperfect due to tritefuct). Tearopsy 'H' X 10 


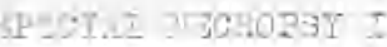

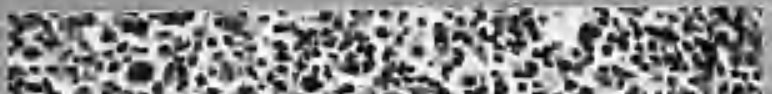
ano. 7 .

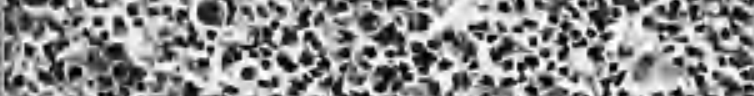

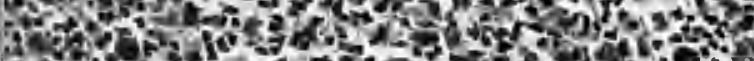

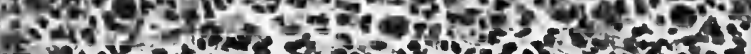

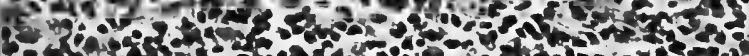

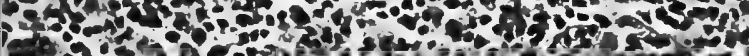
Costantron

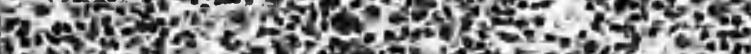

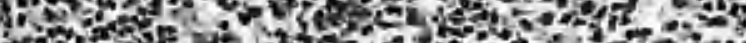

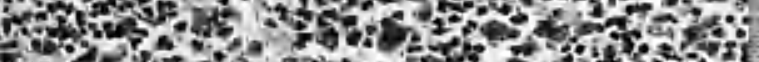

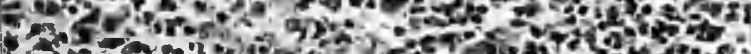
10.0.

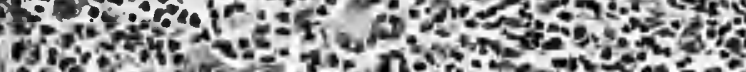

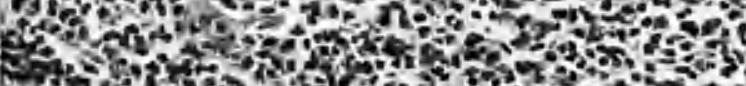
W 4 H

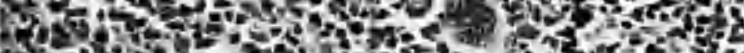

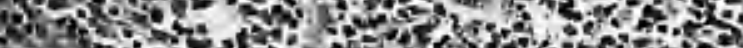

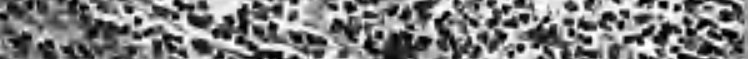

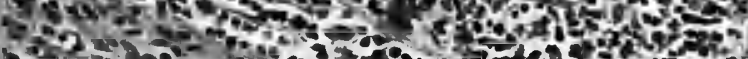

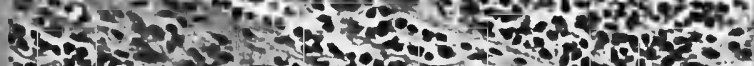

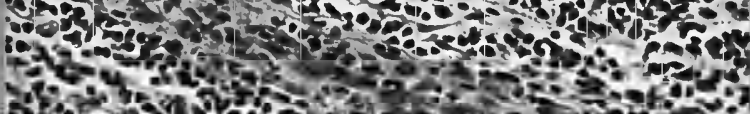

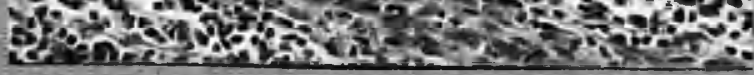

Fig. Biopsy. Field from one of tire

174 larger groin nodes; typical

Hodgkin's diseage.

SeriaI 191. H\&e X 200

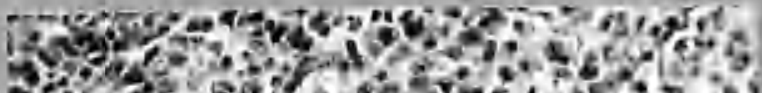
3.20

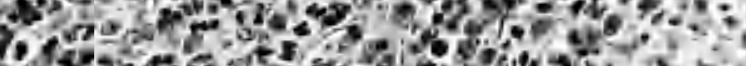

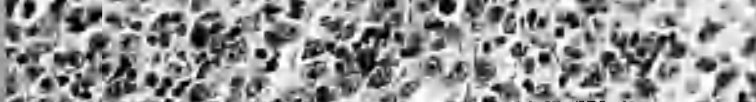

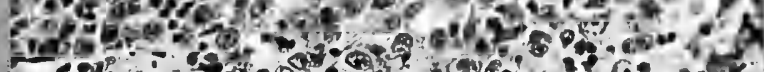

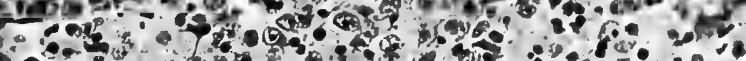

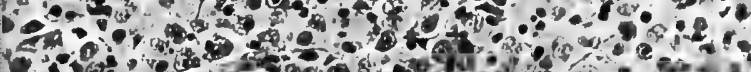

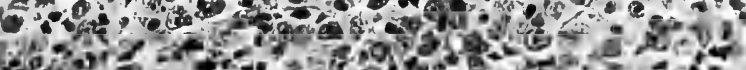

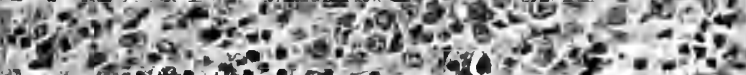

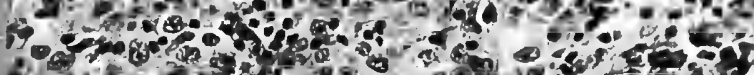

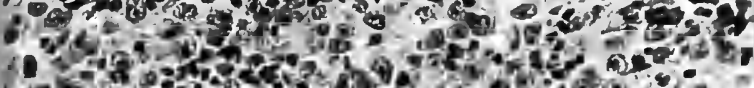

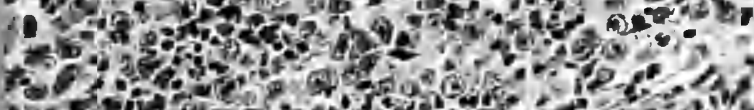

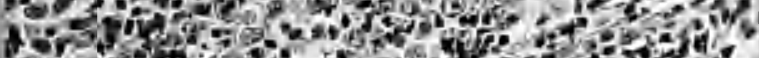

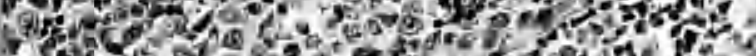

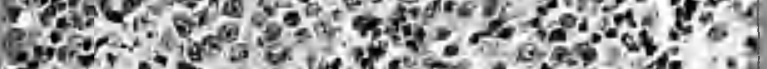
-2.0.

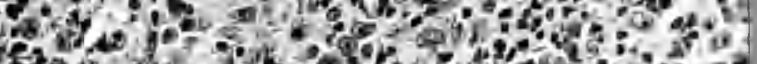
Fas

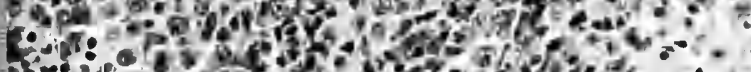
s.3.

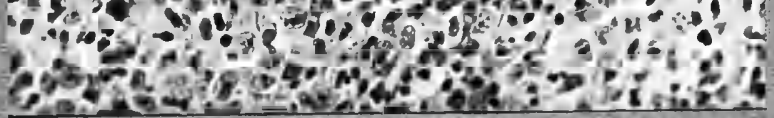

Fig. Biopsy. Field from the andi-

I75 est groin node; reticulum oesl sarcoma.

Serian 191. 


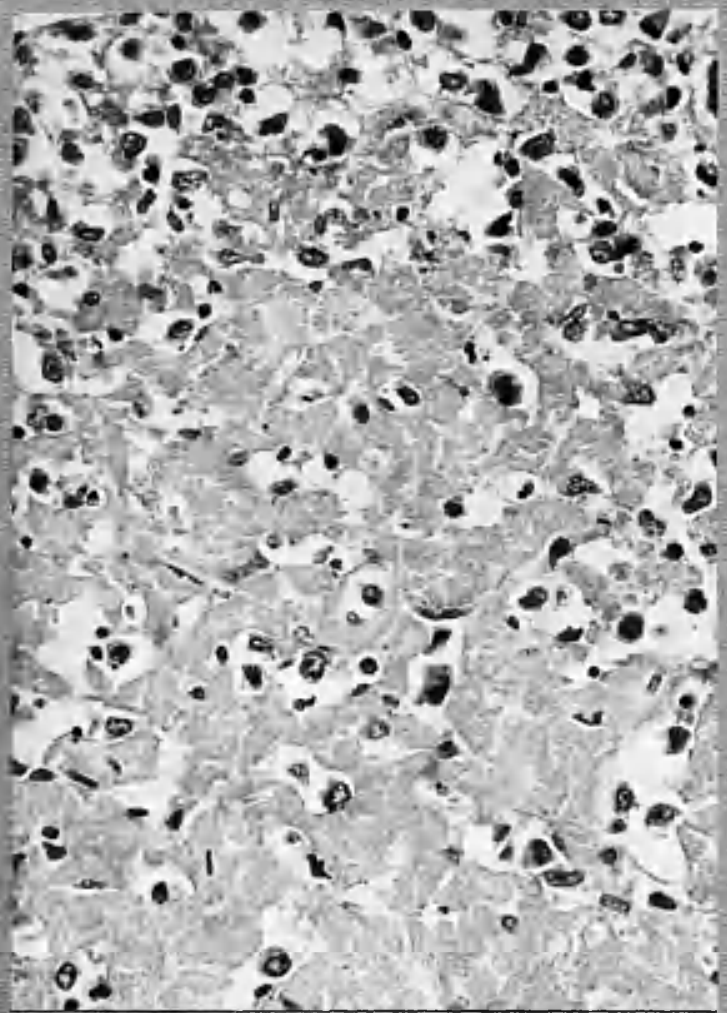

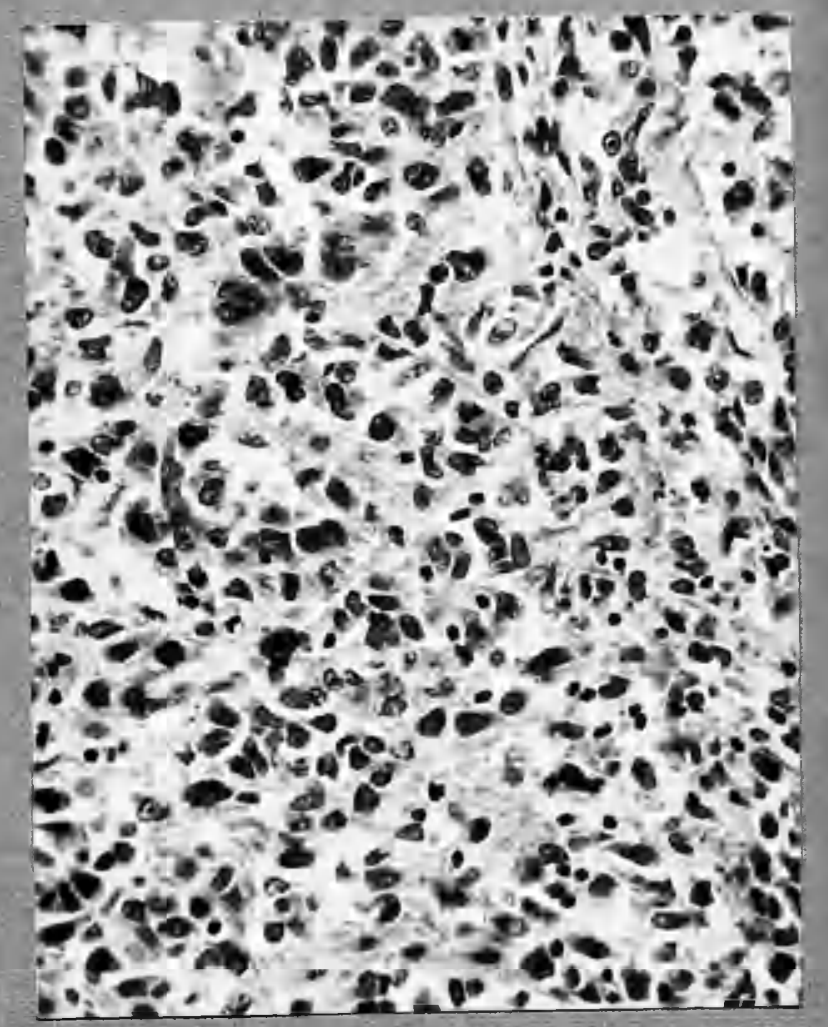

as

Fig. Necropsy. Jerviocil lumph nole. 176 frea of wature Hodgkin's

disectse. Note the groliferim ting reticulum call nucle at the margin of the colizerem. Iymphocytes are in aboymce.

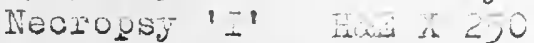

Pis.

177
E. D.

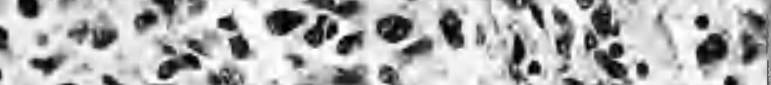

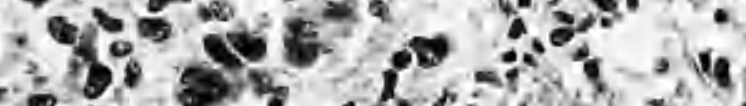

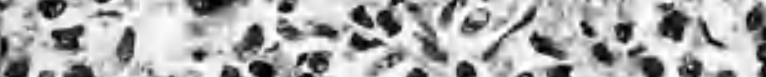

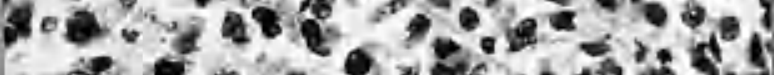

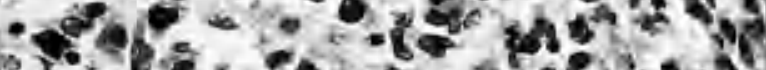

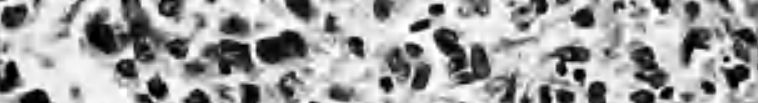

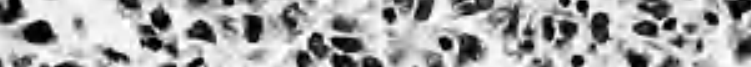

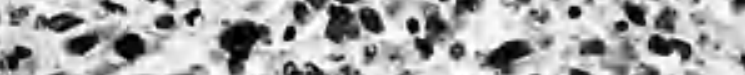

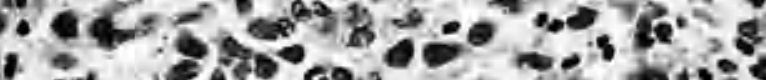

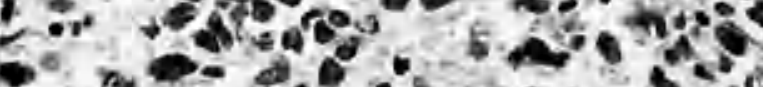

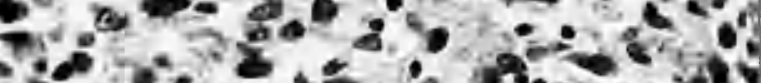
Siacos.

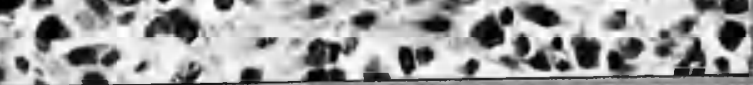


$70 \% 190 \%$ - aporesos is $: 2, y$ $060 \%$ 3. 30 into

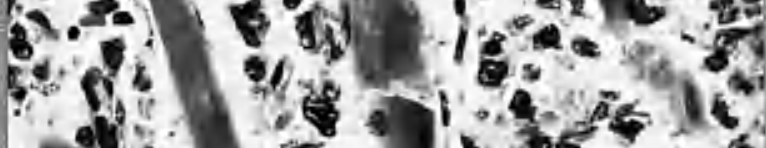

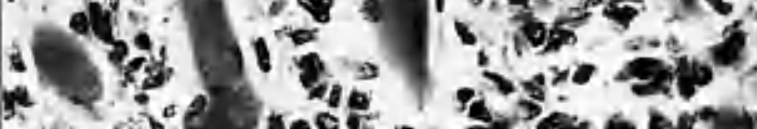

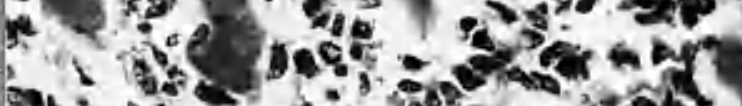

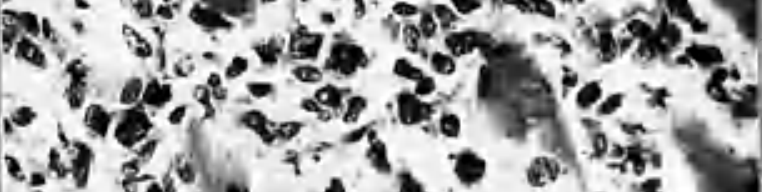
$, 47,2$.

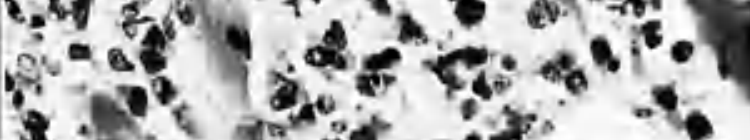
1.

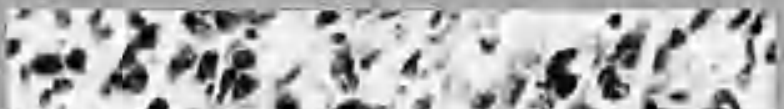
$\therefore$ os ar 150

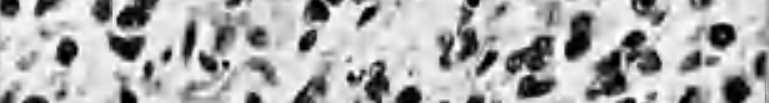
lo to 0 \%

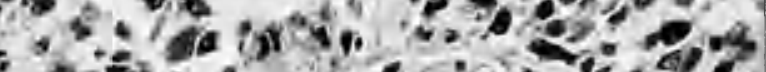

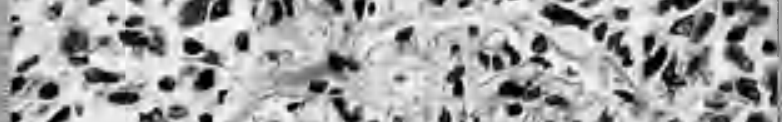

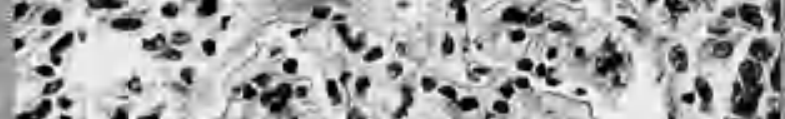

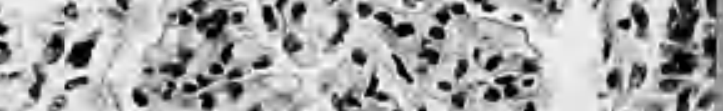

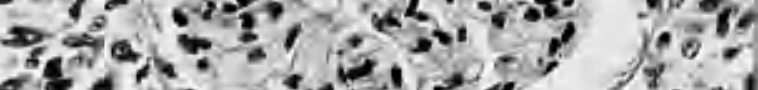

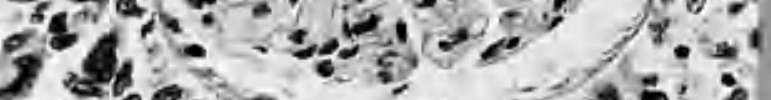

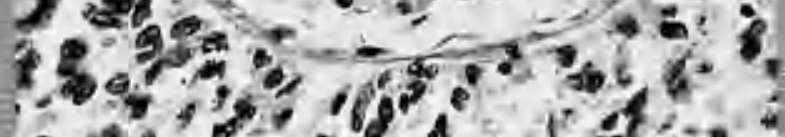

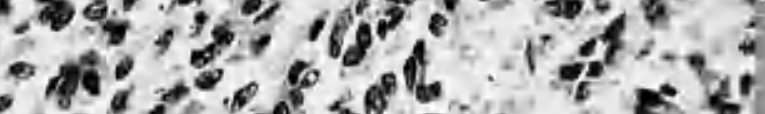
40 and

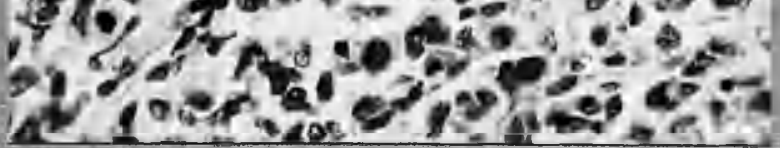

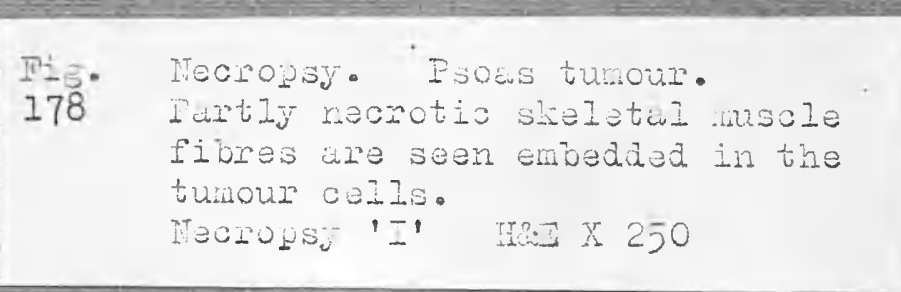

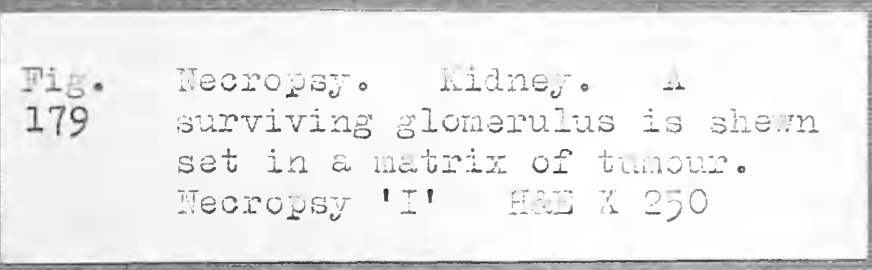




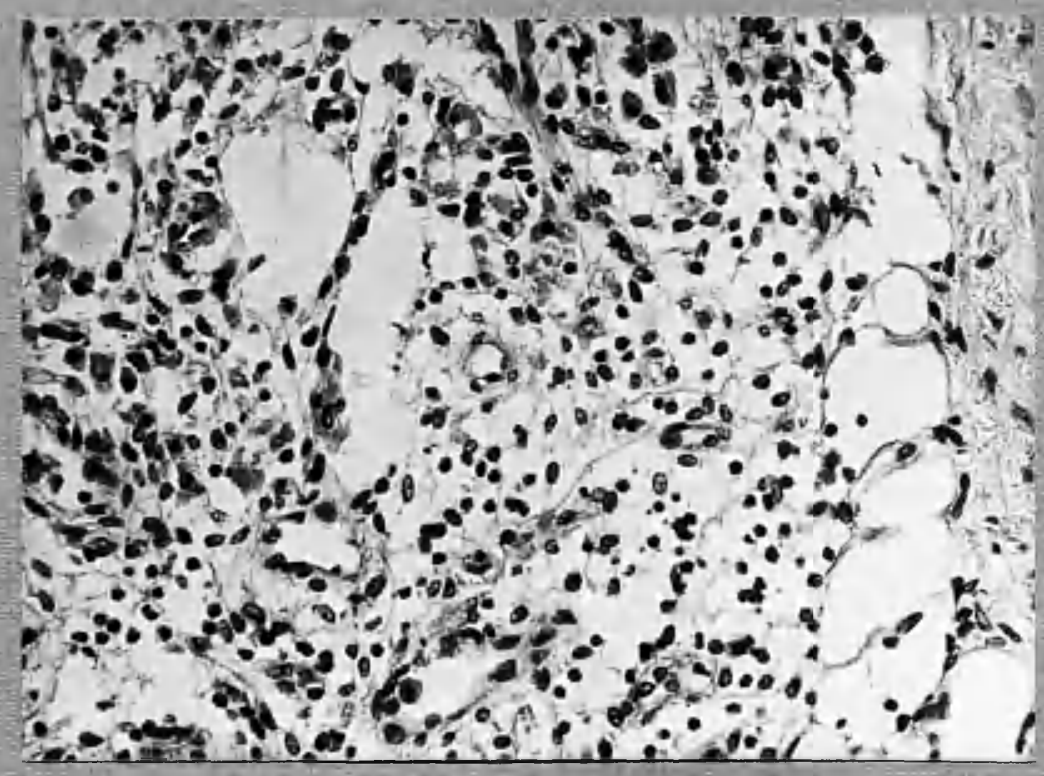

$$
\begin{aligned}
& \text { Fig. Necropsy. Ivmph node from } \\
& 180 \text { right sroin. The structure.is } \\
& \text { not morbid beyond shenling extreme } \\
& \text { lymphocyte depletion. } \\
& \text { Necropsy 'I' BVE } \mathrm{A} 250
\end{aligned}
$$




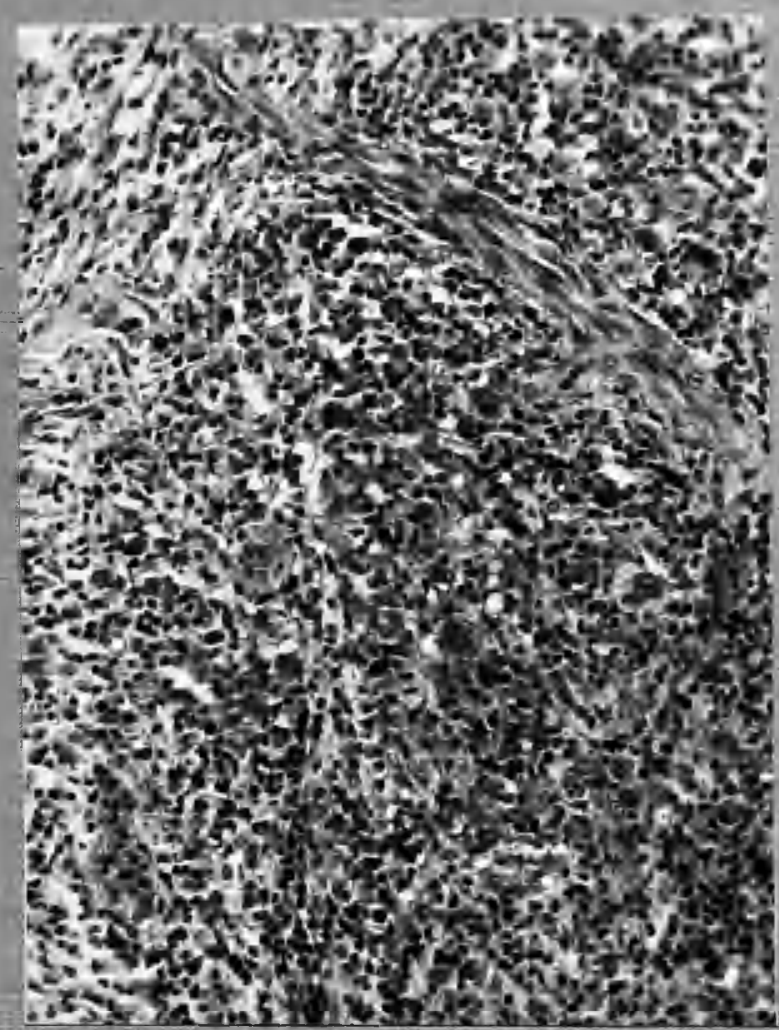

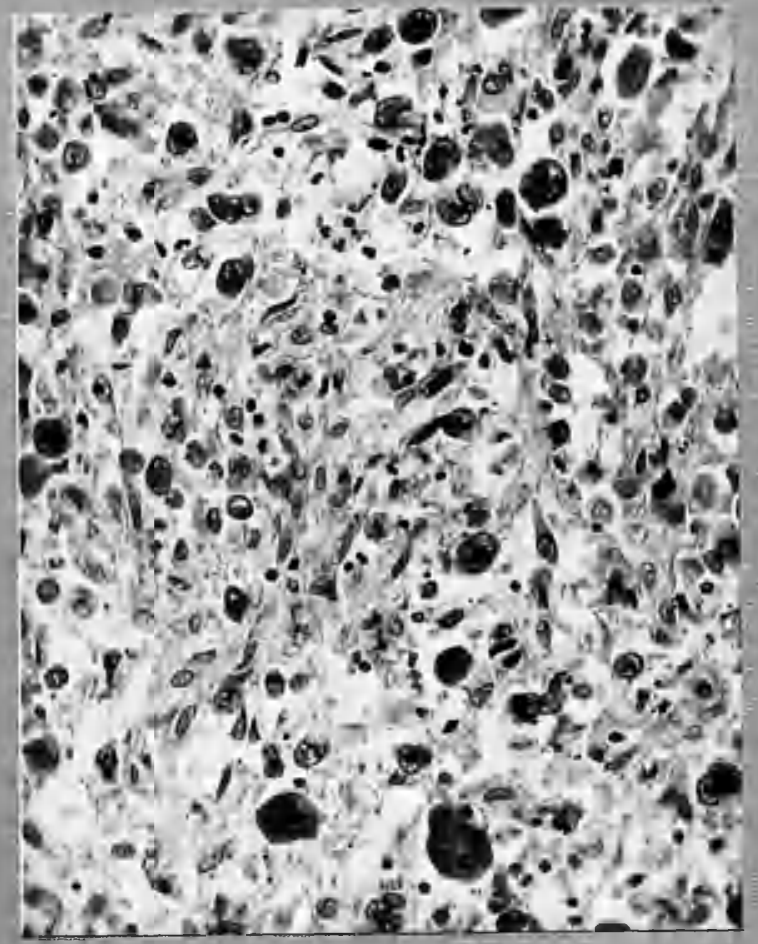

Fig. Necropsy. Right inferior 182 tracheo-broncinial node. A random field of the tunour now present. (All the lesions present tinis apearance). Fodginin's sarconi, note the numerous Hodgkin giant-cells, sclerosis and general pleiomorphism. Necropsy ' $\mathrm{J}$ ' HEI X 250 


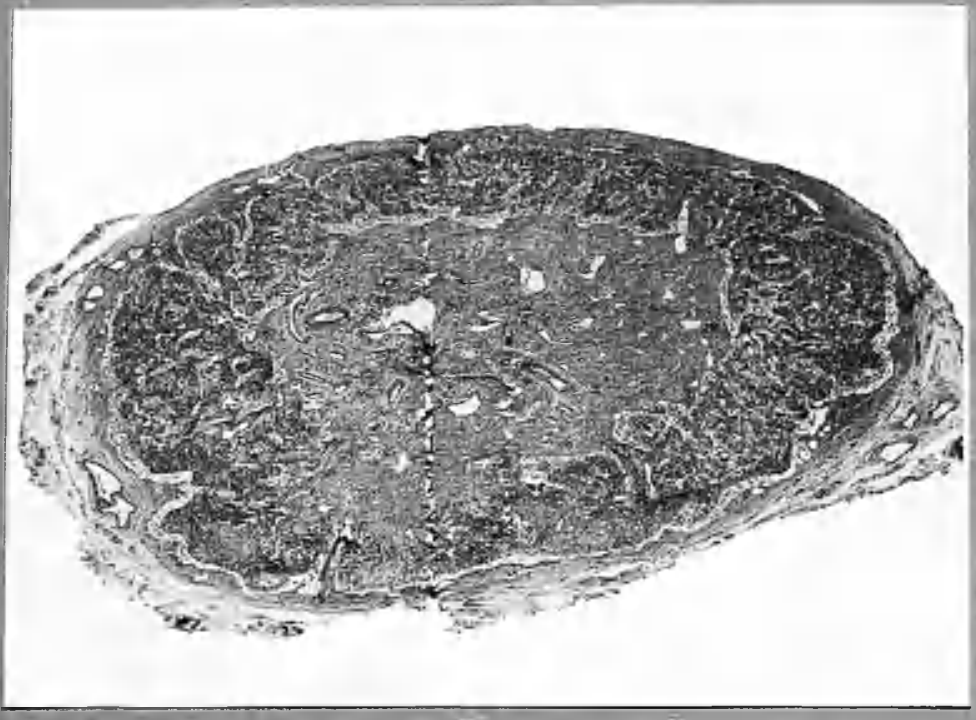

Fig. Necropsy. Iymph node from 183 left groin. This node is physiological. Note the retention of sinus structure; the central fibro-vascular tissue is the hilar intrusion. (This type of fibrosis is common in groin nodes). Necropsy ' $J$ ' H\&d X 15

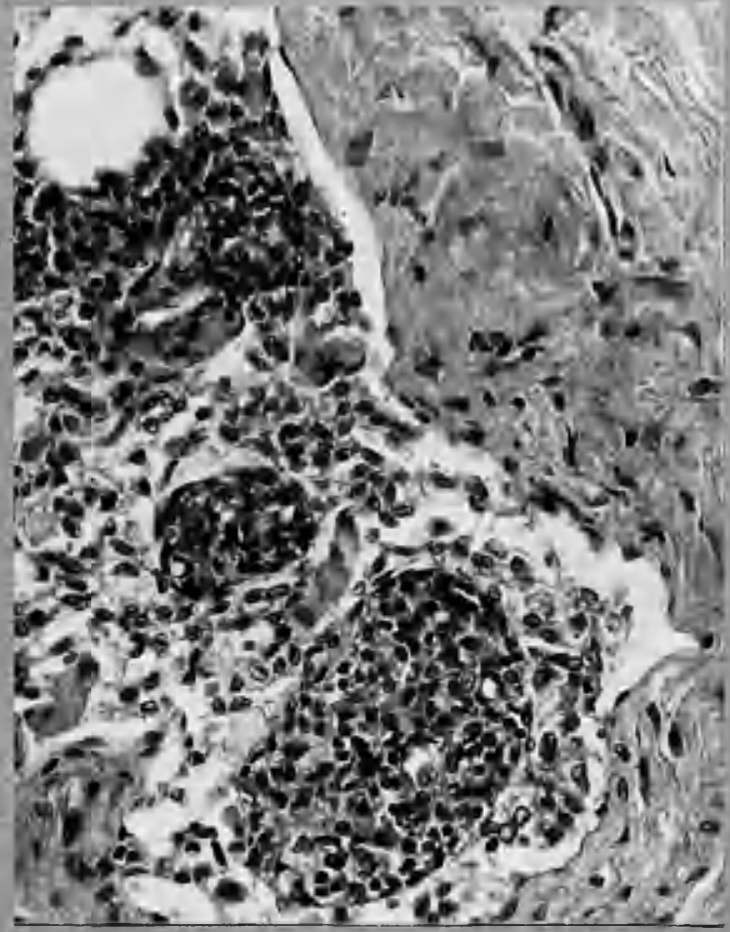

Fig. Necropsy. Lymph node from 184 right popliteal fossa. Ihis node is physiological. An high power view to shew normal marginal and trajectorial sinuses together with lymphatic cords. The capsule is seen, top right. Necropsy ' $J$ ' Hods X 250 


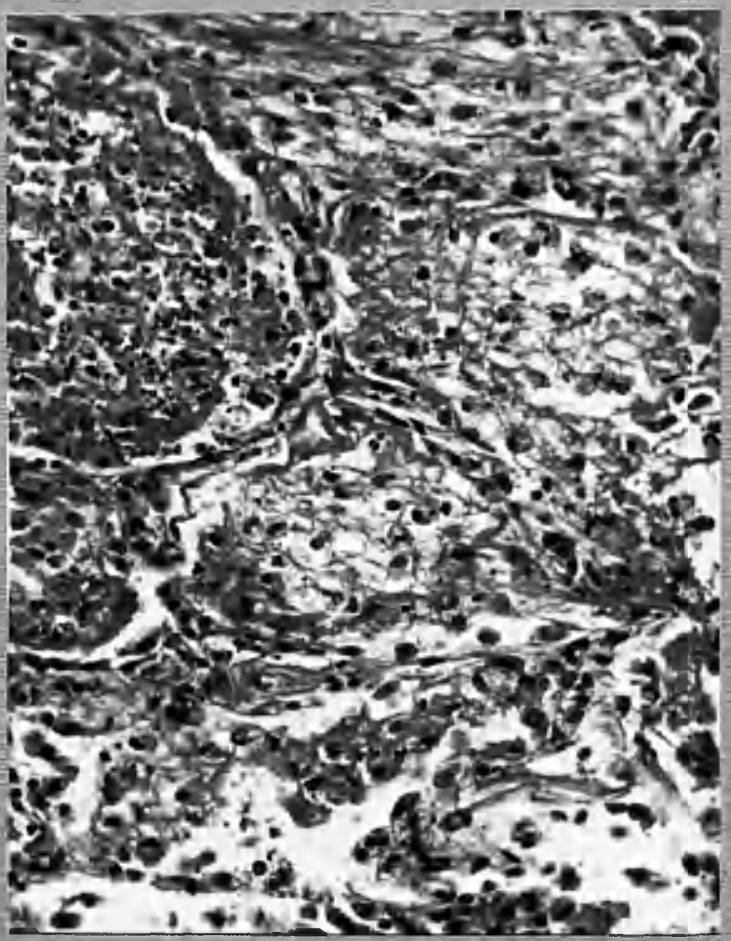

Fig. Necropsy. Lung; right upper

lobe. scute exuative and

casating tuberculosis. (Hyco.

tuberculosis is demonstrable

with ZiehI-ivealsen stiins).

Necropsy ' $\mathrm{J}$ ' $\mathrm{H}$ \& $\mathrm{X} 250$ 

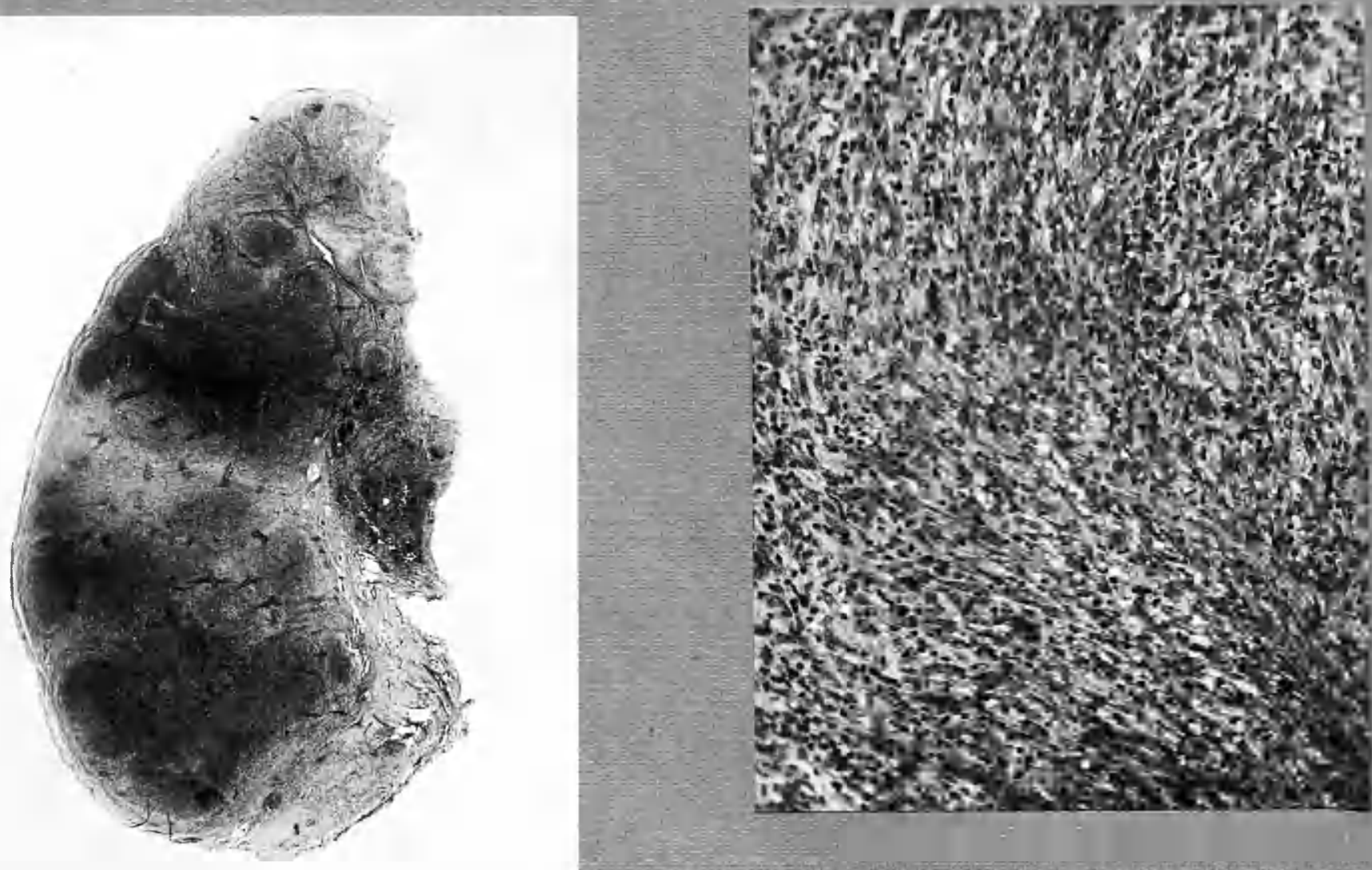

Fig. Biopsy, Cervical Iymph

186 node. The cellular new

tissue is disposed in

irregular folliculoid or

segmented fashion with

paler stainine connective

tissue between the foci.

Serial 219 How X 6

Fig. Biopsy. Cervical node.

187 Diffuse fibroblastic Hodgkin's disease. Swathes of young

connective tissue are seen

arranged in a sinuous manner. Note the relative homogeneity of the growth.

Serial 219 H\&E X 150 


\section{IPJDTEL TQROPSY K.}

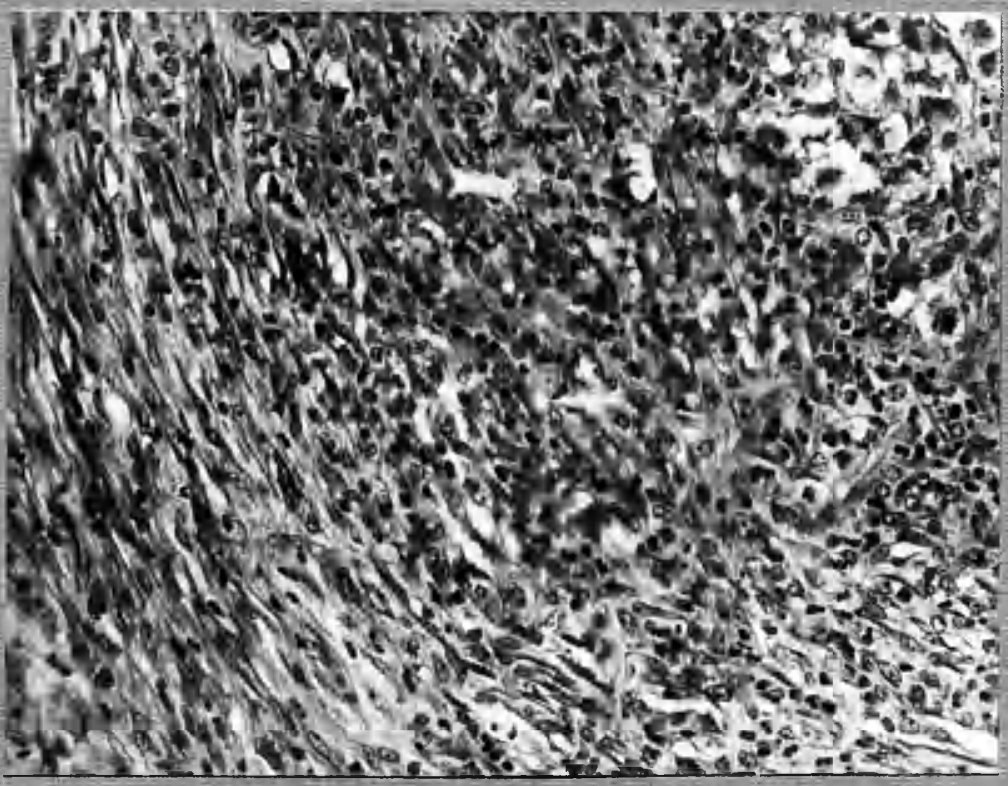

Fig. Biopsy. A high power view of

188 the edge of one of tine nodules.

lworbid mononuclear and Hodgkin

giant-cells can be made out.

The small deeply staining

binucleate cells are eosinophils.

Serial 219

Irow $\times 250$ 


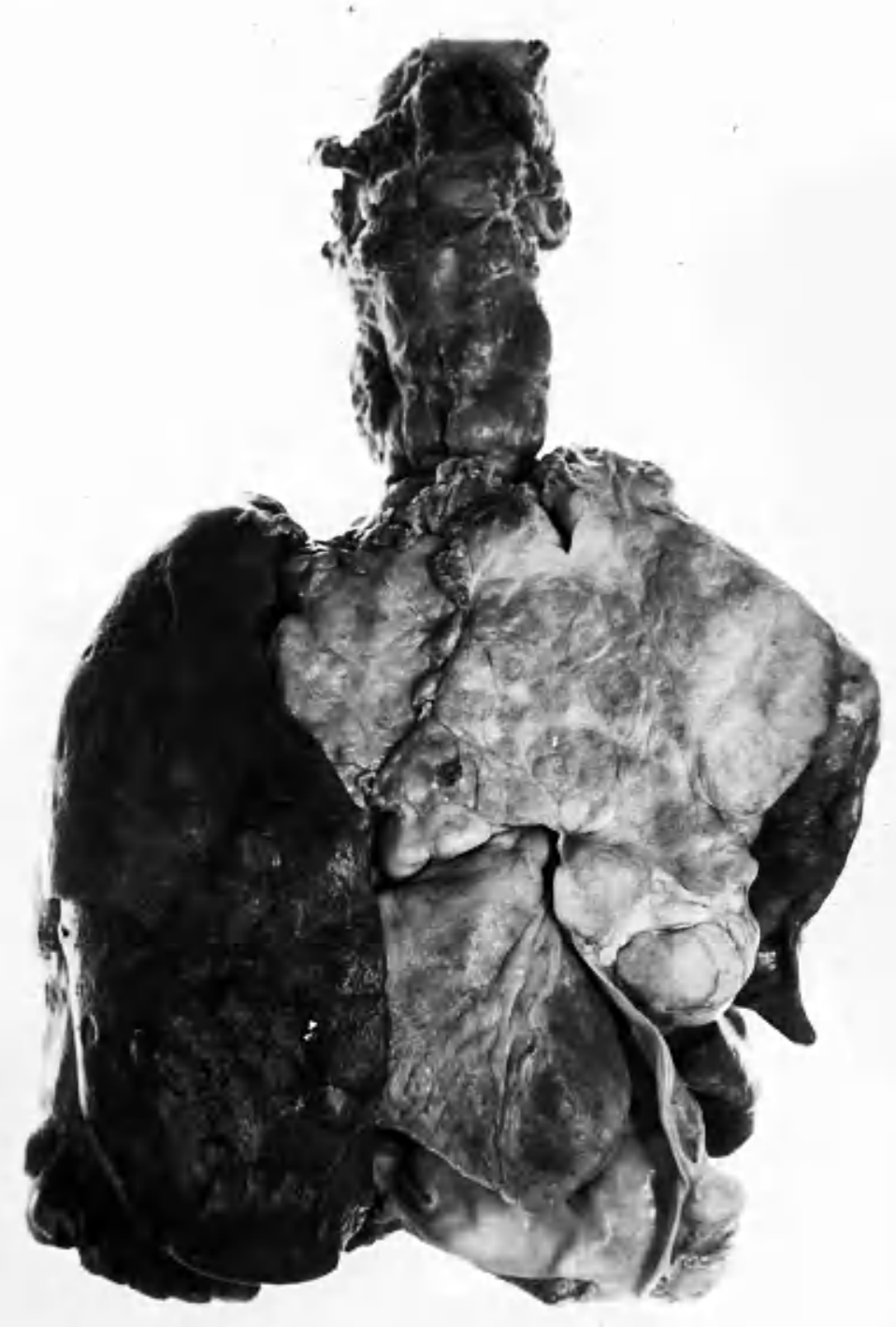

Fig.189... Necropsy.

Cervical and thoracic

viscera, interior rspect.

Note Iarge tumour-like mass

occupying position of

thymus gland, and nolular

masses in the pericurdial

sac. Intra-pulmonary

nodules are just

Biscernible.

Necropsy' 'K' Xe Natural Size. 
Fig.189.3. Necropsy.

Posterion view of cervicul and thoracic visarid d sagittal slice hus been made. Hote tha remarkdibla resentidice to

carcinonato

Nacropsy ' $K$ ' X Titural

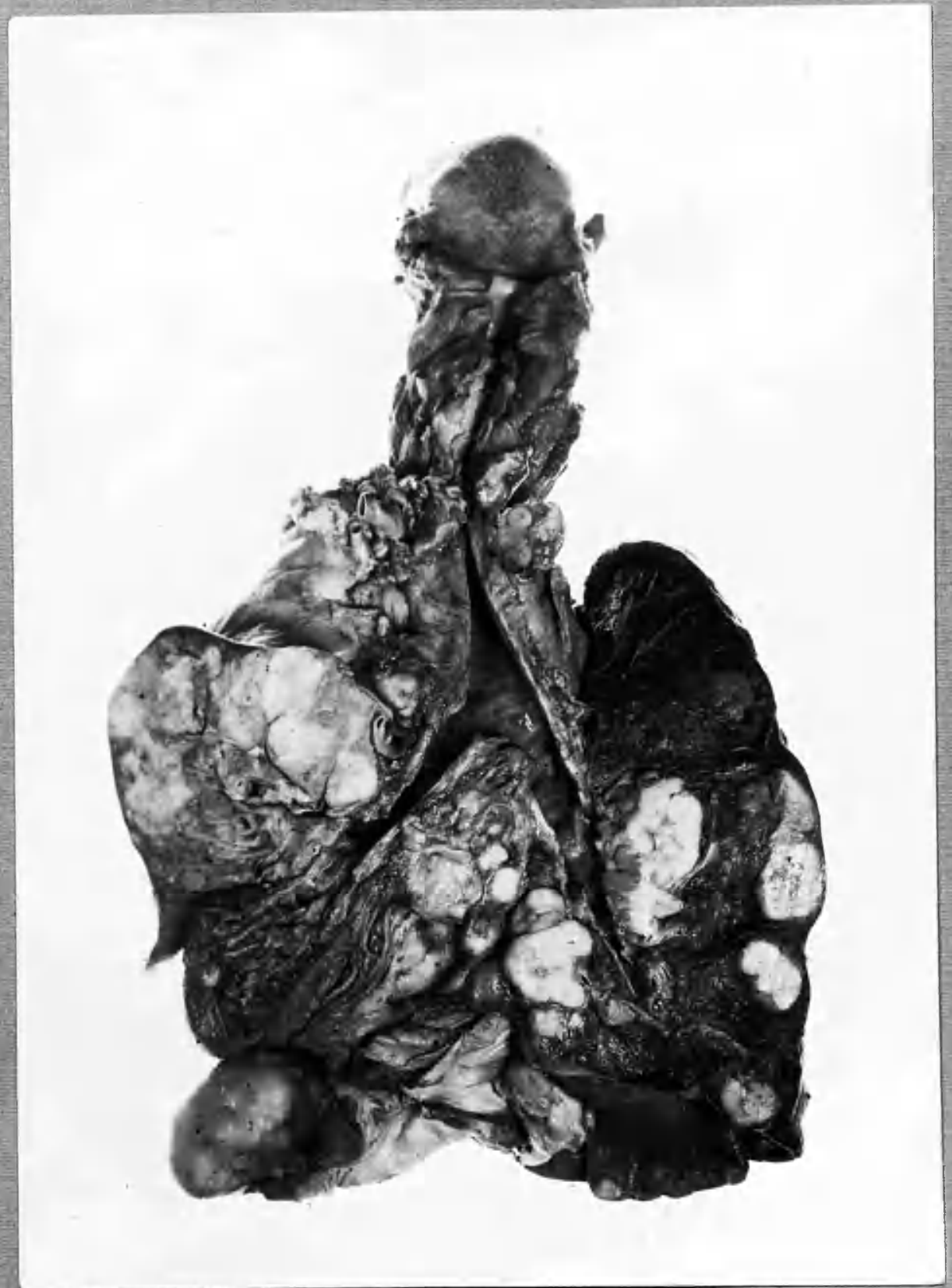




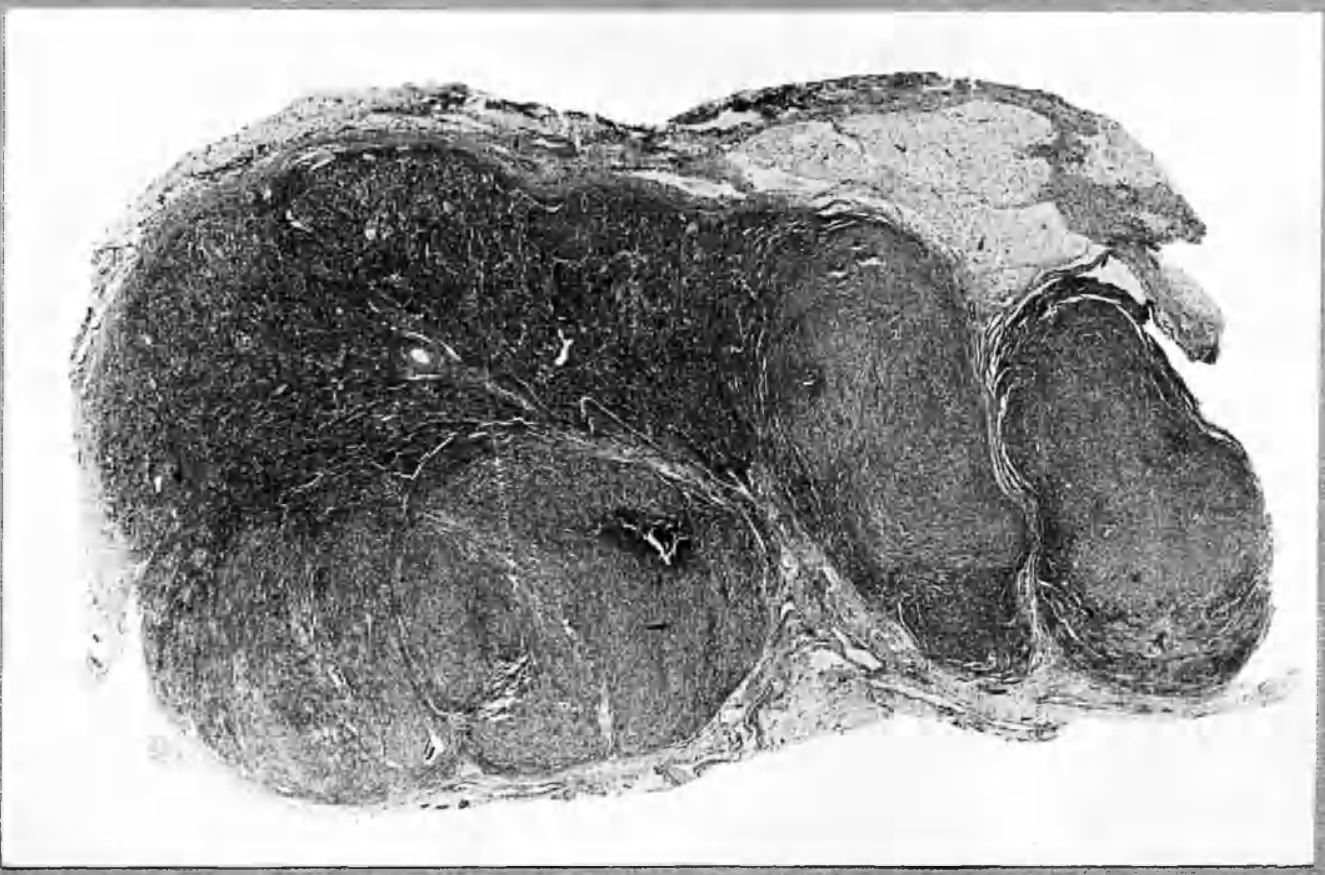

Fig. Necropsy. Ifediastinal ljmph node shewing focal 190 Hodgkin's disease. The pale nodules are the morbid tissue. Necropsy ' $K$ ' Heis $X 8$ 


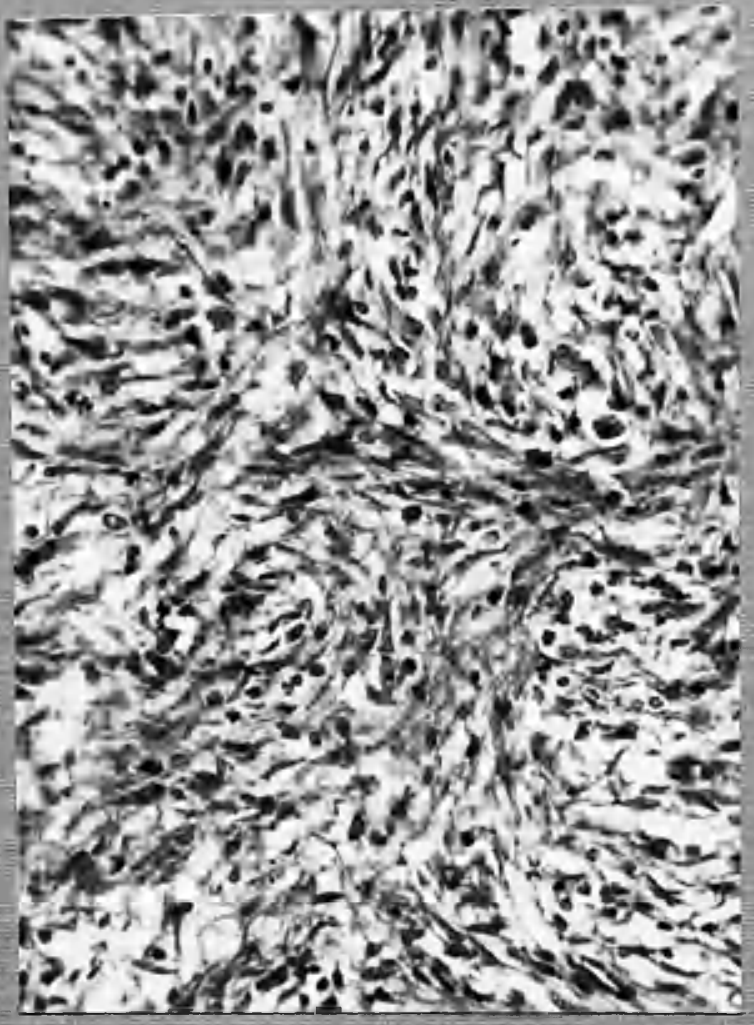

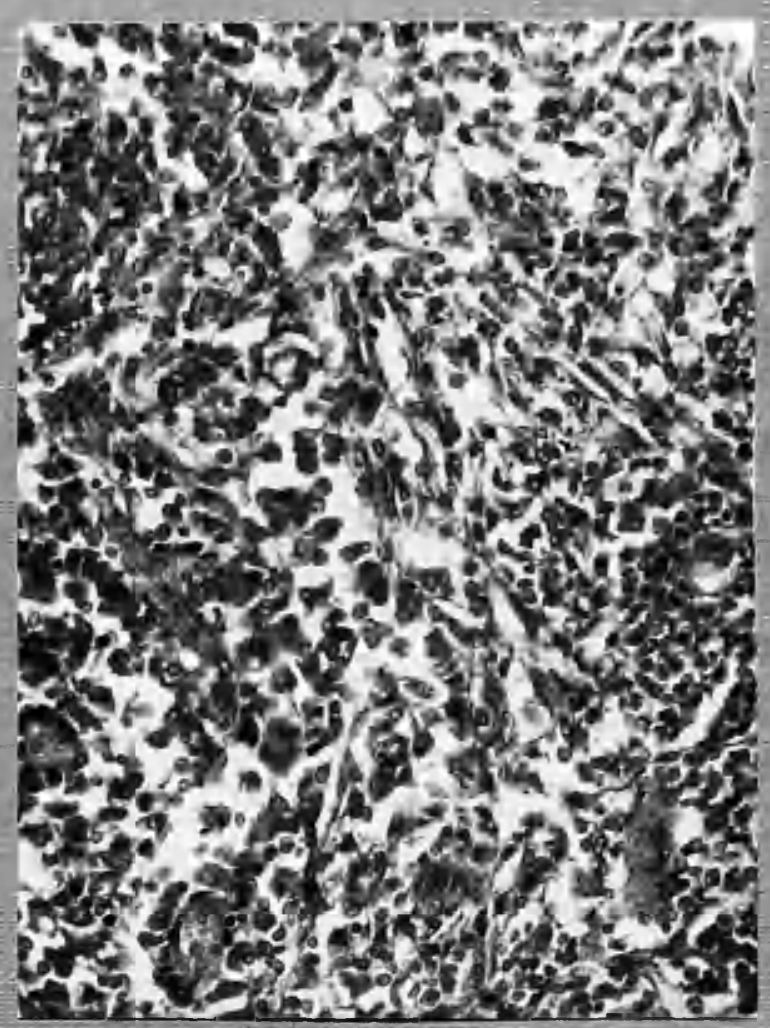

Fig. Necropsy. Wediastinal lymph
191 node. Diffuse fibroblastic
Hodgkin's disease. This field
is typical of most sites, there
is general failure to iorm dense
deposits of collagen.
NiCBOPY 'K'

Fig. Necropsy. is cellular îous

192 from the sane node victured in Fig. 191 These foci are small and rare, eosinophils are present in them.

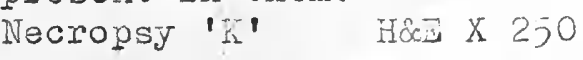




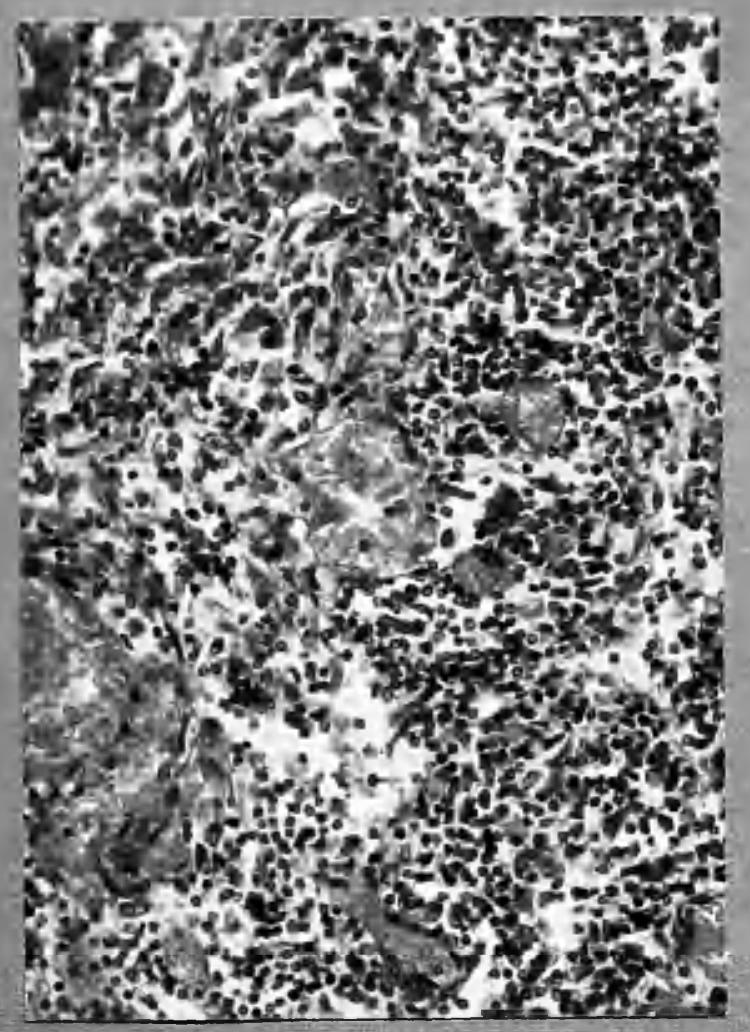

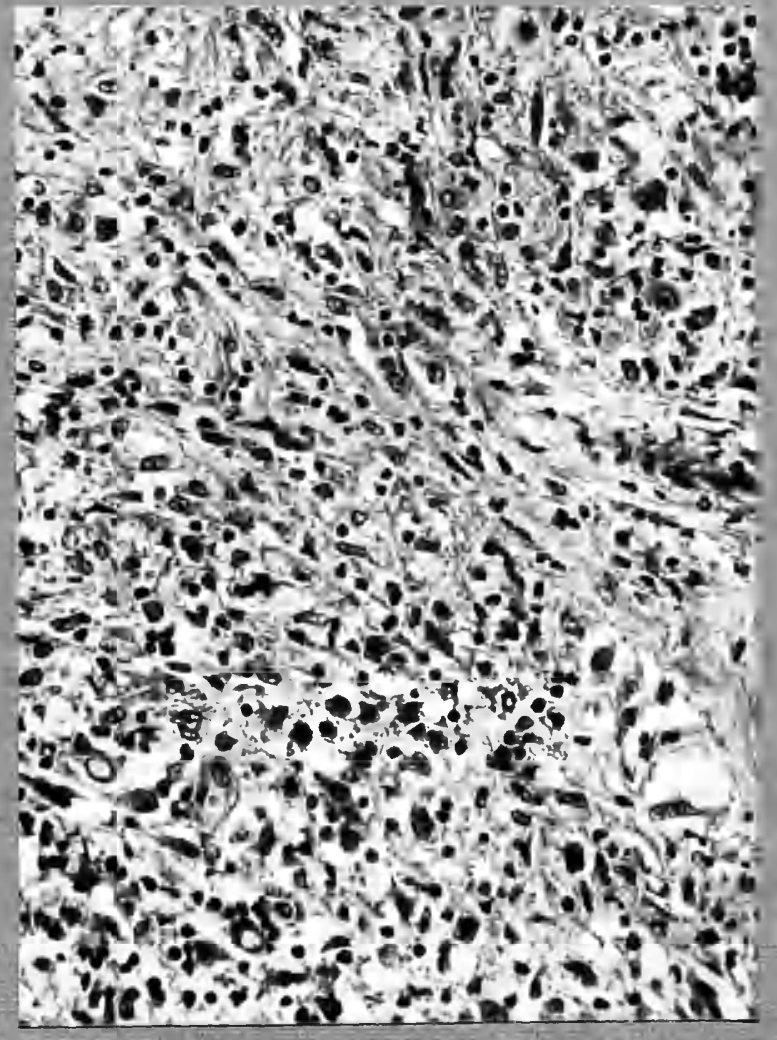
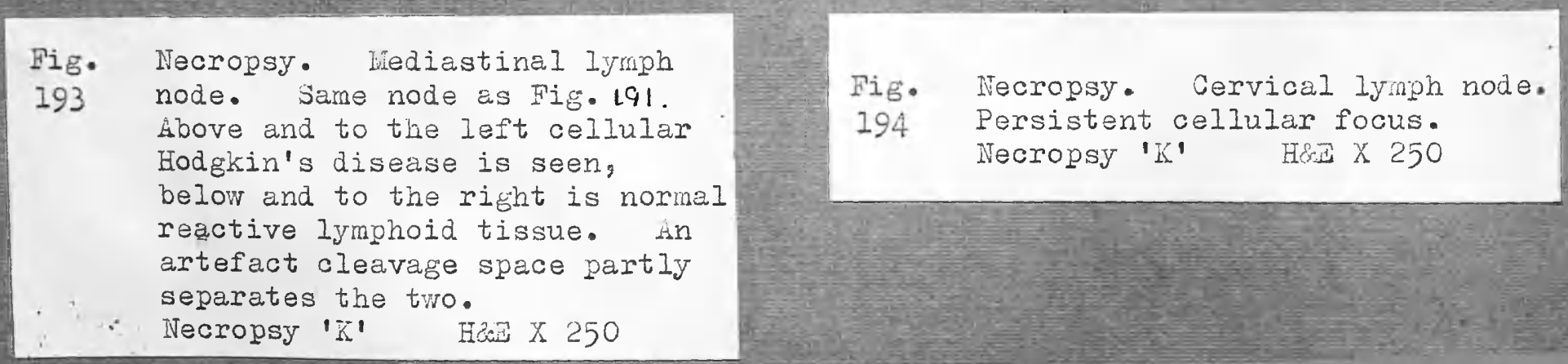


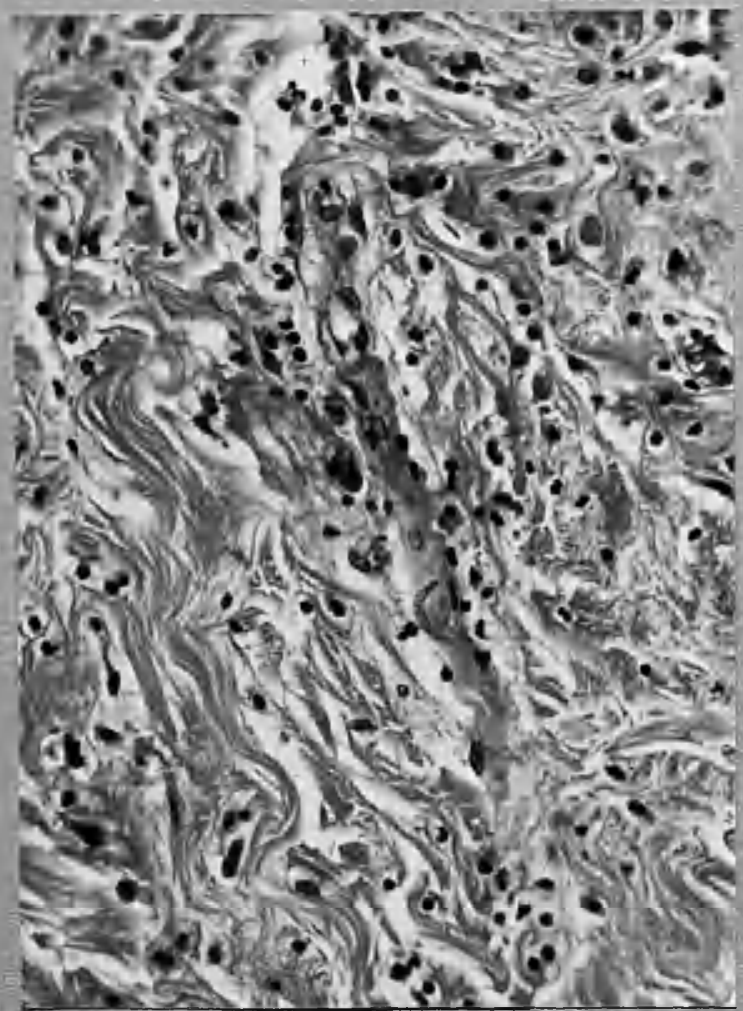

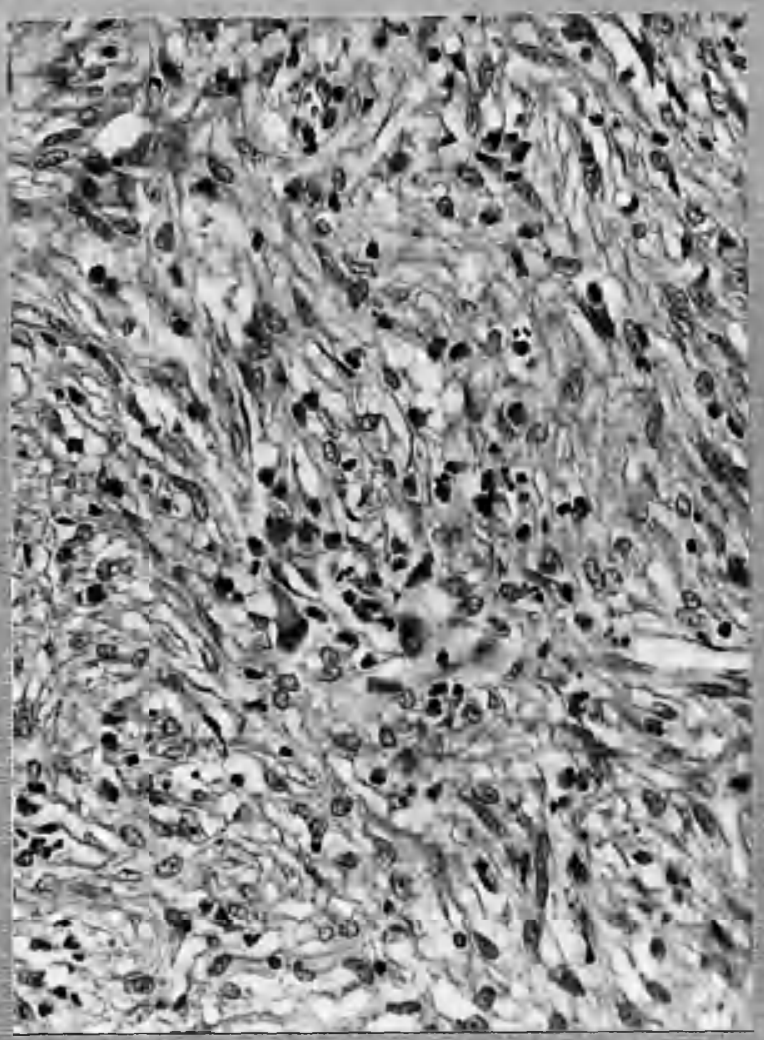

\begin{tabular}{|c|c|}
\hline Fig. & Servical Irmph node. \\
\hline 195 & Wiature extensively collagenised \\
\hline & $\begin{array}{l}\text { Hodgkin's disease. Josinophils } \\
\text { can still be wade out. } \\
\text { Necropsy ' } K \text { ' H\&E X } 250\end{array}$ \\
\hline
\end{tabular}

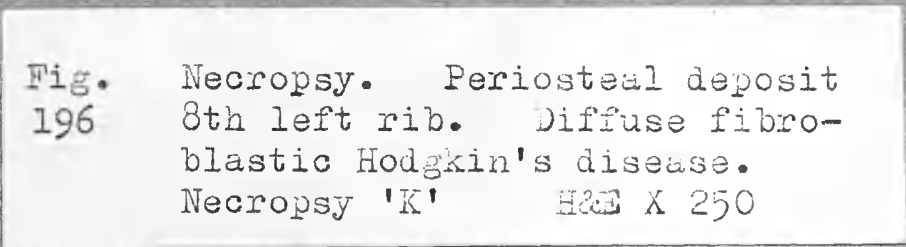




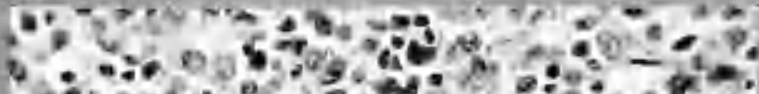

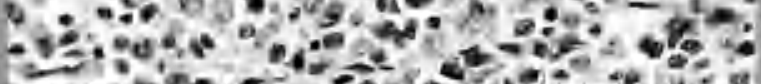

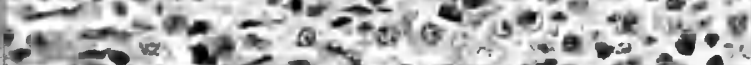

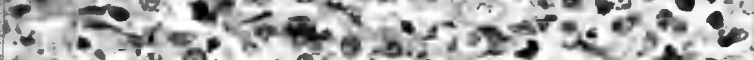

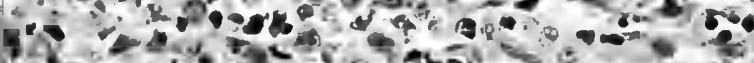

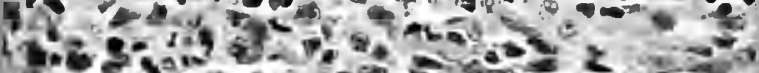

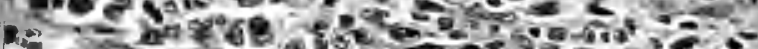

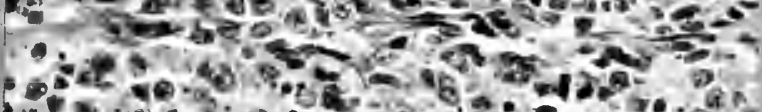

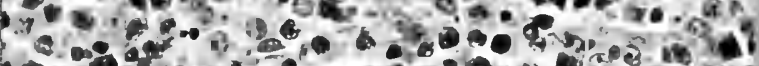

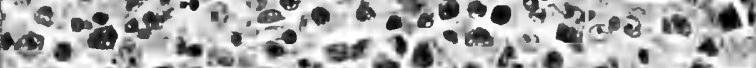

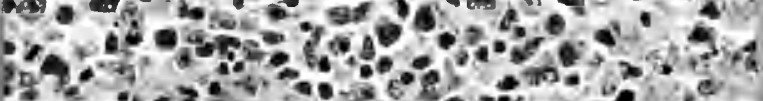

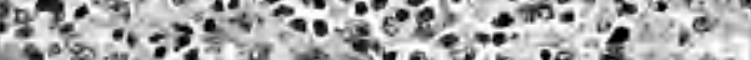

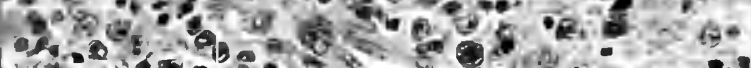

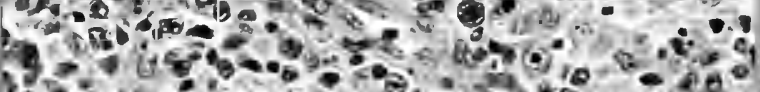

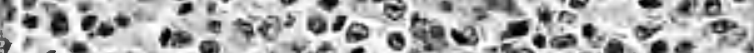

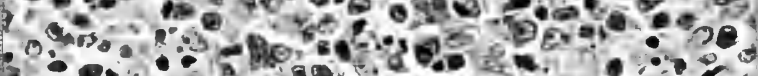
3

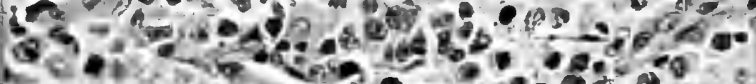

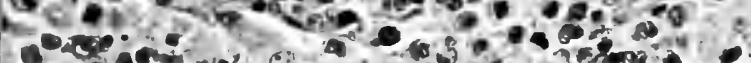

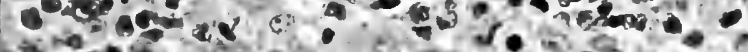

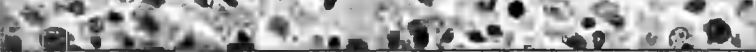

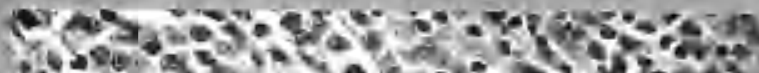

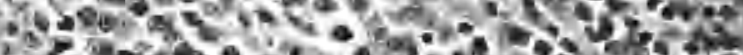
20.5.

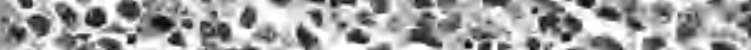

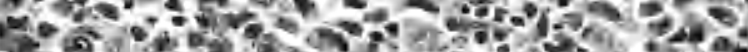

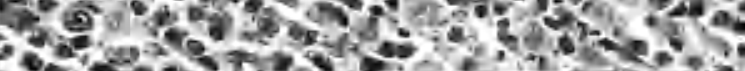

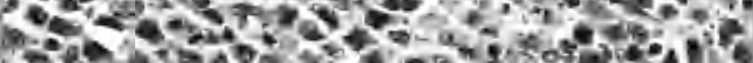

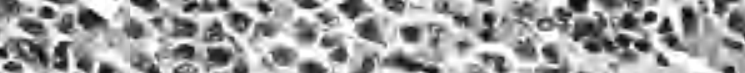

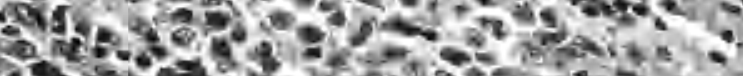

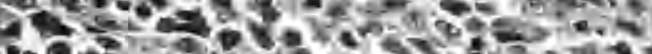

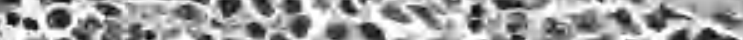

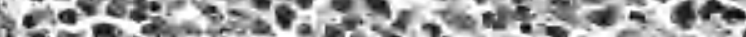

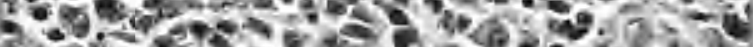

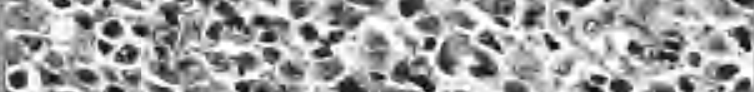

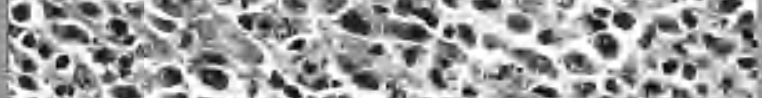

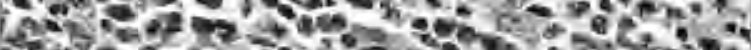
5 sar. 30 .

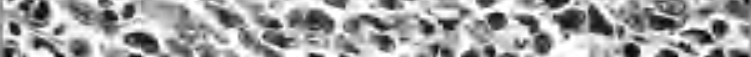

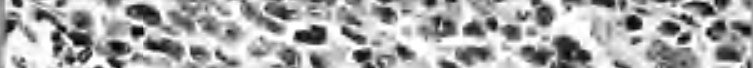

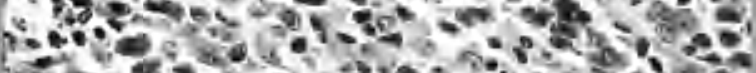
-

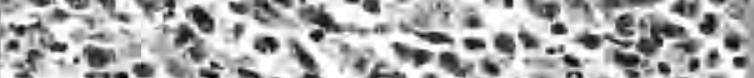

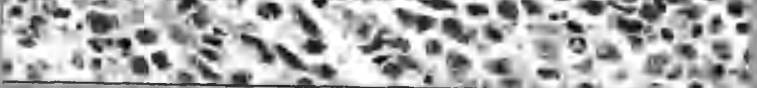

Fig. Biopsy. Cervical lywh node.

197 Highly cellular tumour-like

Hodgkin's disease. Piorosis is

scanty, pleiomorphism restricted

and rnitoses numarous. several

binucleate eosinophils are

discernible.

Serial 209.

Exis $\times 250$
Fig. Ifecropsy. Axillary lymph node.

198 Preservation is not so good as in the biopsy specimen but the growth is essentially similar. Necropsy ' $I$ ' H\&d X 250 


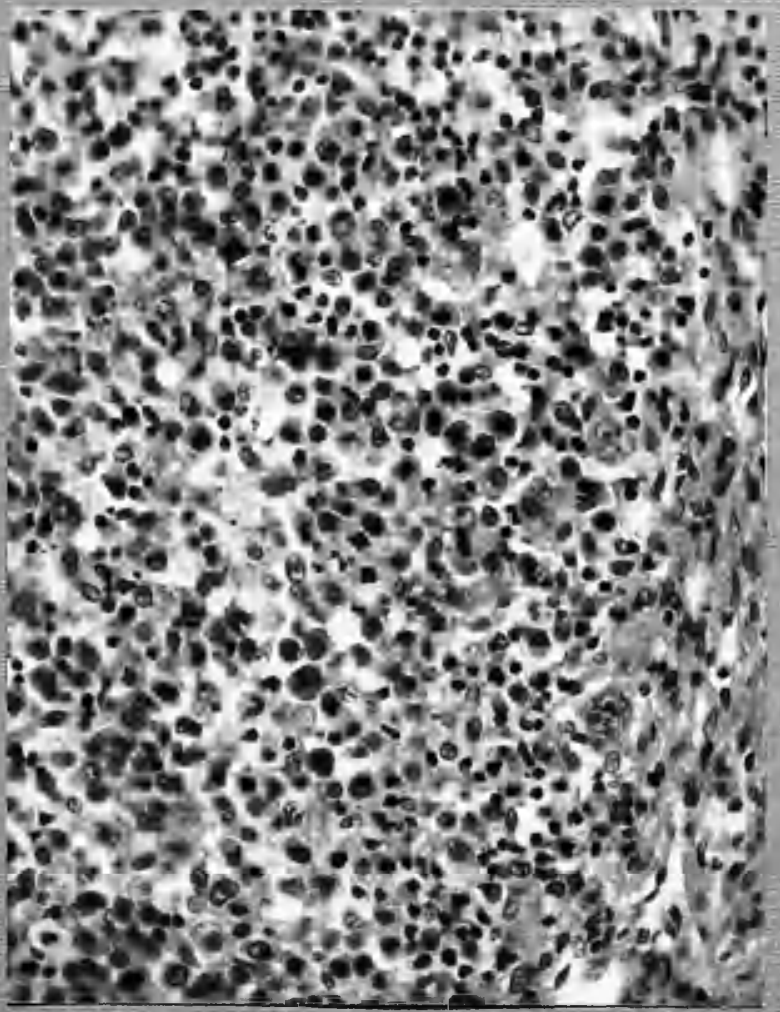

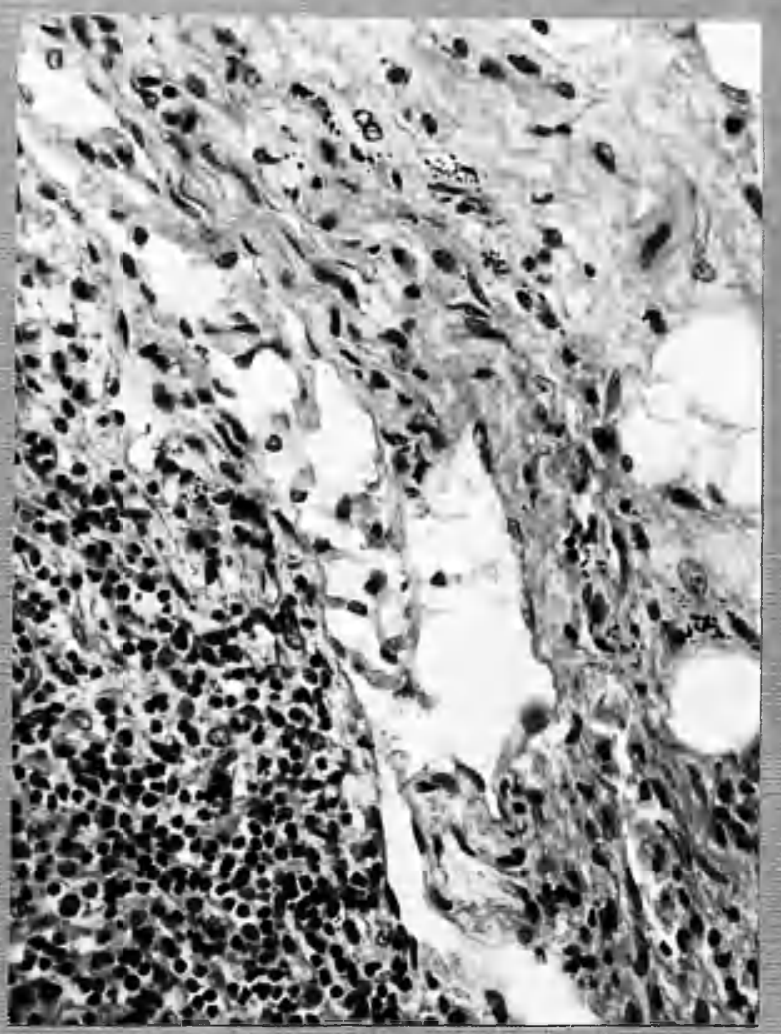

Fig. Necropsy. Antecubital lymph 200 node. Field includes capsule and marginal sinus. The structure is normal. The small black granules are haemosiderin deposit. Note the relatively small size of the muclei. Necropsy 'L' Hin $\mathrm{A} 250$ 
$B \times 1938 \%$ $140,027 \% 20$ y vor on. 1060

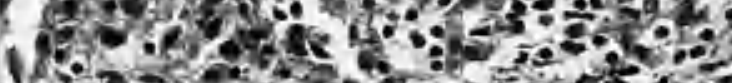

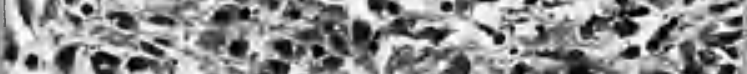

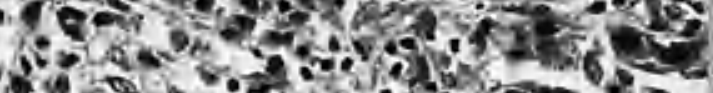
it on 3020

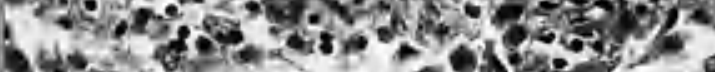
3.1. $\rightarrow 20 \%$

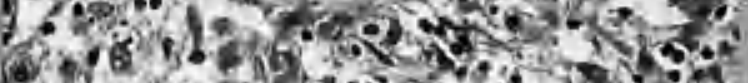

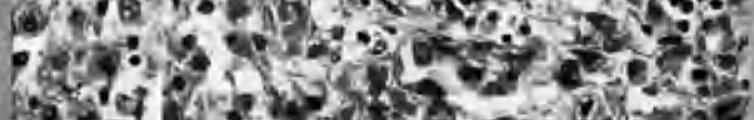

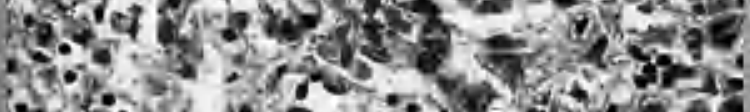

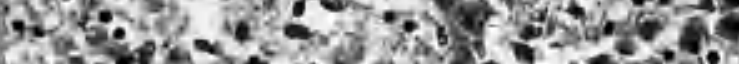

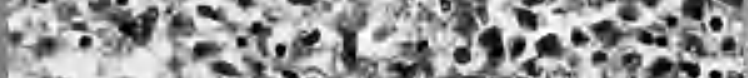

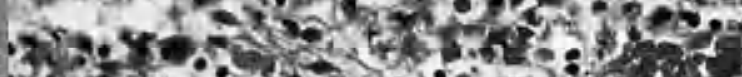

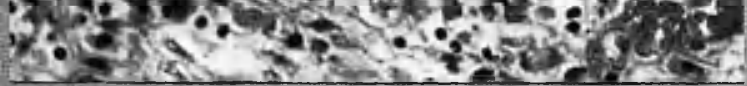

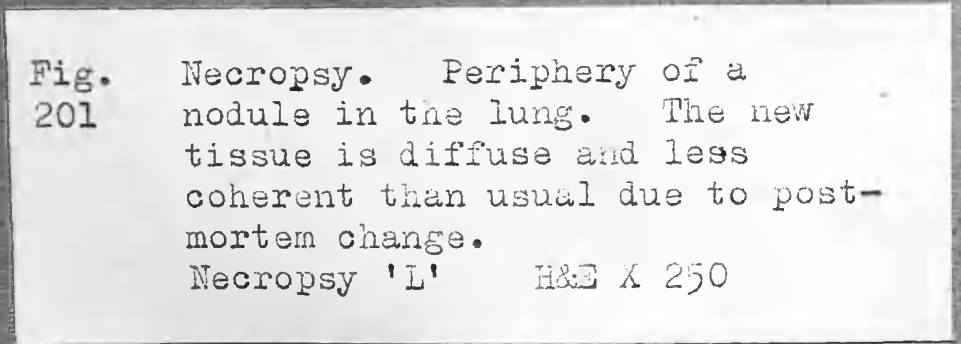

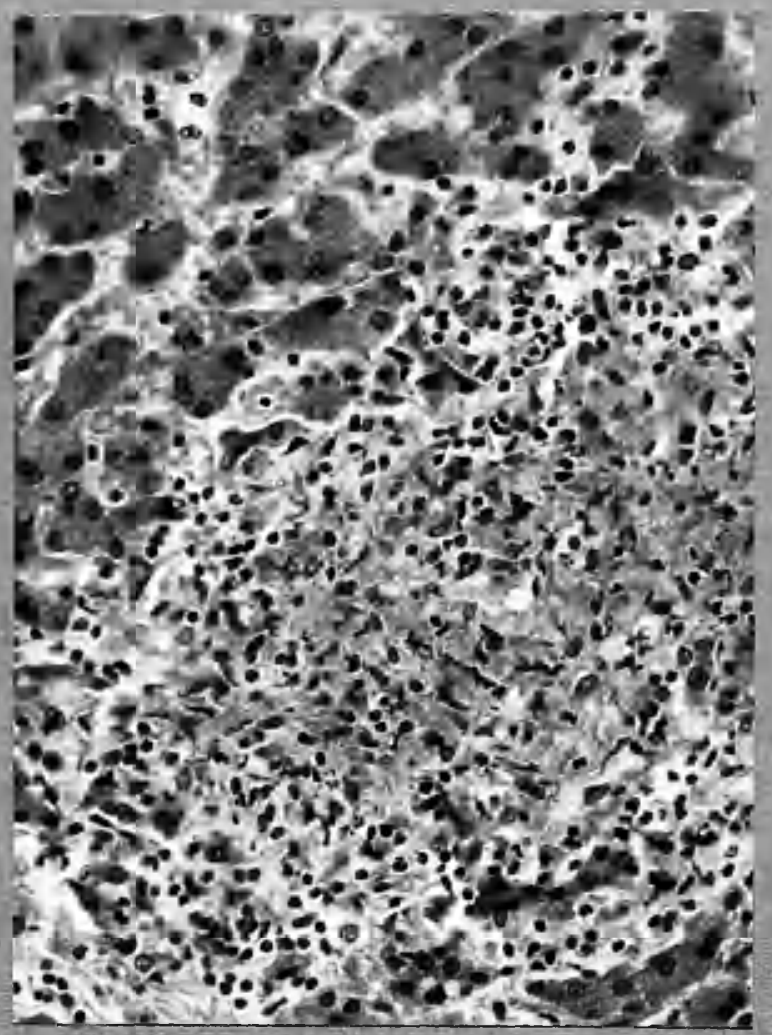

Fig. Necropsy. Iiver. Indeter-

202

minate lesion; probably tuberculous. liost of the cells and. nuclei in the focus are small. Sarly necrosis is present. Necropsy ' $L$ ' H\&: $K 250$ 


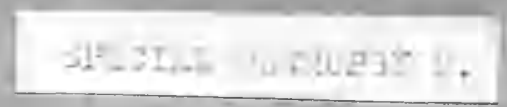

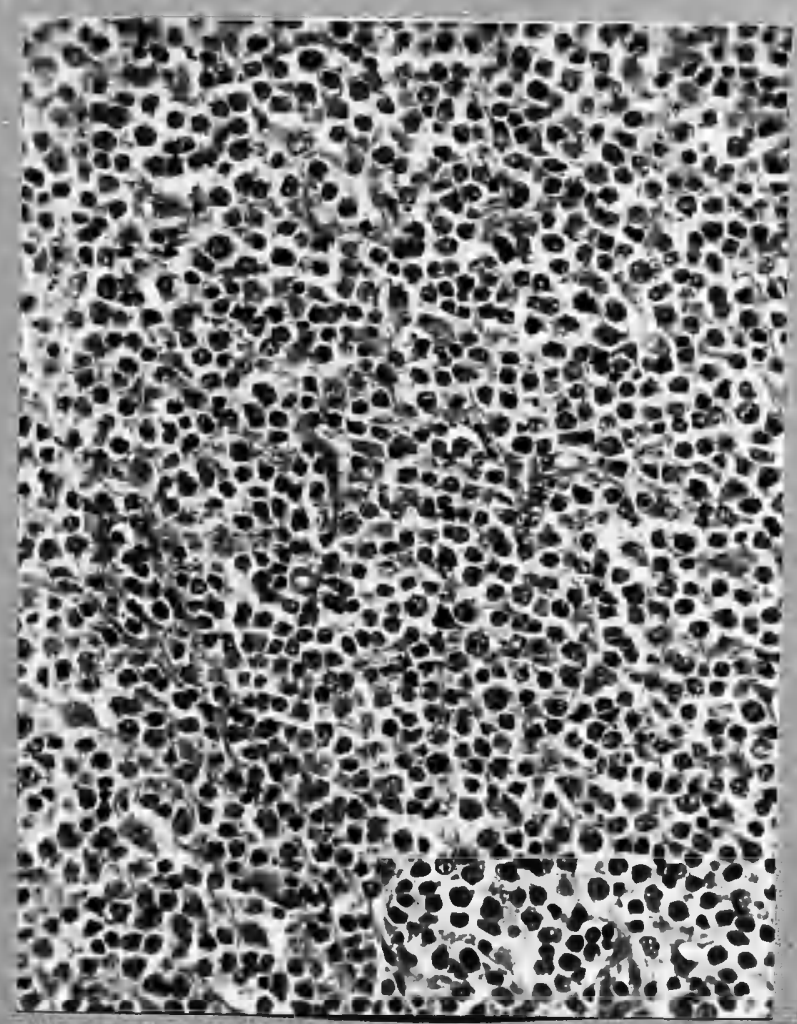

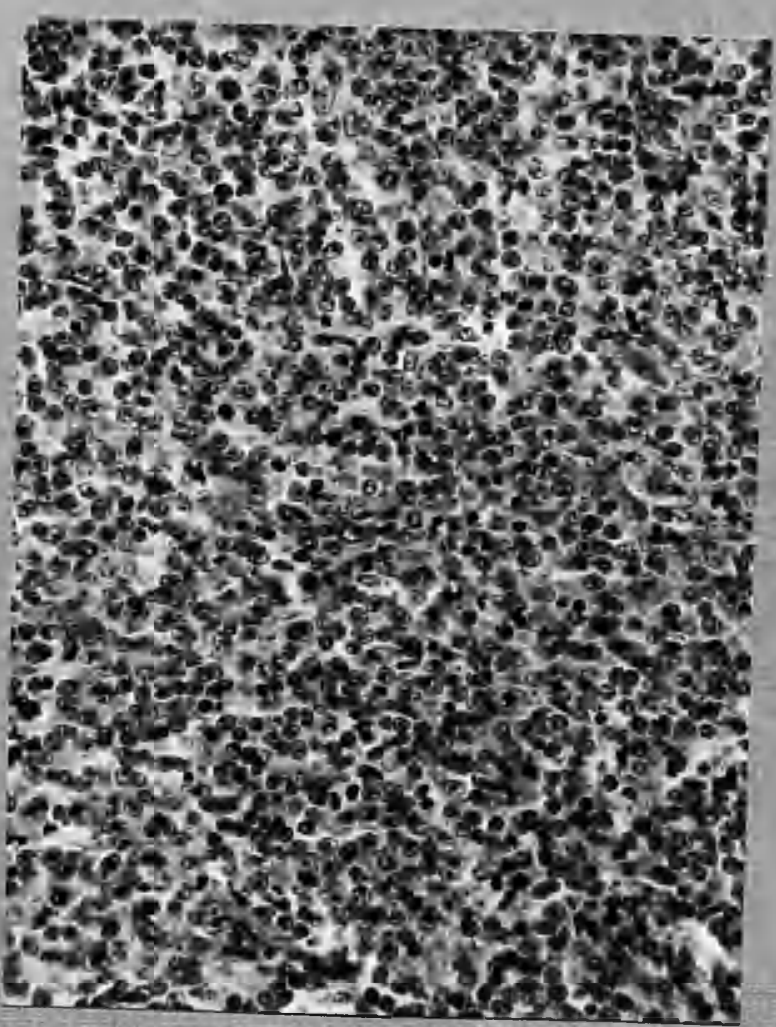

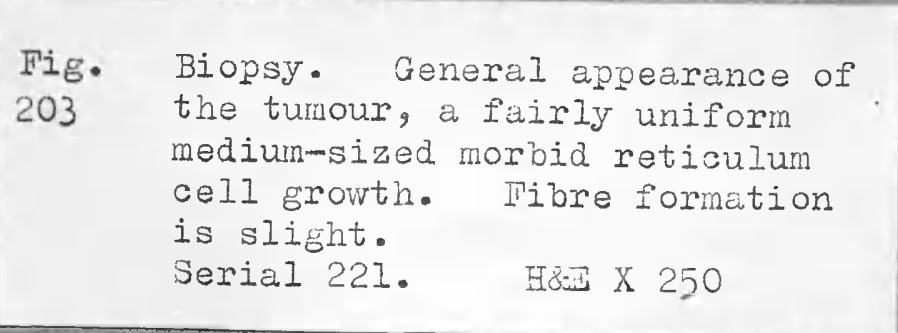

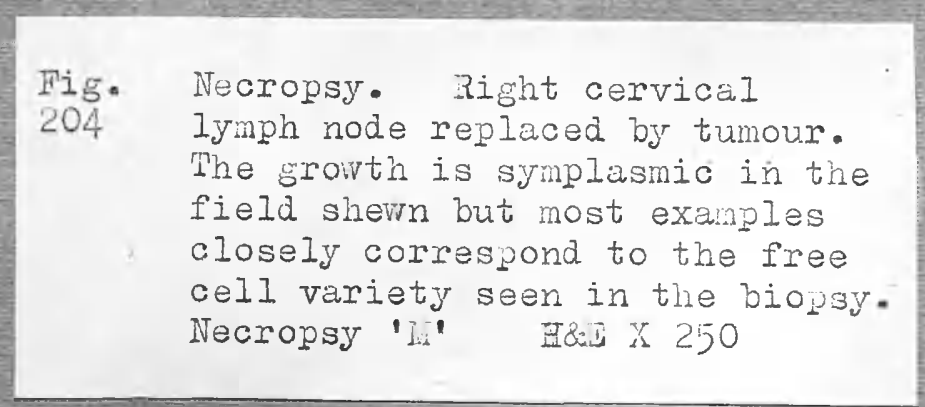



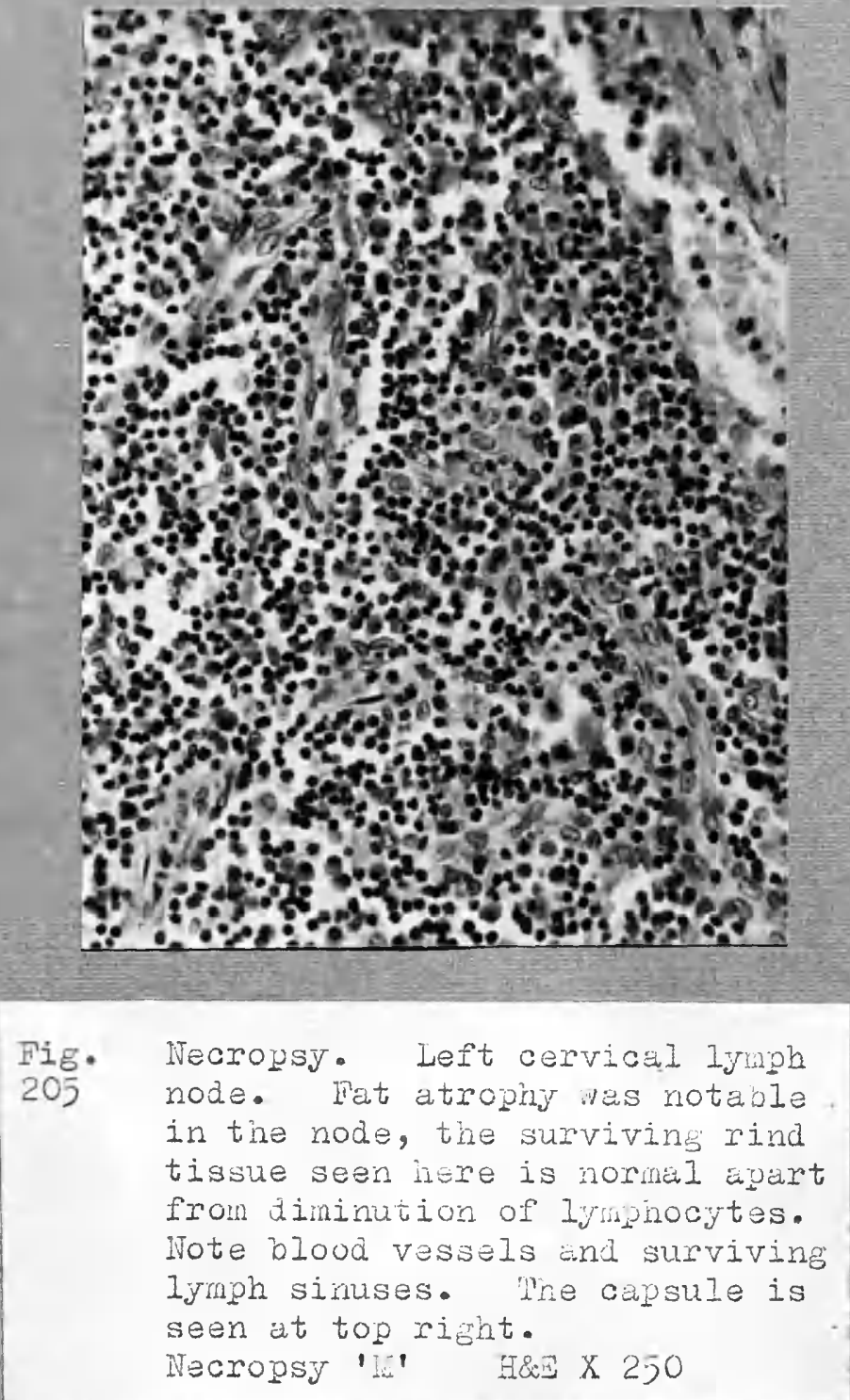

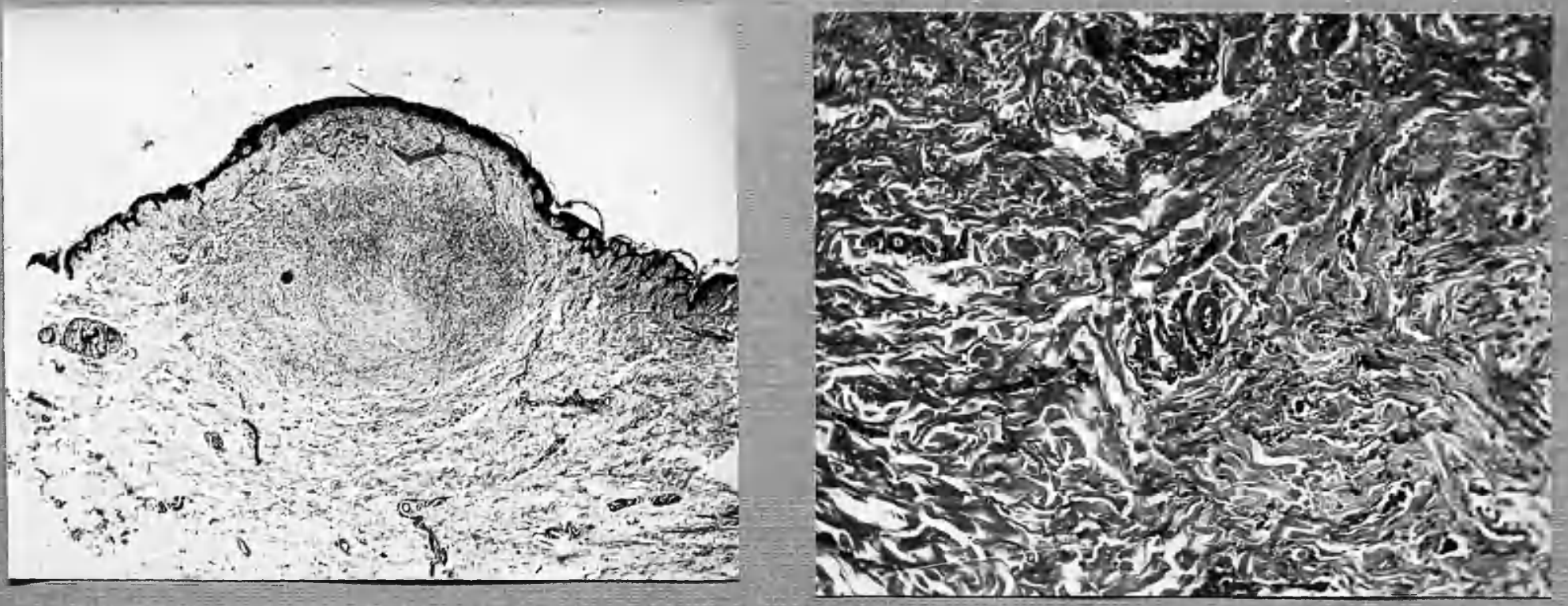

Fig. Low power vien of cheloid,

206 followine sur ical vound. Note indistinct encapsulation.

Fig. Hign power view of nommal

207 (leit), cheloid (rignt) Fis $\times 5$. 


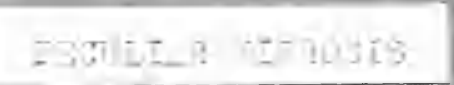

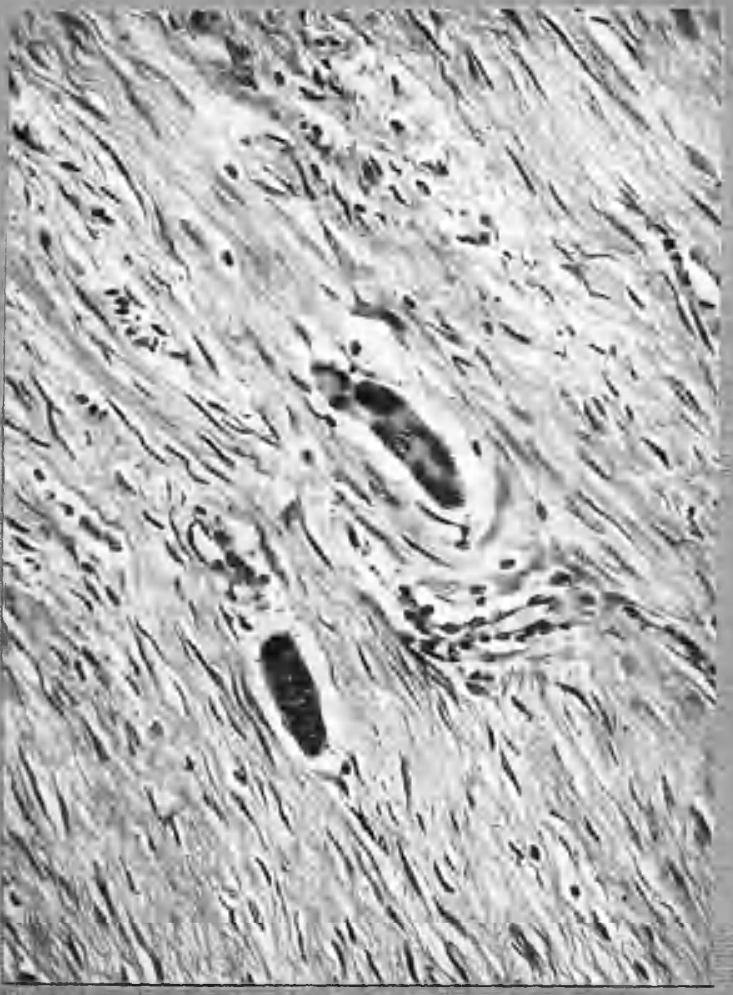

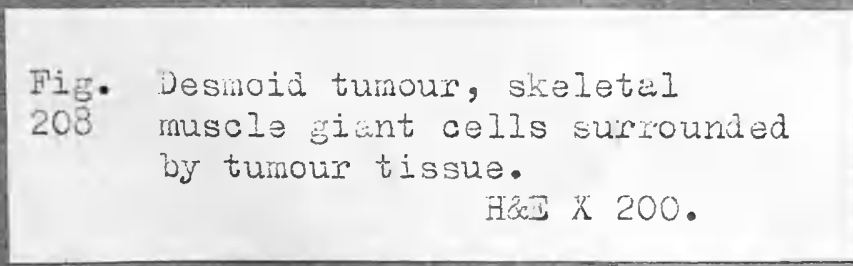

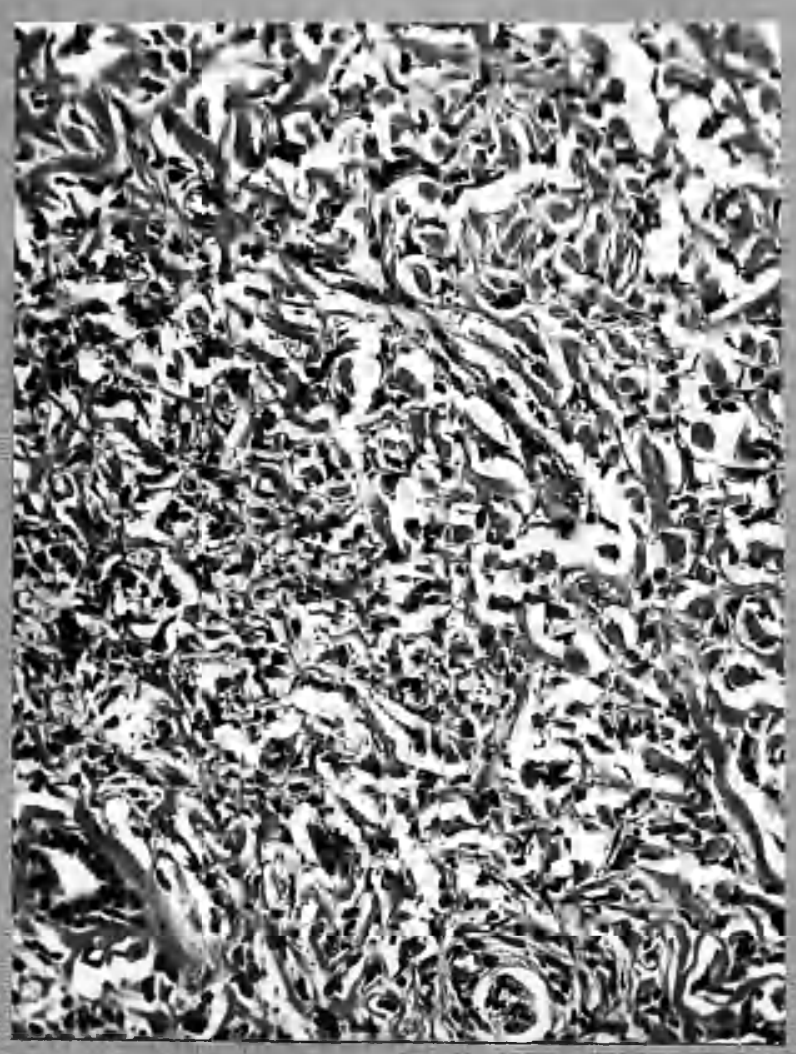

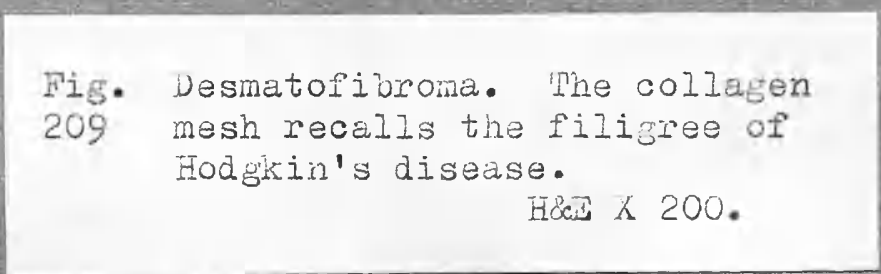




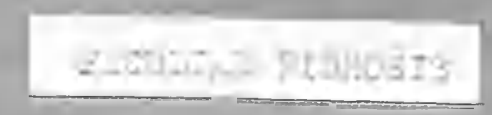

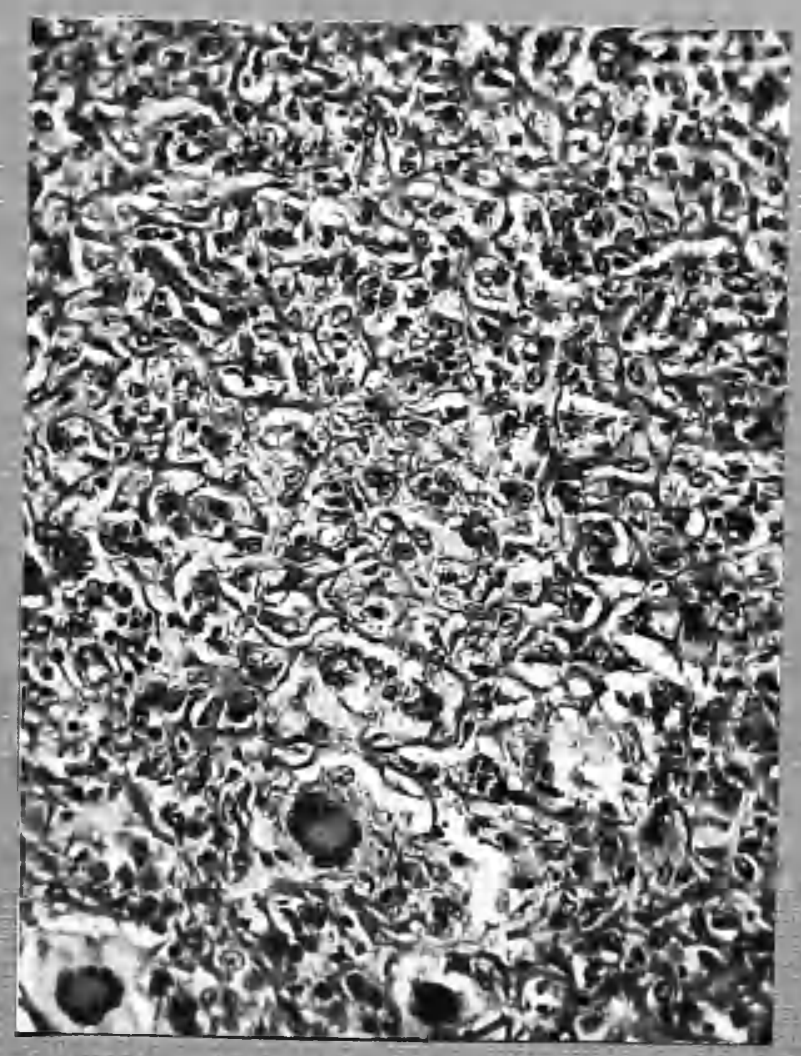

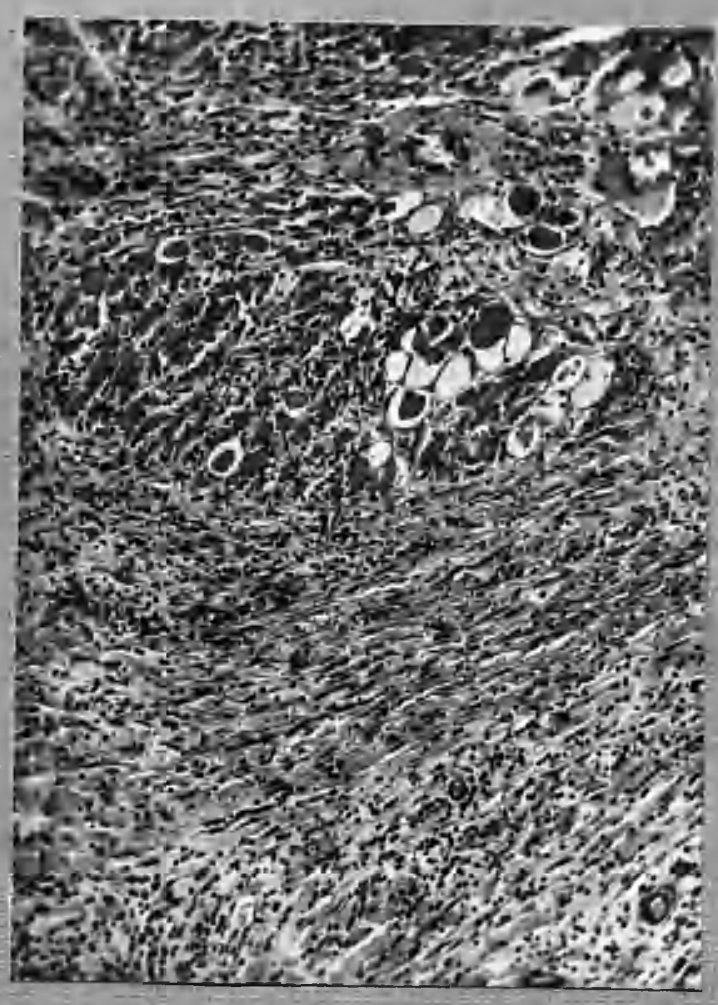

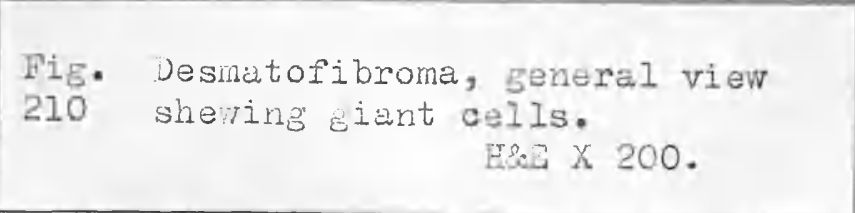

$$
\begin{aligned}
& \text { Fig. Reidel's struma bleletal } \\
& 211 \text { muscle overwhelmed by peri- } \\
& \text { glandular fibrous tissue growth. } \\
& \text { Hoi } \mathrm{x} 100 .
\end{aligned}
$$



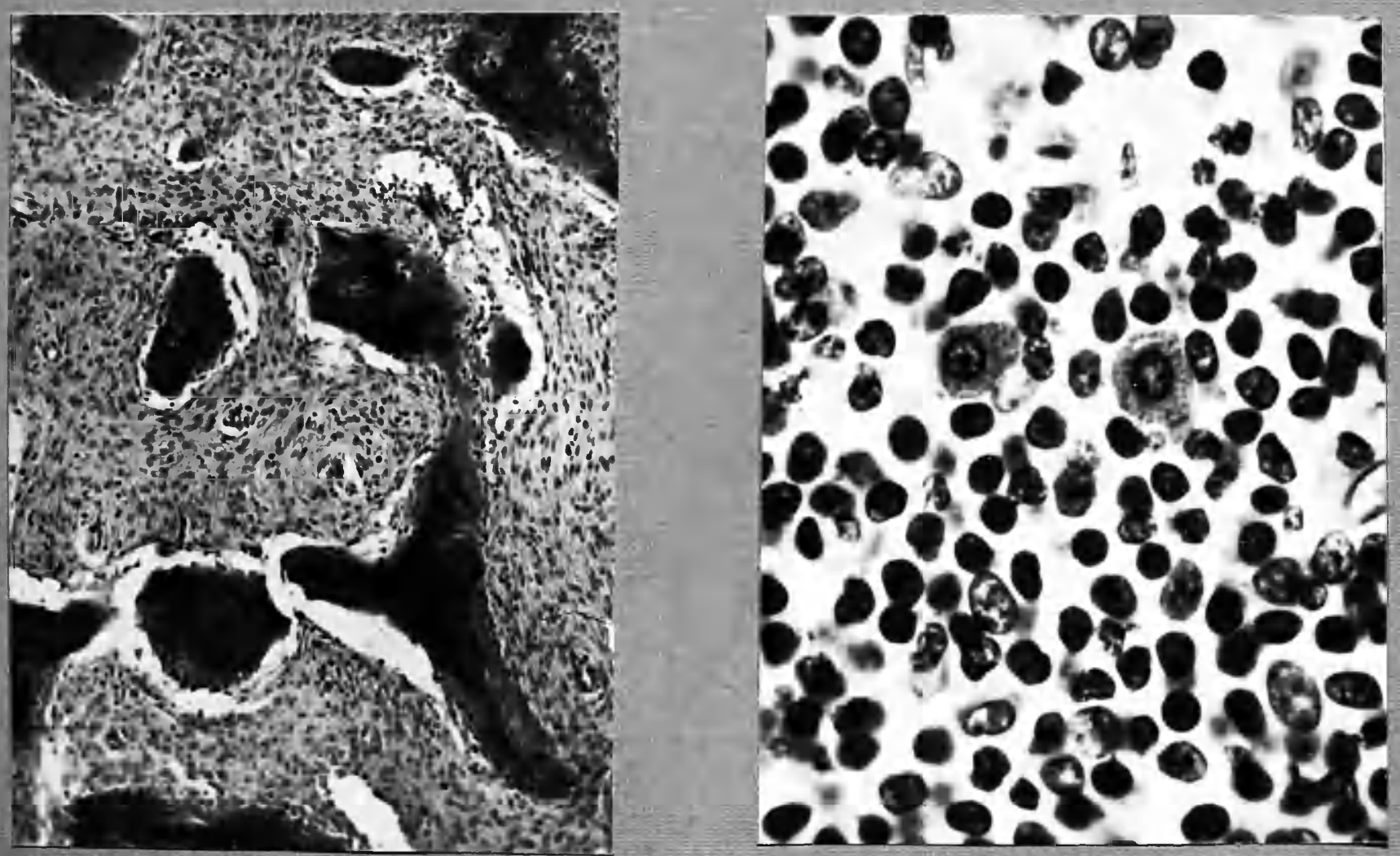

Fig. Lyelofibrosis. Uniform young 212 connective tissue replaces the marrow.
Fig. Iymphocytoid eosinophiles in 213 lymph node shewing early Hodghin's àisease. Doninici $\times 600$. 

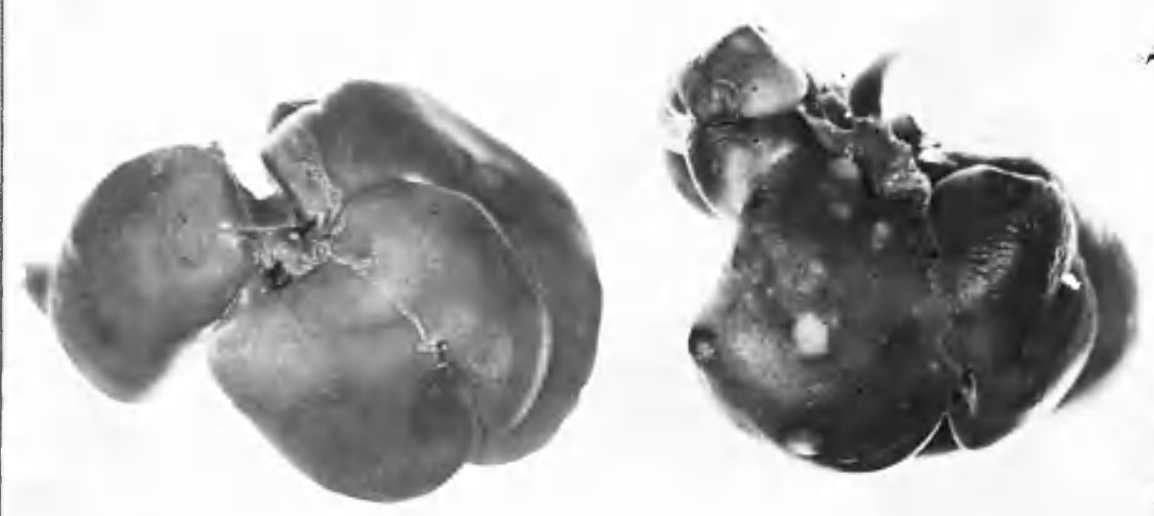

Fig. Liver and spleen of rat 215 D.3. in section. After weekly I.P.I. $10 \mathrm{mgm}$. trypan blue over 125 days. $\frac{1}{2}$.

Fig. Left. Liver of rat A.5. after fortnightly I.P.I. $10 \mathrm{mgm}$. trypan 214 blue over 330 days. Tumour-like nodules are not present. Right. Liver of rat 4.4 . after fortnightly I.P.I. $10 \mathrm{mgm}$. trypan blue over 200 days. Numerous spheroidal tumour-like nodules. 1/1 


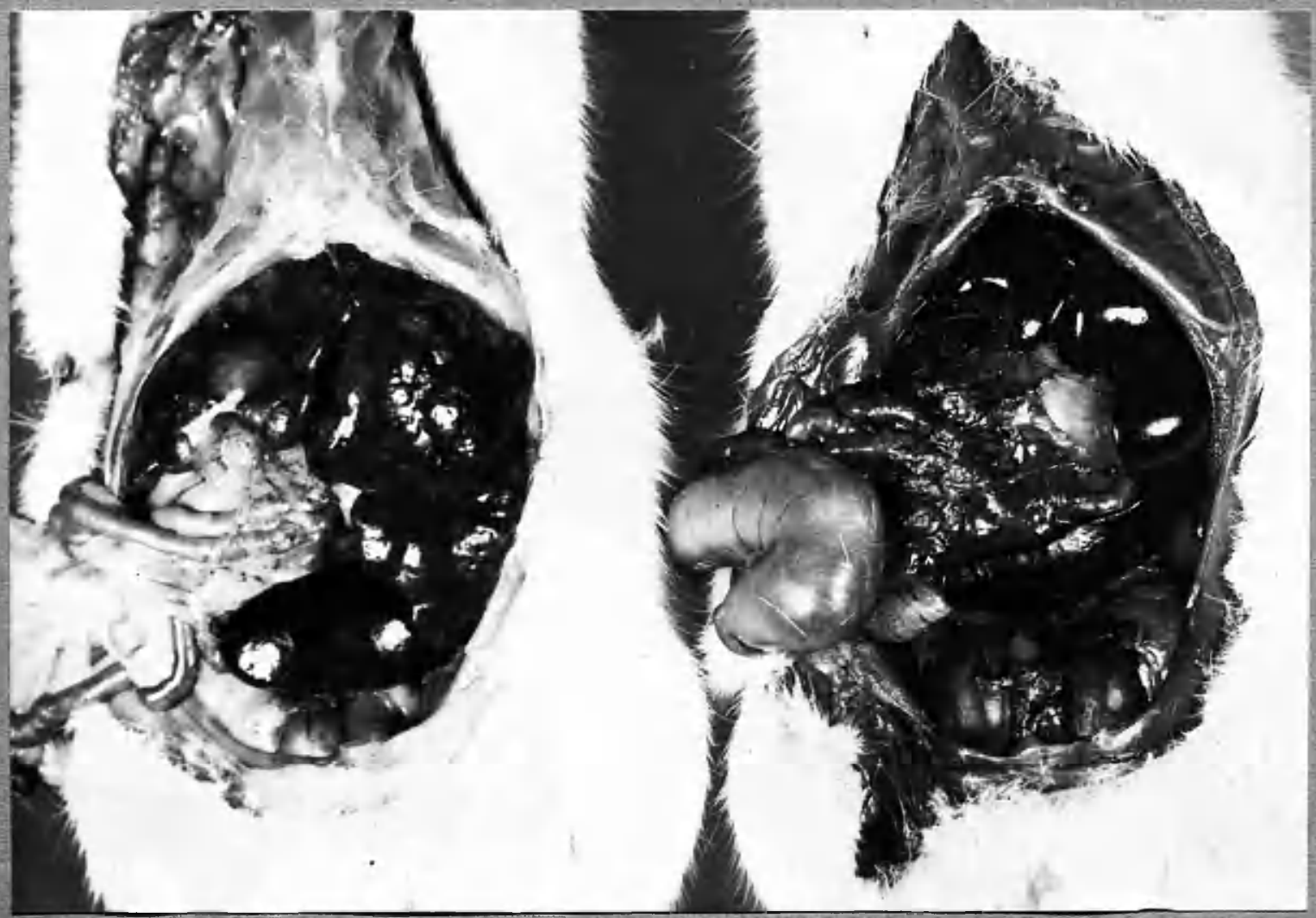

Fig. Necropsy of rats 3.9. (left), E.10. (right). Nodules are seen in 216 the liver of $\mathrm{E} .9$. but not in $\mathrm{E.10}$. (In both the wedge-shaped deficiencies are due to antecedent biopsies). Highlights on the spleen give a false impression of nodules. $1 / 1$. 


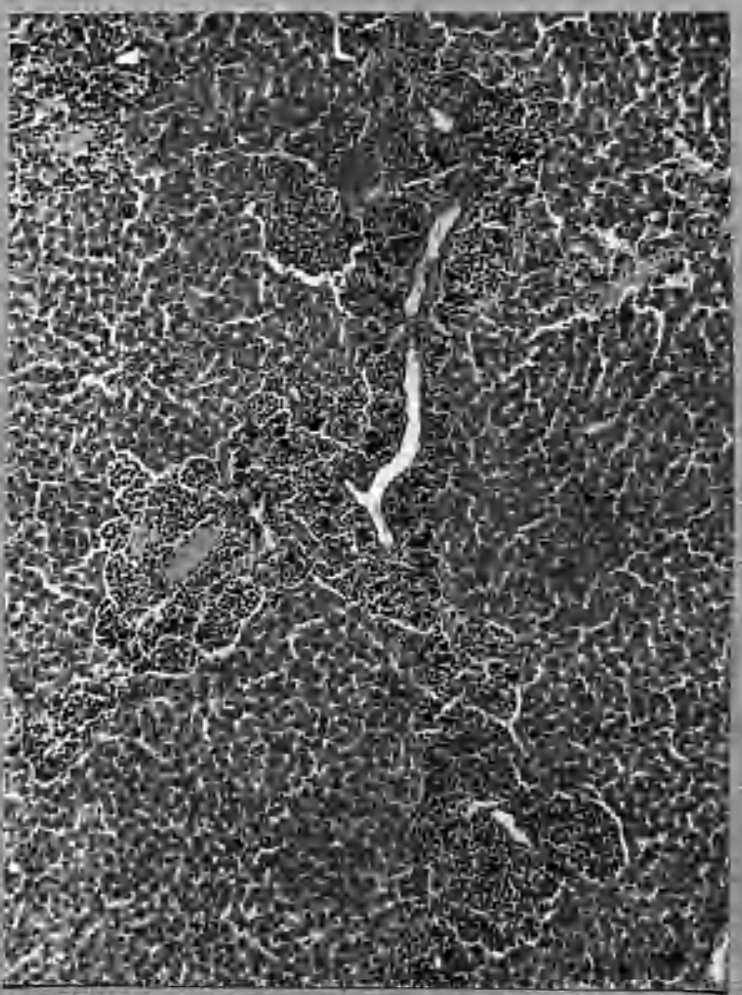

Fig. Rat A.2. Trypan blue I.P.I.

217182 diys. Iiver. Nodular deposits of cells along portal tracts. The dye-laden cells appeir black. H\&a X 65

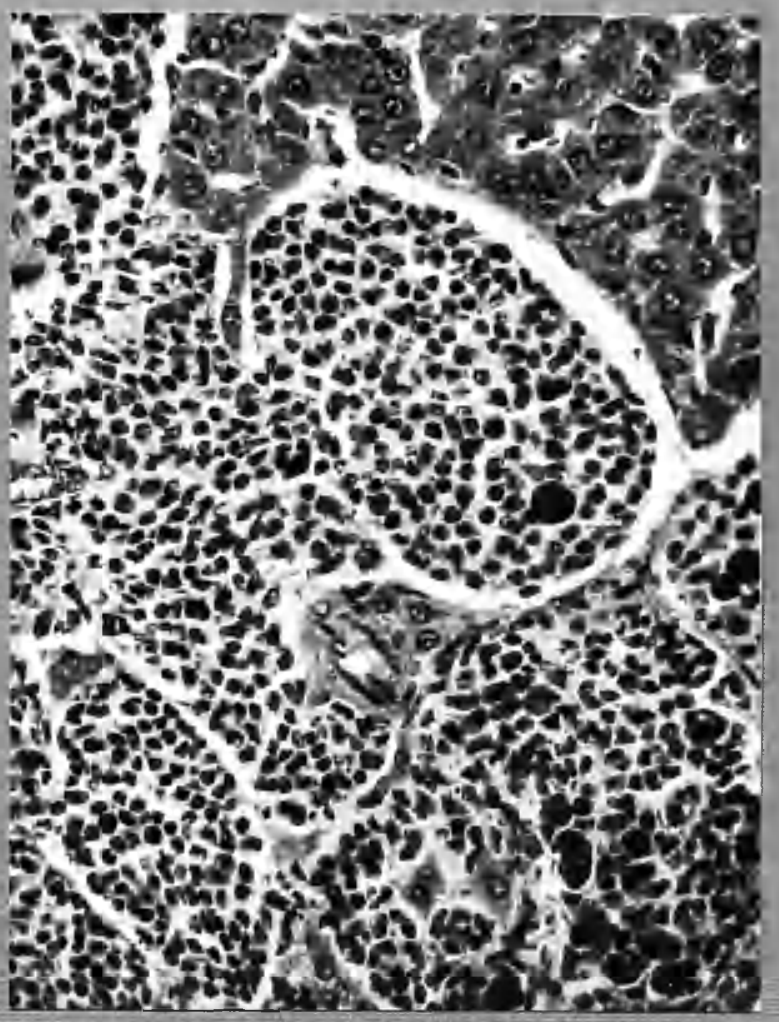
Fig.
Rat A.2. Liver. HP View of 218 previous figure. Note uni- formity of cell type except for dye and iron holding cells. H\& X 250 

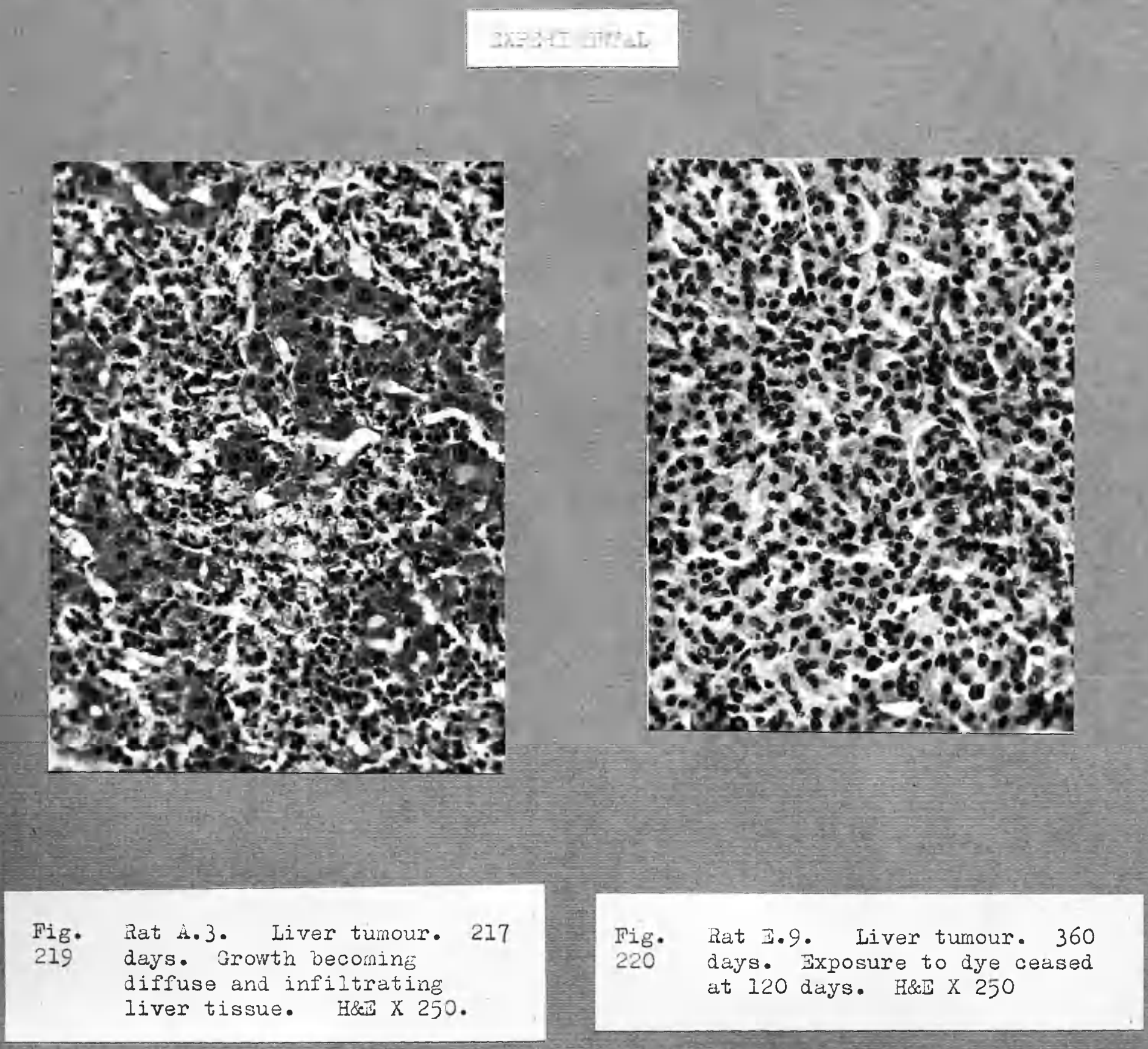


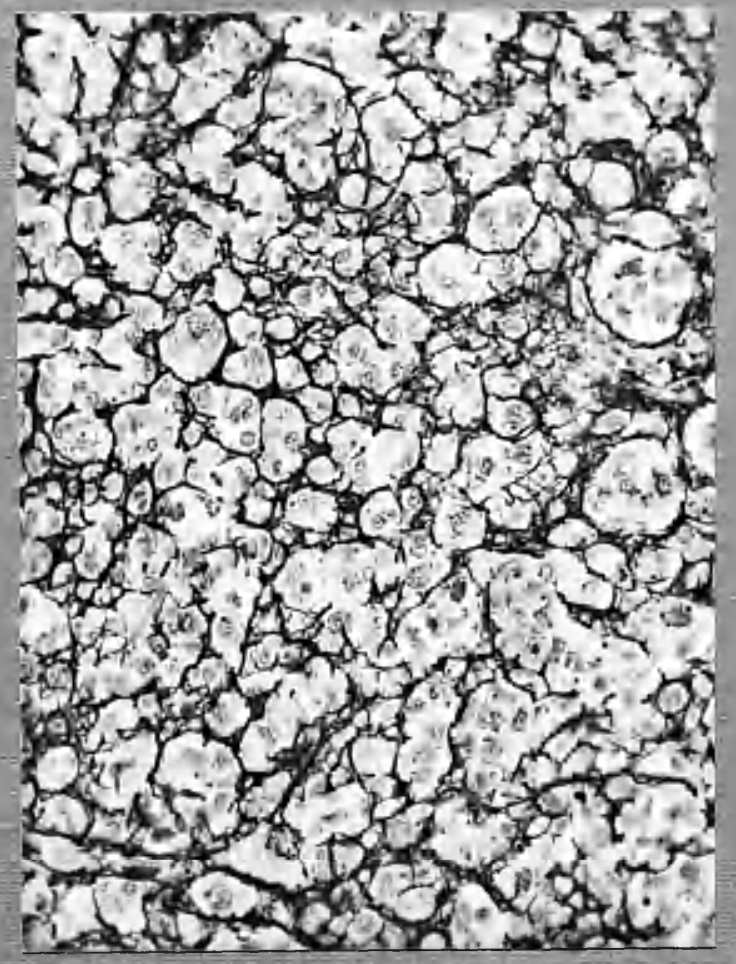

Fig. Rat $\mathrm{E} .9 . \quad$ Liver tumour, silver

221 formation. silver $\times 250$.

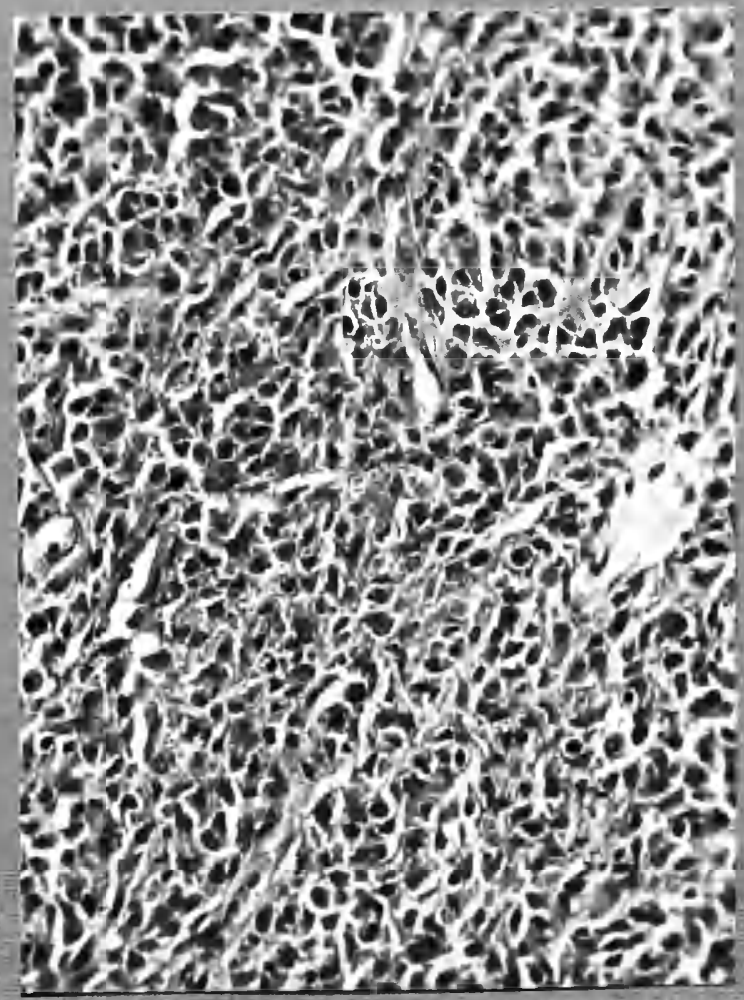

Fig. Rat A.4. Liver tumour.

222 Incipient fibrosis. Hed X 250. 


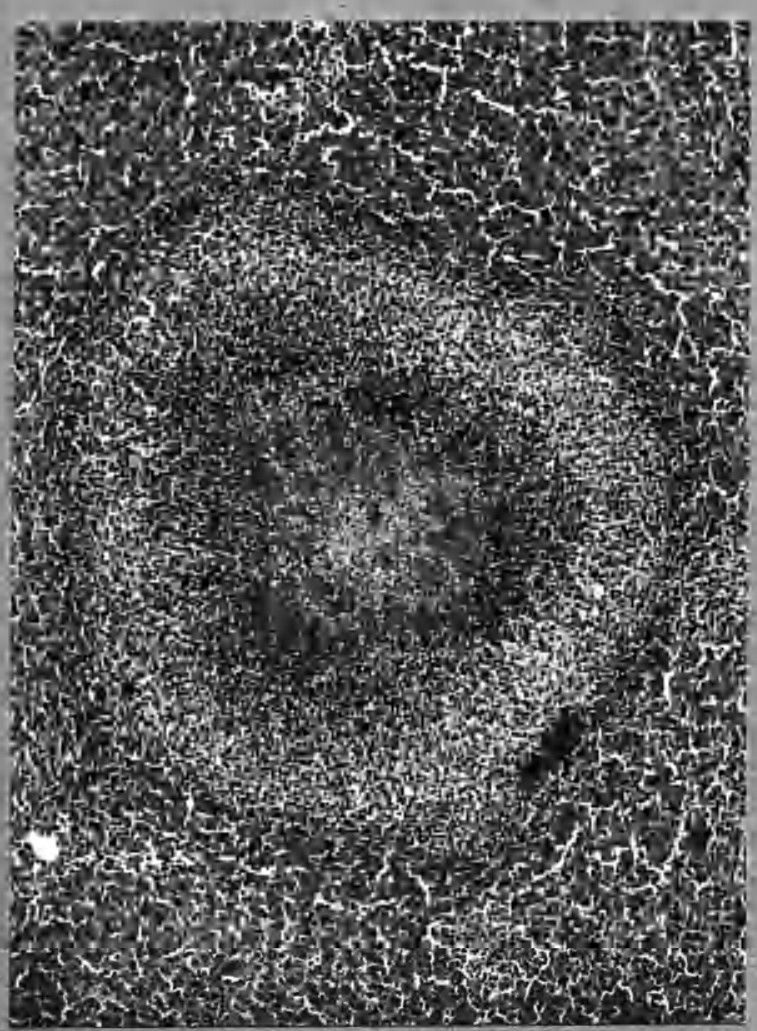

Fig. Rat A.7. Liver tumour with

223 central necrosis (280 days). H8: X 65.

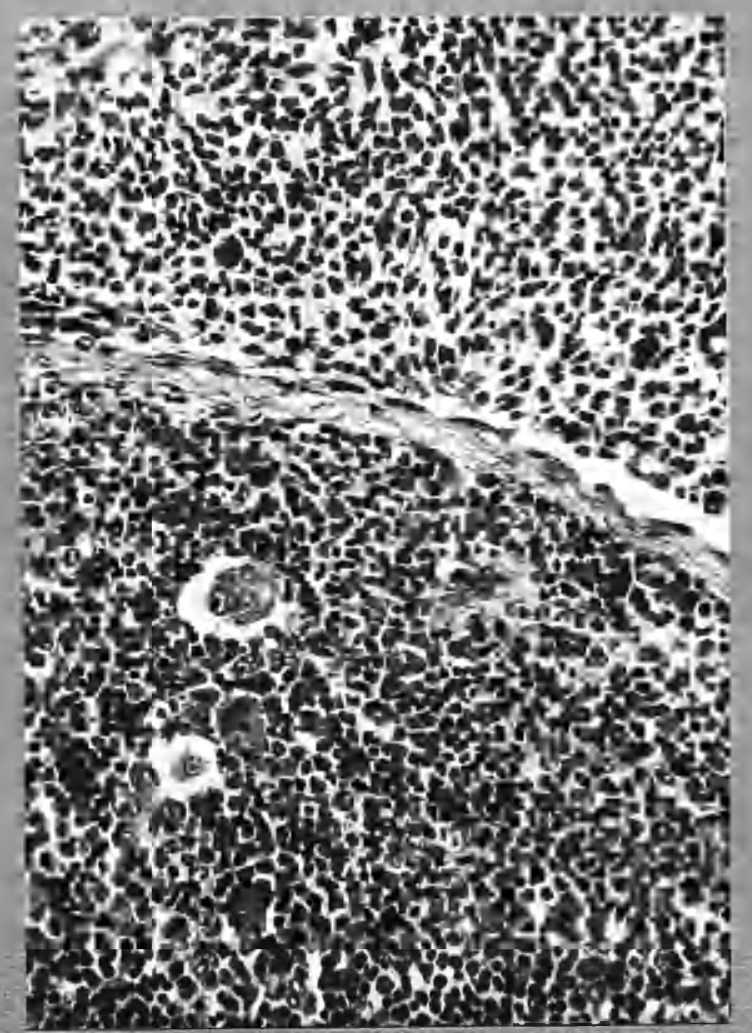

Fig. Rat. A.2. Spleen with capsule 224 and omental tag above. Reactive hyperplasia and megakaryocytes. Omental tag shews similar picture to hepatic tumour. Ho X 250. 


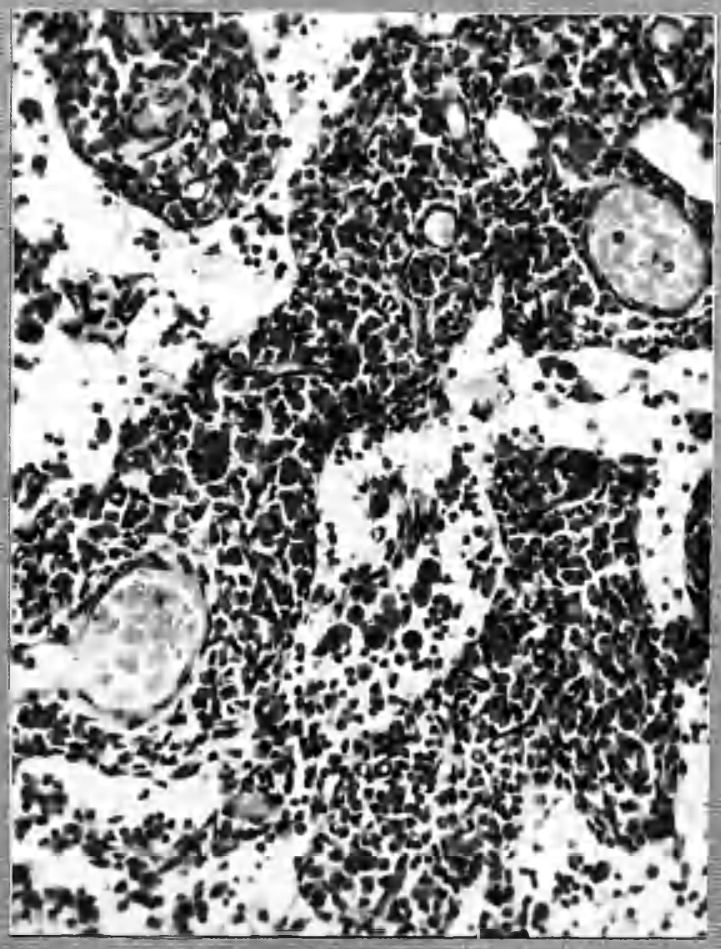
Fig. Rat A.8. 300 days. Axillary
225 lymph node. Haemosiderin and dye in macrophages, sinus oedema. H\&E X 250.

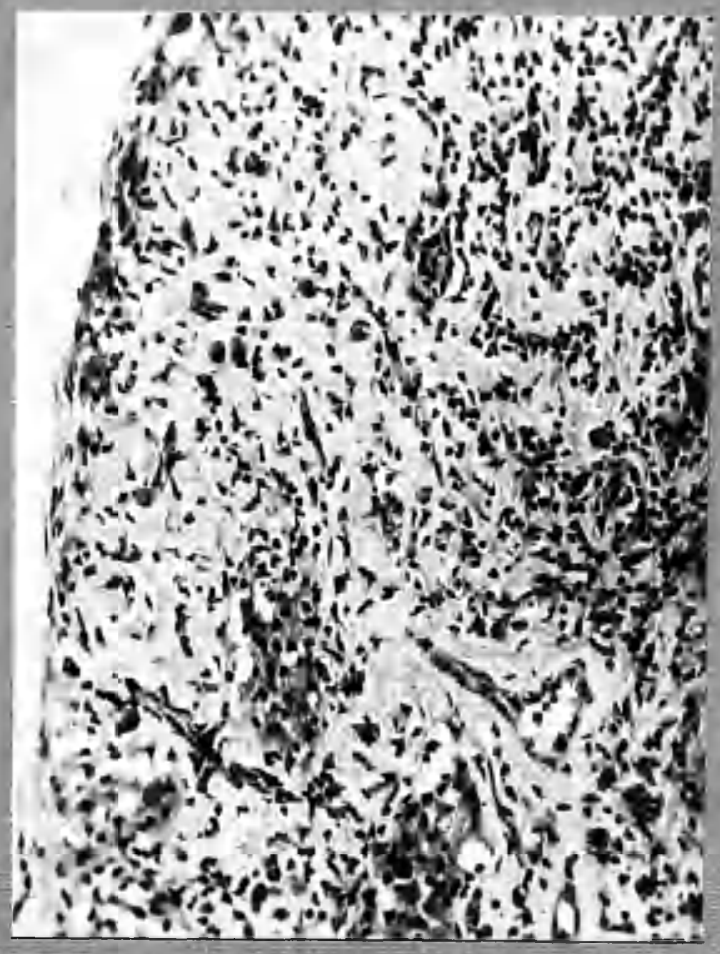




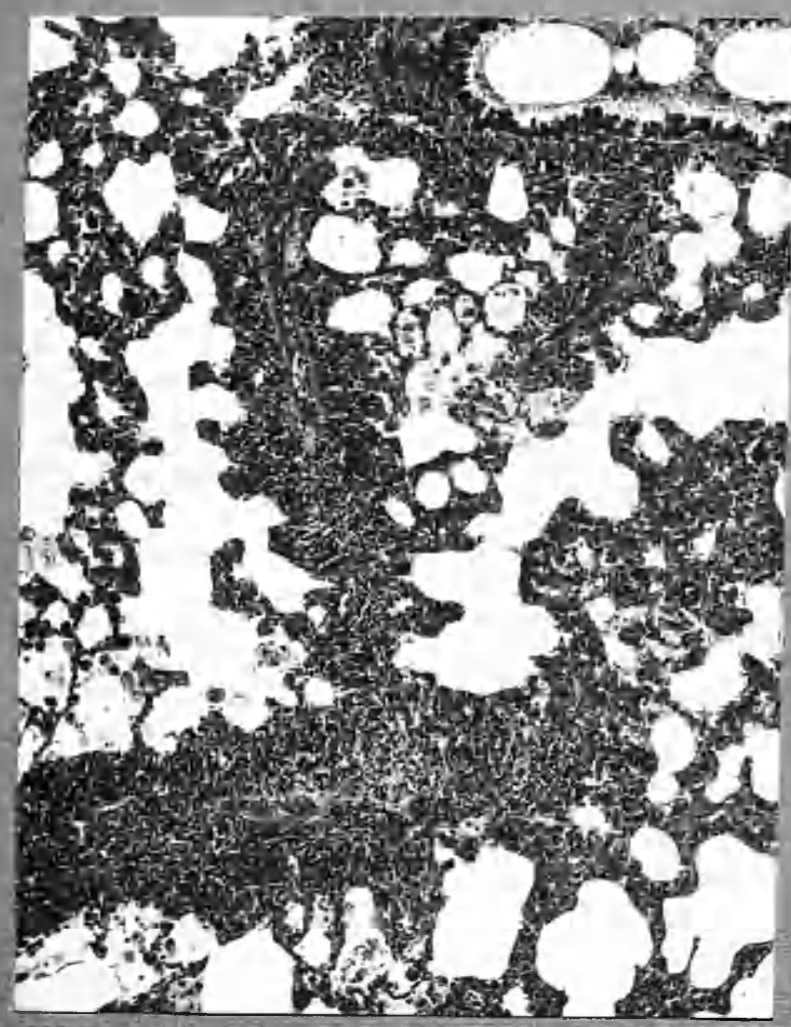

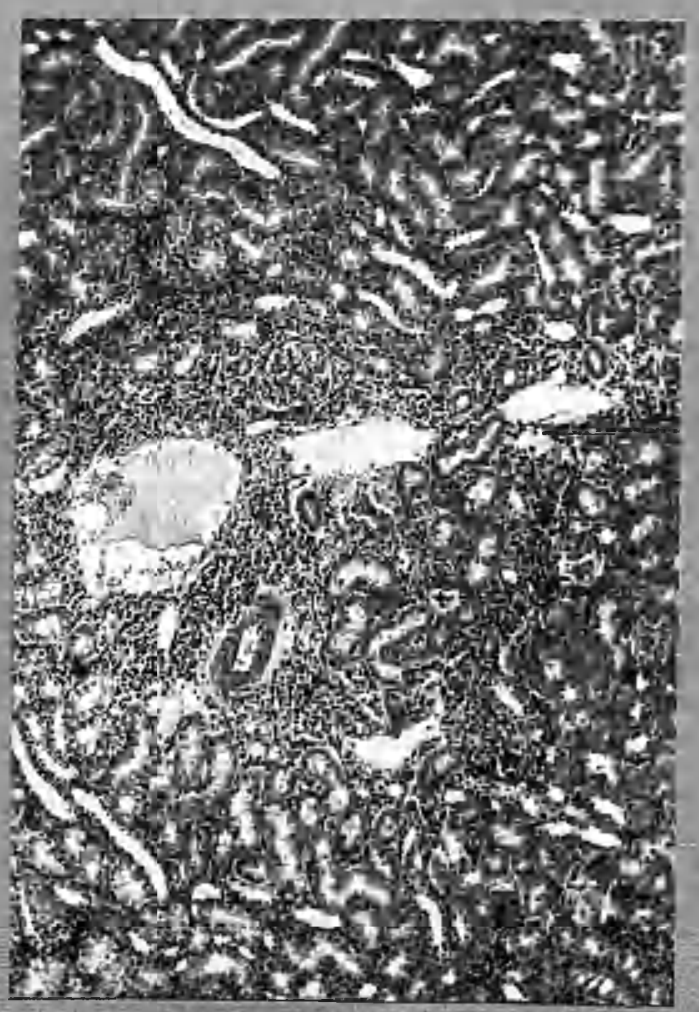

Fig. Rat.A.2. Kidney. Difiuse macro228 phage focus, simulates pyelonephritis. H\&6 X 60.
Fig. Rat d.2. Lung. Perivascular 227 and peri-bronchiolar cuffing with nacropinages. H\&i $\times 65$. 


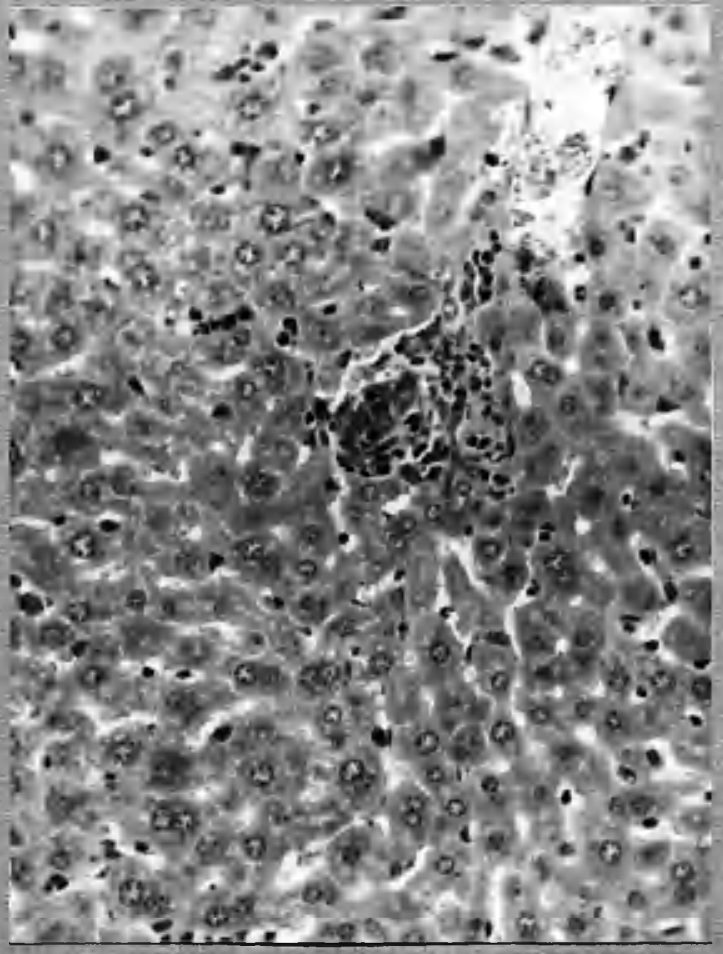

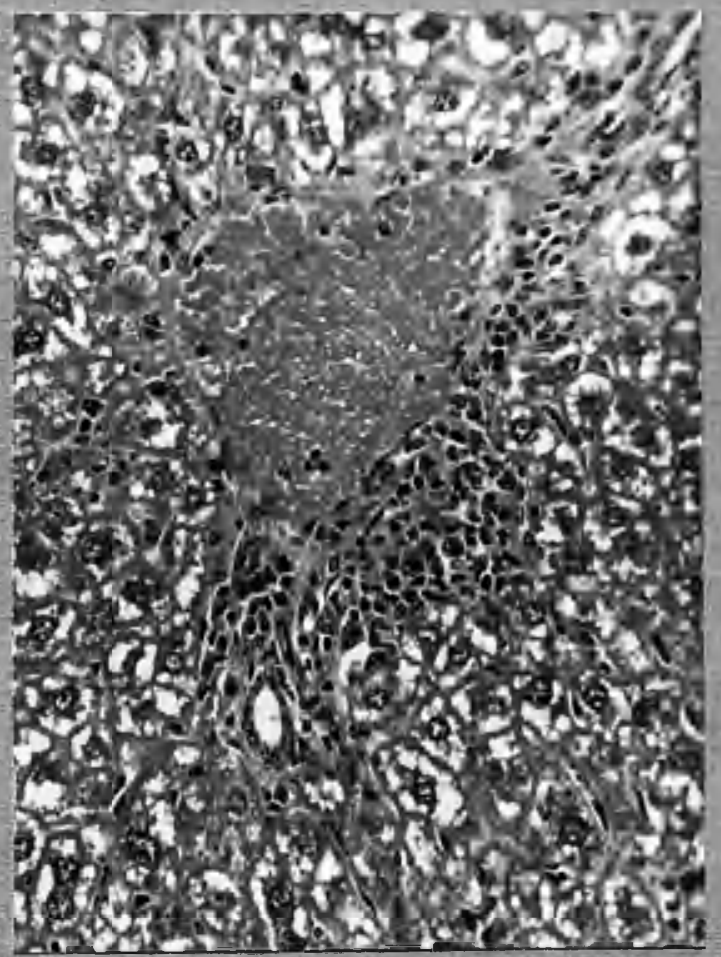

Fig. House F.j. 1.0 ngm. trypan

229 blue I.P.I. weekly. 250 days. liver. Doubtful nodule in portal tract. Kuppier cells prominent due to dye content. F\&: $\times 250$.
Fig. Wouse F.20. $1.0 \mathrm{mg}$. trypan

230 blue I.P.I. weekly. 360 days. Iiver. Portal tract. jlight periportal proliferation. स8्य X 250. 


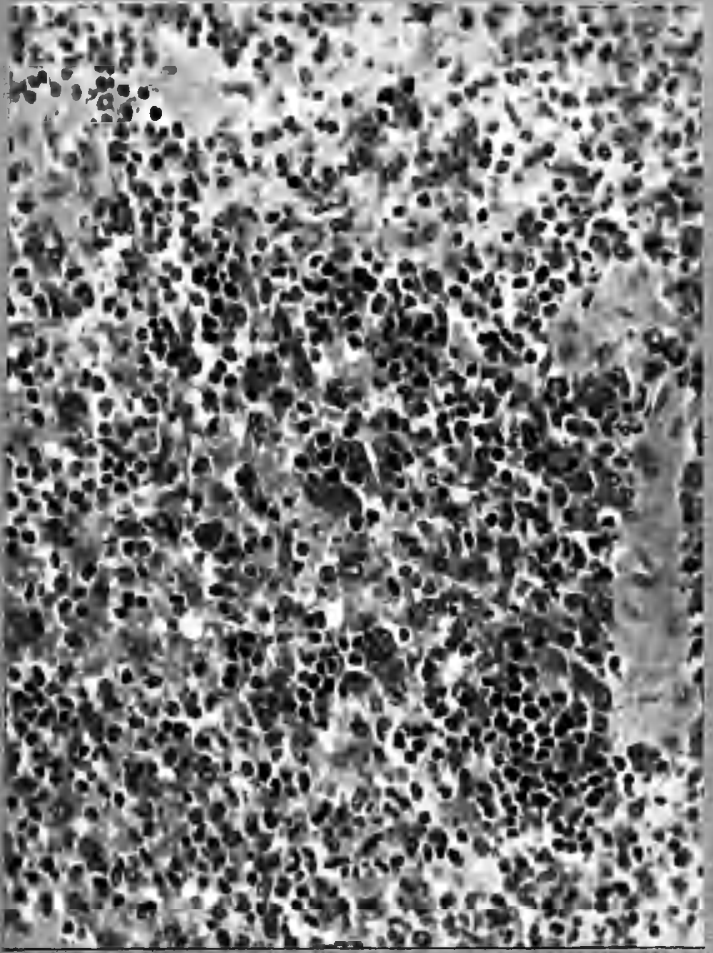

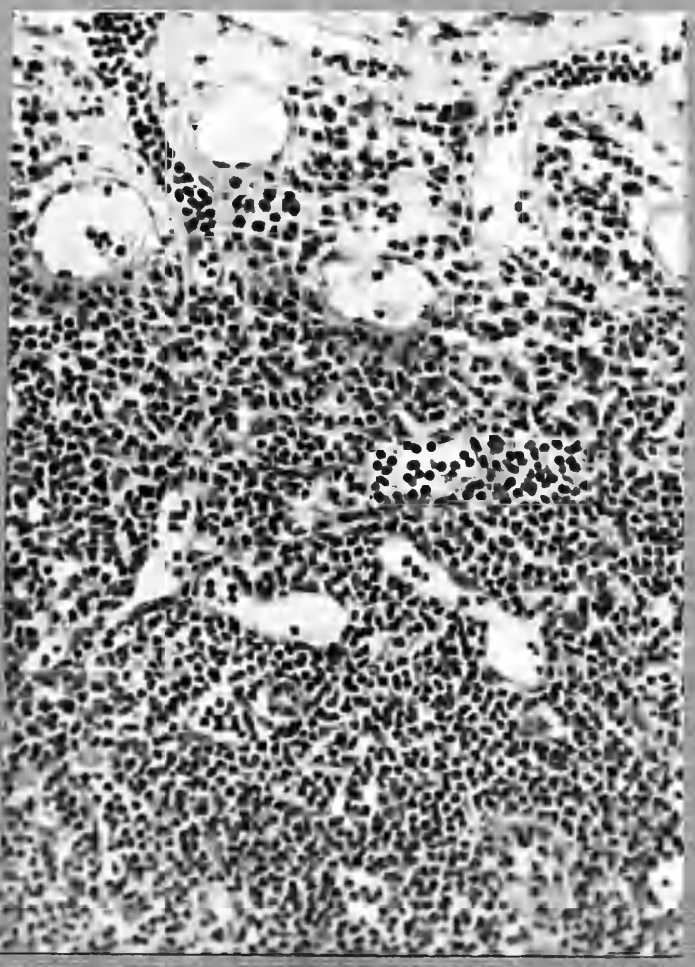

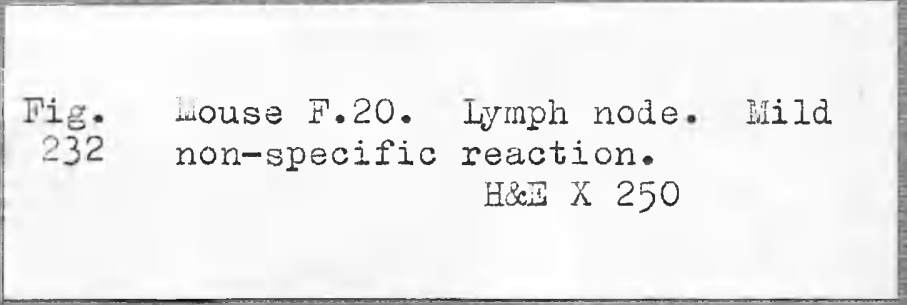

Pig. House F.5. Spleen. Red pulp

231 hyperplasia anà mild haenosiderosis. Cells with dark cytoplasm contain iron. H\&E X 250 . 

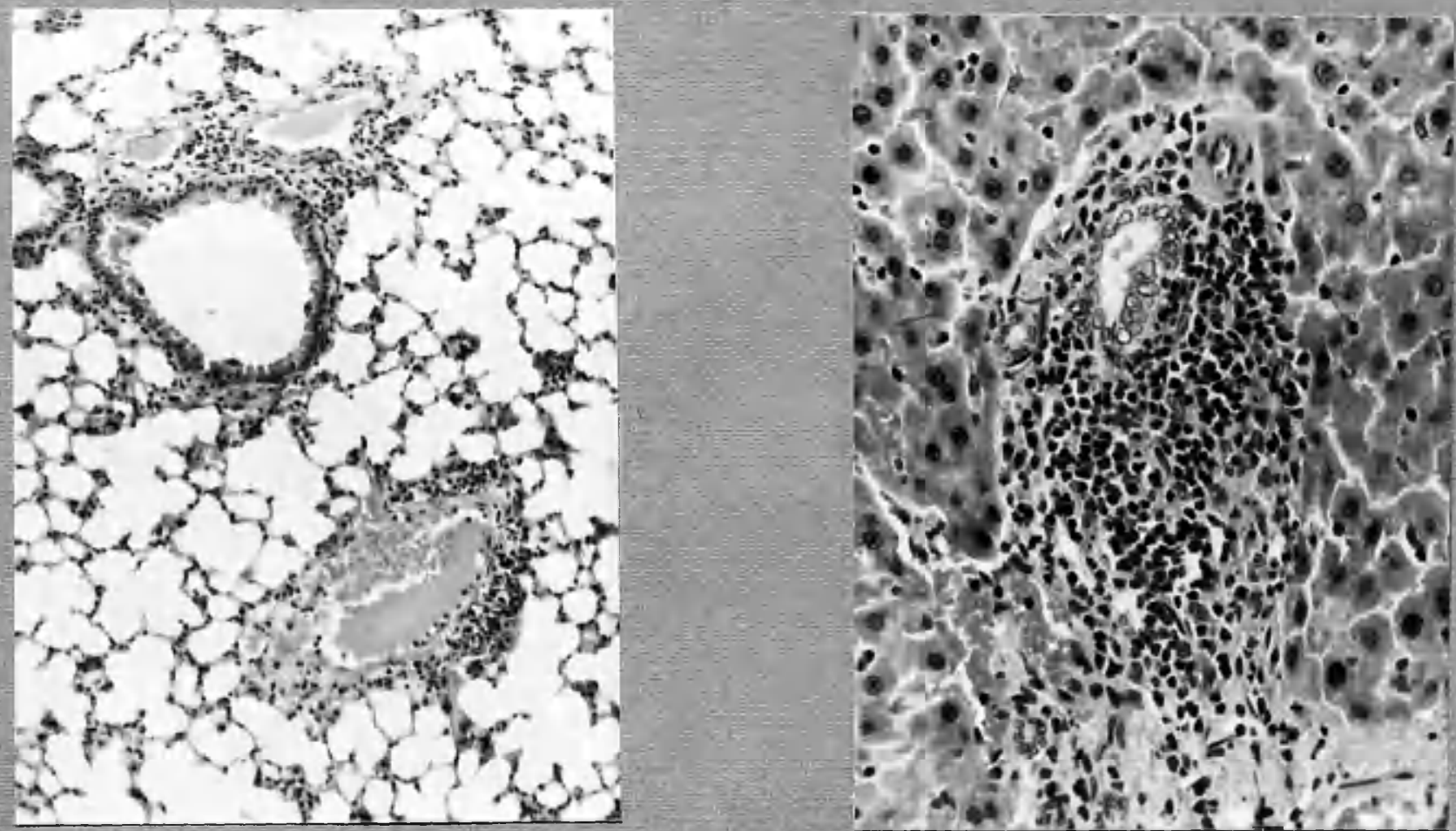

Fig. WouseF.18. Iung. Light peri-

233 vascular and peribronchial cuffing with proliferated macrophages. Hici X 150.

Fig. Rat C.8. Vital new red. 10

mgm. I.P.I. fortnightly 360

days. This is the largest periportal lesion found in the series. HiE X 250. 


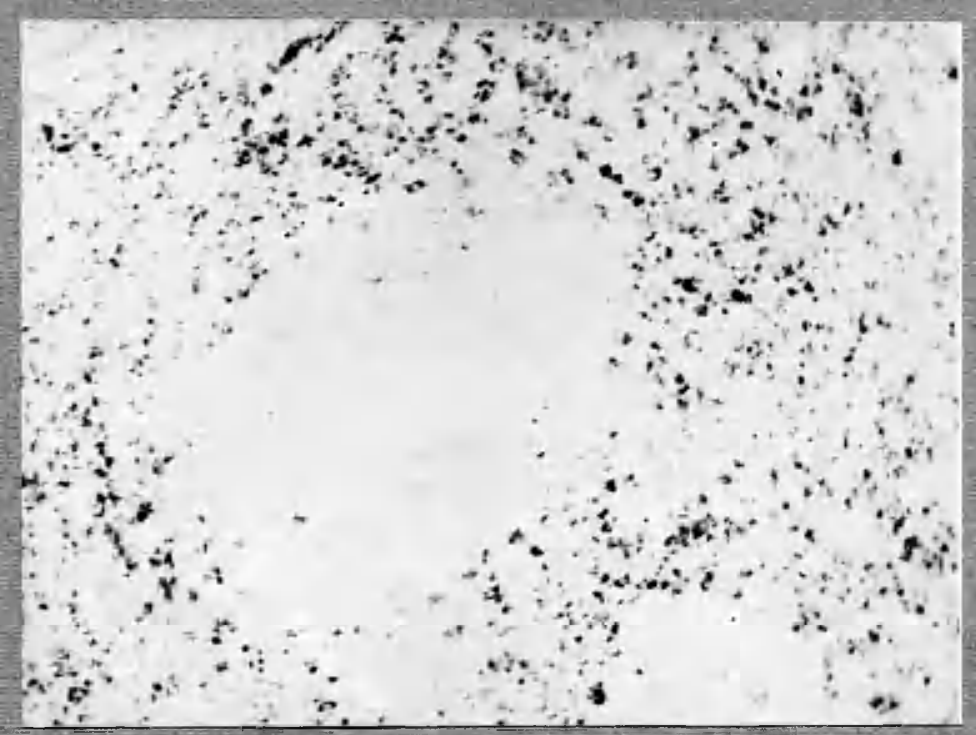

Fig. Rat 0.8 . Jpleen, to show 235 haemosiderosis. Prussian blue \& aaffranin. Red filter a cju. 
Photographs Fig.236 to Fig.245 illustrute the Iymph nodes recovered by dissection from cases 2. 3.5.6. 8. 9. 10. 11. 14. 20. Fig.246 depiots nodes recovered from the left axilla in an untreated case of Hodgkin's disease, 46 masses dre shewn but if the lobulated nodes are considered fusion products the total is 55 .

Text reference p.109 et seq.

E.149.

LYMPH NODES RECOVERED FROM THE LEFT AXILLAOF AN INFANT AGED 6 DAYS. TOTAL NUMBER 10.

\section{Pig. 236}

$+3 \cdots \ldots$

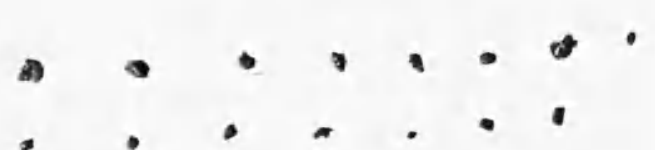

B.147.

LYMPH NODES RECOVERED FROM THE LEFT AXILLAOF AN INFANT AGED 30 DAYS. TOTAL NUMBER 23.

\section{Sopopont.

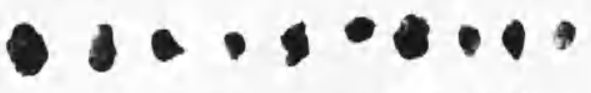 \\ - •...... \\ 150.}

LYMPH NODES RECOVERED FROM THE LEFT AXILLA OF A BOY AGED 5. YEARS. TOTAL NUMBER 32.

\section{Fig. 238}

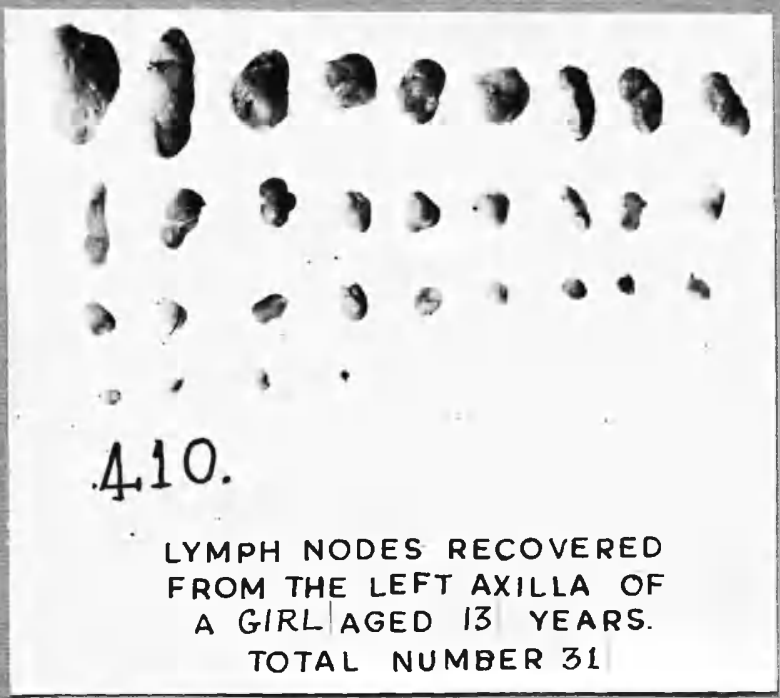




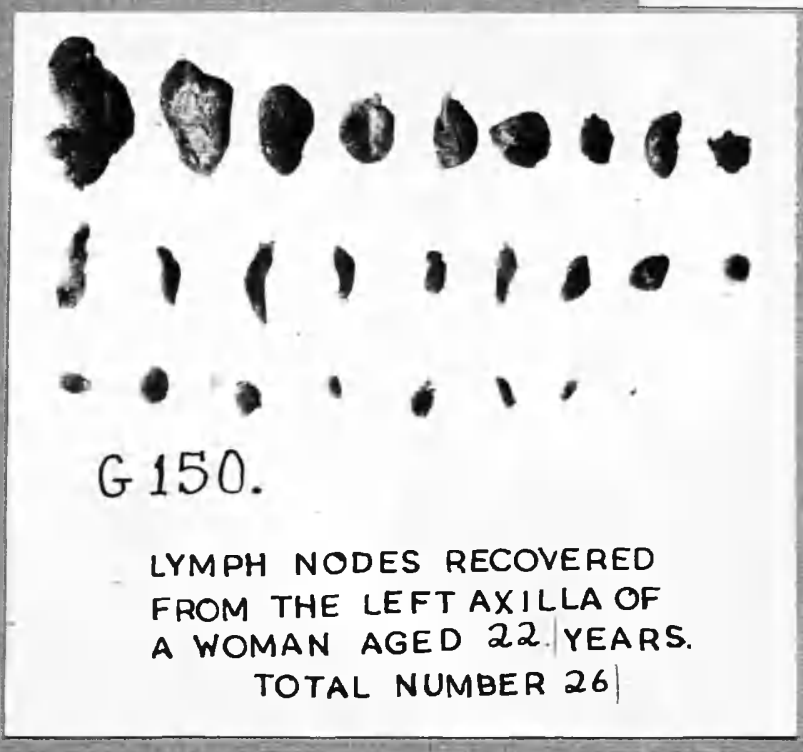

\section{Fig. 240}

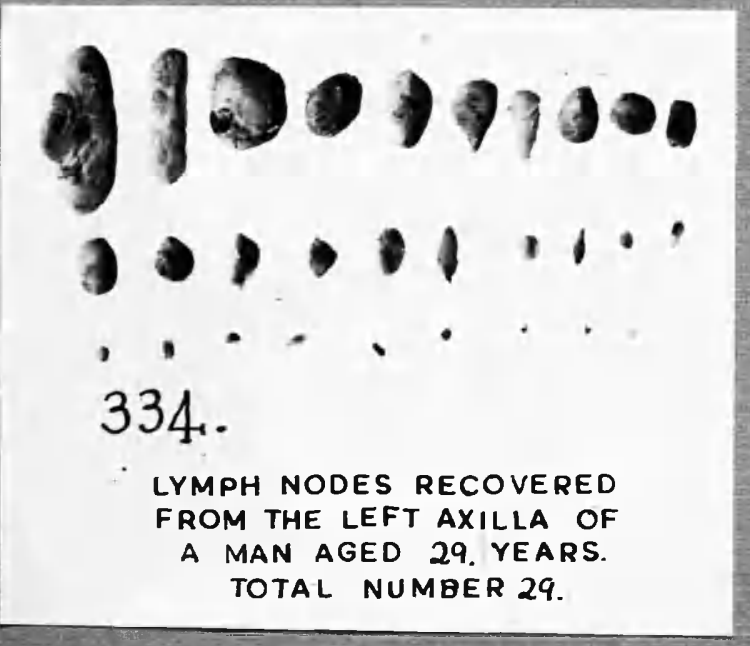

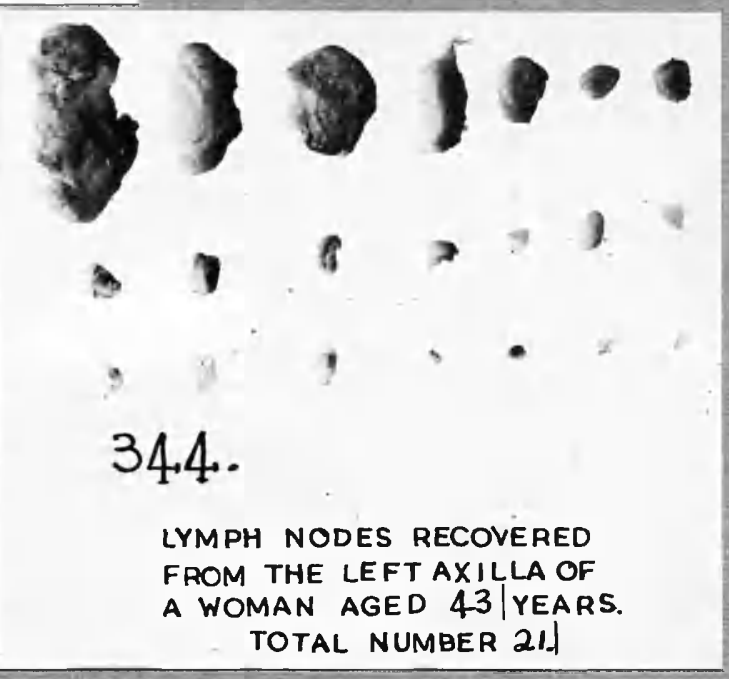

Fig. 242

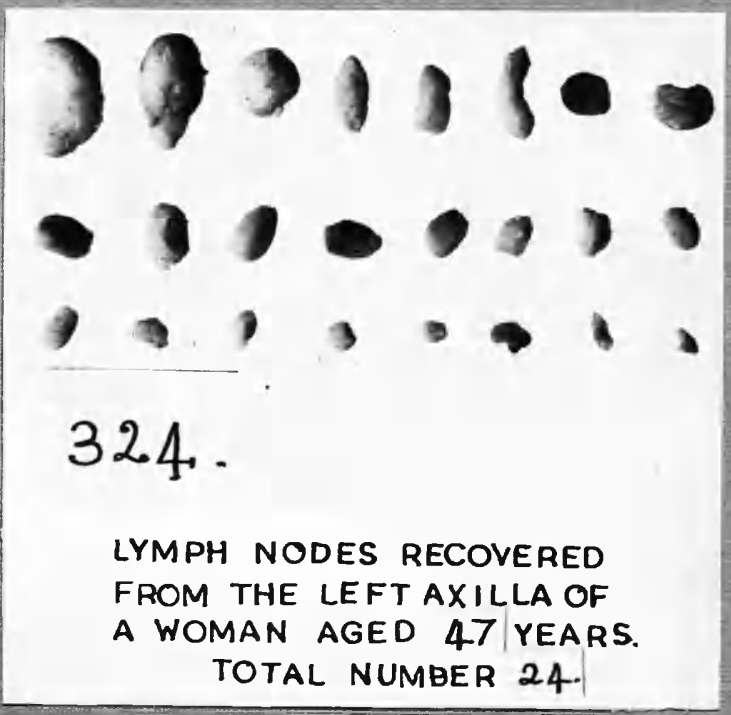

\section{Fig. 241}

\section{Fig. 243}


LYMPH NODES RECOVERED FROM THE

IEFT AXILIA OF A MAN AGED $6 I$ YEARS TOTAI 12 .

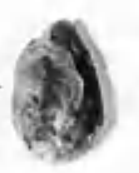

,
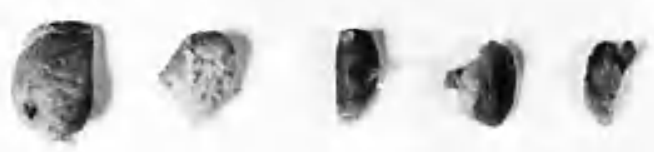

कำ

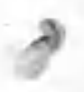

4

$$
\text { Fig. } 244
$$

LYMPH NODES RECOVERED FRON

THE LEFT AXILLA OF A WOMAN

AGED 81 YEARS. TOTAL 11.

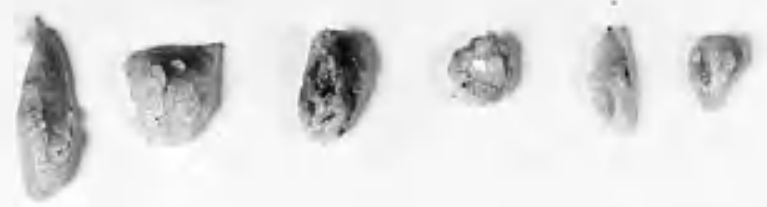

क क क 8 .

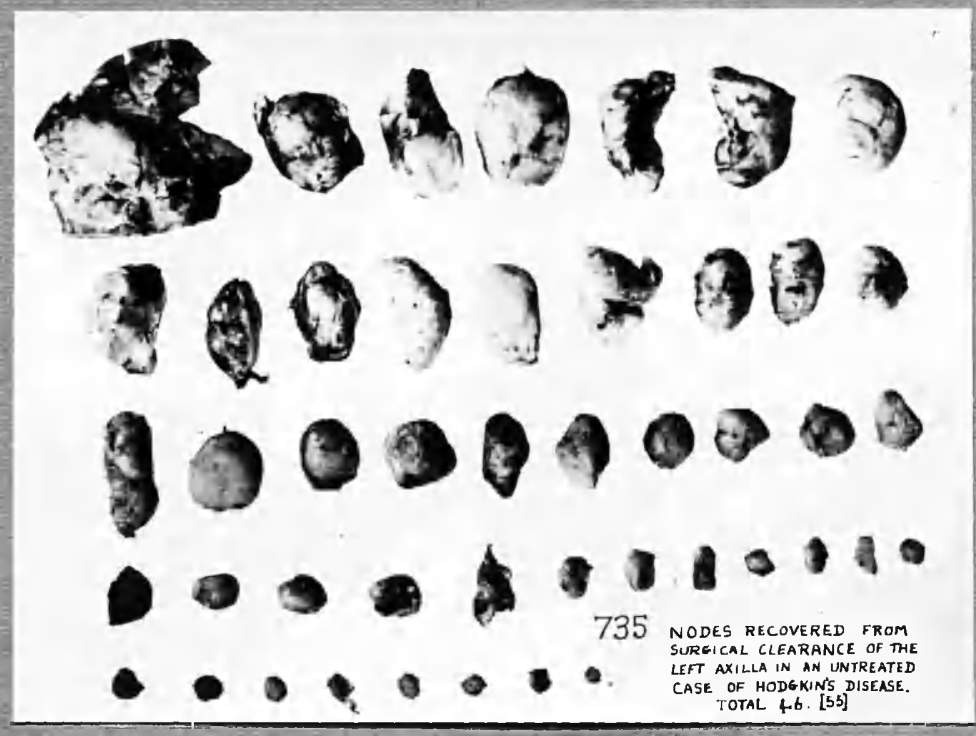

Fi.j. 21:6 


\section{VOLUTUE IV}

DRAWINGS, DIAGRAIIS AND GRAPHS 


\section{DIAGRALIS AND DRA INNGS}

\section{INDEX}

Subject

Pages

1. Maximow' schema of lymph node cell genealogy.

2. General relations of the lymphatic system.

3. Schematic Iymph node.

4. Structure of a lymph node (semi diagramatic).

5. Fat atrophy of lymoh nodes.

6. Vascular hyalinisation fibrosis in a lymph node.

7. Typical Hodgkin's disease.

8. Basic expressions of fibrosis in Hodgkin's disease.

$20-21$

9. Morbid reticulum cells.

10. Fibrosis from morbid reticulum cells.

11. Lymphocytoid eosinophiles.

12. Immature lymphocytes in Hodgkin's disease.

13. Folliculoid habit of Hodgkin's disease.

14. Physiological lymphoid tissue in Hodgkin's disease.

15. Illustrations of morphological affinities in lymphoid tissue sarcoma.

i Hodgkin's disease.

ii Hodgkin's disease, cellular type.

iii Hodgkin's disease, tumour-like type.

iv Hodgkin's disease, diffuse fibroblastic type.

v Hodgkin's disease, peculiar variant.

vi Hodgkin's sarcoma. 
viii Lympho-reticulo sarcoma.

ix Large celled lympho-sarcoma

16. The axillary lymph nodes. 58. 59.

17. Homunculus diagrams to shew distribution of lesions in Necropsy Series A - M.

18. Experimental Records.

19. Drawing of rat liver shewing appearance after 101 proloned trypan blue injection. 
MAXINOW' SCHEMA OP IYNAPI NODE (AND SPLEEN) CEIL GBNGAIOGY, SLIGHTTY IRODIRIED. 
$\Longrightarrow=\frac{\text { Inine of development with potencies }}{\text { uncestricted. }}$

$\Longrightarrow=$ Line of development ith potencies

(2) = Haenocytobiasts.

(3) = rree histiocytes = histiogenoms

(4) = Monoeytes.

(6) = Secondary erythrooyte semies (Definitive).

(7) $=$ Granulocytes.

O = Snall Iymphocytes.

$A=$ Common fixed mesenchymal cell.

AA = CeII lineage of Pixed synoitial histiocitic elements in the lumph nodes.

BB = CeII Iineage of free independentIy proliferating haenocytoblasts in the Iymph nodes.

CC = CoII lineage of tibroblasts in the Iymph nodes.

NOTES.

The Iarce Iymphocyte of Naximow is identified with the lymphoid wanderine cell and the haemocytoblast, (of Ferrato. et alii).

There is not any indication of megakaryocytes being formed in lymph nodes, $1 . e$. from haernocytoblasts, nor is there any reference to mast cell oricin. The polyblast of Maximow is conveniently regarded as an "indeterminate" cell which has several possible origins, and several possible development lines, and thus represents a kind of neutral phase.

In extra embryonic life, fibrosis can arise from reticular syncytium, both directly and via a polyblast metamorphosis. 
LYMPH NODES AND SPLEEN.

FIBROBLASTS. RETICULAR HAEMOCYTOBLÁSTS.

SYNCYTIUM.

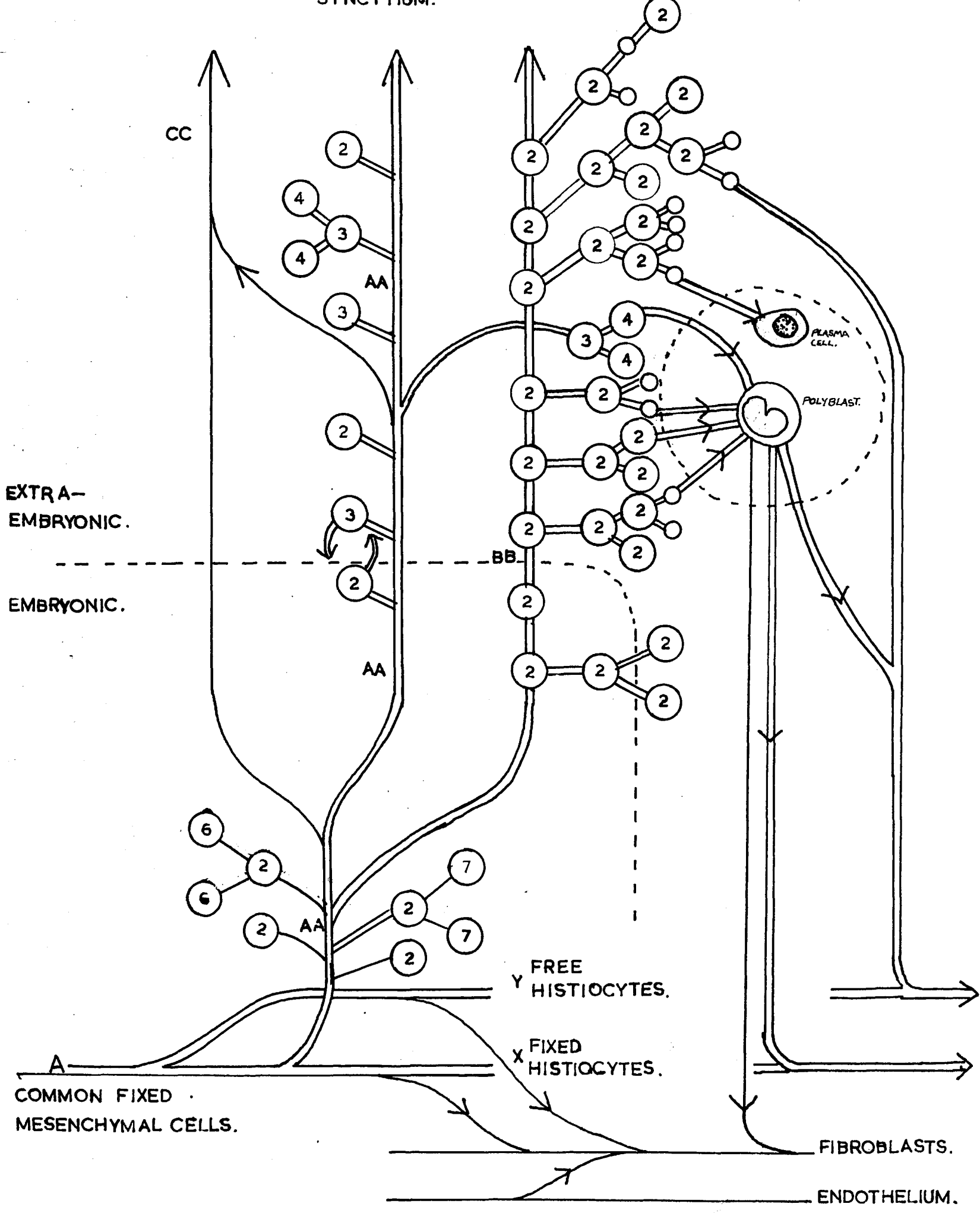


6.

\section{GRNARAL REIATIONS OF THE LYMPHATIC SYSTAM}

Arterial distribution is in red, the venous in blue. The vertical blue/red line represents the blood capillary mesh. The lymph stream is shewn in yellow.

Most lymphocytes entering the lymph stream are derived from the para-enteronic lymphoid tissue and related lymph nodes. The main lymphatic duct effects junction with the termination of the venous system. (The lymphatic drainage from the lungs is omitted in this diagram). 


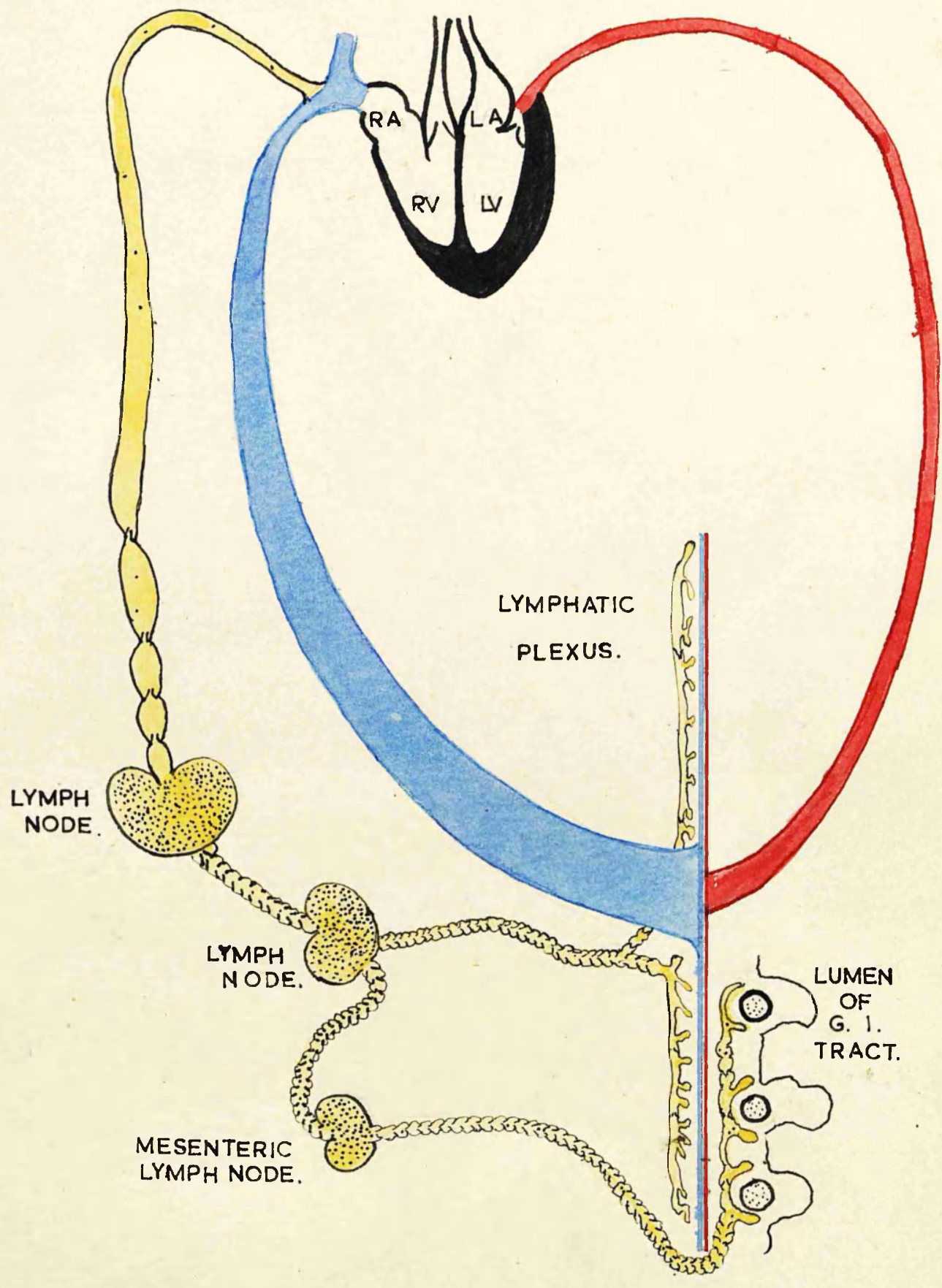

R.9.8A. 
8.

\section{SCHEILATIC LYHPH NODE}

A. Afferent lymphatic piercing capsule to reach marginal sinus.

$\mathrm{A}^{I}$. Efferent lymphatic leaving in hilar intrusion.

B. Capsule.

C. Marginal (sub-capsular) lymphatic sinus.

D. Cortical germinal lymphoid follicle, active.

E. Inactive germinal follicle.

F. Solid (secondary) lymphoid follicle.

G. Trabecula in section, note cuff of trajectorial lymph sinus.

H. Pulp of lymphoid tissue (loose lymphatic tissue, functional medulla).

I. Vessels of hilar intrusion.

J. Medullary lymphatic sinuses. 


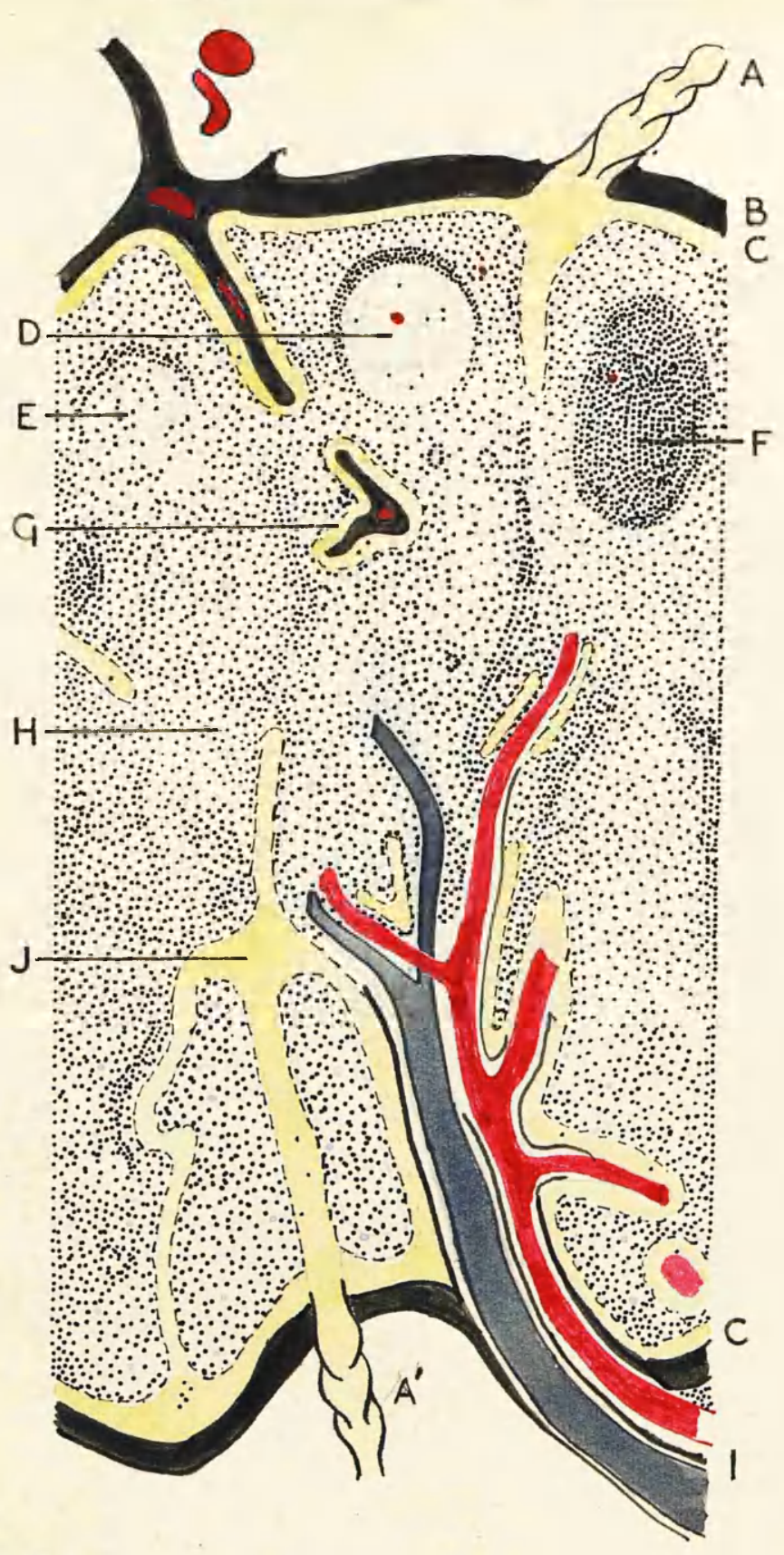

R.9.92 


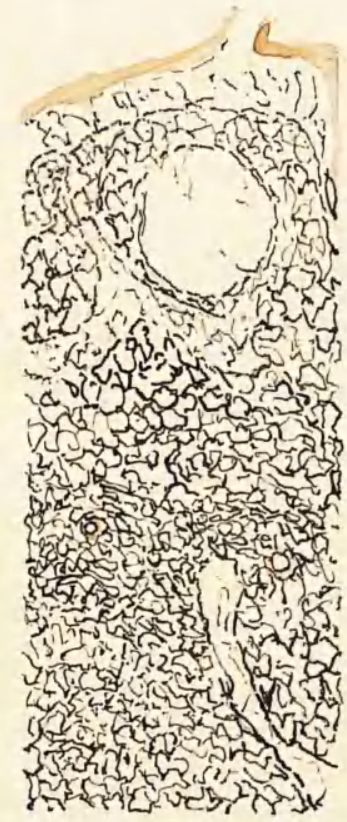

i.

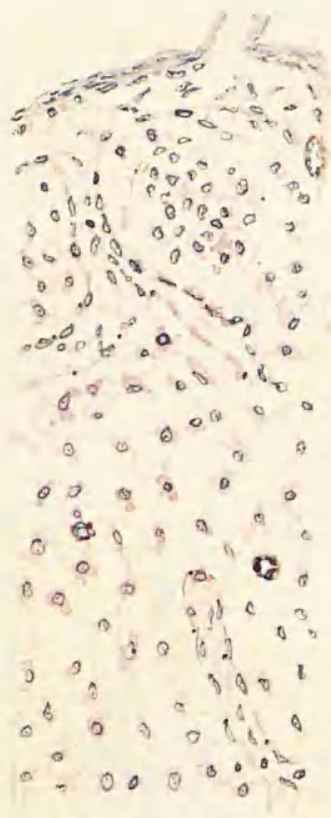

ii.

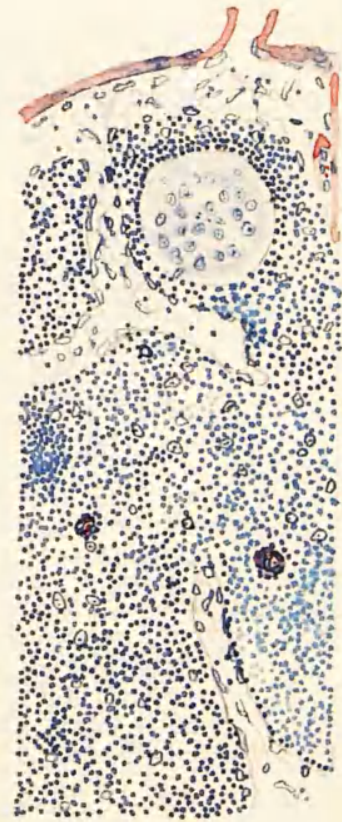

iii.

\section{STRUCTURE OF LYUPH NODE - SMMI DIAGRAMATIC}

Three hypothetical sections differently treated.

Fig. i Silver impregnation.

Fig. ii Ideal pencilled preparation.

Fig. iii Routine haematoxylin and eosin stained paraffin section.

In Fig. i. the argyrophile fibres - reticulin is shewn, note the absence from the pale centre. In Fig.ii. the reticulum cell plasmodium is shewn, pervasive continuity is maintained. Fig.iii depicts the familiar appearance of lymph node sections. 


\section{FAT REPLLICDNENT OF LYHFH NODES}

Figs. 1 and 2 represent low-power and high-power views of the process.

In Fig.l. Note that the fat of the fat cone is composed of larger cells than the fat of the adnate external areolar tissue. The lymphoid tissue has fallen back onto the persisting capsule and some marginal sinus tissue persists. Below the fat cone has reached the capsule and this membrane is disintegrating.

In Fig.2. Note the attenuation and complete lack of reaction of the lymphoid tissue, at this point the tissue resembles marrow. Lymphocytes tend to persist between the fat cells. 

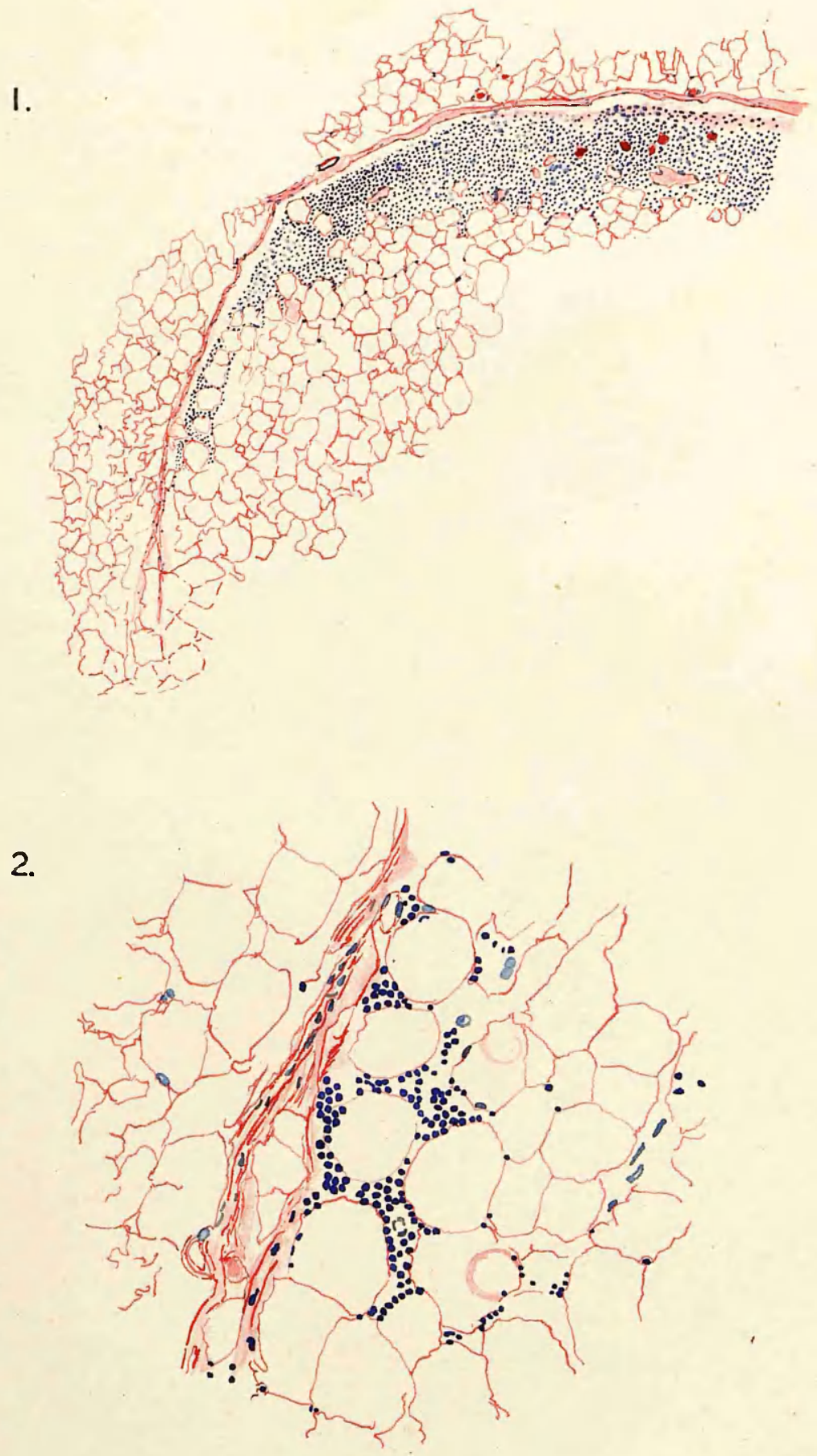

R.9.P. 


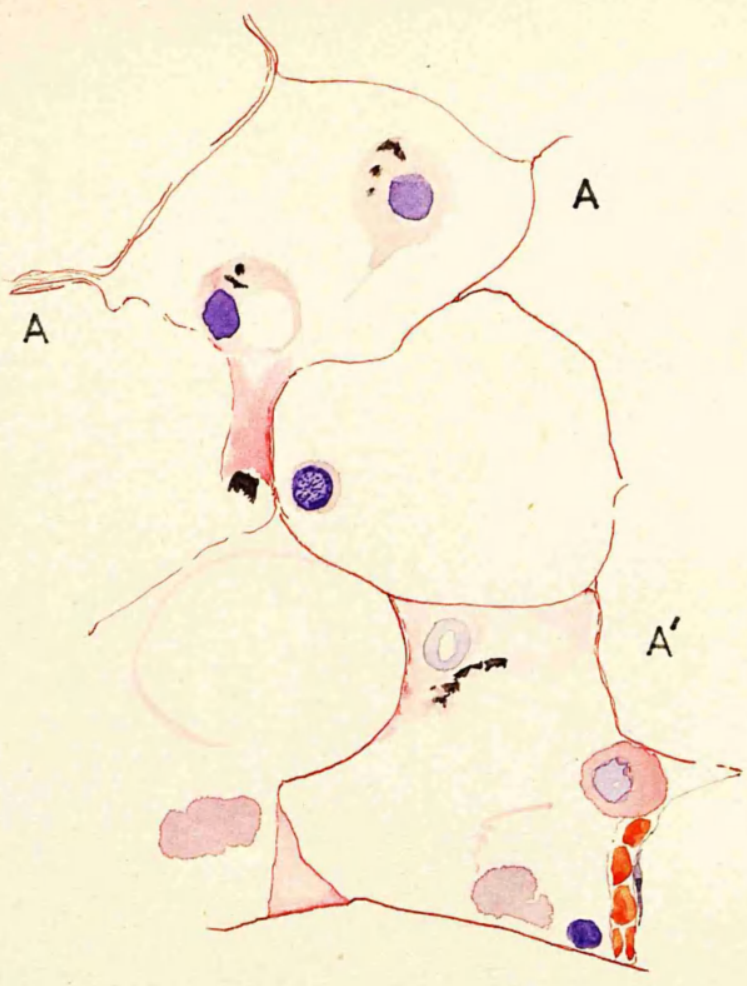

R9.SA).

FAT REPLACEUSNT OF LYHPHOID TISSUE IN A LYMPH HODE

This high power drawing illustrates an interesting point. The node was recovered from an elderly man who had been tattooed when he was a soldier fifty-two jears previously. Residual granules of insoluble dye-stuff are seen in the cytoplasm of macrophages at $\mathrm{A}, \mathrm{A}$ and in a fat-cell cytoplasm at $\mathrm{A}^{1}$. Note the minute epinuclear vacuole in the nucleus of $A^{I}$ - this is seen only in fat cells. The finding suggests that a process of metamorphosis from reticulum cell to fat cell is part of the mechanism of fat atrophy. (The arawing is from the edge of the fat $\operatorname{cons}$ ). 
16. 


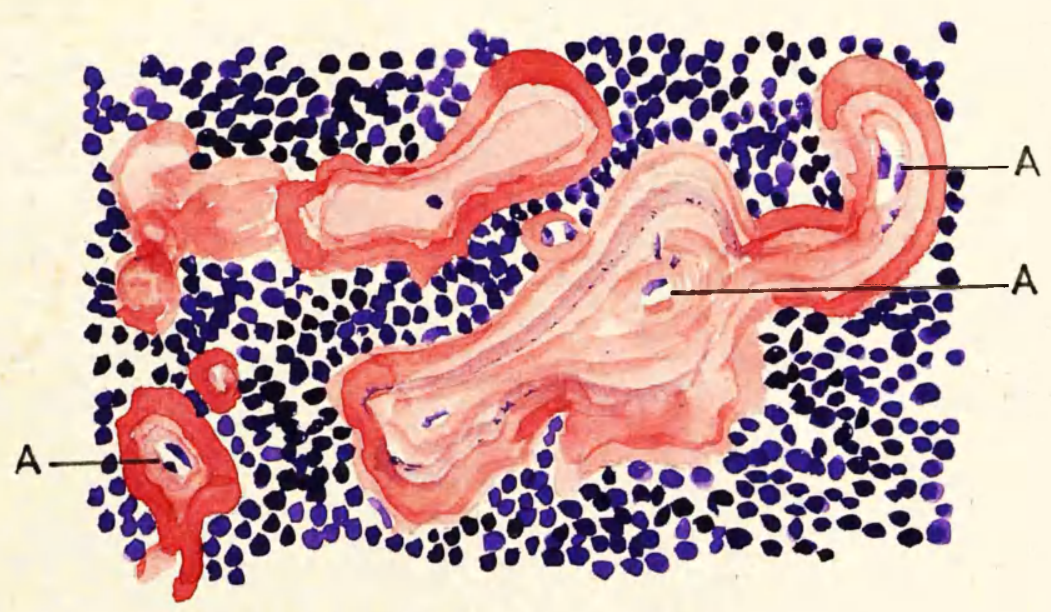

$\operatorname{Reft}$

\section{VASGULAR HYALINISATION FIBROSIS IN A ITUPH NODE}

A small field from the cortex is snewn. Capillary blood vessels in different stages of hyalinisation. At $A, A, A$, note persistent stenosed lumina. In the thickest vessel depicted the laminated appecrance is well seen and necrotic nuclear material can be made out in the interstices. From a very elderly woman. About $300 x$. 
18

s.

$-$ 
Ris PS.

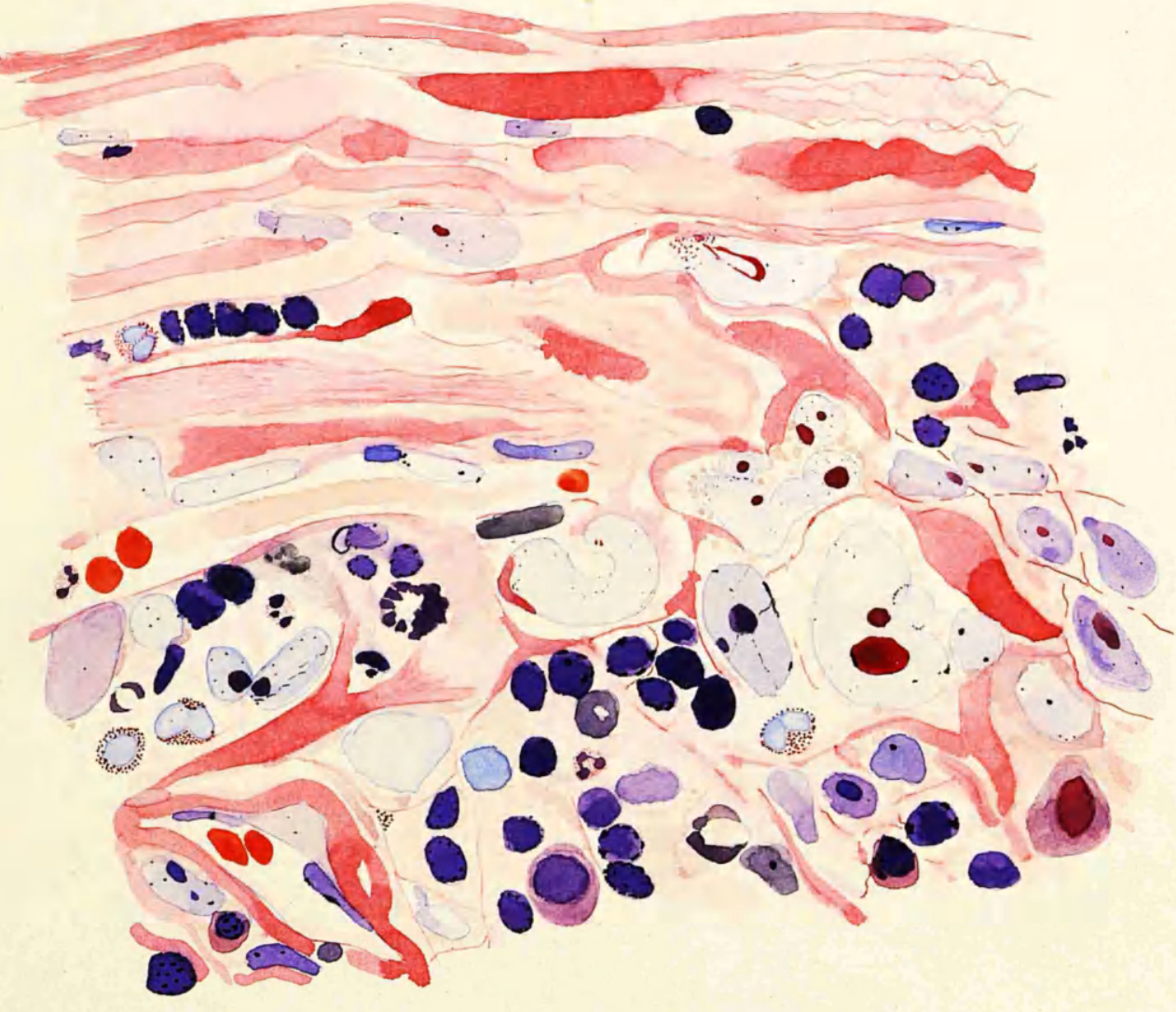

TYPICAL HODGKIN'S DISIASE - LYIPH NODE

Field drawn from sub-capsular periphery. The full thickness of the capsule is not depicted.

Note - Fibrosis - Capsule, fibrils, fibrillae, and coralline deposit.

Hodgkin giant-cells.

ilorbid mononuclear reticulum cells.

Bosinophiles, plasma cells, neutrophiles.

Rare individual cells are necrotic.

Variation in size and staining of lymphocytes.

Serial 89. About 700X. H\&i. 


\section{BASIC EXPRESSIONS OF FIBROSIS IN HODGKIN'S DISEASE}

Fig. 1. Fibrillae. From a dissociated (macerated) specimen; fine collagenous filaments are seen. ibout $350 \mathrm{X}$ Van Gieson.

Fig. 2. Fibres. About $350 \times \operatorname{Van}$ Gieson.

Fig. 3. Coralline deposits. (Compacted fibres; sometimes vascular hyalinisation also produces these fornations). About $350 \times$ Van Gieson.

Fig. 4. Symplasmic transformation. Collagen fibrillae and small angular deposits form in the peripheral cytoplasm of epithelioid type reticulum cells. About $350 \mathrm{X} \operatorname{Van}$ Gieson. 


\section{BASIC EXPRESSIONS OF FIBROSIS IN HODGKIN'S DIDEADE}

1.

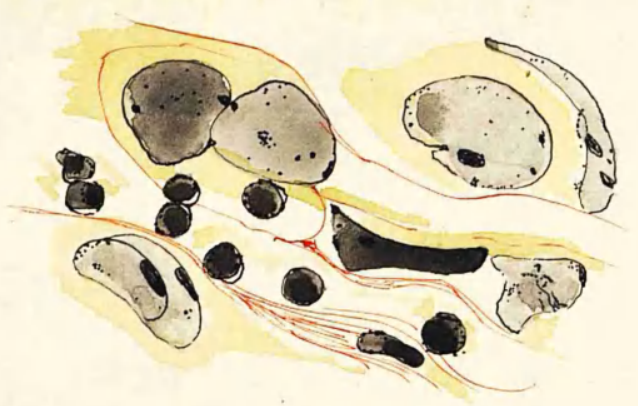

2.

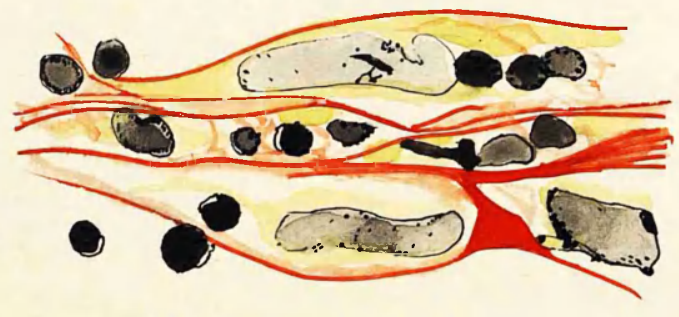

3.

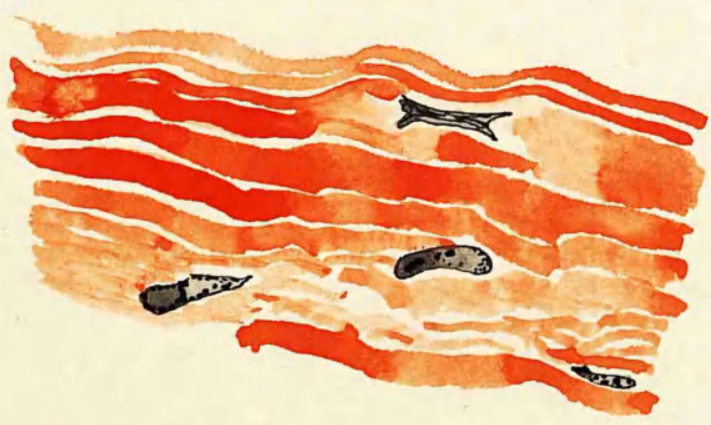

4.

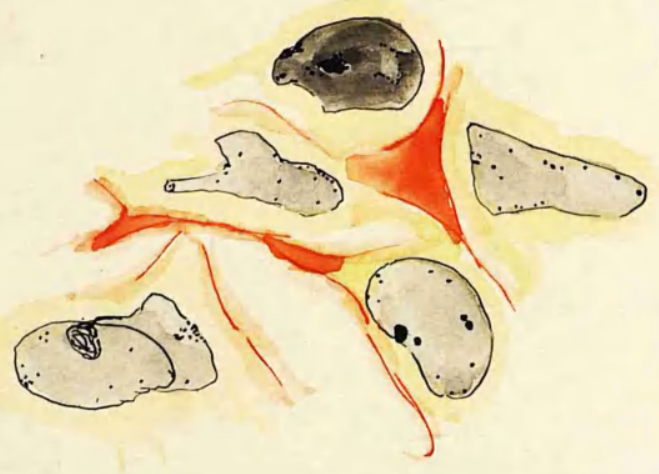

R.r. 9 . . 


\section{IORBID RTPICULUH OELLS IN HODGKIN'S DISEASE}

Fig. 1. Normal reticulum cell, nucleus and cytoplasm. (A small lymphocyte is drawn to scale for comparison).

Fig. 2. Normal reticulum cell; from a germinal centre, notching of the nuclear membrane is usual in this situation.

Figs. 3-9. Horbid reticulum cells which are still uninucleate. Increase of size of the nucleus is the most constant feature. Plasmasomes are fairly common, also evidences of necrosis.

Fig. 8. depicts two adnate epithelioid types, collagen formation by symplasmic transformation is indicated.

Figs. 10-12. Binucleate morbid reticulum cells, mirror-image form is common.

Figs. 13-24. Hodgkin giant-cells. These are all derived from a single section and shew the remarkable pleiomorphism of these cells. 
1.

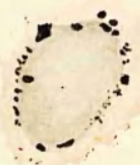

5.

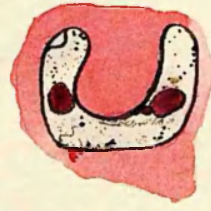

9.

R.P.P.
2.

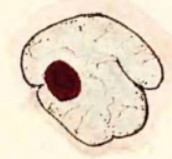

6.

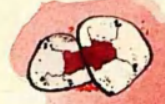

10.

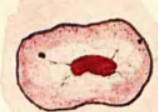

3.

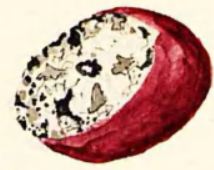

7.

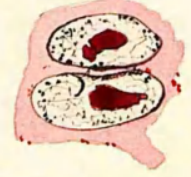

11.

12.

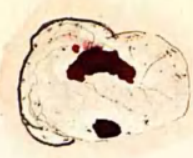

4.

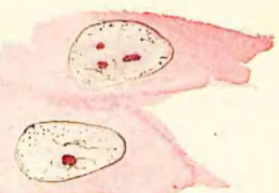

8.

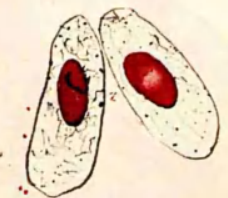

MORBID RETICULUII CELLS IN HODGKIN'S DISEASE 
24. 


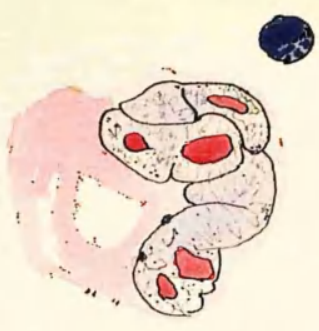

13.

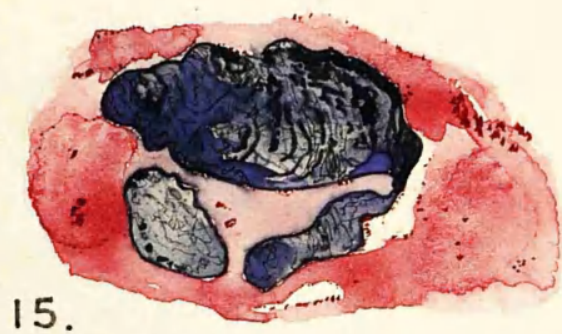

17.

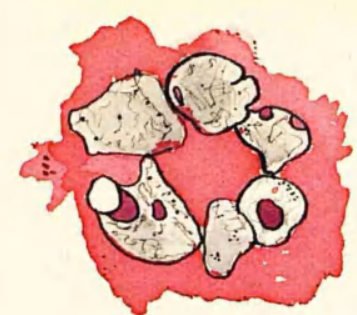

14.
16.

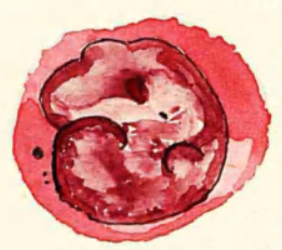

18.

R.9.2. 
26. 

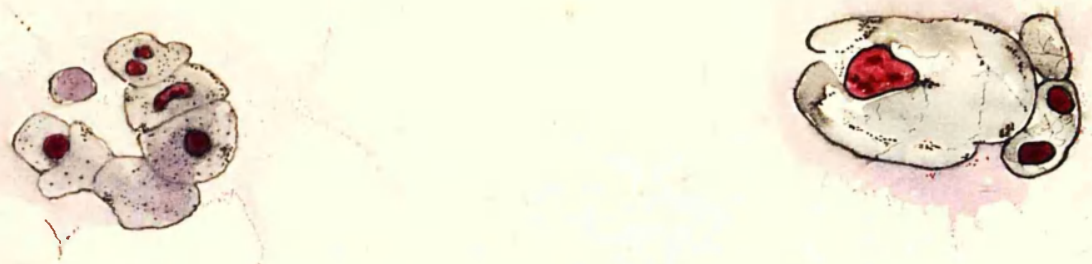

19.

20.

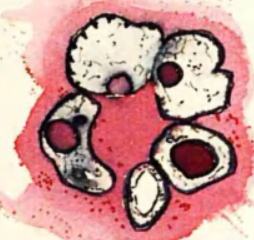

21.

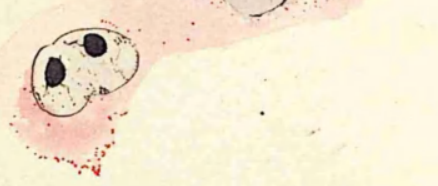

23.

R.r. 解.

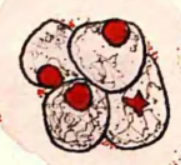

22.

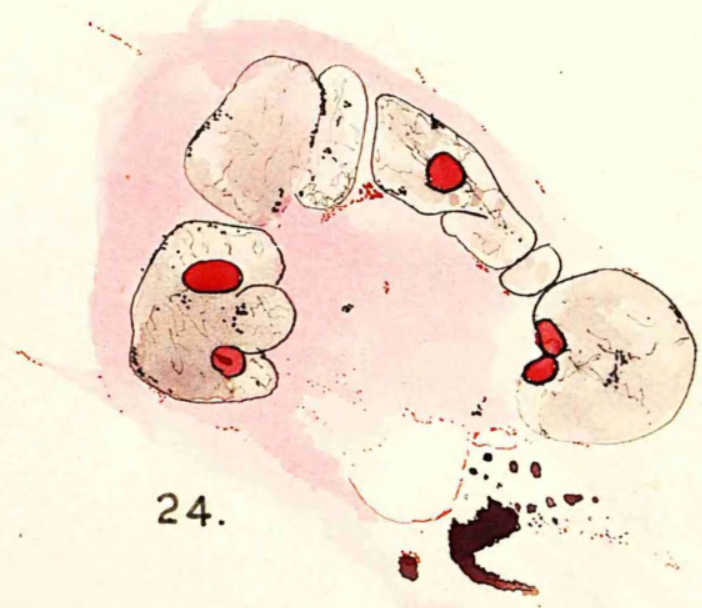


28.

. 

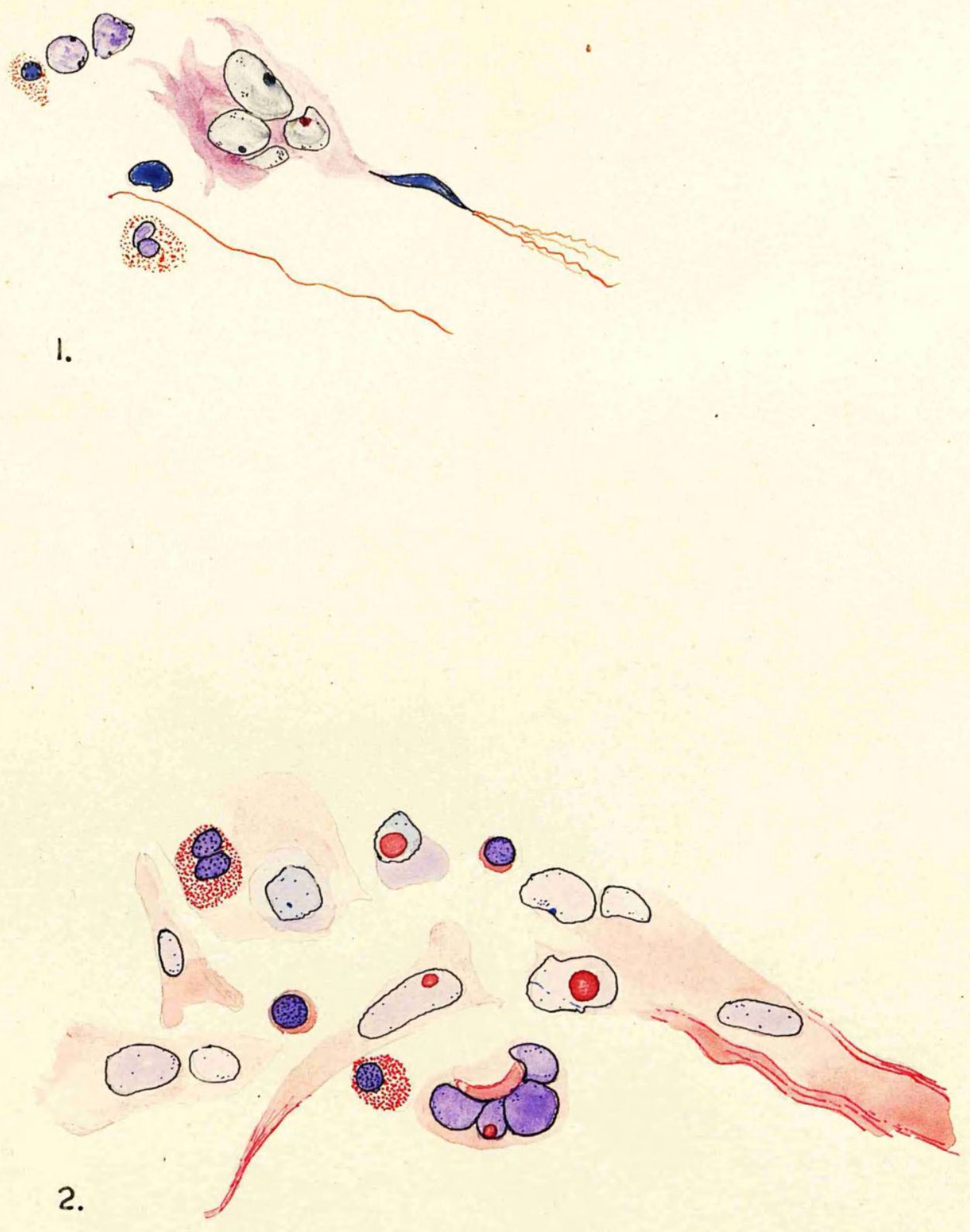

R.9.9. .

FORUATION OF COLLAGIN FIBRتS BY HODGKIN GIATT CELLS AIDD WORBID WONONUCLEAR RITICULUN CELLS

1. From Serial 101.

2. From Serial 122. 


$$
30 .
$$




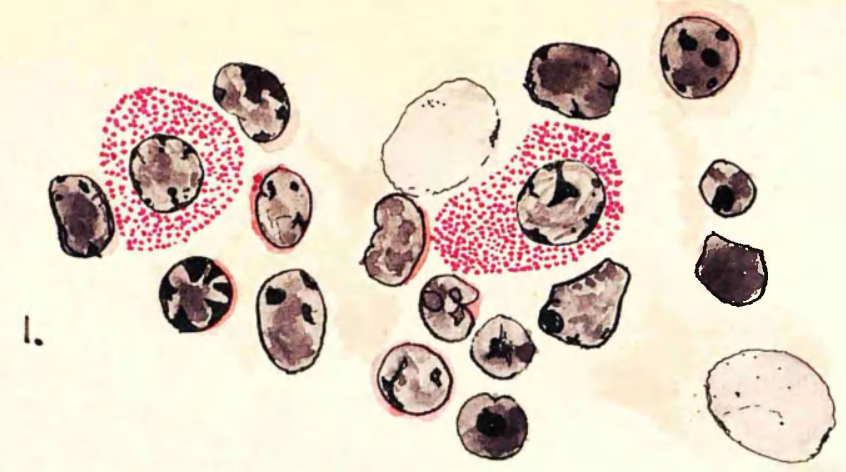

2.

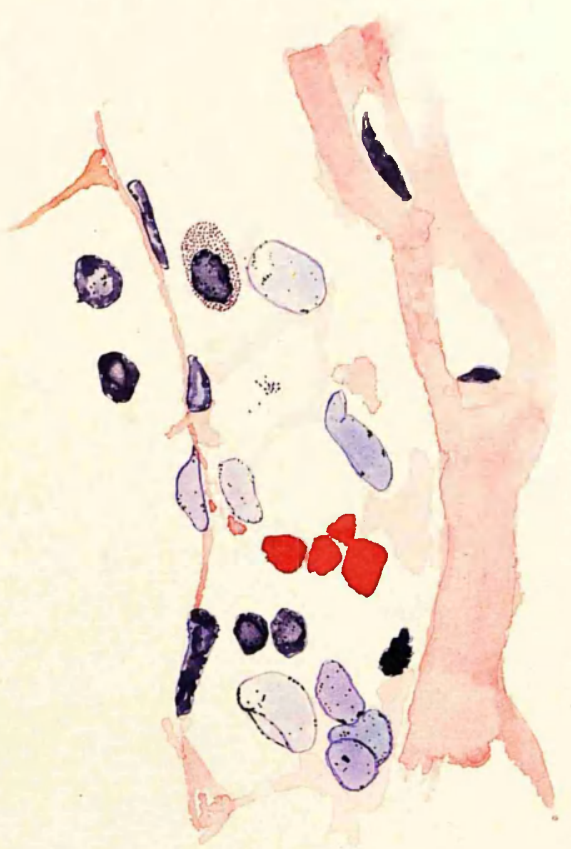

R.9๑八.

\section{LYIPHOCYIOID DOSINOPHILDS}

1. Direct metamorphosis in loose lymphatic tissue. The nuclei of the eosinophiles are sinete and correspond closely to large lymphocytic nuclei.

$$
\text { sbout } 750 \mathrm{X} \text { H\&w }
$$

2. Lymphooytoid eosinophiles in the markinal sinus of a normal lymph node. 
32. 


\section{Q)}
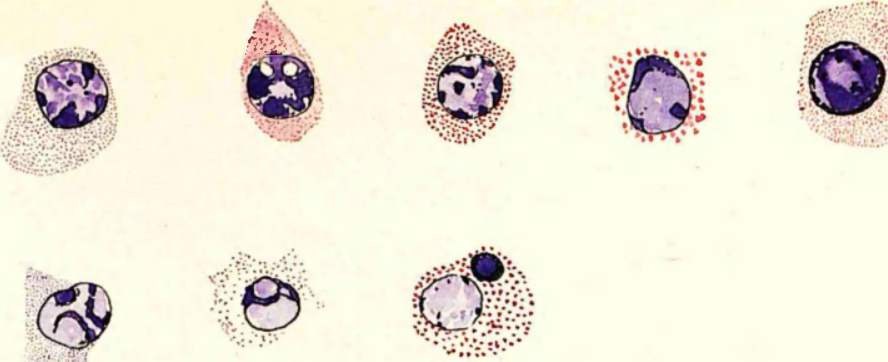

R.s.

SELECTION OF LYMPHOCYYOID EOSINOPHILAS

Note the amphophilic character of granules also the disparity of size and density. A small lymphocyte is shewn, top left. Serial 79. H.P. H\&. 
34. 


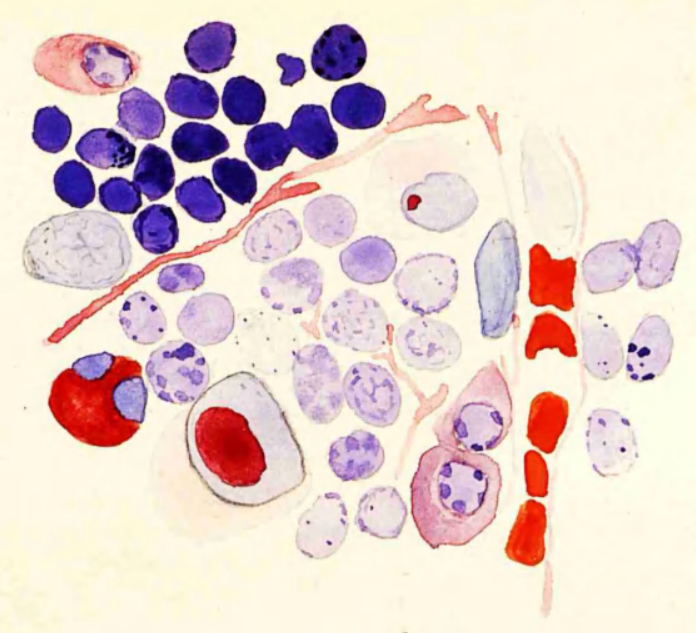

R.s.s.

\section{INIATURE LYHPHOCYTES IN HODGKIN'S DISEASE}

A survival islet of mature small lymphocytes is seen top left. It is separated from the new tissue by a fibril of collagen. The lymphocyte series cells in the tumour are larger and their nuclei are leptochromatic and tending to a vesicular type. (Lymphocyte cytoplasm was not discernible in this preparation). Serial 110. About 600 X Hor. 
FOLLICULOID DISPOSITION OF LATE HODGKIN'S DISRASE

Half-tone drawing. The black tissue is the cellular moiety, the pale grey the collagenous.

Serial 156. Camera lucida X 5 . 


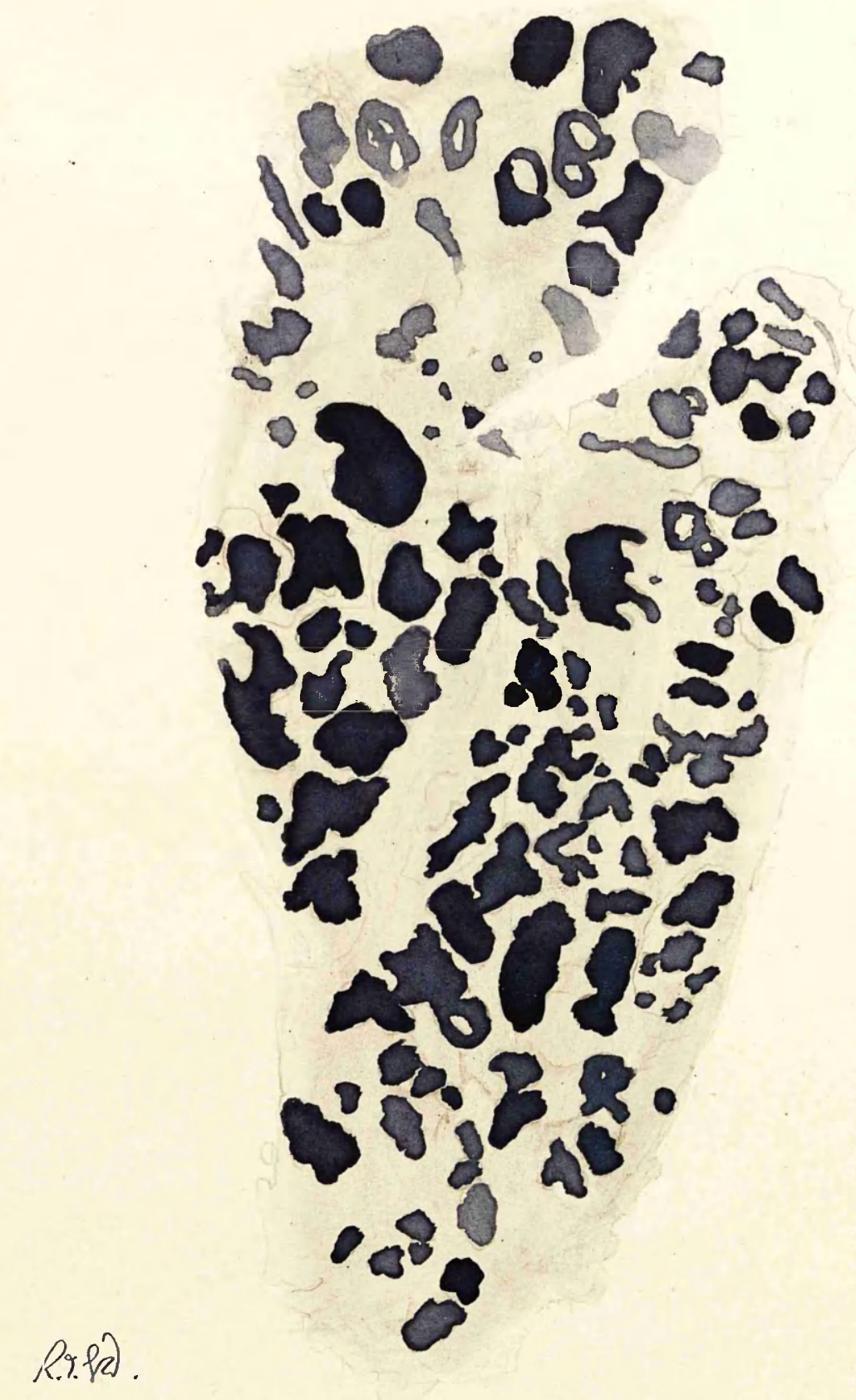


38. 


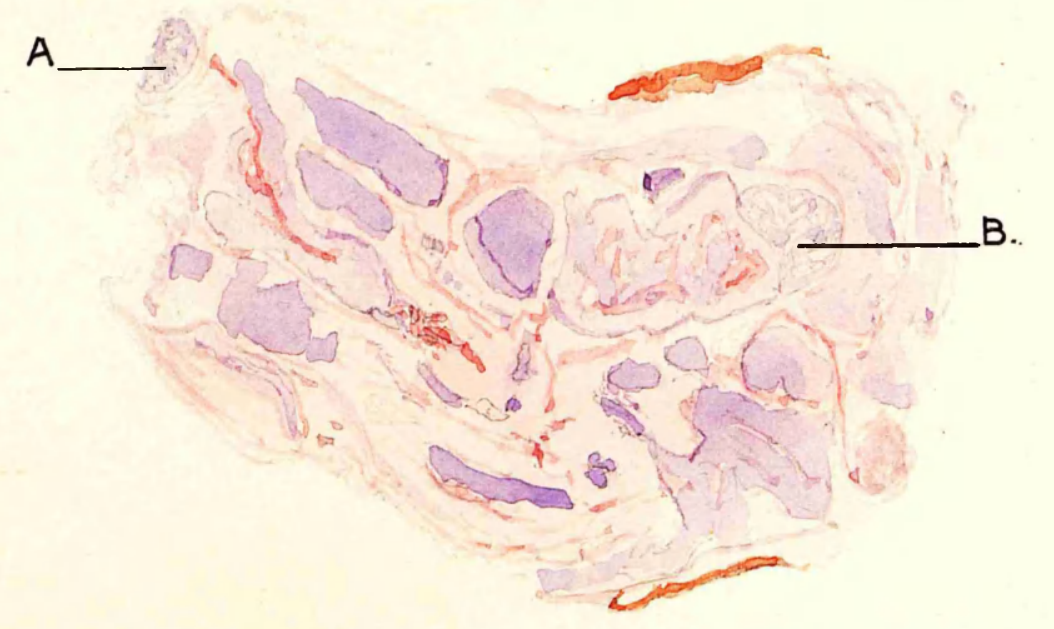

R. 92 .

INOLUSION OE PEYSIOLOGIOAL LYMPHOID PISSUE IN USTABIISAED EODGKIN'S DISIASE

A late lesion is depicted; at $A$ and $B$ small unaffected lymph nodes are present.

Serial 199. Camera lucida X 5. H\&E. 


$$
40
$$




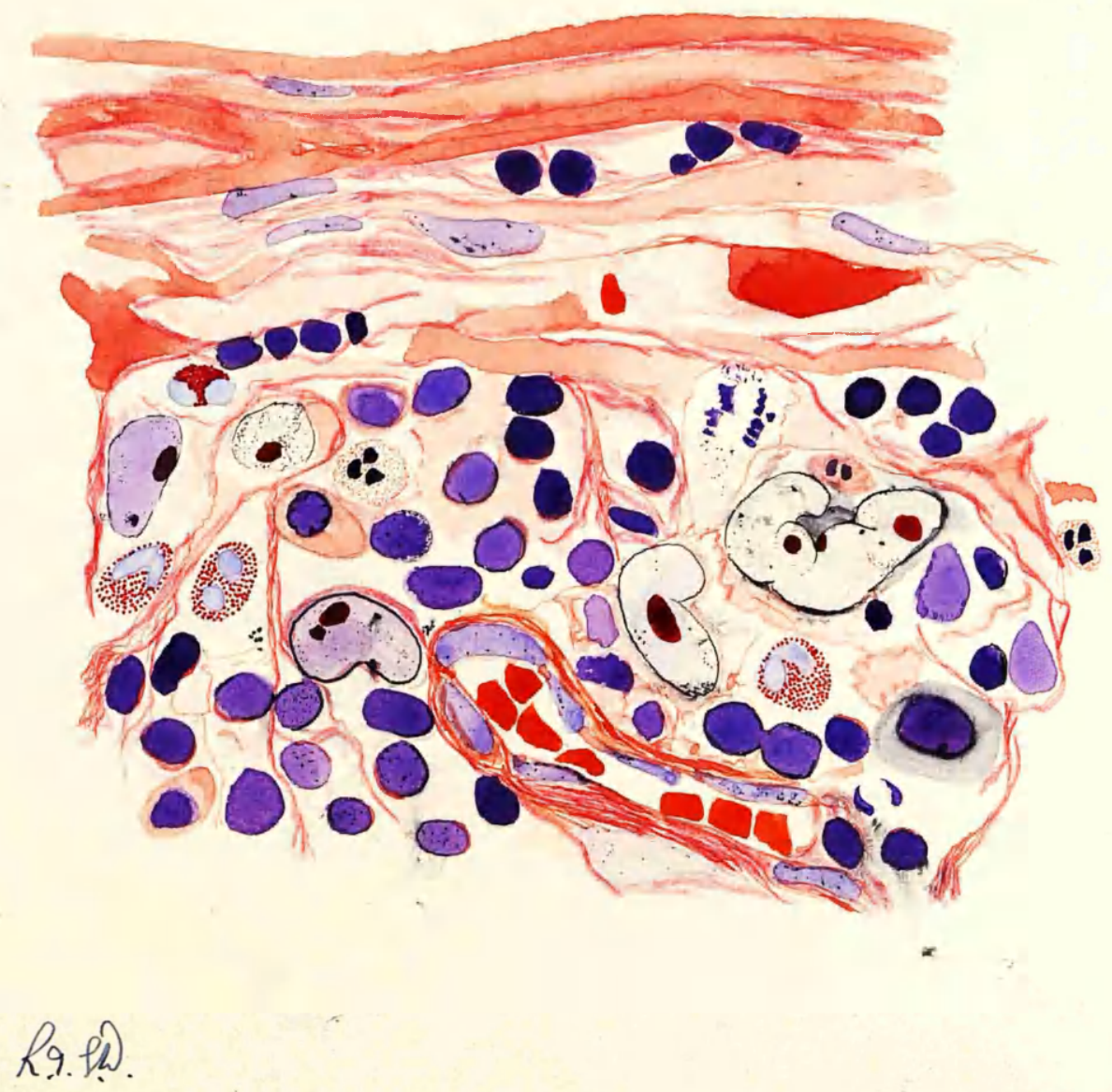

\section{HODGKIN'S DISEASE, TYPICAL. (EARLY)}

Cervical lymph node, at the periphery; the full thickness of the capsule is not drawn. All cells of the corplex are represented, necrosis is limited to individual cells. Note the large (imnature) lymphocyte nuclei. 
42 


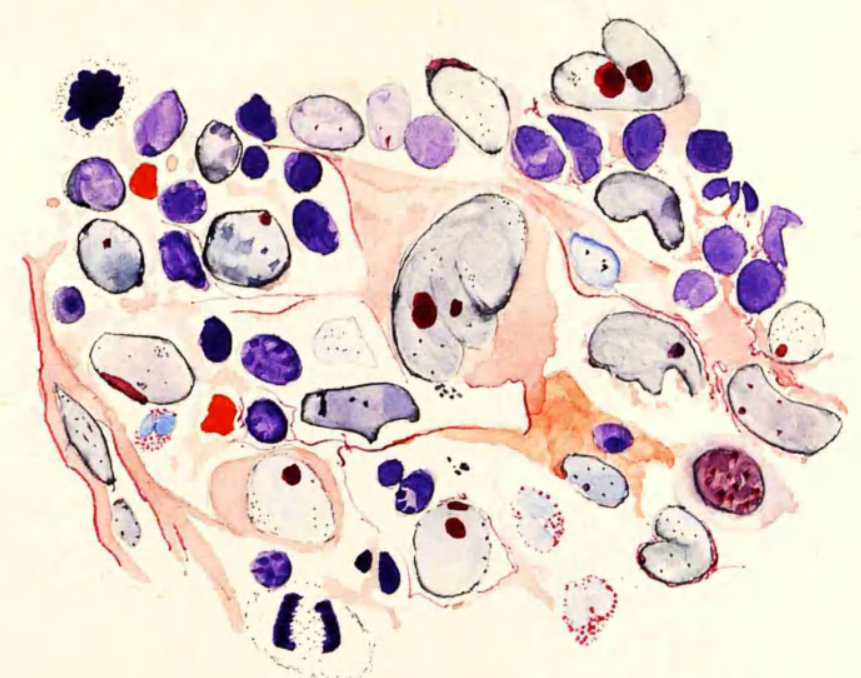

$\operatorname{Ra}(1)$.

HODGKII'S DISEASE, CELLULAR TYPE

The general pleionoppism is retained or enhanced. Worbid reticulum cells are relatively increased but generally remain somewhat small. Hodgkin giant-celis are retained but large forms are unusual. Fosinophiles persist, fibrosis is tenuous and Eenerally uniform. Litoses vary but are often increased in number. 
44. 


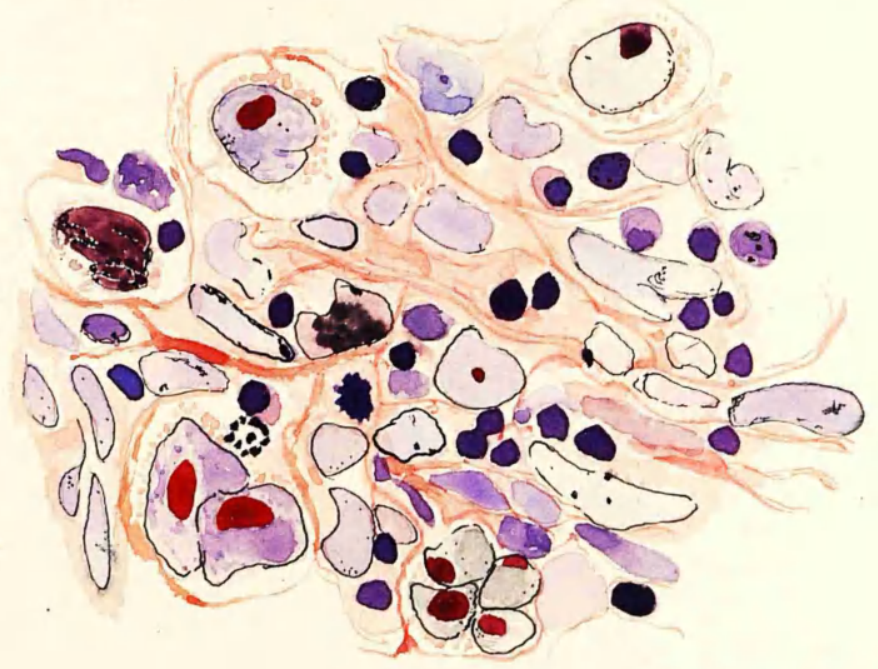

R.9. f.

\section{APYPICAL HODGKIN'S DISAASA, TUNOUR-LIKE PYPE}

The drawing is representative of alnost the entire node. Iforbid mononuclear reticulum cells are numerous, they are more uniformly scattered than in the typical lesion, Hodgkin giantcells are small. Bosinophiles are absent, the fibrosis is chiefly in fibrillary form and is uniform. The general uniformity of the pattern is tumour-like. Serial 154. About $500 \times$ H\&E. 
46.

$v$

$-$ 


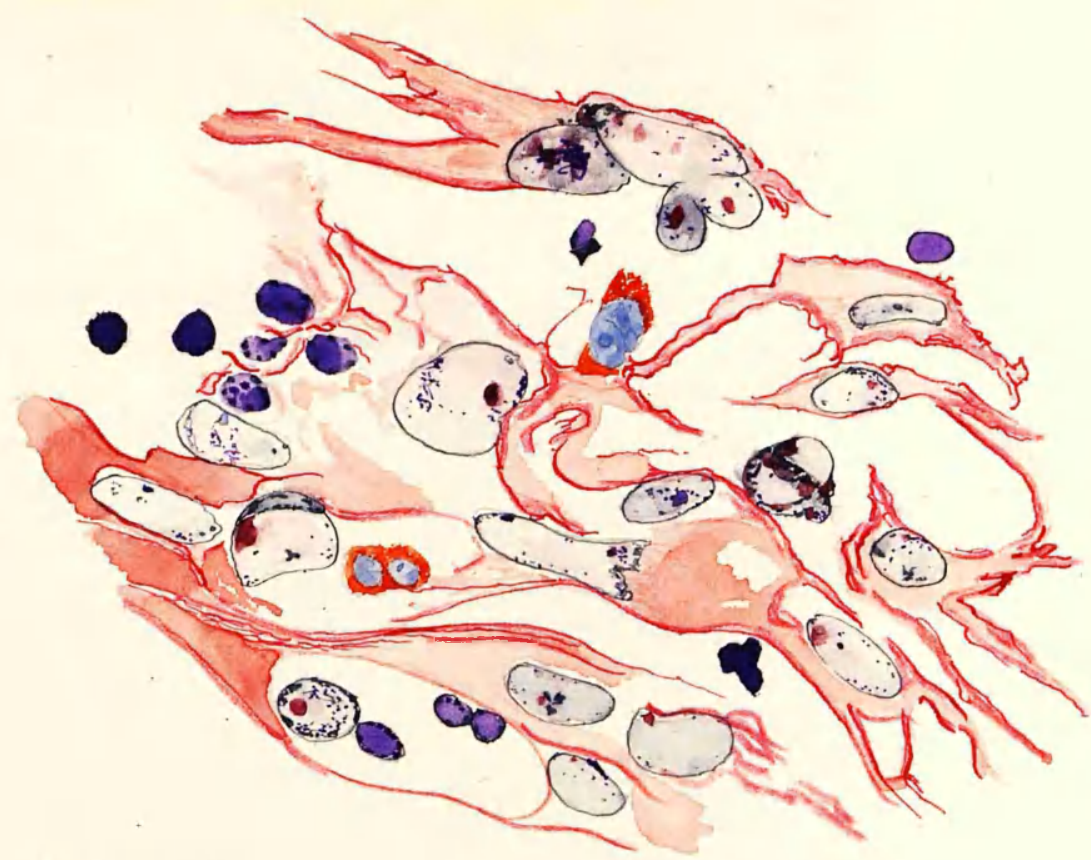

Ra.pis.

\section{DIFHUST FIBROBLASTIC HODGKIN'S DISEASE}

The field is from the most cellular portions of the lesion. The majority of the cells are fibroblastic and their nuclei correspond to the range between morbid mononuclear reticulum cells and fibrocjtes. The collagen in fibrils and fibrillae is forming by symplasmic transformation and is more densely oxyphile than the ground cytoplasm. The unstained spaces are shrinkage phenomena and are almost universal in this specimen. At the top an Hodgkin giant-cell is seen, these are rare in this example. Bosinophiles, small lymphocytes, (some of them imnature, complete the picture.

Serial 168. About 600 x Ho: 


\section{8 \\ 8.}

$-$ 


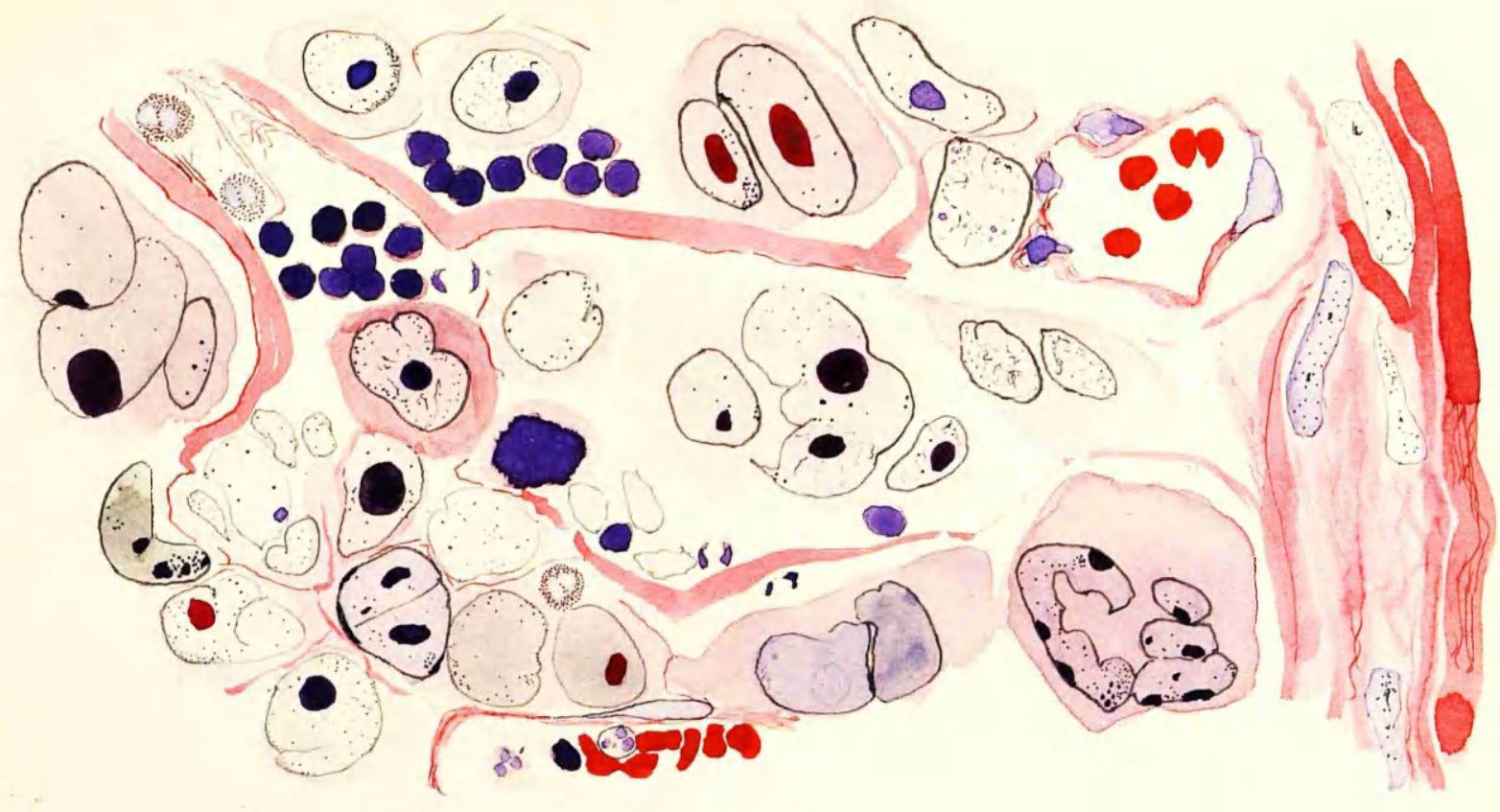

R. . F.

$\frac{\text { HODGKIN'S DISEASA, PJCULIAR VARIAIT }}{\text { (LINK TO RITICULUI OILI SAROONA) }}$

This growth is quite peculiar. All features of typical Hodgkin's disease can be made out but the overgrowth of large morbid nononuclear reticulum cells and Hodgkin giant-cells is extra-ordinarily florid. Note the retention of eosinophiles. Serial 44. About $600 \mathrm{X}$ H虫. 
50 


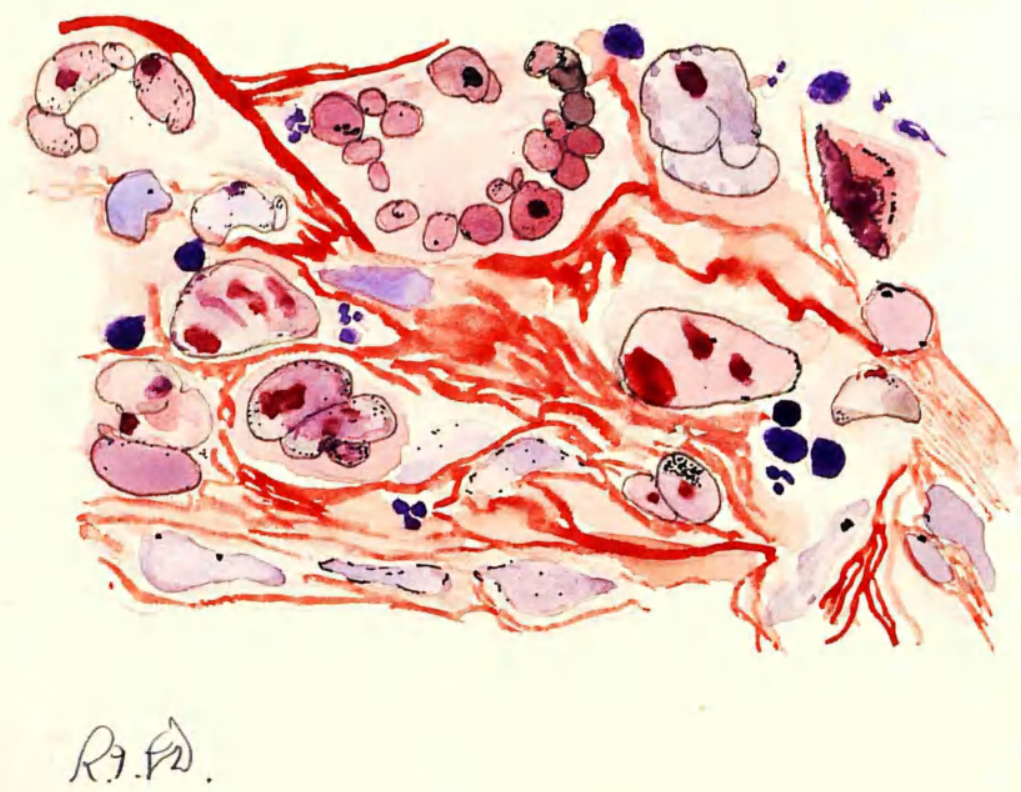

HODGKIN'S SAPCONA

Cellular portion of lesion, very numerous Hodgkin giant-cells including many bizarre forms, rare lymphocytes, and pyknotic neutrophiles, no eosinophiles, morbid mononuclear reticulum cells and ribroblasts; the whole set in a fibrous mesh. Serial 202. About $500 \mathrm{x}$ Heir. 
52. 


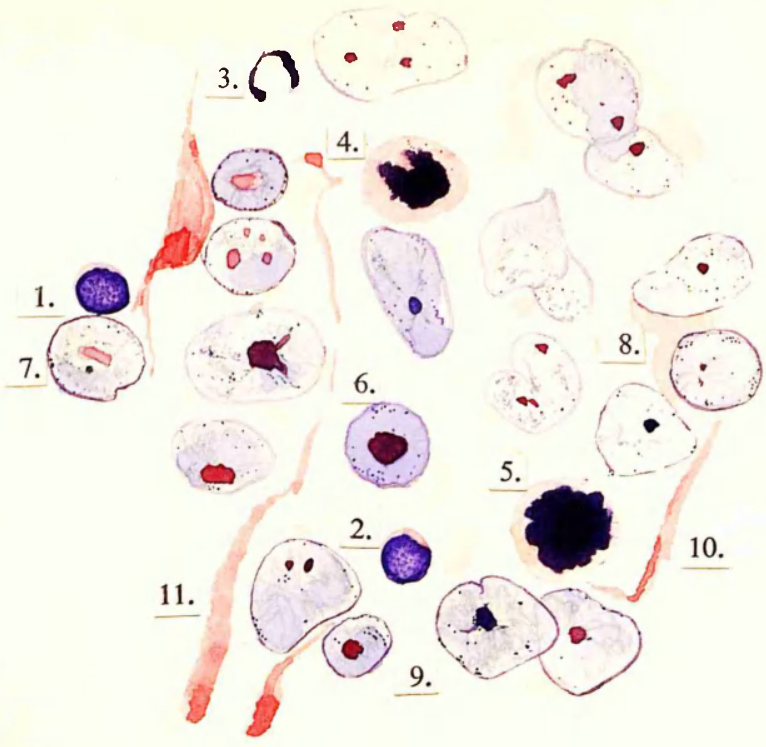

Ragid.

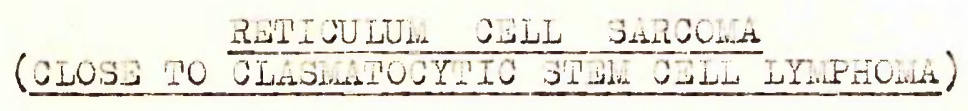

The turnour is unilorm. The type-cell. nucleus has a norbid reticuluin cell structure. Phe cytoplasin is sparse and feebly stained (visible only round a sall proportion of nuclei).

1, 2. Small lymphocytes. 3. Necrotic Iymphocyte nucleus.

4, 5. ilitoses.

6-9 Tumour reticuluri cell nuclei. Hote karjosomes and piasmasomes.

10,II. Collagen ribrils.

Serial 109. Hbout 600 X H\&a. 
54. 


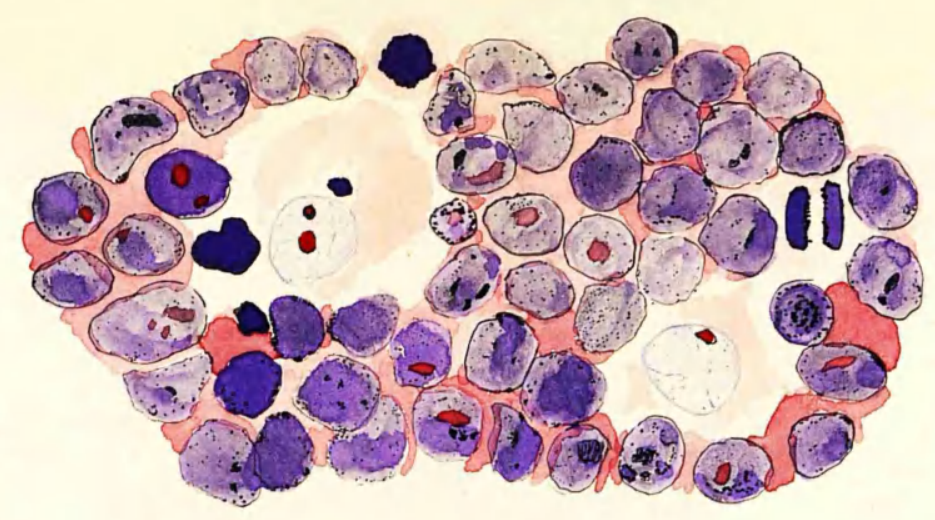

R.9.8.

\section{LYILPO-RITICULO SMROOHA \\ (Intermediate type reticulum cell sarcoma)}

Dichotomy of cell type characterises this turour. Cells identical with the morbid reticulum cells of Hodgkin's disease are scattered uniformly through the growth. The majority of the cells are imperfectly differentiated to lymphocyte precursors. (Although not depicted here fibrosis and rare Hodgkin giant-cells were present).

1. Iypical pathological reticulum cells.

2. Reticulum cells shewing affinities to lymphocyte series.

3. Lymphocyte precursors.

liote - Plasmesomes in some cells which are predominantly lymphocytic in character. Serial 4l. About $600 \times \mathrm{H} 2 \mathrm{P}$ 
56. 


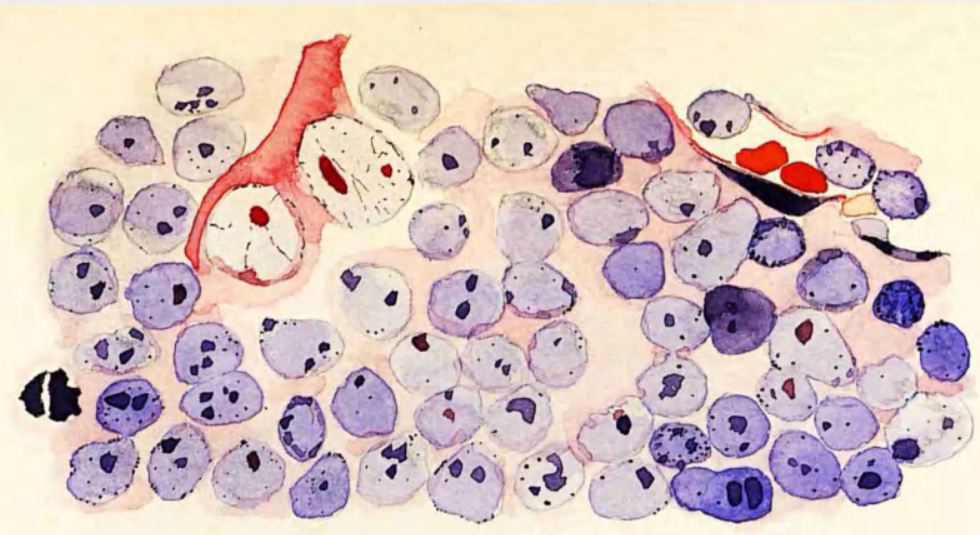

R.9.

\section{LARGI CILI IYIPHOGARCONA}

Under low power the tumour eppears an honogeneous cellular neoplasm in which the type cell is a young linhocyte series cell, (Iymphoblast). Under high power scrutiny this uniformity is lost, typical pathological reticulum cells are revealed, sore of these cells are engaged in abortive fibre fornations.

Note how most of the lymphocyte urecursors have karyosomes but some have plasmasomes. Several tumour cells are shewn in a capillary. 
A DIAGRIA OF A DISSTCTION OF THE LAFT AXILIA TO SHO THE LOCATION AND DISPOSITION OF, TUE AXILLARY LMIPH NODES (TAYLOR AND TAPHANSON TONTNCLATURT).

1. Axillary vein group.

2. Subscapular vein group.

3. Lateral thoracic vein group.

4. Thoracico-acromial vein group.

(5. Deep inferior cervical group). 


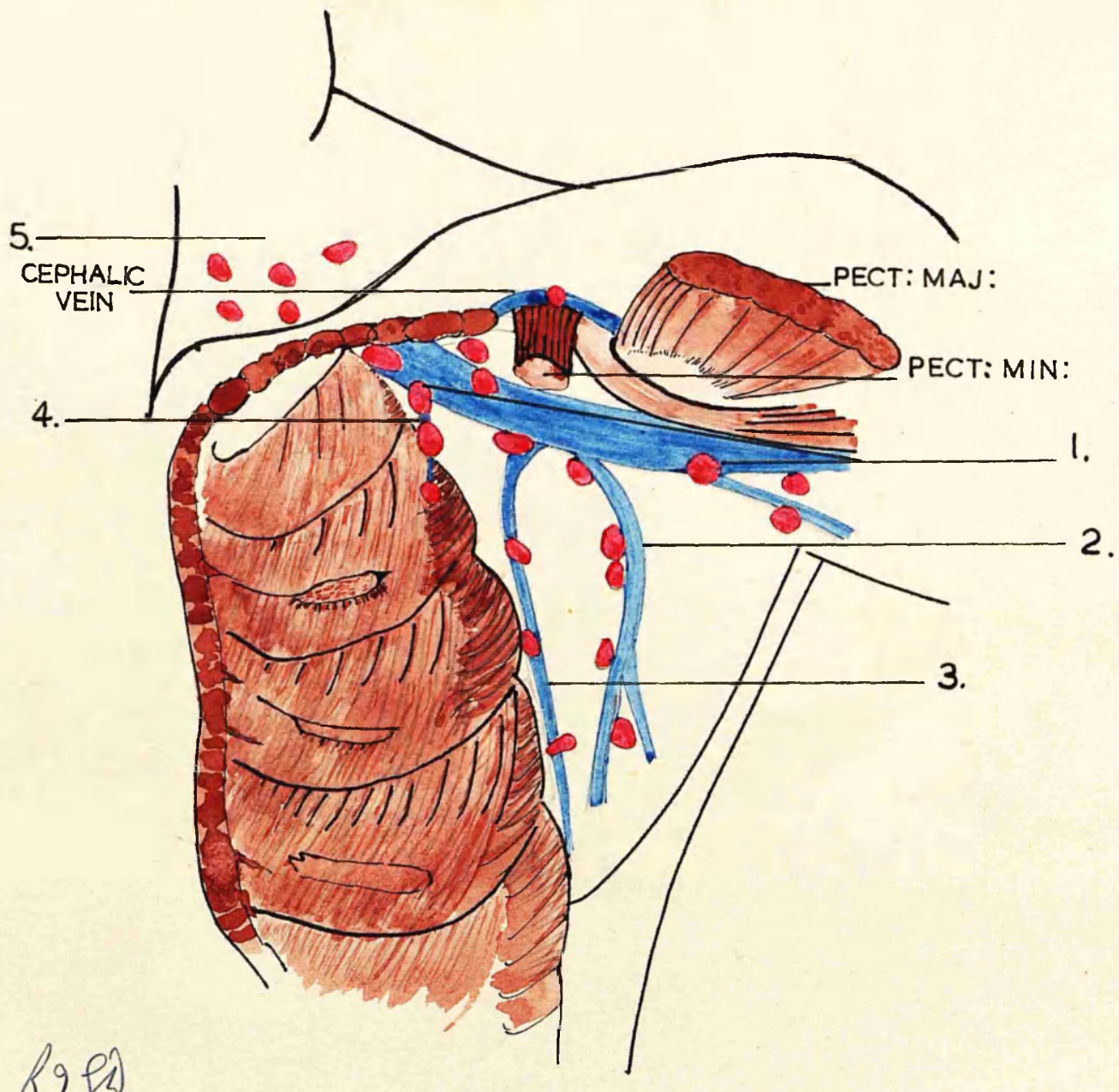


60. 


\section{SPBCIAI NECROPSY STRIAS}

\section{LOCALISATIN OP LESIONS}

\section{KPY TO THE DIAGRAIS}

1. Morakl lymph nodes, including those which are atrophic or shering physiological reactive change.

2. Nodes absent, or at least not found.

3. Hodes shewing recognisable Hodgkin's disetse (not necessarily typical).

4. Nodes shewing Fodgkin's surcoma or reticulum cell sarcoma.

ijote. Liver, spleen or other organs way be outlined in blue, purple, or red, indicating absence of lesion or participation in the morbid process. Important extranodal loci are similarly indicated. 
62. 
63.

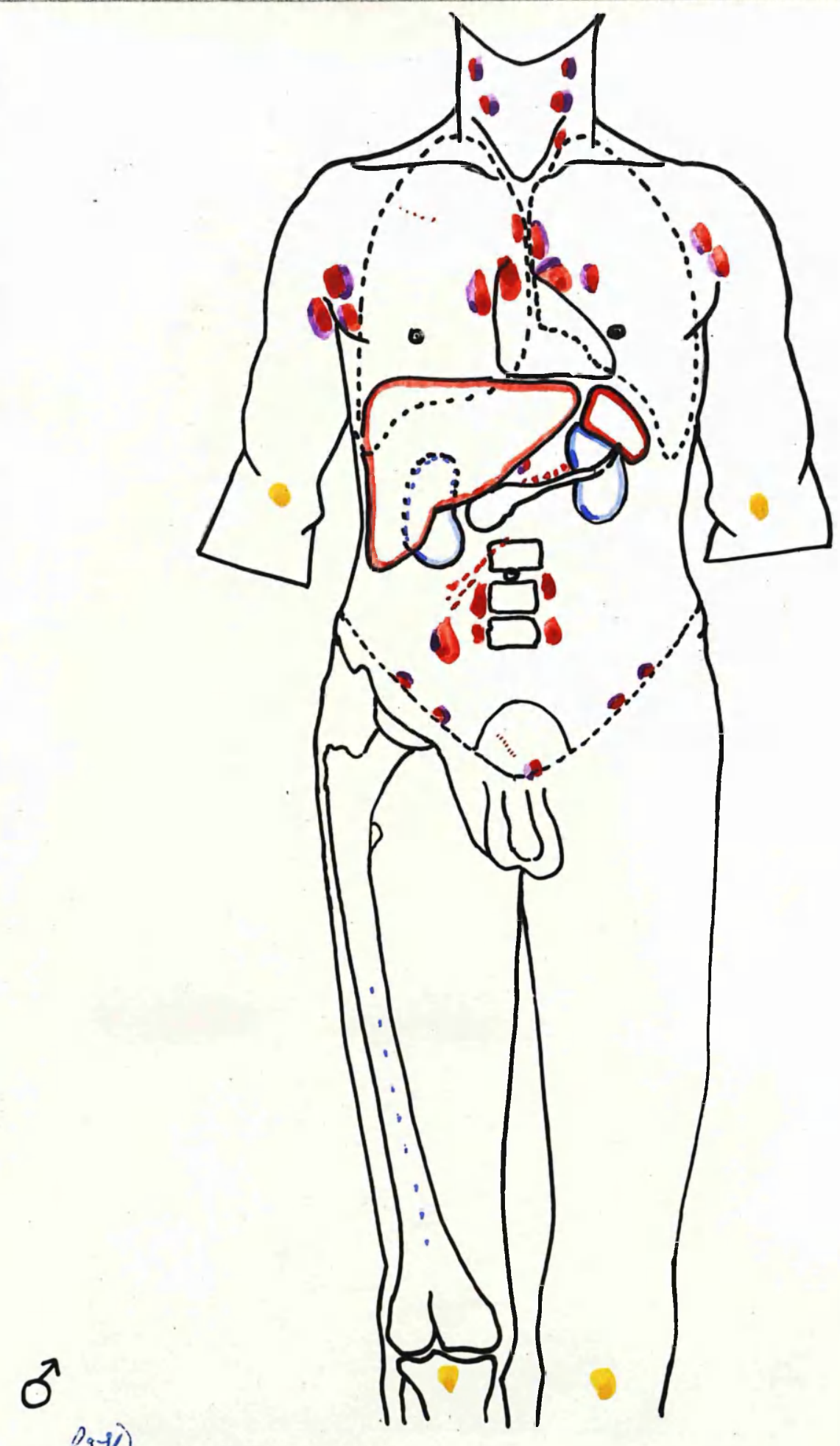

R.9.10).

MUROPSY 'A' JALES RAESIDJ J.I6:49. 
64.

. 


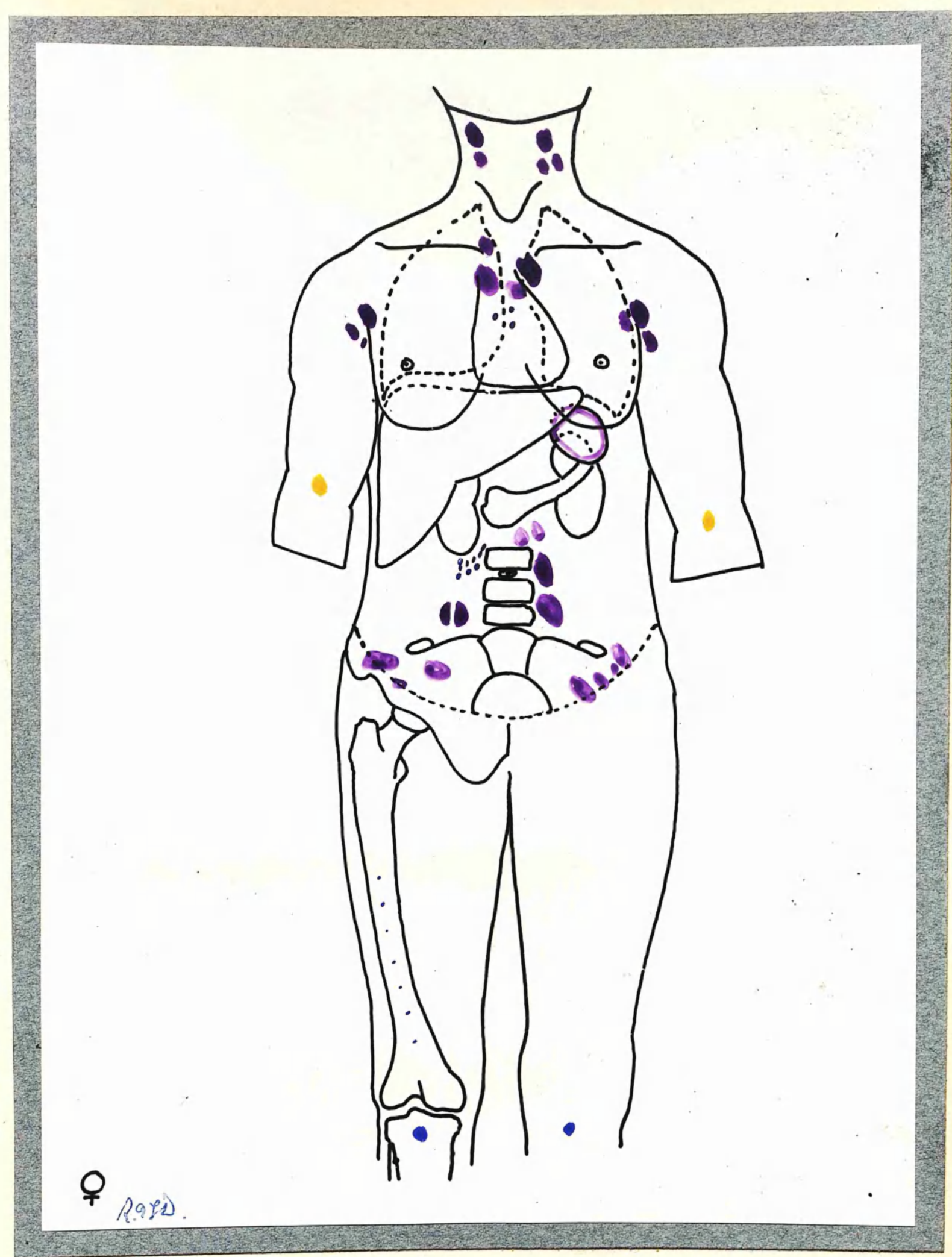

NOCROPST 'B' AGNDS LCNIVEN P.I. 201:49 G.R.I. 
66.

i 
67.

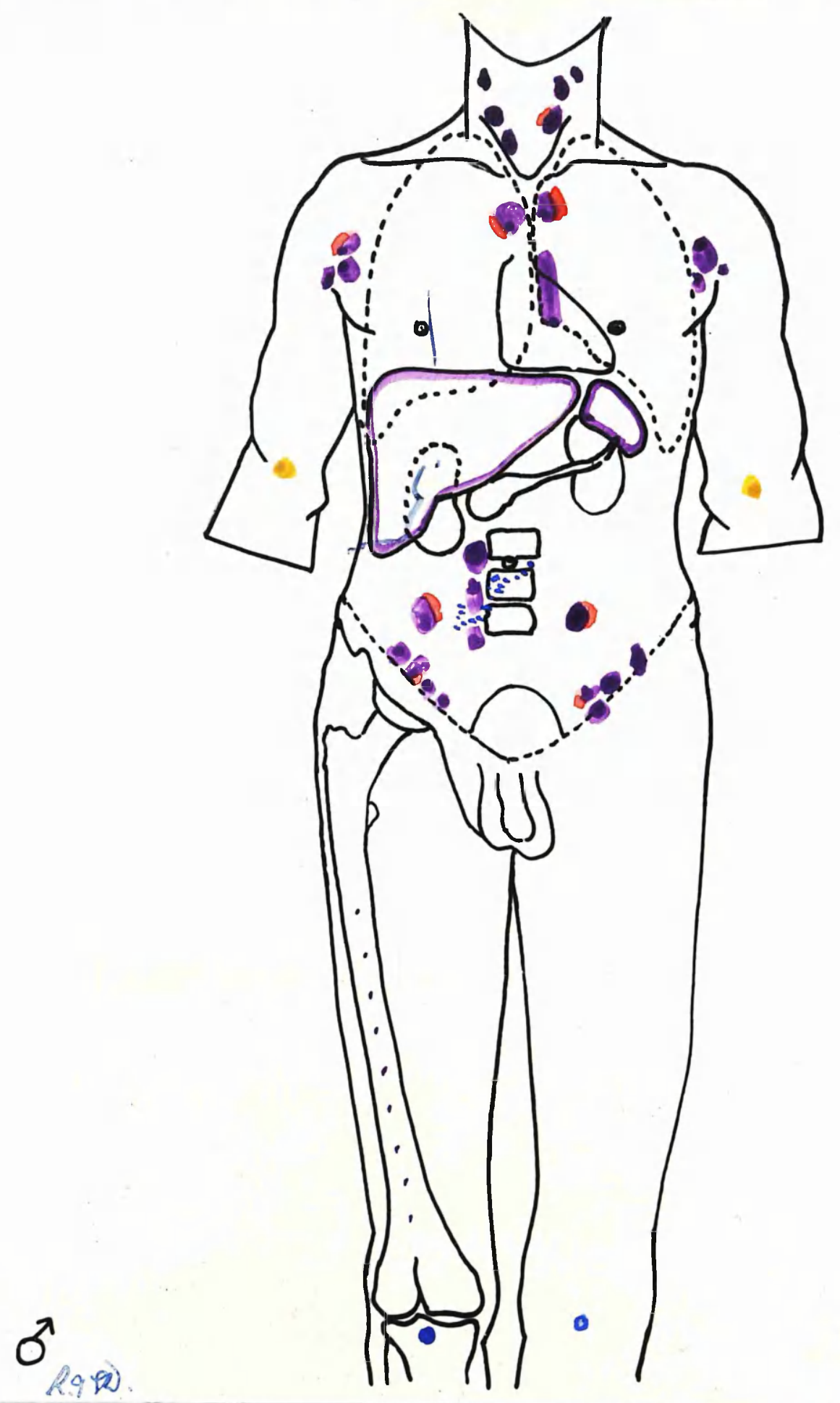

NECROPSY 'O' ROBERT LAT P.W. 193:50. G.R.I. 
68

\section{8}




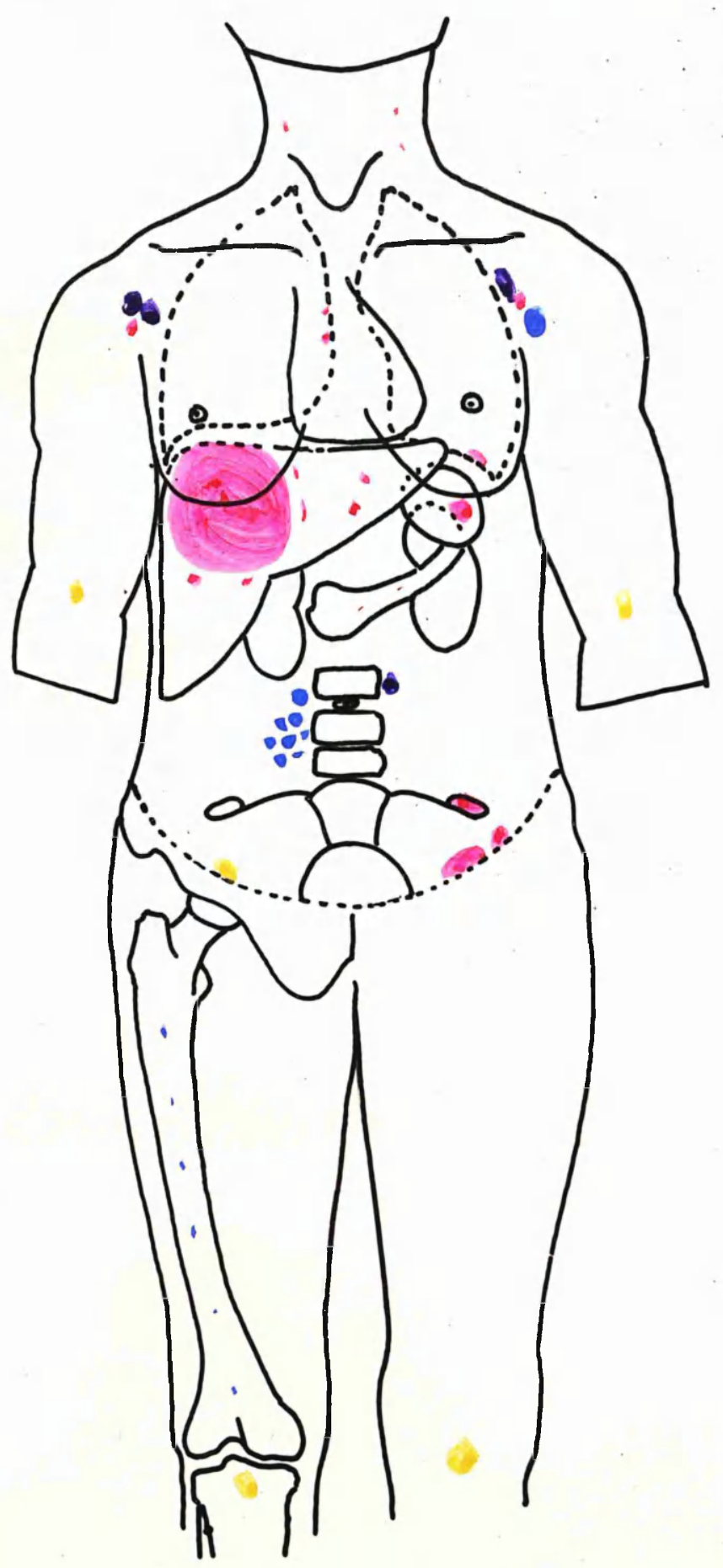

우

NACROPSY 'D' WRS. HARGARET LRONARD P.II. 332:50. G.R.I. 
70.

-

8 


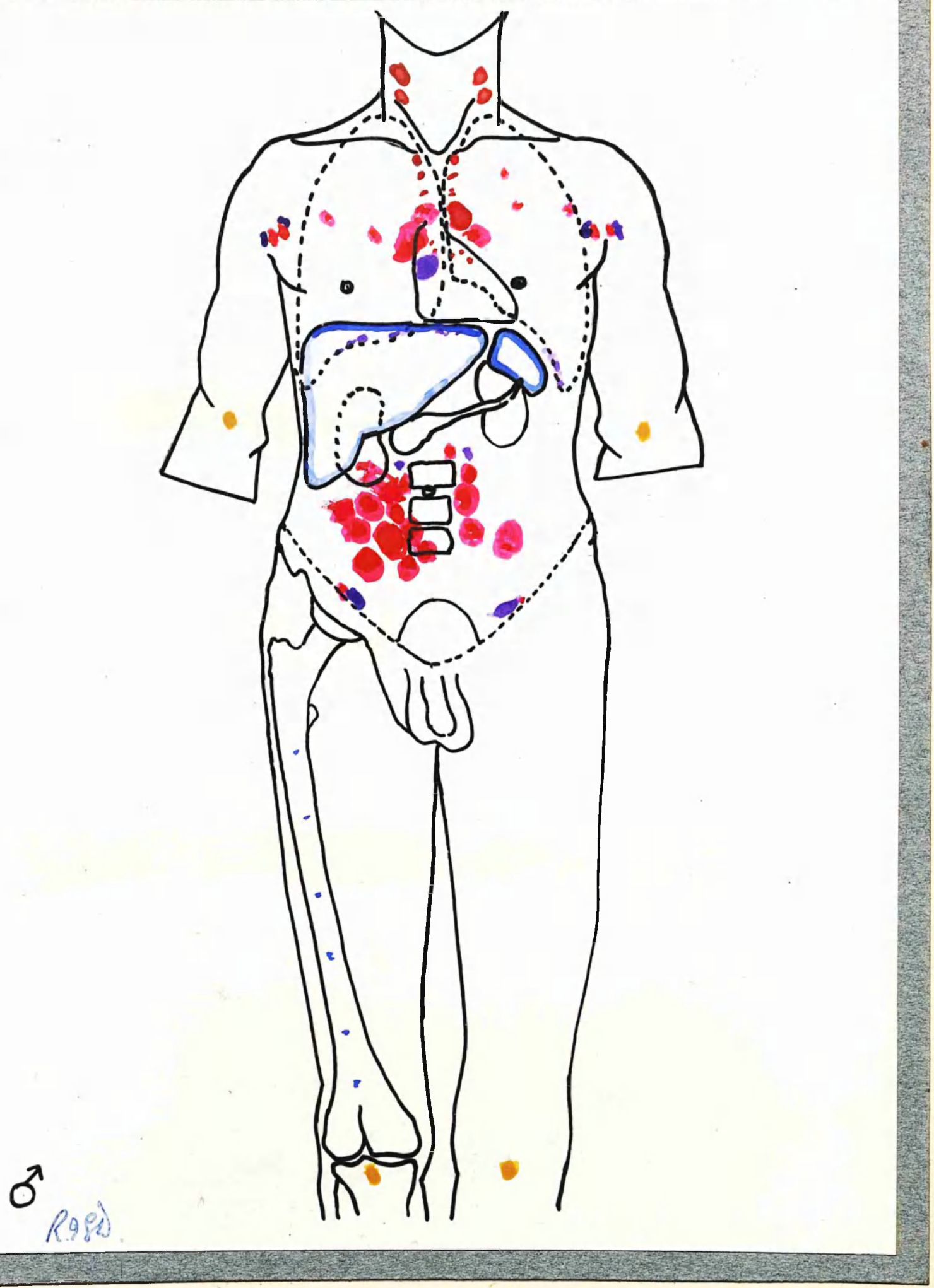

NBCROPSY 'I' AINDREN YOUITG P.H. B.35:51. 
72. 


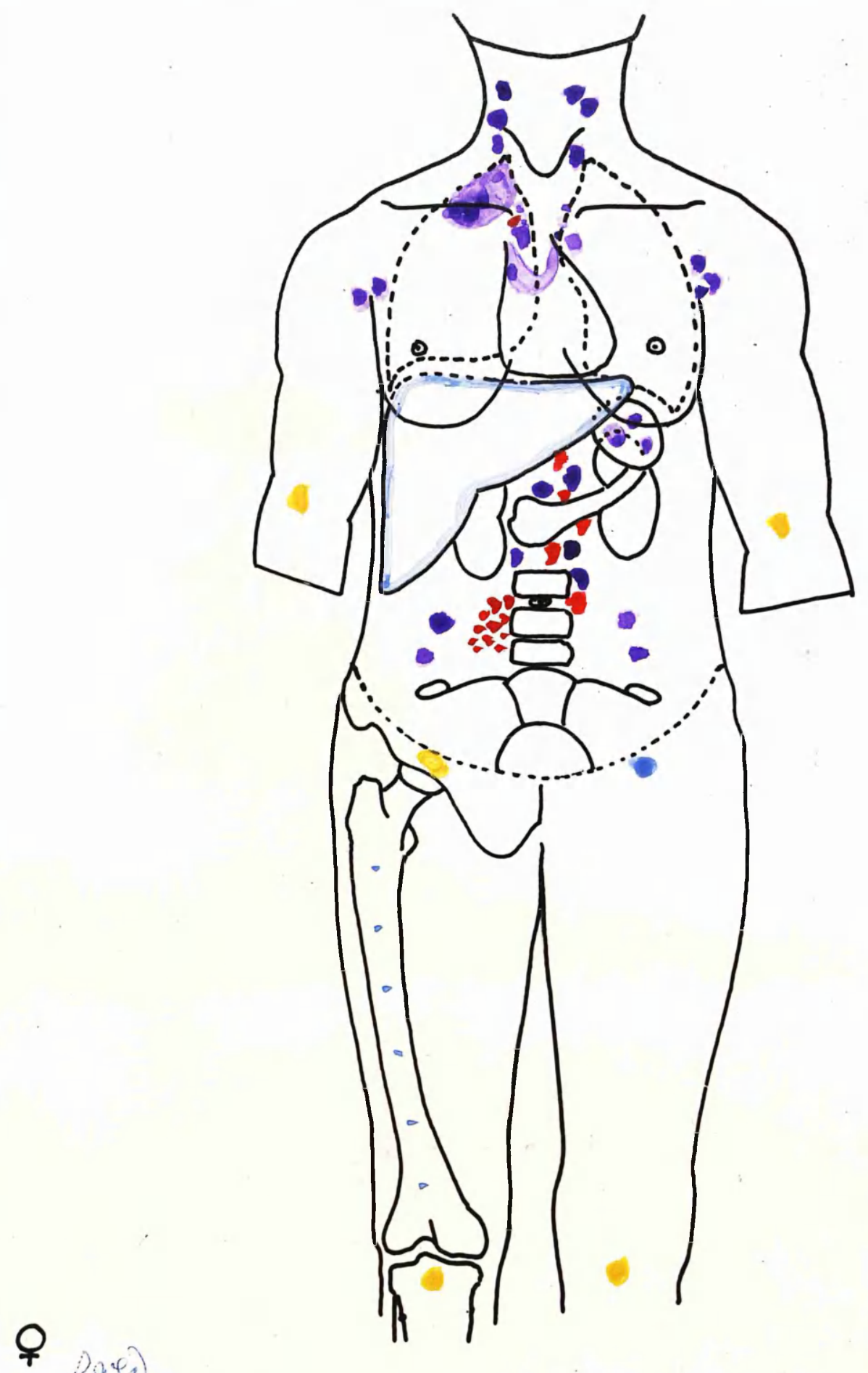


74.

I

- ir

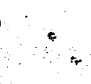

-
$*$

$+$.

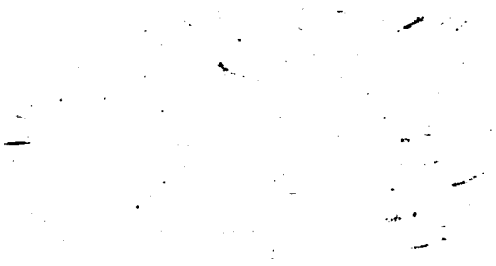




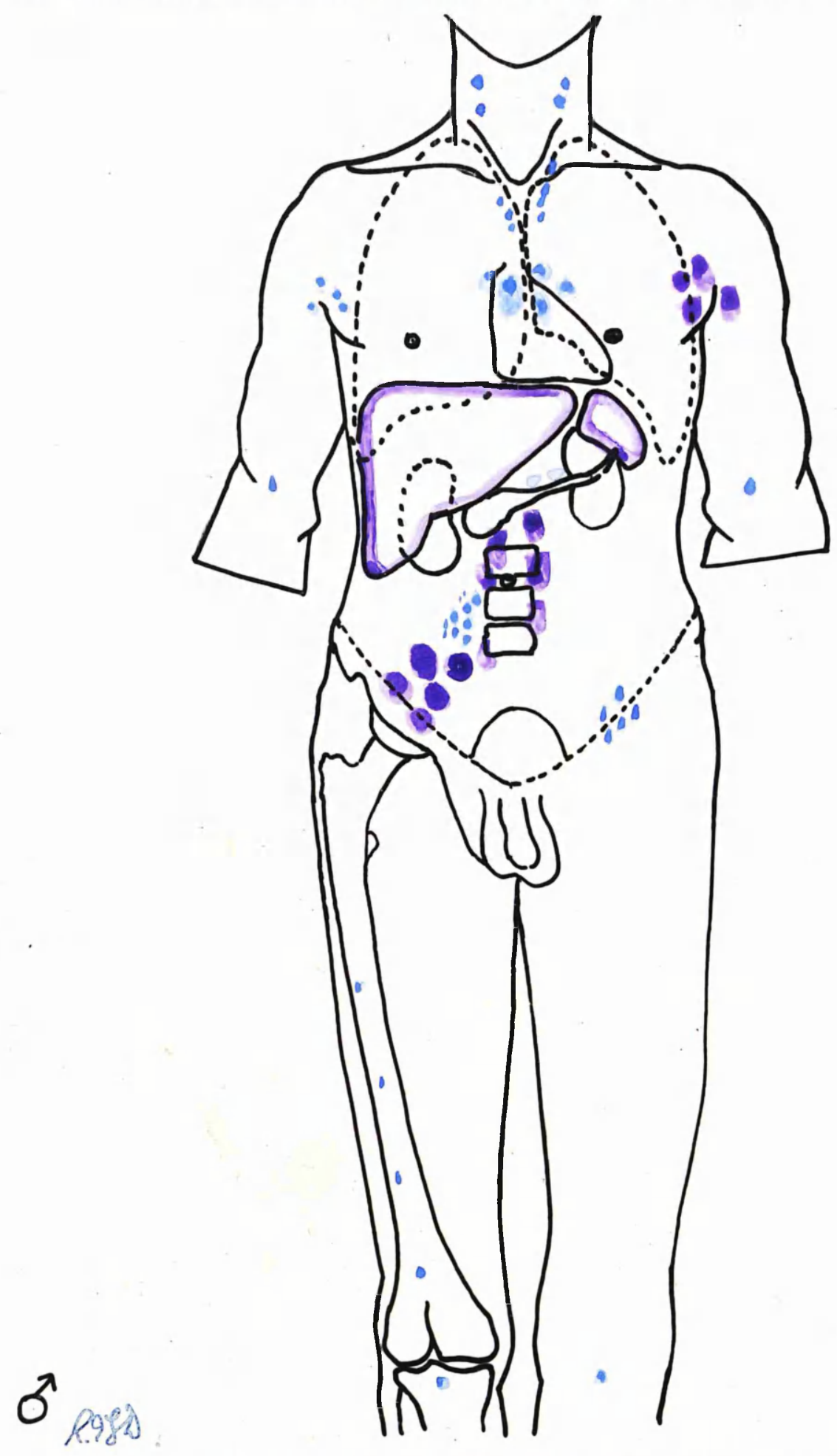

MECROPSY 'G' DAVID EATTOF P.M. L.131:52. 
76. 


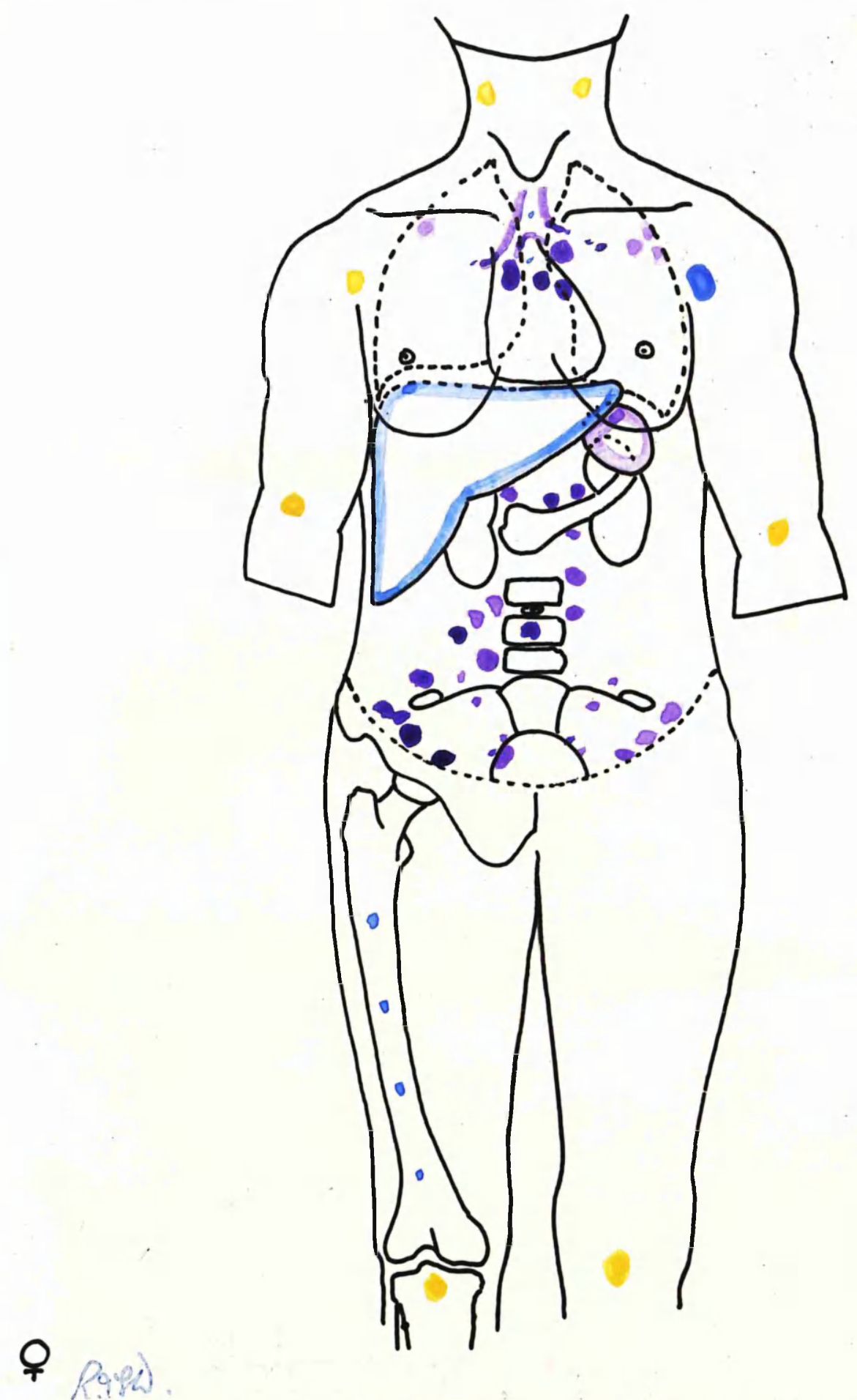


78

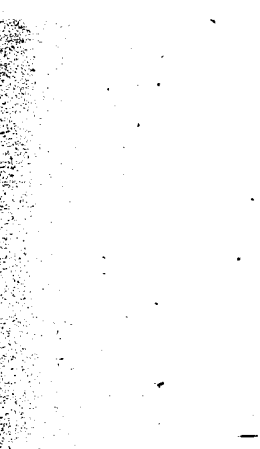




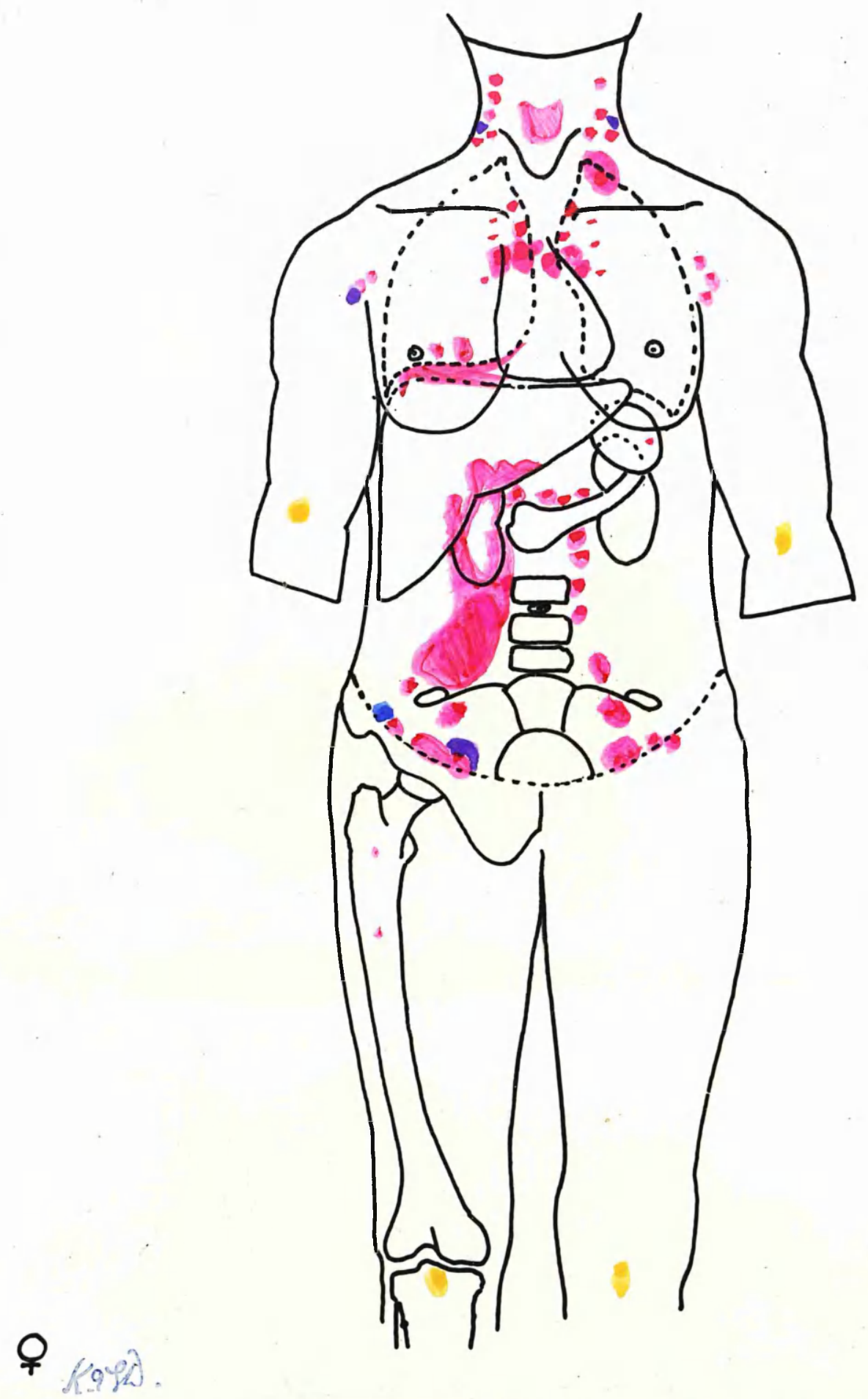


80. 


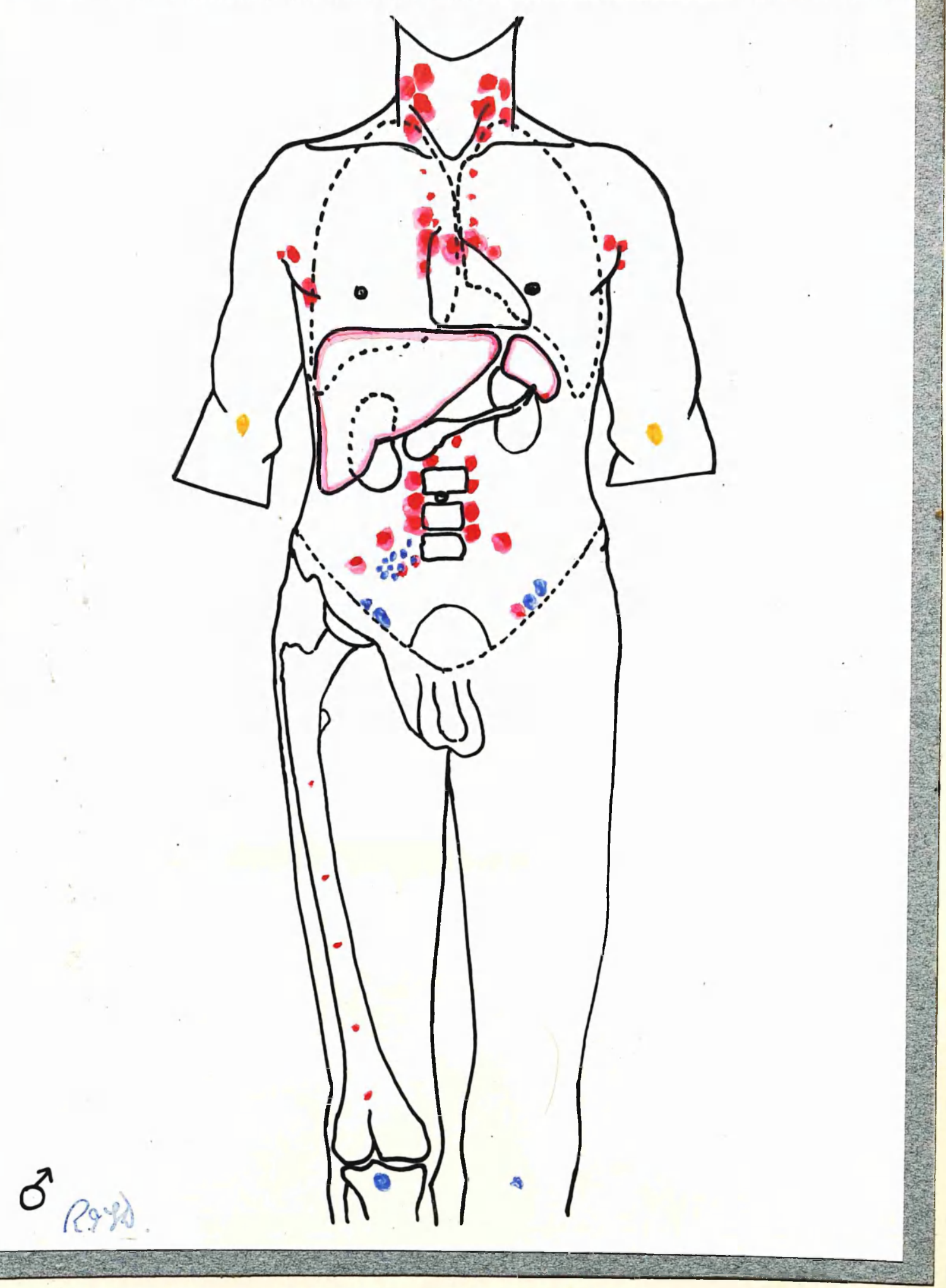


82. 
83.

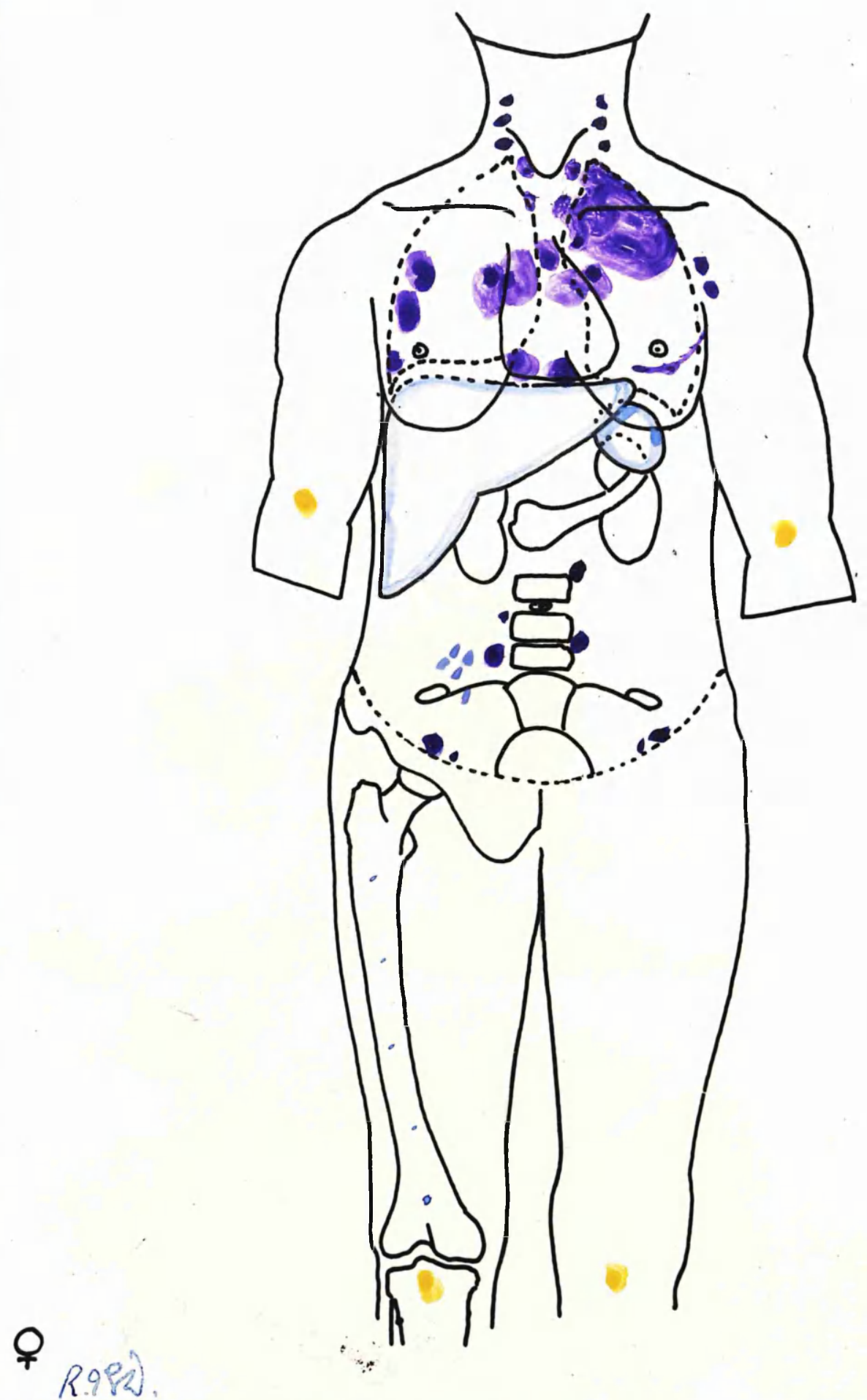


84. 


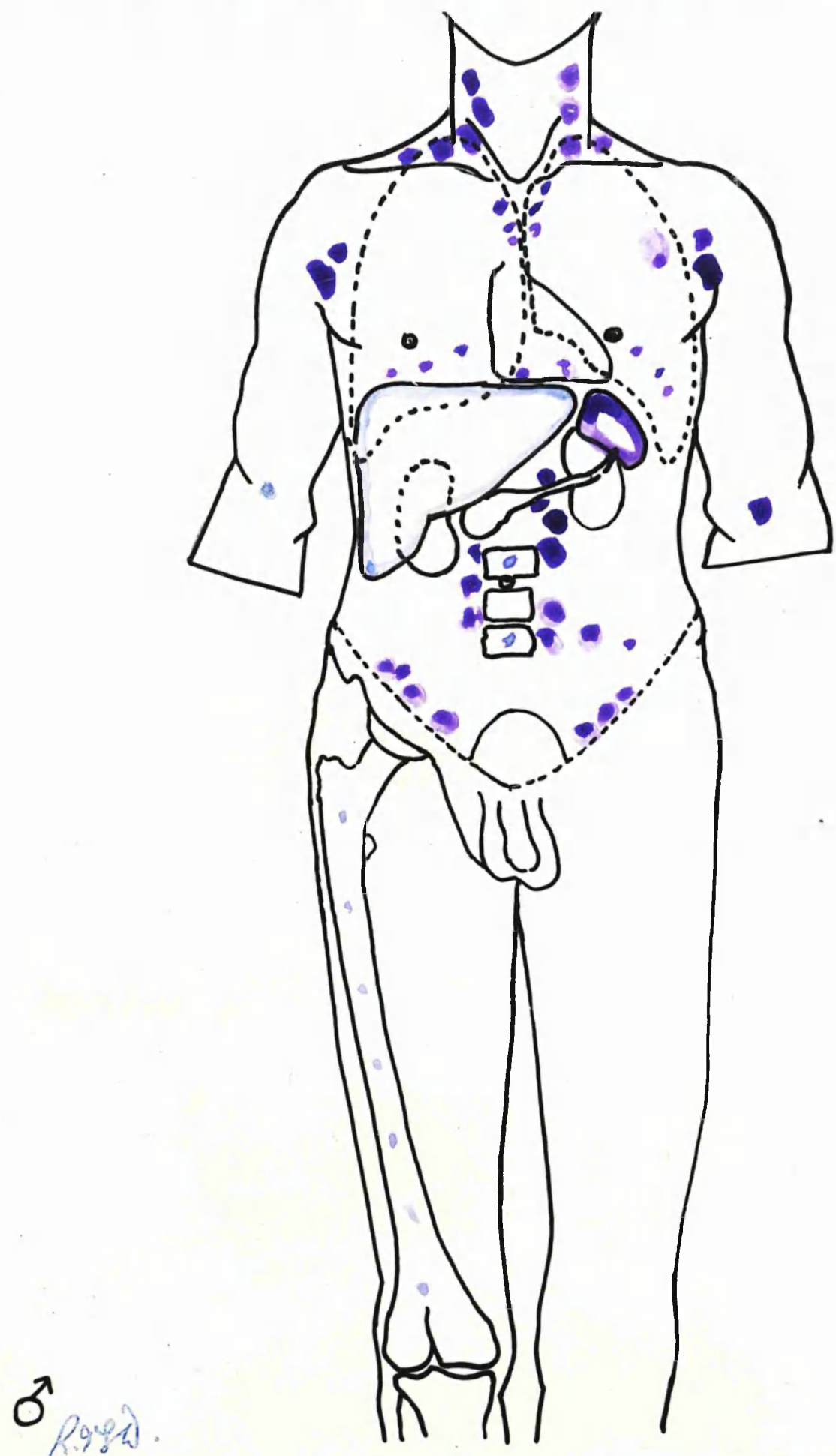

NECROPSY ' $L$ ' JOHI YOUNG P.M. 418:53. G.R.I. 
86 


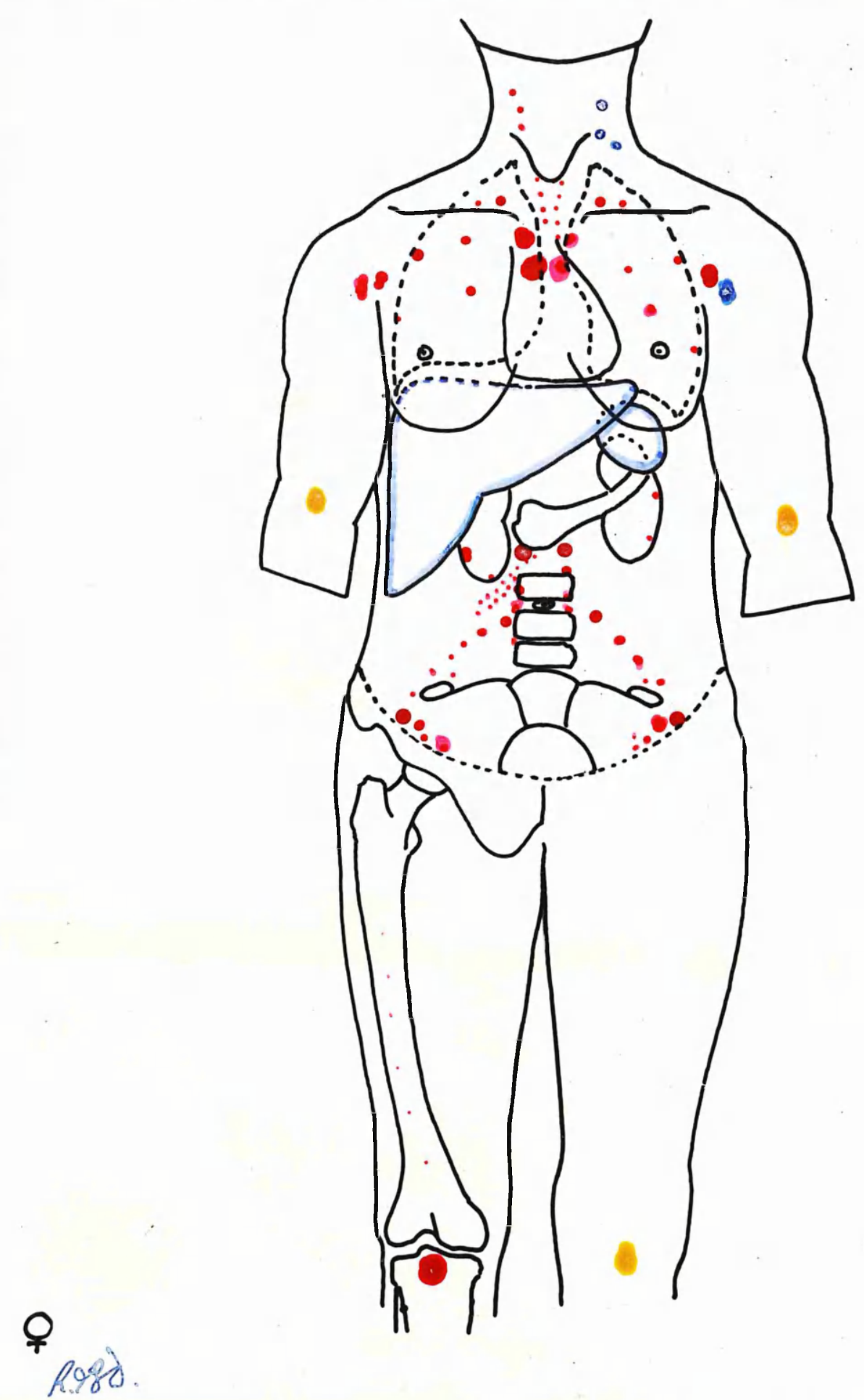


88 


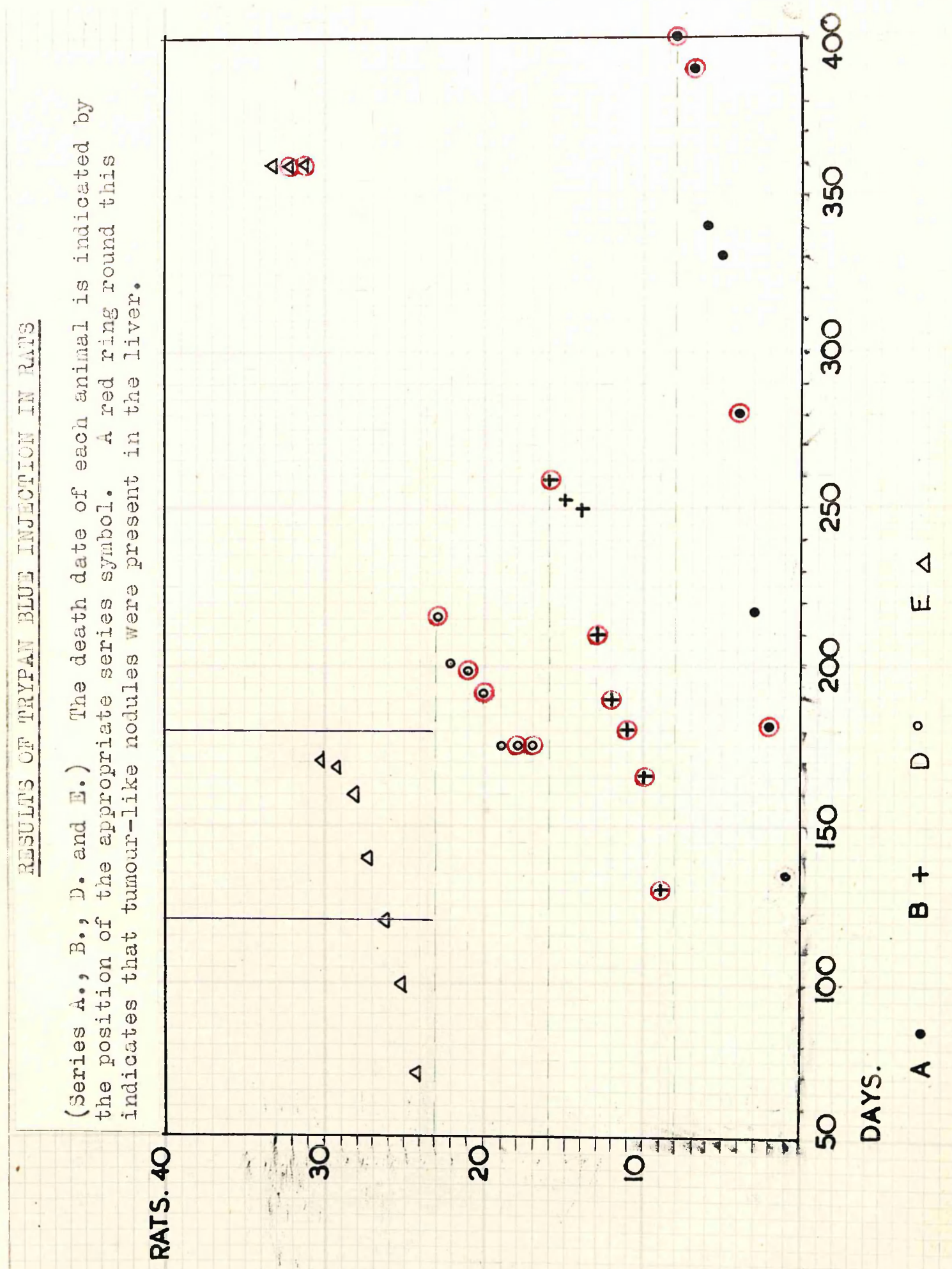


90. 
ค.

\begin{tabular}{l|l}
\hline I.PI. & OOSE \\
MGM & TRYPAN BLUE.
\end{tabular}
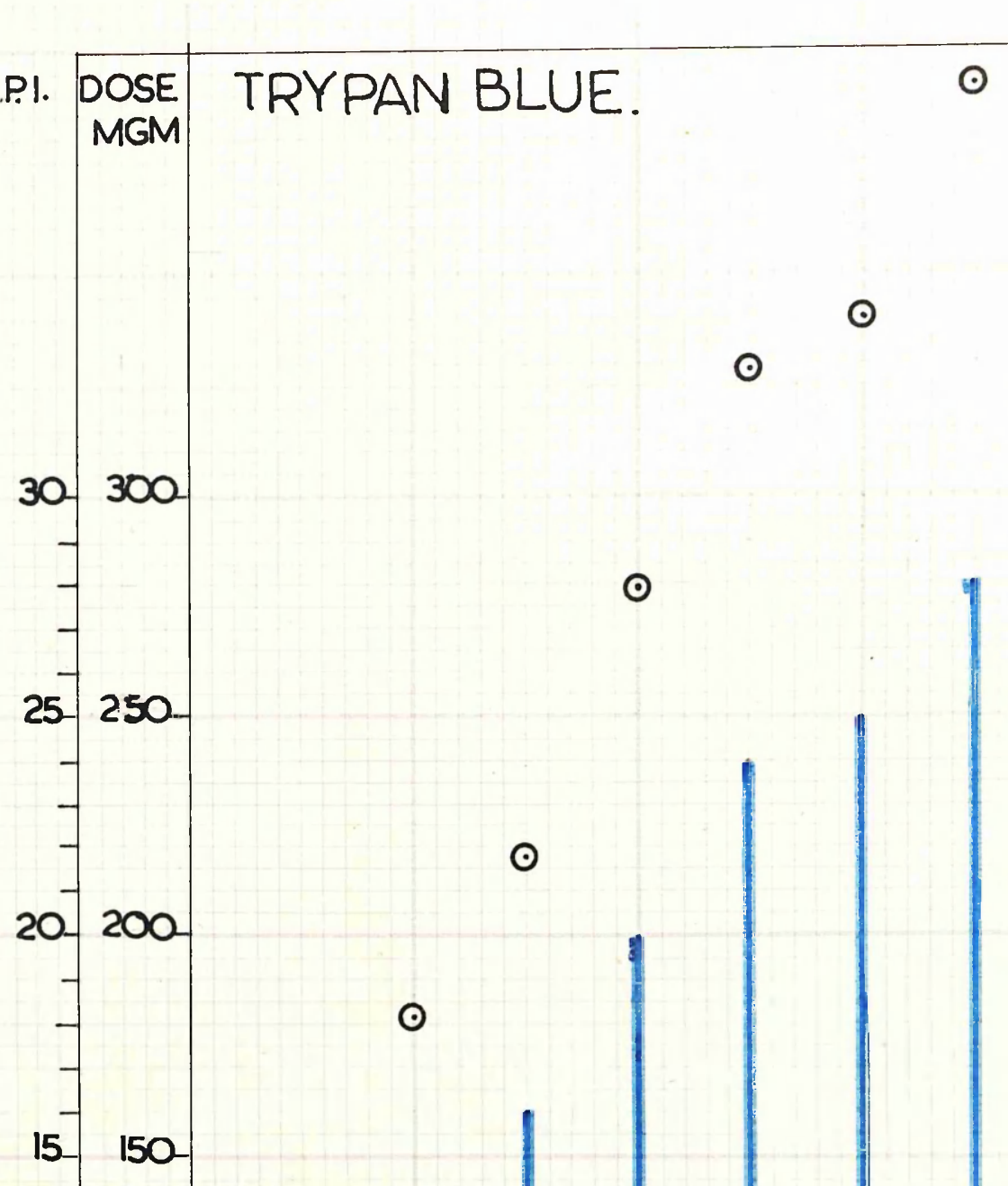

○

$\odot$

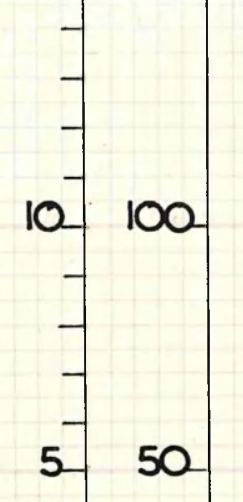

$\odot$

$\odot$
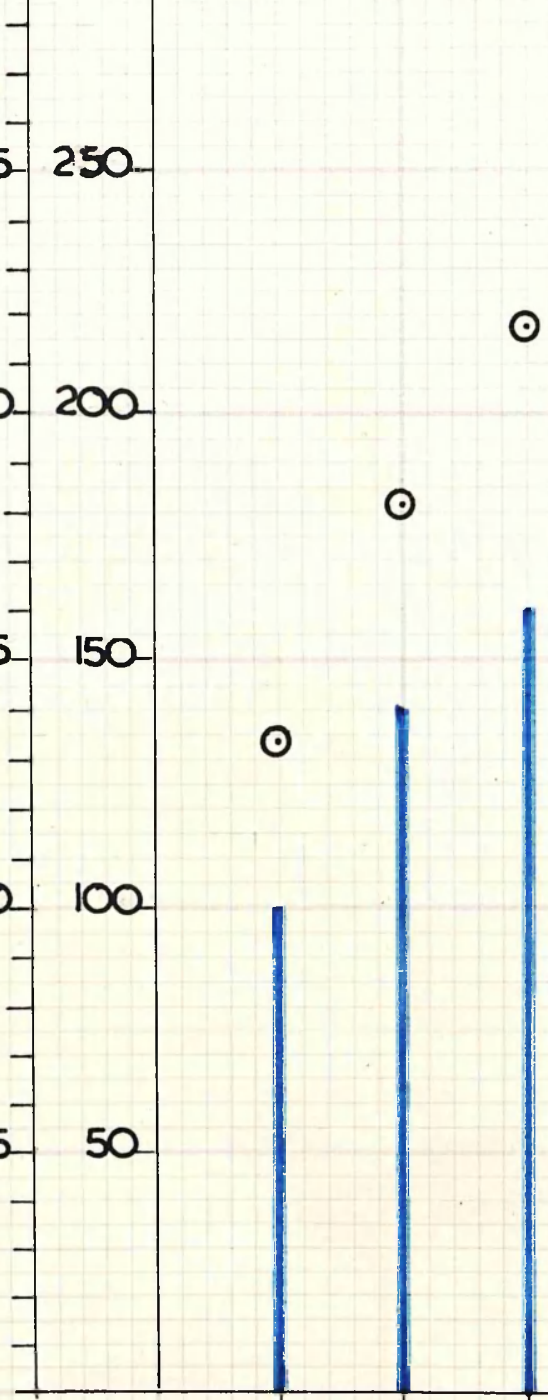
92. 


\section{GerIJS B.}

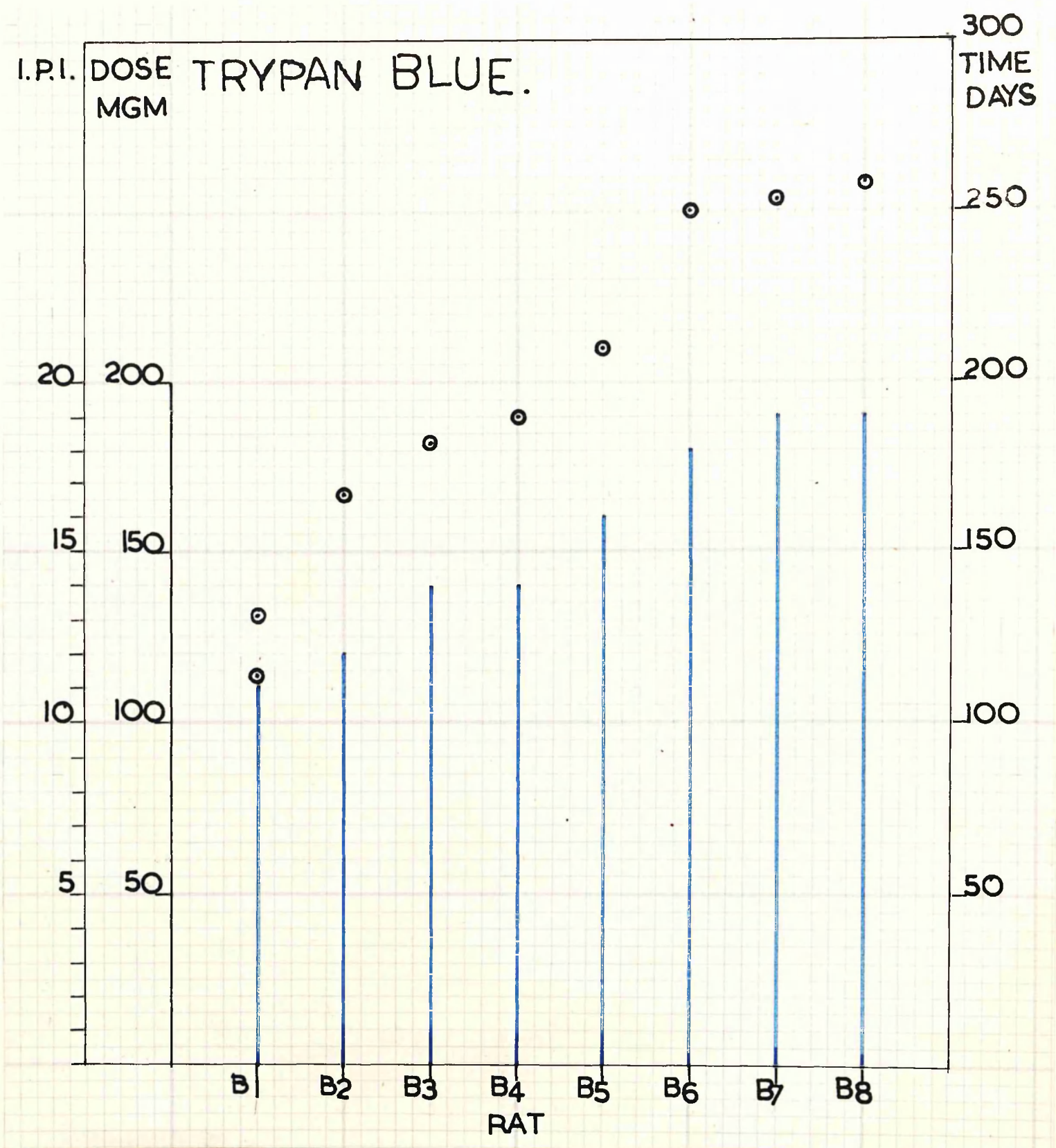




$$
94 .
$$




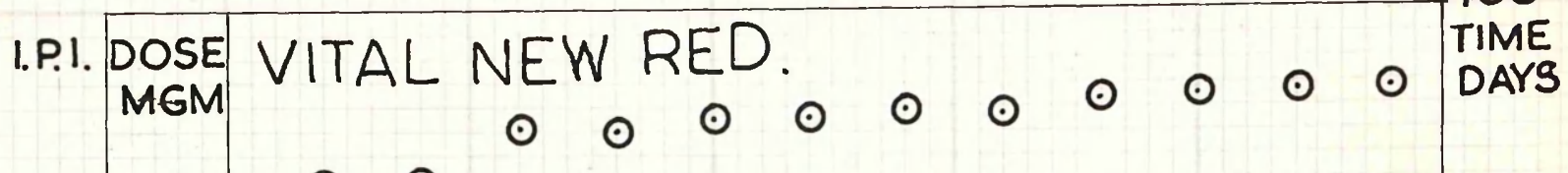
$\odot \odot$

$30 \quad 300$

300
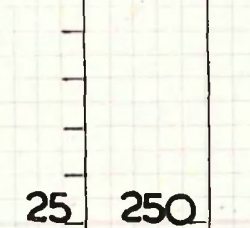

$$
20
$$

25.250

$15 \quad 150$

$10 \cdot 100$
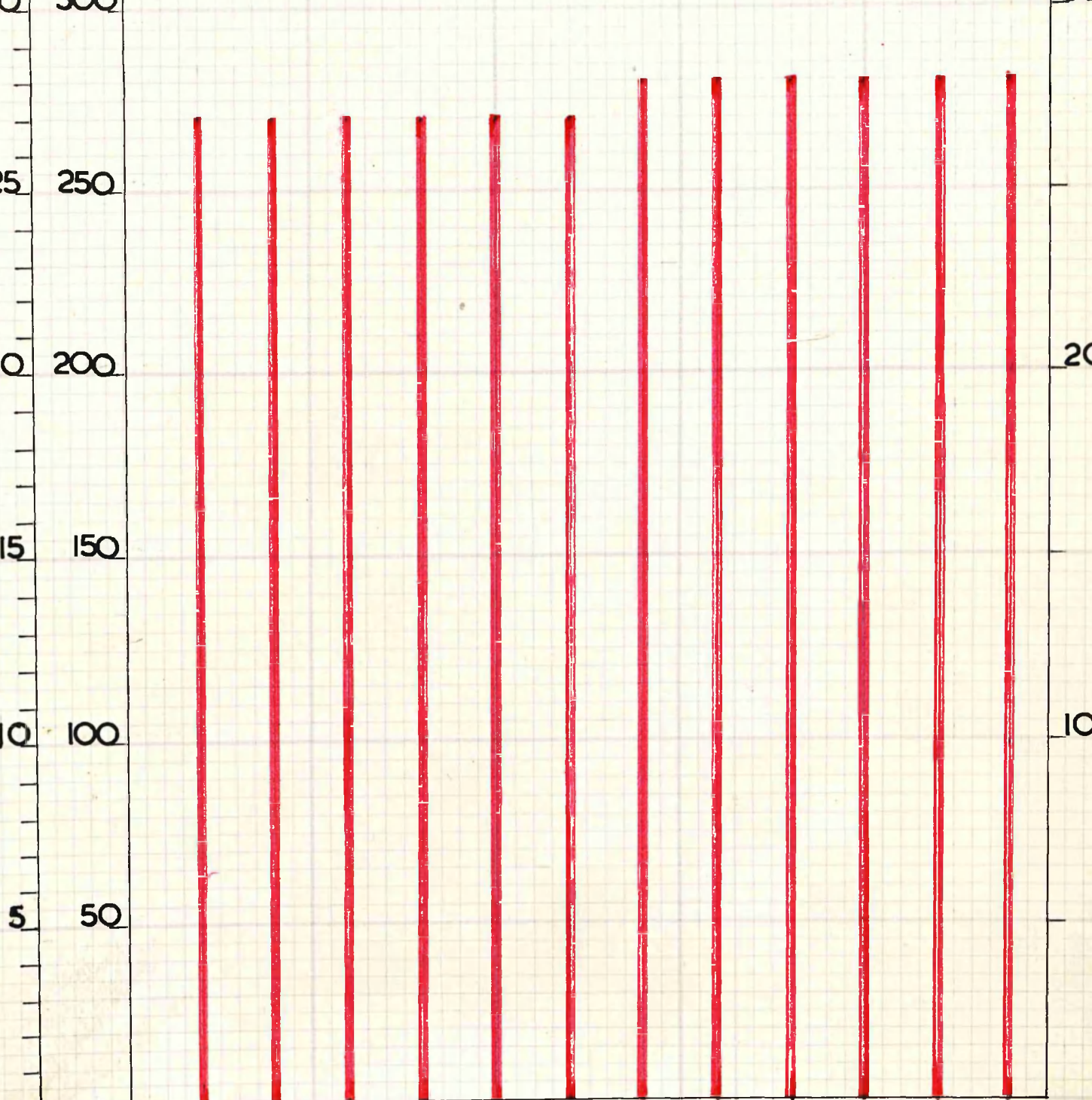
96. 


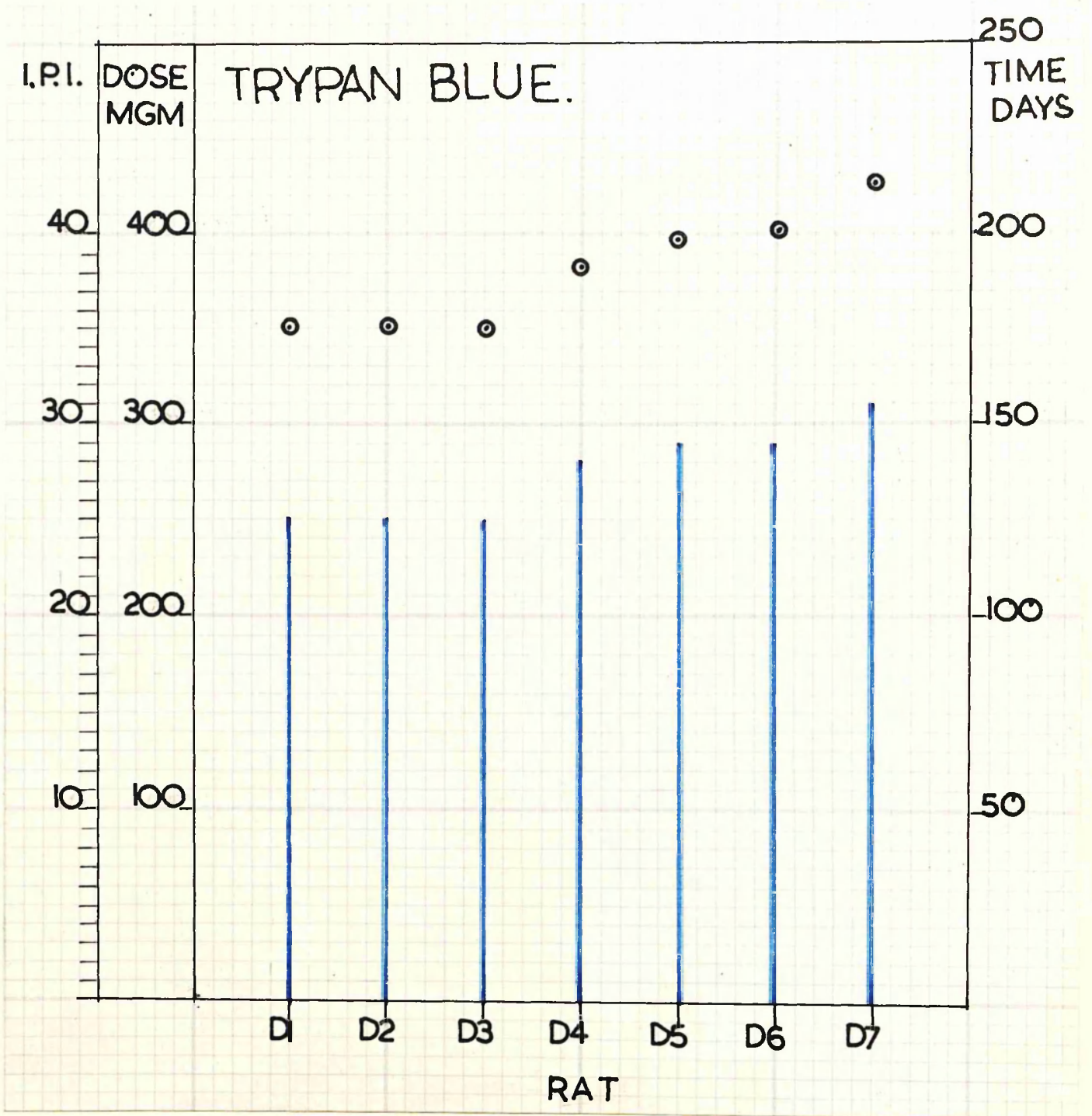


98. 


\section{RESULPS}

\section{VEIGHT RECORDS OF}

GERTES A., B., O., D. and COMTROLS

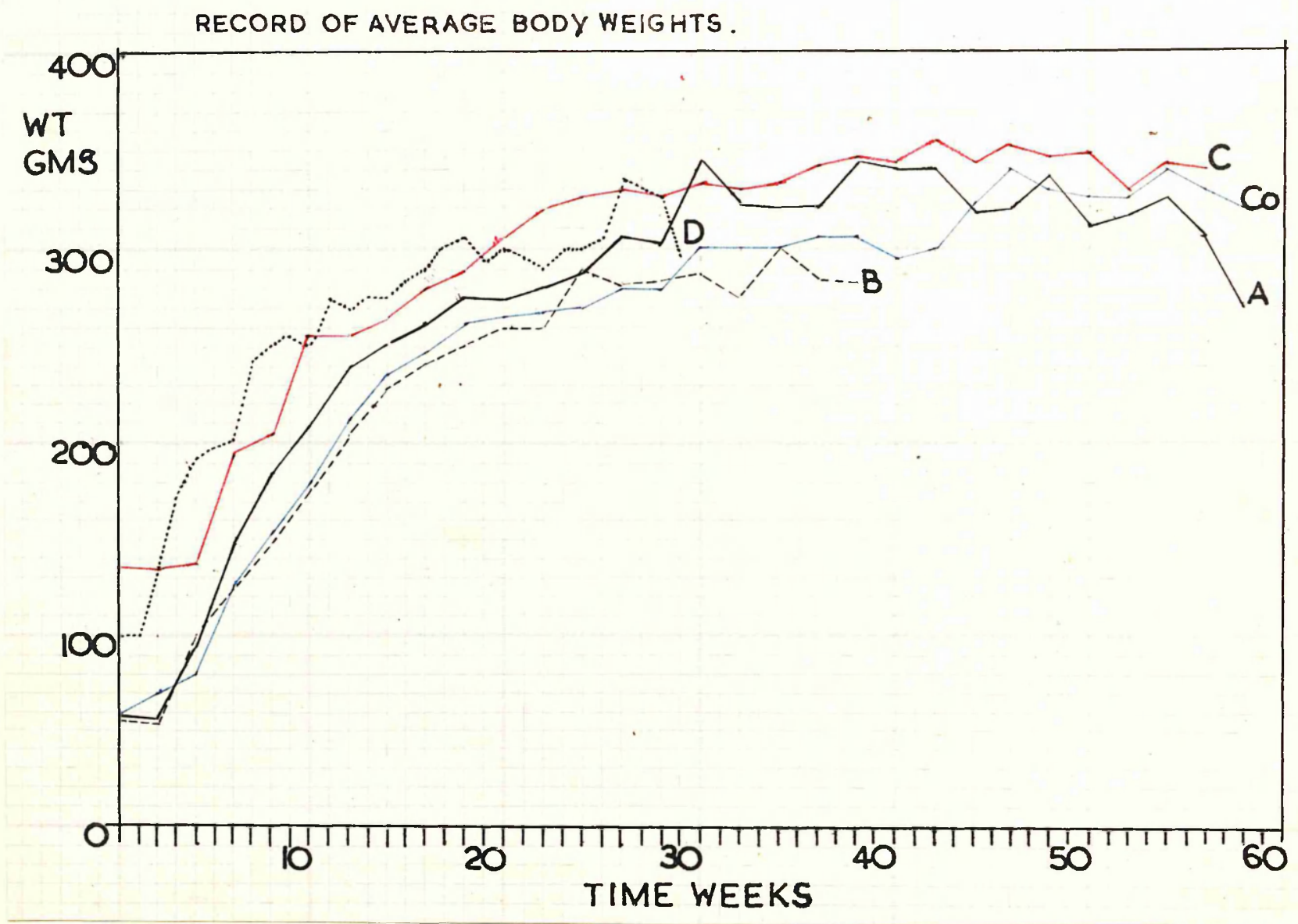


100. 


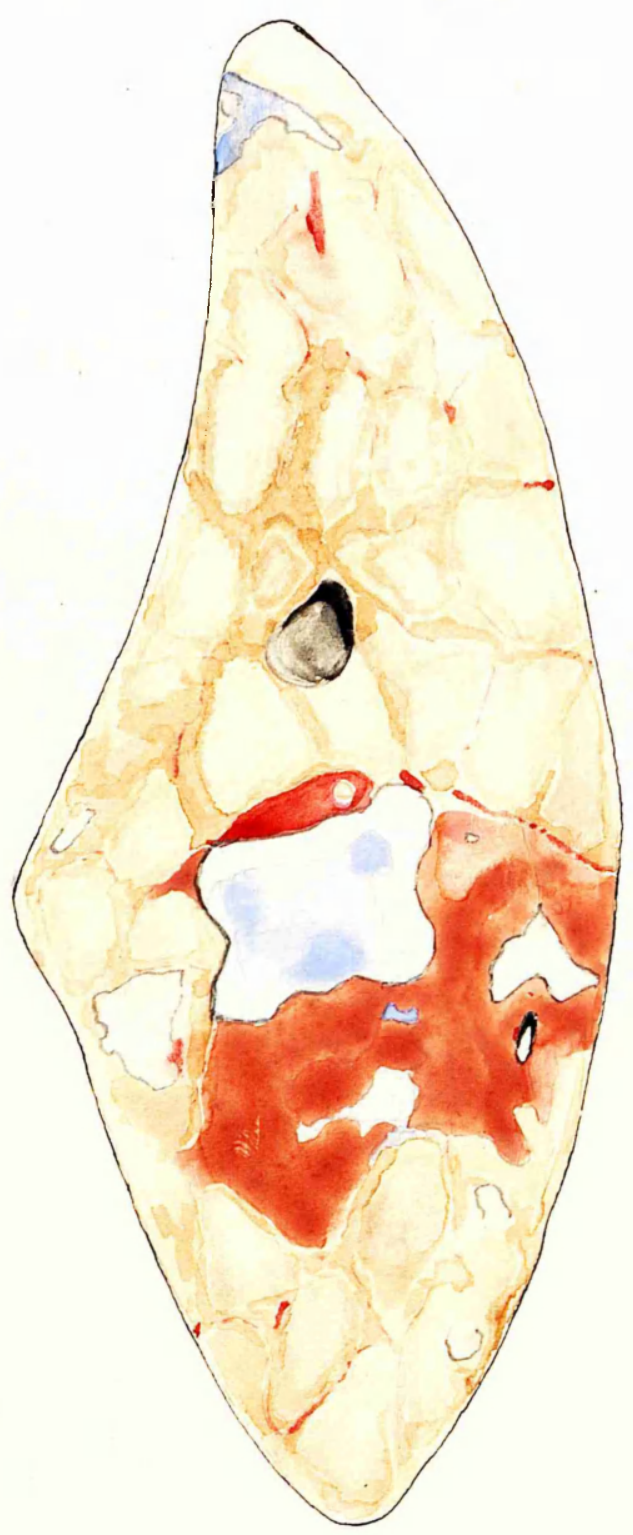

R.940.

Drawing of cut surface of rat liver shewing tumour-like nodules and areas of necrosis.

Rat A.4 282 Days. X4. 
102.

$\%$ 